\title{
Chemical Characteristics
}

\section{Of Delaware River Water}

Trenton, New Jersey, to

Marcus Hook

Pennsylvania

$B y$ C. N. DURFOR and W. B. KEIGHTON

GE OLOGICAL SURVEY WATER-S UPPLY PAPER 1262

Prepared in cooperation with

the City of Philadelphia

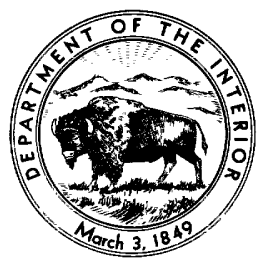


UNITED STATES DEPARTMENT OF THE INTERIOR Douglas McKay, Secretary

\section{GEOLOGICAL SURVEY}

W. E. Wrather, Director 


\section{CONTENTS}

\begin{tabular}{|c|c|}
\hline \multicolumn{2}{|l|}{ Ahstract } \\
\hline 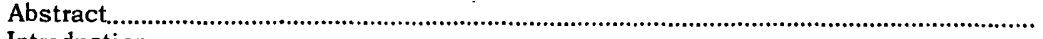 & \\
\hline 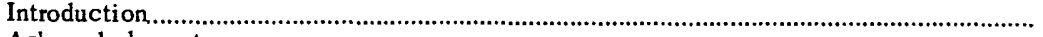 & \\
\hline Acknowledgments.................... & \\
\hline Earlier investigations............. & \\
\hline 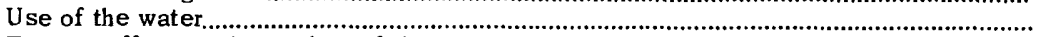 & \\
\hline 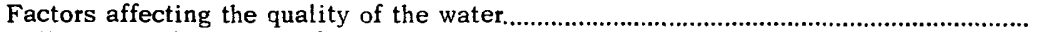 & 6 \\
\hline Collection and analysis of water samples... & 14 \\
\hline 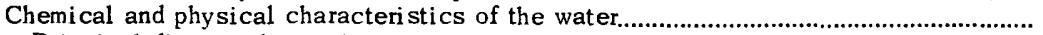 & 16 \\
\hline 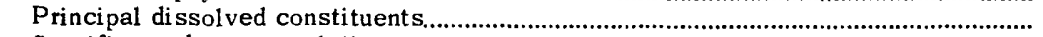 & 17 \\
\hline Specific conductance relations.................... & 18 \\
\hline 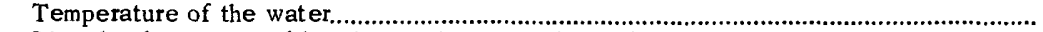 & 26 \\
\hline Dissolved oxygen and biochemical oxygen demand.. & 29 \\
\hline 1ded sediment, by J. P. Eiler..................................... & 33 \\
\hline Summ & 34 \\
\hline Glossa: & 38 \\
\hline Methods & 40 \\
\hline Bibliograpl & 42 \\
\hline blos & \\
\hline
\end{tabular}

\section{ILLUSTRATIONS}

Plate 1. Map of Delaware River basin showing sampling locations for chemical quality and sediment.

Figure 1. Location of monthly sampling stations between Bristol and Marcus Hook, Pa. In pocket

2. The relation between streamflow at Trenton, and precipitation above Trenton, 1950

3. Streamflow frequency curve at Trenton.

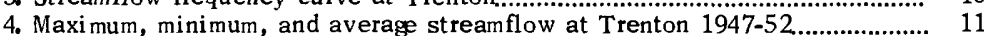

5. Cross sections of Delaware River channel at sampling stations...................... 15

6. Effect of streamflow on the quality of water at Bristol, Camden, and Marcus Hook

7. Chloride concentration at sampling stations for high, medium, and low flow rates.

8. The relation between electrical conductivity and dissolved solids $(0-5,000 \mathrm{ppm})$

9. The relation between electrical conductivity and dissolved solids (0-200 ppm)

ectrical conductivity at sampling stations for high, medium, and low

\section{Electrical con
flow rates.}

11. The relation between electrical conductivity and chloride concentration (0-2,000 ppm).

12. The relation between electrical conductivity and chloride concentration (60 ppm and above).

13. The relation between electrical conductivity and chloride concentration (20 to $60 \mathrm{ppm}$ ). 
Figure 14. The relation between electrical conductivity and chloride concentration $(0-20 \mathrm{ppm})$.

15. The relation between electrical conductivity and chloride concentration at three ranges of bicarbonate concentration.

.

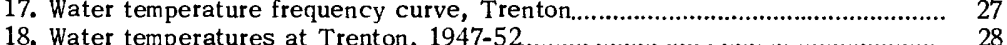

19. Dissolved oxygen as percent saturation at sampling stations for selected days.

20. Relation between dissolved oxygen and biochemical oxygen demand, August 1949 to December 1952 .

\section{TABLES}

Table 1. Drainage areas and discharge rates of the main tributaries of the Delaware River between Trenton, N. J. and Marcus Hook, Pa

2. Location of sampling stations...

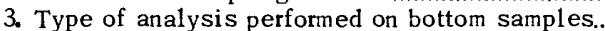

4. Analyses of dissolved oxygen from sampling stations between Torresdale and Marcus Hook, 1950-52, and the average monthly flow rate.

5. Maximum and minimum values in the specific conductance and chemical analyses of water of the Delaware River, Trenton, N. J. to Marcus Hook, Pa

6. Chemical quality of water at Trenton, N. J.

7. Chemical quality of water at 3ristol-Burlington station

8. Chemical quality of water at Torresdale Intake station.

. Chemical quality of water at Lehigh Avenue station.

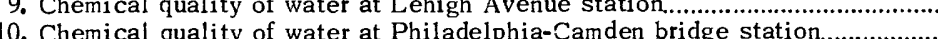

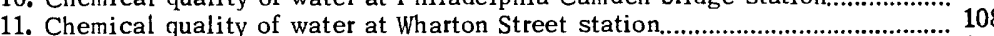

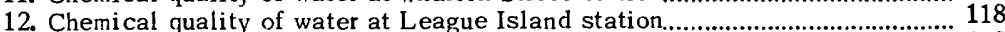

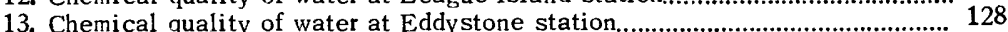

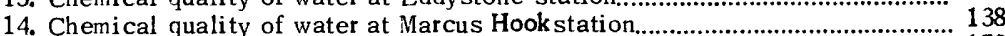

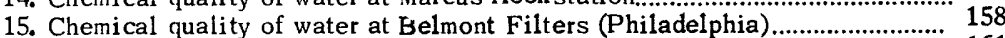

16. Suspended sediment at Trenton, N. J

17. Suspended sediment at Manayunk (Philadelphia) ......................................... 167

18. Particle size of sediment at Trenton, N. J., and Manayunk (Philadelphia).. 173 


\title{
GHEMICAL GHARACTERISTIGS OF DELAWARE RIVER WATER, TRENTON, N. J., TO MARGUS HOOK, PA.
}

\author{
By C. N. Durfor and W. B. Keighton
}

\begin{abstract}
This progress report gives the results of an investigation of the quality of water in the Delaware River from Trenton, N. J. to Marcus Hook, $\mathrm{Pa}_{\text {, }}$, for the period August 1949 to December 1952. The Delaware River is the principal source of water for the many industries and municipal water supplies along this reach of the river and both industries and municipalities use it for the disposal of their wastes. Consequently, a study of the quality of the water and variations in the quality caused by changes in streamflow, tidal effects, pollution and other factors is important to the many users. In both New Jersey and Pennsylvania steps are being taken to abate pollution, thus it is of more than passing interest to measure the effects of waste treatment on the quality of the Delaware River water.
\end{abstract}

At average or higher rates of streamflow the mineral content of the water increases slightly from Trenton to Marcus Hook. There is little variation in the concentration of dissolved minerals from bank to bank or from top to bottom of the river. At times of protracted low rates of flow the effect of ocean water mixing with the river water may be noted as far upstream as Philadelphia. At such times the salinity is often greater near the bottom of the river than near the top. The increase in chloride concentration upstream from Philadelphia is small compared to the rapid increase downstream from Philadelphia. Temperatures of offshore water vary with the season, but on a given day are substantially uniform throughout the reach of the river from Trenton to Marcus Hook.

The water contains less dissolved oxygen as it flows downstream indicating that oxygen is being consumed by oxidizable matter. From Philadelphia downstream there are periods, especially in late summer, when the dissolved oxygen is barely sufficient to meet the oxygen demands of the pollution load. 


\section{INTRODUCTION}

This progress report summarizes the results of a quality-ofwater investigation of the Delaware River near Philadelphia, Pa., made cooperatively by the city of Philadelphia and the U. S. Geological Survey. Chemical quality and sanitary data at various locations on the Delaware River between Trenton, N. J., and Marcus Hook, Pa., collected in the period from August 1949 to December 1952, are presented, and the usefulness and significance of these data are discussed. The report will serve as a comprehensive source of data on the chemical characteristics of the Delaware River and will show the use made to date of data available from this continuing investigation.

The region adjacent to the 53 miles of river from Trenton to Marcus Hook is one of the great industrial regions of the United States. During 1952, more than 8,900 ships from 42 countries arrived in its port. A vessel going from Trenton to the sea would pass 25 cities and towns, thousands of manufacturing plants, 348 wharves, and 190, 000 lineal feet of berthing space. Three trunkline railroads serve this area and provide such rail-to-ship services as the operation of piers, tugs, car floats, cranes, terminals, elevators, and freight yards; the largest privately owned tidewater terminal is at Port Richmond, Philadelphia. Philadelphia is a large oil-refining center, and the port handles large quantities of petroleum and petroleum products. Many firms are building or planning to expand facilities, and a large steel plant recently located in this area.

Industries along the Delaware River between Trenton and Marcus Hook used over 1,939 cfs $(3,000 \mathrm{mgd})^{4}$ of water in 1951. The average flow in the Delaware River past Trenton, for the 36-year period from $1913-49$ was 11,780 cfs $(7,614 \mathrm{mgd})$, but in the late summer months the flow may decrease to less than 2,000 cfs $(1,292 \mathrm{mgd})$. These factors of water use and streamflow make it important to have continuing data available on the quantity and quality of the water in this reach of the Delaware River. This information will be useful in solving such problems as the cost of water treatment for municipal and industrial uses, the availability of water suitable for new industries that may consider locating in this area, the influence of natural forces (such as tides, rainfall, and river flow) on the character of the river water, the assessing of the possible effects of channel dredging, the determination of the qualitative effects of infiltration of ground water, and the effects of pollution and waste treatment on the character of the river water. During the 41 months covered by this report, a start has been made in alleviating the pollution load on the river through more extensive and adequate waste-treatment methods. Data for this period,will

'Abbreviations and technical terms are defined in the glossary, page 38 . 
be of value for comparison with that of later years when further progress in waste treatment is expected.

The first part of this report presents basic data on the geographic and economic environment of the Delaware River, its physical characteristics, the drainage areas and flow rates of this section of the river and its tributaries. Then the factors causing variations in electrical conductance, concentration of chemical constituents, water temperature, dissolved oxygen, and biochemical oxygen demand in several reaches and locations in the river from Bristol to Philadelphia, and from Philadelphia to Marcus Hook, are discussed. Next, the variation of electrical conductance and chemical constituents with the rate of flow of the river, the frequency of occurrence of selected water temperatures, the monthly variation of water discharge rates, water temperature, dissolved oxygen, and biochemical oxygen demand are considered. The report concludes with a tabular compilation of the data on which it is based.

\section{ACKNOWLEDGMENTS}

This investigation was conducted by the U. S. Geological Survey in cooperation with the city of Philadelphia, through its Water Department: Samuel S. Baxter, commissioner, and J. S. Reich, industrial waste engineer. It was under the general supervision of W. F. White, district chemist, 1948-52, and N. H. Beamer, district chemist of Pennsylvania, 1952-.

M. J. McGonigle assisted in the analytical work on the chemical quality of the water. The biological analyses were supervised by J. H. Thorpe, city chemist, Philadelphia.

Records of suspended sediments were obtained by personnel of the U. S. Geological Survey office at Schuylkill Haven, Pa., under the supervision of J. P. Eiler, engineer in charge.

Records of water discharge and drainage area in Pennsylvania and New Jersey were furnished by district offices of the U. S. Geological Survey at Harrisburg, Pa. and Trenton, N. J., respectively.

Data on the river cross section soundings were furnished by C. F. Wicker, chief, Engineering Division of Corps of Engineers, U. S. Army, Philadelphia, Pa.

\section{EARLIER INVESTIGATIONS}

More than 25 years ago a proposal was made by the city of New York to augment its water supply by impounding the water of certain 
New York intrastate streams tributary to the upper Delaware River. During the last six months of 1930 the natural flow of the Delaware River was unusually low, and the salinity of the river increased markedly in the reach from Chester, $\mathrm{Pa}$., to Marcus Hook, Pa. This reach is in an industrial area where the river water was used as a supply for the city of Chester, and then, as now, was used as process water, and for steam generation.

Officials of industries in this region that were affected became concerned about this unusual increase in salinity of the water and formed the Delaware River Conservation Association, primarily to investigate salinity of the stream. A study of the salinity data collected by this Association and cooperating industries in 1930 and 1931 has been published with charts for estimating the effect of river-flow on salinity conditions (Mason and Pietsch, 1940). Some industries in the Chester area still carry on their own salinity investigations.

In 1928 the U. S. Army Engineers began observations of salinity in the Delaware River and Delaware Bay and correlated such data with streamflow. These data were incorporated in a report published by the Commonwealth of Pennsylvania in 1935. These salinity observations were discontinued in 1934. Most of the infrequent data obtained since 1934 primarily consist of the results of chloride determinations and tide-gage records are unpublishea, and are in the files of the U. S. Army Engineers.

In 1931, the Commonwealth of Pennsylvania authorized a comprehensive salinity survey, the results of which are described in the "Final Report to the Sanitary Water Board by the Bureau of Engineering on the Salinity Survey of the Delaware River". This report contains data for the years 1931-33 concerning the relation of salinity to flow rate of the river, and to the stage of the tide for the Delaware River below Marcus Hook.

The city of Philadelphia has been making daily chemical determinations at their Torresdale Filter Station since its inception in 1900. Some determinations, as that for chloride, are made daily, and others are made monthly. Analyses are also made daily of the water of the Schuylkill River (a tributary of the Delaware River) at the Belmont Filter Plant. Most of the resulting data are unpublished, and in the files of the city of Philadelphia.

Since October 1, 1944, the U. S. Geological Survey has maintained a daily sampling station at Morrisville, $\mathrm{Pa}$., in cooperation with the Pennsylvania Department of Commerce, State Planning Board. Publications 17 and 23 of the State Planning Board contain complete chemical analyses of the water in the Delaware River at Morrisville, for the years 1944-46 (publication 17) and 1946-49 
(publication 23). Also at Morrisville, sediment determinations have been made daily since September 1, 1949.

Although not conducting salinity investigations, several agencies have been instrumental in developing interest and concern for improving the quality of water in this reach of the Delaware River.

The Interstate Commission on the Delaware River (INCODEL) is a joint governmental agency created by the States of New York, New Jersey, Pennsylvania, and Delaware to formulate, adopt, and execute policies for the development and utilization of the resources of the Delaware River watershed. This agency has proposed the construction of specific dams and reservoirs in order that all States concerned may have an adequate supply of water of good quality from the Delaware River. However, these States must pass joint legislation in order to adopt the policies suggested.

The Pennsylvania Sanitary Water Board is an enforcement agency established by the Commonwealth of Pennsylvania to control pollution of the stream waters necessary for public water supply, preservation of fish and aquatic life, recreational purposes, agricultural, and industrial use.

The Water Resources Committee of the Greater PhiladelphiaDelaware-South Jersey Council is composed of business men and local governmental officials who are interested in the economic and social advancement of the people of Delaware River basin. Realizing that the se can be advanced only if an adequate supply of water of good quality is available, they have been making studies of the pollution of the local streams, policies advocated by INCODEL, public water supplies, and legislation affecting the water resources of the basin.

\section{USE OF THE WATER}

In the Delaware Valley between Trenton and Marcus Hook the re are many different types of industry: power-generating stations which supply electricity for Pennsylvania, New Jersey, and Delaware; large oil refineries; chemical industries; textile and yarn mills; synthetic fiber and resin plants; distilleries; sugar refineries; many nationally-known plants manufacturing metal goods; branch factories of national food-processing companies; and shipbuilding concerns. In addition, there are several military installations.

The Delaware River provides over 97 percent of the water used by these industries. Most of this water is used for cooling or quenching purposes-especially in stations generating electricity, 
the metal industries, and oil refineries-and needs little or no treatment before it is returned to the river. A smaller but still substantial quantity is used for process purposes by other manufacturing industries, chemical industries, sugar refineries, foodprocessing plants, and textile plants. Such water often requires treatment before it is pure enough to be returned to the river. Most of the water withdrawn from the river is non-consumptive and returned to it after use, but small quantities are lost through evaporation in cooling or quenching, or in use as boiler makeup water, or through incorporation into the product in the process industries.

Seldom can the raw river water be usedindustrially without some type of water treatment. The treatment depends upon the physical location of the plant and the intended use of the water. Industries in this area treat the river water in one or more of the following ways. Chlorine is added to prevent the growth of algae or other slime-producing materials in the water-distribution system. Alum or ferrous sulfate is added to aid in settling or flocculation of suspended solids. The water may be strained or filtered without the addition of any flocculant. The sodafash and lime process, or demineralizers are used at some plants to soften the water. Phosphate salts are added to aid in preventing the formation of scale. Sodium sulfite is added to aid in the removal of residual oxygen. The $\mathrm{pH}$ is adjusted. In the lower reaches of the river, where considerable oil may be present, the water is passed through oil separators. In certain industries it is necessary to reduce the amount of color.

Invasion of salty water from Delaware Baycauses another problem for industries and municipalities along the lower reaches of the river. The increase in concentration of salt in the river water requires more frequent blowdowns of steam boilers and increases the amount of chemicals required to soften the water. To decrease the amount of treatment required, some industries withdraw and store water from the river only on the ebb tide, when the salinity is less than on the floodtide. However, impounding of river water is required only when the salinity is high, as when the flow of river water is low.

\section{FACTORS AFFECTING THE QUALITY OF THE WATER}

A number of factors may influence the quality of water in a stream. The topography of the land and composition of minerals in the area drained by the river have much to do with the kind of dissolved materials in the water and their concentration. Also farmlands, mining regions, and industrial areas each affect the composition of the water differently. Variations in the quantity of 
precipitation influence the quantities of dissolved and suspended matter carried into the river as well as the rate of discharge of the river. The several tributary streams are likewise influenced by these same factors and affect the quality of the main stem of the river differently depending upon their relative flows and the proportions of dissolved and suspended solids characteristic of each of them. Ground water, when it reaches the river, has an effect on quality of the river water. Industrial and sewage wastes that enter the river will also affect the quality of the river water. In addition to these factors, sea water has some tendency to mix with the river water, particularly in the reach of the river downstream from Philadelphia.

The East Branch and the West Branch Delaware River originate in the Catskill Mountains and flows in a southwestwardly direction to their junction at Hancock, N. Y. From there, the Delaware River flows southwardly 326 miles to the Atlantic Ocean as a boundary water first separating New York and Pennsylvania, then New Jersey and Pennsylvania, and finally New Jersey and Delaware. The river widens and deepens as it meanders through the Atlantic Coastal Plain to the sea. At its mouth, the Delaware River has a drainage area of 12,765 square miles which includes the area of the bay. The reach of the river from Trenton, N. J. to Marcus Hook, Pa., (fig. 1) is 53 miles long and drains an area of 3,606 square miles (32 percent of the total drainage area) and has a water area of 34 square miles. Plate 1 is a map showing the relation of the area included in this report to the total drainage area of the Delaware River.

The precipitation in the basin of the Delaware River, one of the factors influencing the amount of water in the river, is fairly evenly distributed throughout the year with maximum amounts occurring during the late summer months. Figure 2 is a hydrograph showing mean daily discharge of Delaware River at Trenton, N. J., for 1950. Figure 2 also illustrates the daily precipitation recorded at the Port Jervis, N. Y.; Equinunk, N. Y.; Portland, Pa.; and Phillipsburg, N. J. weather stations combined into one graph. These stations are located in the drainage basin of the Delaware River above Trenton. The peaks in the hydrograph curve usually lag behind the peaks in the precipitation curve by one to three days, depending on the distribution of rainfall on the watershed and time of travel from source of precipitation to runoff measuring site. A large increase in flow generally follows heavy rains (see fig. 2, November 26-27). However, even a relatively light rain may result in a heavy flow if augmented by melting snow as in the early spring months (see fig. 2, April 4-6). During the winter months the level of the ground water generally reaches its peak for the year and the gradient to the river is greatest; therefore, ground water will infiltrate to the river at a faster rate than during the summer months when the level of the ground water is lower. When the 


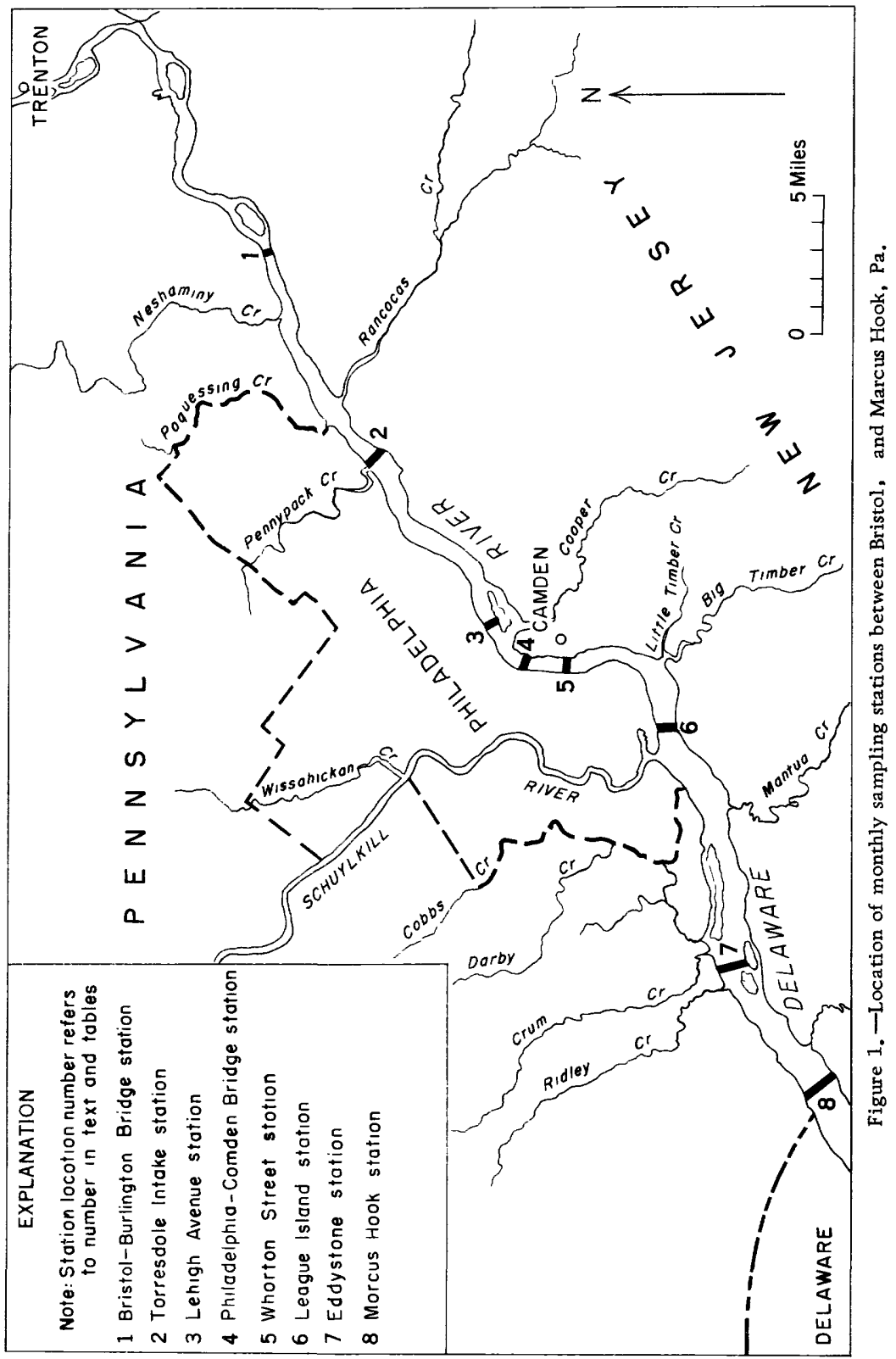




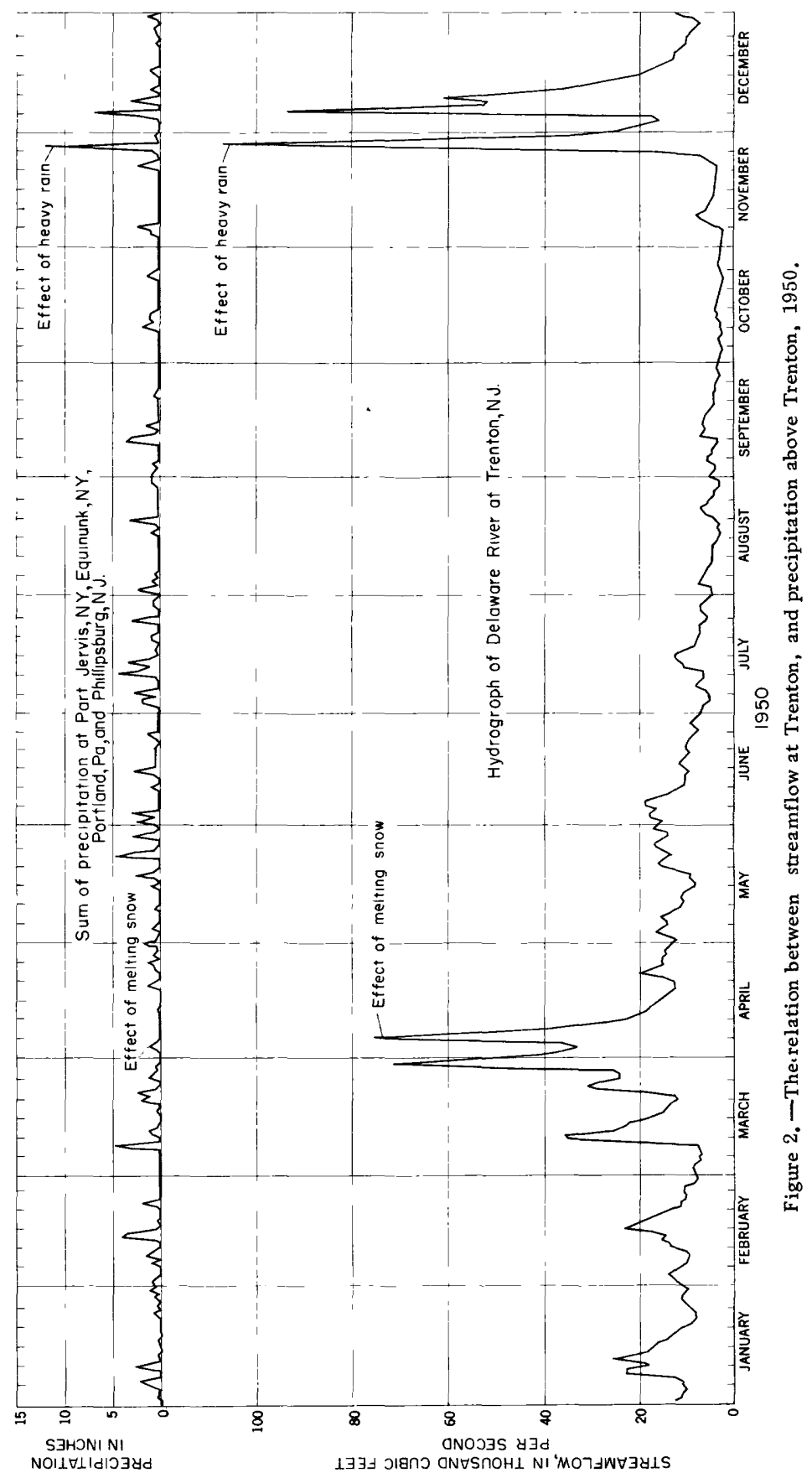


ground is frozen, rain has difficulty penetrating the ground, and runoff to the stream is rapid (compare the parts of hydrograph for Jaıuary and August 1950 in fig. 2). During the late spring months evapotranspiration consumes much of the water which falls on the ground; a smaller fraction of the rainfall runs off or seeps through the ground into the river.

A frequency curve for the streamflow at the gaging station at Trenton ${ }^{2}$ for 1942-52 has been constructed, (fig. 3). This curve

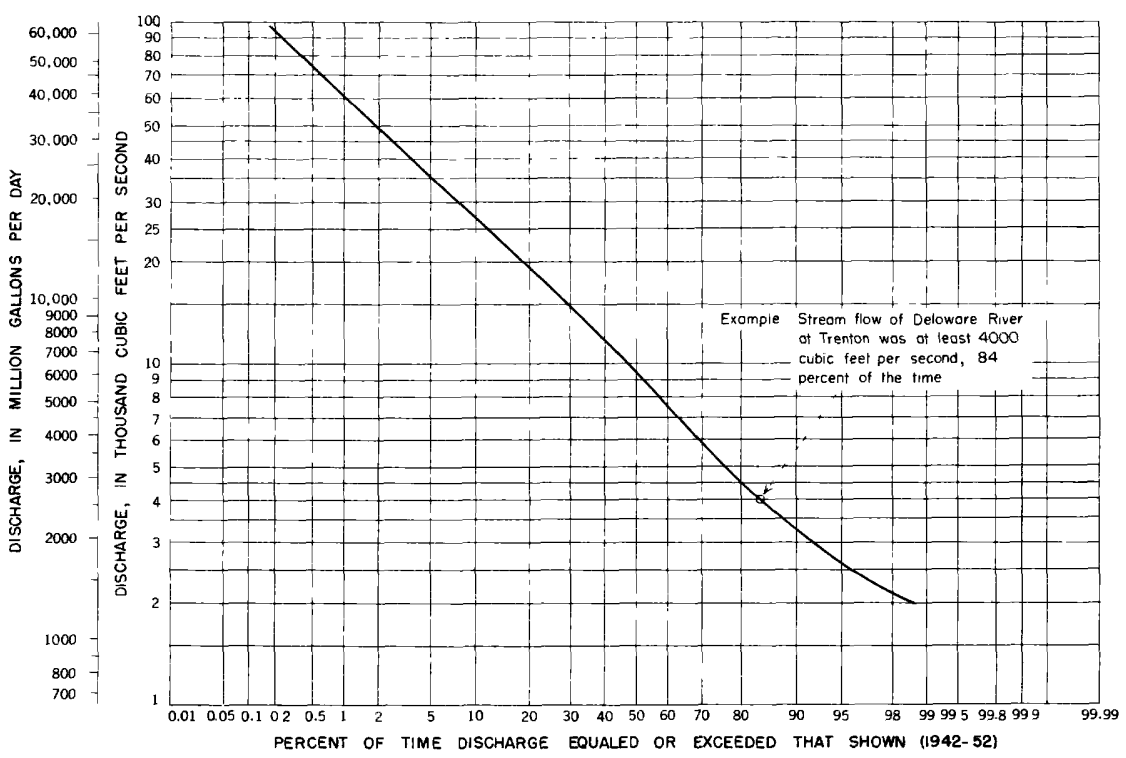

Figure 3. - Streamflow frequency curve at Trenton.

indicates the frequency of various flow rates, irrespective of the chronological sequence. For example, the flow rate was at least $4,000 \mathrm{cfs}(2,585 \mathrm{mgd})$ during 84 percent of the time.

The discharge rate at Trenton for each month from October 1947 to September 1952 is illustrated in figure 4. For each month are plotted four data: the maximum daily flow, the minimum daily flow, the average monthly flow, and the range in readings of the middle 50 percent of the daily flows. The maximum daily flow is the highest point on the vertical line, the minimum daily flow is the lowest point on the vertical line, and the average monthly flow is repre-

${ }^{2}$ Average monthly and the annual discharge of Delaware River at Trenton, N. J. from 191349 are reported by Mangan, Graham, and White (1951), who also describe the Delaware River in Bucks County. 


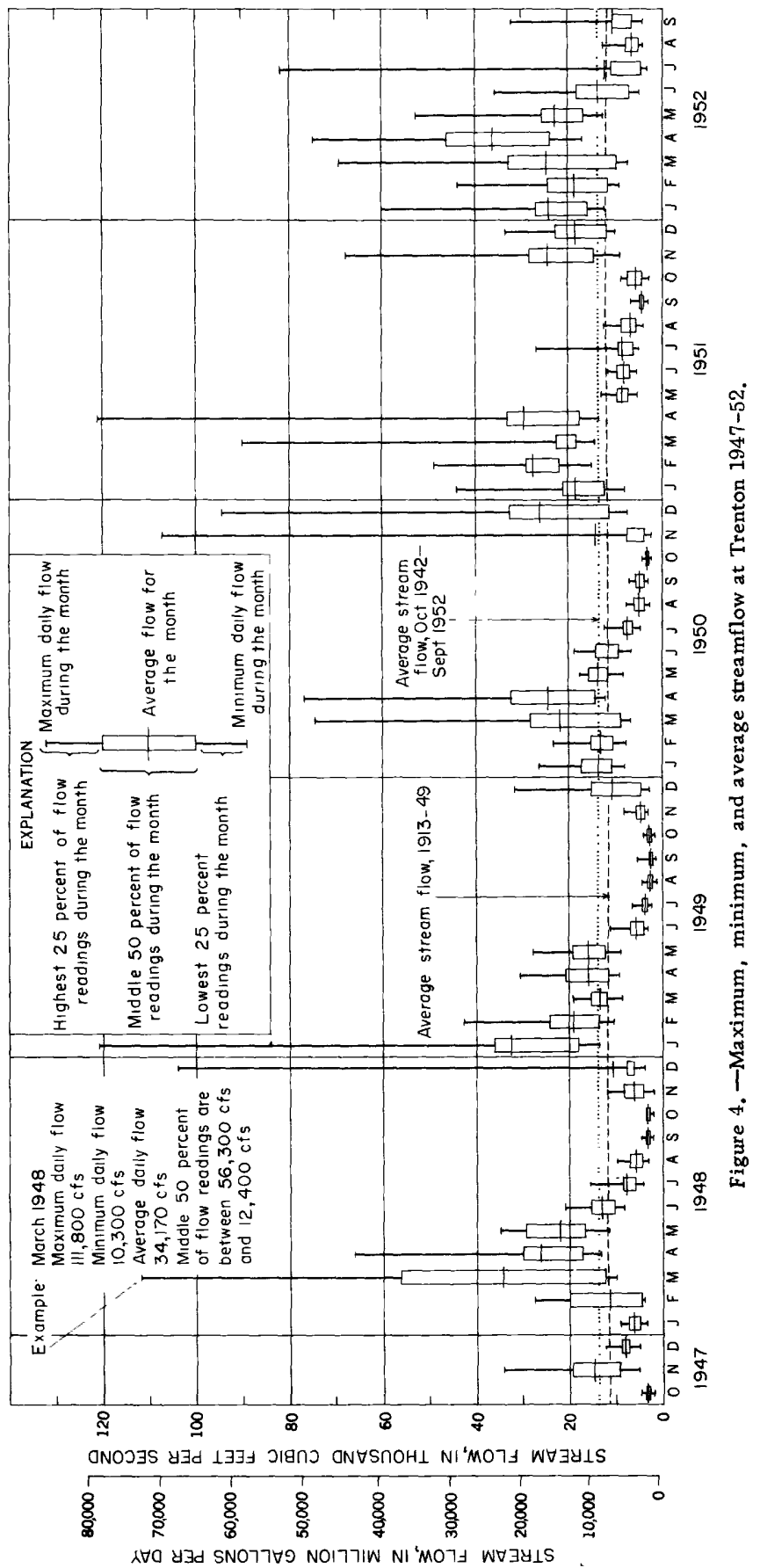


sented by a short horizontal line. The highest 25 percent of readings of the daily flows fall between the top of the rectangle and the top of the vertical line, the lowest 25 percent fall between the bottom of the rectangle and the bottom of the vertical line, the rectangle itself represents the middle 50 percent of the daily flows during the month. For comparison, the average flow for the 10year period, October 1942 to September 1952, 13, 392 cfs $(8,655$ mgd)-dotted line; and the average flow for the 36-year period, 1913 to $1949,11,780 \mathrm{cfs}(7,614 \mathrm{mgd})$-dashed line; are shown in the figure. The rate of flow in the Delaware River and its tributaries is characterized by a typical yearly pattern. The greatest streamflow occurs in the period from late fall to early spring; during the summer and early fall months there is usually an extended period of low rate of flow.

The tributaries of the Delaware River affect the quality of the water in the mainstem, in proportion to the volume of water added from the tributaries, and the amounts of the dissolved or suspended matter in the tributary waters. In table 1 are the tabulated drain-

Table 1. - Drainage areas and discharge rates of the main tributaries of the Delaware River between Trenton, N. J. and Marars Hook, Pa.

\begin{tabular}{|c|c|c|c|c|c|}
\hline Tributary & $\begin{array}{l}\text { Location } \\
\text { of gaging } \\
\text { station on } \\
\text { tributary }\end{array}$ & $\begin{array}{l}\text { Drainage } \\
\text { area above } \\
\text { gaging sta. } \\
\text { (sq miles) }\end{array}$ & $\begin{array}{l}\text { Drainage } \\
\text { area of } \\
\text { tributary } \\
\text { (sq miles) }\end{array}$ & $\begin{array}{c}\text { Period } \\
\text { of record } \\
\text { for which } \\
\text { average dis- } \\
\text { charge rate } \\
\text { is computed }\end{array}$ & $\begin{array}{l}\text { Average } \\
\text { discharge } \\
\text { rate at } \\
\text { gaging sta. } \\
\text { (cfs) }\end{array}$ \\
\hline $\begin{array}{l}\text { New Jersey } \\
\text { Assunpink Cr...... } \\
\text { Rancocas Cr....... } \\
\text { Big Timber Cr..... } \\
\text { Pensauken Cr...... } \\
\text { Mantua Cr.......... } \\
\text { Pennsylvania } \\
\text { Common Cr........ } \\
\text { Neshaminy Cr..... } \\
\text { Pennypack Cr...... } \\
\text { Schuylkill River... } \\
\\
\text { Darby Cr............ } \\
\text { Crum Cr............ } \\
\text { Ridley Cr.......... } \\
\text { Chester Cr......... } \\
\text { Marcus Hook Cr... }\end{array}$ & 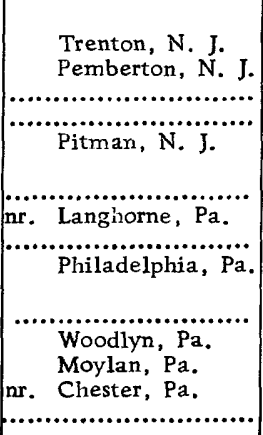 & 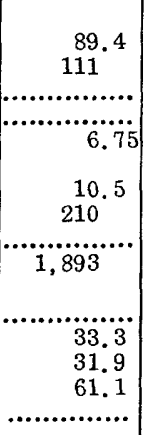 & $\begin{array}{c}341.4 \\
c_{59.3} \\
c_{35.4} \\
c_{51.2} \\
11.9 \\
233 \\
56.0 \\
1,909 \\
\\
78.6 \\
37.9 \\
38.2 \\
66.2 \\
\text { b5.5 }\end{array}$ & 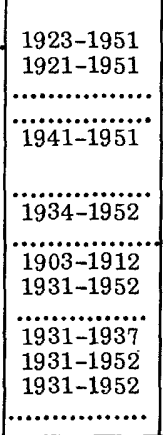 & 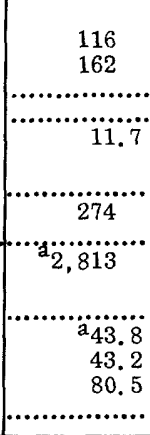 \\
\hline
\end{tabular}

a Adjusted for diversion.

bee bibliography: Pennsylvania Water Supply Commission, 1917.

c See bibliography: Vermeule, 1894.

age areas in square miles, average discharge rate in cubic feet per second, and the years for which the average flow was computed for the main tributaries of the Delaware River between Trenton and Marcus Hook.

Ground water occurs in abundance in the valley fill on both sides of the Delaware River between Trenton and Marcus Hook. It seems 
probable that both seepage of ground water into the river and infiltration of river water into the ground-water aquifers occur between Trenton and Marcus Hook. Analyses of ground water show it to be much higher in mineral content than the water in the river adjacent to it. Infiltration of a large volume of ground water into the water of the Delaware River would increase its mineral content; a considerable flow of river water into the ground-water aquifers could reduce the mineral content of the ground water, although much depends upon the rate of flow and the kind of soil through which the water percolates.

Another factor influencing the quality of the river water is pollution by industries and municipalities. Industrial wastes, in general, are treated in some manner before they are returned to the sewage systems or directly to the river. Such treatment may consist of cooling the water to river temperature, neutralizing the acids, removing sediment or oil, or decomposing oxidizable or putrescent material by bacterial action. Unless all the pollutant is removed by treatment the river is depended upon to dilute and carry away the remaining waste material. Wastes added to the river, of course, will change its character, the ultimate effect upon the stream depending upon the total volume of waste, its nature and concentration, and the volume of water available for dilution. The growth in population and in industries will add more pollution to an already polluted Delaware River. Fortunately, both industries and governmental agencies in this area are aware of the importance of an adequate supply of water for continuous economic growth in this area. Municipalities and industries that had no treatment or inadequate treatment facilities before 1949 are presently treating or planning to treat waste waters. On April 6, 1951, the city of Philadelphia put into operation its new Northeast Sewage Treatment Works, now treating 97 millions of gallons per day. Two other plants, the Southeast and the Southwest Treatment Works, are under construction and when completed the 3 plants will serve an area having a population of $1,884,000$. Industries, too, have improved their waste-treatment procedures, and newly established industries are giving special attention to the design of adequate treatment facilities.

In its lower reaches the Delaware River becomes a tidal stream with the head of tide at the Pennsylvania Railroad bridge at Trenton Falls. Fresh water from the upper Delaware River flows past Trenton, and is augmented by fresh water from the Schuylkill River and from other tributaries on both sides of the river between Trenton and Marcus Hook, and by precipitaíion directly on its surface, and possibly by ground-water infiltration. Salt water from the ocean tends to mix with the fresh water; on the flooding tide the salt water is carried upstream and on the ebbing tide, downstream. The percentage of oceansalt in the river is greatest near its mouth and diminishes upstream. Very little salt water is 
ever found upstream from central Philadelphia. The salinity increases when the discharge rate is low, and when a low discharge rate persists, the salinity encroaches progressively upstream from Marcus Hook toward Philadelphia, and also into the tidal tributaries.

\section{COLLECTION AND ANALYSES OF WATER SAMPLES}

Water samples for chemical and sanitary analyses are taken monthly from a boat furnished by the city of Philadelphia. The locations at which samples are taken are described in table 2 and

Table 2-Location of sampling stations 1

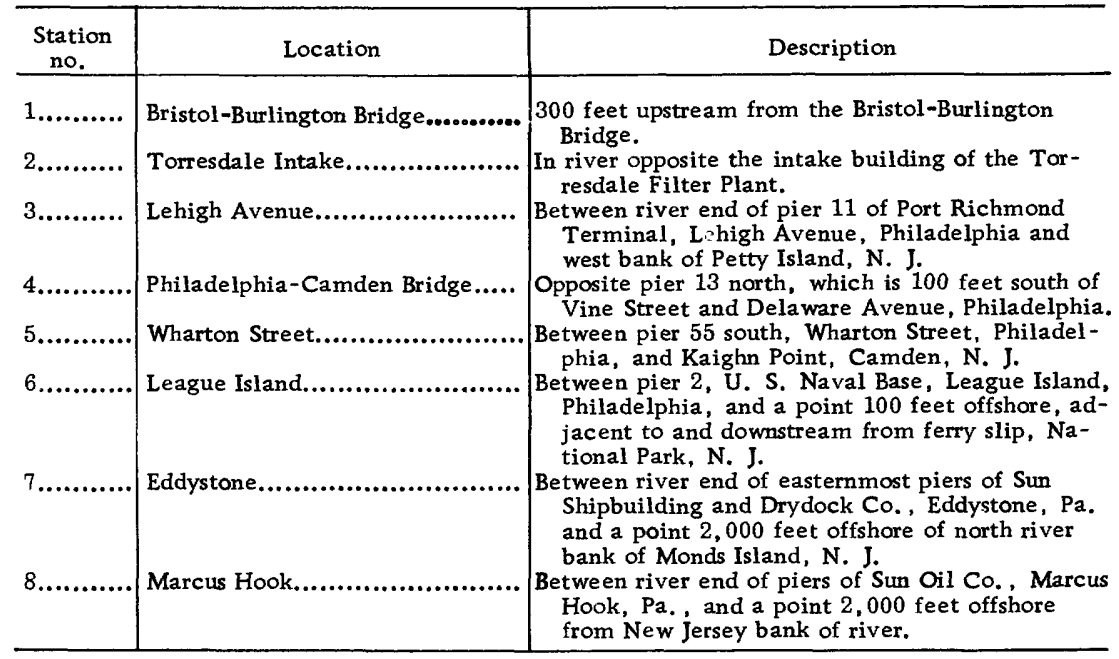

1 Refer to figure 1.

these sampling stations are also shown on figure 1. Samples are taken on one day in the early part of each month at the 4 upstream stations (stations 1,2,3, and 4). Usually on the following or preceding day samples are taken at the 4 downstream stations (stations $5,6,7$, and 8). At each station a five-point traverse of the river is made. River cross sections at each station are shown in figure 5.

The content of dissolved oxygen in the water is determined when the sample is taken, and water is also collected for the determination of the five-day at $20^{\circ} \mathrm{C}$ biochemical oxygen demand (B.O.D.) These samples are taken at approximately 3 feet below the surface of the river. Additional samples are taken with a Foerst sampler at approximately 3 feet above the bottom of the river at most locations. The time of sampling and the temperature of the water are recorded immediately. Samples are collected in 370-cc capacity, pressure-closure bottles for the determination of $\mathrm{pH}$, specific 
Table 3. - Type of analysis performed on bottom samples ${ }^{1}$ at indicated locations on stations

[ $c=$ comprehensive analysis consists of determination of color, $\mathrm{pH}$, specific conductance, silica, iron, calcium, magnesium, sodium, bicarbonate, sulfate, chloride, fluoride, nitrate, dissolved solids, and hardness. $\mathrm{p}$ =partial analysis consisting of determination of $\mathrm{pH}$, specific conductance, chloride, D. O., B. O. D. , and suspended sediment]

\begin{tabular}{|c|c|c|c|c|c|c|}
\hline \multirow[b]{2}{*}{$\begin{array}{c}\text { Station } \\
\text { no. }\end{array}$} & \multirow[b]{2}{*}{ Station } & \multicolumn{5}{|c|}{ Location on station } \\
\hline & & Pa. side & $\begin{array}{c}\text { West } \\
\text { center }\end{array}$ & Center & $\begin{array}{c}\text { East } \\
\text { center }\end{array}$ & $\begin{array}{c}\text { New Jersey } \\
\text { side }\end{array}$ \\
\hline$\cdots$ & Bristol-Burlington Bridge............. & c & $\mathrm{p}$ & c & $\mathrm{p}$ & c \\
\hline 2......... & $\begin{array}{l}\text { Torresdale Inlet, Philadelphia...... } \\
\text { Lehigh Avenue, Philadelphia........ }\end{array}$ & (n............... & $\cdots$ & $\mathrm{p}$ & $p$ & 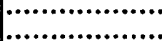 \\
\hline $4 \ldots \ldots \ldots$ & Philadelphia-Camden Bridge........... & ${ }^{\cdots} \mathrm{c}$ & $\mathrm{p}$ & c & $\mathrm{p}$ & c \\
\hline $5 . . . . .$. & Wharton Street, Philadelphia........ & $\ldots$ & $\mathrm{p}$ & $\mathrm{p}$ & $\mathrm{p}$ &.. \\
\hline & League Island, Philadelphia.......... & & $\mathrm{p}$ & $\mathrm{p}$ & $\mathrm{p}$ & .... \\
\hline .... & Eddystone $\ldots \ldots \ldots \ldots \ldots \ldots \ldots \ldots \ldots \ldots$ & ............. & $\mathrm{p}$ & $\mathrm{p}$ & $\mathrm{p}$ & ................... \\
\hline & Marcus Hook.............................. & & $\mathrm{p}$ & c & $\mathrm{p}$ & 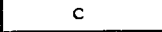 \\
\hline
\end{tabular}

${ }^{1}$ The table applies to samples collected from January 1951 to December 1952. From August 1949 to December 1950 comprehensive analysis were made of all bottom samplestaken at Bristol-Burlington Bridge, Philadelphia-Camden Bridge, and Marcus Hook.
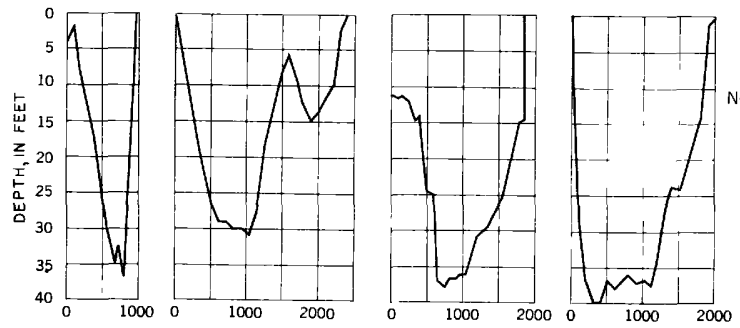

Note Horizontal scale for Philadelphı-Camden Bridge is distonce in feet fram Pennsylvania side

BRISTOL- TORRESDALE BURLINGTON INTAKE

\section{LEHIGH AVENUE

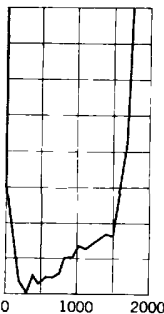

WHARTON STREET

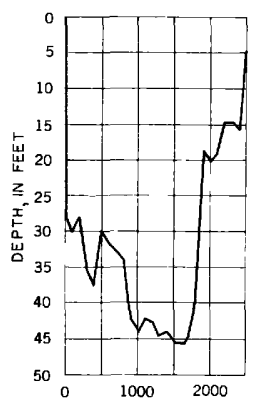

LEAGUE ISLAND

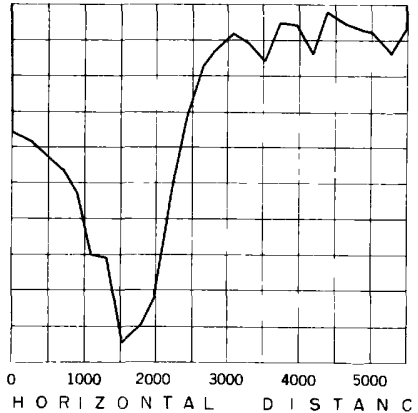
EDDYSTONE

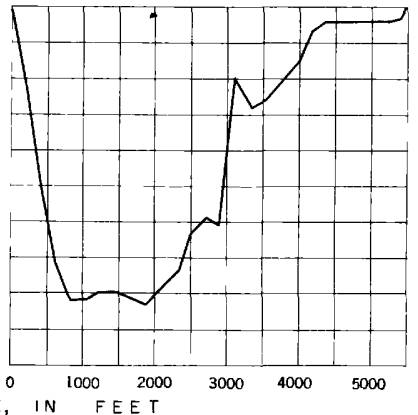

MARCUS HOOK

Soundıngs made by U.S. Army Corps of Engineers, 1942

Figure 5. - Cross sections of Delaware River channel at sampling stations. 
conductance, concentration of chloride, and suspended sediment. For more complete analyses, gallon-samples are collected in Pyrex bottles. Table 3 indicates the type of analysis performed for each sample from the bottom of the river. A partial analysis is performed on all top samples.

\section{CHEMICAL AND PHYSICAL CHARACTERISTICS OF THE WATER}

The water in the Delaware River above Philadelphia is fresh water and usually contains less than $200 \mathrm{ppm}$ of dissolved solids. During the late summer months of 1949, when the average monthly flow rates were less than $3,000 \mathrm{cfs}$, the concentration of dissolved solids at the Philadelphia-Camden Bridge reached $248 \mathrm{ppm}$. Below Philadelphia, during the periods of low water flow, the concentration of dissolved solids has reached $5,000 \mathrm{ppm}$, due to the invasion of salt water.

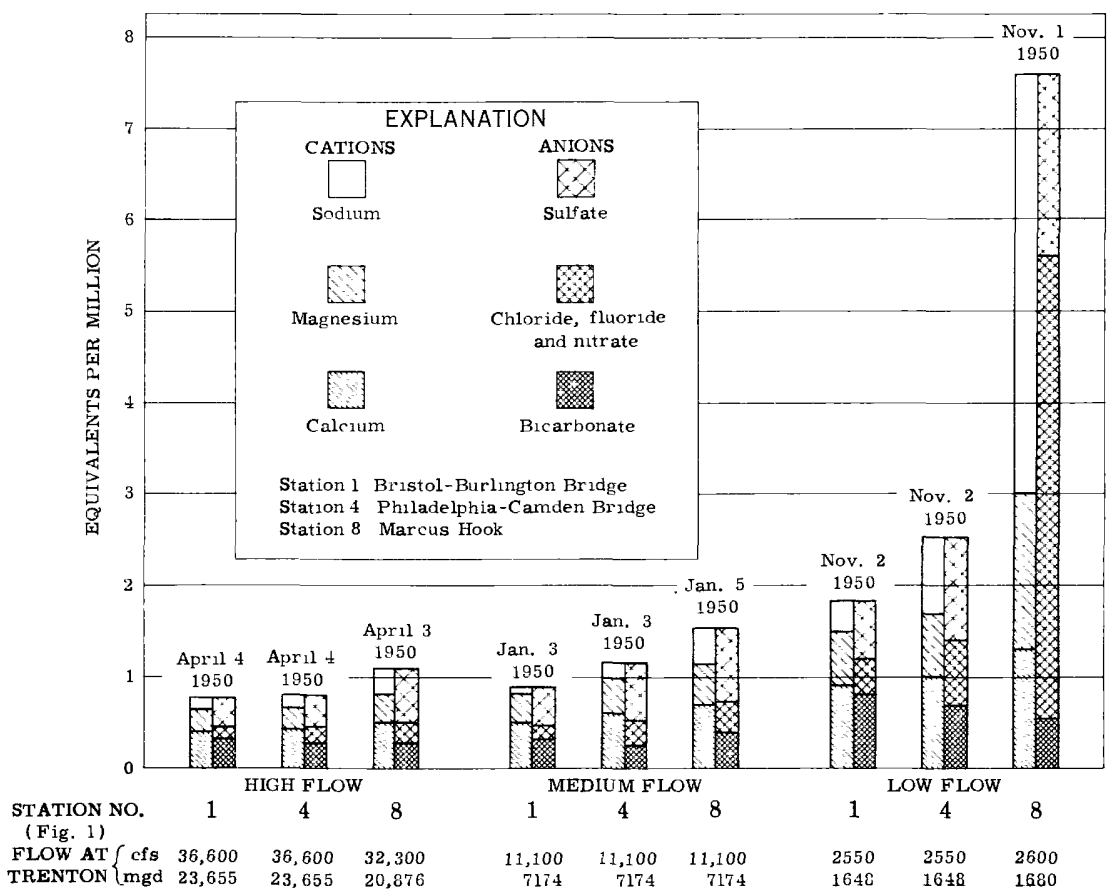

Figure 6. -Effect of streamflow on the quality of water at Bristol, Camden, and Marcus Hook. 
Principal dissolved constituents. - The principal dissolved constituents present in the Delaware River are sulfate, chloride, fluoride, nitrate, bicarbonate, sodium, calcium, and magnesium ions. The relative quantities of these ions at the Bristol-Burlington Bridge, Philadelphia-Camden Bridge, and Marcus Hook stations are shown infigure 6 for high, medium, and low flow rates at Trenton. Minor quantities of other constituents such as aluminum, manganese, iron, silica, etc., are also present. Regardless of the amount of water flowing past Trenton, the equivalents per million of sodium ion and chloride ion increase downstream from Bristol to Marcus Hook. These increases in concentration are due principally to the increased mixing of ocean water with the fresh water as the river water moves toward the bay. The increase in the dissolved solids is more pronounced as the rate of flow decreases.

The amount of each individual chemical element present in the Delaware River will vary with the physical location and the rate of flow of the river. Figure 7 shows how the chloride content increases from Trenton to Marcus Hook. The chloride concentration in parts

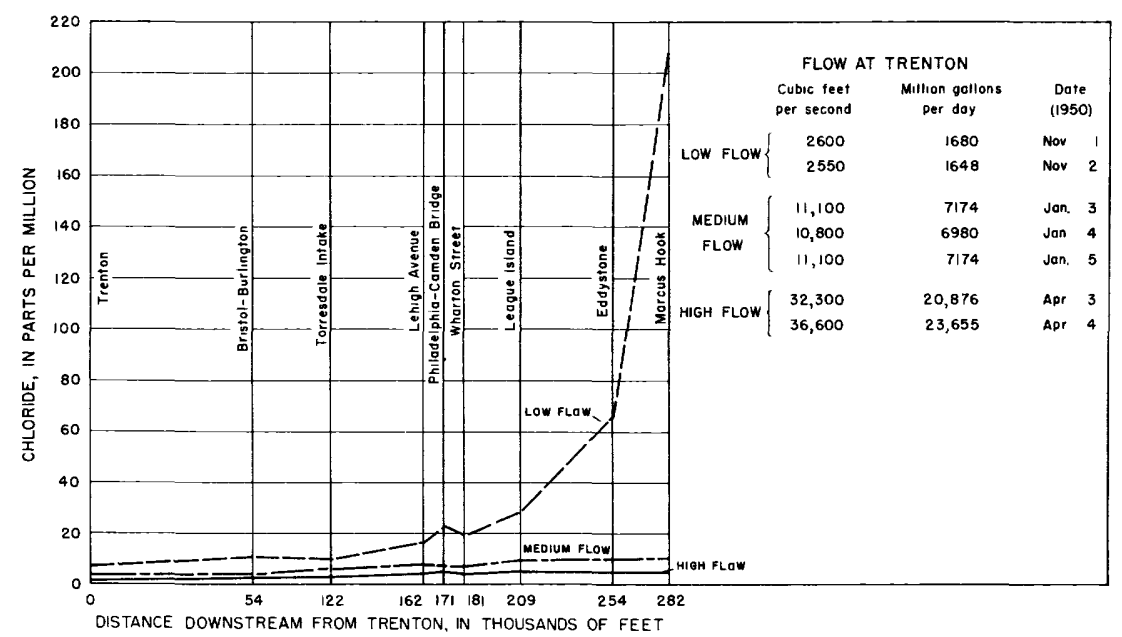

Figure 7. - Chloride concentration at sampling stations for high, medium, and low flow rates.

per million is plotted for each sampling station for typical medium, high, and low streamflow rates. In the example, typical of the river at medium flow rate, the chloride content of the water increases slightly from Trenton to Marcus Hook. When four to five times as much water flows in the river, the concentration of chloride may be reduced to as little as half these quantities. At low-flow 
rates the chloride content increases sharply at Eddystone and Marcus Hook. At the se locations there is a tendency for the ocean water to move upstream along the river bottom at low flow.

Specific conductance relations. - The specific conductance relations of natural waters are due to the ionization of salts in the water. The specific electrical conductance (or electrical conductivity) of water is the reciprocal of the electrical resistance of a cubic contimeter of the water, between electrodes each 1 -square centimeter in area, and is expressed in a unit, the micromho. Because the electrical conductivity increases in direct proportions to the number of ions present, the specific electrical conductance is used as a measure of the concentration of dissolved salts. The measurement of electrical conductivity is more rapid and convenient than chemical analyses of water; however it gives only a fraction of the information obtained from a chemical analysis. The relation between electrical conductivity and dissolved solids for this part of the Delaware River is shown in figures 8 and 9 . For this reach of the river the numerical value of specific conductance in micromhos measured at $25^{\circ} \mathrm{C}$ is approximately 1.5 times the weight of dissolved solids, in parts per million (fig. 9). Because the concentration of dissolved salts present in the water increases as more ocean water mixes with the fresh water during the ebb and flow of the tide, the electrical conductivity of the water increases downstream. This is demonstrated in figure 10 in which the electrical conductance in micromhos is plotted for each sampling station for typical high, medium, and low rates of flow.

The measurement of electrical conductivity is a convenient and rapid method for detecting sea-water intrusion or the presence of slugs of pollution,3 for a change in the specific electrical conductance of a stream demonstrates a change in the amount or kind of dissolved material in the water. Where measurements of specific conductance are not accompanied by chemical analysis, it is convenient to have a means of estimating chloride or sulfate concentration from the specific electrical conductance. The relation between electrical conductivity and chloride is shown in figures $11,12,13,14$, and 15 , and that between electrical conductivity and parts per million sulfate in figure 16 .

Above a chloride concentration of $60 \mathrm{ppm}$, the relation between the chloride concentration and electrical conductivity is approximately a straight line when plotted on a logarithmic scale (fig. 12). In this range of chloride concentration, the majority of anions present are chloride ions, and the conductivity of the water is greatly dependent upon the chloride ion. Below $60 \mathrm{ppm}$ chloride

\footnotetext{
${ }^{3} \mathrm{~A}$ slug of pollution is a compact mass of polluted water that remains in the stream for a time as a detectable unit.
} 


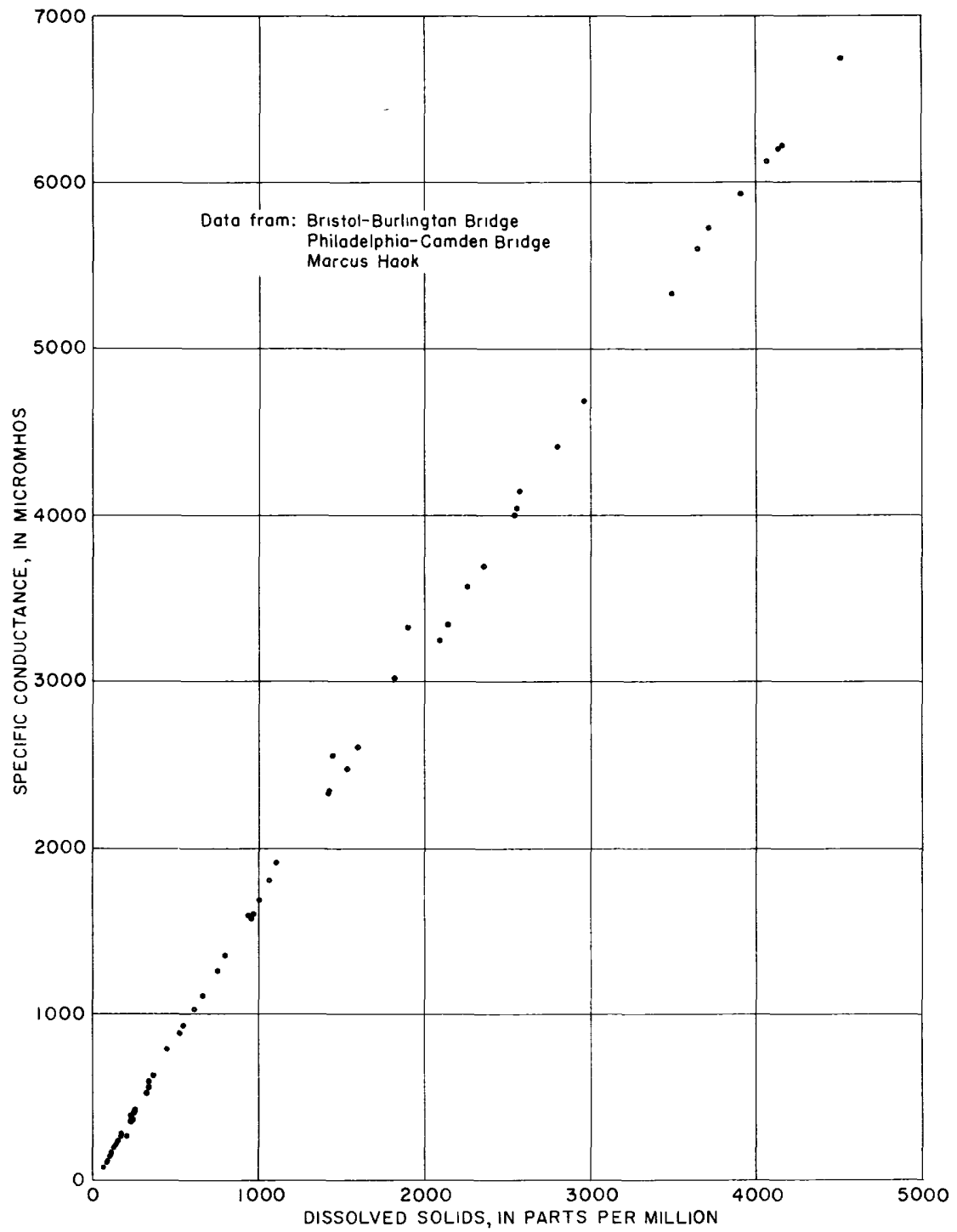

Figure 8. - The relation between electrical conductivity and dissolved solids $(0-5,000 \mathrm{ppm})$. 


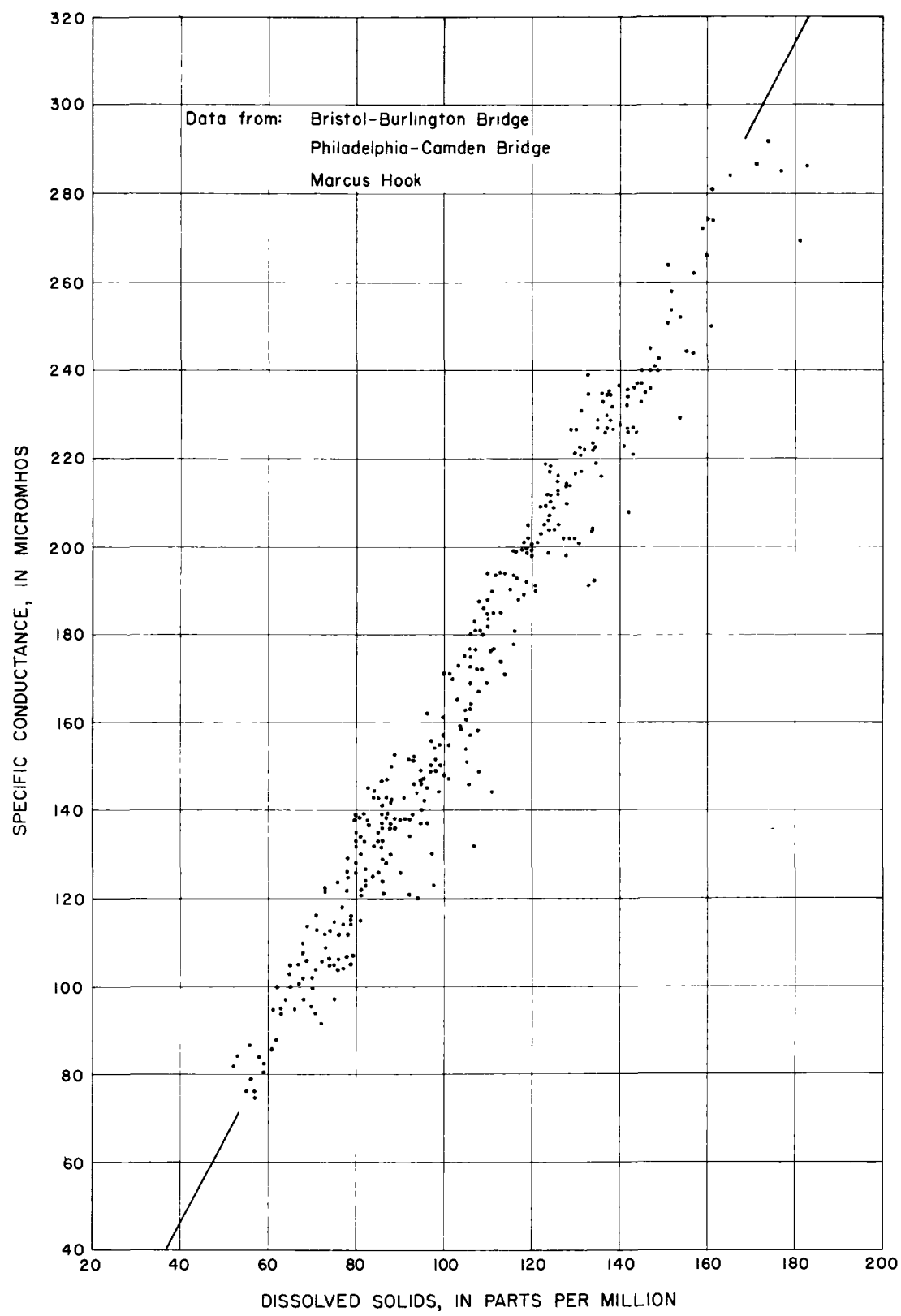

Figure 9. - The relation between electrical conductivity and dissolved solids (0-200 ppm). 


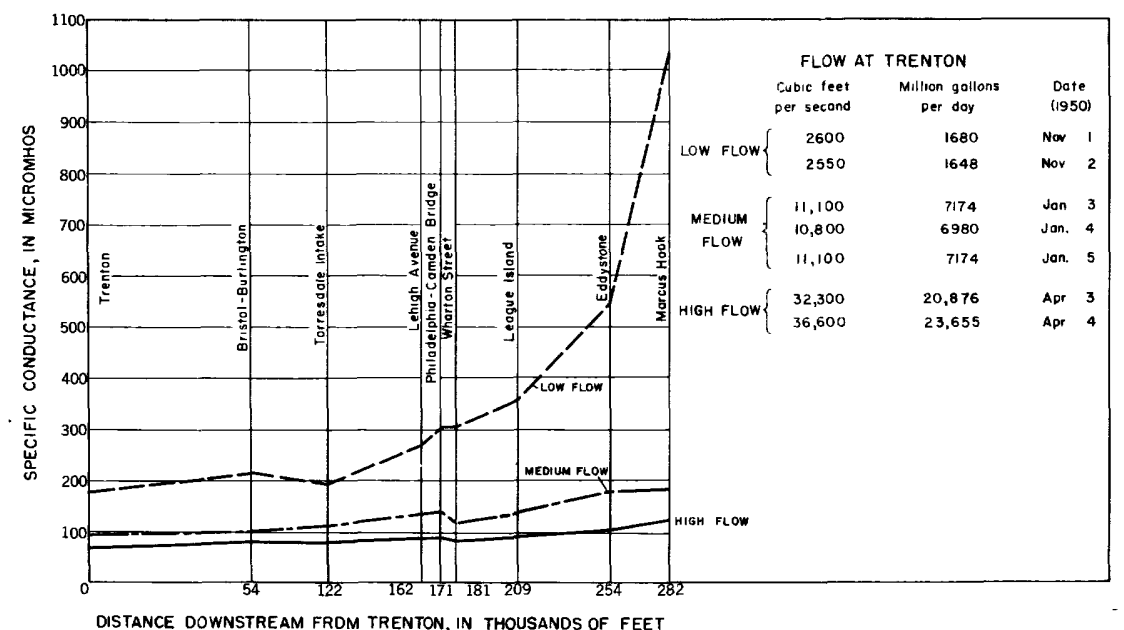

Figure 10. -Electrical conductivity at sampling stations for high, medium, and low flow rates.

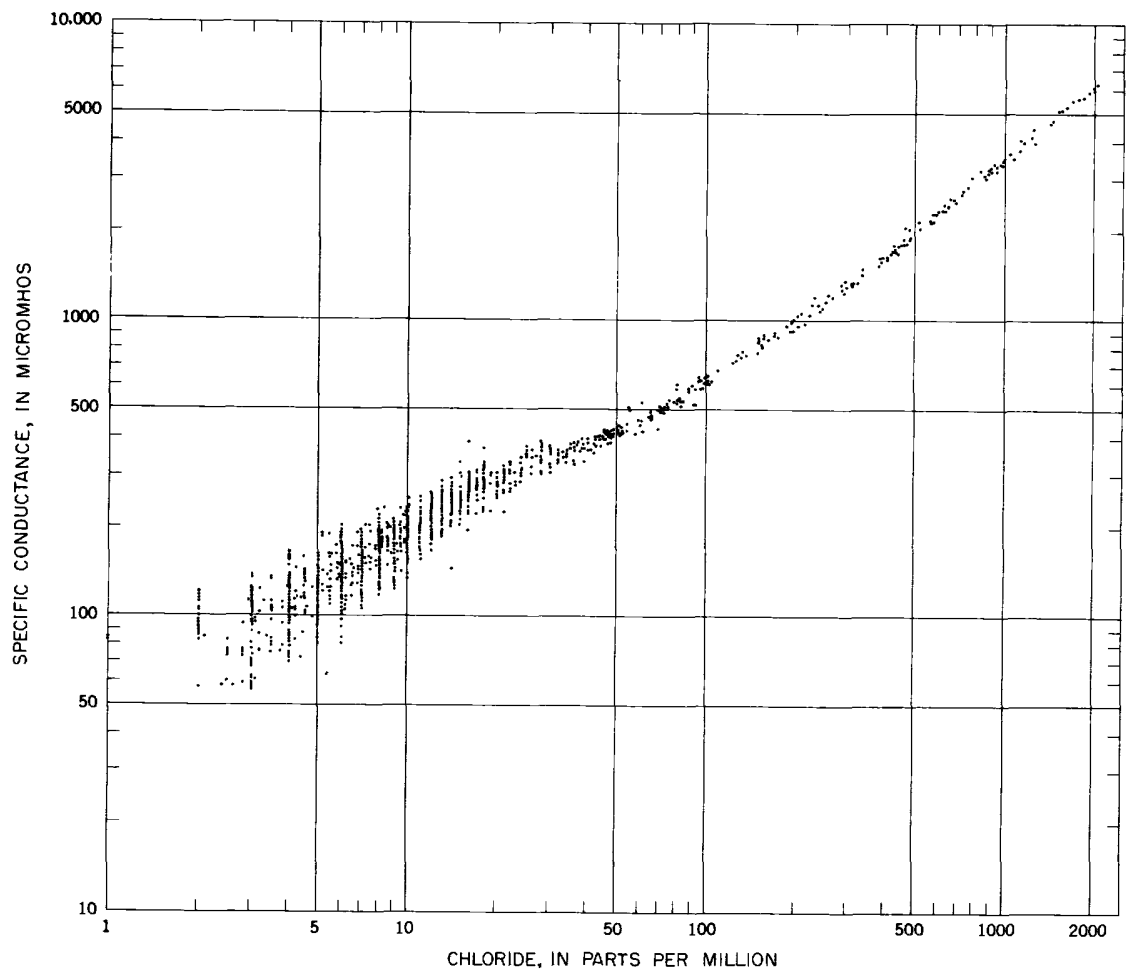

Figure 11. - The relation between electrical conductivity and chloride concentration $(0-2,000 \mathrm{ppm})$. 


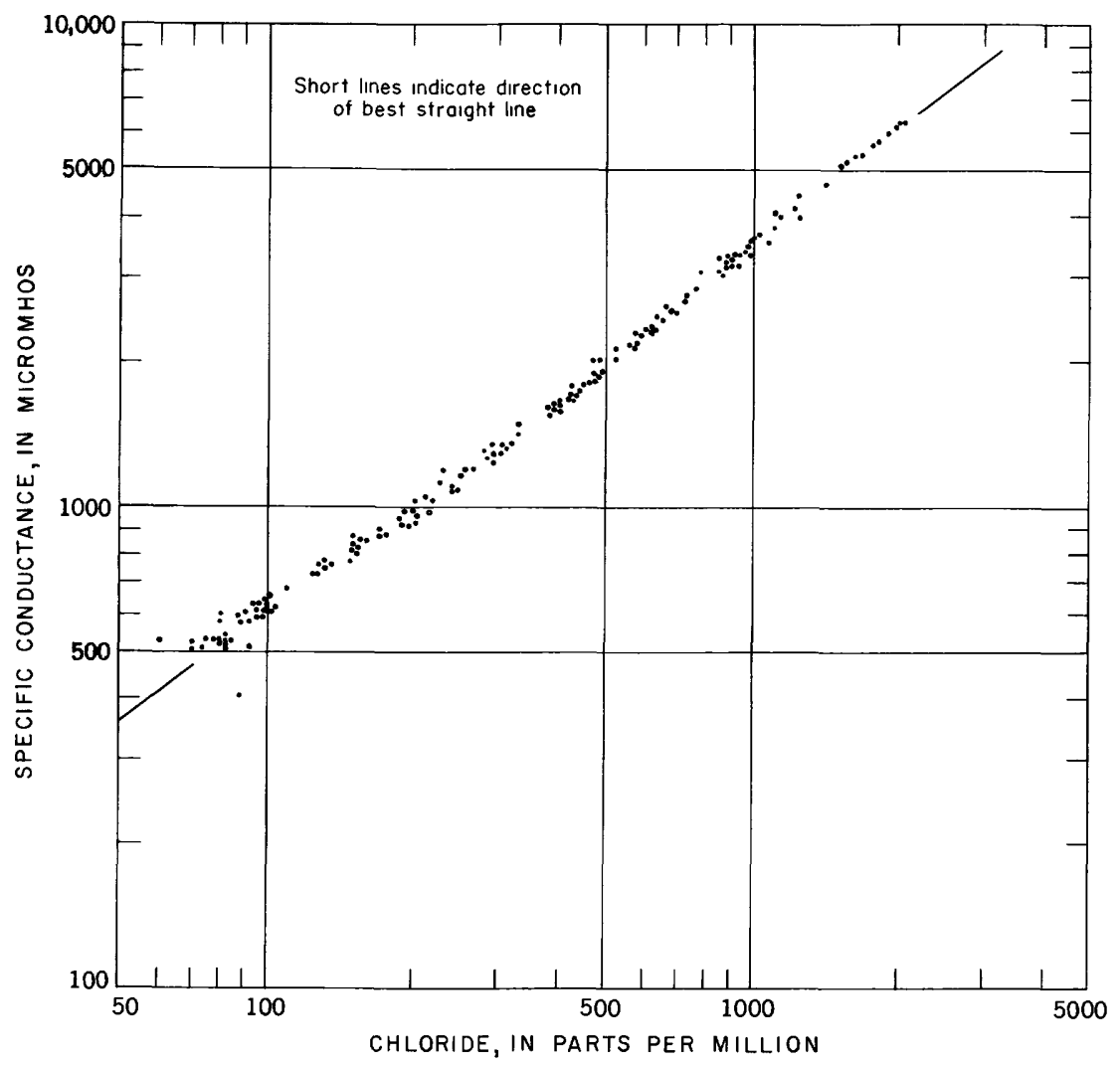

Figure 12. - The relation between electrical conductivity and chloride concentration ( $60 \mathrm{ppm}$ and above).

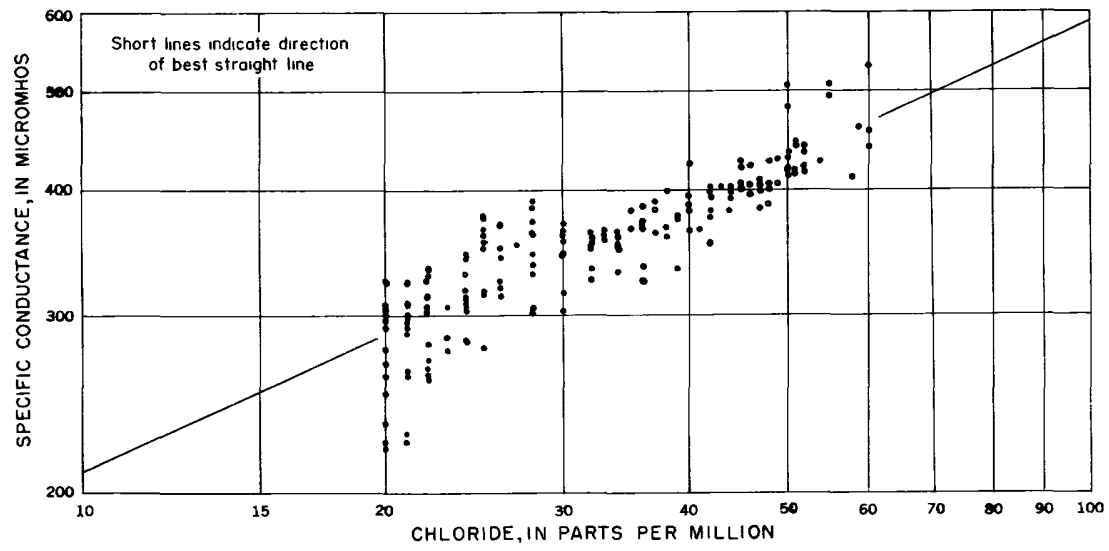

Figure 13. - The relation between electrical conductivity and chloride concentration (20 to $60 \mathrm{ppm})$. 


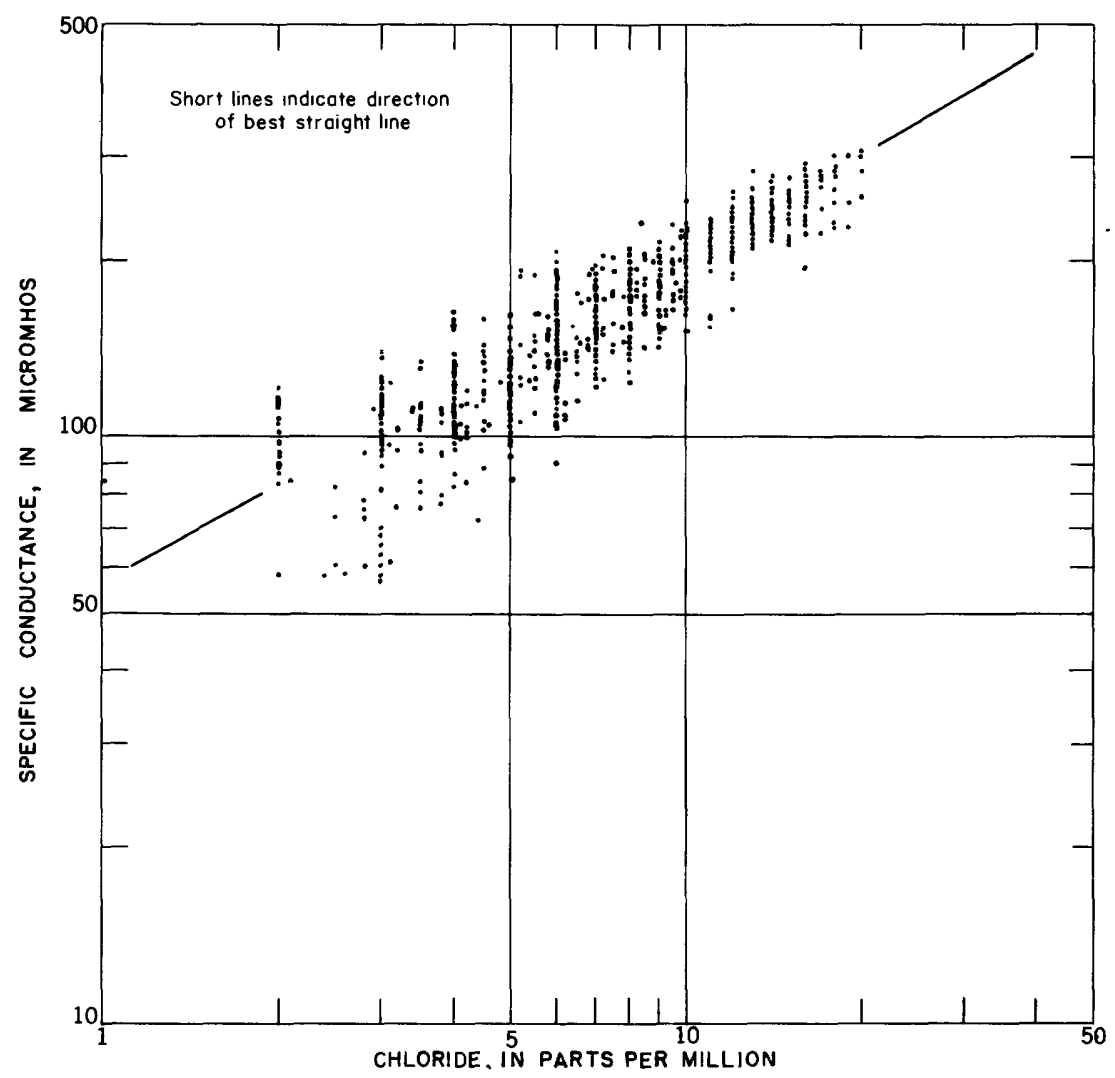

igure 14. - The relation between electrical conductivity and chloride concentration $(0-20 \mathrm{ppm})$.

'he relation between chloride concentration and conductivity is nore complex. The chloride ions no longer predominate; sulfate and bicarbonate ions are present in greater proportions, and their individual effect on conductivity is slightly different from that of the chloride ion. The experimental data for chloride concen"rations from 20 to $60 \mathrm{ppm}$ are plotted in figure 13, for concen"rations of less than $20 \mathrm{ppm}$, in figure 14. In each case, the direction of the best straight line has been indicated. If concentra"ion of chloride is below $20 \mathrm{ppm}$, the relation between electrical conductivity and chloride concentration depends largely on the icarbonate concentration. This is demonstrated in figure 15 for "hree ranges of bicarbonate concentration. As the concentration 


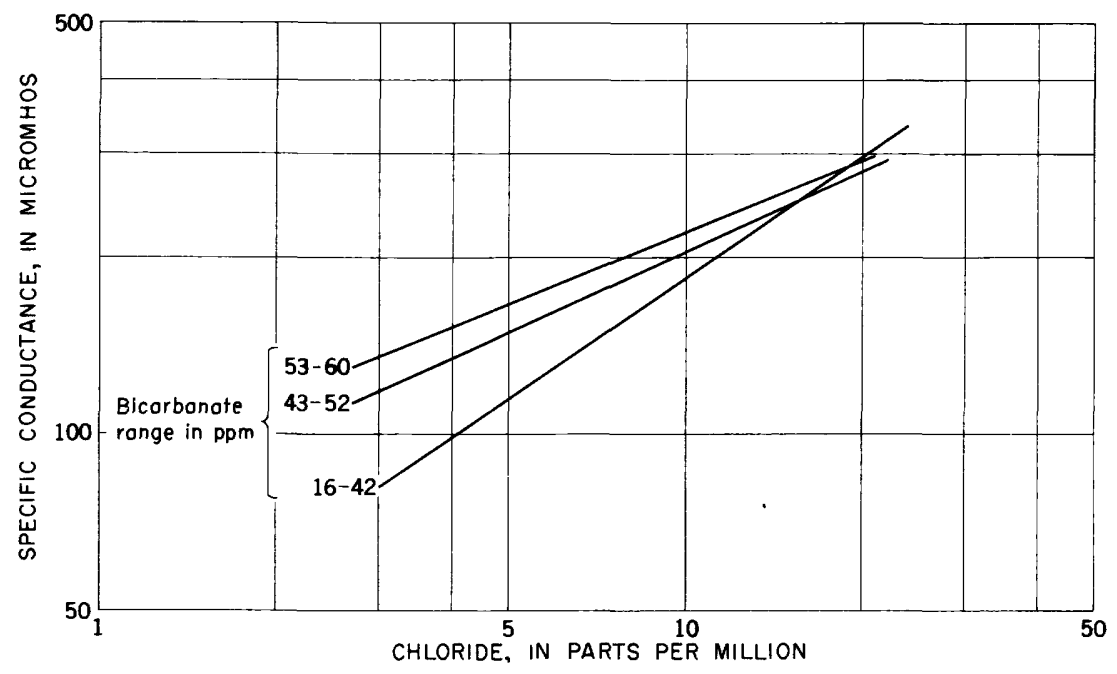

Figure 15. - The relation between electrical conductivity and chloride concentration at three ranges of bicarbonate concentration.

of chloride ions nears 20 ppm the effect of the bicarbonate ions diminishes.

The concentration of sulfate (ppm) has been plotted against the specific conductance (micromhos) (fig. 16). The conductivity of water increases as the concentration of sulfate ion increases. The bar graph of cations and anions indicates that the sulfate concentration increases downstream from Trenton to Marcus Hook at all flow rates (see fig. 6). This increase in sulfate concentration is especially evident at low-flow rate. In the Delaware River above Philadelphia, the concentration of sulfate expressed in equivalents per million usually exceeds that of chloride. As a result, the concentration of sulfate here has a greater effect upon the conductivity than does the concentration of chloride. In the lower reaches of the Delaware River when saline invasion upstream takes place, the concentration of chloride ions, exceeds the equivalent concentration of sulfate ions because there is approximately 10 times as many equivalents of chloride in the ocean water as of sulfate.

It is important to understand that all these relations pertain to the Delaware River under normal conditions. Should a large slug of pollution be introduced into the river, the relation such as shown in figures 6 to 16 would not apply. In any case, they merely represent the experience of the period from August 1919 to December 1952, and estimates based on them will not have the reliability of actual chemical analyses. 


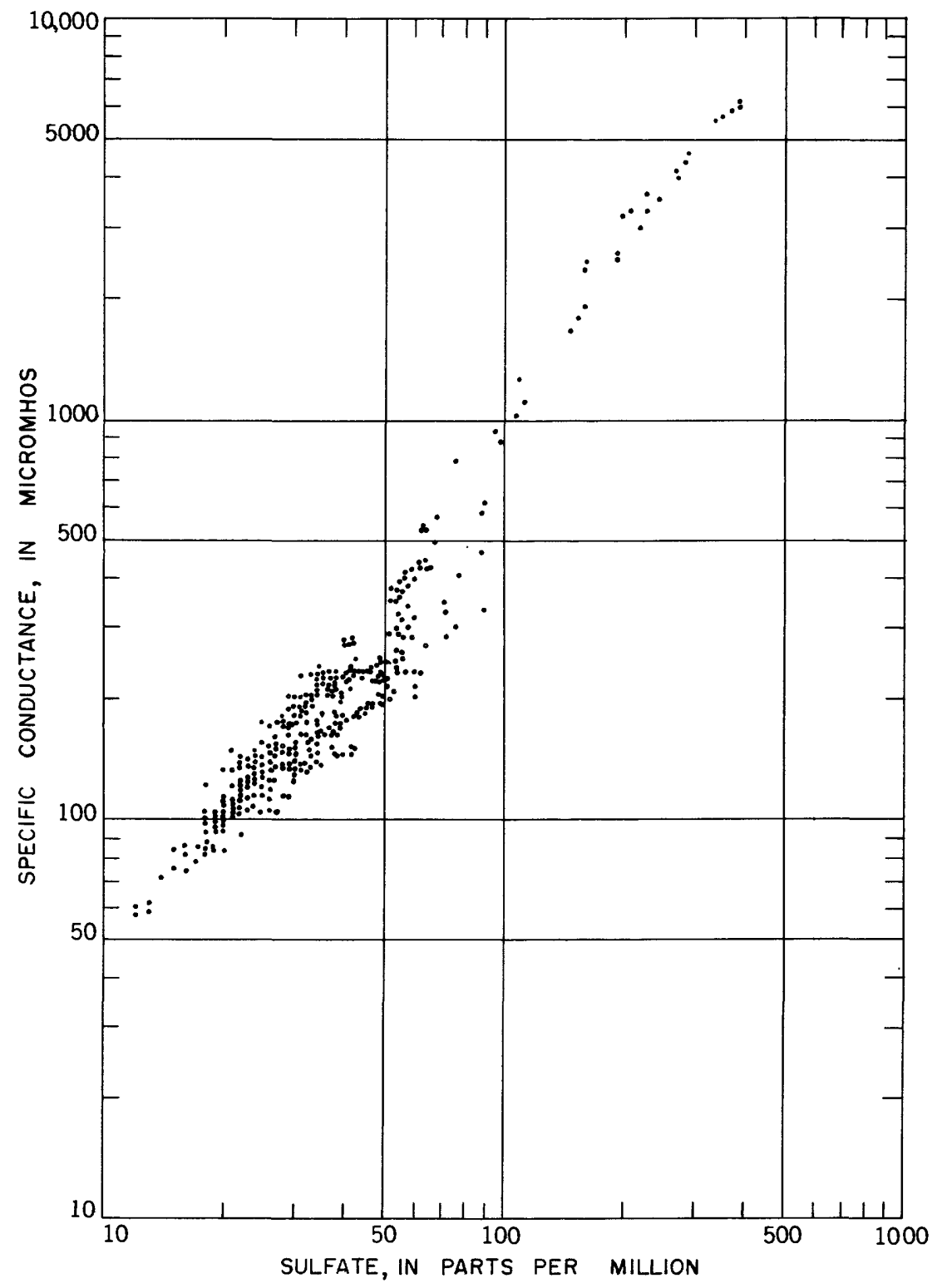

Figure 16. - The relation between electrical conductivity and sulfate concentration. 


\section{TEMPERATURE OF THE WATER}

An adequate supply of water to be used for cooling purposes is often one of the essential considerations in selecting the site for an industrial plant. A survey by INCODEL in 1951 found that 91 percent of the water used in industries in this region was used for cooling purposes. This large percentage of use for cooling is due to the many stations generating electricity and the many oil refineries along the banks of the Delaware River in this area. The temperature of the water to be used in any plant is a critical design factor. Ground water, when available, is often a more suitable source of cooling-water because its temperature is low and uniform throughout the year. River water, however, is of ten the cheapest and most available source of cooling-water, but its average temperature is close to the temperature of the air. The temperature of water, of the Delaware River has exceeded $80^{\circ} \mathrm{F}$ in the summer months; thus industries have found it advantageous to use ground water in the summer and river water in the winter. In many industrial plants the use of cooling towers makes it possible to reuse the cooling-water many times before it is returned to the river; here smaller treatment facilities and waste disposal systems are adequate. In some plants it is efficient to use water for cooling purposes and afterwards to use the warmed water as a heating agent or in process work to save heating costs.

From October 1944 to June 1950, the temperature of the water in the Delaware River was measured daily at Morrisville, $\mathrm{Pa}$. (and/or Trenton, N. J.). In June 1950 a Stevens continuous recording thermograph was installed in the Trenton waterworks. The sensing element of the thermograph was located in the intake pipe of the waterworks, which pumps continuously. In March 1953, a continuous-recording resistance thermometer was installed in the Delaware River offshore from the Bristol waterworks. In addition, once each month the water temperature of all samples from the cross section at each sampling station is determined.

Figure 17 shows the frequencies with which various water temperatures occur and is based on random one-a-day readings during the period 1947-50 and on averages of thermograph readings during 1950-52. The curve shows that the water temperature was $75^{\circ} \mathrm{F}$ or above 20 percent of the time. On any one day, the temperature of the water at Trenton is generally within $2^{\circ} \mathrm{F}$ of the water temperature recorded at any of the eight sampling stations between Bristol and Marcus Hook. When the temperature of any sample from a cross section of the river is compared with the temperature of the water in the center of the river just below the surface, the difference is less than $1^{\circ} \mathrm{F}$ in 92 percent of the samples.

The temperatures of the water in the Delaware River at Trenton from October 1947 to September 1952 are illustrated in figure 18. 


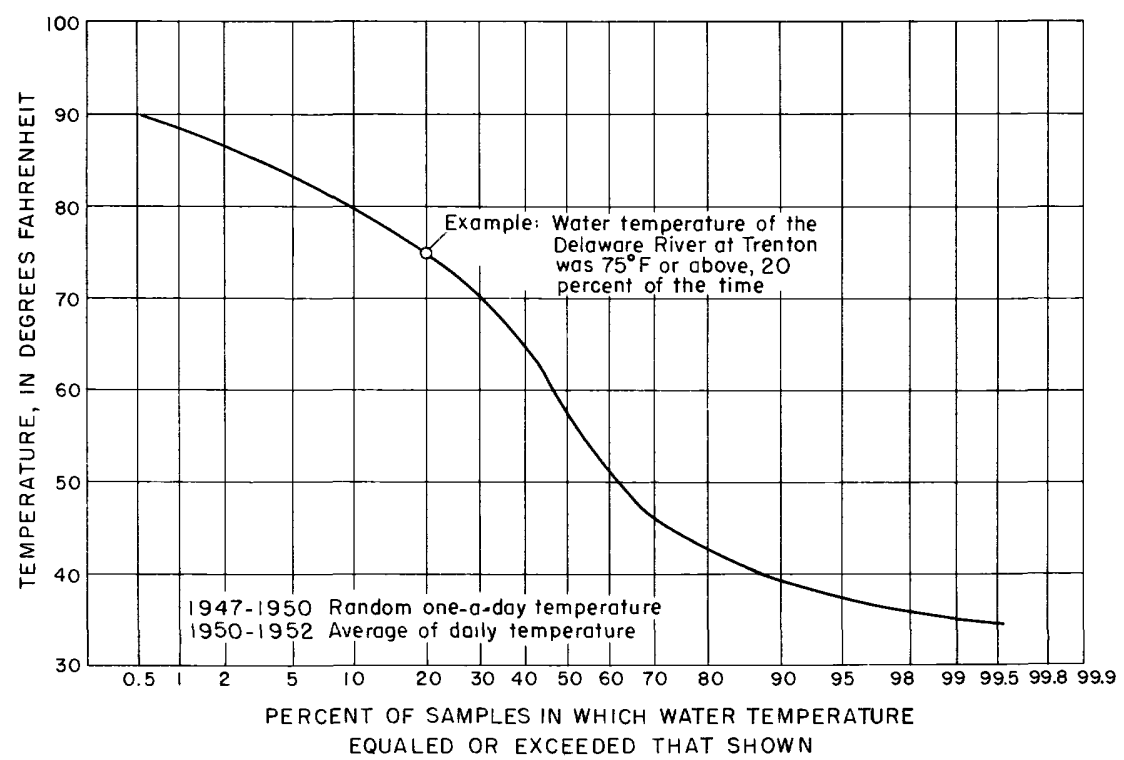

Figure 17. -Water temperature frequency curve, Trenton.

For each month are plottedfour data: the maximum daily temperature, the minimum daily temperature, the average temperature for the month, and the range in temperature of the middle 50 percent of the readings for the month. The maximum daily temperature is the highest point on the vertical line, the minimum daily temperature is the lowest point on the vertical line, and the average temperature of the water for the month is represented by a short horizontal line. The highest 25 percent of the readings of the daily temperatures are between the top of the rectangle and the highest point on the vertical line, the lowest 25 percent are between the bottom of the rectangle and the bottom of the vertical line, the rectangle itself represents the middle 50 percent of the readings of the temperature during a month. The average temperature of the water in the Delaware River at Trenton from October 1947 to September 1952 is shown as a dashed horizontal line across the graph. 


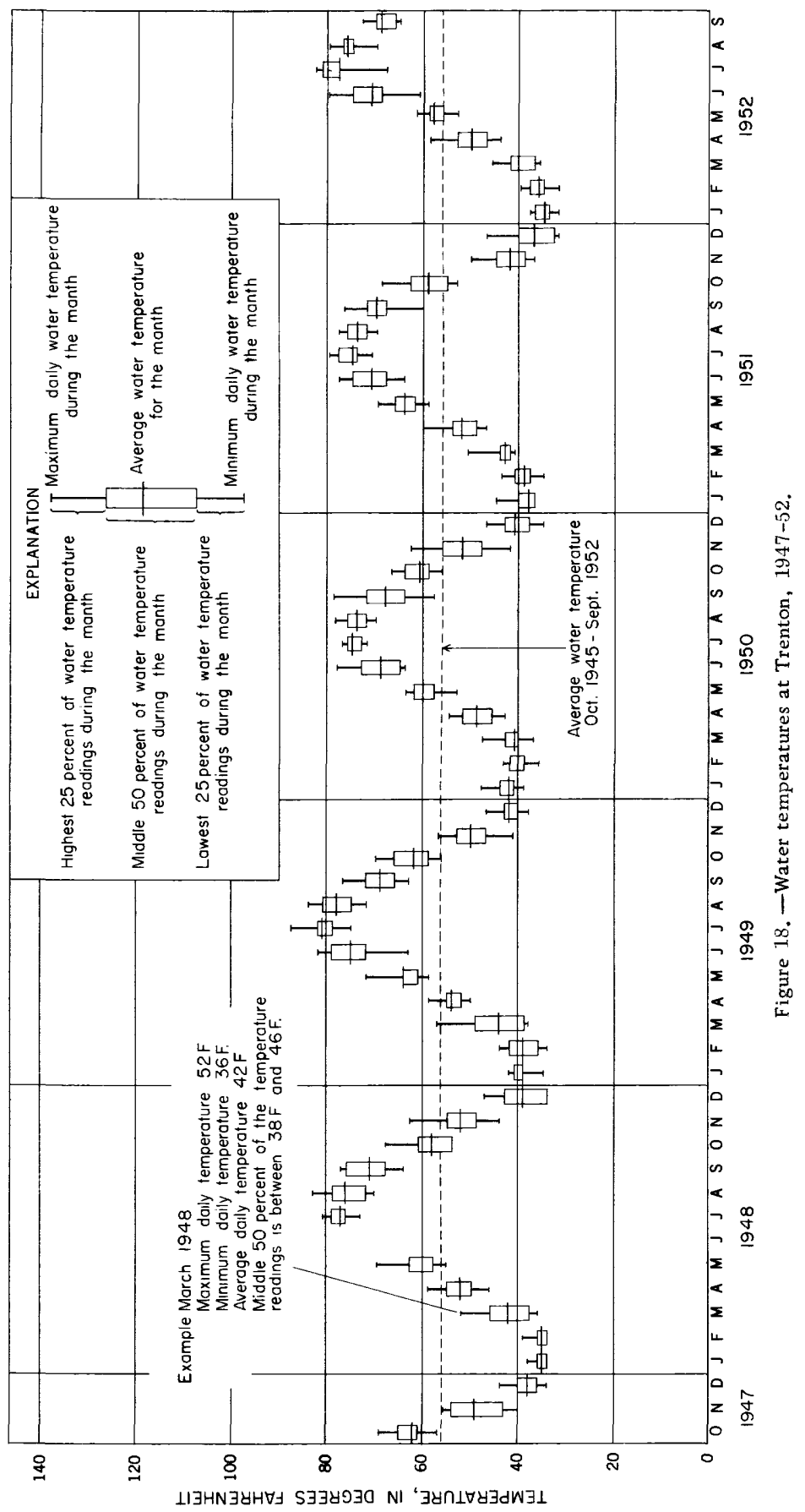




\section{DISSOLVED OXYGEN AND BIOCHEMICAL OXYGEN DEMAND}

A shallow stream containing pure water brought into contact with the air through turbulence will be saturated with oxygen, that is, no more oxygen will dissolve in the water when air is bubbled through it. Green plants require some oxygen to live; in the light they produce more oxygen than they consume, but in the dark they consume more oxygen than they produce. The dissolved oxygen may be consumed in respiration by fish and other animals and also by bacteria which decompose organic impurities in the water. Thus, organic pollutants require oxygen for decomposition. The dissolved oxygen content of a stream depends upon the balance between (1) consumption of oxygen by animals and plants and by organic pollutants and (2) replenishment of oxygen from the air and through the action of aquatic plants. Thus the dissolved oxygen content (D.O.) of water, in parts per million, or its percentage saturation with oxygen is one index of the stream's ability to destroy polluting material. If the amount of oxygen required to oxidize the pollutants exceeds the quantity of dissolved oxygen present, and additional oxygen is not supplied to the stream by aeration or plant activity, the oxygen content of the water may be so depleted that putrefaction occurs, with accompanying odors and destruction of fish and green plantlife. The restoration of oxygen to a deep, quiet-flowing stream, such as the Delaware River below Trenton, is less rapid than to a shallow, fast-moving, turbulent stream, both because of the smaller surface of contact of water and air and because of smaller effect of green plantlife in a deep river as compared to a shallower stream in which the sunlight can penetrate to the bottom.

The biochemical oxygen demand (B. O. D.) is a measure of the quantity of oxygen required to decompose organic wastes through bacterial oxidation. In this test the water sample to be analyzed is mixed with water containing an excess of oxygen and bacteria of the type present in the stream and incubated for 5 days at $20^{\circ} \mathrm{C}$, during which time the bacteria use the dissolved oxygen to oxidize the organic matter. Analysis of the sample before and after the 5 -day period shows the amount of oxygen consumed under these conditions and is therefore a measure of the rate at which the organic material consumes oxygen.

Figure 19 shows that the dissolved oxygen content of the water is depleted as it moves downstream. The percent of saturation of the water with oxygen in the Delaware River is plotted for each of the sampling stations for March 1-2, May 1-2, and September $5-6,1951$. In each case the percent of saturation with oxygen decreases as the water moves downstream. For example, in May, when the rate of flow of the river was approximately average, the water at Bristol-Burlington was 90 percent saturated; the oxygen was rapidly consumed in the next 25 miles of river, falling to 28 


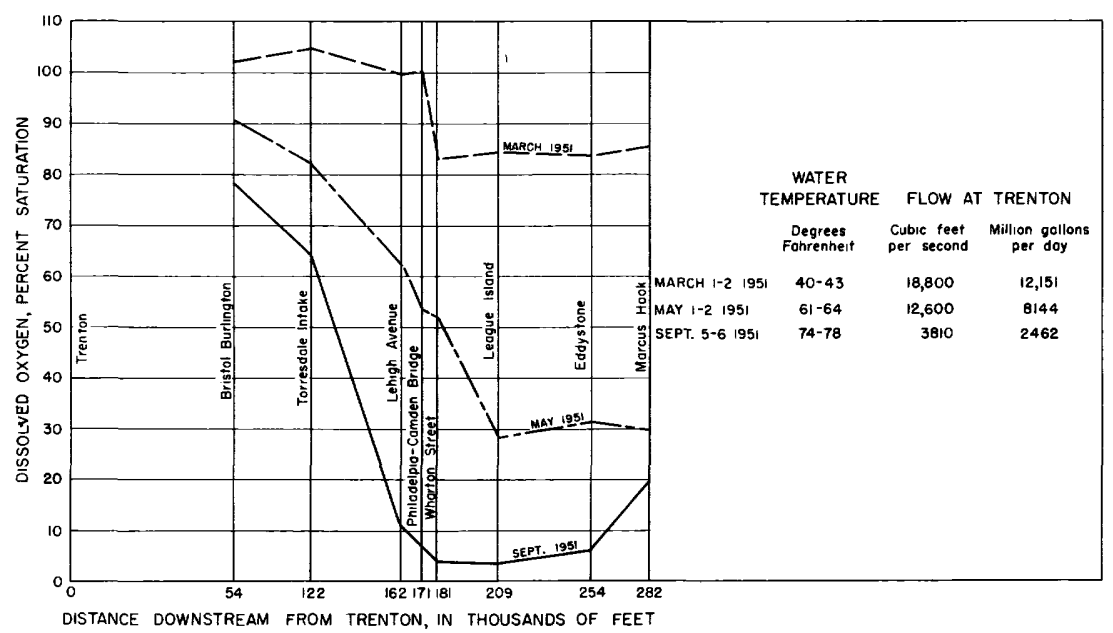

Figure 19. - Dissolved oxygen as percent saturation at sampling stations for selected days.

percent of saturation at League Island. In March when the flow rate was about 60 percent above the average, the river remained completely saturated from Bristol-Burlington to the PhiladelphiaCamden Bridge, but decreased to 83 percent of saturation in the 2 miles between the Philadelphia-Camden Bridge and Wharton Street, with little further change from there to Marcus Hook. In September when the flow rate was about one-third of the average, the dissolved oxygen content at $\mathrm{Bristol-Burlington}$ was 78 percent of saturation, falling to 11 percent at Lehigh Avenue and 4 percent at Wharton Street. From there some increase took place (19 percent saturation at Marcus Hook). In considering these data, it should be noted that as the temperature of the water increases, less oxygen is required to saturate the water 100 percent. For example, water at $41^{\circ} \mathrm{F}$ contains $12.8 \mathrm{ppm}$ or 107 pounds of dissolved oxygen per million gallons when saturated, whereas water at $76^{\circ} \mathrm{F}$ requires only $8.5 \mathrm{ppm}$ or 71 pounds per million gallons for 100 percent of saturation, because warmer water can dissolve less oxygen.

The New Jersey State Board of Health and the Pennsylvania Sanitary Water Board are attempting to restore to 50 percent of saturation the waters of the portion of the Delaware River from the Pennypack Creek (Torresdale Intake) to Marcus Hook. In table 4 are the average percentage saturation with oxygen and the percentage of samples which have less than 50 percentsaturation with oxygen for the calendar years 1950, 1951, and 1952 for this portion of the river. Examination of the data reveals that the average con- 
tent of dissolved oxygen has been increasing and the percentage of samples under 50 percent saturation has been decreasing for each of the three years. This apparent improvement in the river may be due to the more thorough treatment of wastes or to the increasing rate of flow of the river-especially from August to October-during the period covered by this report (see table 4). A greater volume of water flowing in the river would be expected to bring about an improvement both because of the greater quantity of oxygen available and because of increased dilution.

In figure 20 are plotted the once-a-month determinations of dissolved oxygen and biochemical oxygen demand for each of the eight sampling stations for the period August 1949 to December 1952. If the amount of dissolved oxygen exceeds the biochemical oxygen demand, sufficient oxygen is present to oxidize the organic and inorganic matter present in water. At all times during the period of record sufficient oxygen was present to satisfy the biochemical oxygen demand at $\mathrm{Bristol,} \mathrm{Pa}$. At all other stations there were occasions when the biochemical oxygen demand exceeded the dis solved oxygen. These periods of oxygen deficiency (illustrated by shaded areas on the graph) occurred between May and December.

Table 4. - Analyses of dissolved oxygen from sampling stations between Torresdale and Marcus Hook, $1950-52$, and the average monthly flow rate

\begin{tabular}{|c|c|c|c|}
\hline & $\begin{array}{c}\text { Jan, to Dec. } \\
1950\end{array}$ & $\begin{array}{c}\text { Jan, to Dec. } \\
1951\end{array}$ & $\begin{array}{c}\text { Jan. to Dec. } \\
1952 \\
\end{array}$ \\
\hline 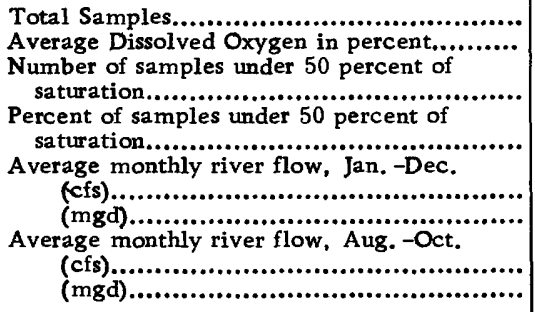 & $\begin{array}{r}82 \\
45.9 \\
40 \\
49 \\
13,397 \\
8,659 \\
3,842 \\
2,483\end{array}$ & $\begin{array}{r}77 \\
51.4 \\
36 \\
47 \\
15,190 \\
9,818 \\
5,345 \\
3,455\end{array}$ & $\begin{array}{r}84 \\
55.2 \\
27 \\
32 \\
17,557 \\
13,474 \\
6,662 \\
4,306\end{array}$ \\
\hline
\end{tabular}

There are several possible reasons for the oxygen deficiency in the warmer months of the year: (1) because the water is warmer, less oxygen is dissolved in it, (2) the chemical and biological reactions that consume oxygen proceed faster at the higher temper atures so that the oxygen is more rapidly consumed, and (3) in the late summer months less waterflows in the river and consequently the total oxygen carrying capacity is less. 


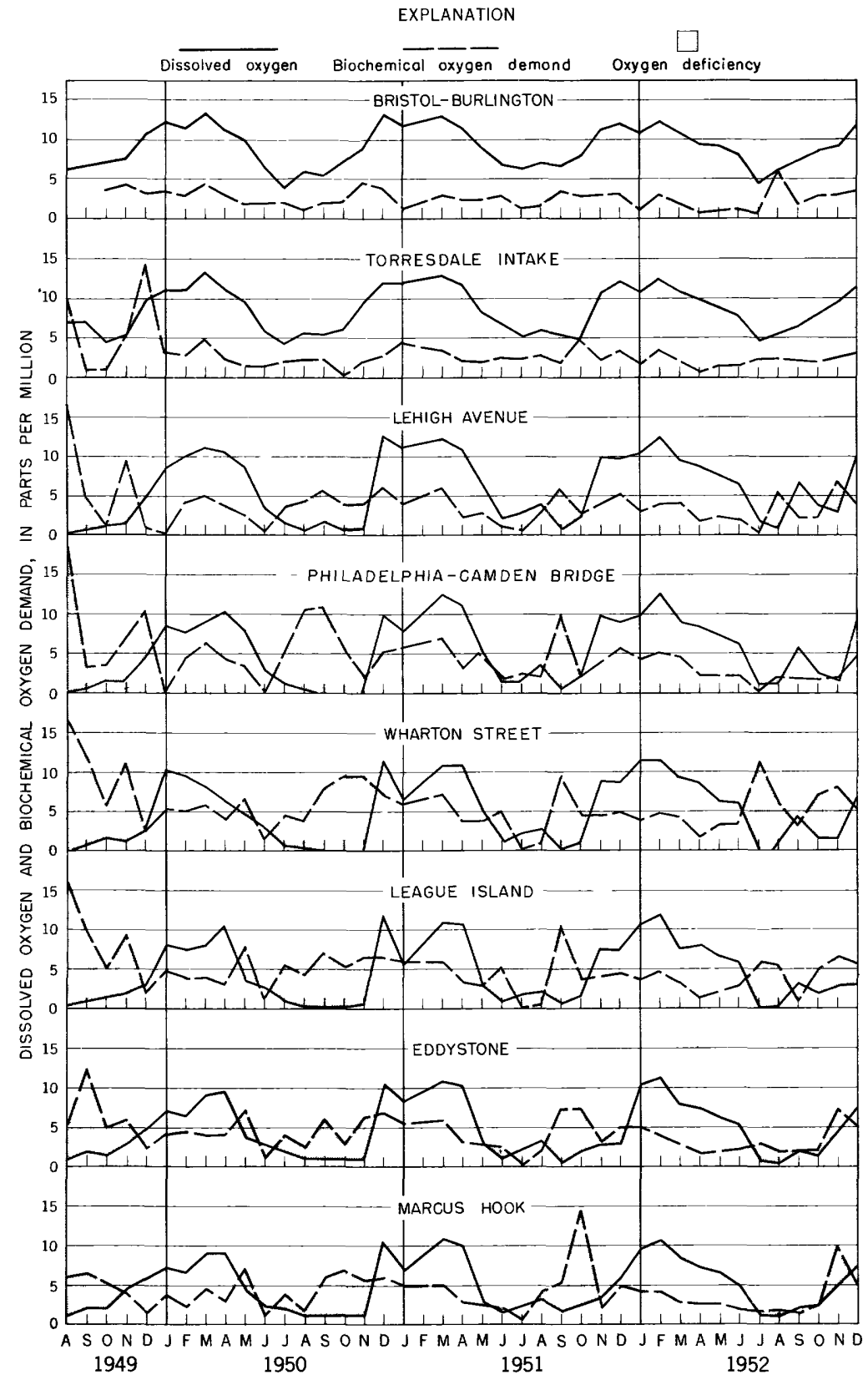

Figure 20. -Relation between dissolved oxygen and biochemical oxygen demand, August 1949 to December 1952. 


\section{SUSPENDED SEDIMENT}

By J. P. Eiler

The measurement of suspended-sediment discharge in the Delaware River at Trenton, was begun by the U. S. Geological Survey on September 1, 1949. The sampling station is located near the midpoint of the Calhoun Street Bridge, which connects Trenton, N. J., and Morrisville, $\mathrm{Pa}$. The measurement of suspendedsediment discharge is dependent on a continuous record of water discharge on which to base calculations of the amount of sediment in transport at all times. The streamflow recording station from which this continuous record of water discharge is obtained is located 200 feet upstream from the bridge.

The measurement of suspended-sediment discharge in the Schuylkill River, which joins the Delaware River at Philadelphia, was begun in the fall of 1947 as a part of the Schuylkill River Restoration Project of the Pennsylvania Department of Forests and Waters. The downstream station at Philadelphia, located near midpoint of the Green Lane Avenue bridge in the Manayunk section of Philadelphia, is currently being operated, and its record extends without break from November 16,1947 . The streamflow recording station is located approximately 5 miles downstream and just above Fairmount Dam.

The sediment-sampling equipment consists of a U. S. D-43 suspended-sediment sampler, which weighs 50 pounds; a reel for lowering and raising the sampler; and a housing to protect the sampler and reel. The samples are collected by a resident observer.

Each suspended-sediment sample represents only the concentration of sediment in the stream at the time of sampling and in the vertical in which the sample is taken. The concentration is, however, continuously varying with time in natural streams. If a continuous-concentration-time relation of reasonable accuracy is to be developed, samples must be taken at intervals frequent enough to define the pattern of variation of the concentration. If the streamflow is changing slowly, the sediment concentration also is likely to be changing slowly. Conversely, if the streamflow is changing rapidly, the sediment concentration usually is changing rapidly also, and frequent sampling will be required. The greatest rate of change of sediment concentration very often occurs at the beginning of a rise or shortly thereafter. Samples were collected once daily at the stations when the flow was uniform. During periods of rapidly changing flow samples were collected at intervals of 2 hours on the rise and 4 to 6 hours on the fall. 
It is recognized that a sample taken in a single vertical may not represent the average concentration in the cross section of the stream. In order to determine cross-sectional differences additional samples were collected at monthly intervals and at times of high flow at five or six verticals in the cross section, each sample representing an equal part of flow. The ratio of the average of these cross-section samples to the concentration obtained at the regular sampling station at the same time was used as a correction factor to apply to the resident observer's samples. The correction factor was applied on the basis of time or gage height, whichever controlled.

The sample concentrations are plotted against the time and a smooth-line curve is drawn through the plotted points following in general the configuration of the streamflow hydrograph. Meandaily values of sediment concentration are computed from this graph which together with values of daily water-discharge are used to compute the daily sediment loads for periods of fairly uniform flow and concentration. For periods of rapidly changing stage and concentration, daily sediment discharges were obtained by adding the discharges computed for subdivisions of the day.

Tables 16 and 17 are tabulations for the Delaware River at Trenton, N. J. and the Schuylkill River at Philadelphia, $\mathrm{Pa}$. of mean-daily water discharge in cubic feet per second and monthly totals of water discharge in cfs-days, mean-daily suspendedsediment concentration in parts per million, daily suspendedsediment load in tons per day, and monthly totals of suspended load in tons. Table 18 is a tabulation of particle-size analyses of selected suspended-sediment samples for these stations.

Composite suspended-sediment concentrations of five grabsamples taken at approximately 3 feet below the surface of the river at each of the eight monthly sampling stations between Bristol and Marcus Hook are given in tables 7-14. These concentrations are representative of only the surface water for this reach of the river and as approximations of the overall concentrations should be studied only in relation to each other.

There is a general downstream increase in the sediment concentration particularly at the Eddystone and Marcus Hook stations which are downstream from the junction of the Schuylkill River which carries a heavy load of sediment (table 17). Industrial and municipal wastes may have a cumulative downstream effect on concentration of suspended sediment also.

\section{SUMMARY}

The mineral content of the water in the Delaware River increases downstream from Trenton to Marcus Hook; from Trenton to Philadel - 
phia the increase is uniform and relatively slight at all rates of flow. During protracted periods of low rate of flow, salt water moves up the river along the river bottom and mixes partly with the river water as a result of currents arising from tidal action, river flow and navigation. Under such conditions of saline invasion the chloride content increases sharply at Eddystone and at Marcus Hook, and the composition of the downstream river water tends to approach the composition of the highly mineralized water of the Atlantic Ocean. Saline invasion is often accompanied by a higher concentration of dissolved solids near the bottom of the river than near the surface. Upstream and at higher flow rates

Table 5. - Maximum and minimum values in the specific conductance and chemical analyses of water of the Delaware River, Trenton, N. J. to Marcus Hook, Pa.

[Based on once-a-monthsampling August 1949 to December 1952]

\begin{tabular}{|c|c|c|c|c|c|c|c|c|c|}
\hline & \multicolumn{9}{|c|}{ Maximum values } \\
\hline & \multicolumn{9}{|c|}{ Station 1} \\
\hline & Trenton & 1 & 2 & 3 & 4 & 5 & 6 & 7 & 8 \\
\hline \multirow[t]{4}{*}{ 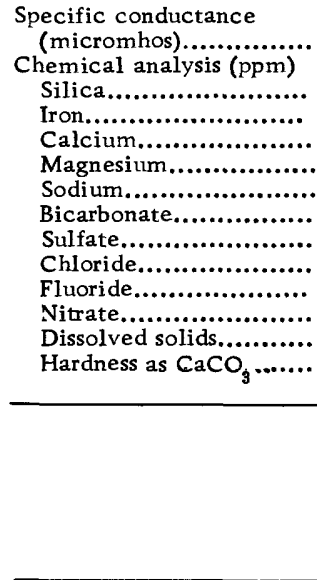 } & $\begin{array}{c}245 \\
9.7 \\
9.23 \\
25 \\
7.4 \\
\ldots \ldots \ldots . . \\
61 \\
31 \\
10 \\
.2 \\
4.4 \\
119 \\
83\end{array}$ & $\begin{array}{c}252 \\
7.3 \\
.28 \\
22 \\
8.9 \\
15 \\
63 \\
42 \\
14 \\
.2 \\
11^{\circ} \\
209 \\
89\end{array}$ & 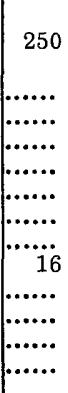 & 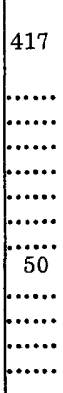 & $\begin{array}{c}442 \\
7.4 \\
2^{.3} \\
11 \\
38 \\
64 \\
64 \\
52 \\
18^{.3} \\
248 \\
105\end{array}$ & 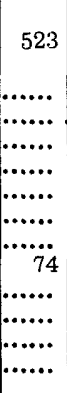 & 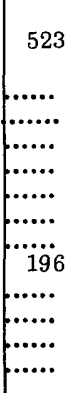 & 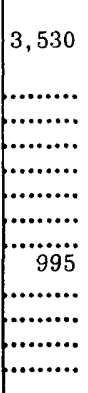 & $\begin{array}{c}6,230 \\
14 \\
.48 \\
75 \\
147 \\
1,080 \\
71 \\
382 \\
2,020 \\
1^{.9} .9 \\
4,150 \\
792\end{array}$ \\
\hline & \multicolumn{9}{|c|}{ Minimum values } \\
\hline & \multicolumn{9}{|c|}{ Station } \\
\hline & Trenton & 1 & 2 & 3 & 4 & 5 & 6 & 7 & 8 \\
\hline 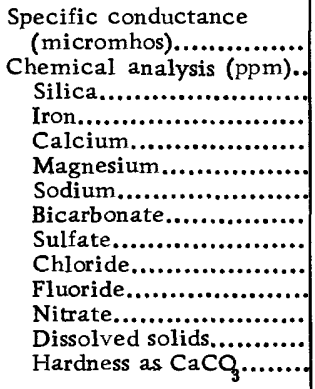 & $\begin{array}{c}1.9 \\
.01 \\
8.3 \\
2.6 \\
2.07 \\
15 \\
12 \\
2.0 \\
.0 \\
1.0 \\
53 \\
25\end{array}$ & $\begin{array}{c}1.4 \\
.00 \\
7.9 \\
2.6 \\
2.6 \\
11 \\
12 \\
1.0 \\
.0 \\
1.2 \\
52 \\
23\end{array}$ & 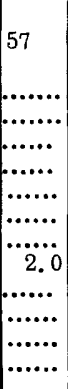 & 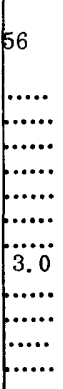 & $\begin{array}{l}58 \\
1.9 \\
.01 \\
8.0 \\
2.6 \\
2.3 \\
10 \\
13 \\
2.8 \\
.0 \\
.1 \\
22 \\
22\end{array}$ & 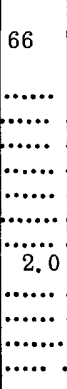 & 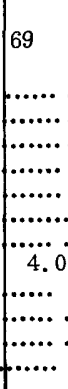 & 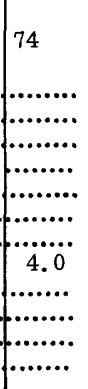 & $\begin{array}{c}82 \\
1.3 \\
.01 \\
10 \\
3.5 \\
22.4 \\
10 \\
18 \\
3.0 \\
.0 \\
.8 \\
73 \\
32\end{array}$ \\
\hline
\end{tabular}

1 Numbered stations are those located on figure 1.

2Less than this value. 
there is little difference in the composition of the water near the surface and near the bottom of the river. The conductivity of the water is usually slightly higher near the Pennsylvania shoreline than near the New Jersey side, indicating a higher mineral content in the water.

Table 5 shows the maximum and minimum concentrations of the chemical constituents of the river water during this investigation. In the Delaware River during normal flow conditions there is more calcium than magnesium and more sulfate than chloride in the water. These conditions are reversed downstream from Philadelphia when the flow is low and the ocean water mixes with the river water. During these periods the amount of magnesium dissolved is greater than the amount of calcium and the amount of chloride is greater than the amount of sulfate, as is the case with ocean water.

Saline invasion occurs only during the late summer months when the volume of water flowing downstream is low. Aside from this phenomenon the water in this reach of the river is of adequate quality, after treatment, to meet the needs of many municipalities and industries. During the period covered by this report the quality of the river water, from a sanitary viewpoint, has improved; the number of samples with a low content of dissolved oxygen has decreased and, toward the end of the period, except at League Island and Wharton Street, there were fewer samples having an oxygen deficiency. Oxygen deficiency at these two stations may occur less frequently after the city of Philadelphia Southwest Sewage Treatment Plant is placed in operation. The apparent improvement in sanitary quality may be due to the more thorough treatment of wastes as well as to the increasing flow rate of the river during the period covered by this report. A greater volume of water flowing in the river would be expected to bring about improved stream conditions both because of the greater quantity of oxygen available and because of dilution of wastes. The relative importance of these two factors can be determined only from an investigation covering a longer period of time.

The temperature of the water in the Delaware River is characterized by a seasonal variation. The maximum temperatures are reached in July and August and the minimum temperatures are reached in January and February. The observed maximum temperature of $88^{\circ} \mathrm{F}$ was recorded in July of 1949 and the minimum of $32^{\circ} \mathrm{F}$ in January 1952 . The temperature of the water from 1947-1952 was $75^{\circ} \mathrm{F}$ or more, 20 percent of the time. The temperature recorded at Trenton is generally within $2^{\circ} \mathrm{F}$ of the water temperature measured elsewhere downstream to Marcus Hook. Cross-sectional sampling indicates that there is little difference in temperature between the Pennsylvania shoreline and the New Jersey shoreline. The temperature of the water at the bottom of 
the river is generally within one degree of the temperature at the surface of the water.

There is a general downstream increase in the concentration of suspended sediment, particularly between League Island and Eddystone (table 17). Industrial and municipal wastes may have a cumulative downstream effect upon the sediment concentration of the Delaware River.

The amount of precipitation in the area above Trenton, the loss or gain of water from ground-water aquifers along the Delaware River between Trenton and Marcus Hook, the amount of water flowing in the Delaware River and its tributaries, the range and stage of tides, and the pollution load of the river are factors which influence the quality of the water. As the natural forces which cause variation in the quality of the water cannot be controlled it will be necessary to make further studies of the river data in order to predict quantitative changes in the quality of the water. The data obtained to date, although they do not supply the answer to many of the above problems, will be of value to industries, agencies, and consultants who have an interest in the quality of the water in the Delaware River between Trenton and Marcus Hook. 


\section{GLOSSARY}

Aquifer: a water-bearing geological stratum, a natural reservoir of ground water.

Biochemical oxygen demand, or B.O.D.: a measure of the amount of oxygen required to destroy organic wastes by bacterial action, thus it is a measure of the degree of pollution of stream water.

cfs, cubic feet per second: see discharge rate. The rate of flow in a stream whose channel is 1 square foot in cross section and whose average velocity is 1 foot per second is $1 \mathrm{cfs}$.

cfsm, cubic feet per second per square mile: the average number of cubic feet of flow per second from each square mile of area drained.

Discharge rate, or flow rate: the rate of flow of water in the stream, us ually expressed in cubic feet per second (cfs) or million gallons per day (mgd). A stream flowing at the rate of 1 cubic foot per second ( $1 \mathrm{cfs}$ ) discharges 646,317 gallons in a 24hour day. The volume of flow is of ten expressed in cfs-days. One cfs-day is the volume of water represented by a flow of a cubic foot of water per second for 24 hours.

Dissolved oxygen, or D.O.: the weight of oxygen actually dissolved in a sample of water, expressed in parts per million.

Drainage area, or catchment area: the area drained by a stream and its tributaries.

epm, equivalents per million: the number of chemical equivalents of a chemical substance per million parts by weight of solution. It is numerically equal to the milliequivalents per liter of water at $4^{\circ} \mathrm{C}$.

Fresh water: water of relatively low dissolved solids content. As used in this report it refers to water flowing down the Delaware River, or flowing into the river from its various tributaries, which contains no ocean water.

Ground water: the water derived from underground sources; it is tapped by springs, wells, shafts, or infiltration galleries.

Hardiness: the calcium carbonate $\left(\mathrm{CaCO}_{3}\right)$ equivalent of calcium and magnesium and of all other individually-determined cations having similar soap-consuming and incrusting properties. Hard water requires an excessive amount of soap to form a lather, and is the source of scale deposits in steam boilers. Hardness expressed as calcium carbonate is 50 times the sum of the number of equivalents of calcium and magnesium.

Hydrograph: a diagram in which the discharge rate of a stream is plotted as ordinate against the time as abscissa (see fig. 2). mgd, million gallons per day: a stream discharging $1 \mathrm{mgd}$ flows at a rate of $1.547 \mathrm{cfs}$.

$\mathrm{pH}$ : a measure of the acidity or alkalinity of water. $\mathrm{A} \mathrm{pH}$ of 7.0 is neutral, an acid solution has a $\mathrm{pH}$ lower than 7.0, while a basic or alkaline solution has a pH higher than 7.0. For example, a solution containing 4 pounds of sulfuric acid in 1, 000 gallons of water has a $\mathrm{pH}$ of 2 , while a solution of $3 \frac{1}{2}$ pounds of caustic soda in 1,000 gallons of water has a $\mathrm{pH}$ of 12 . 
ppm, parts per million: the number of parts by weight of a substance in a million parts by weight of water; the number of milligrams of the substance in 1,000 grams of water.

Saline invasion: the upstream movement of salty water from the ocean or bay, which results in the fresh water becoming saline. In the lower reaches of the Delaware River, as in other estuaries, a saline invasion is most likely to take place at the period of low streamflow (usually in late summer or fall).

Salinity: the degree of saltiness or content of natural salts in the water.

Sediment concentration: the ratio of weight of dry sediment to total weight of the water-sediment mixture, usually expressed in parts per million (ppm).

Sediment load or discharge: the total dry weight of sediment transported past a given point in a given time, usually expressed in tons per day.

Suspended sediment: the material moving in suspension in the water being kept from settling by the upward components of the turbulent currents or by colloidal suspension.

Vertical: an imaginary line extending from a point in a stream surface vertically downward to the bed of the stream.

Water year: a period of 12 months beginning October 1 , and ending September 30 , the following year. 


\section{METHODS OF ANALYSES}

Method

Temperature Read to nearest $1^{\circ} \mathrm{F}$, either with field thermometer or on the thermograph.

$\mathrm{pH}$ Electrometric with glass electrodes. Specific conductance. Wheatstone bridge; cell with platinum electrodes calibrated against standard potassium chloride at $25^{\circ} \mathrm{C}$.

Dissolved oxygen................Titration with sodium thiosulfate in presence of starch.

Biochemical oxygen demand.. Incubation at $20^{\circ} \mathrm{C}$ for 5 days.

Silica............................ Spectrophotometer measurement of color developed by addition of ammonium molybdate.

Iron August 1949 to May 6, 1952, spectrophotometer measurement of color developed by addition of thiocyanate in hydrochloric acid solution. May 6, 1952 to date, spectrophotometer measurement of the color developed by the addition of $2,2^{\prime}$ dipyridine solution.

Calcium.........................Calcium is precipitated as calcium oxalate. The precipitate is redissolved and the oxalate ion is titrated with potassium permanganate.

Magnesium.................... Determined gravimetrically as magnesium pyrophosphate.

Sodium......................... August 1949 to February 2, 1952, determined gravimetrically as the triple salt of sodium zinc uranylzinc acetate. February 2, 1952 to date of report, determined with flame photometer.

Bicarbonate..................... Titration with standard sulfuric acid in presence of methyl red indicator.

Sulfate..........................Determined gravimetrically as barium sulfate.

Chloride........................ Titrated with silver nitrate in the presence of potassium chromate.

Fluoride........................ Compared colorometrically with standards of zirconium nitratealizarin red solution. 
Nitrate.

Spectrophotometer measurement of the color developed by the addition of phenoldisulphonic acid and ammonium hydroxide.

Dissolved solids. Residue upon evaporation of solution and heating at $180^{\circ} \mathrm{F}$ for 1 hour in platinum dish.

Hardness........................Calculated from the equivalent calcium and magnesium.

Sediment concentration.......... Determined by evaporation or filtration.

Particle size..................... Determined by wet sieving or by the decantation method of sedimentary analysis or by a combination of these methods. In some analyses the bottom withdrawal tube method of sedimentary analysis was used in place of the decantation method. 


\section{SELECTED BIBLIOGRAPHY}

California State Water Pollution Control Board, 1952, Water-quality criteria: State Water Pollution Control Bd. Pub No. 3, 512 p.

Federal Security Agency, Public Health Service, 1951, North Atlantic drainage basins: Water Pollution Ser. No. 10, 137 p.

1952, North Atlantic drainage basins, Delaware River Basin - Zone 3: Water

Pollution Ser. No. 41, 69 p.

Graham, J. B., Mangan, J. W., and White, W. F., Jr., 1951, Water resources of southeastern Bucks County, Pa.: U. S. Geol. Survey Circ. 104, 21 p.

Interstate Commission on the Delaware River Basin, 1940, The Delaware River basin, physical facts, a graphic compendium of maps and charts.

Mason, W. D., and Pietsch, W. H., 1940, Salinity movement and its causes in the Delaware River estuary, Am. Geophys. Union Trans., v. 21, pt. 2, p. 457-463.

Pennsylvania Department of Commerce, 1947, Industrial utility of water in Pennsylvania, chemical character of surface water, 1944-46: State Planning Bd. Pub. 17, 172 p.

Pennsylvania Department of Health, 1935, Final report to the Sanitary Water Board by the Bureau of Engineering on the salinity survey of the Delaware River: mi meo. rept., 75 p., supp., app.

Pennsylvania Water Resources Council, 1952, Index of water-resources records in the Delaware River basin to September 30, 1951: U. S. Geol. Survey Circ. 190, 19 p.

Pennsylvania Water Supply Commission, 1917, Water-resources inventory report, part 3 , Gazetteer of streams, 657 p., 20 pls.

United States Geological Survey water-supply papers, issued annually, Surface-water supply of the United States, Pt. 1, North Atlantic slope basins.

United States Public Health Service, 1946, Drinking-water standards: Reprint 2697, p. 14.

United States Weather Bureau, 1950, Monthly meteorological summary, Philadelphia, Pa., J an.-Dec.

1950, Monthly meteorological summary, Trenton, N. J., Jan.-Dec.

Vermeule, C. C., 1894, Report on water supply, New Jersey: N. J. Geol. Survey, vol. 3 of final rept., State Geologist.

White, W. F., Jr., 1951, Chemical character of surface water in Pennsylvania, 1946-1949: Pennsylvania State Planning Board Pub. 23, 169 p.

White, W. F., Jr., and Lindholm, C. F., 1950, Water-resources investigations relating to the Schuylkill River Restoration Project: Pennsylvania Dept. of Forests and Waters, 125 p. 


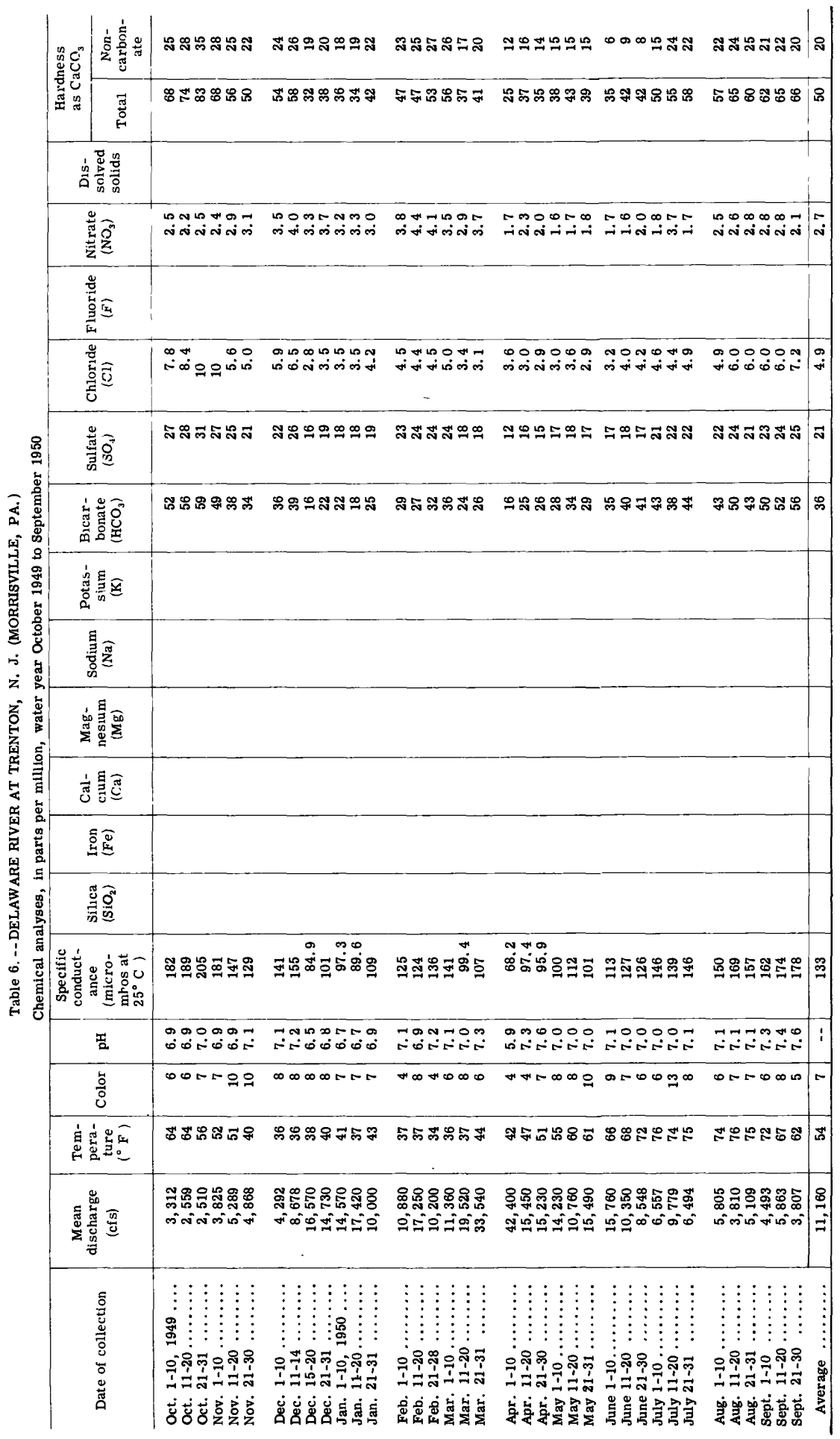




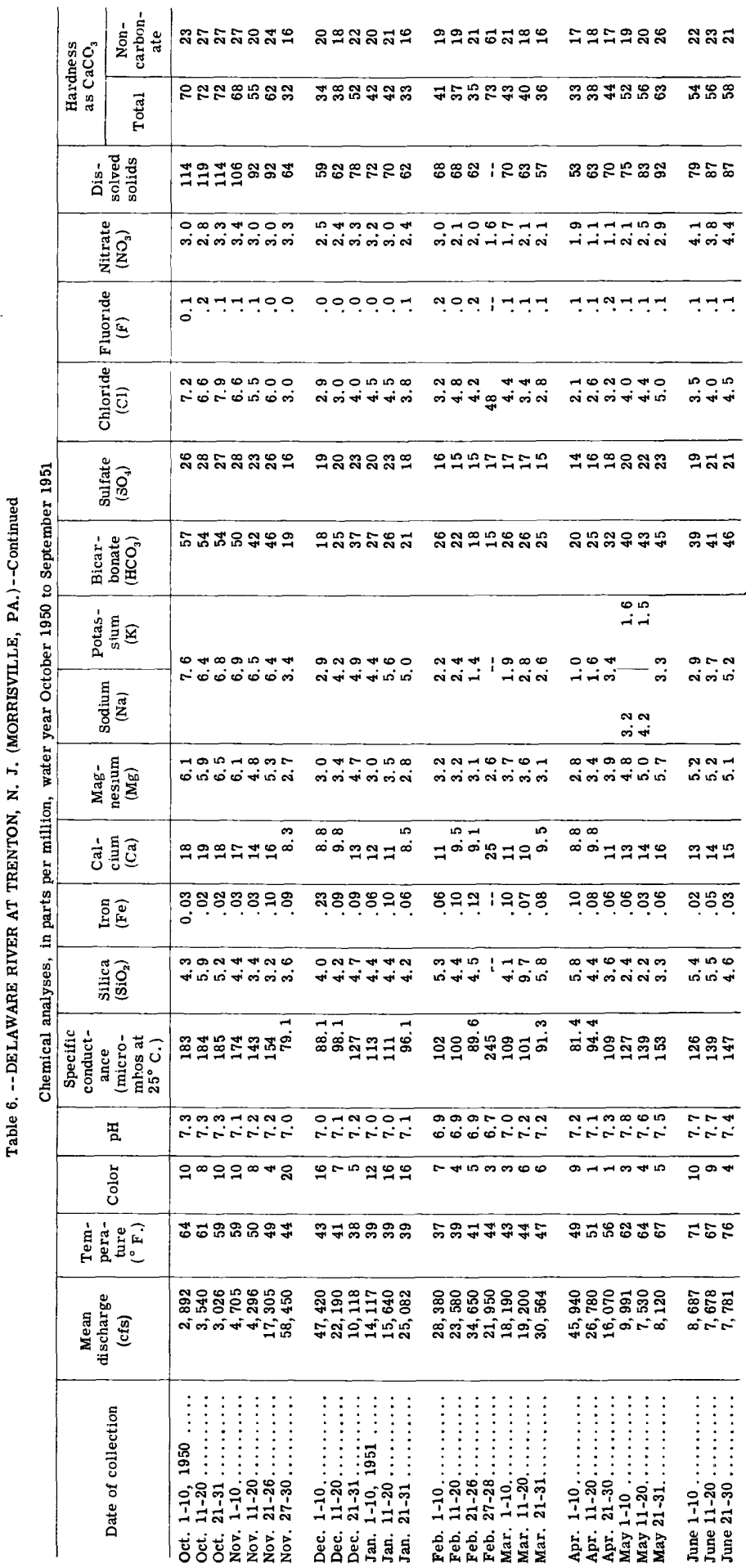




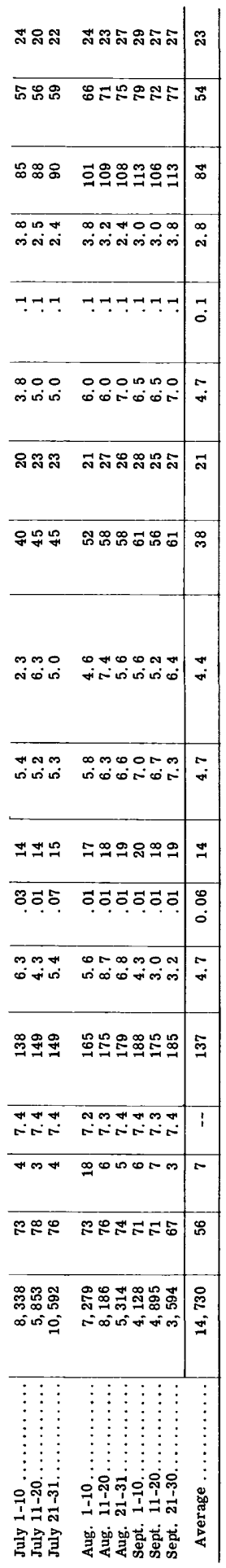




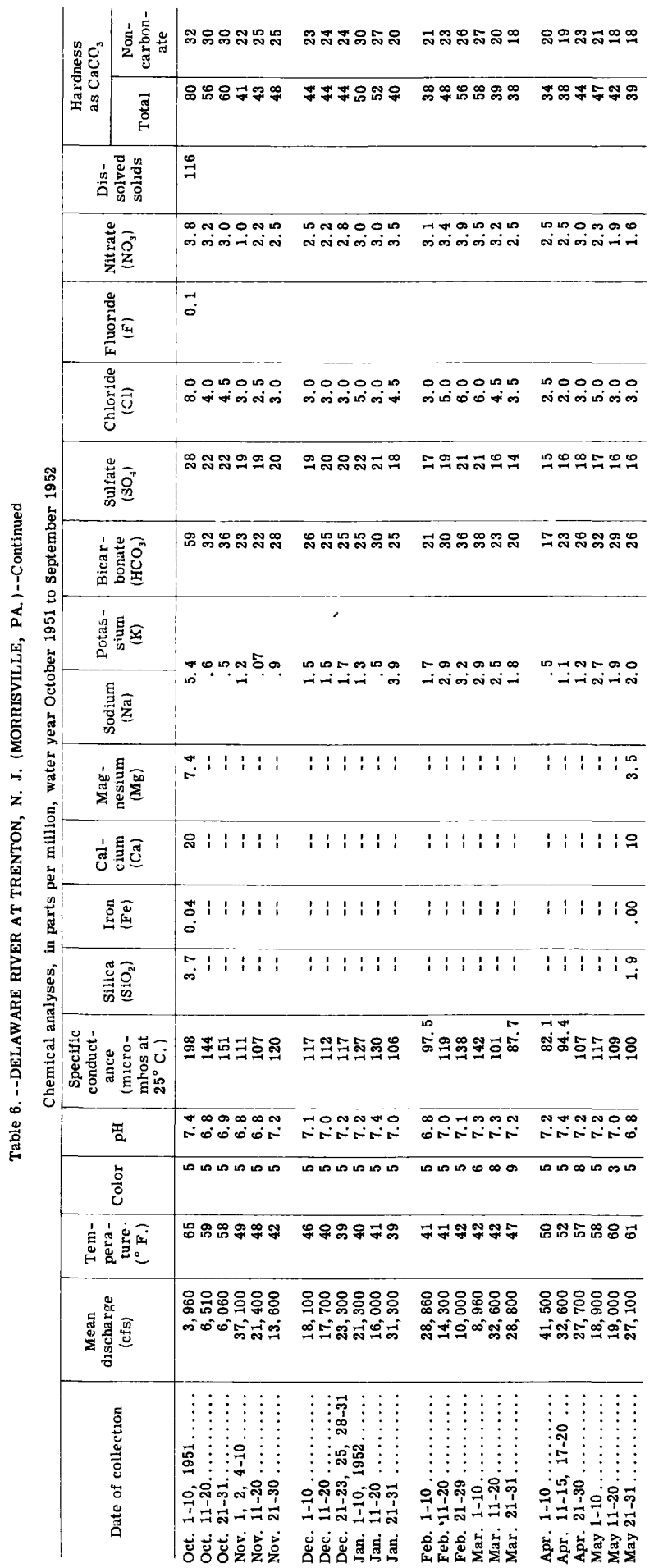




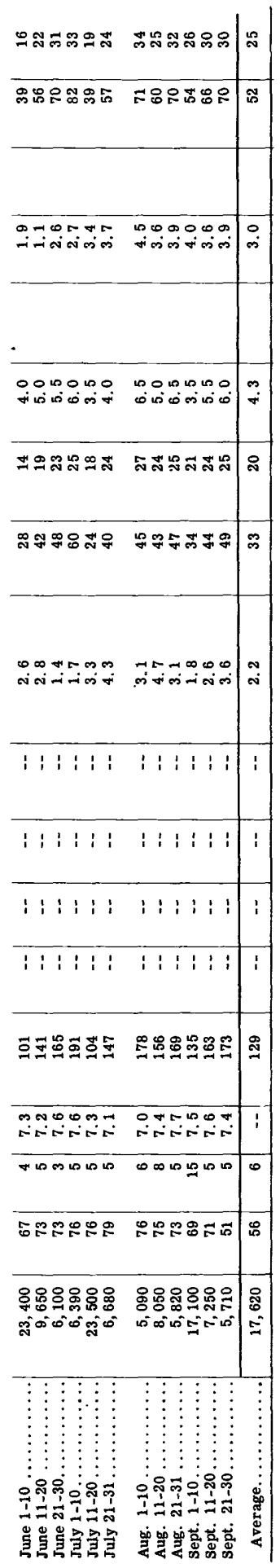


Table 7. -- WATER ANALYSES OF DELAWARE RIVER BETWEEN BRISTOL AND MARCUS HOOK, PA. Analyzed by City of Philadelphia and U. S. Geological Survey; analyses in parts per million

Location Bristol, Pa.-Eurlington, N. J. Bridge Date August 1, 1949 Sampling study No. 1 Weather Cloudy $\quad$ Water discharge at Trenton (cfs) - 3,350

\begin{tabular}{|c|c|c|c|c|c|c|}
\hline & & & & & & \\
\hline & \multicolumn{6}{|c|}{ Station } \\
\hline & $\begin{array}{c}\text { Pennsylvania } \\
\text { side }\end{array}$ & West Center & Center & East Center & \begin{tabular}{|c|}
$\begin{array}{c}\text { New Jersey } \\
\text { side }\end{array}$ \\
\end{tabular} & Average \\
\hline $\begin{array}{l}\text { Time (EST) } \\
\text { Sounding (ft) } \\
\text { Temperature }\left({ }^{\circ} \mathrm{F}\right)-\begin{array}{r}\text { Top } \\
\text { Bottom }\end{array} \\
\text { Color } \\
\text { pH }- \text { Bottom } \\
\text { Bottom }\end{array}$ & $\begin{array}{c}12: 30 \text { p.m. } \\
4 \\
86 \\
86 \\
10 \\
6.1 \\
6.4\end{array}$ & $\begin{array}{c}12: 10 \text { p.m. } \\
16 \\
86 \\
85 \\
8 \\
6.3 \\
6.3\end{array}$ & $\begin{array}{c}12: 00 \mathrm{n} . \\
27 \\
86 \\
85 \\
10 \\
6.2 \\
6.5\end{array}$ & $\begin{array}{c}11: 50 \text { a.m. } \\
35 \\
85 \\
85 \\
15 \\
6.3 \\
6.9\end{array}$ & $\begin{array}{c}11: 45 \text { a.m. } \\
8 \\
84 \\
84 \\
10 \\
6.2 \\
6.5\end{array}$ & $\begin{array}{ll}\text { T. } & 84 \\
\text { B. } & 85 \\
\text { B. } & 11 \\
\text { T. } & 6.2 \\
\text { B. } & 6.5\end{array}$ \\
\hline $\begin{array}{l}\begin{array}{l}\text { Specific conductance } \\
\left(\text { micrombos at } 25^{\circ} \mathrm{C}\right)\end{array} \\
\begin{array}{l}\text { Top } \\
\text { Bottom }\end{array} \\
\text { Dissolved oxygen -2- - Top } \\
\left.\text { B. O.D. (5-day, } 20^{\circ} \mathrm{C}\right)- \text { - Top }\end{array}$ & $\begin{array}{c}206 \\
204 \\
5.7 \\
-\end{array}$ & $\begin{array}{l}194 \\
193 \\
6.0 \\
-\end{array}$ & $\begin{array}{c}193 \\
192 \\
6.7 \\
-\end{array}$ & $\begin{array}{c}193 \\
190 \\
6.9 \\
-\end{array}$ & $\begin{array}{l}192 \\
190 \\
5.7 \\
-\end{array}$ & $\begin{array}{ll} & \\
\text { T. } & 196 \\
\text { B. } & 194 \\
\text { T. } & 6.2 \\
\text { T. } & --\end{array}$ \\
\hline 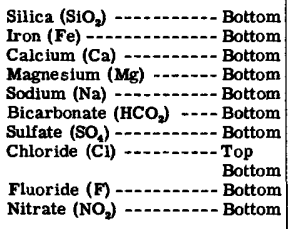 & $\begin{array}{l}3.4 \\
18 \\
6.07 \\
9.0 \\
4.0 \\
36 \\
10 \\
8.5 \\
.1 \\
6.1\end{array}$ & $\begin{array}{l}2.3 \\
.06 \\
18 \\
6.6 \\
8.1 \\
52 \\
32 \\
9.0 \\
7.1 \\
.1 \\
4.7\end{array}$ & $\begin{array}{l}2.4 \\
18.06 \\
6.6 \\
9.1 \\
52 \\
31 \\
8.0 \\
6.9 \\
.1 \\
4.8\end{array}$ & $\begin{array}{l}4.6 \\
19 \\
6.05 \\
7.0 \\
51 \\
31 \\
9.0 \\
7.0 \\
.2 \\
4.1\end{array}$ & $\begin{array}{l}2.9 \\
.07 \\
19 \\
6.4 \\
9.7 \\
53 \\
29 \\
8.0 \\
6.9 \\
.1 \\
4.4\end{array}$ & $\begin{array}{lc}\text { B. } & 3.1 \\
\text { B. } & .06 \\
\text { B. } & 18 \\
\text { B. } & 6.5 \\
\text { B. } & 8.6 \\
\text { B. } & 50 \\
\text { B. } & 32 \\
\text { T. } & 8.8 \\
\text { B. } & 7.3 \\
\text { B. } & .1 \\
\text { B. } & 4.8\end{array}$ \\
\hline $\begin{array}{l}\text { Dissolved solids }-\ldots-\text { Bottom } \\
\text { Hardness as } \mathrm{CaCO}_{3}-\cdots \text { Bottom } \\
\text { Suspended sediment -- Top }\end{array}$ & $\begin{array}{r}125 \\
72 \\
. \\
\end{array}$ & $\begin{array}{l}118 \\
72 \\
. \cdots\end{array}$ & $\begin{array}{l}120 \\
72 \\
. \quad C \text { Con }\end{array}$ & $\begin{array}{r}119 \\
74 \\
\text { ite. . }\end{array}$ & $\begin{array}{r}119 \\
74 \\
. . \\
\end{array}$ & $\begin{array}{lr}\text { B. } & 120 \\
\text { B. } & 73 \\
\text { T. } & \\
\end{array}$ \\
\hline
\end{tabular}

Location Bristol, Pa.-Burlington, N. J. Bridge Date September 6, 1949 Sampling study No. 2

\begin{tabular}{|c|c|c|c|c|c|c|}
\hline Weather Cloudy & & & Wa & $r$ discharge & Trenton (cfs) & 5,370 \\
\hline & & & Statior & & & \\
\hline & $\begin{array}{c}\text { Pennsylvania } \\
\text { side }\end{array}$ & West Center & Center & East Center & $\begin{array}{c}\text { New Jersey } \\
\text { side }\end{array}$ & Average \\
\hline 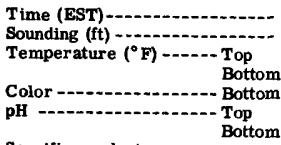 & $\begin{array}{l}- \\
15 \\
77 \\
77 \\
7 \\
6.7 \\
6.9\end{array}$ & $\begin{array}{l}-7 \\
78 \\
77 \\
8 \\
6.7 \\
6.9\end{array}$ & $\begin{array}{c}11: 00 \text { a.m. } \\
46 \\
78 \\
77 \\
10 \\
6.8 \\
7.3\end{array}$ & $\begin{array}{l}-\overline{40} \\
77 \\
76 \\
8 \\
6.8 \\
6.8\end{array}$ & $\begin{array}{c}-7 \\
77 \\
77 \\
5 \\
6.8 \\
7.2\end{array}$ & $\begin{array}{ll}\text { T. } & 77 \\
\text { B. } & 77 \\
\text { B. } & 8 \\
\text { T. } & 6.8 \\
\text { B. } & 7.0\end{array}$ \\
\hline $\begin{array}{l}\text { Specific conductance } \\
\quad\left(\text { micromhos at } 25^{\circ} \mathrm{C}\right) \\
\begin{array}{l}\text { Top } \\
\text { Bottom }\end{array} \\
\begin{array}{c}\text { Dissolved oxygen }-\cdots \\
\left.\text { B. O.D. (5-day, } 20^{\circ} \mathrm{C}\right)\end{array}-\text { Top } \\
\end{array}$ & $\begin{array}{r}231 \\
232 \\
6.3 \\
-\end{array}$ & $\begin{array}{r}229 \\
229 \\
6.6 \\
-\end{array}$ & $\begin{array}{l}224 \\
221 \\
6.8 \\
-\end{array}$ & $\begin{array}{l}224 \\
223 \\
6.6 \\
-\end{array}$ & $\begin{array}{l}224 \\
226 \\
6.6 \\
-\end{array}$ & $\begin{array}{l}\text { T. } 226 \\
\text { B. } 226 \\
\text { T. } 6.6 \\
\text { T. }\end{array}$ \\
\hline 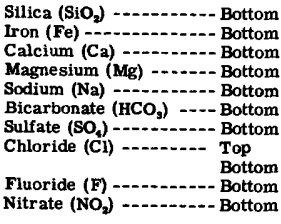 & $\begin{array}{l}2.1 \\
.10 \\
21 \\
8.1 \\
7.2 \\
56 \\
40 \\
11 \\
8.4 \\
.1 \\
6.0\end{array}$ & $\begin{array}{l}2.4 \\
.10 \\
21 \\
8.1 \\
8.7 \\
55 \\
38 \\
12 \\
10 \\
.1 \\
5.6\end{array}$ & $\begin{array}{l}2.7 \\
.06 \\
21 \\
8.0 \\
9.0 \\
56 \\
38 \\
10 \\
9.8 \\
.1 \\
5.3\end{array}$ & $\begin{array}{l}2.3 \\
22.06 \\
8.0 \\
10 \\
58 \\
38 \\
11 \\
9.9 \\
.1 \\
5.6\end{array}$ & $\begin{array}{l}1.8 \\
.06 \\
21 \\
8.1 \\
10 \\
60 \\
37 \\
11 \\
10 \\
.1 \\
5.8\end{array}$ & $\begin{array}{lc}\text { B. } & 2.3 \\
\text { B. } & .08 \\
\text { B. } & 21 \\
\text { B. } & 8.1 \\
\text { B. } & 9.0 \\
\text { B. } & 57 \\
\text { B. } & 38 \\
\text { T. } & 11 \\
\text { B. } & 9.6 \\
\text { B. } & .1 \\
\text { B. } & 5.7\end{array}$ \\
\hline 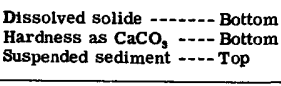 & $\begin{array}{r}142 \\
86 \\
\cdots \\
\end{array}$ & $\begin{array}{r}135 \\
86 \\
. \\
\end{array}$ & $\begin{array}{r}143 \\
85 \\
. \quad \text { Com }\end{array}$ & $\begin{array}{r}147 \\
88 \\
\text { ite . . }\end{array}$ & $\begin{array}{r}142 \\
86 \\
. \cdot .\end{array}$ & $\begin{array}{lr}\text { B. } & 147 \\
\text { B. } & 86 \\
\text { T. } & 27\end{array}$ \\
\hline
\end{tabular}


Table 7. --WATER ANALYSES OF DELAWARE RIVR BETWEEN BRISTOL AND MARCUS HOOK, PA.--Continued Analyzed by City of Philadelphia and U. S. Geologtcal Survey; analyses in parts per million

Location Bristol, Pa.-Burlington, N. J. Bridge Date October 4, 1949 Sampling study No. 3 Weather Cloudy $\quad$ Water discharge at Trenton (cfs) 3.520

\begin{tabular}{|c|c|c|c|c|c|c|}
\hline & \\
\hline & & & & & & \\
\hline & \begin{tabular}{|c} 
Pennsylvania \\
side
\end{tabular} & West Center & Center & East Center & \begin{tabular}{|c|c|c|}
$\begin{array}{c}\text { New Jersey } \\
\text { side }\end{array}$ \\
\end{tabular} & Average \\
\hline 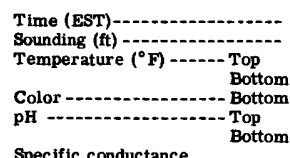 & $\begin{array}{l}\overrightarrow{15} \\
68 \\
65 \\
9 \\
7.1 \\
6.8\end{array}$ & $\begin{array}{l}-23 \\
68 \\
65 \\
8 \\
6.9 \\
6.9\end{array}$ & $\begin{array}{c}11: 00 \mathrm{s.m.} \\
46 \\
68 \\
66 \\
14 \\
6.9 \\
6.9\end{array}$ & $\begin{array}{l}-\overline{46} \\
68 \\
66 \\
10 \\
6.9 \\
7.2\end{array}$ & $\begin{array}{l}-18 \\
68 \\
68 \\
12 \\
6.8 \\
7.0\end{array}$ & $\begin{array}{ll}\text { T. } & 68 \\
\text { B. } & 66 \\
\text { B. } & 11 \\
\text { T. } & 6.9 \\
\text { B. } & 7.0\end{array}$ \\
\hline 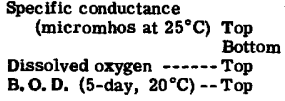 & $\begin{array}{r}229 \\
228 \\
6.8 \\
3.6\end{array}$ & $\begin{array}{r}226 \\
227 \\
7.5 \\
3.3\end{array}$ & $\begin{array}{l}221 \\
216 \\
7.3 \\
2.5\end{array}$ & $\begin{array}{l}221 \\
216 \\
7.0 \\
4.0\end{array}$ & $\begin{array}{r}217 \\
213 \\
7.0 \\
4.6\end{array}$ & $\begin{array}{ll}\text { T. } & 223 \\
\text { B. } & 220 \\
\text { T. } & 7.1 \\
\text { T. } & 3.6\end{array}$ \\
\hline 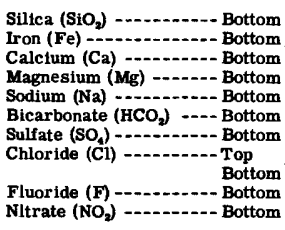 & $\begin{array}{l}2.2 \\
.06 \\
20 \\
7.6 \\
10^{.0} \\
52 \\
38 \\
14 \\
11 \\
5.1\end{array}$ & $\begin{array}{l}2.6 \\
19^{.05} \\
7.5 \\
11 \\
52 \\
37 \\
13 \\
11 \\
5.6\end{array}$ & $\begin{array}{l}2.4 \\
.10 \\
20 \\
7.5 \\
9.6 \\
53 \\
36 \\
12 \\
11 \\
5.5\end{array}$ & $\begin{array}{l}2.6 \\
20.10 \\
7.6 \\
10 \\
56 \\
36 \\
12 \\
10 \\
5.5\end{array}$ & $\begin{array}{l}3.0 \\
20 \\
7.08 \\
.7 \\
.5 \\
36 \\
12 \\
11 \\
5.6\end{array}$ & $\begin{array}{ll}\text { B. } & 2.6 \\
\text { B. } & .08 \\
\text { B. } & 20 \\
\text { B. } & 7.5 \\
\text { B. } & 10 \\
\text { B. } & 54 \\
\text { B. } & 37 \\
\text { T. } & 13 \\
\text { B. } & 11 \\
\text { B. } & .2 \\
\text { B. } & 5.6\end{array}$ \\
\hline $\begin{array}{l}\text { Dissolved solids - }-1-- \text { Bottom } \\
\text { Hardness as } \mathrm{CaCO}_{3}-\text { Bottom } \\
\text { Suspended sediment }-\cdots \text { Top }\end{array}$ & $\begin{array}{r}131 \\
81 \\
. \cdots\end{array}$ & $\begin{array}{c}129 \\
78 \\
\cdots \cdots\end{array}$ & $\begin{array}{r}126 \\
81 \\
. \quad \text { Comy } \\
\end{array}$ & $\begin{array}{r}126 \\
81 \\
\text { Lsite.... }\end{array}$ & $\begin{array}{r}126 \\
81 \\
. \cdots\end{array}$ & $\begin{array}{rr}\text { B. } & 128 \\
\text { B. } & 80 \\
\text { T. } & 33 \\
\end{array}$ \\
\hline
\end{tabular}
Location Bristol, P8.-Burlington, N. J. Bridge
Weather Date $\frac{\text { November I, } 1949}{\text { Cloudy }}$ Sampling study No. $\frac{4}{3,340}$ Water discharge at Trenton (cfs)

\begin{tabular}{|c|c|c|c|c|c|c|}
\hline & \\
\hline & \multicolumn{6}{|c|}{ Station } \\
\hline & \begin{tabular}{|c} 
Pennsylvania \\
slde
\end{tabular} & West Center & Center & East Center & $\begin{array}{c}\text { New Jersey } \\
\text { slds }\end{array}$ & Average \\
\hline 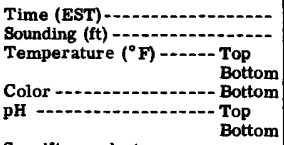 & $\begin{array}{l}- \\
\overline{57} \\
57 \\
6 \\
6.8 \\
7.8\end{array}$ & $\begin{array}{l}-7 \\
57 \\
57 \\
7 \\
6.8 \\
7.6\end{array}$ & $\begin{array}{c}11: 45 \mathrm{~s} . \mathrm{m} . \\
40 \\
54 \\
54 \\
8 \\
6.9 \\
7.8\end{array}$ & $\begin{array}{l}- \\
\overline{57} \\
55 \\
5 \\
6.9 \\
7.7\end{array}$ & $\begin{array}{l}-12 \\
57 \\
55 \\
8 \\
6.9 \\
7.6\end{array}$ & $\begin{array}{ll}\text { T. } & 56 \\
\text { B. } & 56 \\
\text { B. } & 7 \\
\text { T. } & 6.9 \\
\text { B. } & 7.7\end{array}$ \\
\hline $\begin{array}{l}\begin{array}{l}\text { Speciftc conductance } \\
\left.\text { (micrombos at } 25^{\circ} \mathrm{C}\right)\end{array} \\
\begin{array}{l}\text { Top } \\
\text { Bottom }\end{array} \\
\text { Dissolved oxygen --1 - - Top } \\
\text { B. O. D. (5-day, } 20^{\circ} \mathrm{C} \text { ) -- Top }\end{array}$ & $\begin{array}{l}216 \\
217 \\
7.3 \\
4.3\end{array}$ & $\begin{array}{l}215 \\
218 \\
7.3 \\
4.2\end{array}$ & $\begin{array}{l}213 \\
217 \\
7.3 \\
4.2\end{array}$ & $\begin{array}{l}212 \\
214 \\
7.6 \\
4.0\end{array}$ & $\begin{array}{l}210 \\
205 \\
7.3 \\
5.0\end{array}$ & $\begin{array}{lr}\text { T. } & 213 \\
\text { B. } & 214 \\
\text { T. } & 7.4 \\
\text { T. } & 4.3\end{array}$ \\
\hline 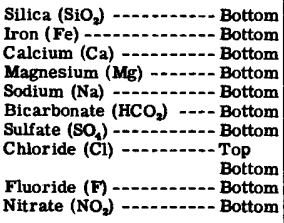 & $\begin{array}{l}1.4 \\
.03 \\
19 \\
7.3 \\
9.8 \\
53 \\
34 \\
12 \\
11 \\
. .2\end{array}$ & $\begin{array}{l}1.6 \\
18^{.03} \\
7.6 \\
10 \\
52 \\
35 \\
11 \\
11 \\
.2 \\
4.4\end{array}$ & $\begin{array}{l}1.6 \\
19 \\
7.04 \\
7.3 \\
9.4 \\
54 \\
34 \\
12 \\
.2 \\
4.2\end{array}$ & $\begin{array}{l}1.8 \\
.03 \\
19 \\
7.1 \\
9.8 \\
54 \\
34 \\
12 \\
10 \\
.2 \\
4.6\end{array}$ & $\begin{array}{l}1.5 \\
19 \\
7.03 \\
7.3 \\
9.3 \\
55 \\
33 \\
12 \\
10 \\
.02 \\
4.4\end{array}$ & $\begin{array}{ll}\text { B. } & 1.6 \\
\text { B. } & .03 \\
\text { B. } & 19 \\
\text { B. } & 7.3 \\
\text { B. } & 9.7 \\
\text { B. } & 54 \\
\text { B. } & 34 \\
\text { T. } & 12 \\
\text { B. } & 10 \\
\text { B. } & .2 \\
\text { B. } & 4.3\end{array}$ \\
\hline $\begin{array}{l}\text { Dissolved solids --:-- Bottom } \\
\text { Hardness as } \mathrm{CaCO}_{3}--- \text { Bottom } \\
\text { Suspended sediment }--- \text { Top }\end{array}$ & $\begin{array}{r}124 \\
77 \\
. . . . .\end{array}$ & $\begin{array}{r}124 \\
76 \\
i \cdot \cdots\end{array}$ & $\begin{array}{l}124 \\
77 \\
. \quad \text { Comp } \\
\end{array}$ & $\begin{array}{r}123 \\
77 \\
\text { site } . \cdots \\
\end{array}$ & $\begin{array}{l}123 \\
77 \\
\cdots\end{array}$ & $\begin{array}{lr}\text { B. } & 124 \\
\text { B. } & 77 \\
\text { T. } & 12 \\
\end{array}$ \\
\hline
\end{tabular}


Table 7. --WATER ANALYSES OF DELAWARE RIVER BETWEEN BRISTOL AND MARCUS HOOK, PA. --Continued Analyzed by City of Philadelphia and U. S. Geological Survey; analyses in parts per million

Location -Bristol, Pa.-Burlington, N. J. Bridge Date December 1, 1949 _Sampling study No. 5 Weather Clear $\quad$ Water discharge at Trenton (cfs) $-4,270$

\begin{tabular}{|c|c|c|c|c|c|c|}
\hline & \multicolumn{6}{|c|}{ Station } \\
\hline & \begin{tabular}{|c}
$\begin{array}{c}\text { Pennsylvania } \\
\text { side }\end{array}$ \\
\end{tabular} & West Center & Center & East Center & $\begin{array}{c}\text { New Jersey } \\
\text { side }\end{array}$ & Average \\
\hline 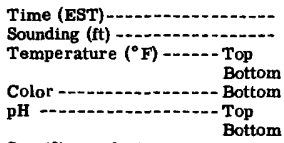 & $\begin{array}{l}15 \\
40 \\
40 \\
8 \\
6.7 \\
6.8\end{array}$ & $\begin{array}{l}\overline{21} \\
43 \\
41 \\
7 \\
6.8 \\
6.6\end{array}$ & $\begin{array}{c}10: 45 \mathrm{a} . \mathrm{m} . \\
21 \\
42 \\
42 \\
6 \\
6.7 \\
6.6\end{array}$ & $\begin{array}{l}- \\
29 \\
42 \\
42 \\
6 \\
6.8 \\
6.8\end{array}$ & $\begin{array}{l}\overline{10} \\
42 \\
42 \\
10 \\
7.1 \\
6.4\end{array}$ & $\begin{array}{ll}\text { T. } & 42 \\
\text { B. } & 41 \\
\text { B. } & 7 \\
\text { T. } & 6.8 \\
\text { B. } & 6.6\end{array}$ \\
\hline 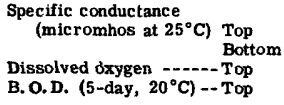 & $\begin{array}{r}145 \\
145 \\
11.0 \\
3.4\end{array}$ & $\begin{array}{r}145 \\
139 \\
10.7 \\
3.0\end{array}$ & $\begin{array}{r}140 \\
138 \\
11.0 \\
3.1\end{array}$ & $\begin{array}{r}139 \\
139 \\
10.5 \\
2.6\end{array}$ & $\begin{array}{r}141 \\
147 \\
10.3 \\
3.0\end{array}$ & $\begin{array}{lr} & \\
\text { T. } & 142 \\
\text { B. } & 142 \\
\text { T. } & 10.7 \\
\text { T. } & 3.0\end{array}$ \\
\hline 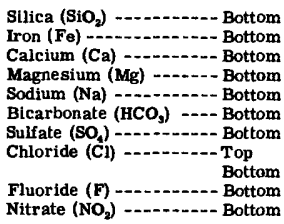 & $\begin{array}{l}3.2 \\
.08 \\
13 \\
5.3 \\
5.7 \\
31 \\
26 \\
7.0 \\
8.0 \\
.0 \\
3.7\end{array}$ & $\begin{array}{l}3.2 \\
.15 \\
13 \\
4.7 \\
5.2 \\
31 \\
24 \\
6.0 \\
7.0 \\
.0 \\
3.5\end{array}$ & $\begin{array}{l}2.6 \\
.14 \\
13 \\
4.5 \\
4.4 \\
30 \\
23 \\
6.0 \\
6.5 \\
.1 \\
3.2\end{array}$ & $\begin{array}{l}3.5 \\
.14 \\
13 \\
5.0 \\
3.9 \\
30 \\
24 \\
6.0 \\
6.5 \\
.0 \\
3.4\end{array}$ & $\begin{array}{l}4.7 \\
1.05 \\
5.1 \\
4.9 \\
36 \\
21 \\
6.0 \\
6.5 \\
.1 \\
1.2\end{array}$ & $\begin{array}{lc}\text { B. } & 3.4 \\
\text { B. } & .11 \\
\text { B. } & 13 \\
\text { B. } & 4.9 \\
\text { B. } & 4.8 \\
\text { B. } & 32 \\
\text { B. } & 24 \\
\text { T. } & 6.2 \\
\text { B. } & 6.9 \\
\text { B. } & .0 \\
\text { B. } & 3.0\end{array}$ \\
\hline $\begin{array}{l}\text { Dissolved solids - }-\cdots \text { Bottom } \\
\text { Hardness as } \mathrm{CaCO}_{3}-\cdots \text { Bottom } \\
\text { Suspended sediment }-\cdots \text { Top }\end{array}$ & $\begin{array}{l}83 \\
54 \\
. \\
\end{array}$ & $\begin{array}{c}80 \\
52 \\
. .\end{array}$ & $\begin{array}{l}80 \\
51 \\
. \mathrm{Co}\end{array}$ & $\begin{array}{r}82 \\
53 \\
\text { site. }\end{array}$ & $\begin{array}{l}87 \\
56 \\
.\end{array}$ & $\begin{array}{ll}\text { B. } & 82 \\
\text { B. } & 53 \\
\text { T. } & 17\end{array}$ \\
\hline
\end{tabular}

Location Bristo1, Pa.-Burlington, N. J. Bridge Date January 3, 1950 Sampling study No. 6 No. 6100 Weather_Cloud

\begin{tabular}{|c|c|c|c|c|c|c|}
\hline \multirow[b]{3}{*}{ 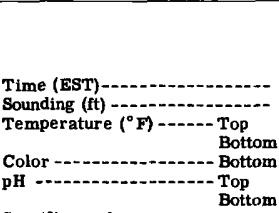 } & \multicolumn{6}{|c|}{ Station } \\
\hline & \begin{tabular}{|c}
$\begin{array}{c}\text { Pennsylvania } \\
\text { side }\end{array}$ \\
\end{tabular} & West Center & Center & East Center & \begin{tabular}{|c|}
$\begin{array}{c}\text { New Jersey } \\
\text { side }\end{array}$ \\
\end{tabular} & Average \\
\hline & $\begin{array}{c}11: \frac{12}{13} \mathrm{a}, \mathrm{m} . \\
- \\
\overline{10} \\
6.6 \\
6.8\end{array}$ & $\begin{array}{c}11: 10 \text { a.m. } \\
8 \\
- \\
11 \\
6.5 \\
6.8\end{array}$ & $\begin{array}{c}11: 06 \text { a.m. } \\
35 \\
- \\
\overrightarrow{12} \\
6.5 \\
6.9\end{array}$ & $\begin{array}{c}11: 03 \text { a.m. } \\
35 \\
- \\
- \\
10 \\
6.5 \\
7.1\end{array}$ & $\begin{array}{c}11: 00 \mathrm{a.m} . \\
11 \\
- \\
- \\
9 \\
6.5 \\
7.0\end{array}$ & $\begin{array}{ll}\text { T. } & - \\
\text { B. } & \overline{10} \\
\text { B. } & 6.5 \\
\text { T. } & 6.9 \\
\text { B. } & 6.9\end{array}$ \\
\hline $\begin{array}{l}\text { Specific conductance } \\
\quad \text { (micromhos at } 25^{\circ} \mathrm{C} \text { ) } \\
\begin{array}{l}\text { Top } \\
\text { Bottom }\end{array} \\
\text { Dissolved oxygen }-10^{\circ} \mathrm{C} \text { - Top } \\
\text { B. O.D. (5-day, } 20^{\circ} \mathrm{Cop}\end{array}$ & $\begin{array}{r}96.4 \\
94.9 \\
12.2 \\
3.8\end{array}$ & $\begin{array}{r}97.6 \\
102 \\
12.3 \\
3.6\end{array}$ & $\begin{array}{r}98.5 \\
94.9 \\
12.5 \\
3.6\end{array}$ & $\begin{array}{r}97.6 \\
95.2 \\
12.2 \\
3.8\end{array}$ & $\begin{array}{r}99.4 \\
97.0 \\
11.9 \\
2.4\end{array}$ & $\begin{array}{lr}\text { T. } & 97.9 \\
\text { B. } & 96.8 \\
\text { T. } & 12.2 \\
\text { T. } & 3.4\end{array}$ \\
\hline 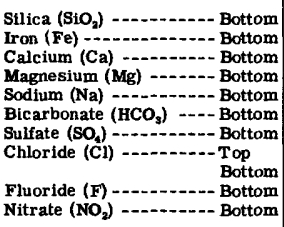 & $\begin{array}{l}5.0 \\
.06 \\
9.6 \\
3.6 \\
2.4 \\
18 \\
20 \\
5.0 \\
3.5 \\
.0 \\
3.3\end{array}$ & $\begin{array}{l}5.2 \\
.12 \\
10 \\
3.9 \\
1.6 \\
19 \\
21 \\
5.0 \\
3.2 \\
.1 \\
5.2\end{array}$ & $\begin{array}{l}4.8 \\
.05 \\
9.7 \\
3.7 \\
1.3 \\
18 \\
19 \\
5.0 \\
3.5 \\
.0 \\
3.4\end{array}$ & $\begin{array}{l}4.8 \\
.05 \\
10 \\
3.6 \\
1.2 \\
19 \\
19 \\
5.0 \\
3.0 \\
.0 \\
3.4\end{array}$ & $\begin{array}{l}5.1 \\
.11 \\
9.9 \\
4.1 \\
1.1 \\
18 \\
20 \\
6.0 \\
3.5 \\
.0 \\
3.9\end{array}$ & $\begin{array}{ll}\text { B. } & 5.0 \\
\text { B. } & .08 \\
\text { B. } & 9.8 \\
\text { B. } & 3.8 \\
\text { B. } & 1.5 \\
\text { B. } & 18 \\
\text { B. } & 20 \\
\text { T. } & 5.2 \\
\text { B. } & 3.3 \\
\text { B. } & .0 \\
\text { B. } & 3.8\end{array}$ \\
\hline $\begin{array}{l}\text { Dissolved solids } \ldots \text {....- Bottom } \\
\text { Hardness as } \mathrm{CaCO}_{3},-. \text { Bottom } \\
\text { Suspended sediment }-\cdots \text { Top }\end{array}$ & $\begin{array}{l}61 \\
39 \\
.\end{array}$ & $\begin{array}{l}68 \\
41 \\
\cdots\end{array}$ & $\begin{array}{l}63 \\
39 \\
.\end{array}$ & $\begin{array}{r}63 \\
40 \\
\end{array}$ & $\begin{array}{l}64 \\
42 \\
\cdots\end{array}$ & $\begin{array}{ll}\text { B. } & 64 \\
\text { B. } & 40 \\
\text { T. } & 16\end{array}$ \\
\hline
\end{tabular}


Table 7. -- WATER ANALYSES OF DELAWARE RIVER BETWEEN BRISTOL AND MARCUS HOOK, PA. --Continued Analyzed by City of Philadelphia and U. S. Geological Survey; analyses in parts per million

Location Bristol, Pa.-Burlingtion, N. L._Bridge Date February 2, 1950 Sampling study No. 7 Weather Cloudy

\begin{tabular}{|c|c|c|c|c|c|c|}
\hline \multirow[b]{3}{*}{$\begin{array}{l}\text { Time (EST) } \\
\text { Sounding (ft) }-10 \\
\text { Temperature }\left({ }^{\circ} \text { F) }-1\right. \\
\text { Color } \\
\text { pH } \begin{array}{c}\text { Top } \\
\text { Bottom }\end{array} \\
\end{array}$} & \multicolumn{6}{|c|}{ Station } \\
\hline & \begin{tabular}{|c|} 
Pennsylvania \\
side
\end{tabular} & West Center & Center & East Center & \begin{tabular}{|c|}
$\begin{array}{c}\text { New Jersey } \\
\text { side }\end{array}$ \\
\end{tabular} & Average \\
\hline & $\begin{array}{c}11: 51 \text { a.m. } \\
8 \\
40 \\
39 \\
18 \\
6.9 \\
6.8\end{array}$ & $\begin{array}{c}11: 47 \text { a.m. } \\
16 \\
40 \\
39 \\
17 \\
6.9 \\
6.9\end{array}$ & $\begin{array}{c}11: 44 \text { a.m. } \\
28 \\
40 \\
39 \\
18 \\
6.9 \\
7.0\end{array}$ & $\begin{array}{c}11: 36 \text { a.m. } \\
48 \\
40 \\
38 \\
19 \\
6.9 \\
7.1\end{array}$ & $\begin{array}{c}11: 32 \text { a.m. } \\
38 \\
40 \\
40 \\
28 \\
6.9 \\
6.8\end{array}$ & $\begin{array}{lc}\text { T. } & 40 \\
\text { B. } & 39 \\
\text { B. } & 20 \\
\text { T. } & 6.9 \\
\text { B. } & 6.9\end{array}$ \\
\hline $\begin{array}{l}\begin{array}{l}\text { Specific conductance } \\
\text { (micromhos at } 25^{\circ} \mathrm{C} \text { ) }\end{array} \\
\begin{array}{l}\text { Top } \\
\text { Bottom }\end{array} \\
\text { Dissolved oxygen - - - Top } \\
\text { B. O. D. (5-day, } 20^{\circ} \mathrm{C} \text { ) - Top }\end{array}$ & $\begin{array}{l}123 \\
125 \\
11.3 \\
3.8\end{array}$ & $\begin{array}{l}117 \\
122 \\
11.5 \\
3.0\end{array}$ & $\begin{array}{l}113 \\
115 \\
11.7 \\
2.8\end{array}$ & $\begin{array}{l}112 \\
114 \\
11.5 \\
2.8\end{array}$ & $\begin{array}{l}113 \\
115 \\
11.0 \\
2.2\end{array}$ & $\begin{array}{l}\text { T. } 116 \\
\text { B. } 118 \\
\text { T. } 11.4 \\
\text { T. } 2.9\end{array}$ \\
\hline 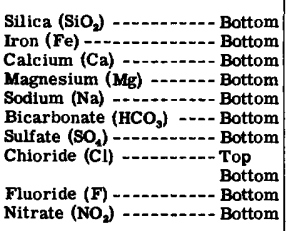 & $\begin{array}{l}5.2 \\
11.14 \\
4.0 \\
7.9 \\
27 \\
26 \\
6.0 \\
6.0 \\
.2 \\
3.7\end{array}$ & $\begin{array}{l}5.1 \\
11.14 \\
3.9 \\
5.1 \\
23 \\
24 \\
4.0 \\
5.2 \\
.1 \\
4.0\end{array}$ & $\begin{array}{l}5.6 \\
11.13 \\
3.8 \\
5.7 \\
26 \\
23 \\
4.0 \\
4.5 \\
.1 \\
4.5\end{array}$ & $\begin{array}{l}5.9 \\
.13 \\
11 \\
3.9 \\
6.1 \\
29 \\
23 \\
4.0 \\
4.2 \\
.1 \\
3.6\end{array}$ & $\begin{array}{l}5.1 \\
11.23 \\
3.8 \\
5.6 \\
25 \\
24 \\
4.0 \\
4.5 \\
.1 \\
3.8\end{array}$ & $\begin{array}{lc}\text { B. } & 5.4 \\
\text { B. } & .15 \\
\text { B. } & 11 \\
\text { B. } & 3.9 \\
\text { B. } & 6.1 \\
\text { B. } & 26 \\
\text { B. } & 24 \\
\text { T. } & 4.4 \\
\text { B. } & 4.9 \\
\text { B. } & .1 \\
\text { B. } & 3.9\end{array}$ \\
\hline 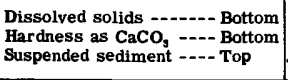 & $\begin{array}{l}84 \\
44 \\
. \quad\end{array}$ & $\begin{array}{l}81 \\
44 \\
\cdots\end{array}$ & $\begin{array}{l}81 \\
43 \\
.\end{array}$ & $\begin{array}{r}79 \\
44 \\
\text { ite. }\end{array}$ & $\begin{array}{l}79 \\
43 \\
\cdots\end{array}$ & $\begin{array}{ll}\text { B. } & 81 \\
\text { B. } & 44 \\
\text { T. } & 13\end{array}$ \\
\hline
\end{tabular}

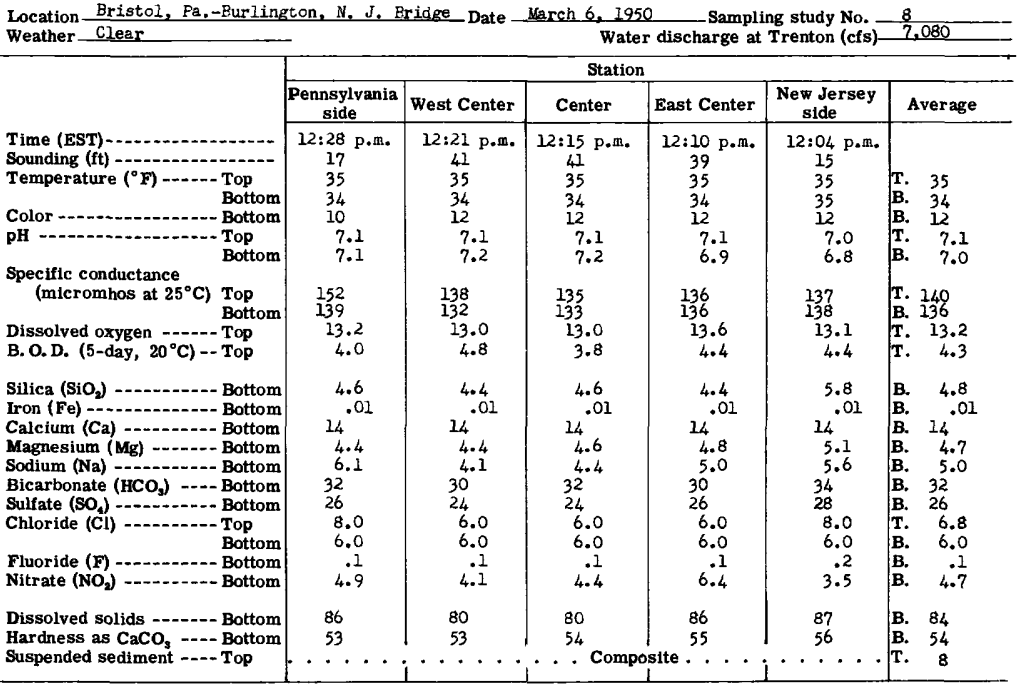


Table 7. -- WATER ANAL YSES OF DELAWARE RIVER BETWEEN BRISTOL AND MARCUS HOOK, PA. --Continued Analyzed by City of Philadelphia and U. S. Geological Survey; analyses in parts per million

\begin{tabular}{|c|c|c|c|c|c|c|}
\hline \multicolumn{7}{|c|}{$\begin{array}{l}\text { Location Bristol, Pa.-Burlington, N. J. Bridge Date } \frac{\text { April }}{\text { 4, } 1950} \text { Water discharge at Trenton (cfs) } \\
\text { Weather Clear }\end{array}$} \\
\hline & \multicolumn{6}{|c|}{ Station } \\
\hline & $\begin{array}{c}\text { Pennsylvania } \\
\text { side }\end{array}$ & West Center & Center & East Center & $\begin{array}{l}\text { New Jersey } \\
\text { side }\end{array}$ & Average \\
\hline $\begin{array}{l}\text { Time (EST) } \\
\text { Sounding (ft) } \\
\left.\text { Temperature ( }{ }^{\circ} \mathrm{F}\right)-\begin{array}{l}\text { Bop } \\
\text { Bottom }\end{array} \\
\text { Color } \\
\text { pH }\end{array}$ & $\begin{array}{c}11: 50 \mathrm{a.m.} \\
10 \\
49 \\
47 \\
8 \\
6.8 \\
6.8\end{array}$ & $\begin{array}{c}11: 45 \text { a.m. } \\
20 \\
48 \\
46 \\
8 \\
6.9 \\
7.0\end{array}$ & $\begin{array}{c}11: 40 \mathrm{a} . \mathrm{m} . \\
30 \\
47 \\
47 \\
6 \\
6.9 \\
7.0\end{array}$ & $\begin{array}{c}11: 35 \mathrm{a} . \mathrm{m} . \\
30 \\
47 \\
47 \\
7 \\
6.9 \\
7.0\end{array}$ & $\begin{array}{c}11.25 \mathrm{a} . \mathrm{m} . \\
15 \\
48 \\
47 \\
6 \\
6.8 \\
7.0\end{array}$ & $\begin{array}{ll}\text { T. } & 48 \\
\text { B. } & 47 \\
\text { B. } & 7 \\
\text { T. } & 6.9 \\
\text { B. } & 7.0\end{array}$ \\
\hline $\begin{array}{l}\text { Specific conductance } \\
\quad \text { (micromhos at } 25^{\circ} \mathrm{C} \text { ) } \\
\begin{array}{l}\text { Top } \\
\text { Bottom }\end{array} \\
\text { Dissolved oxygen }-10^{\circ} \mathrm{C} \text { ) Top } \\
\text { B. O.D. (5-day, } 20^{\circ} \mathrm{Top}\end{array}$ & $\begin{array}{r}81.4 \\
83.9 \\
10.9 \\
4.8\end{array}$ & $\begin{array}{r}74.7 \\
74.9 \\
11.3 \\
2.8\end{array}$ & $\begin{array}{r}73.3 \\
76.0 \\
11.2 \\
2.6\end{array}$ & $\begin{array}{r}73.5 \\
76.3 \\
11.1 \\
2.6\end{array}$ & $\begin{array}{r}77.9 \\
78.9 \\
10.7 \\
2.1\end{array}$ & $\begin{array}{lr}\text { T. } & 76.2 \\
\text { B. } & 78.0 \\
\text { T. } & 11.0 \\
\text { T. } & 3.0\end{array}$ \\
\hline 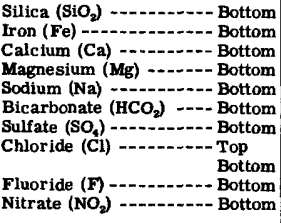 & $\begin{array}{l}4.0 \\
.06 \\
8.2 \\
3.0 \\
3.5 \\
16 \\
19 \\
3.0 \\
3.5 \\
.1 \\
3.0\end{array}$ & $\begin{array}{l}4.1 \\
.05 \\
8.4 \\
2.7 \\
3.7 \\
19 \\
16 \\
2.8 \\
3.5 \\
.1 \\
2.4\end{array}$ & $\begin{array}{l}4.1 \\
.02 \\
8.0 \\
2.9 \\
2.9 \\
19 \\
16 \\
2.8 \\
3.2 \\
.1 \\
1.4\end{array}$ & $\begin{array}{l}4.1 \\
.09 \\
8.0 \\
2.8 \\
3.0 \\
18 \\
15 \\
2.5 \\
3.8 \\
.1 \\
.2 .6\end{array}$ & $\begin{array}{l}4.3 \\
.05 \\
7.9 \\
3.0 \\
4.2 \\
19 \\
17 \\
2.8 \\
3.8 \\
.1 \\
2.8\end{array}$ & $\begin{array}{ll}\text { B. } & 4.1 \\
\text { B. } & .05 \\
\text { B. } & 8.1 \\
\text { B. } & 2.9 \\
\text { B. } & 3.5 \\
\text { B. } & 18 \\
\text { B. } & 17 \\
\text { T. } & 2.8 \\
\text { B. } & 3.6 \\
\text { B. } & .1 \\
\text { B. } & 2.4\end{array}$ \\
\hline $\begin{array}{l}\text { Dissolved solids - } \\
\text { Hardness as } \mathrm{CaCO}_{3}--- \text { Bottom } \\
\text { Suspended sediment } \ldots \text { Top }\end{array}$ & $\begin{array}{l}58 \\
33 \\
.\end{array}$ & $\begin{array}{l}57 \\
32\end{array}$ & $\begin{array}{l}57 \\
32 \\
. \mathrm{C}\end{array}$ & $\begin{array}{l}55 \\
31\end{array}$ & $\begin{array}{r}56 \\
32 \\
\cdots\end{array}$ & $\begin{array}{ll}\text { B. } & 57 \\
\text { B. } & 32 \\
\text { T. } & 18\end{array}$ \\
\hline
\end{tabular}

Location Bristol, Pa,-Burlington, N. J. Bridge Date Nay 1, 1950 Sampling study No. 10

\begin{tabular}{|c|c|c|c|c|c|c|}
\hline ocation CIoudy & & & $w_{a}$ & lischarge & $\begin{array}{l}\text { study No. } \\
\text { renton (cfs) }\end{array}$ & 2.300 \\
\hline & & & Station & & & \\
\hline & \begin{tabular}{|c|}
$\begin{array}{c}\text { Pennsylvania } \\
\text { side }\end{array}$ \\
\end{tabular} & West Center & Center & East Center & $\begin{array}{c}\begin{array}{c}\text { New Jersey } \\
\text { side }\end{array} \\
\end{array}$ & Average \\
\hline $\begin{array}{l}\text { Time (EST) } \\
\text { Sounding (ft) } \\
\text { Temperature }\left({ }^{\circ} \mathrm{F}\right)-\text { Top } \\
\text { Color } \\
\text { pH }\end{array}$ & $\begin{array}{c}11: 35 \mathrm{a} . \mathrm{m} . \\
10 \\
53 \\
52 \\
5 \\
6.9 \\
6.8\end{array}$ & $\begin{array}{c}11: 30 \text { a.m. } \\
19 \\
52 \\
52 \\
6 \\
6.9 \\
7.0\end{array}$ & $\begin{array}{c}11: 26 \text { a.m. } \\
27 \\
54 \\
52 \\
5 \\
7.1 \\
7.1\end{array}$ & $\begin{array}{c}11: 22 \text { a.m. } \\
46 \\
54 \\
52 \\
5 \\
7.1 \\
7.0\end{array}$ & $\begin{array}{c}11: 15 \mathrm{a} . \mathrm{m} . \\
14 \\
54 \\
54 \\
5 \\
7.1 \\
6.6\end{array}$ & $\begin{array}{ll}\text { T. } & 53 \\
\text { B. } & 52 \\
\text { B. } & 5 \\
\text { T. } & 7.0 \\
\text { B. } & 6.9\end{array}$ \\
\hline 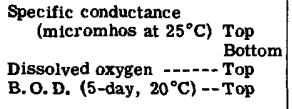 & $\begin{array}{l}111 \\
115 \\
9.6 \\
2.3\end{array}$ & $\begin{array}{l}107 \\
106 \\
9.8 \\
2.1\end{array}$ & $\begin{array}{l}102 \\
101 \\
9.7 \\
1.7\end{array}$ & $\begin{array}{l}103 \\
104 \\
9.7 \\
1.6\end{array}$ & $\begin{array}{l}103 \\
105 \\
9.8 \\
1.7\end{array}$ & $\begin{array}{ll}\text { T. } & 105 \\
\text { B. } & 106 \\
\text { T. } & 9.7 \\
\text { T. } & 1.9\end{array}$ \\
\hline 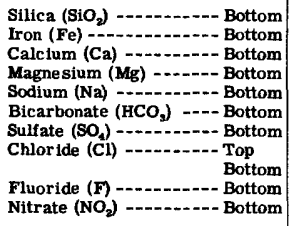 & $\begin{array}{l}3.6 \\
.08 \\
10 \\
3.8 \\
7.1 \\
27 \\
22 \\
6.0 \\
6.0 \\
.1 \\
2.9\end{array}$ & $\begin{array}{l}3.6 \\
10.06 \\
3.6 \\
6.8 \\
28 \\
20 \\
5.0 \\
6.2 \\
.1 \\
2.1\end{array}$ & $\begin{array}{l}3.4 \\
10.06 \\
3.5 \\
6.0 \\
32 \\
18 \\
5.0 \\
4.0 \\
.0 \\
1.9\end{array}$ & $\begin{array}{l}3.7 \\
11.09 \\
3.6 \\
4.0 \\
29 \\
19 \\
4.0 \\
4.0 \\
.1 \\
1.9\end{array}$ & $\begin{array}{l}3.6 \\
10.06 \\
3.8 \\
3.7 \\
26 \\
19 \\
5.0 \\
4.0 \\
.1 \\
2.1\end{array}$ & $\begin{array}{ll}\text { B. } & 3.6 \\
\text { B. } & .07 \\
\text { B. } & 10 \\
\text { B. } & 3.7 \\
\text { B. } & 5.5 \\
\text { B. } & 28 \\
\text { B. } & 20 \\
\text { T. } & 5.0 \\
\text { B. } & 4.8 \\
\text { B. } & .1 \\
\text { B. } & 2.2\end{array}$ \\
\hline $\begin{array}{l}\text { Dissolved solids }-\cdots \text { Bottom } \\
\text { Hardness as CaCO, }-.- \text { Bottom } \\
\text { Suspended sediment }-\cdots \text { Top }\end{array}$ & $\begin{array}{l}75 \\
41 \\
. \quad .\end{array}$ & $\begin{array}{l}72 \\
40\end{array}$ & $\begin{array}{l}67 \\
39 \\
\text {. Con }\end{array}$ & $\begin{array}{l}71 \\
42 \\
\cdots\end{array}$ & $\begin{array}{l}67 \\
4 I \\
\cdots\end{array}$ & $\begin{array}{ll}\text { B. } & 70 \\
\text { B. } & 47 \\
\text { T. } & 21\end{array}$ \\
\hline
\end{tabular}


Table 7. -- WAT ER ANALYSES OF DELAWARE RIVER BETWEEN BRISTOL AND MARCUS HOOK, PA. --Continued Analyzed by City of Philadelphia and U. S. Geological Survey; analyses in parts per million

Location Bristol, Pa.-Burlington, N. J. Bridge Date June 6, 1950 Sampling study No. 11

\begin{tabular}{|c|c|c|c|c|c|c|}
\hline \multirow[t]{3}{*}{ Weather Clear } & \multicolumn{6}{|c|}{ Water discharge at Trenton (cfs) 18,900} \\
\hline & \multicolumn{6}{|c|}{ Station } \\
\hline & \begin{tabular}{|c}
$\begin{array}{c}\text { Pennsylvania } \\
\text { side }\end{array}$ \\
\end{tabular} & West Center & Center & East Center & $\begin{array}{c}\text { New Jersey } \\
\text { side }\end{array}$ & Average \\
\hline 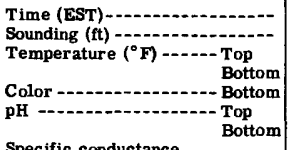 & $\begin{array}{c}12: 04 \text { p.m. } \\
16 \\
69 \\
68 \\
20 \\
6.8 \\
7.0\end{array}$ & $\begin{array}{c}12: 00 \mathrm{n} \\
21 \\
68 \\
68 \\
20 \\
6.7 \\
6.8\end{array}$ & $\begin{array}{c}11: 55 \text { a.m. } \\
41 \\
68 \\
68 \\
20 \\
6.8 \\
6.9\end{array}$ & $\begin{array}{c}11: 50 \text { a.m. } \\
38 \\
69 \\
68 \\
20 \\
6.9 \\
7.2\end{array}$ & $\begin{array}{c}11: 46 \text { a.m. } \\
15 \\
69 \\
68 \\
20 \\
6.9 \\
7.2\end{array}$ & $\begin{array}{ll}\text { T. } & 69 \\
\text { B. } & 68 \\
\text { B. } & 20 \\
\text { T. } & 6.8 \\
\text { B. } & 7.0\end{array}$ \\
\hline $\begin{array}{l}\begin{array}{l}\text { Specific conductance } \\
\text { (micromhos at } 25^{\circ} \mathrm{C} \text { ) }\end{array} \\
\text { Top } \\
\text { Bottom } \\
\text { Dissolved oxygen }-\cdots-\text { Top } \\
\text { B. O. D. (5-day, } 20^{\circ} \mathrm{C} \text { ) -- Top }\end{array}$ & $\begin{array}{r}112 \\
114 \\
6.3 \\
2.6\end{array}$ & $\begin{array}{l}120 \\
106 \\
6.5 \\
2.2\end{array}$ & $\begin{array}{l}106 \\
105 \\
6.6 \\
2.3\end{array}$ & $\begin{array}{l}107 \\
106 \\
6.4 \\
1.3\end{array}$ & $\begin{array}{l}107 \\
106 \\
6.1 \\
1.4\end{array}$ & $\begin{array}{lr}\text { T. } & 110 \\
\text { B. } & 107 \\
\text { T. } & 6.4 \\
\text { T. } & 2.0\end{array}$ \\
\hline 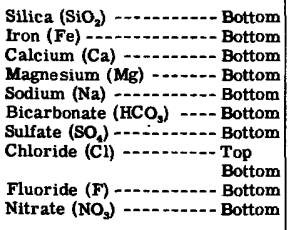 & $\begin{array}{l}5.1 \\
12.03 \\
3.3 \\
3.5 \\
29 \\
21 \\
5.0 \\
4.4 \\
.1 \\
3.1\end{array}$ & $\begin{array}{l}5.0 \\
11.04 \\
2.6 \\
3.4 \\
28 \\
19 \\
6.0 \\
3.8 \\
.1 \\
3.0\end{array}$ & $\begin{array}{l}4.8 \\
12.03 \\
3.1 \\
2.1 \\
28 \\
19 \\
5.0 \\
4.1 \\
.1 \\
3.2\end{array}$ & $\begin{array}{l}5.0 \\
12.02 \\
3.1 \\
2.8 \\
27 \\
18 \\
4.0 \\
3.5 \\
.1 \\
2.7\end{array}$ & $\begin{array}{l}5.1 \\
11.06 \\
3.2 \\
2.9 \\
27 \\
19 \\
4.0 \\
3.5 \\
.1 \\
3.0\end{array}$ & $\begin{array}{lc}\text { B. } & 5.0 \\
\text { B. } & .04 \\
\text { B. } & 12 \\
\text { B. } & 3.1 \\
\text { B. } & 2.9 \\
\text { B. } & 28 \\
\text { B. } & 19 \\
\text { T. } & 4.8 \\
\text { B. } & 3.9 \\
\text { B. } & .1 \\
\text { B. } & 3.0\end{array}$ \\
\hline $\begin{array}{l}\text { Dissolved solids } \ldots \ldots \text { - Bottom } \\
\text { Hardness as } \mathrm{CaCO}_{3} \ldots-\text { Bottom } \\
\text { Suspended sediment }-\cdots \text { Top }\end{array}$ & $\begin{array}{l}77 \\
51 \\
.\end{array}$ & $\begin{array}{l}76 \\
38 \\
. \\
\end{array}$ & $\begin{array}{l}74 \\
47 \\
\cdot \mathrm{Co}\end{array}$ & $\begin{array}{r}72 \\
49 \\
\text { Ite } .\end{array}$ & $\begin{array}{l}74 \\
41 \\
\cdots\end{array}$ & $\begin{array}{ll}\text { B. } & 75 \\
\text { B. } & 45 \\
\text { T. } & 12\end{array}$ \\
\hline
\end{tabular}

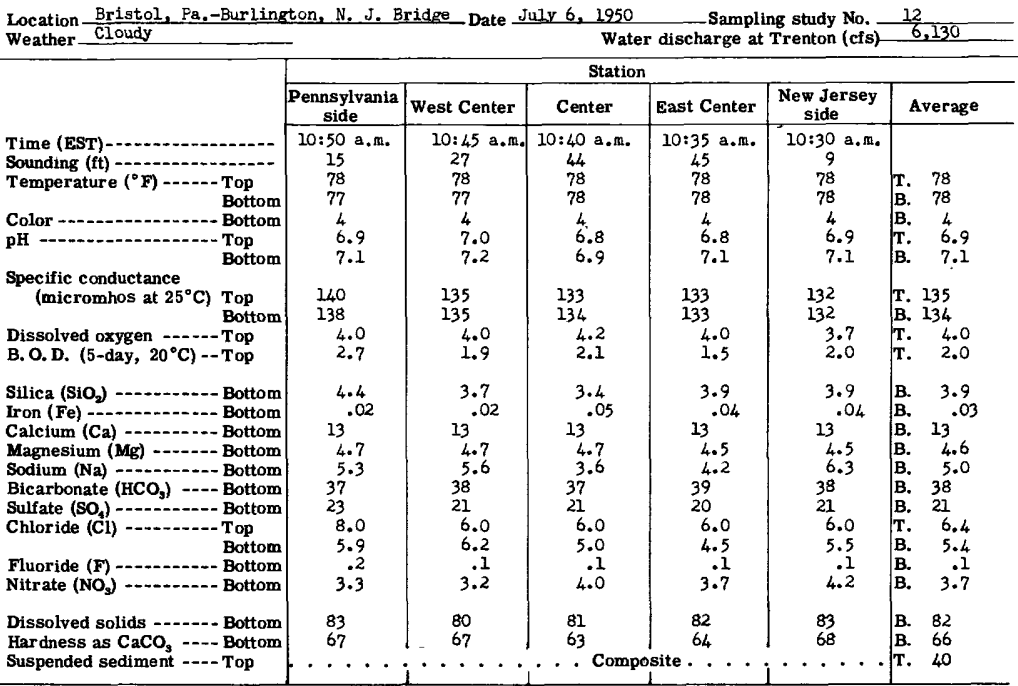


Table 7. -- WATER ANALYSES OF DELAWARE RIVER BETWEEN BRISTOL AND MARCUS HOOK, PA. --CONtinUed Analyzed by City of Philadelphia and U. S. Geological Survey; analyses in parts per million

Location Bristol, PA.-Burlington, N. J. Bridge Date August 2, 1950 Sampling study No. 13 Weather Cloudy $\quad$ Water discharge at Trenton (cfs) $-5,090$

\begin{tabular}{|c|c|c|c|c|c|c|}
\hline \multirow[b]{3}{*}{ 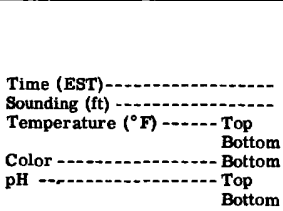 } & \multicolumn{6}{|c|}{ Station } \\
\hline & $\begin{array}{c}\text { Pennsylvania } \\
\text { side }\end{array}$ & West Center & Center & East Center & \begin{tabular}{|c|} 
New Jersey \\
side
\end{tabular} & Average \\
\hline & $\begin{array}{c}11: 40 \mathrm{a.m} . \\
9 \\
79 \\
79 \\
10 \\
6.2 \\
6.5\end{array}$ & $\begin{array}{c}11: 35 \mathrm{a} . \mathrm{m} . \\
19 \\
79 \\
79 \\
8 \\
6.7 \\
6.9\end{array}$ & $\begin{array}{c}11: 30 \mathrm{a} . \mathrm{m} . \\
36 \\
79 \\
79 \\
12 \\
6.7 \\
6.6\end{array}$ & $\begin{array}{c}11: 25 \mathrm{arm} . \\
37 \\
78 \\
79 \\
8 \\
6.7 \\
6.7\end{array}$ & $\begin{array}{c}11: 20 \text { a.m. } \\
16 \\
79 \\
79 \\
12 \\
6.7 \\
6.6\end{array}$ & $\begin{array}{lc}\text { T. } & 79 \\
\text { B. } & 79 \\
\text { B. } & 10 \\
\text { T. } & 6.6 \\
\text { B. } & 6.7\end{array}$ \\
\hline $\begin{array}{l}\text { (micromhos at } 25^{\circ} \mathrm{C} \text { ) } \begin{array}{l}\text { Top } \\
\text { Bottom }\end{array} \\
\text { Dissolved oxygen }-10 \text { - Top } \\
\text { B. O. D. (5-day, } 20^{\circ} \mathrm{C} \text { ) -- Top }\end{array}$ & $\begin{array}{r}163 \\
154 \\
6.0 \\
-\end{array}$ & $\begin{array}{l}140 \\
140 \\
6.4 \\
1.3\end{array}$ & $\begin{array}{l}137 \\
138 \\
6.3 \\
1.0\end{array}$ & $\begin{array}{l}138 \\
137 \\
6.1 \\
1.0\end{array}$ & $\begin{array}{l}137 \\
137 \\
5.8 \\
1.3\end{array}$ & $\begin{array}{lc}\text { T. } & 143 \\
\text { B. } & 141 \\
\text { T. } & 6.1 \\
\text { T. } & 1.1\end{array}$ \\
\hline 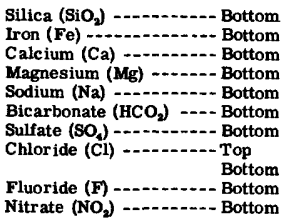 & $\begin{array}{l}4.8 \\
.03 \\
14 \\
4.3 \\
7.3 \\
35 \\
28 \\
8.0 \\
7.2 \\
.1 \\
2.6\end{array}$ & $\begin{array}{l}4.5 \\
.04 \\
14 \\
4.2 \\
5.3 \\
39 \\
23 \\
8.0 \\
5.5 \\
.1 \\
2.4\end{array}$ & $\begin{array}{l}4.2 \\
.07 \\
14 \\
4.2 \\
4.6 \\
38 \\
22 \\
7.0 \\
5.0 \\
.1 \\
3.1\end{array}$ & $\begin{array}{l}4.6 \\
.04 \\
14 \\
4.2 \\
4.5 \\
38 \\
22 \\
7.0 \\
5.0 \\
.1 \\
3.0\end{array}$ & $\begin{array}{l}4.6 \\
.08 \\
14 \\
4.3 \\
4.2 \\
38 \\
22 \\
7.0 \\
5.0 \\
.1 \\
3.1\end{array}$ & $\begin{array}{lc}\text { B. } & 4.5 \\
\text { B. } & .05 \\
\text { B. } & 14 \\
\text { B. } & 4.2 \\
\text { B. } & 5.2 \\
\text { B. } & 38 \\
\text { B. } & 23 \\
\text { T. } & 7.4 \\
\text { B. } & 5.5 \\
\text { B. } & .1 \\
\text { B. } & 2.8\end{array}$ \\
\hline $\begin{array}{l}\text { Dissolved solids - - - - Bottom } \\
\text { Hardness as CaCO, --- Bottom } \\
\text { Suspended sediment ---- Top }\end{array}$ & $\begin{array}{r}105 \\
53 \\
\cdots\end{array}$ & $\begin{array}{r}95 \\
52 \\
. \\
\end{array}$ & $\begin{array}{l}91 \\
52 \\
.\end{array}$ & $\begin{array}{r}95 \\
52 \\
.\end{array}$ & $\begin{array}{l}96 \\
53 \\
.\end{array}$ & $\begin{array}{ll}\text { B. } & 96 \\
\text { B. } & 52 \\
\text { T. } & 33\end{array}$ \\
\hline
\end{tabular}

Location Bristol, Pa.-Burlington, N. J. Bridge Date September 6, 1950 Sampling study No. 14 Weather Clear

\begin{tabular}{|c|c|c|c|c|c|c|}
\hline & & & & & & \\
\hline & \multicolumn{6}{|c|}{ Station } \\
\hline & \begin{tabular}{|c} 
Pennsylvania \\
side
\end{tabular} & West Center & Center & East Center & \begin{tabular}{|c|c|}
$\begin{array}{c}\text { New Jersey } \\
\text { side }\end{array}$ \\
\end{tabular} & Average \\
\hline $\begin{array}{l}\text { Time (EST) } \\
\text { Sounding (ft) } \\
\text { Temperature }\left({ }^{\circ} \mathrm{F}\right) \\
\text { Color } \\
\text { pH }\end{array}$ & $\begin{array}{c}12: 45 \mathrm{p} . \mathrm{m} . \\
24 \\
76 \\
76 \\
8 \\
7.1 \\
7.1\end{array}$ & $\begin{array}{c}12.49 \mathrm{p.m} . \\
32 \\
76 \\
75 \\
8 \\
6.9 \\
6.9\end{array}$ & $\begin{array}{c}12: 52 \mathrm{p} . \mathrm{m} . \\
45 \\
76 \\
76 \\
7 \\
6.9 \\
7.1\end{array}$ & $\begin{array}{c}12: 57 \text { p.m. } \\
22 \\
76- \\
76 \\
9 \\
6.9 \\
6.9\end{array}$ & $\begin{array}{l}\text { l:01 p.m. } \\
9 \\
77 \\
76 \\
9 \\
6.9 \\
7.0\end{array}$ & $\begin{array}{ll}\text { T. } & 76 \\
\text { B. } & 76 \\
\text { B. } & 8 \\
\text { T. } & 6.9 \\
\text { B. } & 7.0\end{array}$ \\
\hline $\begin{array}{l}\text { (micromhos at } 25^{\circ} \mathrm{C} \text { ) Top } \\
\text { Bottom } \\
\text { Dissolved oxygen } \\
\text { B. O. D. (5-day, } 20^{\circ} \mathrm{C} \text { ) - Top }\end{array}$ & $\begin{array}{l}175 \\
178 \\
5.5 \\
6.3\end{array}$ & $\begin{array}{r}178 \\
-174 \\
5.5 \\
3.3\end{array}$ & $\begin{array}{r}176 \\
174 \\
-5.5 \\
3.0\end{array}$ & $\begin{array}{r}173 \\
-172 \\
5.5 \\
2.1\end{array}$ & $\begin{array}{l}173 \\
172 \\
6.0 \\
5.1\end{array}$ & $\begin{array}{lr}\text { T. } & 175 \\
\text { B. } & 174 \\
\text { T. } & 5.6 \\
\text { T. } & 4.0\end{array}$ \\
\hline 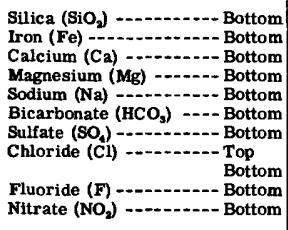 & $\begin{array}{l}4.3 \\
16 \\
6.11 \\
7.0 \\
46 \\
28 \\
9.0 \\
8.2 \\
.1 \\
4.4\end{array}$ & $\begin{array}{l}4.2 \\
17 \\
6.12 \\
4.9 \\
45 \\
25 \\
9.0 \\
8.2 \\
.1 \\
4.9\end{array}$ & $\begin{array}{l}4.2 \\
.07 \\
16 \\
6.1 \\
6.6 \\
46 \\
27 \\
9.0 \\
8.0 \\
.1 \\
4.3\end{array}$ & $\begin{array}{l}4.3 \\
16 \\
6.07 \\
6.4 \\
46 \\
26 \\
8.0 \\
7.8 \\
.1 \\
4.7\end{array}$ & $\begin{array}{l}4.3 \\
1.14 \\
6.3 \\
5.2 \\
47 \\
26 \\
8.0 \\
7.5 \\
.1 \\
4.7\end{array}$ & $\begin{array}{ll}\text { B. } & 4.3 \\
\text { B. } & .10 \\
\text { B. } & 16 \\
\text { B. } & 6.2 \\
\text { B. } & 6.0 \\
\text { B. } & 46 \\
\text { B. } & 26 \\
\text { T. } & 8.6 \\
\text { B. } & 7.9 \\
\text { B. } & .1 \\
\text { B. } & 4.6\end{array}$ \\
\hline $\begin{array}{l}\text { Dissolved solids }---- \text { Bottom } \\
\text { Hardness as } \mathrm{CaCO},--- \text { Bottom } \\
\text { Suspended sediment }--- \text { Top }\end{array}$ & $\begin{array}{r}116 \\
67 \\
. \\
\end{array}$ & $\begin{array}{r}113 \\
68 \\
\cdots \\
\end{array}$ & $\begin{array}{r}113 \\
05 \\
.\end{array}$ & $\begin{array}{r}108 \\
65 \\
\text { te. }\end{array}$ & $\begin{array}{l}108 \\
68 \\
\cdots\end{array}$ & $\begin{array}{lr}\text { B. } & 112 \\
\text { B. } & 67 \\
\text { T. } & 16\end{array}$ \\
\hline
\end{tabular}


Table 7. -- WATER ANALYSES OF DELAWARE RIVER BETWEEN BRISTOL AND MARCUS HOOK, PA. --CONtInUEd Analyzed by City of Philadelphia and U. S. Geological Survey; analyses in parts per million

Location Bristol, Pa.-Burlington, N. J. Bridge Date October 3, 1950 Sampling study No. 15 Weather-Misty Water discharge at Trenton (cfs) $2,8 \mathrm{~L}$

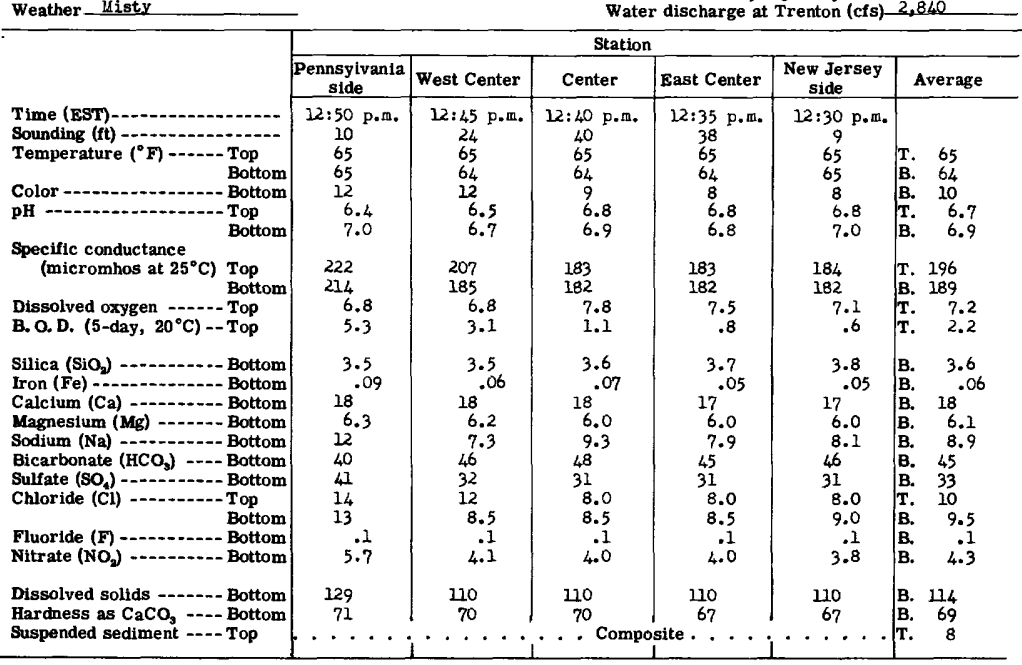

Location Bristol, Pa,-Burlington, N. J. Bridge Date November 2, 1950 Sampling study No. 16 Weather Clear

\begin{tabular}{|c|c|c|c|c|c|c|}
\hline \multirow[b]{3}{*}{ 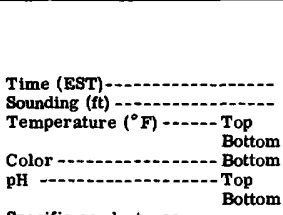 } & \multicolumn{6}{|c|}{ Station } \\
\hline & \begin{tabular}{|c|}
$\begin{array}{c}\text { Pennsylvania } \\
\text { side }\end{array}$ \\
\end{tabular} & West Center & Center & East Center & \begin{tabular}{|c|}
$\begin{array}{c}\text { New Jersey } \\
\text { side. }\end{array}$ \\
\end{tabular} & Average \\
\hline & $\begin{array}{c}12: 20 \text { p.m. } \\
.3 \\
60 \\
61 \\
5 \\
6.7 \\
6.8\end{array}$ & $\begin{array}{c}12: 16 \mathrm{p} . \mathrm{m} . \\
16 \\
60 \\
60 \\
7 \\
6.8 \\
7.1\end{array}$ & $\begin{array}{c}12: 12 \text { p.m. } \\
37 \\
60 \\
60 \\
10 \\
6.9 \\
7.3\end{array}$ & $\begin{array}{c}12=08 \mathrm{p} . \mathrm{m} \\
44 \\
60 \\
60 \\
6 \\
6.9 \\
7.3\end{array}$ & $\begin{array}{c}12: 03 \mathrm{p} . \mathrm{m} \\
9 \\
60 \\
60 \\
8 \\
6.8 \\
7.3\end{array}$ & $\begin{array}{lc}\text { T. } & 60 \\
\text { B. } & 60 \\
\text { B. } & 7 \\
\text { T. } & 6.8 \\
\text { B. } & 7.2\end{array}$ \\
\hline $\begin{array}{l}\text { Specific conductance } \\
\quad \text { (micromhos at } 25^{\circ} \mathrm{C} \text { ) } \begin{array}{l}\text { Top } \\
\text { Bottom }\end{array} \\
\text { Dissolved oxygen }-10^{\circ} \mathrm{C} \text { )- Top } \\
\text { B. O. D. (5-day, }\end{array}$ & $\begin{array}{r}236 \\
235 \\
9.1 \\
8.4\end{array}$ & $\begin{array}{r}219 \\
212 \\
7.9 \\
4.5\end{array}$ & $\begin{array}{l}203 \\
202 \\
8.9 \\
3.4\end{array}$ & $\begin{array}{l}203 \\
202 \\
9.6 \\
3.5\end{array}$ & $\begin{array}{l}203 \\
201 \\
9.0 \\
3.2\end{array}$ & $\begin{array}{ll}\text { T. } & 213 \\
\text { B. } & 210 \\
\text { T. } & 8.9 \\
\text { T. } & 4.6\end{array}$ \\
\hline 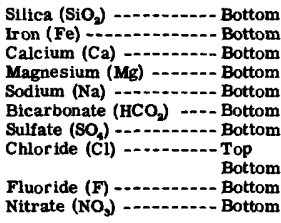 & $\begin{array}{l}1.9 \\
.01 \\
18 \\
7.1 \\
15 \\
48 \\
42 \\
12 \\
14 \\
.1 \\
5.6\end{array}$ & $\begin{array}{l}1.8 \\
18 \\
7.05 \\
11 \\
50 \\
36 \\
12 \\
12 \\
.1 .9\end{array}$ & $\begin{array}{l}2.1 \\
18 \\
7.04 \\
7.9 \\
49 \\
32 \\
10 \\
10 \\
.1 \\
4.9\end{array}$ & $\begin{array}{l}1.8 \\
.04 \\
18 \\
7.1 \\
8.1 \\
49 \\
33 \\
10 \\
10 \\
.1 \\
4.6\end{array}$ & $\begin{array}{l}2.2 \\
18 \\
7.05 \\
7.9 \\
49 \\
33 \\
10 \\
10 \\
.1 \\
4.0\end{array}$ & $\begin{array}{ll}\text { B. } & 2.0 \\
\text { B. } & .04 \\
\text { B. } & 18 \\
\text { B. } & 7.1 \\
\text { B. } & 10 \\
\text { B. } & 49 \\
\text { B. } & 35 \\
\text { T. } & 11 \\
\text { B. } & 11 \\
\text { B. } & .1 \\
\text { B. } & 4.8\end{array}$ \\
\hline $\begin{array}{l}\text { Dissolved solide }-1 .-- \text { Bottom } \\
\text { Hardness as } \mathrm{CaCO}_{3}-\cdots-\text { Bottom } \\
\text { Suspended sediment }-\cdots \text { Top }\end{array}$ & $\begin{array}{r}138 \\
74 \\
.\end{array}$ & $\begin{array}{r}126 \\
74\end{array}$ & $\stackrel{121}{74} \cdot \mathrm{C}$ & $\begin{array}{r}119 \\
74 \\
\text { e. }\end{array}$ & $\begin{array}{r}120 \\
74 \\
\cdots\end{array}$ & $\begin{array}{lr}\text { B. } & 125 \\
\text { B. } & 74 \\
\text { T. } & 17\end{array}$ \\
\hline
\end{tabular}


Table 7. -- WATER ANA LYSES OF DELAWARE RIVER BETWEEN BRISTOL AND MARCUS HOOK, PA. --Continued Analyzed by City of Philadelphia and U. S. Geological Survey; analyses in parts per million

Location Bristcl, Pa.-Burlington, N. J. Bridge Date December 4, 1950 Sampling study No. 17 Weather Rain

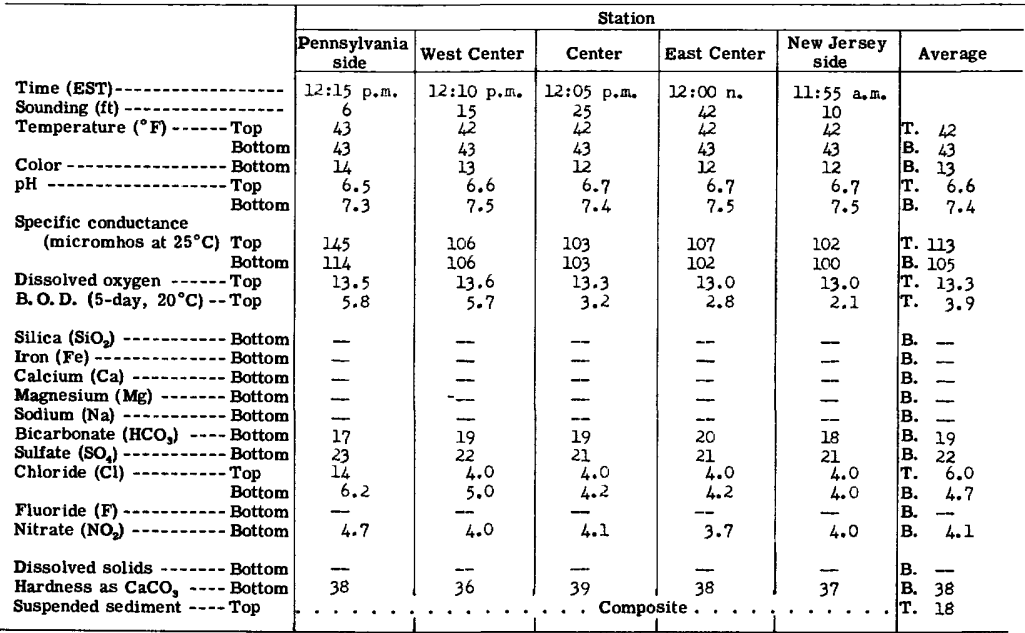

Location Bristol, Pa,-Burlington, N. J. Bridge Date January 3, 1951 Sampling study No, 18

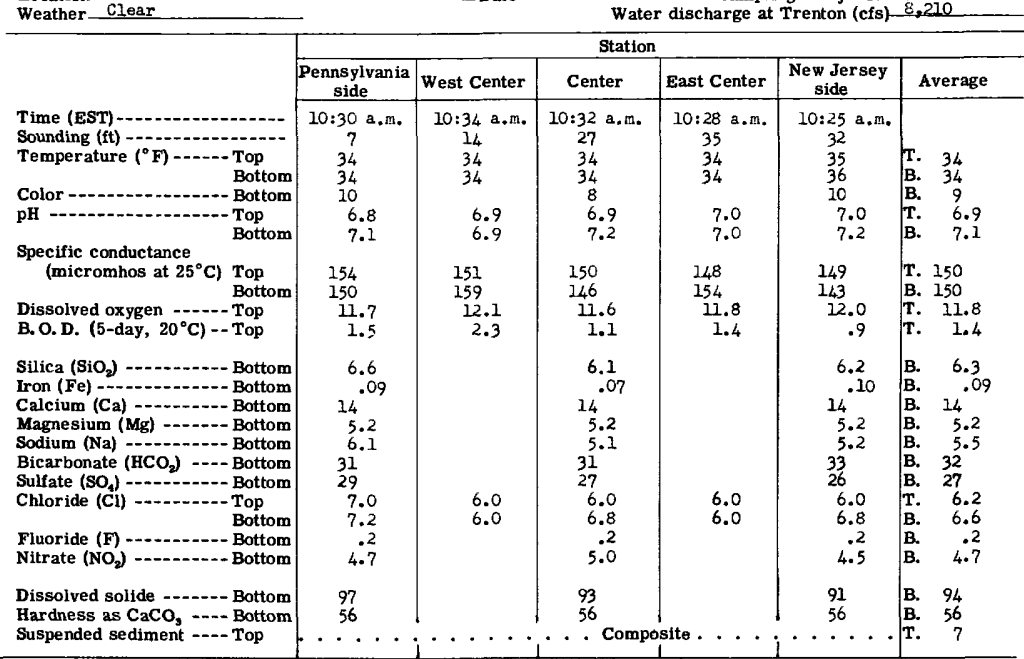


Table 7. --WATER ANALYSES OF DELAWARE RIVER BETWEEN BRISTOL AND MARCUS HOOK, PA. --Continued Analyzed by City of Philadelphia and U. S. Geological Survey; analyses in parts per million

Location Bristol, Pa,-Burlington, N. J. Bridge Date March 2, 1951 Sampling study No. 19 Weather Clear $\quad$ Water discharge at Trenton (cfs) 19,600

\begin{tabular}{|c|c|c|c|c|c|c|}
\hline \multirow[b]{3}{*}{$\begin{array}{l}\text { Time (EST) } \\
\text { Sounding (ft) } \\
\text { Temperature }\left({ }^{\circ} \mathrm{F}\right)-\begin{array}{l}\text { Bop } \\
\text { Bottom }\end{array} \\
\text { Color }-1 \text { Bottom } \\
\text { pH }-\begin{array}{l}\text { Top } \\
\text { Bottom }\end{array}\end{array}$} & \multicolumn{6}{|c|}{ Station } \\
\hline & $\begin{array}{c}\text { Pennsylvania } \\
\text { side }\end{array}$ & West Center & Center & East Center & \begin{tabular}{|c|}
$\begin{array}{c}\text { New Jersey } \\
\text { side }\end{array}$ \\
\end{tabular} & Average \\
\hline & $\begin{array}{c}11: 25 \mathrm{a} . \mathrm{m} . \\
25 \\
43 \\
42 \\
9 \\
6.6 \\
7.2\end{array}$ & $\begin{array}{c}11: 15 \mathrm{a}, \mathrm{m} . \\
28 \\
42 \\
42 \\
6.7 \\
6.6\end{array}$ & $\begin{array}{c}11: 10 \mathrm{a} . \mathrm{m} . \\
40 \\
42 \\
42 \\
10 \\
6.6 \\
7.3\end{array}$ & $\begin{array}{c}11: 05 \text { a.m. } \\
43 \\
43 \\
42 \\
6.7 \\
6.8\end{array}$ & $\begin{array}{c}11: 00 \mathrm{a} . \mathrm{m} . \\
31 \\
43 \\
42 \\
9 \\
6.8 \\
7.3\end{array}$ & $\begin{array}{ll}\text { T. } & 43 \\
\text { B. } & 42 \\
\text { B. } & 9 \\
\text { T. } & 6.7 \\
\text { B. } & 7.0\end{array}$ \\
\hline $\begin{array}{l}\begin{array}{l}\text { Specific conductance } \\
\left.\text { (micromhos at } 25^{\circ} \mathrm{C}\right)\end{array} \\
\begin{array}{l}\text { Top } \\
\text { Bottom }\end{array} \\
\text { Dissolved oxygen --- - Top } \\
\text { B. O. D. (5-day, } 20^{\circ} \mathrm{C} \text { ) - Top }\end{array}$ & $\begin{array}{l}105 \\
103 \\
12.5 \\
3.6\end{array}$ & $\begin{array}{l}102 \\
100 \\
12.9 \\
3.3\end{array}$ & $\begin{array}{r}101 \\
99.7 \\
12.9 \\
3.0\end{array}$ & $\begin{array}{r}101 \\
99.4 \\
13.0 \\
3.3\end{array}$ & $\begin{array}{r}102 \\
99.4 \\
13.0 \\
2.5\end{array}$ & $\begin{array}{l}\text { T. } 102 \\
\text { B. } 100 \\
\text { T. } 12.9 \\
\text { T. } 3.1\end{array}$ \\
\hline 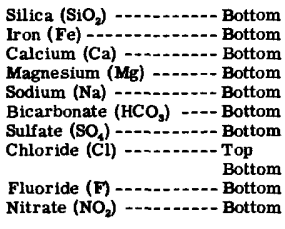 & $\begin{array}{l}4.0 \\
10.15 \\
3.8 \\
3.1 \\
23 \\
19 \\
3.0 \\
4.2 \\
.2 \\
2.9\end{array}$ & $\begin{array}{l}3.0 \\
4.0\end{array}$ & $\begin{array}{l}3.9 \\
.16 \\
9.6 \\
3.7 \\
4.2 \\
25 \\
18 \\
3.0 \\
4.8 \\
.2 \\
2.3\end{array}$ & $\begin{array}{l}3.0 \\
4.0\end{array}$ & $\begin{array}{l}4.1 \\
.18 \\
9.9 \\
3.5 \\
3.4 \\
24 \\
18 \\
3.0 \\
4.2 \\
.1 \\
2.7\end{array}$ & $\begin{array}{ll}\text { B. } & 4.0 \\
\text { B. } & .16 \\
\text { B. } & 9.8 \\
\text { B. } & 3.7 \\
\text { B. } & 3.6 \\
\text { B. } & 24 \\
\text { B. } & 18 \\
\text { T. } & 3.0 \\
\text { B. } & 4.2 \\
\text { B. } & .2 \\
\text { B. } & 2.6\end{array}$ \\
\hline 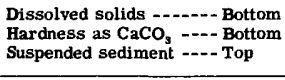 & $\begin{array}{l}65 \\
41 \\
.\end{array}$ & & $\begin{array}{l}62 \\
39 \\
. \quad \text { Cor } \\
\end{array}$ & site... & $\begin{array}{r}65 \\
39 \\
\cdots \cdots \\
\end{array}$ & $\begin{array}{rr}\text { B. } & 64 \\
\text { B. } & 40 \\
\text { T. } & 9 \\
\end{array}$ \\
\hline
\end{tabular}

Location Bristol, Pa.-Burlington, N. J. Bridge_Date April 2, 1951 Sampling study No, 20

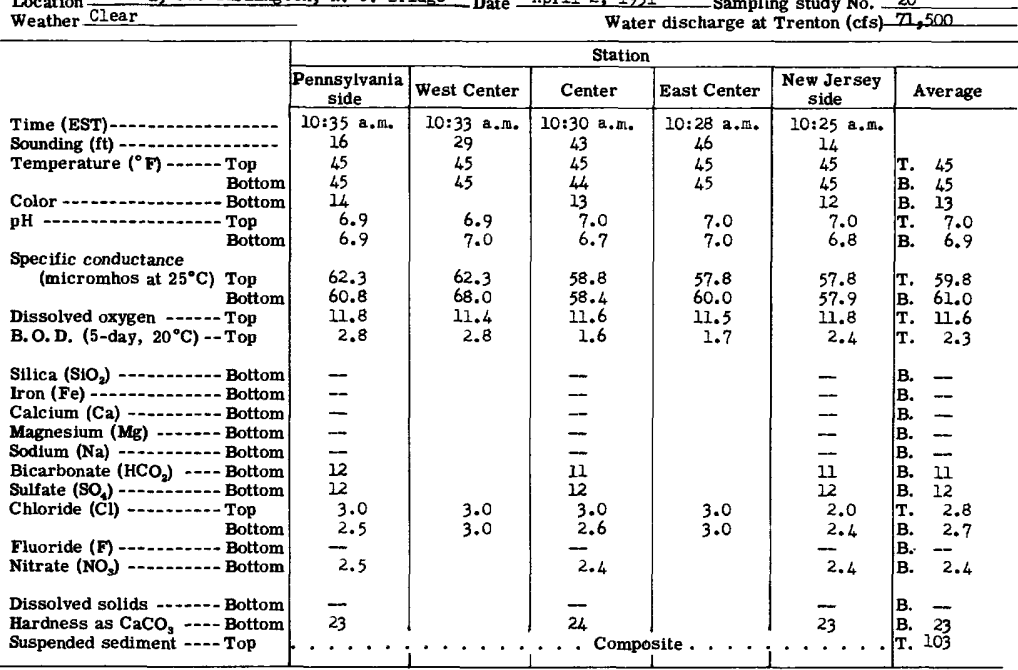


Table 7. --WATER ANALYSES OF DELAWARE RIVER BETWEEN BRISTOL AND MARCUS HOOK, PA. --Continued Analyzed by City of Philadelphia and U. S. Geological Survey; analyses in parts per million

Location Bristol, Pa.-Burlington, N. J. Bridge Date May 1, 1951

Sampling study No. 21 Weather Clear

Water discharge at Trenton (cfs) 13,000

\begin{tabular}{|c|c|c|c|c|c|c|}
\hline & \multicolumn{6}{|c|}{ Station } \\
\hline & \begin{tabular}{|c} 
Pennsylvania \\
side
\end{tabular} & West Center & Center & East Center & $\begin{array}{c}\text { New Jersey } \\
\text { side }\end{array}$ & Aver age \\
\hline 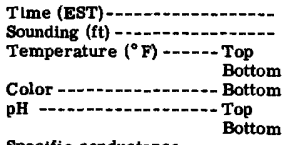 & $\begin{array}{c}10: 24 \text { a.m. } \\
19 \\
63 \\
61 \\
12 \\
6.9 \\
7.4\end{array}$ & $\begin{array}{c}10: 21 \text { a.m. } \\
21 \\
62 \\
62 \\
7.1 \\
7.2\end{array}$ & $\begin{array}{c}10: 19 \mathrm{a} . \mathrm{m} . \\
39 \\
62 \\
61 \\
12 \\
7.1 \\
7.4\end{array}$ & $\begin{array}{c}10: 17 \text { a.m. } \\
43 \\
63 \\
62 \\
7.1 \\
7.3\end{array}$ & $\begin{array}{c}10: 15 \text { a.m. } \\
9 \\
63 \\
62 \\
10 \\
7.0 \\
7.3\end{array}$ & $\begin{array}{ll}\text { T. } & 63 \\
\text { B. } & 62 \\
\text { B. } & 11 \\
\text { T. } & 7.0 \\
\text { B. } & 7.3\end{array}$ \\
\hline $\begin{array}{l}\text { Specific conductance } \\
\quad \text { (micromhos at } 25^{\circ} \mathrm{C} \text { ) } \begin{array}{l}\text { Top } \\
\text { Bottom }\end{array} \\
\begin{array}{c}\text { Dissolved oxygen } \\
\text { B. O.D. (5-day, } 20^{\circ} \mathrm{C} \text { ) - Top }\end{array}\end{array}$ & $\begin{array}{r}128 \\
113 \\
8.9 \\
2.5\end{array}$ & $\begin{array}{r}122 \\
125 \\
8.9 \\
2.6\end{array}$ & $\begin{array}{l}116 \\
116 \\
9.0 \\
2.0\end{array}$ & $\begin{array}{l}114 \\
116 \\
8.9 \\
2.1\end{array}$ & $\begin{array}{r}113 \\
124 \\
8.9 \\
2.2\end{array}$ & $\begin{array}{lr}\text { T. } & 119 \\
\text { B. } & 119 \\
\text { T. } & 8.9 \\
\text { T. } & 2.3\end{array}$ \\
\hline 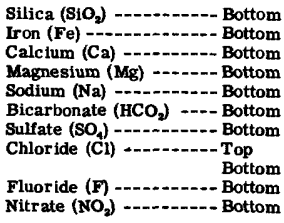 & $\begin{array}{l}5.4 \\
.16 \\
11.0 \\
4.0 \\
30 \\
20 \\
5.0 \\
4.2 \\
.0 \\
2.0\end{array}$ & $\begin{array}{l}5.0 \\
5.0\end{array}$ & $\begin{array}{l}5.7 \\
.12 \\
11.0 \\
4.0 \\
28 \\
20 \\
5.0 \\
4.5 \\
.0 \\
1.7\end{array}$ & $\begin{array}{l}5.0 \\
5.0\end{array}$ & $\begin{array}{l}6.7 \\
.08 \\
21 \\
4.2 \\
4.5 \\
29 \\
22 \\
5.0 \\
4.8 \\
.0 \\
1.7\end{array}$ & $\begin{array}{ll}\text { B. } & 5.9 \\
\text { B. } & .12 \\
\text { B. } & 11 \\
\text { B. } & 4.1 \\
\text { B. } & 4.0 \\
\text { B. } & 29 \\
\text { B. } & 21 \\
\text { T. } & 5.0 \\
\text { B. } & 4.7 \\
\text { B. } & .0 \\
\text { B. } & 1.8\end{array}$ \\
\hline $\begin{array}{l}\text { Dissolved solids }-1 .-- \text { Bottom } \\
\text { Hardness as } \mathrm{CaCO}_{3}-\ldots \text { Bottom } \\
\text { Suspended sediment }-\cdots \text { Top }\end{array}$ & $\begin{array}{l}71 \\
44 \\
.\end{array}$ & & $\begin{array}{l}71 \\
44 \\
\text { C Con }\end{array}$ & 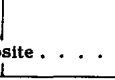 & $\begin{array}{l}76 \\
45 \\
\cdots\end{array}$ & $\begin{array}{lr}\text { B. } & 73 \\
\text { B. } & 44 \\
\text { T. } & 142\end{array}$ \\
\hline
\end{tabular}
Location $\frac{\text { Bristol, Pa,-Burlingtion, N, J. Bridge }}{\text { Cloudy }}$ Date June 7, 1951 Sampling study No. $\frac{22}{\text { water discharge at Trenton (cfs)- } 8,670}$ Location $\frac{\text { Bristol, Pa,-Burlingtion, N, J. Bridge }}{\text { Cloudy }}$ Date June 7, 1951 Sampling study No. $\frac{22}{\text { water discharge at Trenton (cfs)-8,670 }}$

\begin{tabular}{|c|c|c|c|c|c|c|}
\hline Weather Cloudy & & & & harg & ton & 8,670 \\
\hline & & & Statio & & & \\
\hline & \begin{tabular}{|c|} 
Pennsylvania \\
side
\end{tabular} & West Center & Center & East Center & \begin{tabular}{|c|}
$\begin{array}{c}\text { New Jersey } \\
\text { side }\end{array}$ \\
\end{tabular} & Average \\
\hline 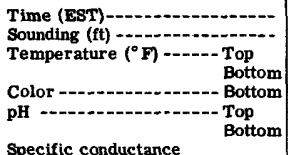 & {$\left[\begin{array}{c}11: 16 \text { a.m. } \\
19 \\
73 \\
72 \\
20 \\
6.8 \\
7.4\end{array}\right.$} & $\begin{array}{c}11: 14 \\
29 \\
73 \\
72 \\
6.9 \\
7.4\end{array}$ & $\begin{array}{c}11: 12 \mathrm{a} . \mathrm{m} \\
36 \\
73 \\
73 \\
23 \\
6.9 \\
7.2\end{array}$ & $\begin{array}{c}11: 10 \text { B.m. } \\
23 \\
73 \\
73 \\
6.9 \\
7.4\end{array}$ & $\begin{array}{c}11: 08 \text { a.m. } \\
14 \\
73 \\
73 \\
20 \\
7.0 \\
7.3\end{array}$ & $\begin{array}{lc}\text { T. } & 73 \\
\text { B. } & 73 \\
\text { B. } & 21 \\
\text { T. } & 6.9 \\
\text { B. } & 7.3\end{array}$ \\
\hline $\begin{array}{l}\begin{array}{l}\text { Specific conductance } \\
\text { (micromhos at } 25^{\circ} \mathrm{C} \text { ) }\end{array} \\
\begin{array}{l}\text { Top } \\
\text { Bottom }\end{array} \\
\text { Dissolved oxygen }-10^{\circ} \mathrm{C} \text { ) - Top } \\
\text { B. O.D. (5-day, } 20^{-} \text {Top }\end{array}$ & $\begin{array}{r}159 \\
144 \\
6.6 \\
4.5\end{array}$ & $\begin{array}{r}1 / 1 \\
142 \\
6.9 \\
2.1\end{array}$ & $\begin{array}{l}141 \\
143 \\
6.8 \\
1.9\end{array}$ & $\begin{array}{l}138 \\
139 \\
7.0 \\
2.2\end{array}$ & $\begin{array}{l}138 \\
138 \\
7.1 \\
2.6\end{array}$ & $\begin{array}{l}\text { T. } 143 \\
\text { B. } 141 \\
\text { T. } \quad 6.9 \\
\text { T. } 2.7\end{array}$ \\
\hline 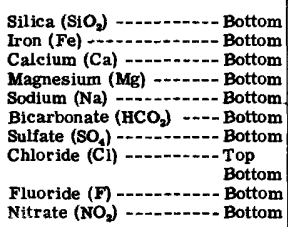 & $\begin{array}{l}4.5 \\
.27 \\
13 \\
5.2 \\
4.5 \\
37 \\
23 \\
7.0 \\
5.2 \\
.0 \\
3.6\end{array}$ & $\begin{array}{l}5.0 \\
5.0\end{array}$ & $\begin{array}{l}4.8 \\
.28 \\
13^{5.2} \\
4.9 \\
38 \\
23 \\
5.0 \\
5.0 \\
.1 \\
3.9\end{array}$ & $\begin{array}{l}5.0 \\
5.0\end{array}$ & $\begin{array}{l}4.4 \\
13.23 \\
5.2 \\
4.0 \\
37 \\
22 \\
5.0 \\
5.0 \\
.1 \\
3.0\end{array}$ & $\begin{array}{lc}\text { B. } & 4.6 \\
\text { B. } & .26 \\
\text { B. } & 13 \\
\text { B. } & 5.2 \\
\text { B. } & 4.5 \\
\text { B. } & 37 \\
\text { B. } & 23 \\
\text { T. } & 5.4 \\
\text { B. } & 5.0 \\
\text { B. } & .1 \\
\text { B. } & 3.5\end{array}$ \\
\hline $\begin{array}{l}\text { Dissolved solids } \ldots \ldots \text { Bottom } \\
\text { Hardness as } \mathrm{CaCO}_{9} \ldots \ldots \text { Botton } \\
\text { Suspended sediment } \ldots \text { Top }\end{array}$ & $\begin{array}{l}34 \\
54 \\
\cdots\end{array}$ & & $\begin{array}{l}84 \\
54 \\
. \quad \mathrm{Cor}\end{array}$ & Nite. & $\begin{array}{r}83 \\
54 \\
. \cdots\end{array}$ & $\begin{array}{ll}\text { B. } & 84 \\
\text { B. } & 54 \\
\text { T. } & 19\end{array}$ \\
\hline
\end{tabular}


Table 7, -- WATER ANALYSES OF DELAWARE RIVER BETWEEN BRISTOL AND MARCUS HOOK, PA. --Continued Analyzed by City of Philadelphia and U. S. Geological Survey; analyses in parts per million

Location Bristol, Pa.-Burlington, N. J. Bridge Date July 5, 1951___ Sampling study No.

No. $\frac{23}{9.190}$

\begin{tabular}{|c|c|c|c|c|c|c|}
\hline \multirow{3}{*}{ Weather Cloudy } & \multicolumn{6}{|c|}{ Water discharge at Trenton (cfs) $\quad 9,190$} \\
\hline & \multicolumn{6}{|c|}{ Station } \\
\hline & \begin{tabular}{|c|} 
Penns ylvania \\
side
\end{tabular} & West Center & Center & East Center & \begin{tabular}{|c|}
$\begin{array}{c}\text { New Jersey } \\
\text { side }\end{array}$ \\
\end{tabular} & Average \\
\hline $\begin{array}{l}\text { Time (EST) } \\
\text { Sounding (ft) } \\
\text { Temperature }\left({ }^{\circ} \mathrm{F} \text { ) }\right. \\
\begin{array}{l}\text { Color } \\
\text { pH }\end{array} \\
\begin{array}{c}\text { Bottom } \\
\text { Bottom }\end{array}\end{array}$ & $\begin{array}{c}11: 33 \text { a.m. } \\
20 \\
76 \\
76 \\
10 \\
7.2 \\
7.2\end{array}$ & $\begin{array}{c}11: 31 \text { a.m. } \\
27 \\
76 \\
76 \\
7.2 \\
7.2\end{array}$ & $\begin{array}{c}11: 29 \text { a.m. } \\
45 \\
76 \\
76 \\
8 \\
7.2 \\
7.4\end{array}$ & $\begin{array}{c}11: 27 \text { a.m. } \\
40 \\
76 \\
76 \\
7.2 \\
7.4\end{array}$ & $\begin{array}{c}21: 25 \text { a.m. } \\
20 \\
76 \\
76 \\
8 \\
4 \\
4\end{array}$ & $\begin{array}{ll}\text { T. } & 76 \\
\text { B. } & 76 \\
\text { B. } & 9 \\
\text { T. } & 7.2 \\
\text { B. } & 7.3\end{array}$ \\
\hline $\begin{array}{l}\text { Specific conductance } \\
\text { (micromhos at } 25^{\circ} \mathrm{C} \text { ) } \begin{array}{l}\text { Top } \\
\text { Bottom }\end{array} \\
\text { Dissolved oxygen ---- Top } \\
\text { B. O.D. (5-day, } 20^{\circ} \mathrm{C} \text { ) -- Top }\end{array}$ & $\begin{array}{l}145 \\
149 \\
6.1 \\
1.8\end{array}$ & $\begin{array}{l}140 \\
141 \\
6.4 \\
1.8\end{array}$ & $\begin{array}{r}145 \\
144 \\
6.7 \\
1.6\end{array}$ & $\begin{array}{r}154 \\
143 \\
6.3 \\
1.1\end{array}$ & $\begin{array}{l}139 \\
144 \\
6.5 \\
1.5\end{array}$ & $\rightarrow \begin{array}{lr}\text { T. } & 145 \\
\text { B. } & 144 \\
\text { T. } & 6.4 \\
\text { T. } & 1.6\end{array}$ \\
\hline 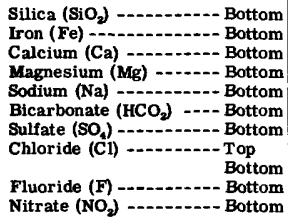 & $\begin{array}{l}4.9 \\
16 \\
.10 \\
5.3 \\
2.7 \\
40 \\
24 \\
5.0 \\
5.0 \\
.0 \\
3.5\end{array}$ & $\begin{array}{l}5.0 \\
5.0\end{array}$ & $\begin{array}{l}5.0 \\
.12 \\
16 \\
5.3 \\
1.2 \\
40 \\
22 \\
5.0 \\
4.2 \\
.0 \\
3.2\end{array}$ & $\begin{array}{l}5.0 \\
5.0\end{array}$ & $\begin{array}{l}5.1 \\
.11 \\
16 \\
5.2 \\
1.4 \\
40 \\
22 \\
5.0 \\
4.5 \\
.0 \\
3.0\end{array}$ & $\begin{array}{lc}\text { B. } & 5.0 \\
\text { B. } & .11 \\
\text { B. } & 16 \\
\text { B. } & 5.3 \\
\text { B. } & 1.8 \\
\text { B. } & 40 \\
\text { B. } & 23 \\
\text { T. } & 5.0 \\
\text { B. } & 4.7 \\
\text { B. } & .0 \\
\text { B. } & 3.2\end{array}$ \\
\hline 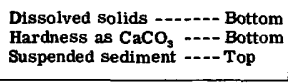 & $\begin{array}{l}98 \\
62 \\
.\end{array}$ & & $\begin{array}{l}99 \\
62 \\
. \quad C \text { Com }\end{array}$ & site. . & $\begin{array}{c}94 \\
61 \\
\cdots\end{array}$ & $\begin{array}{ll}\text { B. } & 97 \\
\text { B. } & 62 \\
\text { T. } & 17\end{array}$ \\
\hline
\end{tabular}

Location Bristol, Pa,-Burlington, N. J, Eridge-Date August 1, 1951 Sampling study No.

No. 24 Weather Cloudy

\begin{tabular}{|c|c|c|c|c|c|c|}
\hline \multirow[b]{3}{*}{ 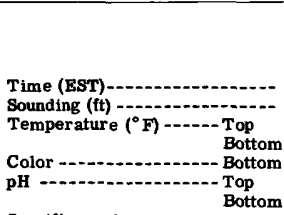 } & \multicolumn{6}{|c|}{ Station } \\
\hline & \begin{tabular}{|c} 
Pennsylvania \\
side
\end{tabular} & West Center & Center & East Center & $\begin{array}{l}\text { New Jer sey } \\
\text { side }\end{array}$ & Average \\
\hline & \begin{tabular}{|c|}
$10: 48$ a.m. \\
22 \\
76 \\
76 \\
6 \\
6.8 \\
7.3
\end{tabular} & $\begin{array}{c}10: 46 \text { a.m. } \\
25 \\
76 \\
76 \\
6.9 \\
7.0\end{array}$ & $\begin{array}{c}10: 44 \mathrm{a} \cdot \mathrm{m} . \\
38 \\
76 \\
76 \\
6 \\
6.9 \\
7.7\end{array}$ & $\begin{array}{c}10: 42 \text { a.m. } \\
44 \\
76 \\
76 \\
6.9 \\
7.0\end{array}$ & $\begin{array}{c}10: 40 \text { a.m. } \\
30 \\
76 \\
76 \\
6 \\
6.9 \\
7.9\end{array}$ & $\begin{array}{ll}\text { T. } & 76 \\
\text { B. } & 76 \\
\text { B. } & 6 \\
\text { T. } & 6.9 \\
\text { B. } & 7.4\end{array}$ \\
\hline $\begin{array}{l}\text { Specific conductance } \\
\quad \text { (micromhos at } 25^{\circ} \mathrm{C} \text { ) Top } \\
\text { Bottom }\end{array}$ & $\begin{array}{l}163 \\
158 \\
7.0 \\
1.3\end{array}$ & $\begin{array}{l}158 \\
161 \\
7.1 \\
1.0\end{array}$ & $\begin{array}{l}157 \\
155 \\
7.4 \\
1.5\end{array}$ & $\begin{array}{l}157 \\
158 \\
7 \cdot 3 \\
2.0\end{array}$ & $\begin{array}{r}157 \\
154 \\
7.2 \\
2.8\end{array}$ & $\begin{array}{lr}\text { T. } & 158 \\
\text { B. } & 157 \\
\text { T. } & 7.2 \\
\text { T. } & 1.7\end{array}$ \\
\hline 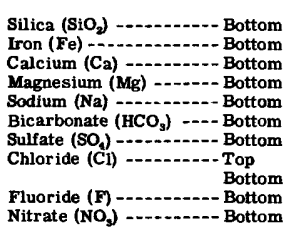 & $\begin{array}{l}3.9 \\
17^{.02} \\
5.2 \\
4.0 \\
40 \\
32 \\
4.0 \\
4.5 \\
. .1 \\
5.1\end{array}$ & $\begin{array}{l}4.0 \\
4.0\end{array}$ & $\begin{array}{l}3.4 \\
17^{.02} \\
5.2 \\
3.3 \\
40 \\
30 \\
4.0 \\
4.0 \\
.1 \\
5.1\end{array}$ & $\begin{array}{l}4.0 \\
4.0\end{array}$ & $\begin{array}{l}4.7 \\
17^{.02} \\
5.2 \\
3.2 \\
40 \\
30 \\
4.0 \\
3.9 \\
.1 \\
4.3\end{array}$ & $\begin{array}{ll}\text { B. } & 4.0 \\
\text { B. } & .02 \\
\text { B. } & 17 \\
\text { B. } & 5.2 \\
\text { B. } & 3.5 \\
\text { B. } & 40 \\
\text { B. } & 31 \\
\text { T. } & 4.0 \\
\text { B. } & 4.1 \\
\text { B. } & .1 \\
\text { B. } & 4.8\end{array}$ \\
\hline $\begin{array}{l}\text { Dissolved solids }-\ldots-\ldots \text { Bottom } \\
\text { Hardness as } \mathrm{CaCO}_{3}-\cdots \text { - Bottom } \\
\text { Suspended sediment }-\cdots-\text { Top }\end{array}$ & $\begin{array}{r}108 \\
64 \\
. \\
\end{array}$ & & $\begin{array}{r}101 \\
64 \\
\cdot C \mathrm{Co}\end{array}$ & te. . & $\begin{array}{r}105 \\
64 \\
\cdots \cdots\end{array}$ & $\begin{array}{rr}\text { B. } & 105 \\
\text { B. } & 64 \\
\text { T. } & 24\end{array}$ \\
\hline
\end{tabular}


Table 7. -- WATER ANALYSES OF DELAWARE RIVR BETWEEN BRISTOL AND MARCUS HOOK, PA. --Continued Analyzed by City of Philadelphia and U. S. Geological Survey; analyses in parts per million

Location Bristol, Pu.-Burlington, N. J. Bridge Date September 6, 1951 Sampling study No. 25 Weather $\mathrm{kain} \quad$ Water discharge at Trenton (cfs) $-3,810$

\begin{tabular}{|c|c|c|c|c|c|c|}
\hline \multirow[b]{3}{*}{ 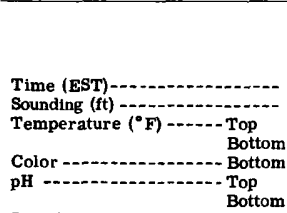 } & \multicolumn{6}{|c|}{ Station } \\
\hline & \begin{tabular}{|c|}
$\begin{array}{c}\text { Pennsylvania } \\
\text { side }\end{array}$ \\
\end{tabular} & West Center & Center & East Center & \begin{tabular}{|c|}
$\begin{array}{c}\text { New Jersey } \\
\text { side }\end{array}$ \\
\end{tabular} & Average \\
\hline & $\begin{array}{c}11: 28 \text { a.m. } \\
9 \\
74 \\
74 \\
5 \\
7.2 \\
7.9\end{array}$ & $\begin{array}{c}11: 26 \text { a.m. } \\
25 \\
74 \\
74 \\
6.9 \\
7.6\end{array}$ & $\begin{array}{c}11: 24 \text { a.m. } \\
41 \\
75 \\
74 \\
5 \\
6.9 \\
8.0\end{array}$ & $\begin{array}{c}11: 22 \mathrm{a} . \mathrm{m} . \\
39 \\
75 \\
75 \\
7.0 \\
7.7\end{array}$ & $\begin{array}{c}11: 20 \mathrm{a} . \mathrm{m} . \\
15 \\
75 \\
75 \\
5 \\
7.2 \\
8.0\end{array}$ & $\begin{array}{lc}\text { T. } & 75 \\
\text { B. } & 74 \\
\text { B. } & 5 \\
\text { T. } & 7.0 \\
\text { B. } & 7.8\end{array}$ \\
\hline $\begin{array}{ll}\left.\text { (micromhos at } 25^{\circ} \mathrm{C}\right) & \begin{array}{l}\text { Top } \\
\text { Bottom }\end{array} \\
\text { Dissolved oxygen - - } & \text { Top } \\
\left.\text { B. O.D. (5-day, } 20^{\circ} \mathrm{C}\right) & - \text { Top }\end{array}$ & $\begin{array}{r}252 \\
232 \\
6.1 \\
5.9\end{array}$ & $\begin{array}{l}207 \\
185 \\
6.8 \\
3.3\end{array}$ & $\begin{array}{l}206 \\
201 \\
6.8 \\
2.9\end{array}$ & $\begin{array}{r}206 \\
194 \\
6.6 \\
2.5\end{array}$ & $\begin{array}{l}206 \\
202 \\
7.3 \\
3.5\end{array}$ & $\begin{array}{lc}\text { T. } & 215 \\
\text { B. } & 203 \\
\text { T. } & 6.7 \\
\text { T. } & 3.6\end{array}$ \\
\hline 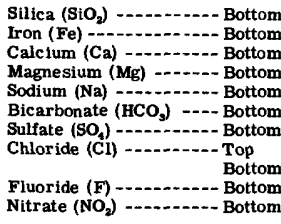 & $\begin{array}{l}3.1 \\
20.02 \\
7.3 \\
13 \\
62 \\
37 \\
10 \\
12 \\
.1 \\
5.4\end{array}$ & $\begin{array}{l}6.0 \\
7.0\end{array}$ & $\begin{array}{l}3.6 \\
.01 \\
20 \\
7.2 \\
7.0 \\
61 \\
31 \\
6.0 \\
7.5 \\
.1 \\
4.9\end{array}$ & $\begin{array}{l}6.0 \\
7.0\end{array}$ & $\begin{array}{l}2.9 \\
.02 \\
20 \\
7.2 \\
6.8 \\
60 \\
32 \\
6.0 \\
7.2 \\
.1 \\
4.8\end{array}$ & $\begin{array}{lc}\text { B. } & 3.2 \\
\text { B. } & .02 \\
\text { B. } & 20 \\
\text { B. } & 7.2 \\
\text { B. } & 8.9 \\
\text { B. } & 61 \\
\text { B. } & 33 \\
\text { T. } & 6.8 \\
\text { B. } & 8.1 \\
\text { B. } & .1 \\
\text { B. } & 5.0\end{array}$ \\
\hline 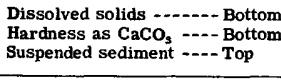 & $\begin{array}{r}138 \\
80 \\
.\end{array}$ & & $\begin{array}{r}131 \\
80 \\
. \quad \text { Cor }\end{array}$ & site . . & $\begin{array}{r}130 \\
80 \\
\cdots\end{array}$ & $\begin{array}{lr}\text { B. } & 133 \\
\text { B. } & 80 \\
\text { T. } & 16\end{array}$ \\
\hline
\end{tabular}

Location Bristol, Pa.-Burlington, N. J. Bridge Date October 2, 1951_. Sampling study No. 26 Sampling study No. $\frac{26}{2,990}$ Weather Clear

\begin{tabular}{|c|c|c|c|c|c|c|}
\hline \multirow[b]{3}{*}{$\begin{array}{l}\text { Time (EST) } \\
\text { Sounding (ft) } \\
\text { Temperature }\left({ }^{\circ} \mathrm{F}\right) \\
\text { Color } \\
\text { pH }-\begin{array}{c}\text { Top } \\
\text { Bottom }\end{array} \\
\begin{array}{c}\text { Bottom } \\
\text { B }\end{array}\end{array}$} & \multicolumn{6}{|c|}{ Station } \\
\hline & \begin{tabular}{|c|}
$\begin{array}{c}\text { Pennsylvania } \\
\text { side }\end{array}$ \\
\end{tabular} & West Center & Center & East Center & \begin{tabular}{|c|}
$\begin{array}{c}\text { New Jersey } \\
\text { slde }\end{array}$ \\
\end{tabular} & Average \\
\hline & $\begin{array}{c}11: 44 \text { a.m. } \\
22 \\
70 \\
68 \\
8 \\
6.7 \\
7.9\end{array}$ & $\begin{array}{c}11: 42 \text { a.m. } \\
30 \\
69 \\
67 \\
6.9 \\
6.8\end{array}$ & $\begin{array}{c}11: 39 \text { a.m. } \\
45 \\
70 \\
67 \\
9 \\
6.8 \\
7.9\end{array}$ & $\begin{array}{c}11: 36 \text { a.m. } \\
30 \\
69 \\
67 \\
6.9 \\
6.8\end{array}$ & $\begin{array}{c}11: 33 \text { a. a. } \\
13 \\
70 \\
68 \\
6 \\
6.8 \\
7.9\end{array}$ & $\begin{array}{ll}\text { T. } & 70 \\
\text { B. } & 67 \\
\text { B. } & 8 \\
\text { T. } & 6.8 \\
\text { B. } & 7.5\end{array}$ \\
\hline $\begin{array}{l}\text { Specific conductance } \\
\quad \text { (micromhos at } 25^{\circ} \mathrm{C} \text { ) } \begin{array}{l}\text { Top } \\
\text { Bottom }\end{array} \\
\text { Dissolved oxygen }-10^{\circ} \mathrm{C} \text {-- Top } \\
\text { B. - Top }\left(5 \text {-day, } 20^{\circ} \text { - Top }\right.\end{array}$ & $\begin{array}{r}217 \\
217 \\
7.5 \\
3.7\end{array}$ & $\begin{array}{r}216 \\
202 \\
8.0 \\
4.1\end{array}$ & $\begin{array}{l}226 \\
209 \\
7.8 \\
2.9\end{array}$ & $\begin{array}{r}212 \\
192 \\
7.8 \\
1.8\end{array}$ & $\begin{array}{l}208 \\
202 \\
7.7 \\
2.3\end{array}$ & $\begin{array}{ll}\text { T. } & 216 \\
\text { B. } & 204 \\
\text { T. } & 7.8 \\
\text { T. } & 3.0\end{array}$ \\
\hline 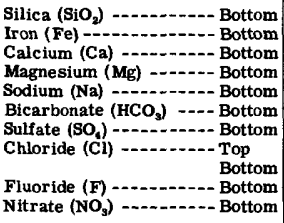 & $\begin{array}{l}3.2 \\
.04 \\
7.2 \\
9.2 \\
60 \\
37 \\
10 \\
11 \\
.0 \\
5.3\end{array}$ & $\begin{array}{l}10 \\
11\end{array}$ & $\begin{array}{l}6.0 \\
.05 \\
21 \\
7.3 \\
8.3 \\
62 \\
37 \\
11 \\
10 \\
.0 \\
5.2\end{array}$ & $\begin{array}{l}10 \\
11\end{array}$ & $\begin{array}{l}3.2 \\
.05 \\
21 \\
7.2 \\
8.3 \\
60 \\
36 \\
9.0 \\
10 \\
.0 \\
8.9\end{array}$ & $\begin{array}{ll}\text { B. } & 4.1 \\
\text { B. } & .05 \\
\text { B. } & 21 \\
\text { B. } & 7.2 \\
\text { B. } & 8.6 \\
\text { B. } & 61 \\
\text { B. } & 37 \\
\text { T. } & 10 \\
\text { B. } & 11 \\
\text { B. } & .0 \\
\text { B. } & 6.5\end{array}$ \\
\hline $\begin{array}{l}\text { Dissolved solids - } \\
\text { Hardness as } \mathrm{CaCO}_{3}--- \text { Bottom } \\
\text { Suspended sediment }-\cdots \text { - Top }\end{array}$ & $\begin{array}{r}130 \\
82 \\
\cdots\end{array}$ & & $\begin{array}{l}209 \\
82 \\
. \quad \mathrm{Con}\end{array}$ & . & $\begin{array}{r}202 \\
82 \\
+. \\
\end{array}$ & $\begin{array}{rr}\text { B. } & 180 \\
\text { B. } & 82 \\
\text { T. } & 25 \\
\end{array}$ \\
\hline
\end{tabular}


Table 7. --WATER ANALYSES OF DELAWARE RIVER BETWEEN BRISTOL, AND MARCUS HOOK, PA. --Continued Analyzed by City of Philadelphia and U. S. Geological Survey; analyses in parts per million

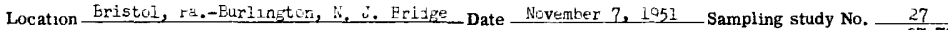
Weather_Cloudy _-_ W Water discharge at Trenton (cfs) 37,700

\begin{tabular}{|c|c|c|c|c|c|c|}
\hline & \multicolumn{6}{|c|}{ Station } \\
\hline & $\begin{array}{c}\text { Pennsylvania } \\
\text { side }\end{array}$ & West Center & Center & East Center & \begin{tabular}{|c}
$\begin{array}{c}\text { New Jersey } \\
\text { side }\end{array}$ \\
\end{tabular} & Average \\
\hline $\begin{array}{l}\text { Time (EST) } \\
\text { Sounding (ft) } \\
\text { Temperature }\left({ }^{\circ} \mathrm{F}\right) \\
\text { Color } \\
\text { pH }\end{array}$ & $\begin{array}{c}12: 55 \text { H. } \pi . \\
15 \\
40 \\
40 \\
8 \\
0.0 \\
7.1\end{array}$ & $\begin{array}{l}12: 5 \mathrm{u} \text { p.m. } \\
24 \\
45 \\
44 \\
\\
0.5 \\
0.9\end{array}$ & $\begin{array}{c}12: 45 \text { F.m. } \\
4 I \\
45 \\
44 \\
8 \\
0.0 \\
7.0\end{array}$ & $\begin{array}{c}12: 40 \text { p.m. } \\
4 e^{2} \\
48 \\
42 \\
0.0 \\
0.7\end{array}$ & $\begin{array}{c}12: 30 \mathrm{p} \cdot \mathrm{n} . \\
15 \\
46 \\
45 \\
8 \\
6.6 \\
7.3\end{array}$ & $\begin{array}{ll}\text { T. } & 46 \\
\text { B. } & 44 \\
\text { B. } & 8 \\
\text { T. } & 6.5 \\
\text { B. } & 7.0\end{array}$ \\
\hline $\begin{array}{l}\begin{array}{l}\text { Specific conductance } \\
\text { (micromhos at } 25^{\circ} \mathrm{C} \text { ) }\end{array} \\
\begin{aligned} \text { Top } \\
\text { Bottom } \\
\text { Dissolved oxygen --- - Top } \\
\text { B. O. D. (5-day, } 20^{\circ} \mathrm{C} \text { ) - - Top }\end{aligned}\end{array}$ & $\begin{array}{r}1<8 \\
124 \\
11.2 \\
8.3\end{array}$ & $\begin{array}{r}95.8 \\
89.9 \\
11.5 \\
2.2\end{array}$ & $\begin{array}{r}94.0 \\
77.2 \\
11.2 \\
1.8\end{array}$ & $\begin{array}{l}76.0 \\
9 ? .2 \\
11.5 \\
1.8\end{array}$ & $\begin{array}{r}93.0 \\
95.6 \\
11.2 \\
1.2\end{array}$ & $\begin{array}{lr}\text { T. } & 101 \\
\text { B. } & 100 \\
\text { T. } & 11.4 \\
\text { T. } & 3.1\end{array}$ \\
\hline 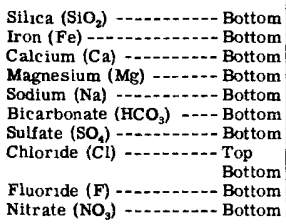 & $\begin{array}{l}4.8 \\
.03 \\
7.6 \\
2.9 \\
7.2 \\
11 \\
27 \\
7.0 \\
7.1 \\
. .1 \\
6.5\end{array}$ & $\begin{array}{l}3.0 \\
3.0\end{array}$ & $\begin{array}{l}5.3 \\
.02 \\
9.8 \\
3.1 \\
2.4 \\
20 \\
19 \\
2.0 \\
3.1 \\
.1 \\
4.6\end{array}$ & $\begin{array}{l}3.0 \\
3.0\end{array}$ & $\begin{array}{l}4.8 \\
.02 \\
9.2 \\
3.1 \\
2.4 \\
20 \\
19 \\
2.0 \\
3.0 \\
.1 \\
4.1\end{array}$ & $\begin{array}{ll}\text { B. } & 5.0 \\
\text { B. } & .02 \\
\text { B. } & 9.5 \\
\text { B. } & 3.0 \\
\text { B. } & 4.0 \\
\text { B. } & 17 \\
\text { B. } & 22 \\
\text { T. } & 3.4 \\
\text { B. } & 3.8 \\
\text { B. } & .1 \\
\text { B. } & 5.1\end{array}$ \\
\hline $\begin{array}{l}\text { Dissolved solids } \\
\text { Hardness as } \mathrm{CaCO}_{3} \ldots \text { Bottom } \\
\text { Suspended sediment } \ldots \text { Top }\end{array}$ & $\begin{array}{l}86 \\
36 \\
.\end{array}$ & 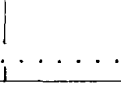 & $\begin{array}{l}75 \\
37 \\
\end{array}$ & 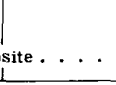 & $\begin{array}{l}70 \\
36 \\
.\end{array}$ & $\begin{array}{ll}\text { B. } & 77 \\
\text { B. } & 36 \\
\text { T. } & 29\end{array}$ \\
\hline
\end{tabular}

Location Bristol, Fa.-Eurlington, N. J. Bridge Date December 5, 1951 _. Sampling study No. 28 Weather Razn

study No. $\frac{28}{12}-1$

Station

\begin{tabular}{|c|c|c|c|c|c|c|}
\hline & \multicolumn{6}{|c|}{ Station } \\
\hline & $\begin{array}{c}\text { Pennsyivania } \\
\text { stde }\end{array}$ & West Center & Center & East Center & $\begin{array}{c}\text { New Jersey } \\
\text { side }\end{array}$ & Average \\
\hline $\begin{array}{l}\text { Time (EST) } \\
\text { Sounding }(\mathrm{ft}) \\
\text { Temperature }\left({ }^{\circ} \mathrm{F}\right) \\
\begin{array}{r}\text { Color } \\
\text { pH }\end{array} \\
\begin{array}{l}\text { Bottom } \\
\text { Bottom }\end{array}\end{array}$ & $\begin{array}{l}1: 14 \\
15 \\
42 \\
42 \\
9 \\
6.9 \\
7.6\end{array}$ & $\begin{array}{l}1: 12 \text { p.m. } \\
25 \\
42 \\
42 \\
\\
7.9 \\
7.3\end{array}$ & $\begin{array}{l}1: 10 \mathrm{p} \cdot \mathrm{m} . \\
41 \\
42 \\
44 \\
8 \\
7.0 \\
7.6\end{array}$ & $\begin{array}{l}1: 08 \mathrm{~F} . \mathrm{m} . \\
34 \\
43 \\
42 \\
7.0 \\
7.5\end{array}$ & $\begin{array}{l}1: 06 \mathrm{p} . \mathrm{m} . \\
26 \\
43 \\
43 \\
8 \\
6.7 \\
7.5\end{array}$ & $\begin{array}{ll}\text { T. } & 42 \\
\text { B. } & 43 \\
\text { B. } & 8 \\
\text { T. } & 6.9 \\
\text { B. } & 7.5\end{array}$ \\
\hline 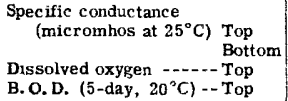 & $\begin{array}{l}131 \\
136 \\
12.0 \\
3.8\end{array}$ & $\begin{array}{l}128 \\
123 \\
11.8 \\
2.6\end{array}$ & $\begin{array}{l}128 \\
128 \\
12.2 \\
2.8\end{array}$ & $\begin{array}{r}129 \\
124 \\
12.1 \\
3.2\end{array}$ & $\begin{array}{r}131 \\
130 \\
12.2 \\
4.0\end{array}$ & $\begin{array}{l}\text { T. } 129 \\
\text { B. } 128 \\
\text { T. } 12.1 \\
\text { T. } 3.3\end{array}$ \\
\hline 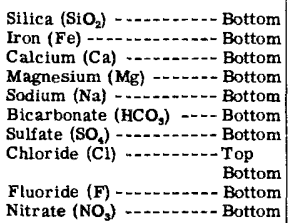 & $\begin{array}{l}5.7 \\
13 \\
4.02 \\
4.0 \\
34 \\
24 \\
4.0 \\
4.9 \\
.0 \\
3.6\end{array}$ & $\begin{array}{l}4.0 \\
3.0\end{array}$ & $\begin{array}{c}5.7 \\
.02 \\
13 \\
4.3 \\
3.4 \\
34 \\
23 \\
4.0 \\
3.8 \\
.0 \\
3.5\end{array}$ & $\begin{array}{l}4.0 \\
3.0\end{array}$ & $\begin{array}{l}6.5 \\
13 \\
4.02 \\
3.6 \\
32 \\
24 \\
4.0 \\
3.8 \\
.0 \\
3.8\end{array}$ & $\begin{array}{ll}\text { B. } & 6.0 \\
\text { B. } & .02 \\
\text { B. } & 13 \\
\text { B. } & 4.2 \\
\text { B. } & 3.9 \\
\text { B. } & 33 \\
\text { B. } & 24 \\
\text { T. } & 4.0 \\
\text { B. } & 3.7 \\
\text { B. } & .0 \\
\text { B. } & 3.6\end{array}$ \\
\hline $\begin{array}{l}\text { Dissolved solids }-\ldots-1 \text { Bottom } \\
\text { Hardness as } \mathrm{CaCO}_{3}-- \text { Bottom } \\
\text { Suspended sediment }-\ldots \text { Top }\end{array}$ & $\begin{array}{r}89 \\
50 \\
. \\
\end{array}$ & & $\begin{array}{l}87 \\
50 \\
\quad \operatorname{Cor}\end{array}$ & 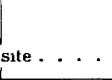 & $\begin{array}{l}97 \\
49 \\
\cdots\end{array}$ & $\begin{array}{ll}\text { B. } & 91 \\
\text { B. } & 50 \\
\text { T. } & 13\end{array}$ \\
\hline
\end{tabular}

$3153910-54-5$ 
Table 7. --WATER ANALYSES OF DELAWARE RIVER BETWEEN BRISTOL AND MARCUS HOOK, PA. --Continued Analyzed by City of Philadelphia and U. S. Geological Survey; analyses in parts per million

Location Bristol, Pa,-Burlington, d, J. Bridge Date January 3, 1952 _ Sampling study No. _ 29 Weather Clear $\quad$ Water discharge at Trenton (cfs) 30,500

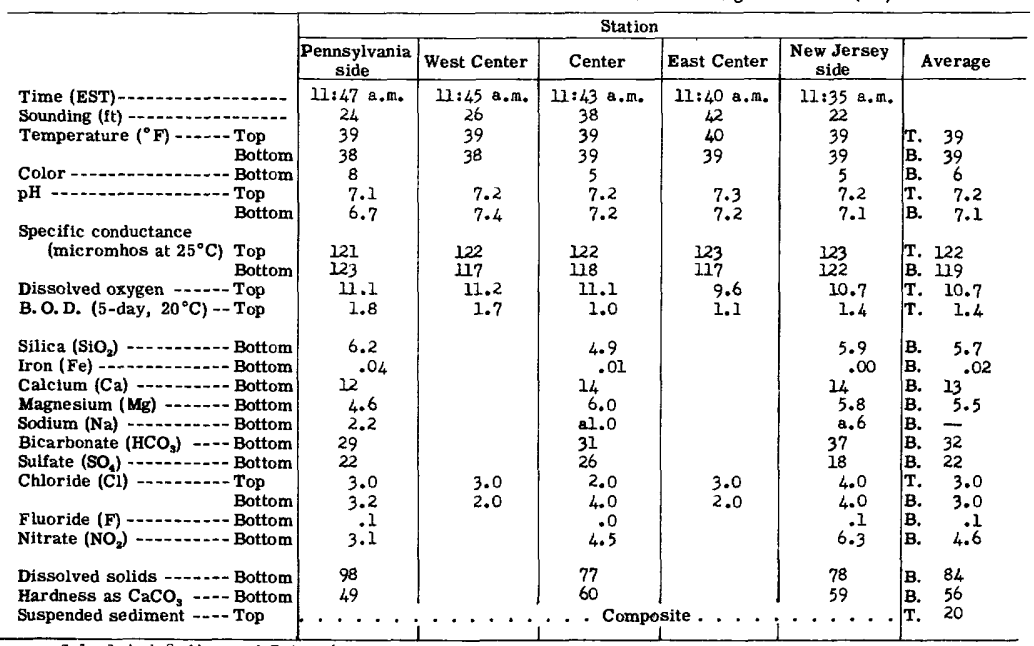

a Calculated Sodium and Potassium.

Location Bristol, Pa.-Burlington, N, J. Bridge Date February 5, 1952 Sampling study No. 30 Weather Clear

\begin{tabular}{|c|c|c|c|c|c|c|}
\hline \multirow[b]{3}{*}{ 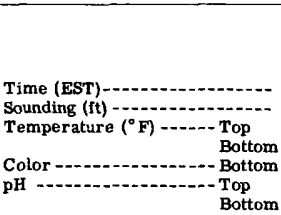 } & \multicolumn{6}{|c|}{ Station } \\
\hline & \begin{tabular}{|c}
$\begin{array}{c}\text { Pennsylvania } \\
\text { side }\end{array}$ \\
\end{tabular} & West Center & Center & East Center & $\begin{array}{c}\text { New Jersey } \\
\text { side }\end{array}$ & Average \\
\hline & $\begin{array}{c}10: 17 \text { a.m. } \\
22 \\
40 \\
40 \\
12 \\
6.6 \\
6.2\end{array}$ & $\begin{array}{c}10: 15 \mathrm{a} . \mathrm{m} . \\
30 \\
41 \\
40 \\
\\
6.4 \\
7.6\end{array}$ & $\begin{array}{c}10: 10 \mathrm{a} . \mathrm{m} \\
48 \\
40 \\
41 \\
12 \\
6.5 \\
6.0\end{array}$ & $\begin{array}{c}10: 02 \text { a.m. } \\
42 \\
40 \\
40 \\
6.5 \\
7.5\end{array}$ & $\begin{array}{c}10: 00 \text { a.m. } \\
18 \\
42 \\
41 \\
12 \\
6.4 \\
5.9\end{array}$ & $\begin{array}{ll}\text { T. } & 47 \\
\text { B. } & 40 \\
\text { B. } & 12 \\
\text { T. } & 6.5 \\
\text { B. } & 6.6\end{array}$ \\
\hline $\begin{array}{l}\text { Specific conductance } \\
\text { (micromhos at } 25^{\circ} \mathrm{C} \text { ) } \begin{array}{l}\text { Top } \\
\text { Bottom }\end{array}\end{array}$ & $\frac{106}{105}$ & $\begin{array}{l}105 \\
102\end{array}$ & $\frac{116}{104}$ & $\begin{array}{l}106 \\
103\end{array}$ & $\begin{array}{l}103 \\
107\end{array}$ & $\begin{array}{l}\text { T. } 107 \\
\text { B. } 104\end{array}$ \\
\hline $\begin{array}{l}\text { Dissolved oxygen - - Top } \\
\left.\text { B. O.D. (5-day, } 20^{\circ} \mathrm{C}\right)- \text { Top }\end{array}$ & $\begin{array}{r}12.5 \\
3.2\end{array}$ & $\begin{array}{r}12.4 \\
3.3\end{array}$ & $\begin{array}{r}12.1 \\
2.7\end{array}$ & $\begin{array}{r}12.4 \\
4.1\end{array}$ & $\begin{array}{r}11.6 \\
2.4\end{array}$ & $\begin{array}{rr}\text { T. } & 12.2 \\
\text { T. } & 3.1\end{array}$ \\
\hline 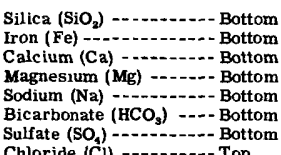 & $\begin{array}{l}5.8 \\
.06 \\
9.8 \\
4.2 \\
2.4 \\
23 \\
20 \\
3.0\end{array}$ & & $\begin{array}{l}5.7 \\
.07 \\
9.2 \\
4.5 \\
1.9 \\
21 \\
20\end{array}$ & & $\begin{array}{l}4.0 \\
.02 \\
9.8 \\
4.0 \\
2.0 \\
20 \\
21\end{array}$ & $\begin{array}{ll}\text { B. } & 5.2 \\
\text { B. } & .05 \\
\text { B. } & 9.6 \\
\text { B. } & 4.2 \\
\text { B. } & 2.1 \\
\text { B. } & 21 \\
\text { B. } & 20 \\
\text { T. }\end{array}$ \\
\hline 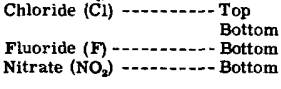 & $\begin{array}{l}3.0 \\
3.5 \\
.1 \\
3.4\end{array}$ & $\begin{array}{l}2.0 \\
3.0\end{array}$ & $\begin{array}{r}6.0 \\
3.5 \\
. .1 \\
3.4\end{array}$ & $\begin{array}{l}2.0 \\
3.0\end{array}$ & $\begin{array}{r}3.0 \\
3.5 \\
.1 \\
3.6\end{array}$ & $\begin{array}{lr}\text { T. } & 3.2 \\
\text { B. } & 3.3 \\
\text { B. } & .1 \\
\text { B. } & 3.5\end{array}$ \\
\hline $\begin{array}{l}\text { Dissolved solids }-\ldots-\text { Bottom } \\
\text { Hardness as } \mathrm{CaCO}_{9} \ldots-\text { Bottom } \\
\text { Suspended sediment } \ldots \text { - Top }\end{array}$ & $\begin{array}{l}79 \\
42 \\
\cdot .\end{array}$ & & $\begin{array}{l}76 \\
4 I \text { Com } \\
\end{array}$ & - & $\begin{array}{l}78 \\
41 \\
.\end{array}$ & $\begin{array}{ll}\text { B. } & 78 \\
\text { B. } & 41 \\
\text { T. } & 73 \\
\end{array}$ \\
\hline
\end{tabular}


Table 7. -- WATER ANALYSES OF DELAWARE RIVR BETWEEN BRISTOL AND MARCUS HOOK, PA. --Continued Analyzed by City of Philadelphia and U. S. Geological Survey; analyses in parts per million

Location Bristol, Pa.-Burlington, N. J. Bridge Date March 5, 1952 Sampling study No. 31

\begin{tabular}{|c|c|c|c|c|c|c|}
\hline \multirow[t]{3}{*}{ Weather Overcast } & \multicolumn{6}{|c|}{ Water discharge at Trenton (cfs) 9,730} \\
\hline & \multicolumn{6}{|c|}{ Station } \\
\hline & $\begin{array}{c}\text { Pennsylvania } \\
\text { side }\end{array}$ & West Center & Center & East Center & $\begin{array}{c}\text { New Jersey } \\
\text { side }\end{array}$ & Average \\
\hline $\begin{array}{l}\begin{array}{l}\text { Time (EST) } \\
\text { Sounding (ft) } \\
\text { Temperature }\left({ }^{\circ} \mathrm{F}\right)-\end{array} \\
\begin{array}{l}\text { Bop } \\
\text { Coltom } \\
\text { pH }\end{array}\end{array}$ & $\begin{array}{c}10: 41 \text { a.m. } \\
12 \\
39 \\
38 \\
4 \\
6.6 \\
7.6\end{array}$ & $\begin{array}{c}10.38 \text { a.m. } \\
40 \\
39 \\
38 \\
\\
6.7 \\
7.8\end{array}$ & $\begin{array}{c}10: 35 \text { a.m. } \\
40 \\
39 \\
38 \\
3 \\
6.7 \\
7.9\end{array}$ & $\begin{array}{c}10: 33 \text { a.m. } \\
35 \\
40 \\
39 \\
6.8 \\
7.7\end{array}$ & $\begin{array}{c}10: 30 \text { a.m. } \\
23 \\
40 \\
39 \\
4 \\
6.8 \\
7.8\end{array}$ & $\begin{array}{ll}\text { T. } & 39 \\
\text { B. } & 38 \\
\text { B. } & 4 \\
\text { T. } & 6.7 \\
\text { B. } & 7.8\end{array}$ \\
\hline 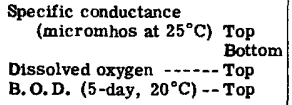 & $\begin{array}{l}155 \\
153 \\
11.2 \\
2.8\end{array}$ & $\begin{array}{l}157 \\
150 \\
11.6 \\
3.0\end{array}$ & $\begin{array}{r}155 \\
147 \\
10.0 \\
.7\end{array}$ & $\begin{array}{l}153 \\
143 \\
10.9 \\
1.6\end{array}$ & $\begin{array}{r}154 \\
143 \\
13.4 \\
1.8\end{array}$ & $\begin{array}{ll} & \\
\text { T. } & 155 \\
\text { B. } & 147 \\
\text { T. } & 11.0 \\
\text { T. } & 2.0\end{array}$ \\
\hline 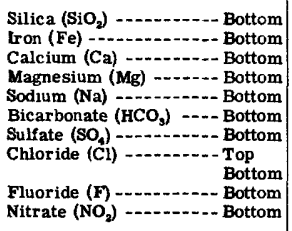 & $\begin{array}{l}5.0 \\
.08 \\
14 \\
5.2 \\
5.8 \\
38 \\
27 \\
6.0 \\
6.4 \\
.0 \\
5.3\end{array}$ & $\begin{array}{l}6.0 \\
7.0\end{array}$ & $\begin{array}{l}4.6 \\
.10 \\
14 \\
5.0 \\
5.8 \\
38 \\
26 \\
6.0 \\
5.9 \\
.1 \\
4.8\end{array}$ & $\begin{array}{l}5.0 \\
6.0\end{array}$ & $\begin{array}{l}4.8 \\
.08 \\
15 \\
4.9 \\
4.0 \\
37 \\
25 \\
5.0 \\
5.0 \\
.1 \\
4.6\end{array}$ & $\begin{array}{lc}\text { B. } & 4.8 \\
\text { B. } & .09 \\
\text { B. } & 14 \\
\text { B. } & 5.0 \\
\text { B. } & 5.2 \\
\text { B. } & 38 \\
\text { B. } & 26 \\
\text { T. } & 5.6 \\
\text { B. } & 6.1 \\
\text { B. } & .1 \\
\text { B. } & 4.9\end{array}$ \\
\hline $\begin{array}{l}\text { Dissolved solids }-1-\text { Bottom } \\
\text { Hardness as } \mathrm{CaCO}_{3}, \ldots-\text { Bottom } \\
\text { Suspended sediment } \ldots \ldots \text { Top }\end{array}$ & $\begin{array}{l}89 \\
56 \\
.\end{array}$ & 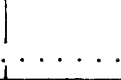 & $\begin{array}{l}86 \\
56 \\
. \quad C o n\end{array}$ & 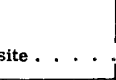 & $\begin{array}{l}85 \\
58 \\
\cdots\end{array}$ & $\begin{array}{lr}\text { B. } & 87 \\
\text { B. } & 57 \\
\text { T. } & 9\end{array}$ \\
\hline
\end{tabular}

Location Bristol, Pa.-Burlington, N. J. Bridge Date April 2, 1952 Sampling study No. 32

\begin{tabular}{|c|c|c|c|c|c|c|}
\hline \multirow[t]{3}{*}{ Weather Overcast } & \multicolumn{6}{|c|}{ Water discharge at Trenton (cfs) 22,700} \\
\hline & \multicolumn{6}{|c|}{ Station } \\
\hline & \begin{tabular}{|c|}
$\begin{array}{c}\text { Pennsylvania } \\
\text { side }\end{array}$ \\
\end{tabular} & West Center & Center & East Center & $\begin{array}{c}\text { New Jersey } \\
\text { side }\end{array}$ & Average \\
\hline $\begin{array}{l}\text { Time (EST) } \\
\text { Sounding (ft) } \\
\text { Temperature }\left({ }^{\circ} \mathrm{F}\right) \\
\begin{array}{l}\text { Color } \\
\text { pH }\end{array}\end{array}$ & $\begin{array}{c}10: 42 \text { a.m. } \\
14 \\
49 \\
48 \\
4 \\
6.5 \\
7.0\end{array}$ & $\begin{array}{c}10: 39 \text { a.m. } \\
29 \\
48 \\
48 \\
\\
6.8 \\
7.5\end{array}$ & $\begin{array}{c}10.36 \text { a.m. } \\
43 \\
49 \\
48 \\
3 \\
6.7 \\
7.1\end{array}$ & $\begin{array}{c}10: 33 \text { a.th. } \\
36 \\
49 \\
48 \\
6.8 \\
7.6\end{array}$ & $\begin{array}{c}10: 30 \mathrm{a} . \mathrm{m} . \\
10 \\
49 \\
48 \\
4 \\
6.8 \\
7.0\end{array}$ & $\begin{array}{ll}\text { T. } & 49 \\
\text { B. } & 48 \\
\text { B. } & 4 \\
\text { r. } & 6.7 \\
\text { B. } & 7.2\end{array}$ \\
\hline $\begin{array}{l}\begin{array}{l}\text { Specific conductance } \\
\text { (micromhos at } 25^{\circ} \mathrm{C} \text { ) }\end{array} \\
\begin{array}{l}\text { Top } \\
\text { Bottom }\end{array} \\
\text { Dissolved oxygen }-\ldots-\text { Top } \\
\text { B. O.D. (5-day, } 20^{\circ} \mathrm{C} \text { ) -- Top }\end{array}$ & $\begin{array}{r}105 \\
97.1 \\
10.0 \\
1.9\end{array}$ & $\begin{array}{r}101 \\
99.7 \\
9.9 \\
.9\end{array}$ & $\begin{array}{r}98.9 \\
94.7 \\
9.7 \\
.5\end{array}$ & $\begin{array}{r}99.2 \\
96.7 \\
9.4 \\
.6\end{array}$ & $\begin{array}{r}98.6 \\
93.9 \\
8.5 \\
.0\end{array}$ & $\begin{array}{rr} & \\
\text { T. } & 101 \\
\text { B. } & 96.4 \\
\text { r. } & 9.5 \\
\text { r. } & .8\end{array}$ \\
\hline 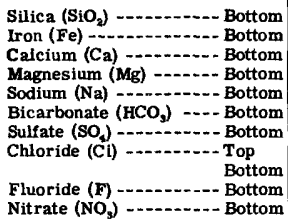 & $\begin{array}{l}4.6 \\
.04 \\
9.7 \\
2.9 \\
4.6 \\
26 . \\
18 \\
4.0 \\
3.5 \\
.0 \\
3.8\end{array}$ & $\begin{array}{l}3.0 \\
4.0\end{array}$ & $\begin{array}{l}4.6 \\
.06 \\
9.8 \\
3.6 \\
2.2 \\
23 \\
19 \\
3.0 \\
3.2 \\
.0 \\
3.5\end{array}$ & $\begin{array}{l}3.0 \\
4.0\end{array}$ & $\begin{array}{l}4.4 \\
.04 \\
9.8 \\
3.1 \\
3.6 \\
26 \\
18 \\
3.0 \\
2.8 \\
.0 \\
3.6\end{array}$ & $\begin{array}{ll}\text { R. } & 4.5 \\
\text { B. } & .05 \\
\text { B. } & 9.8 \\
\text { R. } & 3.2 \\
\text { R. } & 3.5 \\
\text { R. } & 25 \\
\text { B. } & 18 \\
\text { R. } & 3.2 \\
\text { B. } & 3.5 \\
\text { B. } & .0 \\
\text { B. } & 3.6\end{array}$ \\
\hline $\begin{array}{l}\text { Dissolved solids }-\cdots-\text { Bottom } \\
\text { Hardness as } \mathrm{CaCO}_{3}-\cdots-\text { Bottom } \\
\text { Suspended sediment }-\cdots-\text { Top }\end{array}$ & $\begin{array}{r}68 \\
36 \\
. \quad \cdot\end{array}$ & & $\begin{array}{l}66 \\
39 \\
. \quad C o n\end{array}$ & & $\begin{array}{l}71 \\
37 \\
.\end{array}$ & $\begin{array}{ll}\text { A. } & 68 \\
\text { B. } & 37 \\
\text { T. } & 13\end{array}$ \\
\hline
\end{tabular}


Table 7. --WATER ANALYSES OF DELAWARE RIVER BETWEEN BRISTOL AND MARCUS hOOK, PA. --Continued Analyzed by City of Philadelphia and U. S. Geological Survey; analyses in parts per million

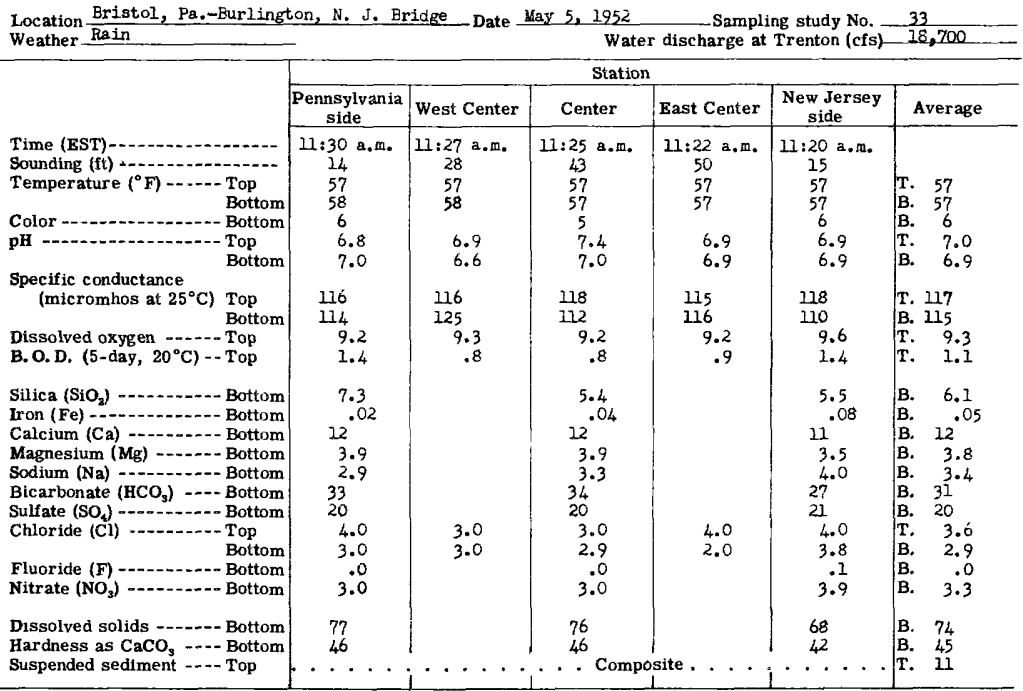

Location Bristol, Pa. -Eurlington, N. J. Bridge_Date June 42 1952 _ Sampling study No. 34 Weather Cloudy $\quad$ Water discharge at Trenton (cfs) 28,600

\begin{tabular}{|c|c|c|c|c|c|c|}
\hline \multicolumn{3}{|l|}{ Weather cloudy } & \multicolumn{4}{|c|}{ Water discharge at Trenton (cfs) 28,600} \\
\hline & \multicolumn{6}{|c|}{ Station } \\
\hline & \begin{tabular}{|c|}
$\begin{array}{c}\text { Pennsylvania } \\
\text { slde }\end{array}$ \\
\end{tabular} & West Center & Center & East Center & $\begin{array}{c}\text { New Jersey } \\
\text { side }\end{array}$ & Average \\
\hline $\begin{array}{l}\text { Time (EST) } \\
\text { Sounding (ft) } \\
\text { Temperature }\left({ }^{\circ} \mathrm{F} \text { ) }\right. \\
\begin{array}{c}\text { Bottom } \\
\text { Color }\end{array} \\
\text { pH } \\
\begin{array}{c}\text { Bottom } \\
\text { Bottom }\end{array}\end{array}$ & $\begin{array}{c}11: 06 \text { a.m. } \\
14 \\
68 \\
69 \\
17 \\
6.8 \\
6.9\end{array}$ & $\begin{array}{c}11: 04 \text { a.m. } \\
25 \\
68 \\
68 \\
\\
6.8 \\
6.8\end{array}$ & $\begin{array}{c}11: 02 \mathrm{a} . \mathrm{m} \\
46 \\
68 \\
67 \\
16 \\
6.7 \\
6.9\end{array}$ & $\begin{array}{c}11: 00 \text { a.m. } \\
31 \\
68 \\
69 \\
6.8 \\
6.8\end{array}$ & $\begin{array}{c}20: 58 \text { a.m. } \\
11 \\
68 \\
68 \\
12 \\
6.8 \\
5.7\end{array}$ & $\begin{array}{lc}\text { T. } & 68 \\
\text { B. } & 68 \\
\text { B. } & 15 \\
\text { T. } & 6.8 \\
\text { B. } & 6.6\end{array}$ \\
\hline 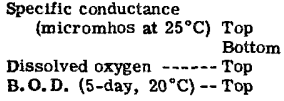 & $\begin{array}{r}86.4 \\
86.6 \\
8.1 \\
1.4\end{array}$ & $\begin{array}{r}86.9 \\
86.6 \\
8.2 \\
1.4\end{array}$ & $\begin{array}{r}83.8 \\
81.6 \\
8.1 \\
1.1\end{array}$ & $\begin{array}{r}82.9 \\
83.6 \\
8.0 \\
1.2\end{array}$ & $\begin{array}{r}87.9 \\
84.3 \\
8.0 \\
1.1\end{array}$ & $\begin{array}{lr}\text { T. } & 85.6 \\
\text { B. } & 84.5 \\
\text { T. } & 8.1 \\
\text { T. } & 1.2\end{array}$ \\
\hline 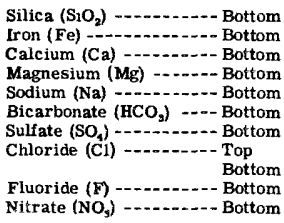 & $\begin{array}{l}5.3 \\
.03 \\
8.8 \\
2.7 \\
3.6 \\
24 \\
16 \\
2.0 \\
3.2 \\
.1 \\
2.2\end{array}$ & $\begin{array}{l}2.0 \\
2.0\end{array}$ & $\begin{array}{l}5.4 \\
.02 \\
8.6 \\
2.6 \\
2.2 \\
20 \\
16 \\
1.0 \\
2.5 \\
. .1 \\
2.1\end{array}$ & $\begin{array}{l}2.0 \\
1.0\end{array}$ & $\begin{array}{l}4.6 \\
.06 \\
8.8 \\
2.8 \\
2.4 \\
24 \\
15 \\
2.0 \\
2.2 \\
.1 \\
2.3\end{array}$ & $\begin{array}{ll}\text { B. } & 5.1 \\
\text { B. } & .04 \\
\text { B. } & 8.7 \\
\text { B. } & 2.7 \\
\text { B. } & 2.7 \\
\text { B. } & 23 \\
\text { B. } & 16 \\
\text { T. } & 1.8 \\
\text { B. } & 2.2 \\
\text { B. } & .1 \\
\text { B. } & 2.2\end{array}$ \\
\hline 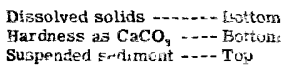 & $\begin{array}{l}56 \\
33\end{array}$ & & $\begin{array}{l}52 \\
32 \\
\cdot \mathrm{Co}\end{array}$ & 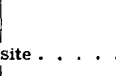 & $\begin{array}{r}53 \\
33 \\
.\end{array}$ & $\begin{array}{ll}\text { B. } & 54 \\
\text { B. } & 33 \\
\text { T. } & 12\end{array}$ \\
\hline
\end{tabular}


Table 7. -- WATER ANALYSES OF DELAWARE RIVER BETWEEN BRISTOL AND MARCUS HOOK, PA. --Continued Analyzed by City of Philadelphia and U. S. Geological Survey; analyses in parts per million

Location-Bristol, Pa.-Burlington, N. J. Bridge_Date July 7, 1952___Sampling study No. 35 Weather-Light Clouds $\quad$ Water discharge at Trenton (cfs) 3.37

\begin{tabular}{|c|c|c|c|c|c|c|}
\hline \multirow[t]{3}{*}{ Weather Light Clouds } & \multicolumn{6}{|c|}{ Water discharge at Trenton (cfs) 3.370} \\
\hline & \multicolumn{6}{|c|}{ Station } \\
\hline & \begin{tabular}{|c|} 
Pennsylvania \\
side
\end{tabular} & West Center & Center & East Center & $\begin{array}{l}\text { New Jersey } \\
\text { side }\end{array}$ & Average \\
\hline $\begin{array}{l}\begin{array}{l}\text { Time (EST) } \\
\text { Sounding }(\mathrm{ft}) \\
\text { Temperature }\left({ }^{\circ} \mathrm{F}\right)\end{array} \\
\begin{array}{r}\text { Bottom } \\
\text { Color }\end{array} \\
\text { pH }\end{array}$ & $\begin{array}{c}11: 34 \text { a.m. } \\
23 \\
80 \\
80 \\
7 \\
6.9 \\
7.3\end{array}$ & $\begin{array}{c}11: 32 \text { a.m. } \\
29 \\
81 \\
81 \\
\\
7.0 \\
6.9\end{array}$ & $\begin{array}{c}11: 30 \mathrm{a} \cdot \mathrm{m} \\
48 \\
81 \\
80 \\
7 \\
7.0 \\
7.3\end{array}$ & $\begin{array}{c}11: 28 \text { a.m. } \\
22 \\
81 \\
81 \\
7.0 \\
6.9\end{array}$ & $\begin{array}{c}11: 26 \text { a.m. } \\
16 \\
81 \\
80 \\
7 \\
7.2 \\
7.2\end{array}$ & $\begin{array}{lc}\text { T. } & 81 \\
\text { B. } & 80 \\
\text { B. } & 7 \\
\text { T. } & 7.0 \\
\text { B. } & 7.1\end{array}$ \\
\hline $\begin{array}{l}\begin{array}{l}\text { Specific conductance } \\
\text { (micromhos at } 25^{\circ} \mathrm{C} \text { ) }\end{array} \\
\begin{array}{l}\text { Top } \\
\text { Bottom }\end{array} \\
\text { Dissolved oxygen }-2-\text { Top } \\
\text { B. O. D. (5-day, } 20^{\circ} \mathrm{C} \text { ) - - Top }\end{array}$ & $\begin{array}{l}192 \\
190 \\
4.4 \\
1.3\end{array}$ & $\begin{array}{r}189 \\
188 \\
4.5 \\
.7\end{array}$ & $\begin{array}{l}188 \\
188 \\
4.7 \\
1.3\end{array}$ & $\begin{array}{r}188 \\
189 \\
4.5 \\
.0\end{array}$ & $\begin{array}{r}197 \\
188 \\
4.5 \\
.4\end{array}$ & $\begin{array}{l}\text { T. } 191 \\
\text { B. } 189 \\
\text { T. } \quad 4.5 \\
\text { T. } \quad .7\end{array}$ \\
\hline 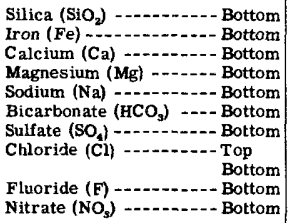 & $\begin{array}{l}5.8 \\
.09 \\
19 \\
7.1 \\
5.6 \\
60 \\
30 \\
6.0 \\
5.2 \\
.01 \\
5.2\end{array}$ & $\begin{array}{l}6.0 \\
6.0\end{array}$ & $\begin{array}{l}5.0 \\
.03 \\
19 \\
7.1 \\
5.4 \\
60 \\
29 \\
6.0 \\
5.2 \\
.1 \\
5.1\end{array}$ & $\begin{array}{l}6.0 \\
6.0\end{array}$ & $\begin{array}{l}5.5 \\
.02 \\
19 \\
7.2 \\
6.4 \\
63 \\
29 \\
6.0 \\
5.5 \\
.1 \\
4.9\end{array}$ & $\begin{array}{lc}\text { B. } & 5.4 \\
\text { B. } & .03 \\
\text { B. } & 19 \\
\text { B. } & 7.1 \\
\text { B. } & 5.8 \\
\text { B. } & 61 \\
\text { B. } & 29 \\
\text { T. } & 6.0 \\
\text { B. } & 5.6 \\
\text { B. } & .1 \\
\text { B. } & 5.1\end{array}$ \\
\hline $\begin{array}{l}\text { Dissolved solids }-\ldots-\text { Bottom } \\
\text { Hardness as } \mathrm{CaCO}_{\mathrm{s}}-\ldots \text { Bottom } \\
\text { Suspended sediment }-\cdots \text { Top }\end{array}$ & $\begin{array}{r}111 \\
77 \\
. \\
\end{array}$ & $\cdots$ & $\stackrel{108}{77} \mathrm{Cor}$ & 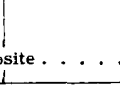 & $\begin{array}{r}110 \\
77\end{array}$ & $\begin{array}{lr}\text { B. } & 110 \\
\text { B. } & 77 \\
\text { T. } & 45\end{array}$ \\
\hline
\end{tabular}

Location Bristol, Pa,-Surlington, N. J. Bridge Date August 6.1952 Sampling study No. 36

\begin{tabular}{|c|c|c|c|c|c|c|}
\hline \multicolumn{3}{|l|}{$\begin{array}{l}\text { Location Bristol } \\
\text { Weather Cloudy }\end{array}$} & \multicolumn{4}{|c|}{$\begin{array}{l}\text { S. } 1952 \text { Sampling study No. }-\frac{36}{4,020} \\
\text { Water discharge at Trenton (cts) }\end{array}$} \\
\hline & \multicolumn{6}{|c|}{ Station } \\
\hline & $\begin{array}{c}\text { Pennsylvania } \\
\text { side }\end{array}$ & West Center & Center & East Center & $\begin{array}{c}\text { New Jersey } \\
\text { side }\end{array}$ & Average \\
\hline 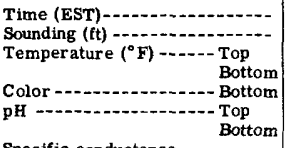 & $\begin{array}{c}11: 05 \mathrm{a} \cdot \mathrm{m} . \\
9 \\
78 \\
78 \\
6 \\
6.7 \\
7.4\end{array}$ & $\begin{array}{l}11: 04 \text { a.m. } \\
22 \\
78 \\
78 \\
6.7 \\
6.9\end{array}$ & $\begin{array}{c}11: 02 \mathrm{a} \cdot \mathrm{m} . \\
30 \\
78 \\
79 \\
5 \\
6.8 \\
7.3\end{array}$ & $\begin{array}{c}11: 00 \text { a.m. } \\
4.1 \\
78 \\
79 \\
6.8 \\
6.9\end{array}$ & $\begin{array}{c}10: 58 \text { a.m. } \\
27 \\
79 \\
79 \\
6 \\
6.8 \\
7.3\end{array}$ & $\begin{array}{ll}\text { T. } & 78 \\
\text { B. } & 79 \\
\text { B. } & 6 \\
\text { T. } & 6.8 \\
\text { B. } & 7.2\end{array}$ \\
\hline $\begin{array}{l}\begin{array}{l}\text { Specific conductance } \\
\quad\left(\text { micromhos at } 25^{\circ} \mathrm{C} \text { ) }\right.\end{array} \\
\begin{array}{l}\text { Top } \\
\text { Bottom }\end{array} \\
\text { Dissolved oxygen }-10^{\circ} \text { - Top } \\
\text { B. O.D. (5-day, } 20^{\circ} \mathrm{C} \text { ) - Top }\end{array}$ & $\begin{array}{l}197 \\
173 \\
5.9 \\
8.2\end{array}$ & $\begin{array}{l}177 \\
171 \\
6.3 \\
5.6\end{array}$ & $\begin{array}{l}173 \\
171 \\
6.3 \\
5.6\end{array}$ & $\begin{array}{l}172 \\
174 \\
6.2 \\
6.3\end{array}$ & $\begin{array}{r}172 \\
171 \\
6.2 \\
6.3\end{array}$ & 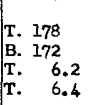 \\
\hline 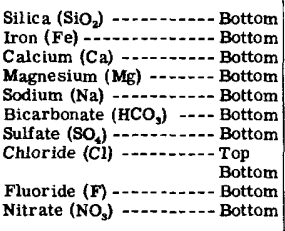 & $\begin{array}{l}4.6 \\
17.01 \\
5.9 \\
6.6 \\
46 \\
30 \\
12 \\
6.8 \\
.1 \\
5.7\end{array}$ & $\begin{array}{l}8.5 \\
7.0\end{array}$ & $\begin{array}{l}4.1 \\
.01 \\
17 \\
6.0 \\
5.6 \\
46 \\
29 \\
7.0 \\
6.6 \\
.1 \\
4.9\end{array}$ & $\begin{array}{l}7.0 \\
7.5\end{array}$ & $\begin{array}{l}4.1 \\
.01 \\
16 \\
5.9 \\
7.0 \\
46 \\
29 \\
7.0 \\
6.8 \\
.1 \\
5.8\end{array}$ & $\begin{array}{lc}\text { B. } & 4.3 \\
\text { B. } & .01 \\
\text { B. } & 17 \\
\text { B. } & 5.9 \\
\text { B. } & 6.4 \\
\text { B. } & 46 \\
\text { B. } & 29 \\
\text { T. } & 8.3 \\
\text { B. } & 6.9 \\
\text { B. } & .1 \\
\text { B. } & 5.5\end{array}$ \\
\hline $\begin{array}{l}\text { Dissolved solids } \ldots . .- \text { Bottom } \\
\text { Hardness as } \mathrm{CaCO}_{3} \ldots-\text { Bottom } \\
\text { Suspended sediment } \ldots-\text { Top }\end{array}$ & $\begin{array}{r}103 \\
67 \\
. \\
\end{array}$ & & $\begin{array}{l}101 \\
67 \\
.\end{array}$ & & $\begin{array}{r}101 \\
64 \\
\cdot \cdot\end{array}$ & $\begin{array}{lr}\text { B. } & 102 \\
\text { B. } & 66 \\
\text { T. } & 18\end{array}$ \\
\hline
\end{tabular}


Table 7. --WATER ANALYSES OF DELAWARE RIVER BETWEEN BRISTOL AND MARCUS HOOK, PA. --ContinUed Analyzed by City of Philadelphia and U. S. Geological Survey; analyses in parts per million

Location Bristol, Fa.-Burlington, N. J. Bridge - Date September 3, 1952 Sampling study No. 37 Weather Clear

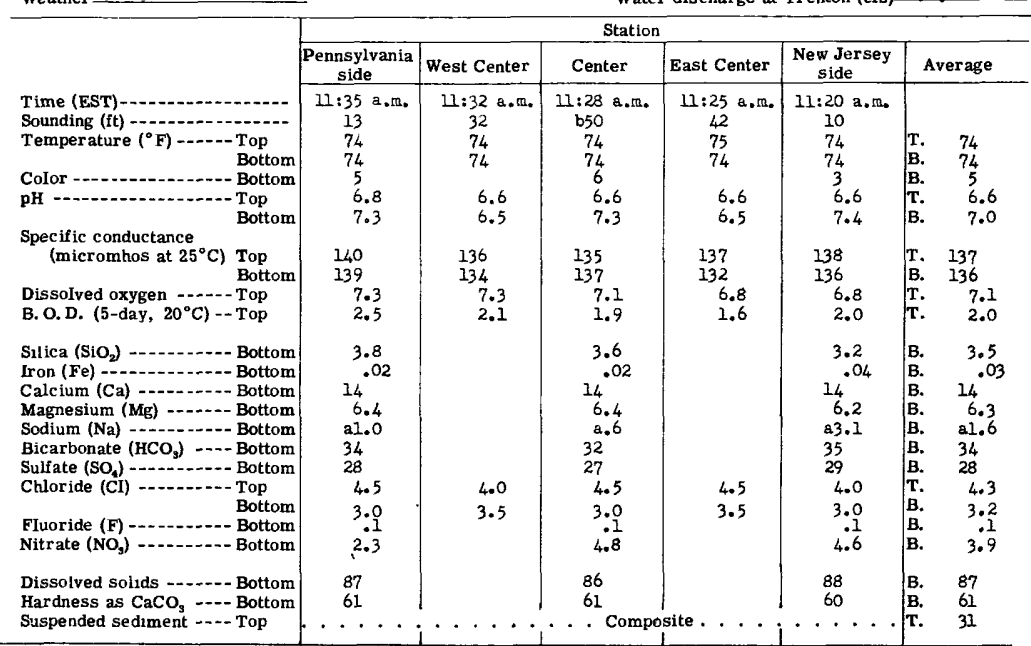

a Calculated Sodium and Potassium.

b Exceeded this value.

Location Bristol, Pa,-Burlington, N. J. Bridge Date October 6, 1952 _Sampling study No. 38 Weather Clear $\quad$ Water discharge at Trenton (cfs) 4,800

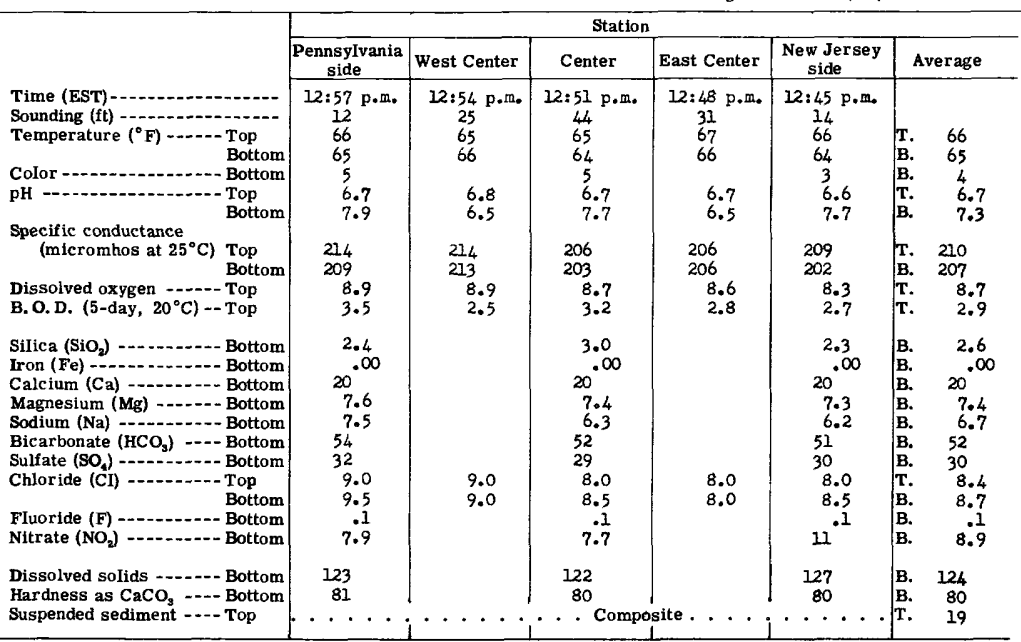


Table 7. --WATER ANALYSES OF DELAWARE RIVR BETWEEN BRISTOL AND MARC US HOOK, PA. --Continued Analyzed by City of Philadelphia and U. S. Geological Survey; analyses in parts per million

Location Bristol. Pa.-Burlington, N. J. Bridge Date November 6, 1952 Sampling study No. 39 Weather Clegr Water discharge at Trenton (cfs) 3,130

\begin{tabular}{|c|c|c|c|c|c|c|}
\hline \multirow[b]{2}{*}{ " } & \multicolumn{6}{|c|}{ Station } \\
\hline & $\begin{array}{c}\text { Pennsylvania } \\
\text { side }\end{array}$ & West Center & Center & East Center & $\begin{array}{l}\text { New Jersey } \\
\text { side }\end{array}$ & Average \\
\hline $\begin{array}{l}\text { Time (EST) } \\
\text { Sounding (ft) } \\
\text { Temperature }\left({ }^{\circ} \mathrm{F}\right) \mathrm{c} \\
\text { Color }-\mathrm{Top} \\
\text { pH }\end{array}$ & $\begin{array}{c}12: 42 \text { p. m. } \\
21 \\
49 \\
49 \\
5 \\
6.4 \\
7.1\end{array}$ & $\begin{array}{c}12: 39 \mathrm{p.m} . \\
26 \\
49 \\
49 \\
6.5 \\
6.5\end{array}$ & $\begin{array}{c}12: 36 \mathrm{p} . \mathrm{m} . \\
29 \\
49 \\
49 \\
8 \\
6.5 \\
7.6\end{array}$ & $\begin{array}{c}12: 32 \mathrm{p} . \mathrm{m} \\
40 \\
49 \\
50 \\
6.5 \\
6.5\end{array}$ & $\begin{array}{c}12: 30 \mathrm{p} . \mathrm{m} . \\
21 \\
49 \\
48 \\
6 \\
6.5 \\
7.6\end{array}$ & $\begin{array}{l}49 \\
49 \\
6 \\
6.5 \\
7.1\end{array}$ \\
\hline $\begin{array}{l}\begin{array}{l}\text { Specific conductance } \\
\quad\left(\text { micrombos at } 25^{\circ} \mathrm{C} \text { ) }\right. \\
\text { Top } \\
\text { Bottom }\end{array} \\
\text { Dissolved oxygen } \\
\text { B. O. D. (5-day, } 20^{\circ} \mathrm{C} \text { ) - Top } \\
\text { - Top }\end{array}$ & $\begin{array}{l}249 \\
235 \\
9.0 \\
4.7\end{array}$ & $\begin{array}{l}232 \\
233 \\
9.3 \\
2.8\end{array}$ & $\begin{array}{l}229 \\
227 \\
9.1 \\
2.6\end{array}$ & $\begin{array}{l}232 \\
235 \\
9.2 \\
2.3\end{array}$ & $\begin{array}{l}232 \\
230 \\
9.5 \\
2.7\end{array}$ & $\begin{array}{ll}\text { T. } & 235 \\
\text { B. } & 232 \\
\text { T. } & 9.2 \\
\text { T. } & 3.0\end{array}$ \\
\hline 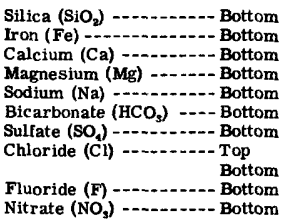 & $\begin{array}{l}2.6 \\
.02 \\
22 \\
8.3 \\
8.6 \\
54 \\
36 \\
13 \\
11 \\
.1 \\
9.7\end{array}$ & $11^{9.5}$ & $\begin{array}{l}2.8 \\
21.03 \\
8.9 \\
7.5 \\
56 \\
33 \\
10 \\
10 \\
11\end{array}$ & $\begin{array}{l}10 \\
10\end{array}$ & $\begin{array}{l}2.3 \\
.02 \\
21 \\
8.9 \\
8.4 \\
56 \\
34 \\
11 \\
10 \\
.1 \\
9.8\end{array}$ & $\begin{array}{l}2.6 \\
.02 \\
21.7 \\
8.7 \\
8.2 \\
55 \\
34 \\
11 \\
10 \\
10.1\end{array}$ \\
\hline $\begin{array}{l}\text { Dissolved solids }-1-1 \text { Bottom } \\
\text { Hardness as } \mathrm{CaCO}_{3}-\cdots \text { Bottom } \\
\text { Suspended sediment }-\cdots-\text { Top }\end{array}$ & $\begin{array}{r}146 \\
89 \\
.\end{array}$ & & $\begin{array}{l}143 \\
89 \\
. \text { Con }\end{array}$ & te. & $\begin{array}{r}137 \\
89 \\
+\cdot .\end{array}$ & $\begin{array}{lr}\text { B. } & 142 \\
\text { B. } & 89 \\
\text { T. } & 13\end{array}$ \\
\hline
\end{tabular}

Location Bristol, Pa,-Burlington, N. J. Bridge Date December 4, 1952 Sampling study No. 40 Weather Clear

\begin{tabular}{|c|c|c|c|c|c|c|}
\hline \multirow{3}{*}{ 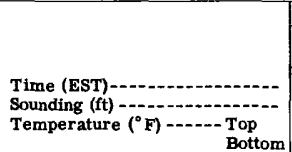 } & \multicolumn{6}{|c|}{ Station } \\
\hline & $\begin{array}{c}\text { Pennsylvania } \\
\text { side }\end{array}$ & West Center & Center & East Center & \begin{tabular}{|c|}
$\begin{array}{c}\text { New Jersey } \\
\text { side }\end{array}$ \\
\end{tabular} & Average \\
\hline & $\begin{array}{c}12: 28 \text { p.m. } \\
14 \\
41 \\
39 \\
5 \\
6.4 \\
6.8\end{array}$ & $\begin{array}{c}12: 31 \mathrm{p.m} . \\
23 \\
39 \\
40 \\
6.4 \\
6.5\end{array}$ & $\begin{array}{c}12: 34 \mathrm{p} . \mathrm{m} . \\
44 \\
39 \\
39 \\
6 \\
6.5 \\
6.9\end{array}$ & $\begin{array}{c}12: 37 \mathrm{p} \cdot \mathrm{m} . \\
43 \\
39 \\
40 \\
\\
6.4 \\
6.6\end{array}$ & $\begin{array}{c}12: 40 \mathrm{p} . \mathrm{m} . \\
9 \\
39 \\
39 \\
5 \\
6.5 \\
7.1\end{array}$ & $\begin{array}{l}39 \\
39 \\
5 \\
6.4 \\
6.8\end{array}$ \\
\hline $\begin{array}{l}\begin{array}{l}\text { Specific conductance } \\
\quad\left(\text { micromhos at } 25^{\circ} \mathrm{C}\right)\end{array} \\
\begin{array}{l}\text { Top } \\
\text { Bottom }\end{array} \\
\text { Dissolved oxygen -...- Top } \\
\text { B. O. D. (5-day, } 20^{\circ} \mathrm{C} \text { ) -- Top }\end{array}$ & $\begin{array}{r}133 \\
130 \\
12.0 \\
3.9\end{array}$ & $\begin{array}{l}127 \\
126 \\
12.3 \\
3.4\end{array}$ & $\begin{array}{l}124 \\
125 \\
12.2 \\
3.0\end{array}$ & $\begin{array}{l}126 \\
125 \\
12.5 \\
3.4\end{array}$ & $\begin{array}{l}138 \\
129 \\
12.1 \\
3.7\end{array}$ & $\begin{array}{ll}\text { T. } & 130 \\
\text { B. } & 127 \\
\text { T. } & 12.2 \\
\text { T. } & 3.5\end{array}$ \\
\hline 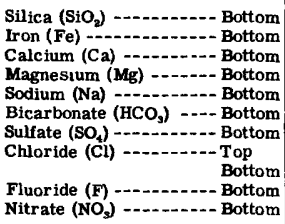 & $\begin{array}{l}5.5 \\
.10 \\
12 \\
4.9 \\
4.2 \\
23 \\
25 \\
6.0 \\
6.0 \\
.1 \\
5.5\end{array}$ & $\begin{array}{l}6.0 \\
4.5\end{array}$ & $\begin{array}{l}4.3 \\
.12 \\
12 \\
4.8 \\
3.2 \\
24 \\
22 \\
5.0 \\
5.5 \\
.1 \\
5.8\end{array}$ & $\begin{array}{l}5.0 \\
5.0\end{array}$ & $\begin{array}{l}4.8 \\
12.15 \\
4.9 \\
3.5 \\
23 \\
24 \\
6.0 \\
6.0 \\
. .1 \\
6.4\end{array}$ & $\begin{array}{l}4.9 \\
12.12 \\
4.9 \\
3.6 \\
23 \\
24 \\
5.6 \\
5.4 \\
.1 \\
5.9\end{array}$ \\
\hline $\begin{array}{l}\text { Dissolved solids } \ldots+.- \text { Bottom } \\
\text { Hardness as } \mathrm{CaCO}_{3}-\ldots-\text { Bottom } \\
\text { Sospended sediment --.- Top }\end{array}$ & $\begin{array}{l}81 \\
50 \\
\cdot .\end{array}$ & & $\begin{array}{l}78 \\
50 \\
. \mathrm{Co}\end{array}$ & e & $\begin{array}{r}78 \\
50 \\
.\end{array}$ & $\begin{array}{l}\text { B. } \\
\text { B. } \\
\text { T. }\end{array}$ \\
\hline
\end{tabular}


Table 8. -- WATER ANALYSES OF DELAWARE RIVER BETWEEN BRISTOL AND MARCUS HOOK, PA. Analyzed by City of Philadelphia and U. S. Geological Survey; analyses in parts per million

Location _Torresdale Intake, Philadelphka, Fa._Date_August 1, 1949 _ Sampling study No._I Weather Cloudy

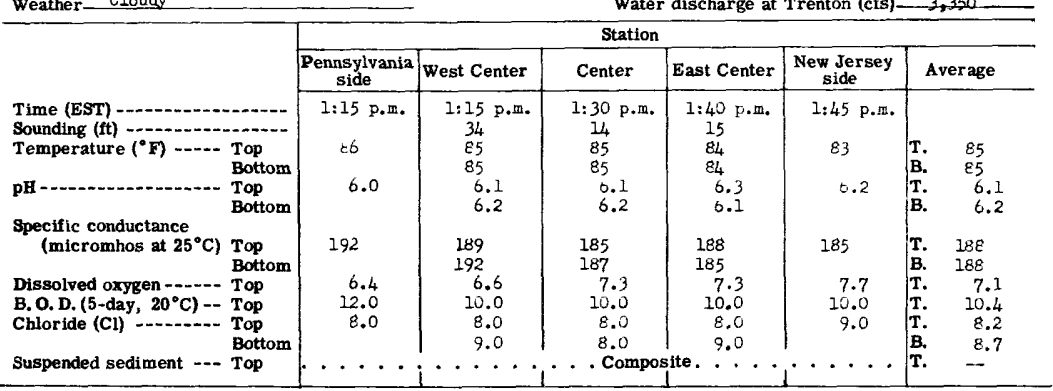

Location Torresiale Intake. Phíladelphia, Pa. Date Sectember 6, 1449 Sampling study No. 2 Weather Cloudy water discharge at Trenton (cfs)- 5 , 300

\begin{tabular}{|c|c|c|c|c|c|c|}
\hline \multicolumn{2}{|c|}{ Weather Cloudy } & \multirow{2}{*}{\multicolumn{5}{|c|}{$\begin{array}{r}\text { Water } \\
\text { Station }\end{array}$}} \\
\hline \multirow[b]{3}{*}{$\begin{array}{ll}\text { Time (EST) } \\
\text { Sounding (ft) } \\
\text { Temperature }\left({ }^{\circ} \mathrm{F}\right) \\
\text { pH }\end{array} \begin{array}{l}\text { Top } \\
\text { Bottom } \\
\text { Bop } \\
\text { Bottom }\end{array}$} & Station & & & & & \\
\hline & \begin{tabular}{|c|} 
Pennsylvania \\
side
\end{tabular} & West Center & Center & East Center & $\begin{array}{c}\text { New Jersey } \\
\text { side }\end{array}$ & Average \\
\hline & $\begin{array}{l}77 \\
6.8\end{array}$ & $\begin{array}{l}-- \\
30 \\
77 \\
78 \\
6.9 \\
6.7\end{array}$ & $\begin{array}{l}9: 45 \mathrm{a} . \mathrm{In} . \\
24 \\
77 \\
78 \\
6.8 \\
6.8\end{array}$ & $\begin{array}{l}-\overline{15} \\
77 \\
76 \\
6.7 \\
6.6\end{array}$ & $\begin{array}{l}-- \\
77 \\
7.0\end{array}$ & $\begin{array}{ll}\text { T. } & 77 \\
\text { B. } & 77 \\
\text { T. } & 6.8 \\
\text { B. } & 6.7\end{array}$ \\
\hline $\begin{array}{ll}\begin{array}{l}\text { Specific conductance } \\
\text { (micromhos at } 25^{\circ} \mathrm{C} \text { ) }\end{array} & \begin{array}{l}\text { Top } \\
\text { Bottom }\end{array} \\
\begin{array}{ll}\text { Dissolved oxygen - }-1 .- & \text { Top } \\
\text { B. O. D. (5-day, } 20^{\circ} \mathrm{C} \text { ) - } & \text { Top } \\
\text { Chloride (Cl) } & \text { Bottom }\end{array}\end{array}$ & $\begin{array}{r}220 \\
7 . ? \\
12^{-5}\end{array}$ & $\begin{array}{l}223 \\
225 \\
0.9 \\
12 \\
11\end{array}$ & $\begin{array}{l}218 \\
205 \\
0.8 \\
11 \\
11\end{array}$ & $\begin{array}{l}196 \\
184 \\
7.1 \\
2.0 \\
12 \\
10\end{array}$ & $\begin{array}{l}204 \\
7.0 \\
2.0 \\
12\end{array}$ & $\begin{array}{lc}\text { T. } & 212 \\
\text { B. } & 205 \\
\text { T. } & 7.0 \\
\text { T. } & .9 \\
\text { T. } & 12 \\
\text { B. } & 11\end{array}$ \\
\hline Suspended sediment $\ldots$ Top & $\cdots$ & . & . Com & ite. . & 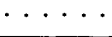 & $\mathbf{T}$ \\
\hline
\end{tabular}

Location - Iorresdato Intake, Ehiladelphis, Pa.__ Date_October 4, 1949 Sampling study No. - - 3 Weather cloudy Water discharge at Trenton (cfs) - 3,520

\begin{tabular}{|c|c|c|c|c|c|c|}
\hline \multicolumn{7}{|c|}{ Weather_clondy } \\
\hline & $\begin{array}{c}\text { Pennsylvania } \\
\text { side }\end{array}$ & West Center & Center & East Center & $\begin{array}{c}\text { New Jersey } \\
\text { side }\end{array}$ & Average \\
\hline $\begin{array}{ll}\text { Time (EST) } & \\
\text { Sounding (ft) } & \\
\text { Temperature }\left({ }^{\circ} \mathrm{F}\right) & \begin{array}{l}\text { Top } \\
\text { Bottom } \\
\text { Top } \\
\text { BH }\end{array} \\
\text { Bottom }\end{array}$ & 69 & $\begin{array}{l}34 \\
69 \\
69 \\
0.6 \\
6.5\end{array}$ & $\begin{array}{c}22: 30 \text { p.m. } \\
38 \\
69 \\
68 \\
6.6 \\
6.6\end{array}$ & $\begin{array}{l}-7 \\
69 \\
66 \\
6.8 \\
6.8\end{array}$ & $\begin{array}{l}-- \\
69 \\
6.8\end{array}$ & $\begin{array}{ll}\text { T. } & 69 \\
\text { B. } & 68 \\
\text { T. } & 6.7 \\
\text { B. } & 6.6\end{array}$ \\
\hline $\begin{array}{ll}\begin{array}{l}\text { Specific conductance } \\
\text { (micromhos at } 25^{\circ} \mathrm{C} \text { ) }\end{array} & \begin{array}{l}\text { Top } \\
\text { Bottom }\end{array} \\
\begin{array}{ll}\text { Dissolved oxygen -...- Top } \\
\text { B. O. D. (5-day, 20 }\end{array} \\
\text { Chloride (C) - } & \text { Top } \\
\text { Suspended sediment }-\cdots & \text { Top }\end{array}$ & $\begin{array}{l}250 \\
3.2 \\
1.5 \\
16 \\
\ldots . . .\end{array}$ & $\begin{array}{l}246 \\
242 \\
2.8 \\
2.0 \\
15 \\
14 \\
\therefore\end{array}$ & $\begin{array}{l}238 \\
246 \\
3.8 \\
14 \\
13 \\
13 \\
. \text { Compo }\end{array}$ & $\begin{array}{l}230 \\
228 \\
5.3 \\
12 \\
12 \\
12 \\
\text { ite. . . }\end{array}$ & $\begin{array}{l}225 \\
6.7 \\
1.3 \\
13\end{array}$ & $\begin{array}{ll}\text { T. } & 238 \\
\text { B. } & 239 \\
\text { T. } & 4.4 \\
\text { T. } & 1.0 \\
\text { T. } & 14 \\
\text { B. } & 13 \\
\text { T. } & 16\end{array}$ \\
\hline
\end{tabular}

Location Toreasde le Intake, Philadelphia, E2, Date-November 1, 1949 Sampling study No. -

\begin{tabular}{|c|c|c|c|c|c|c|}
\hline \multicolumn{7}{|c|}{ Weather Cloudy _ _ C } \\
\hline & \begin{tabular}{|c|} 
Pennsylvania \\
side
\end{tabular} & West Center & Center & East Center & $\begin{array}{c}\text { New Jersey } \\
\text { side }\end{array}$ & Average \\
\hline $\begin{array}{ll}\text { Time (EST) } & \\
\text { Sounding (ft) } & \text { Top } \\
\text { Temperature }(\bullet \mathrm{F}) & \text { Bottom } \\
\text { pH } & \text { Top } \\
& \text { Bottom }\end{array}$ & $\begin{array}{l}-- \\
58 \\
6.8\end{array}$ & $\begin{array}{l}-- \\
24 \\
57 \\
56 \\
6.8 \\
0.8\end{array}$ & $\begin{array}{l}2: 00 \mathrm{p}+\mathrm{m} . \\
40 \\
57 \\
50 \\
6.9 \\
6.8\end{array}$ & $\begin{array}{l}-78 \\
54 \\
56 \\
6.9 \\
6.9\end{array}$ & $\begin{array}{l}-- \\
56 \\
6.9\end{array}$ & $\begin{array}{lc}\text { T. } & 56 \\
\text { B. } & 56 \\
\text { T. } & 6.9 \\
\text { B. } & 6.8\end{array}$ \\
\hline 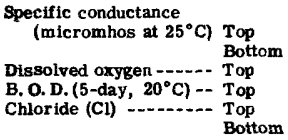 & $\begin{array}{l}227 \\
4.3 \\
5.5 \\
24\end{array}$ & $\begin{array}{l}220 \\
219 \\
4.7 \\
6.0 \\
15 \\
14\end{array}$ & $\begin{array}{l}209 \\
216 \\
4.9 \\
5.7 \\
12 \\
12\end{array}$ & $\begin{array}{l}198 \\
209 \\
0.5 \\
4.6 \\
12 \\
12\end{array}$ & $\begin{array}{l}215 \\
\quad 6.3 \\
2.8 \\
13\end{array}$ & $\begin{array}{lc}\text { T. } & 214 \\
\text { B. } & 215 \\
\text { T. } & 5.3 \\
\text { T. } & 4.9 \\
\text { T. } & 13 \\
\text { B. } & 13\end{array}$ \\
\hline Suspended sediment _.. Top & 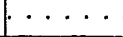 & 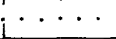 & . Compo & site. . . & $\cdot \cdot \cdot$ & T. 9.2 \\
\hline
\end{tabular}


Table 8. -- WATER ANALYSES OF DELAWARE RIVER BETWEEN BFISTOL AND MARCUS HOOK, PA.--Continued Analyzed by City of Philadelphia and U. S. Geological Survey, analyses in parts per million

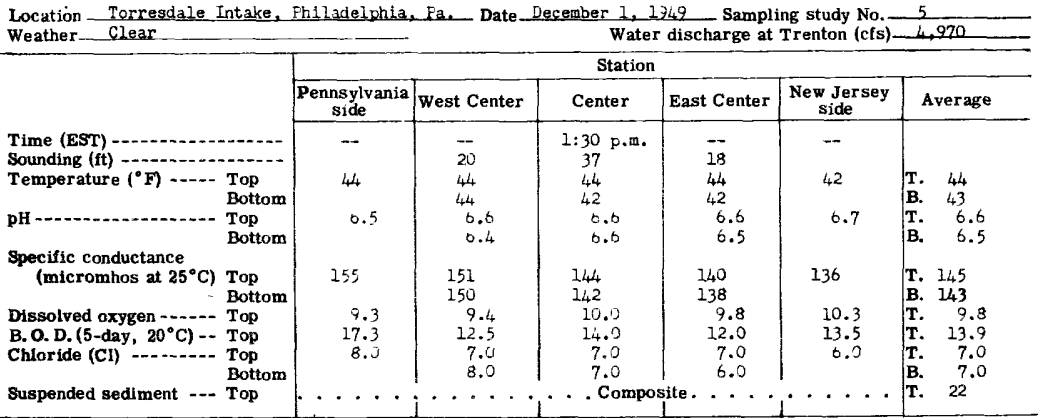

Location Torresdale Intake, Ehilladelpbia, Fa._- Date Januaxy 3, 2950 Sampling study No. 6 Weather_Light Rain _ W Water discharge at Trenton (cfs) - 11,100

\begin{tabular}{|c|c|c|c|c|c|c|}
\hline \multirow[b]{3}{*}{$\begin{array}{l}\text { Time (EST) } \\
\text { Sounding (ft) } \\
\text { Temperature }\left({ }^{\circ} \mathrm{F}\right) \\
\text { pH }\end{array}$} & \multicolumn{6}{|c|}{ Station } \\
\hline & $\begin{array}{c}\begin{array}{c}\text { Pennsylvania } \\
\text { side }\end{array} \\
\end{array}$ & West Center & Center & East Center & $\begin{array}{l}\text { New Jersey } \\
\text { side }\end{array}$ & Aver age \\
\hline & $\begin{array}{c}12: 55 \text { p.m. } \\
-- \\
5.3\end{array}$ & $\begin{array}{l}1: 00 \text { p. I. } \\
37 \\
-- \\
-- \\
6.5 \\
0.4\end{array}$ & $\begin{array}{l}1: 04 \text { p.m. } \\
36 \\
-. \\
- \\
6.5 \\
6.3\end{array}$ & $\begin{array}{c}1: 08 \mathrm{p} . \mathrm{m} . \\
18 \\
- \\
\cdots \\
6.5 \\
b .3\end{array}$ & $\begin{array}{c}1: 13 \text { p.m. } \\
- \\
6.7\end{array}$ & $\begin{array}{l}\text { T. } \\
\text { B. } \\
\text { T. } \\
\text { B. }\end{array}$ \\
\hline 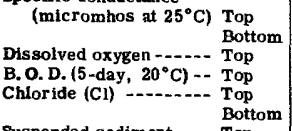 & $\begin{array}{r}115 \\
11.2 \\
5.4 \\
7.0\end{array}$ & $\begin{array}{r}104 \\
104 \\
11.1 \\
2.8 \\
6.0 \\
7.0\end{array}$ & $\begin{array}{r}100 \\
102 \\
11.1 \\
2.8 \\
7.0 \\
6.0\end{array}$ & $\begin{array}{r}99.4 \\
99.4 \\
11.4 \\
3.2 \\
6.0 \\
5.0\end{array}$ & $\begin{array}{r}102 \\
11.0 \\
1.8 \\
6.0\end{array}$ & $\begin{array}{lr}\text { T. } & 104 \\
\text { B. } & 102 \\
\text { T. } & 11.2 \\
\text { T. } & 3.2 \\
\text { T. } & 6.4 \\
\text { B. } & 0.0\end{array}$ \\
\hline Suspended sediment -.- Top & 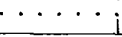 & $\cdots \cdot$ & . Comp & ite. . - & 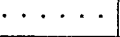 & T. \\
\hline
\end{tabular}

Location Forresdale Intake, Fhiladelphia, Pa... Date February 2, 1750 Sampling study No. 7

\begin{tabular}{|c|c|c|c|c|c|c|}
\hline \multicolumn{3}{|c|}{ Weather Cloudy } & \multicolumn{4}{|c|}{ Water discharge at Trenton (cfs) - 13,200} \\
\hline & \multicolumn{6}{|c|}{ Station } \\
\hline & $\begin{array}{c}\text { Pennsylvania } \\
\text { side }\end{array}$ & West Center & Center & East Center & $\begin{array}{c}\text { New Jersey } \\
\text { side }\end{array}$ & Average \\
\hline $\begin{array}{ll}\text { Time (EST) } & \\
\text { Sounding (ft) } \\
\text { Temperature }\left({ }^{\circ} \mathrm{F}\right) \\
\text { pH } & \begin{array}{l}\text { Top } \\
\text { Bottom } \\
\text { Top } \\
\text { Bottom }\end{array}\end{array}$ & $\begin{array}{c}10: 45 \text { a.m. } \\
40 \\
6.9\end{array}$ & $\begin{array}{c}10: 47 \text { a.m. } \\
34 \\
40 \\
40 \\
0.7 \\
0.7\end{array}$ & $\begin{array}{c}10: 50 \text { a.m. } \\
35 \\
40 \\
40 \\
6.9 \\
6.8\end{array}$ & $\begin{array}{c}10: \\
55 \text { a.m. } \\
15 \\
40 \\
40 \\
6.8 \\
6.9\end{array}$ & $\begin{array}{c}10: 59 \text { a.m. } \\
40 \\
0.7\end{array}$ & $\begin{array}{ll}\text { T. } & 40 \\
\text { B. } & 40 \\
\text { T. } & 6.8 \\
\text { B. } & 6.8\end{array}$ \\
\hline $\begin{array}{l}\text { Specific conductance } \\
\text { (micromhos at } 25^{\circ} \mathrm{C} \text { ) Top } \\
\text { Bottom }\end{array}$ & 124 & $\begin{array}{l}117 \\
118\end{array}$ & $\begin{array}{l}110 \\
110\end{array}$ & $\begin{array}{l}108 \\
109\end{array}$ & 108 & $\begin{array}{l}\text { T. } 113 \\
\text { B. } 112\end{array}$ \\
\hline $\begin{array}{l}\text { Dissolved oxygen }-1 .- \text { Top } \\
\left.\text { B. O. D. (5-day, } 20^{\circ} \mathrm{C}\right)- \text { Top } \\
\text { Chioride (C1) }-1 \text { Top } \\
\end{array}$ & $\begin{array}{r}12.1 \\
2.6 \\
6.0\end{array}$ & $\begin{array}{r}10.8 \\
2.4 \\
5.0 \\
6.0\end{array}$ & $\begin{array}{r}11.4 \\
3.0 \\
4.0 \\
5.0\end{array}$ & $\begin{array}{r}10.8 \\
2.2 \\
5.3 \\
4.0\end{array}$ & $\begin{array}{r}11.0 \\
3.2 \\
6.0\end{array}$ & $\begin{array}{lr}\text { T. } & 11.0 \\
\text { T. } & 2.7 \\
\text { T. } & 5.2 \\
\text { B. } & 5.0\end{array}$ \\
\hline Suspended sediment $\ldots$ Top & & & . Com & ite. . . & 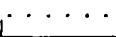 & T. 15 \\
\hline
\end{tabular}

Location Torresdale Intake, Phfladelohla, Fa. Date Karch 6. 1950_ Sampling study No. 8. Water discharge at Trenton (cfs) 7,080

\begin{tabular}{|c|c|c|c|c|c|c|}
\hline \multicolumn{3}{|c|}{ Weather Clear } & \multicolumn{4}{|c|}{ Water discharge at Trenton (cfs) -7.080} \\
\hline & \multicolumn{6}{|c|}{ Station } \\
\hline & $\begin{array}{c}\text { Pennsylvania } \\
\text { side }\end{array}$ & West Center & Center & East Center & $\begin{array}{c}\text { New Jersey } \\
\text { side }\end{array}$ & Average \\
\hline $\begin{array}{ll}\text { Time (EST) } & \\
\text { Sounding (ft) } \\
\text { Temperature }\left({ }^{\circ} \mathrm{F}\right) \\
\text { pH }\end{array}$ & $\begin{array}{c}11: 14 \text { a.m. } \\
35 \\
7.1\end{array}$ & $\begin{array}{c}11: 10 \mathrm{a}+\mathrm{m} . \\
32 \\
35 \\
35 \\
7.0 \\
7.2\end{array}$ & $\begin{array}{c}11: 07 \text { a.m. } \\
38 \\
35 \\
35 \\
7.1 \\
7.2\end{array}$ & $\begin{array}{c}11: 00 \text { a.m. } \\
6 \\
35 \\
35 \\
7.2 \\
7.4\end{array}$ & $\begin{array}{c}11: 05 \text { a.m. } \\
35 \\
7.4\end{array}$ & $\begin{array}{ll}\text { T. } & 35 \\
\text { B. } & 35 \\
\text { T. } & 7.2 \\
\text { B. } & 7.3\end{array}$ \\
\hline 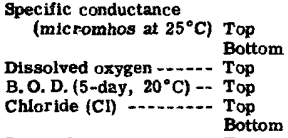 & $\begin{array}{r}147 \\
13.7 \\
5.0 \\
7.0\end{array}$ & $\begin{array}{r}145 \\
144 \\
13.1 \\
4.9 \\
7.0 \\
7.0\end{array}$ & $\begin{array}{r}145 \\
143 \\
13.4 \\
5.2 \\
7.0 \\
7.0\end{array}$ & $\begin{array}{r}129 \\
122 \\
14.0 \\
4.3 \\
0.0 \\
6.0\end{array}$ & $\begin{array}{r}123 \\
12.7 \\
5.0 \\
6.0\end{array}$ & $\begin{array}{lr}\text { T. } & 138 \\
\text { B. } & 136 \\
\text { T. } & 13.4 \\
\text { T. } & 4.9 \\
\text { T. } & 6.6 \\
\text { B. } & 6.7\end{array}$ \\
\hline Suspended sediment -.- Top & 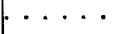 & $\cdots$ & . Com & te. . . & $\cdot \cdot$ & T. 25 \\
\hline
\end{tabular}


Table 8. -- WATER ANALYSES OF DELAWARE RIVER BETWEEN BRISTOL AND MARCUS HOOK, PA. - ContinUed Analyzed by City of Philadelphia and U. S. Geological Survey; analyses in parts per million

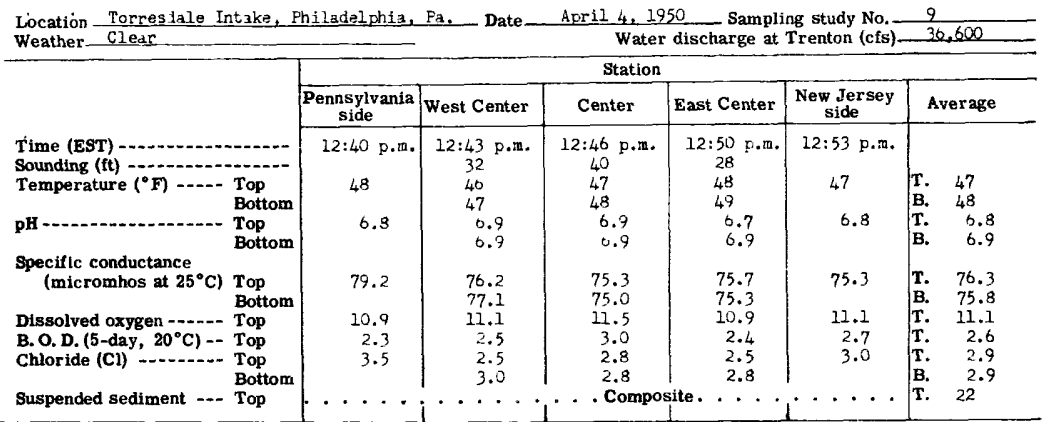

Location Torresdale Intake, Fhiladelphia, Pa, Date May 1, 1950____ Sampling study No. 10

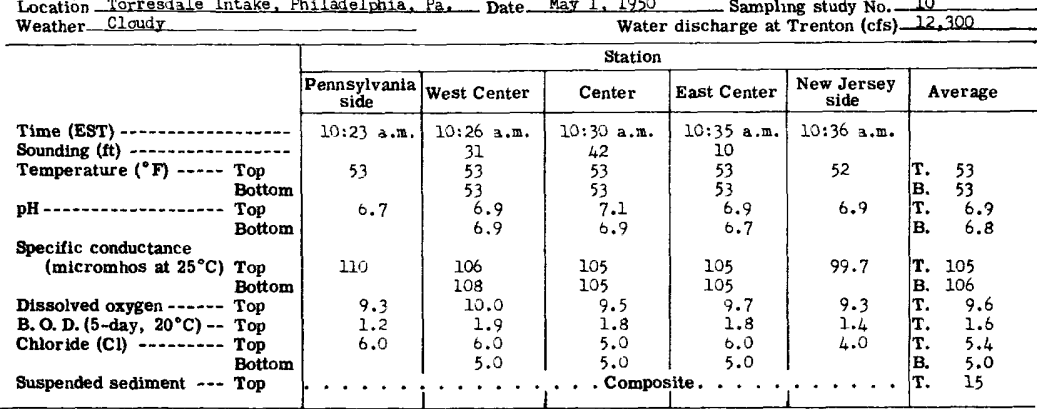

Location Torresdale Intake, Phifadelphia, Pa, Date June 6, 1950 Sampling study No. 11

\begin{tabular}{|c|c|c|c|c|c|c|}
\hline \multicolumn{3}{|c|}{ Weather Misty } & \multicolumn{4}{|c|}{ Water discharge at Trenton (cfs) $-18,900$} \\
\hline & \multicolumn{6}{|c|}{ Station } \\
\hline & $\begin{array}{c}\text { Pennsylvania } \\
\text { side }\end{array}$ & West Center & Center & East Center & \begin{tabular}{|c|} 
New Jersey \\
side
\end{tabular} & Average \\
\hline $\begin{array}{ll}\text { Time (EST) } & \\
\text { Sounding }(\mathrm{ft}) & \\
\text { Temperature }\left({ }^{\circ} \mathrm{F}\right) & \begin{array}{l}\text { Top } \\
\text { Bottom }\end{array} \\
\text { pH } & \begin{array}{l}\text { Top } \\
\text { Bottom }\end{array}\end{array}$ & $\begin{array}{c}10: 27 \text { a.m. } \\
68 \\
6.7\end{array}$ & $\begin{array}{c}10: 30 \text { a.m. } \\
31 \\
67 \\
70 \\
6.8 \\
6.7\end{array}$ & $\begin{array}{c}10: 35 \mathrm{a} . \mathrm{m} . \\
28 \\
69 \\
70 \\
6.8 \\
6.5\end{array}$ & $\begin{array}{c}10: 37 \text { a.m. } \\
17 \\
69 \\
70 \\
6.7 \\
6.7\end{array}$ & $\begin{array}{c}10: 42 \mathrm{a} \cdot \mathrm{m} . \\
70 \\
6.6\end{array}$ & $\begin{array}{ll}\text { T. } & 69 \\
\text { B. } & 70 \\
\text { T. } & 6.7 \\
\text { B. } & 6.6\end{array}$ \\
\hline 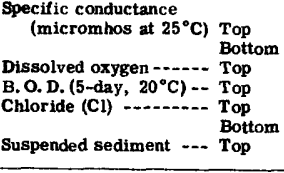 & $\begin{array}{l}112 \\
5.8 \\
1.1 \\
5.0\end{array}$ & $\begin{array}{l}108 \\
108 \\
5.9 \\
.6 \\
5.0 \\
5.0 \\
.\end{array}$ & $\begin{array}{r}105 \\
107 \\
6.1 \\
1.2 \\
4.0 \\
4.0 \\
. \quad \text { Com }\end{array}$ & $\begin{array}{r}105 \\
105 \\
6.0 \\
1.5 \\
5.0 \\
4.0 \\
\text { te. }\end{array}$ & $\begin{array}{r}102 \\
5.8 \\
1.7 \\
5.0 \\
\end{array}$ & $\begin{array}{lc}\text { T. } & 106 \\
\text { B. } & 107 \\
\text { T. } & 5.9 \\
\text { T. } & 1.2 \\
\text { T. } & 4.8 \\
\text { B. } & 4.3 \\
\text { T. } & 19\end{array}$ \\
\hline
\end{tabular}

Location - Torresdale Intake, Fhiladelphia, Pa. Date_July 6, 1950 _ Sampling study No. 12 Weather Cloudy Water discharge at Trenton (cfs) 6.130

\begin{tabular}{|c|c|c|c|c|c|c|}
\hline \multicolumn{3}{|c|}{ Weather_. Cloudy } & \\
\hline & \multicolumn{6}{|c|}{ Station } \\
\hline & $\begin{array}{c}\text { Pennsylvania } \\
\text { side }\end{array}$ & West Center & Center & East Center & $\begin{array}{l}\text { New Jersey } \\
\text { side }\end{array}$ & Average \\
\hline $\begin{array}{ll}\text { Time (EST) } & \\
\text { Sounding (ft) } \\
\text { Temperature }\left({ }^{\circ} \mathrm{F}\right)\end{array} \begin{array}{l}\text { Top } \\
\text { Bottom } \\
\text { pH }\end{array}$ & $\begin{array}{c}11: 35 \mathrm{a} . \mathrm{m} . \\
78 \\
6.7\end{array}$ & $\begin{array}{c}11: 40 \text { a.m. } \\
33 \\
78 \\
78 \\
6.7 \\
6.7\end{array}$ & $\begin{array}{c}11: 45 \mathrm{a} . \mathrm{m} \\
30 \\
78 \\
78 \\
6.7 \\
6.6\end{array}$ & $\begin{array}{l}11: 50 \text { a.m. } \\
17 \\
78 \\
78 \\
6.7 \\
6.6\end{array}$ & $\begin{array}{c}11: 55 \mathrm{a} . \mathrm{m} . \\
78 \\
6.7\end{array}$ & $\begin{array}{ll}\text { T. } & 78 \\
\text { B. } & 78 \\
\text { T. } & 6.7 \\
\text { B. } & 6.6\end{array}$ \\
\hline $\begin{array}{ll}\text { Specific conductance } & \\
\left.\text { (micromhos at } 25^{\circ} \mathrm{C}\right) & \text { Top } \\
& \text { Bottom } \\
\text { Dissolved oxygen - - - - - } & \text { Top } \\
\text { B. O. D. (5-day, 20 } & \text { Top } \\
\text { Chloride (C1) - } & \text { Top } \\
& \text { Bottom }\end{array}$ & $\begin{array}{l}129 \\
4.0 \\
1.7 \\
6.0\end{array}$ & $\begin{array}{l}125 \\
130 \\
4.3 \\
2.0 \\
6.0 \\
6.0\end{array}$ & $\begin{array}{l}125 \\
125 \\
4.5 \\
2.0 \\
6.0 \\
6.0\end{array}$ & $\begin{array}{r}124 \\
126 \\
4.5 \\
2.0 \\
6.0 \\
6.0\end{array}$ & $\begin{array}{r}124 \\
4.6 \\
1.5 \\
7.0\end{array}$ & $\begin{array}{ll}\text { T. } & 125 \\
\text { B. } & 127 \\
\text { T. } & 4.4 \\
\text { T. } & 1.8 \\
\text { T. } & 6.2 \\
\text { B. } & 6.0\end{array}$ \\
\hline Suspended sediment --- Top & & & Com & te. .. & 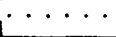 & T. \\
\hline
\end{tabular}


Table 8. -- WATER ANALYSES OF DELAWARE RIVER BETWEEN BRISTOL AND MARCUS HOOK, PA.--Continued Analyzed by City of Philadelphia and U. S. Geological Survey; analyses in parts per million

Location Torresilale Intake, Fhiladelphiz, Fa. Date Augnist 2, 1950 Sampling study No. 13 Weather Overcast _ Water discharge at Trenton (cfs) - 5.090

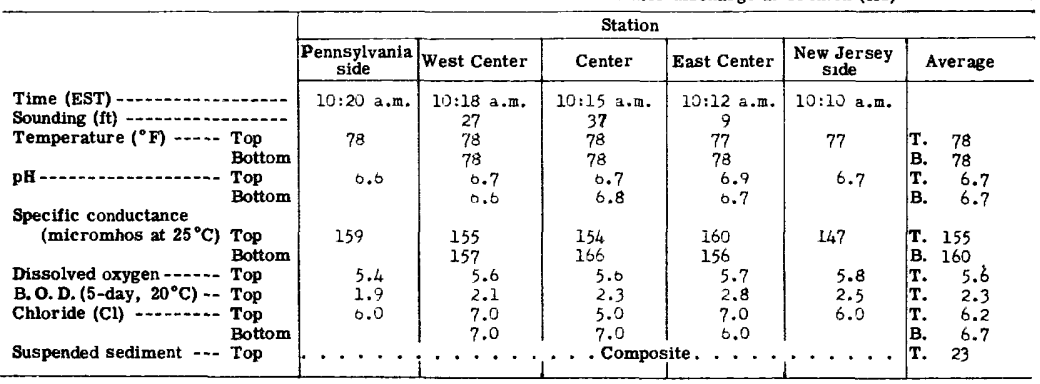

Location Torresdale Intake, Philadelphia, Pa. Date September 6, $125 \mathrm{~V}$ Sampling study No. 14 Weather Clear $\quad$ Water discharge at Trenton (cis) - 4,860

\begin{tabular}{|c|c|c|c|c|c|c|}
\hline \multirow[b]{3}{*}{$\begin{array}{l}\text { Time (EST) } \\
\text { Sounding (ft) } \\
\text { Temperature }\left({ }^{\circ} \mathrm{F}\right) \\
\text { pH }\end{array} \begin{array}{l}\text { Top } \\
\text { Bottom } \\
\text { Top } \\
\text { Bottom }\end{array}$} & \multicolumn{6}{|c|}{ Station } \\
\hline & \begin{tabular}{|c|}
$\begin{array}{c}\text { Pennsylvania } \\
\text { side }\end{array}$ \\
\end{tabular} & West Center & Center & East Center & $\begin{array}{c}\text { New Jersey } \\
\text { side }\end{array}$ & Aver age \\
\hline & $\begin{array}{c}1: 40 \text { p.m. } \\
76 \\
7.1\end{array}$ & $\begin{array}{l}1: 44 \text { p.m. } \\
23 \\
76 \\
76 \\
7.0 \\
7.1\end{array}$ & $\begin{array}{l}1: 47 \text { p.m. } \\
40 \\
76 \\
76 \\
6.9 \\
7.1\end{array}$ & $\begin{array}{c}1: 51 \mathrm{p} . \mathrm{m} . \\
13 \\
76 \\
76 \\
6.8 \\
7.0\end{array}$ & $\begin{array}{c}1: 54 \text { p.m. } \\
76 \\
6.9\end{array}$ & $\begin{array}{lc}\text { T. } & 76 \\
\text { B. } & 76 \\
\text { T. } & 6.9 \\
\text { B. } & 7.1\end{array}$ \\
\hline 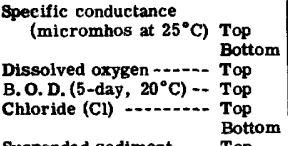 & $\begin{array}{r}172 \\
5.0 \\
.9 \\
9.0\end{array}$ & $\begin{array}{l}172 \\
173 \\
5.0 \\
1.5 \\
9.0 \\
9.0\end{array}$ & $\begin{array}{l}169 \\
170 \\
5.5 \\
1.5 \\
9.0 \\
9.0\end{array}$ & $\begin{array}{r}169 \\
168 \\
5.8 \\
5.1 \\
9.0 \\
9.0\end{array}$ & $\begin{array}{r}168 \\
6.0 \\
2.7 \\
9.0\end{array}$ & $\begin{array}{lr} & \\
\text { T. } & 170 \\
\text { B. } & 170 \\
\text { T. } & 5.5 \\
\text { T. } & 2.3 \\
\text { T. } & 9.0 \\
\text { B. } & 9.0\end{array}$ \\
\hline Suspended sediment $\ldots$ Top & - & $\cdots \cdot \cdot$ & . Compo & ite. . * & $\cdots \cdots \cdot$ & T. 16 \\
\hline
\end{tabular}

Location Torcesdale Intake, Phyladel phia, Pa. Date October 3, 1950 Sampling study No. 15 Water discharge at Trenton (cfs) 2.840

\begin{tabular}{|c|c|c|c|c|c|c|}
\hline \multicolumn{3}{|c|}{ 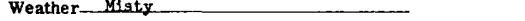 } & \multicolumn{4}{|c|}{ Water discharge at Trenton (cfs) $-2,840$} \\
\hline & \multicolumn{6}{|c|}{ Station } \\
\hline & $\begin{array}{c}\text { Pennsylvania } \\
\text { side }\end{array}$ & West Center & Center & East Center & $\begin{array}{c}\text { New Jersey } \\
\text { side }\end{array}$ & Average \\
\hline $\begin{array}{l}\text { Time (EST) } \\
\text { Sounding (ft) } \\
\text { Temperature }\left({ }^{\circ} \mathrm{F}\right) \\
\text { pH }-\begin{array}{l}\text { Top } \\
\text { Bottom }\end{array} \\
\begin{array}{l}\text { Top } \\
\text { Bottom }\end{array}\end{array}$ & $\begin{array}{l}1: 40 \text { p.m. } \\
68 \\
6.7\end{array}$ & $\begin{array}{c}1: 45 \mathrm{p} . \mathrm{m} \\
30 \\
66 \\
67 \\
6.7 \\
6.7\end{array}$ & $\begin{array}{c}1: 50 \mathrm{p} . \mathrm{m} . \\
33 \\
66 \\
67 \\
6.8 \\
6.8\end{array}$ & $\begin{array}{l}1: 55 \mathrm{p} . \mathrm{m} . \\
19 \\
66 \\
66 \\
6.7 \\
6.7\end{array}$ & $\begin{array}{l}2: 00 \mathrm{p} \cdot \mathrm{m} . \\
66 \\
6.7\end{array}$ & $\begin{array}{ll}\text { T. } & 66 \\
\text { B. } & 67 \\
\text { T. } & 6.7 \\
\text { B. } & 6.7\end{array}$ \\
\hline 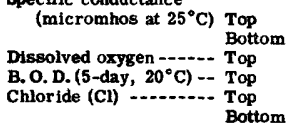 & $\begin{array}{r}180 \\
6.0 \\
.0 \\
8.0\end{array}$ & $\begin{array}{r}181 \\
181 \\
6.1 \\
.4 \\
8.0 \\
8.0\end{array}$ & $\begin{array}{r}171 \\
181 \\
6.4 \\
.5 \\
7.0 \\
8.0\end{array}$ & $\begin{array}{r}170 \\
179 \\
6.1 \\
.0 \\
7.0 \\
7.0\end{array}$ & $\begin{array}{r}170 \\
6.2 \\
.0 \\
7.0\end{array}$ & $\begin{array}{lr}\text { T. } & 174 \\
\text { B. } & 180 \\
\text { T. } & 6.2 \\
\text { T. } & .2 \\
\text { T. } & 7.4 \\
\text { B. } & 7.7\end{array}$ \\
\hline Suspended sediment $-\ldots$ Top & & & . Com & ite. . & & $\mathrm{T}$. \\
\hline
\end{tabular}

Locatiòn Terresdale Intake, Philadelpbla. Pa. Date__oxember 2, 1950 Sampling study No. 16 Weather_Overcagt _ Water discharge at Trenton (cis) $\frac{16}{2.550}$

\begin{tabular}{|c|c|c|c|c|c|c|}
\hline \multirow[b]{3}{*}{ 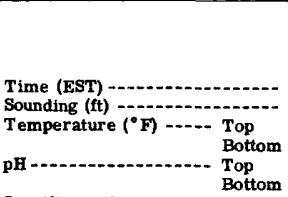 } & \multicolumn{6}{|c|}{ Station } \\
\hline & \begin{tabular}{|c|} 
Pennsylvania \\
side
\end{tabular} & West Center & Center & East Center & $\begin{array}{c}\text { New Jersey } \\
\text { side }\end{array}$ & Average \\
\hline & $\begin{array}{c}1: 17 \text { p.m. } \\
63 \\
6.8\end{array}$ & $\begin{array}{l}1: 21 \text { p.m. } \\
29 \\
62 \\
63 \\
6.9 \\
6.9\end{array}$ & $\begin{array}{l}1: 24 \mathrm{p} \cdot \mathrm{m} . \\
31 \\
62 \\
62 \\
6.9 \\
6.9\end{array}$ & $\begin{array}{c}1: 27 \text { p.m. } \\
9 \\
62 \\
62 \\
0.9 \\
6.9\end{array}$ & $\begin{array}{c}1: 29 \text { p.m. } \\
62 \\
6.9\end{array}$ & $\begin{array}{lc}\text { T. } & 62 \\
\text { B. } & 62 \\
\text { T. } & 6.9 \\
\text { B. } & 6.9\end{array}$ \\
\hline 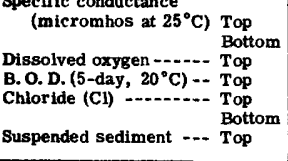 & 200 & $\begin{array}{c}197 \\
197 \\
8.4 \\
1.3 \\
10 \\
10 \\
\ldots\end{array}$ & $\begin{array}{l}192 \\
192 \\
8.4 \\
1.6 \\
10 \\
10 \\
. \text { Com }\end{array}$ & $\begin{array}{c}174 \\
171 \\
9.9 \\
2.6 \\
10 \\
9.0 \\
1 \\
\text { ite. }\end{array}$ & $\begin{array}{l}181 \\
10.4 \\
2.9 \\
10\end{array}$ & $\begin{array}{lc}\text { T. } & 189 \\
\text { B. } & 187 \\
\text { T. } & 9.3 \\
\text { T. } & 2.1 \\
\text { T. } & 10 \\
\text { B. } & 9.7 \\
\text { T. } & 22\end{array}$ \\
\hline
\end{tabular}


Table 8. -- WATER ANALYSES OF DELAWARE RIVER BETWEEN BRISTOL AND MARCUS HOOK, PA. --Continued Analyzed by City of Philadelphia and U. S. Geological Survey; analyses in parts per million

Location Torresdale Intake, Pniladelphı, Pa, Date_December 4, 1950 Sampling study No. 17 Weather Light Rain Water discharge at Trenton (cfs) 78,000

\begin{tabular}{|c|c|c|c|c|c|c|}
\hline & \multicolumn{6}{|c|}{ Station } \\
\hline & $\begin{array}{c}\text { Pennsylvania } \\
\text { side }\end{array}$ & West Center & Center & East Center & $\begin{array}{c}\text { New Jersey } \\
\text { side }\end{array}$ & Average \\
\hline $\begin{array}{l}\text { Time (EST) } \\
\text { Sounding (ft) } \\
\text { Temperature }\left({ }^{\circ} \mathrm{F}\right) \\
\begin{array}{l}\text { Bottom } \\
\text { pH }\end{array}\end{array}$ & $\begin{array}{c}11: 20 \text { a.m. } \\
42 \\
0.5\end{array}$ & $\begin{array}{c}11: 15 \text { a.m. } \\
32 \\
42 \\
42 \\
0.5 \\
0.4\end{array}$ & $\begin{array}{c}11: 10 \text { a.m. } \\
33 \\
43 \\
43 \\
0.5 \\
0.5\end{array}$ & $\begin{array}{c}11: 05 \text { a.m. } \\
18 \\
43 \\
43 \\
0.5 \\
6.5\end{array}$ & $\begin{array}{c}11: 00 \mathrm{a} \cdot \mathrm{m} \\
43 \\
6.4\end{array}$ & $\begin{array}{ll}\text { T. } & 43 \\
\text { B. } & 43 \\
\text { T. } & 6.5 \\
\text { B. } & 6.5\end{array}$ \\
\hline 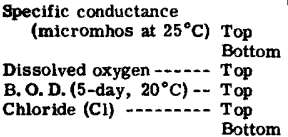 & $\begin{array}{r}108 \\
12.1 \\
3.7 \\
4.0\end{array}$ & $\begin{array}{r}105 \\
106 \\
12.2 \\
4.0 \\
4.0 \\
4.0\end{array}$ & $\begin{array}{r}99.4 \\
101 \\
12.0 \\
2.3 \\
2.0 \\
4.0\end{array}$ & $\begin{array}{r}100 \\
101 \\
12.0 \\
2.2 \\
4.0 \\
4.0\end{array}$ & $\begin{array}{r}101 \\
12.4 \\
2.5 \\
4.0\end{array}$ & $\begin{array}{lr}\text { T. } & 103 \\
\text { B. } & 103 \\
\text { T. } & 12.1 \\
\text { T. } & 2.9 \\
\text { T. } & 3.6 \\
\text { B. } & 4.0\end{array}$ \\
\hline Suspended sediment -.- Top & . . & ( & . Com & $\cdots \cdot$ & • & $\mathbf{T}$ \\
\hline
\end{tabular}

Location _Torresdale Intake, Fh1ladelphia, Fa. Date January 3, 1951 _ Sampling study No. ..... 18 Weather Clear W Water discharge at Trenton (cfs) - 8,210

\begin{tabular}{|c|c|c|c|c|c|c|}
\hline \multicolumn{3}{|c|}{ Weather Clear } & \multicolumn{4}{|c|}{ Water discharge at Trenton (cfs) $-8,210$} \\
\hline & \multicolumn{6}{|c|}{ Station } \\
\hline & \begin{tabular}{|c} 
Pennsylvania \\
side \\
\end{tabular} & West Center & Center & East Center & $\begin{array}{c}\text { New Jersey } \\
\text { side }\end{array}$ & Average \\
\hline $\begin{array}{l}\text { Time (EST) } \\
\text { Sounding }(\mathrm{ft}) \\
\text { Temperature }\left({ }^{\circ} \mathrm{F}\right) \\
\text { pH }-\begin{array}{l}\text { Top } \\
\text { Bottom }\end{array} \\
\begin{array}{l}\text { Top } \\
\text { Bottom }\end{array}\end{array}$ & $\begin{array}{c}9: 53 \text { a..n. } \\
34 \\
6.7\end{array}$ & $\begin{array}{l}7: 50 \text { a.m. } \\
24 \\
35 \\
35 \\
6.6 \\
0.6\end{array}$ & $\begin{array}{c}9: 4 b \text { a.m. } \\
39 \\
35 \\
35 \\
7.0 \\
6.6\end{array}$ & $\begin{array}{l}9: 43 \mathrm{a} . \mathrm{m} . \\
30 \\
35 \\
36 \\
6.7 \\
6.7\end{array}$ & $\begin{array}{l}9: 40 \mathrm{a} . \mathrm{m} . \\
35 \\
6.9\end{array}$ & $\begin{array}{ll}\text { T. } & 35 \\
\text { B. } & 35 \\
\text { T. } & 6.8 \\
\text { B. } & 6.6\end{array}$ \\
\hline 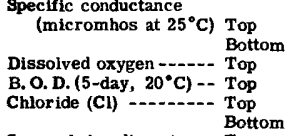 & $\begin{array}{r}164 \\
12.0 \\
5.4 \\
6.0\end{array}$ & $\begin{array}{r}166 \\
166 \\
12.0 \\
6.0 \\
6.0 \\
6.0\end{array}$ & $\begin{array}{r}168 \\
162 \\
12.5 \\
0.2 \\
6.0 \\
6.0\end{array}$ & $\begin{array}{r}148 \\
149 \\
12.0 \\
3.0 \\
4.0 \\
4.0\end{array}$ & $\begin{array}{r}147 \\
12.0 \\
1.9 \\
4.0\end{array}$ & $\begin{array}{lr}\text { T. } & 159 \\
\text { B. } & 159 \\
\text { T. } & 12.1 \\
\text { T. } & 4.5 \\
\text { T. } & 5.2 \\
\text { B. } & 5.3\end{array}$ \\
\hline Suspended sediment -.- Top & & & . Com & ite. . . & $\cdots \cdot$ & T. 13 \\
\hline
\end{tabular}

Location Torregdale Intake, Thiladelphia, Pa. Date-March 2, 195

Sampling study No. 19 Weather Clear Water discharge at Trenton (cfs) $-19,600$

\begin{tabular}{|c|c|c|c|c|c|c|}
\hline & \multicolumn{6}{|c|}{ Station } \\
\hline & \begin{tabular}{|c|}
$\begin{array}{c}\text { Pennsylvania } \\
\text { side }\end{array}$ \\
\end{tabular} & West Center & Center & East Center & $\begin{array}{c}\text { New Jersey } \\
\text { side }\end{array}$ & Average \\
\hline $\begin{array}{l}\text { Time (EST) } \\
\text { Sounding (ft) } \\
\text { Temperature ('F) } \\
\text { pH }\end{array} \begin{array}{l}\text { Top } \\
\text { Bottom }\end{array}$ & $\begin{array}{c}\text { 12:05 p.m. } \\
43 \\
6.5\end{array}$ & $\begin{array}{c}12: 10 \text { p.m. } \\
36 \\
42 \\
42 \\
6.6 \\
6.7\end{array}$ & $\begin{array}{c}12: 15 \text { p.m. } \\
28 \\
42 \\
43 \\
6.7 \\
6.8\end{array}$ & $\begin{array}{c}12: 20 \mathrm{p} \cdot \mathrm{m} . \\
20 \\
43 \\
43 \\
6.7 \\
6.8\end{array}$ & $\begin{array}{c}12: 25 \text { p.m. } \\
43 \\
6.8\end{array}$ & $\begin{array}{ll}\text { T. } & 43 \\
\text { B. } & 43 \\
\text { T. } & 6.7 \\
\text { B. } & 6.8\end{array}$ \\
\hline $\begin{array}{ll}\begin{array}{l}\text { Specific conductance } \\
\left.\text { (micromhos at } 25^{\circ} \mathrm{C}\right)\end{array} & \begin{array}{l}\text { Top } \\
\text { Bottom }\end{array} \\
\begin{aligned} \text { Dissolved oxygen -...- Top } \\
\text { B. O. D. (5-day, 20 }\end{aligned} \\
\text { Chloride (Cl) - } & \text { Top } \\
& \text { Bottom }\end{array}$ & $\begin{array}{r}109 \\
12.9 \\
3.0 \\
4.0\end{array}$ & $\begin{array}{r}105 \\
104 \\
13.2 \\
3.9 \\
4.0 \\
4.0\end{array}$ & $\begin{array}{r}101 \\
102 \\
12.6 \\
3.0 \\
4.0 \\
4.0\end{array}$ & $\begin{array}{r}103 \\
101 \\
12.9 \\
3.3 \\
4.0 \\
4.0\end{array}$ & $\begin{array}{r}97.5 \\
12.8 \\
3.3 \\
3.0\end{array}$ & $\begin{array}{lr} & \\
\text { T. } & 103 \\
\text { B. } & 102 \\
\text { T. } & 12.9 \\
\text { T. } & 3.4 \\
\text { T. } & 3.8 \\
\text { B. } & 4.0\end{array}$ \\
\hline Suspended sediment --- Top & . & $\cdots$ & - Comp & te.... & $\cdots$ & T. \\
\hline
\end{tabular}

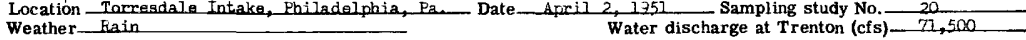

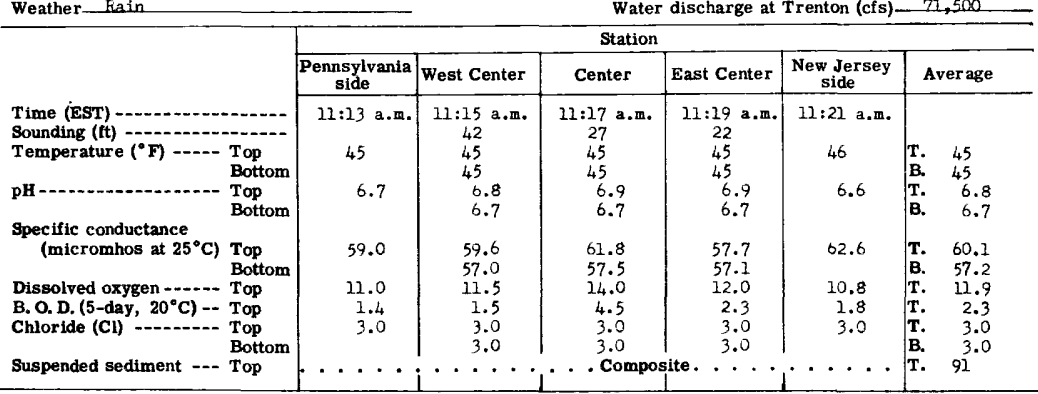


Table 8. -- WATER ANALYSES OF DELAWARE RIVER BETWEEN BRISTOL AND MARCUS HOOK, PA. --Continued Analyzed by City of Philadelphia and U. S. Geological Survey; analyses in parts per million Location Torrestale Intake, Philadel phia, Pa,_- Date_Nay 1, 1951 Sampling study No. - 21
Weather_clear

\begin{tabular}{|c|c|c|c|c|c|c|}
\hline & \multicolumn{6}{|c|}{ Station } \\
\hline & $\begin{array}{c}\text { Pennsylvania } \\
\text { side }\end{array}$ & West Center & Center & East Center & $\begin{array}{c}\text { New Jersey } \\
\text { side }\end{array}$ & Average \\
\hline $\begin{array}{ll}\text { Time (EST) } & \\
\text { Sounding (ft) } \\
\text { Temperature }\left({ }^{\circ} \mathrm{F}\right)\end{array}$ & $\begin{array}{c}9: 40 \mathrm{a} . \mathrm{m} . \\
01 \\
7.0\end{array}$ & $\begin{array}{l}9: 38 \text { a.m. } \\
33 \\
61 \\
\text { b1 } \\
7.0 \\
7.0\end{array}$ & $\begin{array}{l}9: 36 \text { a.m. } \\
37 \\
61 \\
61 \\
7.1 \\
7.0\end{array}$ & $\begin{array}{c}9: 33 \text { a.m. } \\
18 \\
01 \\
61 \\
7.1 \\
7.1\end{array}$ & $\begin{array}{l}9: 30 \mathrm{a} . \mathrm{m} . \\
61 \\
7.1\end{array}$ & $\begin{array}{ll}\text { T. } & 61 \\
\text { B. } & 61 \\
\text { T. } & 7.1 \\
\text { B. } & 7.0\end{array}$ \\
\hline 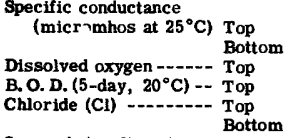 & $\begin{array}{l}119 \\
8.7 \\
3.0 \\
6.0\end{array}$ & $\begin{array}{r}115 \\
115 \\
8.7 \\
2.7 \\
6.0 \\
0.0\end{array}$ & $\begin{array}{l}119 \\
118 \\
8.3 \\
2.5 \\
6.0 \\
0.0\end{array}$ & $\begin{array}{l}109 \\
109 \\
8.1 \\
1.1 \\
0.0 \\
0.0\end{array}$ & $\begin{array}{r}109 \\
7.0 \\
.0 \\
6.0\end{array}$ & $\begin{array}{lr}\text { T. } & 114 \\
\text { B. } & 114 \\
\text { T. } & 8.2 \\
\text { T. } & 1.9 \\
\text { T. } & 6.0 \\
\text { B. } & 6.0\end{array}$ \\
\hline Suspended sediment --- Top & $\cdots \cdot \cdot$ & $\cdot \cdot \cdot$ & . Com & site. . . & - & T. $\quad 31$ \\
\hline
\end{tabular}

Location Torregdale Intake, Fhiladelphia, Pa, Date_June 7, 1951 __ Sampling study No.__22_- 22 Weather_Clear $\quad$ Water discharge at Trenton (cfs) $-\varepsilon, 070$

\begin{tabular}{|c|c|c|c|c|c|c|}
\hline \\
\hline & \multicolumn{6}{|c|}{ Station } \\
\hline & \begin{tabular}{|c|}
$\begin{array}{c}\text { Pennsylvania } \\
\text { side }\end{array}$ \\
\end{tabular} & West Center & Center & East Center & $\begin{array}{c}\text { New Jersey } \\
\text { side }\end{array}$ & Average \\
\hline $\begin{array}{ll}\text { Time (EST) } & \\
\text { Sounding }(\mathrm{ft}) & \\
\text { Temperature }\left({ }^{\circ} \mathrm{F}\right) & \begin{array}{l}\text { Top } \\
\text { Bottom }\end{array} \\
\text { pH } & \begin{array}{l}\text { Top } \\
\text { Bottom }\end{array} \\
\text { Specific conductance } & \end{array}$ & $\begin{array}{c}12: 06 \text { p.m. } \\
74 \\
6.9\end{array}$ & $\begin{array}{c}12: 08 \text { p.m. } \\
30 \\
74 \\
74 \\
7.0 \\
7.0\end{array}$ & $\begin{array}{c}12: 10 \mathrm{p} . \mathrm{m} \\
28 \\
74 \\
74 \\
6.7 \\
0.8\end{array}$ & $\begin{array}{c}12: 15 \mathrm{p} \cdot \mathrm{m} . \\
9 \\
74 \\
74 \\
7.0 \\
6.9\end{array}$ & $\begin{array}{l}12: 17 \text { p.m. } \\
74 \\
0.8\end{array}$ & $\begin{array}{lc}\text { T. } & 74 \\
\text { B. } & 74 \\
\text { T. } & 6.9 \\
\text { B. } & 6.9\end{array}$ \\
\hline $\begin{array}{ll}\left.\text { (micromhos at } 25^{\circ} \mathrm{C}\right) & \begin{array}{l}\text { Top } \\
\text { Bottom }\end{array} \\
\text { Dissolved oxygen - } & \text { Top } \\
\text { B. O. D. (5-day, } 20^{\circ} \mathrm{C} \text { ) - } & \text { Top } \\
\text { Chloride (C) }-10 & \text { Top } \\
& \text { Bottom }\end{array}$ & $\begin{array}{l}133 \\
6.5 \\
2.3 \\
6.0\end{array}$ & $\begin{array}{l}133 \\
133 \\
6.5 \\
2.5 \\
5.0 \\
6.0\end{array}$ & $\begin{array}{l}130 \\
127 \\
6.8 \\
2.9 \\
0.0 \\
5.0\end{array}$ & $\begin{array}{l}126 \\
123 \\
6.8 \\
1.8 \\
0.0 \\
6.0\end{array}$ & $\begin{array}{l}118 \\
6.7 \\
2.3 \\
5.0\end{array}$ & $\begin{array}{lr}\text { T. } & 128 \\
\text { B. } & 128 \\
\text { T. } & 6.7 \\
\text { T. } & 2.4 \\
\text { T. } & 5.6 \\
\text { B. } & 5.7\end{array}$ \\
\hline Suspended sediment --- Top & & $\cdots$ & Com & te. . . & 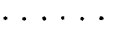 & T. \\
\hline
\end{tabular}

Location Torresdale Intake, Ehiladelohla, Pa._Date July 5, 1951 _ Sampling study No.___23 Weather_clouds Water discharge at Trenton (cfs)_- 9,190

\begin{tabular}{|c|c|c|c|c|c|c|}
\hline & \multicolumn{6}{|c|}{ Station } \\
\hline & $\begin{array}{c}\text { Pennsylvania } \\
\text { side }\end{array}$ & West Center & Center & East Center & $\begin{array}{l}\text { New Jersey } \\
\text { side }\end{array}$ & Aver age \\
\hline $\begin{array}{ll}\text { Time (EST) } & \\
\text { Sounding (ft) } \\
\text { Temperature }\left({ }^{\circ} \mathrm{F}\right)\end{array} \quad \begin{array}{l}\text { Top } \\
\text { Bottom } \\
\text { pH }\end{array}$ & $\begin{array}{c}10: 15 \text { a.m. } \\
77 \\
7.0\end{array}$ & $\begin{array}{c}10: 17 \text { a.m. } \\
32 \\
76 \\
77 \\
7.1 \\
7.2\end{array}$ & $\begin{array}{c}10: 21 \text { a.m. } \\
27 \\
76 \\
76 \\
7.1 \\
7.0\end{array}$ & $\begin{array}{c}20: 25 \text { a.m. } \\
8 \\
76 \\
76 \\
7.0 \\
7.1\end{array}$ & $\begin{array}{c}10: 27 \text { a.m. } \\
77 \\
0.9\end{array}$ & $\begin{array}{ll}\text { T. } & 76 \\
\text { B. } & 76 \\
\text { T. } & 7.0 \\
\text { B. } & 7.1\end{array}$ \\
\hline 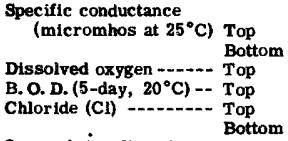 & $\begin{array}{r}135 \\
5.5 \\
1.0 \\
6.0\end{array}$ & $\begin{array}{r}153 \\
150 \\
5.4 \\
.9 \\
6.0 \\
0.0\end{array}$ & $\begin{array}{r}152 \\
151 \\
5.4 \\
6.6 \\
6.0 \\
6.0\end{array}$ & $\begin{array}{r}154 \\
142 \\
5.2 \\
1.5 \\
6.0 \\
0.0\end{array}$ & $\begin{array}{r}132 \\
5.4 \\
1.6 \\
0.0\end{array}$ & $\begin{array}{lr} & \\
\text { T. } & 145 \\
\text { B. } & 148 \\
\text { T. } & 5.4 \\
\text { T. } & 2.3 \\
\text { T. } & 6.0 \\
\text { B. } & 6.0\end{array}$ \\
\hline Suspended sediment --- Top & & & Com & e... & & $\mathrm{T}$. \\
\hline
\end{tabular}

Location Torresdale Intake, Philadelphia, Pa, Date_August 1, 1951 Sampling study No. - 24 - C Weather Cloudy Water discharge at Trenton (cfs) - Date 12,400

\begin{tabular}{|c|c|c|c|c|c|c|}
\hline Weather Cloudy & & & Wat & $\operatorname{arge}$ & enton (cfs) & $-12,40$ \\
\hline & \multicolumn{6}{|c|}{ Station } \\
\hline & $\begin{array}{c}\text { Pennsylvania } \\
\text { side }\end{array}$ & West Center & Center & East Center & $\begin{array}{l}\text { New Jersey } \\
\text { side }\end{array}$ & Average \\
\hline $\begin{array}{l}\text { Time (EST) } \\
\text { Sounding (ft) } \\
\text { Temperature ('F) } \\
\text { pH }\end{array}$ & $\begin{array}{c}12: 00 \mathrm{n} \\
76 \\
6.8\end{array}$ & $\begin{array}{c}12: 02 \text { p.m. } \\
35 \\
70 \\
70 \\
6.7 \\
0.9\end{array}$ & $\begin{array}{c}12: 04 \mathrm{p.m} \\
30 \\
76 \\
70 \\
7.0 \\
0.9\end{array}$ & $\begin{array}{c}12: 06 \mathrm{p.m} . \\
17 \\
76 \\
76 \\
7.0 \\
7.0\end{array}$ & $\begin{array}{c}12: 08 \mathrm{p} \cdot \mathrm{m} . \\
76 \\
6.9\end{array}$ & $\begin{array}{ll}\text { T. } & 76 \\
\text { B. } & 76 \\
\text { T. } & 6.9 \\
\text { B. } & 6.9\end{array}$ \\
\hline 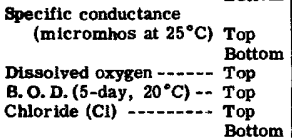 & $\begin{array}{r}164 \\
5.7 \\
4.1 \\
4.0\end{array}$ & $\begin{array}{r}166 \\
162 \\
6.1 \\
2.7 \\
4.0 \\
4.0\end{array}$ & $\begin{array}{l}158 \\
160 \\
6.4 \\
2.8 \\
4.0 \\
4.0\end{array}$ & $\begin{array}{r}162 \\
159 \\
0.0 \\
.9 \\
4.0 \\
4.0\end{array}$ & $\begin{array}{l}157 \\
6.1 \\
3.1 \\
4.0\end{array}$ & $\begin{array}{lr}\text { T. } & 161 \\
\text { B. } & 160 \\
\text { T. } & 6.1 \\
\text { T. } & 2.7 \\
\text { T. } & 4.0 \\
\text { B. } & 4.0\end{array}$ \\
\hline Suspended sediment.-- Top & & & 40 & e... & $\cdots \cdot \cdot$ & 35 \\
\hline
\end{tabular}


Table 8. --WATER ANALYSES OF DELAWARE RIVER BETWEEN BRISTOL AND MARCUS HOOK, PA, --Continued Analyzed by City of Philadelphia and U. S. Geological Survey; analyses in parts per million

Location Torresdale Intake, Philadelphia, Pa. Date September 6, 1951 Sampling study No. 25

$\frac{25}{3,10}$

\begin{tabular}{|c|c|c|c|c|c|c|}
\hline \multicolumn{7}{|c|}{ Weather Rain } \\
\hline \multirow[b]{2}{*}{$\begin{array}{l}\text { Time (EST) } \\
\text { Sounding (ft) } \\
\text { Temperature }\left({ }^{\circ} \mathrm{F}\right) \\
\text { pH }\end{array} \begin{array}{l}\text { Top } \\
\text { Bottom } \\
\text { Top } \\
\text { Bottom }\end{array}$} & $\begin{array}{c}\text { Pennsylvania } \\
\text { side }\end{array}$ & West Center & Center & East Center & $\begin{array}{c}\text { New Jersey } \\
\text { side }\end{array}$ & Average \\
\hline & $\begin{array}{c}12: 20 \text { p.m. } \\
75 \\
7.9\end{array}$ & $\begin{array}{c}12: 22 \mathrm{p} . \mathrm{m} . \\
29 \\
75 \\
75 \\
7.1 \\
7.1\end{array}$ & $\begin{array}{c}12: 24 \text { p.m. } \\
38 \\
75 \\
75 \\
7.1 \\
7.1\end{array}$ & $\begin{array}{c}12: 26 \mathrm{p.m} . \\
15 \\
75 \\
75 \\
7.0 \\
7.0\end{array}$ & $\begin{array}{l}12: 28 \mathrm{p} . \mathrm{m} . \\
75 \\
6.9\end{array}$ & $\begin{array}{c}75 \\
75 \\
7.0 \\
7.1\end{array}$ \\
\hline \multirow[t]{2}{*}{ 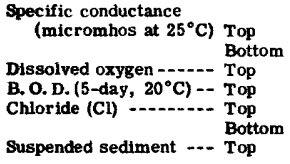 } & $\begin{array}{l}202 \\
\\
5.6 \\
1.8 \\
6.0\end{array}$ & $\begin{array}{r}202 \\
202 \\
5.5 \\
1.9 \\
6.0 \\
7.0\end{array}$ & $\begin{array}{r}202 \\
199 \\
5.3 \\
1.9 \\
6.0 \\
7.0\end{array}$ & $\begin{array}{r}196 \\
195 \\
5.4 \\
1.9 \\
5.0 \\
6.0\end{array}$ & $\begin{array}{r}185 \\
5.6 \\
1.7 \\
0.0\end{array}$ & $\begin{array}{lr}\text { T. } & 197 \\
\text { B. } & 199 \\
\text { T. } & 5.5 \\
\text { T. } & 1.8 \\
\text { T. } & 5.8 \\
\text { B. } & 6.7\end{array}$ \\
\hline & & & & & & T. 11 \\
\hline
\end{tabular}

Location Torresdale Intake, Philadelphia, Pa. Date October 2, 1951 Sampling study No. 26

\begin{tabular}{|c|c|c|c|c|c|c|}
\hline \multicolumn{3}{|c|}{ Weather Clear } & \multicolumn{4}{|c|}{ Water discharge at Trenton (cfs) 2,990} \\
\hline & \multicolumn{6}{|c|}{ Station } \\
\hline & $\begin{array}{c}\text { Pennsylvania } \\
\text { side }\end{array}$ & West Center & Center & East Center & \begin{tabular}{|c}
$\begin{array}{c}\text { New Jersey } \\
\text { side }\end{array}$ \\
\end{tabular} & Average \\
\hline $\begin{array}{l}\text { Time (EST) } \\
\text { Sounding (ft) } \\
\text { Temperature }\left({ }^{\circ} \mathrm{F}\right)\end{array} \begin{array}{l}\text { Top } \\
\text { Bottom } \\
\text { Top } \\
\text { Bottom }\end{array}$ & $\begin{array}{l}1: 19 \text { p.m. } \\
71 \\
0.5\end{array}$ & $\begin{array}{l}1: 20 \mathrm{p} \cdot \mathrm{m} . \\
40 \\
71 \\
70 \\
6.6 \\
6.5\end{array}$ & $\begin{array}{l}1: 23 \mathrm{p} . \mathrm{m} \text {. } \\
35 \\
70 \\
70 \\
6.7 \\
7.3\end{array}$ & $\begin{array}{c}1: 26 \text { p.m. } \\
15 \\
70 \\
70 \\
0.6 \\
0.5\end{array}$ & $\begin{array}{l}1: 28 \text { p.m. } \\
70 \\
6.6\end{array}$ & $\begin{array}{lc}\text { T. } & 70 \\
\text { B. } & 70 \\
\text { T. } & 6.6 \\
\text { B. } & 6.8\end{array}$ \\
\hline 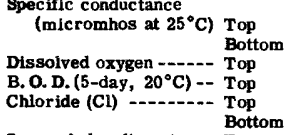 & $\begin{array}{l}235 \\
3.6 \\
8.1 \\
11\end{array}$ & $\begin{array}{l}214 \\
210 \\
4 \cdot 4 \\
3.9 \\
10 \\
10\end{array}$ & $\begin{array}{l}208 \\
207 \\
4.7 \\
4.2 \\
10 \\
9.0\end{array}$ & $\begin{array}{r}205 \\
205 \\
5.5 \\
2.7 \\
9.0 \\
9.0\end{array}$ & $\begin{array}{l}215 \\
0.1 \\
2.6 \\
10\end{array}$ & $\begin{array}{lc}\text { T. } & 215 \\
\text { B. } & 207 \\
\text { T. } & 4.9 \\
\text { T. } & 4.3 \\
\text { T. } & 10 \\
\text { B. } & 9.3\end{array}$ \\
\hline Suspended sediment --- Top & & & . Con & site. . & 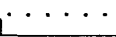 & $\mathbf{T}$ \\
\hline
\end{tabular}

Location Torrasdale Intake, Philadelphia, Pa. Date November 7, 1951 Sampling study No. 27

\begin{tabular}{l|r|c|c|c|c|c|c} 
Location \\
Weather_Cloudy
\end{tabular}

Locatiön - Torresdale Intake, Philadelphia, Pa... Date December 5, 1951 _ampling study No. 28

\begin{tabular}{|c|c|c|c|c|c|c|}
\hline \multicolumn{3}{|l|}{ Weathe } & Wa & scharge & Trenton (c & $-12,500$ \\
\hline & \multicolumn{6}{|c|}{ Station } \\
\hline & \begin{tabular}{|c}
$\begin{array}{c}\text { Pennsylvania } \\
\text { side }\end{array}$ \\
\end{tabular} & West Center & Center & East Center & \begin{tabular}{|c}
$\begin{array}{c}\text { New Jersey } \\
\text { side }\end{array}$ \\
\end{tabular} & Average \\
\hline $\begin{array}{l}\text { Time (EST) } \\
\text { Sounding (ft) } \\
\text { Temperature }\left({ }^{\circ} \mathrm{F}\right) \\
\text { pH }\end{array} \begin{array}{l}\text { Top } \\
\text { Bottom } \\
\text { Top } \\
\text { Bottom }\end{array}$ & $\begin{array}{c}2: 11 \text { p.m. } \\
43 \\
6.9\end{array}$ & $\begin{array}{l}2: 14 \text { p.m. } \\
30 \\
41 \\
44 \\
7.2 \\
6.9\end{array}$ & $\begin{array}{l}2: 16 \text { p.m. } \\
31 \\
41 \\
42 \\
6.9 \\
6.9\end{array}$ & $\begin{array}{l}2: 18 \mathrm{p} . \mathrm{m} \\
37 \\
42 \\
42 \\
6.9 \\
6.9\end{array}$ & $\begin{array}{l}2: 20 \text { p.m. } \\
42 \\
6.8\end{array}$ & $\begin{array}{ll}\text { T. } & 42 \\
\text { B. } & 43 \\
\text { T. } & 6.9 \\
\text { B. } & 0.9\end{array}$ \\
\hline 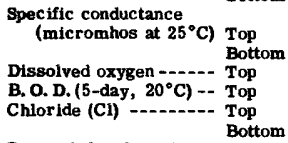 & $\begin{array}{r}132 \\
12.2 \\
3.6 \\
5.0\end{array}$ & $\begin{array}{r}133 \\
135 \\
12.2 \\
3.1 \\
5.0 \\
0.0\end{array}$ & $\begin{array}{r}133 \\
134 \\
12.4 \\
3.6 \\
4.0 \\
5.0\end{array}$ & $\begin{array}{r}136 \\
132 \\
12.2 \\
3.4 \\
0.0 \\
4.0\end{array}$ & $\begin{array}{r}124 \\
12.1 \\
3.8 \\
6.0\end{array}$ & $\begin{array}{lr} & \\
\text { T. } & 132 \\
\text { B. } & 134 \\
\text { T. } & 12.2 \\
\text { T. } & 3.5 \\
\text { T. } & 5.2 \\
\text { B. } & 5.0\end{array}$ \\
\hline Suspended sediment $\ldots$... Top & & & Con & & & T. \\
\hline
\end{tabular}


Table 8. --WATER ANALYSES OF DELAWARE RIVER BETWEEN BRISTOL AND Marcus hoOK, PA. --Continued Analyzed by City of Philadelphia and U. S. Geological Survey; anaiyses in parts per mulion

Location Iorresdale Intaks, Fniladelpnia, Fa. Date January 3, 1952 Sampling study No. 29

\begin{tabular}{|c|c|c|c|c|c|c|}
\hline \multicolumn{3}{|c|}{ Weather Clear } & \multicolumn{4}{|c|}{ Water discharge at Trenton (cfs) 30,500} \\
\hline & \multicolumn{6}{|c|}{ Station } \\
\hline & \begin{tabular}{|c|} 
Pennsylvania \\
side
\end{tabular} & West Center & Center & East Center & $\begin{array}{c}\text { New Jersey } \\
\text { side }\end{array}$ & Average \\
\hline $\begin{array}{l}\text { Time (EST) } \\
\text { Sounding (ft) } \\
\text { Temperature ( }{ }^{\circ} \mathrm{F} \text { ) } \\
\text { pH }\end{array}$ & $\begin{array}{c}20: 30 \text { a. } 1.1 . \\
38 \\
7.1\end{array}$ & $\begin{array}{c}10: 35 \mathrm{a} . \mathrm{m} . \\
31 \\
39 \\
39 \\
7.2 \\
7.1\end{array}$ & $\begin{array}{c}10: 37 \text { a.n. } \\
27 \\
39 \\
38 \\
7.2 \\
7.2\end{array}$ & $\begin{array}{c}10: 40 \text { a.m. } \\
21 \\
39 \\
38 \\
7.1 \\
7.1\end{array}$ & $\begin{array}{c}20: 42 \text { a.m. } \\
39 \\
6.9\end{array}$ & $\begin{array}{ll}\text { T. } & 39 \\
\text { B. } & 38 \\
\text { T. } & 7.1 \\
\text { B. } & 7.1\end{array}$ \\
\hline $\begin{array}{l}\text { Spectfic conductance } \\
\text { (micromhos at } 25^{\circ} \mathrm{C} \text { ) } \begin{array}{l}\text { Top } \\
\text { Bottom }\end{array}\end{array}$ & 136 & $\begin{array}{l}133 \\
132\end{array}$ & $\begin{array}{l}127 \\
128\end{array}$ & $\begin{array}{l}122 \\
123\end{array}$ & 117 & $\begin{array}{l}\text { T. } 127 \\
\text { B. } 128\end{array}$ \\
\hline $\begin{array}{l}\text { Dissolved oxygen }-\ldots-\text { Top } \\
\left.\text { B. O. D. (5-day, } 20^{\circ} \mathrm{C}\right)- \text { Top } \\
\text { Chloride (Cl) }\end{array}$ & $\begin{array}{r}11.2 \\
1.7 \\
4.0\end{array}$ & $\begin{array}{r}11.4 \\
3.5 \\
5.0 \\
4.0\end{array}$ & $\begin{array}{r}10.5 \\
.9 \\
4.0 \\
4.0\end{array}$ & $\begin{array}{r}20.6 \\
1.0 \\
4.0 \\
4.0\end{array}$ & $\begin{array}{r}10.8 \\
1.7 \\
4.0\end{array}$ & $\begin{array}{lr}\text { T. } \\
\text { T. } & 10.9 \\
\text { T. } & 1.8 \\
\text { T. } & 4.2 \\
\text { B. } & 4.0\end{array}$ \\
\hline Suspended sediment --- Top & 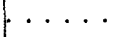 & $4 \cdot 0$ & 40m & ite. . . & 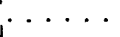 & T. 19 \\
\hline
\end{tabular}

Location Torresdale Intake, Philadelphia, Pa. Date February 5, 1952 Sampling study No. 30

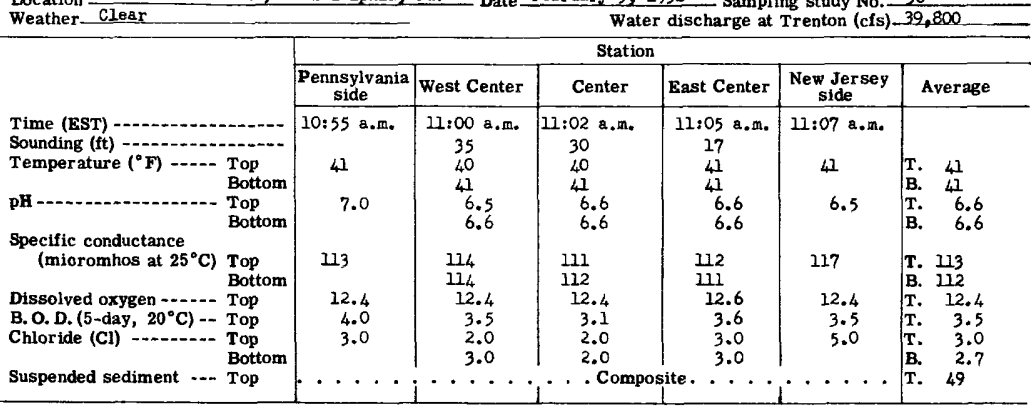

Location Torresdale Intake, Fhiladelnhia, $\mathrm{Pa}$

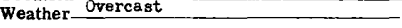

Sampling study No, 31

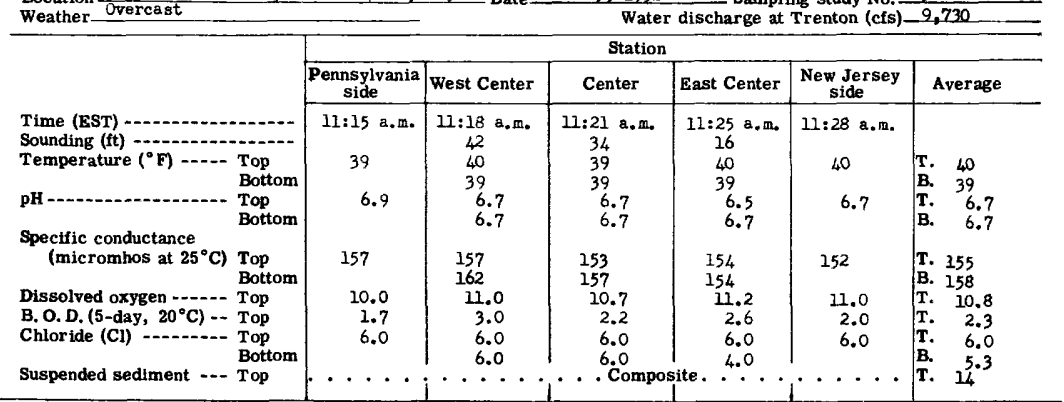

Location Torresdale Intake, Ph11adelphis, Ps. Date Apri1 2, 1952 Sampling study No. 32

\begin{tabular}{|c|c|c|c|c|c|c|}
\hline $\begin{array}{l}\text { Location Torres } \\
\text { Weather Clear }\end{array}$ & & & Wa & Samp & $\begin{array}{l}\text { ng study No.- } \\
\text { Trenton (cfs). }\end{array}$ & 22,700 \\
\hline & & & Station & & & \\
\hline & \begin{tabular}{|c|} 
Pennsylvania \\
side
\end{tabular} & West Center & Center & East Center & \begin{tabular}{|c|}
$\begin{array}{c}\text { New Jersey } \\
\text { side }\end{array}$ \\
\end{tabular} & Average \\
\hline $\begin{array}{l}\text { Time (EST) } \\
\text { Sounding (ft) } \\
\text { Temperature }(" \mathrm{~F}) \\
\text { pH }\end{array}$ & $\begin{array}{c}11: 15 \text { a.m. } \\
49 \\
6.7\end{array}$ & $\begin{array}{c}11: 18 \text { a.m. } \\
34 \\
49 \\
49 \\
6.6 \\
7.1\end{array}$ & $\begin{array}{c}11: 21 \text { a.m. } \\
38 \\
49 \\
49 \\
6.7 \\
6.7\end{array}$ & $\begin{array}{c}11: 24 \mathrm{a} . \mathrm{m} . \\
25 \\
49 \\
50 \\
6.7 \\
6.7\end{array}$ & $\begin{array}{c}11: 27 \text { a.m. } \\
50 \\
6.8\end{array}$ & $\begin{array}{ll}\text { T. } & 49 \\
\text { B. } & 49 \\
\text { T. } & 6.7 \\
\text { B. } & 6.8\end{array}$ \\
\hline 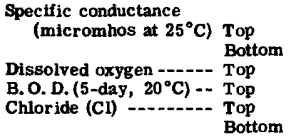 & $\begin{array}{r}99.2 \\
10.5 \\
.9 \\
3.0\end{array}$ & $\begin{array}{c}99.2 \\
103 \\
9.9 \\
.9 \\
3.0 \\
3.0\end{array}$ & $\begin{array}{r}96.5 \\
97.0 \\
9.6 \\
.8 \\
3.0 \\
2.0\end{array}$ & $\begin{array}{r}94.5 \\
95.7 \\
9.2 \\
.3 \\
3.0 \\
3.0\end{array}$ & $\begin{array}{r}94.5 \\
9.8 \\
1.0 \\
3.0\end{array}$ & $\begin{array}{lr}\text { T. } & 96.8 \\
\text { B. } & 98.6 \\
\text { T. } & 9.8 \\
\text { T. } & .8 \\
\text { T. } & 3.0 \\
\text { B. } & 2.7\end{array}$ \\
\hline Suspended sediment -.. Top & ・ & & Com & ite. . . & - & T. 10 \\
\hline
\end{tabular}


Table 8, -- WATER ANALYSES OF DELAWARE RIVER BETWEEN BRISTOL AND MARCUS HOOK, PA. --Continued Analyzed by City of Philadelpha and U. S. Geological Survey; analyses in parts per million

Location Torresdale Intake, Philadelphza, Fa._Date May 5, 1952 ___ Sampling study No. 33

\begin{tabular}{|c|c|c|c|c|c|c|}
\hline \multicolumn{7}{|c|}{ eather Overcast } \\
\hline & $\begin{array}{c}\text { Pennsylvania } \\
\text { side }\end{array}$ & West Center & Center & East Center & $\begin{array}{c}\text { New Jersey } \\
\text { side }\end{array}$ & Average \\
\hline $\begin{array}{l}\text { Time (EST) } \\
\begin{array}{l}\text { Bounding (ft) } \\
\text { Temperature }\left({ }^{\circ} \mathrm{F}\right)\end{array} \\
\text { pH } \\
\begin{array}{l}\text { Top } \\
\text { Bottom } \\
\text { Bop } \\
\text { Bottom }\end{array}\end{array}$ & $\begin{array}{c}10: 12 \text { a.m. } \\
58 \\
6.7\end{array}$ & $\begin{array}{c}10: 15 \text { a.m. } \\
33 \\
58 \\
58 \\
6.7 \\
7.4\end{array}$ & $\begin{array}{c}10: 18 \text { a.m. } \\
40 \\
57 \\
58 \\
0.8 \\
6.8\end{array}$ & $\begin{array}{c}10: 20 \text { a.m. } \\
18 \\
57 \\
58 \\
6.7 \\
6.8\end{array}$ & $\begin{array}{c}10: 22 \text { a.m. } \\
58 \\
6.7\end{array}$ & $\begin{array}{ll}\text { T. } & 58 \\
\text { B. } & 58 \\
\text { T. } & 6.7 \\
\text { B. } & 7.0\end{array}$ \\
\hline 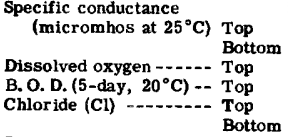 & $\begin{array}{r}124 \\
8.6 \\
1.0 \\
3.0\end{array}$ & $\begin{array}{l}120 \\
120 \\
8.8 \\
1.0 \\
4.0 \\
3.0\end{array}$ & $\begin{array}{r}116 \\
115 \\
8.8 \\
.8 \\
3.0 \\
3.0\end{array}$ & $\begin{array}{l}111 \\
111 \\
8.8 \\
2.0 \\
2.0 \\
2.0\end{array}$ & $\begin{array}{l}106 \\
\\
9.0 \\
2.2 \\
4.0\end{array}$ & $\begin{array}{lr} & \\
\text { T. } & 215 \\
\text { B. } & 115 \\
\text { T. } & 8.8 \\
\text { T. } & 1.4 \\
\text { T. } & 3.2 \\
\text { B. } & 2.7\end{array}$ \\
\hline Suspended sediment --- Top & & $\cdots \cdot$ &. Com & site. . & $\cdot \cdot \cdot$ & T. 18 \\
\hline
\end{tabular}
Location Torresdale Intake, Philadelphia, Pa, Date June $4, \frac{1952}{\text { Weather_Cloudy }}$ Water discharge at Trenton (cis) $-28,600$
... Sampling study No.

\begin{tabular}{|c|c|c|c|c|c|c|}
\hline & \multicolumn{6}{|c|}{ Station } \\
\hline & $\begin{array}{c}\begin{array}{c}\text { Pennsylvania } \\
\text { side }\end{array} \\
\end{array}$ & West Center & Center & East Center & $\begin{array}{c}\text { New Jersey } \\
\text { side }\end{array}$ & Average \\
\hline $\begin{array}{ll}\text { Time (EST) } & \\
\text { Sounding }(\mathrm{ft}) & \\
\text { Temperature }\left({ }^{\circ} \mathrm{F}\right) & \begin{array}{l}\text { Top } \\
\text { Bottom } \\
\text { pH }\end{array} \\
\text { Specific conductance } & \begin{array}{l}\text { Bottom } \\
\text { Spen }\end{array}\end{array}$ & $\begin{array}{c}9: 52 \text { a.m. } \\
67 \\
6.8\end{array}$ & $\begin{array}{l}9: 54 \text { a.m. } \\
35 \\
66 \\
69 \\
6.8 \\
6.6\end{array}$ & $\begin{array}{c}9: 56 \text { a.m. } \\
37 \\
66 \\
69 \\
6.7 \\
6.7\end{array}$ & $\begin{array}{l}9: 58 \text { a.ja. } \\
15 \\
67 \\
68 \\
6.8 \\
6.7\end{array}$ & $\begin{array}{l}10: 00 \mathrm{a} \cdot \mathrm{m} . \\
68 \\
6.6\end{array}$ & $\begin{array}{ll}\text { T. } & 67 \\
\text { B. } & 69 \\
\text { T. } & 6.7 \\
\text { B. } & 6.7\end{array}$ \\
\hline 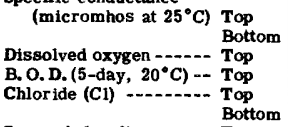 & $\begin{array}{r}77.5 \\
7.7 \\
2.2 \\
2.0\end{array}$ & $\begin{array}{r}92.0 \\
94.8 \\
7.7 \\
1.6 \\
2.0 \\
2.0\end{array}$ & $\begin{array}{r}90.4 \\
90.2 \\
7.9 \\
1.4 \\
2.0 \\
2.0\end{array}$ & $\begin{array}{r}89.7 \\
89.1 \\
7.8 \\
1.3 \\
2.0 \\
2.0\end{array}$ & $\begin{array}{r}89.1 \\
7.5 \\
1.4 \\
2.0\end{array}$ & $\begin{array}{lr}\text { T. } & 91.7 \\
\text { B. } & 91.4 \\
\text { T. } & 7.7 \\
\text { T. } & 1.6 \\
\text { T. } & 2.0 \\
\text { B. } & 2.0\end{array}$ \\
\hline Suspended sediment --- Top & & & . Com & te. . . & $\cdots \cdot$ & T. 16 \\
\hline
\end{tabular}

Location Torresdale Intake, Fhiledelohja, Pe. Date July?, 195

Sampling study No. 35

Weather Light Clouds $\quad$ Water discharge at Trenton (cfs) 3,37

\begin{tabular}{l|c|c|c|c|c|c|c} 
Weather Light Clouds \\
\hline
\end{tabular}

Location Torresdale Intake, Philadelphia, Pa. Date August 6, 1952 Sampling study No. 36 Weather Cloudy

\begin{tabular}{|c|c|c|c|c|c|c|}
\hline \multirow[b]{3}{*}{$\begin{array}{ll}\text { Time (EST) } \\
\text { Sounding (ft) } \\
\text { Temperature }\left({ }^{\circ} \mathrm{F}\right) \\
\text { pH } & \begin{array}{l}\text { Tottom } \\
\text { Top } \\
\text { Bottom }\end{array} \\
& \begin{array}{l}\text { Top } \\
\text { Bot }\end{array}\end{array}$} & \multicolumn{6}{|c|}{ Station } \\
\hline & \begin{tabular}{|c|}
$\begin{array}{c}\text { Pennsylvania } \\
\text { stde }\end{array}$ \\
\end{tabular} & West Center & Center & East Center & \begin{tabular}{|c|}
$\begin{array}{c}\text { New Jersey } \\
\text { side }\end{array}$ \\
\end{tabular} & Average \\
\hline & $\begin{array}{c}12: 15 \text { p.m. } \\
8 . \\
6.6\end{array}$ & $\begin{array}{c}12: 17 \text { p.m. } \\
34 \\
80 \\
80 \\
6.7 \\
6.7\end{array}$ & $\begin{array}{c}12: 20 \mathrm{p} . \mathrm{ml} \\
40 \\
80 \\
80 \\
6.7 \\
6.7\end{array}$ & $\begin{array}{c}12: 22 \mathrm{p} \cdot \mathrm{m} . \\
18 \\
80 \\
80 \\
6.7 \\
6.8\end{array}$ & $\begin{array}{c}12: 25 \text { p.m. } \\
80 \\
6.8\end{array}$ & $\begin{array}{ll}\text { T. } & 80 \\
\text { B. } & 80 \\
\text { T. } & 6.7 \\
\text { B. } & 6.7\end{array}$ \\
\hline 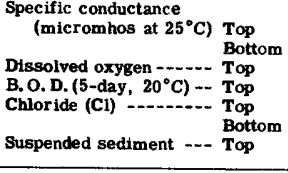 & $\begin{array}{r}178 \\
5.1 \\
11.6 \\
8.0 \\
\ldots\end{array}$ & $\begin{array}{l}168 \\
169 \\
5.1 \\
8.0 \\
8.0 \\
7.0 \\
\cdots\end{array}$ & $\begin{array}{r}164 \\
163 \\
5.2 \\
6.1 \\
7.0 \\
7.0 \\
. \text { Com }\end{array}$ & $\begin{array}{r}159 \\
160 \\
6.5 \\
6.2 \\
7.0 \\
7.0\end{array}$ & $\begin{array}{r}157 \\
5.7 \\
5.2 \\
7.0\end{array}$ & $\begin{array}{ll}\text { T. } & 165 \\
\text { B. } & 164 \\
\text { T. } & 5.5 \\
\text { T. } & 7.4 \\
\text { T. } & 7.4 \\
\text { B. } & 7.0 \\
\text { T. } & 39\end{array}$ \\
\hline
\end{tabular}


Table 8. --WATER ANALYSES OF DELAWARE RIVER BETWEEN BRISTOL AND MARCUS HOOK, PA, --Continued Analyzed by City of Philadelphia and U. S. Geological Survey; analyses in parts per million

\begin{tabular}{|c|c|c|c|c|c|c|}
\hline \multicolumn{3}{|c|}{ Weather Clear } & \multicolumn{4}{|c|}{ Water discharge at Trenton (cfs) 32,000} \\
\hline & \multicolumn{6}{|c|}{ Station } \\
\hline & $\begin{array}{c}\begin{array}{c}\text { Pennsylvania } \\
\text { side }\end{array} \\
\end{array}$ & West Center & Center & East Center & $\begin{array}{c}\text { New Jersey } \\
\text { side }\end{array}$ & Average \\
\hline $\begin{array}{l}\text { Time (EST) } \\
\text { Sounding (ft) } \\
\text { Temperature }\left({ }^{\circ} \mathrm{F}\right) \\
\text { pH }\end{array}$ & $\begin{array}{c}10: 20 \mathrm{a} . \mathrm{m} . \\
74 \\
6.3\end{array}$ & $\begin{array}{c}10: 22 \text { a.m. } \\
30 \\
74 \\
75 \\
6.3 \\
6.3\end{array}$ & $\begin{array}{c}10: 25 \text { a.m. } \\
35 \\
74 \\
75 \\
6.3 \\
6.3\end{array}$ & $\begin{array}{c}10: 27 \text { a.m. } \\
18 \\
74 \\
75 \\
6.2 \\
6.3\end{array}$ & $\begin{array}{c}10: 29 \mathrm{a} . \mathrm{m} . \\
75 \\
6.3\end{array}$ & $\begin{array}{ll}\text { T. } & 74 \\
\text { B. } & 75 \\
\text { T. } & 6.3 \\
\text { B. } & 6.3\end{array}$ \\
\hline 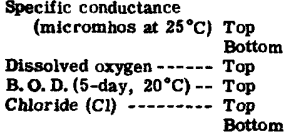 & $\begin{array}{r}162 \\
6.1 \\
2.2 \\
7.0\end{array}$ & $\begin{array}{l}160 \\
161 \\
6.9 \\
2.4 \\
5.0 \\
5.0\end{array}$ & $\begin{array}{l}160 \\
160 \\
6.8 \\
2.3 \\
5.5 \\
5.0\end{array}$ & $\begin{array}{r}148 \\
153 \\
6.3 \\
2.1 \\
5.5 \\
6.0\end{array}$ & $\begin{array}{l}143 \\
6.2 \\
2.0 \\
6.0\end{array}$ & $\begin{array}{lr}\text { T. } & 155 \\
\text { B. } & 158 \\
\text { T. } & 6.5 \\
\text { T. } & 2.2 \\
\text { T. } & 5.8 \\
\text { B. } & 5.3\end{array}$ \\
\hline Suspended sediment --- Top & & & . Comp & ite. . . & 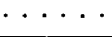 & T. 18 \\
\hline
\end{tabular}

Location Torresuale Intake, Philadelphia, Pa._Date October 6, 1952 _ Sampling study No. 38

\begin{tabular}{|c|c|c|c|c|c|c|}
\hline \multicolumn{3}{|c|}{ Weather Clear } & \multicolumn{4}{|c|}{ Water discharge at Trenton (cfs) $\frac{29}{4,800}$} \\
\hline & \multicolumn{6}{|c|}{ Station } \\
\hline & $\begin{array}{c}\text { Pennsylvania } \\
\text { side }\end{array}$ & West Center & Center & East Center & $\begin{array}{l}\text { New Jersey } \\
\text { side }\end{array}$ & Average \\
\hline $\begin{array}{l}\text { Time (EST) } \\
\text { Sounding (ft) } \\
\text { Temperature }\left({ }^{\circ} \mathrm{F}\right) \\
\text { pH }\end{array}$ & $\begin{array}{c}11: 45 \text { a.m. } \\
67 \\
6.4\end{array}$ & $\begin{array}{l}11: 48 \text { a.m. } \\
27 \\
66 \\
67 \\
6.4 \\
6.5\end{array}$ & $\begin{array}{c}11: 51 \text { a.m. } \\
34 \\
66 \\
67 \\
6.4 \\
6.4\end{array}$ & $\begin{array}{c}11: 54 \text { a.m. } \\
20 \\
67 \\
66 \\
6.4 \\
6.4\end{array}$ & $\begin{array}{c}12: 57 \text { a.m. } \\
66 \\
6.4\end{array}$ & $\begin{array}{lc}\text { T. } & 66 \\
\text { B. } & 67 \\
\text { T. } & 6.4 \\
\text { B. } & 6.4\end{array}$ \\
\hline 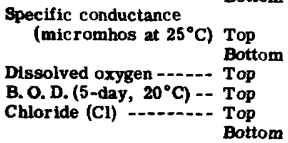 & $\begin{array}{l}212 \\
7.4 \\
1.5 \\
10\end{array}$ & $\begin{array}{r}201 \\
198 \\
7.6 \\
1.9 \\
8.5 \\
8.0\end{array}$ & $\begin{array}{l}199 \\
201 \\
8.1 \\
2.0 \\
8.5 \\
8.5\end{array}$ & $\begin{array}{r}184 \\
185 \\
8.0 \\
2.2 \\
8.5 \\
8.5\end{array}$ & $\begin{array}{r}170 \\
7.9 \\
2.5 \\
8.5\end{array}$ & $\begin{array}{lr}\text { T. } & 193 \\
\text { B. } & 195 \\
\text { T. } & 7.8 \\
\text { T. } & 2.0 \\
\text { T. } & 8.8 \\
\text { B. } & 8.3\end{array}$ \\
\hline Suspended sediment ... Top & $\cdot \cdot$ & & Com & ite. . . & & $\mathrm{T}$ \\
\hline
\end{tabular}

Location Torreadale Intake, Philadelphia, Pa.

\begin{tabular}{l|r|c|c|c|c|c} 
Location Torresdale Intake, Philadelphia, Pa. \\
Weather Clear.
\end{tabular}

Location Torresdale Intake, Philadelphia, Pa. Date December 4, 1952 Sampling study No. 40

\begin{tabular}{|c|c|c|c|c|c|c|}
\hline \multicolumn{3}{|c|}{ Weather Clear } & \multicolumn{4}{|c|}{ Water discharge at Trenton (cfs) 10,300} \\
\hline & \multicolumn{6}{|c|}{ Station } \\
\hline & $\begin{array}{c}\begin{array}{c}\text { Pennsylvania } \\
\text { side }\end{array} \\
\end{array}$ & West Center & Center & East Center & $\begin{array}{c}\text { New Jersey } \\
\text { side }\end{array}$ & Average \\
\hline $\begin{array}{ll}\text { Time (EST) } & \\
\text { Sounding }(\mathrm{ft}) & \\
\text { Temperature }\left({ }^{\circ} \mathrm{F}\right) & \\
\text { pH } & \begin{array}{l}\text { Top } \\
\text { Bottom }\end{array} \\
& \begin{array}{l}\text { Top } \\
\text { Bottom }\end{array}\end{array}$ & $\begin{array}{c}\text { I: } 53 \text { p.m. } \\
41 \\
6.4\end{array}$ & $\begin{array}{l}1: 50 \mathrm{p.m} . \\
35 \\
41 \\
47 \\
6.4 \\
6.5\end{array}$ & $\begin{array}{l}1: 47 \text { p.m. } \\
40 \\
41 \\
41 \\
6.4 \\
6.4\end{array}$ & $\begin{array}{c}1: 44 \mathrm{p} \cdot \mathrm{m} . \\
24 \\
41 \\
42 \\
6.4 \\
6.5\end{array}$ & $\begin{array}{c}1: 41 \text { p.m. } \\
41 \\
6.4\end{array}$ & $\begin{array}{ll}\text { T. } & 41 \\
\text { B. } & 41 \\
\text { T. } & 6.4 \\
\text { B. } & 6.5\end{array}$ \\
\hline 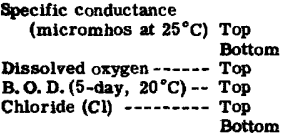 & $\begin{array}{r}134 \\
10.8 \\
2.7 \\
5.0\end{array}$ & $\begin{array}{r}122 \\
121 \\
11.7 \\
2.2 \\
5.0 \\
5.0\end{array}$ & $\begin{array}{l}119 \\
119 \\
11.9 \\
3.3 \\
4.5 \\
5.0\end{array}$ & $\begin{array}{l}117 \\
120 \\
11.9 \\
3.2 \\
5.0 \\
5.0\end{array}$ & $\begin{array}{r}110 \\
11.5 \\
2.5 \\
5.0\end{array}$ & $\begin{array}{lr}\text { T. } & 122 \\
\text { B. } & 120 \\
\text { T. } & 11.6 \\
\text { T. } & 2.8 \\
\text { T. } & 4.9 \\
\text { B. } & 5.0\end{array}$ \\
\hline Suspended sediment -.- Top & . & & . Comp & te. . . & $\cdot{ }^{*} \cdot$ & T. 35 \\
\hline
\end{tabular}


Table 9, --WATER ANALYSES OF DELAWARE RIVER BETWEEN BRISTOL AND MARCUS HOOK, PA. Analyzed by City of Philadelphia and U. \$. Geological Survey; analyses in parts per miltion

Location Lehigh Avenue, Ph1ladelphia, Pa._Date August, 2, 1949_ Sampling study No. 1

\begin{tabular}{|c|c|c|c|c|c|c|}
\hline \multicolumn{7}{|c|}{$\begin{array}{l}\text { Location Lehigh Avenue, Philadelphia, Pa, } \\
\text { Weather-Cloudy }\end{array}$} \\
\hline \multirow[b]{3}{*}{$\begin{array}{l}\text { Time (EST) } \\
\text { Sounding (ft) } \\
\text { Temperature }\left({ }^{\circ} \mathrm{F}\right) \\
\text { pH }\end{array}$} & \multicolumn{6}{|c|}{ Station } \\
\hline & \begin{tabular}{|c|}
$\begin{array}{c}\text { Pennsylvania } \\
\text { side }\end{array}$ \\
\end{tabular} & West Center & Center & East Center & $\begin{array}{l}\text { New Jersey } \\
\text { side }\end{array}$ & Average \\
\hline & 84 & $\begin{array}{l}-- \\
34 \\
84 \\
83 \\
6.1 \\
6.0\end{array}$ & $\begin{array}{l}8: 30 \text { a.m. } \\
34 \\
83 \\
83 \\
6.1 \\
6.1\end{array}$ & $\begin{array}{l}-- \\
28 \\
82 \\
83 \\
6.1 \\
6.0\end{array}$ & 84 & $\begin{array}{ll}\text { T. } & 83 \\
\text { B. } & 83 \\
\text { T. } & 6.1 \\
\text { B. } & 6.0\end{array}$ \\
\hline $\begin{array}{l}\text { Specific conductance } \\
\text { (micromhos at } 25^{\circ} \mathrm{C} \text { ) Top }\end{array}$ & 264 & $\begin{array}{l}271 \\
281\end{array}$ & $\begin{array}{l}277 \\
275\end{array}$ & $\begin{array}{l}274 \\
273\end{array}$ & 266 & $\begin{array}{l}\text { T. } 270 \\
\text { B. } 276\end{array}$ \\
\hline $\begin{array}{ll}\text { Dissolved oxygen }-. .- & \text { Top } \\
\left.\text { B. O. D. (5-day, } 20^{\circ} \mathrm{C}\right)- & \text { Top } \\
\text { Chloride (Cl) } & \text { Bottom }\end{array}$ & 19.0 & $\begin{array}{l}17.1 \\
16 \\
17\end{array}$ & $\begin{array}{l}16.0 \\
17 \\
16\end{array}$ & $\begin{array}{l}15.0 \\
17 \\
16\end{array}$ & $\begin{array}{l}.0 \\
18.0\end{array}$ & $\begin{array}{ll}\text { T. } & .2 \\
\text { T. } & 17.0 \\
\text { T. } & 16 \\
\text { B. } & 16\end{array}$ \\
\hline Suspended sediment $\ldots$ Top & 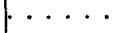 & $\cdot \cdot \cdot$ &. Com & site. . . & $\cdots \cdot \cdot$ & T. -- \\
\hline
\end{tabular}

Location Lehigh Averue, Philadelphia, Pe. Weather Cloudy

Date_September 6, 1949 Sampling study No._- 2

\begin{tabular}{|c|c|c|c|c|c|c|}
\hline Weather Cloudy & & & Wate & discharge & Trenton (cfs) & 5,320 \\
\hline & & & Station & & & \\
\hline & \begin{tabular}{|c}
$\begin{array}{c}\text { Pennsylvania } \\
\text { side }\end{array}$ \\
\end{tabular} & West Center & Center & East Center & \begin{tabular}{|c|}
$\begin{array}{c}\text { New Jersey } \\
\text { side }\end{array}$ \\
\end{tabular} & Aver age \\
\hline $\begin{array}{ll}\text { Time (EST) } & \\
\text { Sounding (ft) } \\
\text { Temperature }\left({ }^{\circ} \mathrm{F}\right)\end{array} \begin{array}{l}\text { Top } \\
\text { Bottom }\end{array}$ & $\begin{array}{l}-- \\
0.6\end{array}$ & $\begin{array}{l}34 \\
78 \\
7 ? \\
0.5 \\
6.6\end{array}$ & $\begin{array}{c}12: 45 \text { p.m. } \\
34 \\
78 \\
78 \\
6.6 \\
6.4\end{array}$ & $\begin{array}{l}-- \\
23 \\
78 \\
78 \\
6.5 \\
6.4\end{array}$ & $\begin{array}{l}- \\
78 \\
6.6\end{array}$ & $\begin{array}{ll}\text { T. } & 78 \\
\text { B. } & 78 \\
\text { T. } & 6.6 \\
\text { B. } & 6.5\end{array}$ \\
\hline 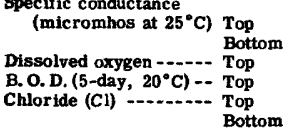 & $\begin{array}{l}318 \\
6.9 \\
24\end{array}$ & $\begin{array}{l}354 \\
357 \\
.4 . \\
34 \\
32\end{array}$ & $\begin{array}{l}360 \\
365 \\
5.0 \\
33 \\
33\end{array}$ & $\begin{array}{l}363 \\
357 \\
1.0 \\
4.0 \\
33 \\
33\end{array}$ & $\begin{array}{l}347 \\
1.0 \\
2.0 \\
30\end{array}$ & $\begin{array}{ll}\text { T. } & 348 \\
\text { B. } & 360 \\
\text { T. } & .7 \\
\text { T. } & 4.7 \\
\text { T. } & 31 \\
\text { B. } & 33\end{array}$ \\
\hline Suspended sediment $\ldots$ Top & $\cdots \cdot$ & & . Comp & ite... & $\cdot \cdot \cdot$ & T. 22 \\
\hline
\end{tabular}

Location Lehigh Avenue, Philadel phia, Pa. Weather. Rain

Date_October 4, 1949 Sampling study No. - 3

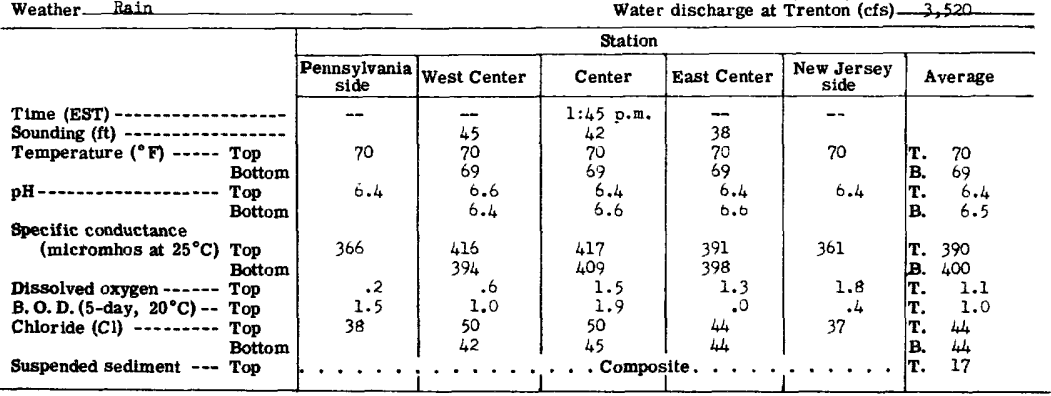

Location Lehigh_Avenue, Philadelphia, Pa. Weather cloudy

Date November 1,1949 Sampling study No. $-\frac{h}{3,340}$

\begin{tabular}{|c|c|c|c|c|c|}
\hline \multicolumn{6}{|c|}{ Station } \\
\hline $\begin{array}{c}\text { Pennsylvania } \\
\text { side }\end{array}$ & West Center & Center & East Center & $\begin{array}{c}\text { New Jersey } \\
\text { side }\end{array}$ & Average \\
\hline $\begin{array}{l}- \\
58 \\
6.6\end{array}$ & $\begin{array}{l}-- \\
44 \\
58 \\
58 \\
6.6 \\
6.6\end{array}$ & $\begin{array}{c}2: 30 \mathrm{p} \cdot \mathrm{m} . \\
44 \\
58 \\
58 \\
5.6 \\
5.6\end{array}$ & $\begin{array}{l}- \\
20 \\
56 \\
56 \\
6.6 \\
6.7\end{array}$ & $\begin{array}{l}-- \\
61 \\
6.8\end{array}$ & $\begin{array}{ll}\text { T. } & 58 \\
\text { B. } & 57 \\
\text { T. } & 6.6 \\
\text { B. } & 6.6\end{array}$ \\
\hline $\begin{array}{l}402 \\
1.0 \\
10.0 \\
48\end{array}$ & $\begin{array}{c}406 \\
412 \\
1.3 \\
9.0 \\
49 \\
50\end{array}$ & $\begin{array}{l}407 \\
404 \\
1.7 \\
9.1 \\
48 \\
47\end{array}$ & $\begin{array}{l}366 \\
365 \\
2.0 \\
8.4 \\
41 \\
40\end{array}$ & $\begin{array}{l}357 \\
2.0 \\
9.0 \\
38\end{array}$ & $\begin{array}{lc}\text { T. } & 388 \\
\text { B. } & 394 \\
\text { T. } & 1.6 \\
\text { T. } & 9.1 \\
\text { T. } & 45 \\
\text { B. } & 46\end{array}$ \\
\hline$\cdots$ & & - Comp & ite. . . & $\cdots \cdot$ & T. $\quad 15$ \\
\hline
\end{tabular}


Table 9, -- WATER ANALYSES OF DELAWARE RIVER BETWEEN BRISTOL AND MARCUS HOOK, PA. --COntinued Analyzed by City of Philadelphia and U. S. Geological Survey; analyses in parts per million

Location Lehlgh Avenue, Phlladel phia, Pa.

Date_December 1, 1949 Sampling study No. 5

Weather Clear

Water discharge at Trenton (cfs) $ـ \quad 4.970$

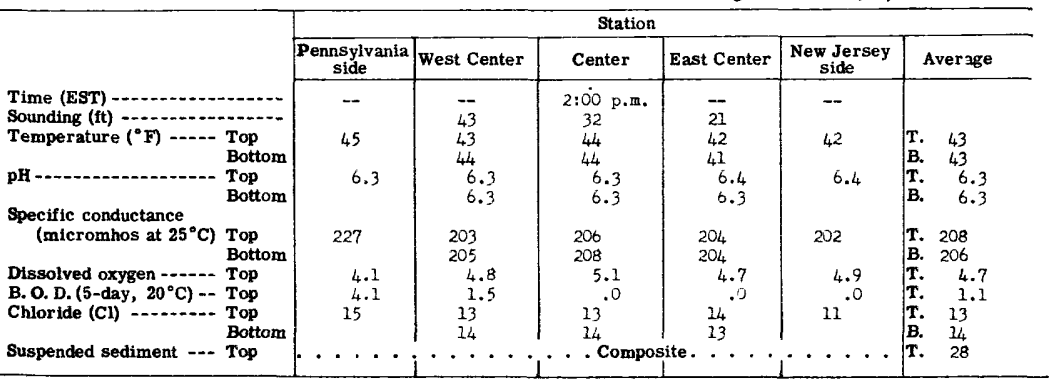

Location Lehigh Avenue, Fhiladelphia, Pa. Weather Light Rain

Date January 3, 1950 Sampling study No.

\begin{tabular}{|c|c|c|c|c|c|c|}
\hline \multicolumn{3}{|c|}{ Weather Light Rain } & \multicolumn{4}{|c|}{ Water discharge at Trenton (cfs) 11,100} \\
\hline & \multicolumn{6}{|c|}{ Station } \\
\hline & $\begin{array}{c}\text { Pennsylvania } \\
\text { side }\end{array}$ & West Center & Center & East Center & $\begin{array}{c}\text { New Jersey } \\
\text { side }\end{array}$ & Average \\
\hline $\begin{array}{l}\text { Time (EST) } \\
\text { Sounding (ft) } \\
\text { Temperature ( }{ }^{\circ} \mathrm{F} \text { ) } \\
\text { pH }\end{array}$ & $\begin{array}{c}1: 45 \text { p.m. } \\
-- \\
6.2\end{array}$ & $\begin{array}{l}1: 48 \text { p.m. } \\
45 \\
-- \\
-- \\
6.5 \\
6.4\end{array}$ & $\begin{array}{l}1: 52 \text { p.r. } \\
39 \\
-- \\
- \\
6.5 \\
0.4\end{array}$ & $\begin{array}{c}1: 55 \mathrm{p} \cdot \mathrm{m} . \\
23 \\
-- \\
-- \\
6.5 \\
6.3\end{array}$ & $\begin{array}{c}1: 58 \text { p.m. } \\
-- \\
6.6\end{array}$ & $\begin{array}{ll}\text { T. } & -- \\
\text { B. } & - \\
\text { T. } & 6.5 \\
\text { B. } & 6.4\end{array}$ \\
\hline 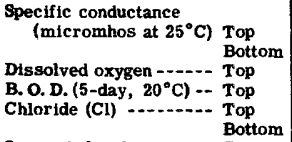 & $\begin{array}{r}130 \\
9.0 \\
.5 \\
7 . .\end{array}$ & $\begin{array}{r}131 \\
1 \geq 1 \\
9.0 \\
8.5 \\
8.0\end{array}$ & $\begin{array}{r}130 \\
128 \\
8.2 \\
.0 \\
7.0 \\
7.0\end{array}$ & $\begin{array}{r}127 \\
128 \\
8.4 \\
.0 \\
6.0 \\
8.0\end{array}$ & $\begin{array}{r}123 \\
8.6 \\
.0 \\
9.0\end{array}$ & $\begin{array}{lr}\text { T. } & 128 \\
\text { B. } & 129 \\
\text { T. } & 8.6 \\
\text { T. } & .2 \\
\text { T. } & 7.4 \\
\text { B. } & 7.7\end{array}$ \\
\hline Suspended sediment --- Top & & &. Com & & $\cdots \cdots \cdot$ & T. \\
\hline
\end{tabular}

Location Lehigh svenue, Philadelphia, Pa. Weather_Cloudy

Date February 2, 1950 Sampling study No. 7

\begin{tabular}{|c|c|c|c|c|c|c|}
\hline Weather- & & & Wa & ischarge & renton (cf & 13,200 \\
\hline & & & Station & & & \\
\hline & $\begin{array}{c}\text { Pennsylvania } \\
\text { side }\end{array}$ & West Center & Center & East Center & $\begin{array}{c}\text { New Jersey } \\
\text { side }\end{array}$ & Aver age \\
\hline 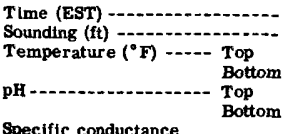 & $\begin{array}{c}0: 55 \mathrm{a} . \mathrm{m} . \\
43 \\
6.5\end{array}$ & $\begin{array}{c}10: 00 \mathrm{a} . \mathrm{m} \\
45 \\
41 \\
40 \\
6.8 \\
6.8\end{array}$ & $\begin{array}{c}10: 02 \mathrm{a} . \mathrm{m} \\
37 \\
41 \\
40 \\
6.8 \\
6.8\end{array}$ & $\begin{array}{c}10: 05 \text { a.m. } \\
24 \\
40 \\
40 \\
6.8 \\
0.8\end{array}$ & $\begin{array}{c}10: 10 \mathrm{a} . \mathrm{m} \\
40 \\
0.9\end{array}$ & $\begin{array}{ll}\text { T. } & 41 \\
\text { B. } & 40 \\
\text { T. } & 6.8 \\
\text { B. } & 6.8\end{array}$ \\
\hline $\begin{array}{ll}\quad \text { (micromhos at } 25^{\circ} \mathrm{C} \text { ) } & \begin{array}{l}\text { Top } \\
\text { Bottom }\end{array} \\
\text { Dissolved oxygen }-1-2 & \text { Top } \\
\text { B, O. D. (5-day, 20 } \\
\text { Chloride }(\mathrm{Cl})- & \text { Top } \\
& \text { Bottom }\end{array}$ & $\begin{array}{l}155 \\
8.4 \\
8.8 \\
9.0\end{array}$ & $\begin{array}{r}147 \\
114 \\
10.2 \\
3.0 \\
0.0 \\
5.0\end{array}$ & $\begin{array}{r}110 \\
1109 \\
11.2 \\
2.8 \\
5.0 \\
5.0\end{array}$ & $\begin{array}{r}109 \\
109 \\
10.0 \\
2.0 \\
5.0 \\
5.0\end{array}$ & $\begin{array}{r}111 \\
10.5 \\
2.0 \\
5.0\end{array}$ & $\begin{array}{lr}\text { T. } & 126 \\
\text { B. } & 111 \\
\text { T. } & 10.2 \\
\text { T. } & 4.0 \\
\text { T. } & 6.0 \\
\text { B. } & 5.0\end{array}$ \\
\hline Buspended sediment --- Top & -1 & & Com & ite. . & 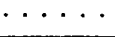 & T. \\
\hline
\end{tabular}

Locatior, Lehigh Avenue, Philadelphia, Pa. Weather Clear

Date_ March 0, 1950 Sampling study No. 8

\begin{tabular}{|c|c|c|c|c|c|c|}
\hline \multicolumn{3}{|c|}{ Weather Clear } & \multicolumn{4}{|c|}{ Water discharge at Trenton (cfs) $-7,080$} \\
\hline & \multicolumn{6}{|c|}{ Station } \\
\hline & \begin{tabular}{|c|}
$\begin{array}{c}\text { Pennsylvania } \\
\text { side }\end{array}$ \\
\end{tabular} & West Center & Center & East Center & $\begin{array}{c}\text { New Jersey } \\
\text { side }\end{array}$ & Average \\
\hline $\begin{array}{ll}\text { Time (EST) } & \\
\text { Sounding (ft) } \\
\text { Temperature }\left({ }^{\circ} \mathrm{F}\right)\end{array}$ & $\begin{array}{c}10: 05 \mathrm{a} . \mathrm{m} . \\
35 \\
7.2\end{array}$ & $\begin{array}{c}10: 01 \text { a.m. } \\
31 \\
34 \\
35 \\
7.0 \\
7.0\end{array}$ & $\begin{array}{c}9: 58 \text { a.m. } \\
19 \\
35 \\
35 \\
6.9 \\
0.7\end{array}$ & $\begin{array}{c}9: 55 \text { a.m. } \\
17 \\
35 \\
35 \\
7.1 \\
\text { b.7 }\end{array}$ & $\begin{array}{c}9: 50 \mathrm{a} . \mathrm{m} . \\
36 \\
6.9\end{array}$ & $\begin{array}{ll}\text { T. } & 35 \\
\text { B. } & 35 \\
\text { T. } & 7.0 \\
\text { B. } & 6.8\end{array}$ \\
\hline $\begin{array}{ll}\begin{array}{l}\text { Specific conductance } \\
\left.\text { (micromhos at } 25^{\circ} \mathrm{C}\right)\end{array} & \begin{array}{l}\text { Top } \\
\text { Bottom }\end{array} \\
\text { Dissolved oxygen }- & \text { Top } \\
\text { B. O. D. }\left(5-\text { day, } 20^{\circ} \mathrm{C}\right)- & \text { Top } \\
\text { Chloride }(\mathrm{Cl})-\ldots & \text { Top } \\
& \text { Bottom }\end{array}$ & $\begin{array}{c}163 \\
10.5 \\
8.3 \\
10\end{array}$ & $\begin{array}{r}144 \\
144 \\
12.3 \\
5.2 \\
7.0 \\
7.0\end{array}$ & $\begin{array}{r}144 \\
146 \\
10.3 \\
2.1 \\
7.0 \\
7.0\end{array}$ & $\begin{array}{r}144 \\
148 \\
10.7 \\
4.0 \\
7.0 \\
7.0\end{array}$ & $\begin{array}{r}148 \\
11.8 \\
4.6 \\
7.0\end{array}$ & $\begin{array}{lr} & \\
\text { T. } & 149 \\
\text { B. } & 146 \\
\text { T. } & 11.2 \\
\text { T. } & 4.8 \\
\text { T. } & 7.6 \\
\text { B. } & 7.0\end{array}$ \\
\hline Suspended sediment --- Top & & & . Com & $\cdots$ & 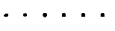 & 18 \\
\hline
\end{tabular}


Table 9. --WATER ANALYSES OF DELAWARE RIVER BETWEEN BRISTOL AND MARCUS HOOK, PA. --Continued Analyzed by City of Philadelphia and U. S. Geological Survey; analyses in parts per million

Location Lehigh Avenue, Philadelchia, Pa. Date April 4, 1950 Sampling study No. 9

\begin{tabular}{l|r|r|r|r|r|r} 
Location \\
Weather Llear
\end{tabular}

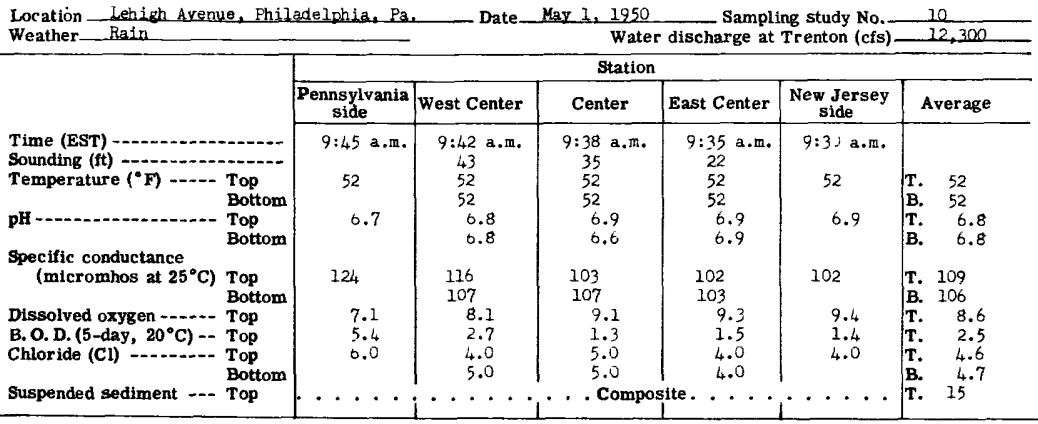

Location Lehigh Avenue, Phil adelphiz, Pa, Weather Cloudy

Date June 6. 1950

Sampling study No. $\ldots$ II

\begin{tabular}{|c|c|c|c|c|c|c|}
\hline \multicolumn{3}{|c|}{ Weather Cloudy } & \multicolumn{4}{|c|}{ Water discharge at Trenton (cfs) $\_18,900$} \\
\hline & \multicolumn{6}{|c|}{ Station } \\
\hline & \begin{tabular}{|c|} 
Pennsylvania \\
side
\end{tabular} & West Center & Center & East Center & $\begin{array}{c}\text { New Jersey } \\
\text { side }\end{array}$ & Average \\
\hline $\begin{array}{l}\text { Time (EST) } \\
\text { Sounding (ft) } \\
\text { Temperature }\left({ }^{\circ} \mathrm{F}\right) \\
\text { pH }\end{array} \begin{array}{l}\text { Top } \\
\text { Bottom } \\
\text { Top } \\
\text { Bottom }\end{array}$ & $\begin{array}{c}9: 19 \text { a.m. } \\
68 \\
6.4\end{array}$ & $\begin{array}{l}9: 10 \mathrm{a} . \mathrm{m} . \\
42 \\
68 \\
68 \\
6.5 \\
6.4\end{array}$ & $\begin{array}{c}9: 13 \text { a.m. } \\
37 \\
68 \\
68 \\
6.5 \\
6.6\end{array}$ & $\begin{array}{l}9: 10 \mathrm{a} . \mathrm{m} . \\
27 \\
67 \\
68 \\
7.2 \\
6.4\end{array}$ & $\begin{array}{l}9: 07 \text { a.m. } \\
67 \\
6.6\end{array}$ & $\begin{array}{ll}\text { T. } & 68 \\
\text { B. } & 68 \\
\text { T. } & 6.6 \\
\text { B. } & 6.5\end{array}$ \\
\hline 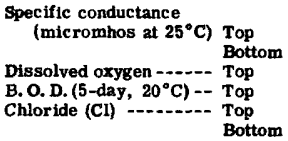 & $\begin{array}{r}140 \\
\\
2.3 \\
2.2 \\
8.0\end{array}$ & $\begin{array}{r}126 \\
135 \\
3.1 \\
.0 \\
6.0 \\
7.0\end{array}$ & $\begin{array}{r}124 \\
126 \\
3.6 \\
.0 \\
7.0 \\
6.0\end{array}$ & $\begin{array}{r}122 \\
123 \\
4.3 \\
.0 \\
6.0 \\
6.0\end{array}$ & $\begin{array}{r}117 \\
4.8 \\
.0 \\
6.0\end{array}$ & $\begin{array}{lr} & \\
\text { T. } & 126 \\
\text { B. } & 128 \\
\text { T. } & 3.6 \\
\text { T. } & .4 \\
\text { T. } & 6.6 \\
\text { B. } & 6.3\end{array}$ \\
\hline Suspended sediment $-\ldots$ Top & & $\cdots \cdot \cdot$ & . Comp & ite. . . & $\cdots \cdot$ & $\mathbf{T}$ \\
\hline
\end{tabular}

Location Lehigh Avenue, Philadelphia, Fa. Weather Light hain

Date July 0, 1950

Sampling study No. 1.2

\begin{tabular}{|c|c|c|c|c|c|c|}
\hline \multicolumn{2}{|l|}{ Weather light kain } & & \multicolumn{4}{|c|}{ Water discharge at Trenton (cfs) 6.130} \\
\hline & \multicolumn{6}{|c|}{ Station } \\
\hline & $\begin{array}{c}\text { Pennsylvania } \\
\text { side }\end{array}$ & West Center & Center & East Center & $\begin{array}{c}\text { New Jersey } \\
\text { side }\end{array}$ & Average \\
\hline $\begin{array}{l}\text { Time (EST) } \\
\text { Sounding (ft) } \\
\text { Temperature }\left({ }^{\circ} \mathrm{F}\right) \\
\text { pH }\end{array}$ & $\begin{array}{c}12: 35 \text { p.m. } \\
78 \\
0.4\end{array}$ & $\begin{array}{c}12: 40 \mathrm{p} . \mathrm{m} \\
47 \\
78 \\
78 \\
0.6 \\
0.4\end{array}$ & $\begin{array}{c}12: 45 \text { p.m. } \\
32 \\
78 \\
78 \\
6.0 \\
6.5\end{array}$ & $\begin{array}{c}12: 48 \text { p.m. } \\
18 \\
78 \\
78 \\
6.6 \\
0.6\end{array}$ & $\begin{array}{c}12: 50 \mathrm{p} \cdot \mathrm{m} \\
77 \\
6.5\end{array}$ & $\begin{array}{lc}\text { T. } & 78 \\
\text { B. } & 78 \\
\text { T. } & 6.5 \\
\text { B. } & 6.5\end{array}$ \\
\hline 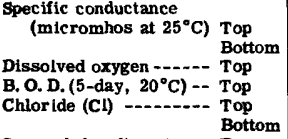 & $\begin{array}{l}181 \\
0.2 \\
10^{.9}\end{array}$ & $\begin{array}{l}153 \\
149 \\
1.2 \\
2.9 \\
8.0 \\
8.0\end{array}$ & $\begin{array}{r}144 \\
142 \\
2.2 \\
2.5 \\
8.0 \\
7.0\end{array}$ & $\begin{array}{l}149 \\
153 \\
2.2 \\
2.5 \\
8.0 \\
7.0\end{array}$ & $\begin{array}{r}146 \\
2.3 \\
3.6 \\
8.0\end{array}$ & $\begin{array}{lr}\text { T. } & 155 \\
\text { B. } & 148 \\
\text { T. } & 1.8 \\
\text { T. } & 3.5 \\
\text { T. } & 8.4 \\
\text { B. } & 7.3\end{array}$ \\
\hline Suspended sediment --- Top & & & 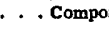 & $\therefore$ & & 22 \\
\hline
\end{tabular}


Table 9. -- WATER ANALYSES OF DELAWARE RIVER BETWEEN BRISTOL AND MARC US HOOK, PA. --Continued Analyzed by City of Philadelphia and U. S. Geological Survey; analyses in parts per million

Location Lehigh Avenue, Fhiladelphia, Pa. Date August ?, 1950 Sampling study No.....13 Weather Cloudy Water discharge at Trenton (cis) - 5.09

\begin{tabular}{|c|c|c|c|c|c|c|}
\hline & \multicolumn{6}{|c|}{ Station } \\
\hline & $\begin{array}{c}\text { Pennsylvania } \\
\text { side }\end{array}$ & West Center & Center & East Center & $\begin{array}{c}\text { New Jersey } \\
\text { side }\end{array}$ & Average \\
\hline $\begin{array}{l}\text { Time (EST) } \\
\text { Sounding (ft) } \\
\left.\text { Temperature ( }{ }^{\circ} \mathrm{F}\right) \mathrm{Top} \\
\text { pH }\end{array}$ & $\begin{array}{c}9: 00 \text { a.m. } \\
79 \\
6.4\end{array}$ & $\begin{array}{l}9: 02 \text { a.m. } \\
41 \\
79 \\
79 \\
6.3 \\
0.4\end{array}$ & $\begin{array}{l}9: 05 \text { a.m. } \\
35 \\
79 \\
70 \\
6.3 \\
0.4\end{array}$ & $\begin{array}{l}9: 00 \mathrm{a} . \mathrm{m} . \\
21 \\
79 \\
79 \\
6.3 \\
0.5\end{array}$ & $\begin{array}{l}8: 55 \text { a.m. } \\
79 \\
0.3\end{array}$ & $\begin{array}{lc}\text { T. } & 79 \\
\text { B. } & 79 \\
\text { T. } & 6.3 \\
\text { B. } & 6.4\end{array}$ \\
\hline 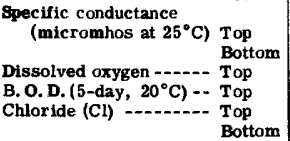 & 225 & $\begin{array}{l}225 \\
229 \\
5.4 \\
11 \\
11\end{array}$ & $\begin{array}{l}217 \\
217 \\
4.5 \\
11 \\
11\end{array}$ & $\begin{array}{l}227 \\
227 \\
3.9 \\
10 \\
10\end{array}$ & $\begin{array}{l}231 \\
1.0 \\
11\end{array}$ & \begin{tabular}{ll} 
T. & 225 \\
B. & 224 \\
T. & .8 \\
T. & \multicolumn{4}{c}{4.2} \\
T. & 11 \\
B. & 11
\end{tabular} \\
\hline Suspended sediment --- Top & $\cdots$ & & . . Com & ite. . . & $\cdots \cdot$ & T. 33 \\
\hline
\end{tabular}

Location LLhigh Avenue, Fhiladelphia, Fa. Weather-Clear. Date September 6, 1950 Sampling study No._-14

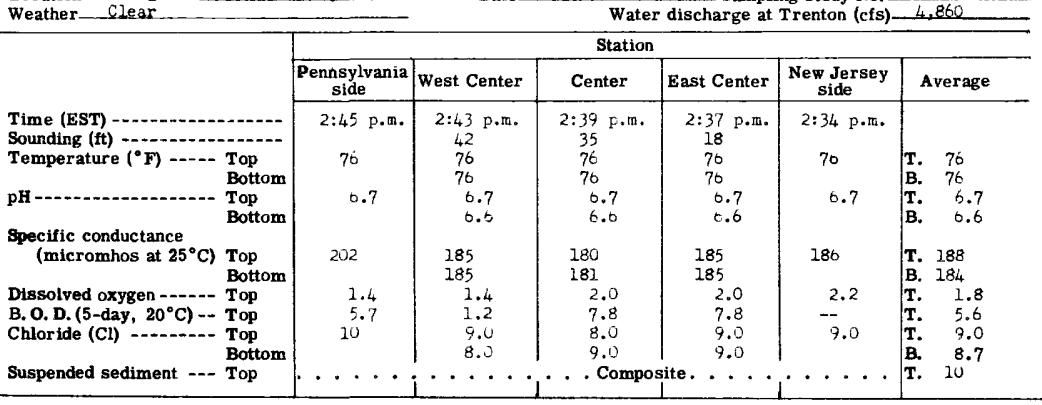

Location Lehigh Averue, Fhiladelphia, $\mathrm{Fa}$.

Date October 3, 1950 Sampling study No. 15

\begin{tabular}{|c|c|c|c|c|c|c|}
\hline \multicolumn{2}{|c|}{ Weather Misty } & \multicolumn{5}{|c|}{ Water discharge at Trenton (cfs) $-2,842$} \\
\hline & \multicolumn{6}{|c|}{ Station } \\
\hline & \begin{tabular}{|c|}
$\begin{array}{c}\text { Pennsylvania } \\
\text { side }\end{array}$ \\
\end{tabular} & West Center & Center & East Center & \begin{tabular}{|c|} 
New Jersey \\
side
\end{tabular} & Aver age \\
\hline $\begin{array}{l}\text { Time (EST) } \\
\text { Sounding (ft) } \\
\text { Temperature }\left({ }^{\circ} \mathrm{F}\right)-l \\
\text { pH } \\
\begin{array}{l}\text { Bottom } \\
\text { Top } \\
\text { Bottom }\end{array}\end{array}$ & $\begin{array}{c}3: 05 \text { p.m. } \\
69 \\
6.3\end{array}$ & $\begin{array}{l}3: 10 \text { p.m. } \\
34 \\
68 \\
69 \\
0.4 \\
0.2\end{array}$ & $\begin{array}{l}3: 15 \mathrm{p} . \mathrm{m} . \\
37 \\
68 \\
68 \\
0.3 \\
0.4\end{array}$ & $\begin{array}{l}3: 20 \text { p.m. } \\
23 \\
68 \\
68 \\
6.5 \\
6.3\end{array}$ & $\begin{array}{c}3: 25 \text { p.m. } \\
68 \\
6.4\end{array}$ & $\begin{array}{lc}\text { T. } & 68 \\
\text { B. } & 68 \\
\text { T. } & 6.4 \\
\text { B. } & 6.3\end{array}$ \\
\hline 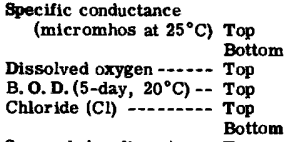 & $\begin{array}{r}240 \\
.6 \\
5.7 \\
14\end{array}$ & $\begin{array}{l}234 \\
226 \\
4.6 \\
1.5 \\
14\end{array}$ & $\begin{array}{l}227 \\
219 \\
3.8 \\
12 \\
12\end{array}$ & $\begin{array}{l}245 \\
226 \\
.7 \\
2.8 \\
10 \\
14\end{array}$ & $\begin{array}{l}231 \\
14^{3.3}\end{array}$ & $\begin{array}{ll}\text { T. } & 235 \\
\text { B. } & 224 \\
\text { T. } & .7 \\
\text { T. } & 4.0 \\
\text { T. } & 14 \\
\text { B. } & 13\end{array}$ \\
\hline Suspended sediment --- Top & $\cdot \cdot \cdot$ & & - Con & ite. . & 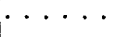 & T. \\
\hline
\end{tabular}

Location Lehigh Avenue, Fhiladelphia, Fa. Weather Cloudy.

Date November 2,1950 Sampling study No. 16

\begin{tabular}{|c|c|c|c|c|c|c|}
\hline Weather Cloudy. & & & Water & discharge at & Trenton (cfs) & 2,550 \\
\hline & & & Station & & & \\
\hline & \begin{tabular}{|c|} 
Pennsylvania \\
side
\end{tabular} & West Center & Center & East Center & $\begin{array}{c}\text { New Jersey } \\
\text { side }\end{array}$ & Average \\
\hline $\begin{array}{l}\text { Time (EST) } \\
\text { Sounding (ft) } \\
\text { Temperature }\left({ }^{\circ} \mathrm{F}\right) \\
\text { pH }\end{array} \begin{array}{l}\text { Top } \\
\text { Bottom } \\
\text { Top } \\
\text { Bottom }\end{array}$ & $\begin{array}{c}2: 44 \text { p.m. } \\
64 \\
0.6\end{array}$ & $\begin{array}{l}2: 42 \mathrm{p} \cdot \mathrm{m} . \\
40 \\
65 \\
65 \\
7.0 \\
6.4\end{array}$ & $\begin{array}{l}2: 39 \mathrm{p} \cdot \mathrm{r} . \\
34 \\
65 \\
65 \\
0.6 \\
6.4\end{array}$ & $\begin{array}{l}2: 36 \mathrm{p} \cdot \mathrm{m} . \\
24 \\
64 \\
65 \\
6.4 \\
6.4\end{array}$ & $\begin{array}{l}2: 33 \text { p.m. } \\
64 \\
0.6\end{array}$ & $\begin{array}{ll}\text { T. } & 64 \\
\text { B. } & 65 \\
\text { T. } & 6.6 \\
\text { B. } & 6.4\end{array}$ \\
\hline 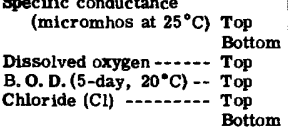 & $\begin{array}{r}266 \\
.5 \\
16\end{array}$ & $\begin{array}{l}300 \\
247 \\
8.6 \\
24 \\
14\end{array}$ & $\begin{array}{l}266 \\
249 \\
1.1 \\
2.3 \\
16 \\
14\end{array}$ & $\begin{array}{l}251 \\
251 \\
1.2 \\
3.3 \\
14 \\
14\end{array}$ & $\begin{array}{l}262 \\
1.4 \\
2.4 \\
16\end{array}$ & $\begin{array}{ll}\text { T. } & 270 \\
\text { B. } & 249 \\
\text { T. } & .9 \\
\text { T. } & 3.9 \\
\text { T. } & 17 \\
\text { B. } & 14\end{array}$ \\
\hline Suspended sediment & 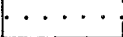 & $\because$ & . Comp & te. . & $\cdots$ & T. \\
\hline
\end{tabular}


Table 9. -- WATER ANALYSES OF DELAWARE RIVER BETWEEN BRISTOL AND MARCUS hOOK, PA. --Continued Analyzed by City of Philadelphia and U. S. Geological Survey; analyses in parts per million

Location Lehigh Avenue, Philadel ghia, Pa._Date Decenger b, 1950 Sampling study No. 17

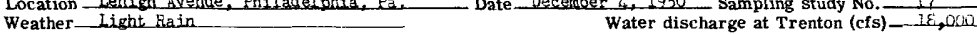

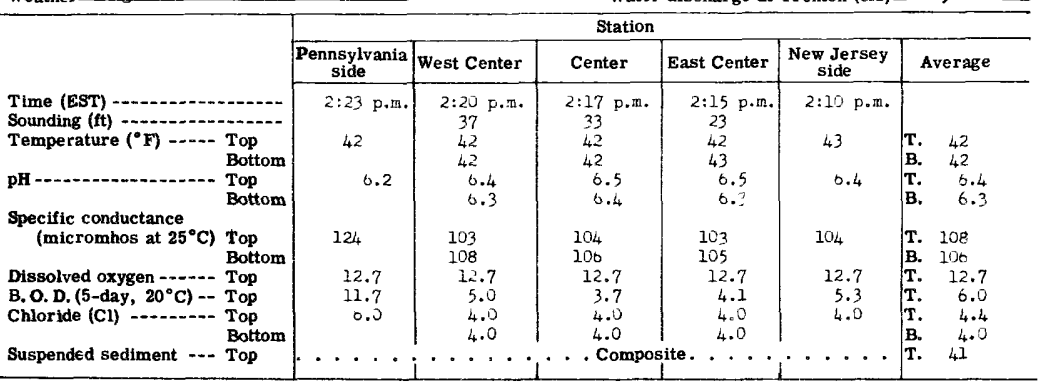

Location L. Lehigh Avenue, Philadel phia, $\mathrm{Pa}$

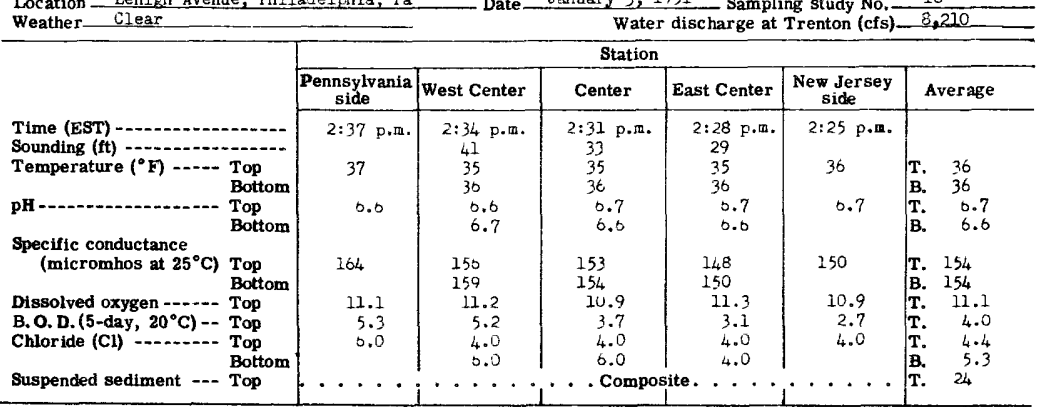

Location,Lehigh_Avenue, Fhiladelphia, Fa. Date_Narch 2, 1951___. Sampling study No. _ I9

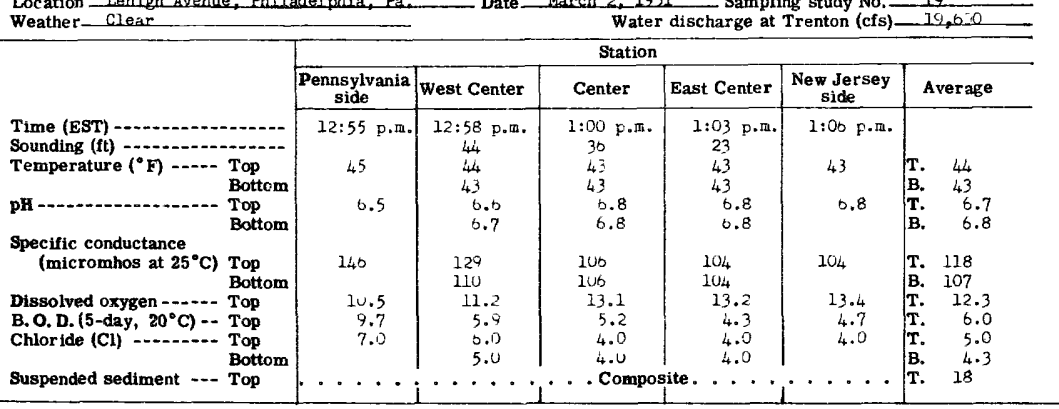

Location Lehigh Avenue, Philadelphia, Fa, Weather Rain Date Apri1 2, 1 1 151 Sampling study No. 20

\begin{tabular}{|c|c|c|c|c|c|c|}
\hline \multicolumn{2}{|c|}{ Weather Rain } & & Wate & discharge at & Trenton (cfs) & $-71,500$ \\
\hline & \multicolumn{6}{|c|}{ Station } \\
\hline & \begin{tabular}{|c|}
$\begin{array}{c}\text { Pernsylvania } \\
\text { side }\end{array}$ \\
\end{tabular} & West Center & Center & East Center & \begin{tabular}{|c|}
$\begin{array}{c}\text { New Jersey } \\
\text { side }\end{array}$ \\
\end{tabular} & Average \\
\hline $\begin{array}{l}\text { Time (EST) } \\
\text { Sounding }(\mathrm{ft}) \\
\text { Temperature }\left({ }^{\circ} \mathrm{F}\right)-\begin{array}{l}\text { Top } \\
\text { Bottom }\end{array} \\
\text { pH }\end{array}$ & $\begin{array}{c}11: 56 \text { a.m. } \\
46 \\
6.8\end{array}$ & $\begin{array}{c}11: 59 \text { a.t. } \\
48 \\
46 \\
46 \\
6.7 \\
0.7\end{array}$ & $\begin{array}{c}12: 01 \text { p.m. } \\
39 \\
46 \\
40 \\
0.8 \\
6.7\end{array}$ & $\begin{array}{c}12: 03 \mathrm{p} . \mathrm{m} . \\
26 \\
40 \\
40 \\
0.8 \\
0.7\end{array}$ & $\begin{array}{c}12: 05 \text { p.m. } \\
46 \\
6.8\end{array}$ & $\begin{array}{ll}\text { T. } & 46 \\
\text { B. } & 46 \\
\text { T. } & 0.8 \\
\text { B. } & 6.7\end{array}$ \\
\hline 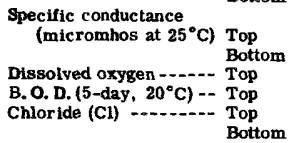 & $\begin{array}{r}00.0 \\
10.6 \\
2.1 \\
3.0\end{array}$ & $\begin{array}{r}59.5 \\
57.9 \\
11.0 \\
2.3 \\
3.0 \\
3.0\end{array}$ & $\begin{array}{r}57.7 \\
56.1 \\
11.2 \\
2.4 \\
3.0 \\
3.0\end{array}$ & $\begin{array}{r}57.3 \\
56.0 \\
11.1 \\
2.0 \\
3.0 \\
3.0\end{array}$ & $\begin{array}{r}56.5 \\
11.3 \\
2.3 \\
3.0\end{array}$ & $\begin{array}{lr}\text { T. } & 58.2 \\
\text { B. } & 56.9 \\
\text { T. } & 11.0 \\
\text { T. } & 2.3 \\
\text { T. } & 3.0 \\
\text { B. } & 3.0\end{array}$ \\
\hline Suspended sediment --- Top & & $\cdot \cdot$ & . Con & te... & & T. 101 \\
\hline
\end{tabular}


Table 9. -- WATER ANALYSES OF DELAWARE RIVER BETWEEN BRISTOL AND MARCUS HOOK, PA. --Continued Analyzed by City of Philadelphia and U. S. Geological Survey; analyses in parts per million

\begin{tabular}{|c|c|c|c|c|c|c|}
\hline \multicolumn{2}{|c|}{$\begin{array}{l}\text { Location Lehigh nvenue, Fhiladel phia, Pa. } \\
\text { Weather Clear }\end{array}$} & \multicolumn{5}{|c|}{$\frac{\text { y } 1,1951}{\text { Water discharge at Trenton (cfs) }-13,000}$} \\
\hline \multicolumn{7}{|c|}{ Station } \\
\hline & \begin{tabular}{|c|} 
Pennsylvania \\
side
\end{tabular} & West Center & Center & East Center & $\begin{array}{c}\text { New Jersey } \\
\text { side }\end{array}$ & Average \\
\hline $\begin{array}{l}\text { Time (EST) } \\
\begin{array}{l}\text { Sounding (ft) } \\
\text { Temperature }\left({ }^{\circ} \mathrm{F}\right)\end{array} \\
\begin{array}{l}\text { Top } \\
\text { Bottom } \\
\text { pH }\end{array} \\
\begin{array}{l}\text { Top } \\
\text { Bottom }\end{array}\end{array}$ & $\begin{array}{c}8: 50 \text { a.m. } \\
6 ? \\
6.8\end{array}$ & $\begin{array}{l}8: 52 \text { a.m. } \\
45 \\
61 \\
61 \\
6.8 \\
6.8\end{array}$ & $\begin{array}{c}8: 54 \text { a.m. } \\
39 \\
61 \\
61 \\
6.7 \\
6.9\end{array}$ & $\begin{array}{c}8: 50 \text { a.m. } \\
26 \\
61 \\
01 \\
0.9 \\
0.9\end{array}$ & $\begin{array}{l}8: 59 \mathrm{a} \cdot \mathrm{m} . \\
61 \\
0.8\end{array}$ & $\begin{array}{lc}\text { T. } & 61 \\
\text { B. } & 61 \\
\text { T. } & b .8 \\
\text { B. } & 6.9\end{array}$ \\
\hline 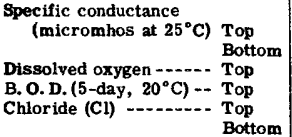 & $\begin{array}{r}134 \\
5.0 \\
3.6 \\
7.0\end{array}$ & $\begin{array}{r}130 \\
129 \\
5.7 \\
3.3 \\
7.0 \\
6.0\end{array}$ & $\begin{array}{l}121 \\
123 \\
6.9 \\
2.3 \\
0.0 \\
0.0\end{array}$ & $\begin{array}{r}119 \\
120 \\
6.9 \\
2.2 \\
0.0 \\
0.0\end{array}$ & $\begin{array}{r}122 \\
6.5 \\
=.3 \\
6.0\end{array}$ & $\begin{array}{ll}\text { T. } & 125 \\
\text { B. } & 124 \\
\text { T. } & 6.2 \\
\text { T. } & 2.7 \\
\text { T. } & 7.4 \\
\text { B. } & 0.0\end{array}$ \\
\hline Suspended sediment --- Top & & & Comp & ... & $\cdots \cdot$ & T. \\
\hline
\end{tabular}

Location Lehigh Avenue, Fniladelphiz, ra. Date_June ?, 1 - 51

Sampling study No. 22

\begin{tabular}{|c|c|c|c|c|c|c|}
\hline \multicolumn{3}{|c|}{ Weather Cloudy } & \multicolumn{4}{|c|}{ Water discharge at Trenton (cis) $-\varepsilon, 670$} \\
\hline & \multicolumn{6}{|c|}{ Station } \\
\hline & \begin{tabular}{|c|}
$\begin{array}{c}\text { Pennsylyania } \\
\text { side }\end{array}$ \\
\end{tabular} & West Center & Center & East Center & \begin{tabular}{|c|} 
New Jersey \\
side
\end{tabular} & Average \\
\hline $\begin{array}{ll}\text { Time (EST) } & \\
\text { Sounding (ft) } \\
\text { Temperature ( }\end{array}$ & $\begin{array}{c}1: 25 \text { p.m. } \\
74 \\
0.6\end{array}$ & $\begin{array}{l}1: 27 \text { p.r. } \\
44 \\
74 \\
75 \\
0.0 \\
0.0\end{array}$ & $\begin{array}{l}1: 29 \text { p.m. } \\
28 \\
74 \\
74 \\
0.7 \\
0.6\end{array}$ & $\begin{array}{l}1: 31 \text { p.m. } \\
30 \\
74 \\
74 \\
0.7 \\
0.6\end{array}$ & $\begin{array}{l}1: 33 \text { p.m. } \\
74 \\
6.7\end{array}$ & $\begin{array}{ll}\text { T. } & 74 \\
\text { B. } & 74 \\
\text { T. } & 6.7 \\
\text { B. } & 6.6\end{array}$ \\
\hline 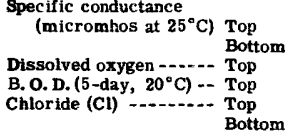 & $\begin{array}{r}181 \\
4.9 \\
9.0\end{array}$ & $\begin{array}{r}175 \\
159 \\
1.3 \\
1.4 \\
9.0 \\
8.0\end{array}$ & $\begin{array}{r}152 \\
150 \\
2.7 \\
7.0 \\
7.0\end{array}$ & $\begin{array}{l}155 \\
160 \\
2.9 \\
.2 \\
7.0 \\
8.0\end{array}$ & $\begin{array}{r}153 \\
3.4 \\
.0 \\
7.0\end{array}$ & $\begin{array}{lr}\text { T. } & 163 \\
\text { B. } & 150 \\
\text { T. } & 2.2 \\
\text { T. } & 1.2 \\
\text { T. } & 7.8 \\
\text { B. } & 7.7\end{array}$ \\
\hline Suspended sediment $\ldots$-.- Top & & & Con & te. . . & 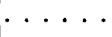 & T. 29 \\
\hline
\end{tabular}

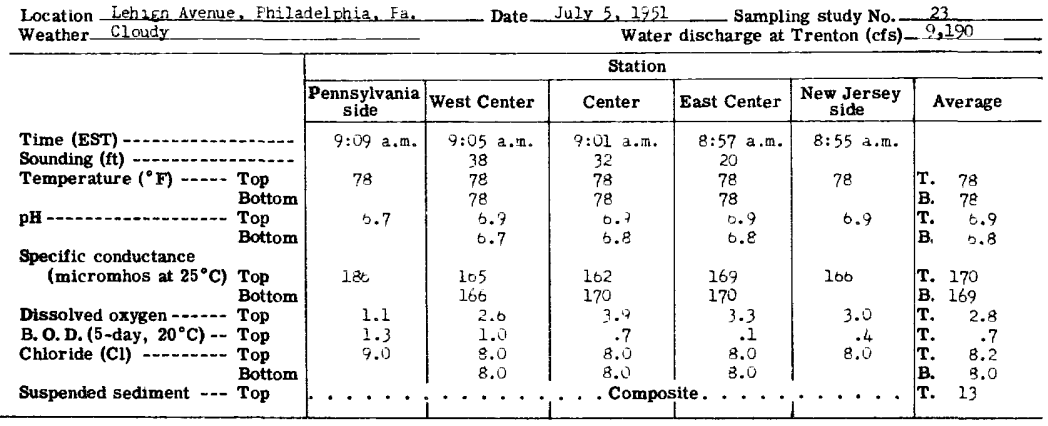

Location Lehigh Avenue, Fhiladelphia, $\mathrm{Fa}$. Weather_Cloudy Date August 1, 195 Sampling study No. -24

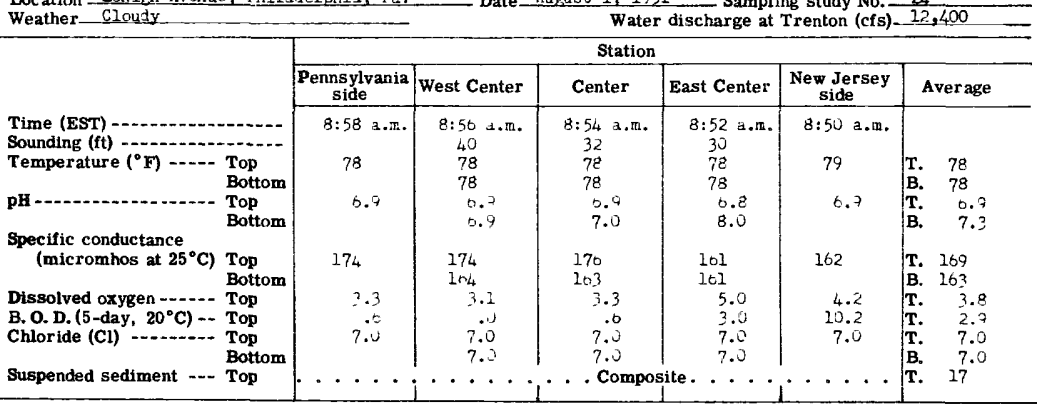


Table 9. -- WATER ANALYSES OF DELAWARE RIVER BETWEEN BRISTOL AND MARCUS HOOK, PA. --Continued Analyzed by City of Philadelphia and U. S. Geological Survey; analyses in parts per million

Location iehigh Avenue, Fhiladelphia, Fa. Weather-Rain

Date_September 0 , 1951 Sampling study No. . 25

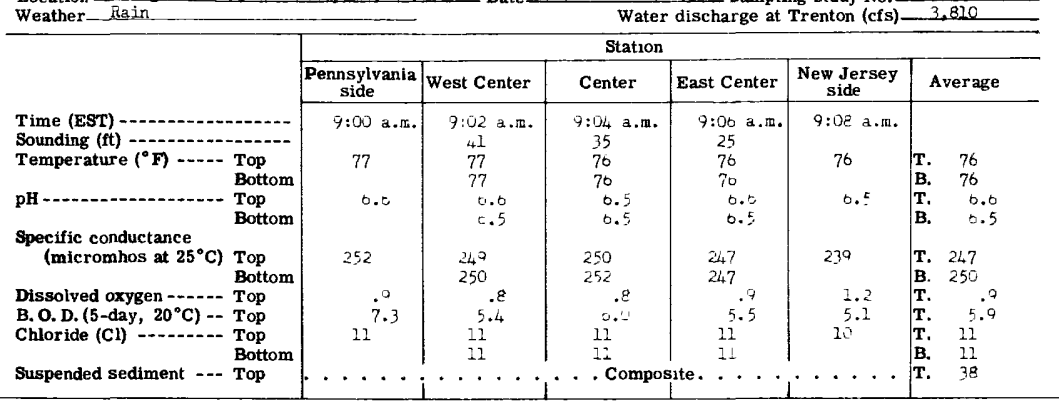

Location Lehigh Avenue, Fhiladel phia, Pa. Weather Clear

Date Uctober $\therefore, 1751$ Sampling study No. _. 26

\begin{tabular}{|c|c|c|c|c|c|c|}
\hline \multicolumn{2}{|l|}{ Weather Clear } & & \multicolumn{4}{|c|}{ Water discharge at Trenton (cfs) - 2.992} \\
\hline & \multicolumn{6}{|c|}{ Station } \\
\hline & $\begin{array}{c}\text { Pennsylvania } \\
\text { side }\end{array}$ & West Center & Center & East Center & $\begin{array}{c}\text { New Jersey } \\
\text { side }\end{array}$ & Average \\
\hline $\begin{array}{ll}\text { Time (EST) } & \\
\text { Sounding (ft) } \\
\text { Temperature }\left({ }^{\circ} \mathrm{F}\right) \\
\text { pH }\end{array} \begin{array}{l}\text { Top } \\
\text { Bottom } \\
\text { Top } \\
\text { Bottom }\end{array}$ & $\begin{array}{c}9: 55 \mathrm{a} . \mathrm{m} . \\
71 \\
0.3\end{array}$ & $\begin{array}{l}9: 52 \text { a.m. } \\
40 \\
7 \mathrm{~J} \\
7 \mathrm{~J} \\
\mathrm{c.3} \\
\text { b.2. }\end{array}$ & $\begin{array}{l}9: 49 \text { a.m. } \\
33 \\
69 \\
70 \\
0.4 \\
0.3\end{array}$ & $\begin{array}{l}9: 47 \mathrm{a} . \mathrm{m} . \\
18 \\
69 \\
67 \\
0.4 \\
0.3\end{array}$ & $\begin{array}{l}3: 44=0 . \mathrm{m} . \\
70 \\
n .3\end{array}$ & $\begin{array}{ll}\text { T. } & 70 \\
\text { B. } & 70 \\
\text { T. } & t .3 \\
\text { B. } & 6.3\end{array}$ \\
\hline 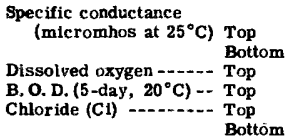 & $\begin{array}{l}272 \\
2.4 \\
7.2 \\
1.4\end{array}$ & $\begin{array}{l}244 \\
240 \\
1.0 \\
1.9 \\
73 \\
12\end{array}$ & $\begin{array}{l}229 \\
229 \\
4.0 \\
12 \\
12\end{array}$ & $\begin{array}{l}234 \\
235 \\
2.7 \\
12 \\
12\end{array}$ & $\begin{array}{l}247 \\
2.0 \\
2.4 \\
23\end{array}$ & $\begin{array}{lc}\text { T. } & 245 \\
\text { B. } & 237 \\
\text { T. } & 2.5 \\
\text { T. } & 2.4 \\
\text { T. } & 12 \\
\text { B. } & 11\end{array}$ \\
\hline Suspended sediment --- Top & $\cdot$ & & . Com & e. . . & . & $\mathbf{T}$ \\
\hline
\end{tabular}

Location Lehigh avenue, Philadelchia, $\mathrm{P}_{3}$.

Date Novemker 7, 2,51__ Sampling study No. 27 Weather Cloudy Water discharge at Trenton (cfs) $-37,700$

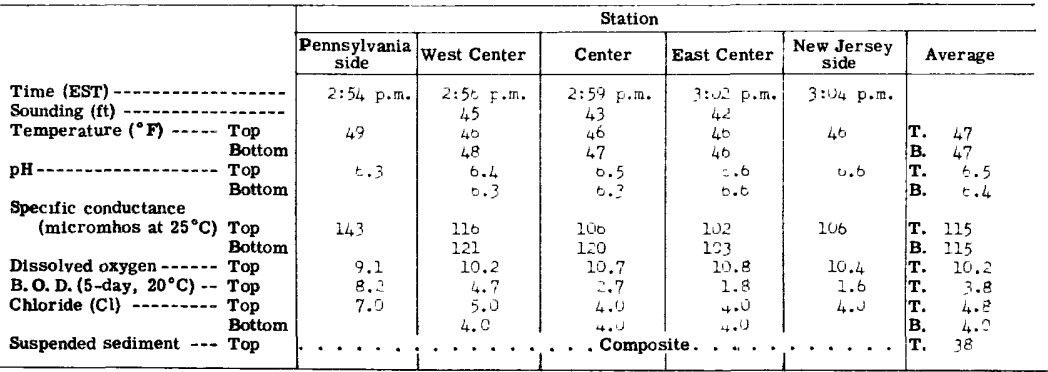

Location Lehigh Avenue, Fhiladelohia, Fa. Weather Rain

Date Decenter 5, 1951 Sampling study No. -28

\begin{tabular}{|c|c|c|c|c|c|c|}
\hline \multicolumn{2}{|c|}{ Weather Rain } & \multicolumn{5}{|c|}{ Water discharge at Trenton (cfs) - 12,500} \\
\hline & \multicolumn{6}{|c|}{ Station } \\
\hline & $\begin{array}{c}\text { Pennsylvanla } \\
\text { side }\end{array}$ & West Center & Center & East Center & $\begin{array}{c}\text { New Jersey } \\
\text { side }\end{array}$ & Average \\
\hline $\begin{array}{l}\text { Time (EST) } \\
\begin{array}{l}\text { Sounding (ft) } \\
\text { Temperature }\left({ }^{\circ} \mathrm{F}\right)\end{array} \\
\begin{array}{l}\text { Top } \\
\text { BH }\end{array} \\
\text { Top } \\
\text { Bottom }\end{array}$ & $\begin{array}{c}10: 18 \text { a.m. } \\
4.4 \\
6.3\end{array}$ & $\begin{array}{c}10: 10 \text { a.r. } \\
44 \\
43 \\
43 \\
\text { b. } \\
\text { b. } 3\end{array}$ & $\begin{array}{c}10: 14 \mathrm{a} . \mathrm{mi} \\
39 \\
42 \\
42 \\
0.5 \\
0.4\end{array}$ & $\begin{array}{l}1 \cup: 12 \text { a.m. } \\
25 \\
42 \\
43 \\
0.5 \\
0.4\end{array}$ & $\begin{array}{c}10: 10 \mathrm{a} . \mathrm{m} . \\
45 \\
0.5\end{array}$ & $\begin{array}{ll}\text { T. } & 43 \\
\text { B. } & 47 \\
\text { T. } & 0.4 \\
\text { B. } & t .4\end{array}$ \\
\hline 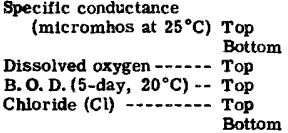 & $\begin{array}{l}174 \\
8.7 \\
0.0 \\
10\end{array}$ & $\begin{array}{l}155 \\
160 \\
7.1 \\
5.7 \\
8.0 \\
8.9\end{array}$ & $\begin{array}{r}146 \\
147 \\
9.2 \\
3.4 \\
7.2 \\
7.4\end{array}$ & $\begin{array}{r}142 \\
144 \\
11.7 \\
7.2 \\
7.1 \\
7.2\end{array}$ & $\begin{array}{r}139 \\
10.4 \\
3.7 \\
0.0\end{array}$ & $\begin{array}{lr}\text { T. } & 151 \\
\text { B. } & 150 \\
\text { T. } & 9.9 \\
\text { T. } & 5.2 \\
\text { T. } & 7.5 \\
\text { B. } & 7.3\end{array}$ \\
\hline Suspended sediment -.- Top & $\cdots \cdot$ & & . Com & ite. . . & $\cdots$ & T. 27 \\
\hline
\end{tabular}


Table 9. --WATER ANALYSES OF DELAWARE RIVER BETWEen BRISTOL AND MARC US HOOK, PA, --Continued Analyzed by City of Philadelphia and U. S. Geological Survey; analyses in parts per million

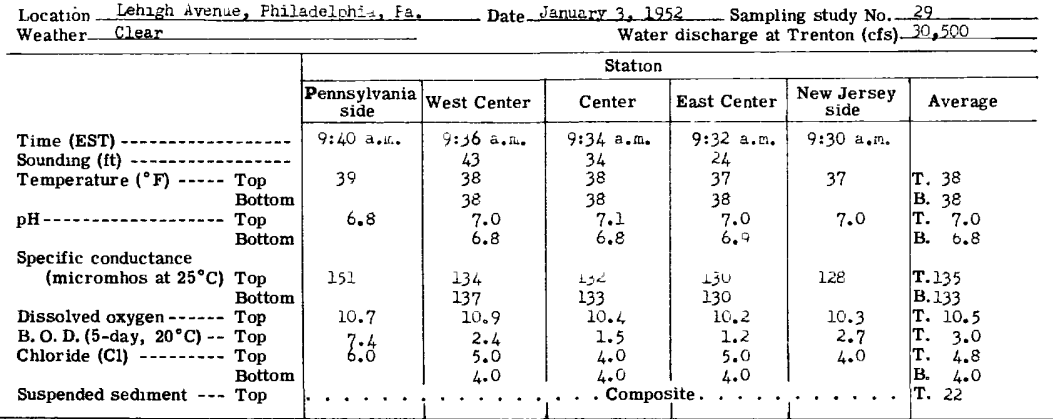

Location Lehigh Avende, Philadelphia, Fa. Weather uvercast

Date February 521952 - Sampling study No. 30

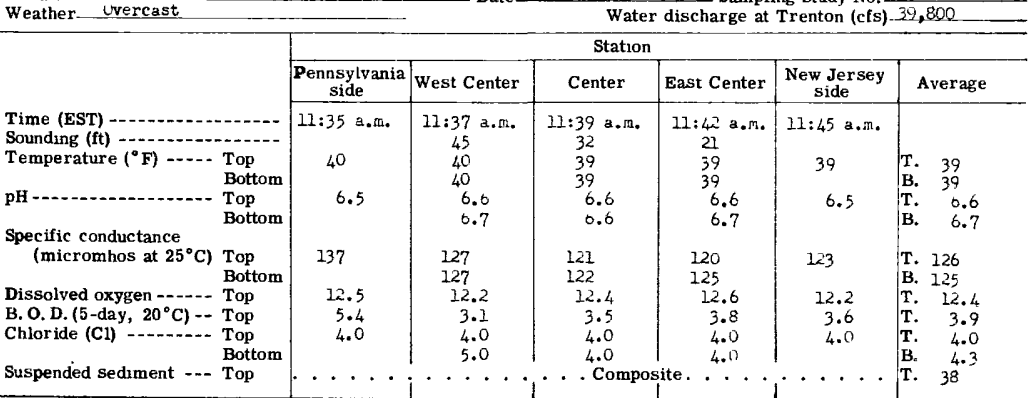

Location Lehigh Avenue, Philadelphia, Fa. Weather Slightly Cloudy

Date March 5, 1952

Sampling study No. 31

\begin{tabular}{|c|c|c|c|c|c|c|}
\hline \multicolumn{3}{|c|}{ Weather Slightli Cloudy } & \multicolumn{4}{|c|}{ Water discharge at Trenton (cfs) 9,730 . } \\
\hline & \multicolumn{6}{|c|}{ Station } \\
\hline & $\begin{array}{c}\text { Pennsylvania } \\
\text { side }\end{array}$ & West Center & Center & East Center & $\begin{array}{l}\text { New Jersey } \\
\text { side }\end{array}$ & Average \\
\hline $\begin{array}{l}\text { Time (EST) } \\
\text { Sounding (ft) } \\
\text { Temper ature }\left({ }^{\circ} \mathrm{F}\right)-\begin{array}{l}\text { Top } \\
\text { Bottom }\end{array} \\
\text { pH } \\
\text { Bop } \\
\text { Bottom }\end{array}$ & $\begin{array}{c}\text { 12:03 F.M. } \\
40 \\
7.4\end{array}$ & $\begin{array}{c}12: 00 \mathrm{n} \\
35 \\
40 \\
40 \\
0.4 \\
6.4\end{array}$ & $\begin{array}{c}11: 56 \text { a.m. } \\
25 \\
40 \\
40 \\
6.5 \\
0.9\end{array}$ & $\begin{array}{c}11: 53 \text { a.m. } \\
20 \\
39 \\
40 \\
6.5 \\
6.5\end{array}$ & $\begin{array}{c}11: 50 \text { a.m. } \\
39 \\
6.4\end{array}$ & $\begin{array}{ll}\text { T. } & 40 \\
\text { B. } & 40 \\
\text { T. } & 6.6 \\
\text { B. } & 6.6\end{array}$ \\
\hline $\begin{array}{ll}\begin{array}{l}\text { Specific conductance } \\
\text { (micromhos at } 25^{\circ} \mathrm{C} \text { ) }\end{array} & \begin{array}{l}\text { Top } \\
\text { Bottom }\end{array} \\
\text { Dissolved oxygen - } & \text { Top } \\
\text { B. O. D. (5-day, } 20^{\circ} \mathrm{C} \text { ) - - Top } & \text { Top } \\
\text { Chloride (Cl) }--1 & \text { Top } \\
& \text { Bottom }\end{array}$ & $\begin{array}{l}178 \\
7.0 \\
5.2 \\
8.0\end{array}$ & $\begin{array}{r}176 \\
178 \\
9.2 \\
5.2 \\
8.0 \\
8.0\end{array}$ & $\begin{array}{r}180 \\
170 \\
9.3 \\
3.3 \\
9.0 \\
7.0\end{array}$ & $\begin{array}{r}169 \\
171 \\
9.7 \\
4.0 \\
6.0 \\
8.0\end{array}$ & $\begin{array}{l}170 \\
9.6 \\
3.4 \\
8.0\end{array}$ & $\begin{array}{lr}\text { T. } & 175 \\
\text { B. } & 173 \\
\text { T. } & 9.4 \\
\text { T. } & 4.2 \\
\text { T. } & 7.8 \\
\text { B. } & 7.7\end{array}$ \\
\hline Suspended sediment & 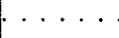 & & Com & ite. . . & $\cdots$ & T. $23^{10}$ \\
\hline
\end{tabular}

Location Lehigh Avenue, Philadelphia, Pa.

Date April 2, 1952

Sampling study No. 32

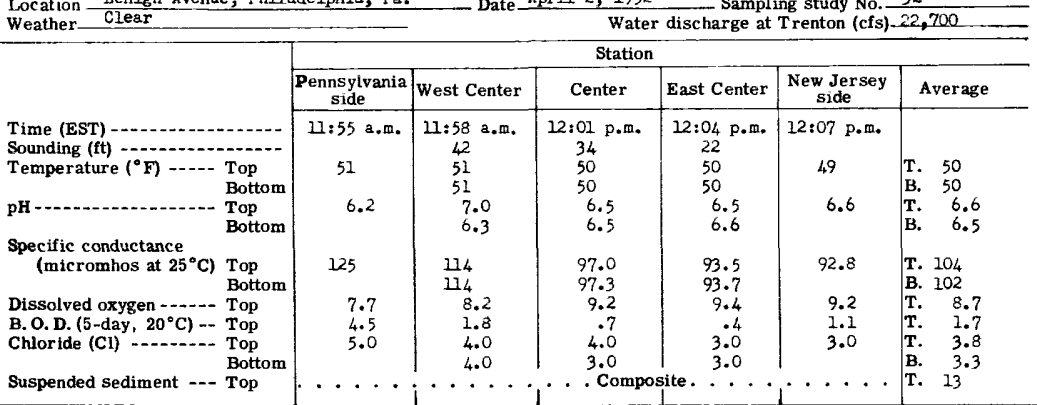


Table 9. - - WATER ANA LYSES OF DELAWARE RIVER BETWEEN BRISTOL AND MARCUS HOOK, PA. --Continued Analyzed by City of Philadelphia and U. S. Geological Survey; analyses in parts per million

Location Lehigh Avenue, Fhiladelphia, Pa. __ Date_Yay 5, 1952 Sampling study No. 33 Weather Cloudy_... Water discharge at Trenton (cfs) $-7 E, 700$

\begin{tabular}{|c|c|c|c|c|c|c|}
\hline \multicolumn{3}{|l|}{ Weather Cloudy ... } & \multicolumn{4}{|c|}{ Water discharge at Trenton (cfs) $-1 E, 700$} \\
\hline & \multicolumn{6}{|c|}{ Station } \\
\hline & $\begin{array}{c}\text { Pennsylvania } \\
\text { side }\end{array}$ & West Center & Center & East Center & $\begin{array}{c}\text { New Jersey } \\
\text { side }\end{array}$ & Average \\
\hline $\begin{array}{l}\text { Time (EST) } \\
\begin{array}{l}\text { Sounding }(\mathrm{ft}) \\
\text { Temperature }\left({ }^{\circ} \mathrm{F}\right)\end{array} \\
\text { pH }\end{array} \begin{array}{l}\text { Top } \\
\text { Bottom }\end{array}$ & $\begin{array}{c}9: 05 \text { a.d. } \\
59 \\
0.3\end{array}$ & $\begin{array}{l}9: 08 \text { a.r. } \\
46 \\
58 \\
59 \\
0.5 \\
6.4\end{array}$ & $\begin{array}{l}9: 11 \text { a.m. } \\
43 \\
58 \\
58 \\
6.5 \\
6.5\end{array}$ & $\begin{array}{l}a: 14 \text { a.m. } \\
34 \\
58 \\
58 \\
6.6 \\
6.6\end{array}$ & $\begin{array}{c}9: 17 \text { a.m. } \\
58 \\
6.5\end{array}$ & $\begin{array}{ll}\text { T. } & 58 \\
\text { B. } & 58 \\
\text { T. } & 5.5 \\
\text { B. } & 6.5\end{array}$ \\
\hline 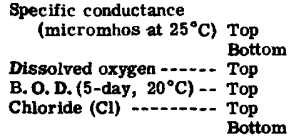 & $\begin{array}{l}126 \\
\\
0.6 \\
1.3 \\
4.0\end{array}$ & $\begin{array}{r}120 \\
122 \\
7.1 \\
1.4 \\
4.0 \\
4.0\end{array}$ & $\begin{array}{r}113 \\
118 \\
7.6 \\
2.0 \\
4.0 \\
3.0\end{array}$ & $\begin{array}{l}113 \\
111 \\
8.0 \\
2.3 \\
4.0 \\
3.0\end{array}$ & $\begin{array}{l}109 \\
8.2 \\
2.8 \\
3.0\end{array}$ & $\begin{array}{lr}\text { T. } & 116 \\
\text { B. } & 117 \\
\text { T. } & 7.5 \\
\text { T. } & 2.2 \\
\text { T. } & 3.8 \\
\text { B. } & 3.3\end{array}$ \\
\hline Suspended sediment -- Top & & & & . . & & $\mathrm{T}$ \\
\hline
\end{tabular}

Location Lehigh Avenue, Philadelphia, $\mathrm{Pa}$.

\begin{tabular}{|c|c|c|c|c|c|c|}
\hline Weather Cloudy & & & Wate & discharge a & Trenton (cf & 28,600 \\
\hline & & & Station & & & \\
\hline & \begin{tabular}{|c|}
$\begin{array}{c}\text { Pennsylvania } \\
\text { side }\end{array}$ \\
\end{tabular} & West Center & Center & East Center & \begin{tabular}{|c|}
$\begin{array}{c}\text { New Jersey } \\
\text { side }\end{array}$ \\
\end{tabular} & Average \\
\hline $\begin{array}{ll}\begin{array}{l}\text { Time (EST) } \\
\text { Sounding (ft) } \\
\text { Temperature }\left({ }^{\circ} \mathrm{F}\right) \\
\text { pH }\end{array} & \begin{array}{l}\text { Top } \\
\text { Bottom }\end{array} \\
& \begin{array}{l}\text { Top } \\
\text { Bottom }\end{array} \\
\text { Specific conductance } & \end{array}$ & $\begin{array}{c}8: 57 \text { a.m. } \\
67 \\
6.6\end{array}$ & $\begin{array}{c}8: 59 \text { a. } 12 . \\
44 \\
66 \\
68 \\
6.7 \\
6.7\end{array}$ & $\begin{array}{l}9: 01 \mathrm{a} . \mathrm{m} . \\
40 \\
67 \\
67 \\
6.8 \\
6.7\end{array}$ & $\begin{array}{l}9: 02 \mathrm{a} \cdot \mathrm{m} . \\
33 \\
67 \\
68 \\
6.8 \\
6.7\end{array}$ & $\begin{array}{c}9: 04 \text { a.m. } \\
67 \\
6.8\end{array}$ & $\begin{array}{ll}\text { T. } & 67 \\
\text { B. } & 68 \\
\text { T. } & 6.7 \\
\text { B. } & 6.7\end{array}$ \\
\hline 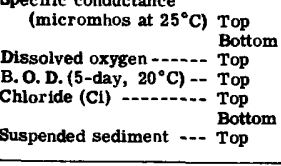 & $\begin{array}{r}123 \\
5.9 \\
3.8 \\
5.0\end{array}$ & $\begin{array}{r}114 \\
115 \\
6.3 \\
2.0 \\
4.0 \\
4.0 \\
.\end{array}$ & $\begin{array}{r}112 \\
110 \\
6.7 \\
.5 \\
4.0 \\
3.0\end{array}$ & $\begin{array}{r}107 \\
108 \\
7.1 \\
1.6 \\
3.0 \\
3.0 \\
.\end{array}$ & $\begin{array}{r}107 \\
7.3 \\
1.9 \\
3.0\end{array}$ & $\begin{array}{lr}\text { T. } & 113 \\
\text { B. } & 111 \\
\text { T. } & 6.7 \\
\text { T. } & 2.0 \\
\text { T. } & 3.8 \\
\text { B. } & 3.3 \\
\text { T. } & 23\end{array}$ \\
\hline
\end{tabular}

Location Lehigh Avenue, Fhiladelphia, Pa. Weather Light Clouds

Sampling study No. 35

\begin{tabular}{|c|c|c|c|c|c|c|}
\hline \multicolumn{3}{|c|}{ Weather Light Clouds } & \multicolumn{4}{|c|}{ Water discharge at Trenton (cfs) 3.370} \\
\hline & \multicolumn{6}{|c|}{ Station } \\
\hline & $\begin{array}{c}\text { Pennsylvania } \\
\text { side }\end{array}$ & West Center & Center & East Center & $\begin{array}{l}\text { New Jersey } \\
\text { side }\end{array}$ & Average \\
\hline $\begin{array}{l}\text { Time (EST) } \\
\text { Sounding (ft) } \\
\text { Temperature }\left({ }^{\circ} \mathrm{F} \text { ) }\right. \\
\text { pH }\end{array}$ & $\begin{array}{c}9: 20 \text { a.m. } \\
80 \\
6.6\end{array}$ & $\begin{array}{l}9: 22 \mathrm{a}, \mathrm{m} . \\
40 \\
80 \\
80 \\
6.8 \\
6.8\end{array}$ & $\begin{array}{l}9: 24 \text { a.m. } \\
33 \\
80 \\
81 \\
6.8 \\
6.8\end{array}$ & $\begin{array}{l}9: 26 \mathrm{a} . \mathrm{m} \\
27 \\
80 \\
80 \\
6.8 \\
6.9\end{array}$ & $\begin{array}{l}9: 28 \mathrm{a} . \mathrm{m} . \\
80 \\
6.8\end{array}$ & $\begin{array}{ll}\text { T. } & 80 \\
\text { B. } & 80 \\
\text { T. } & 6.8 \\
\text { B. } & 6.8\end{array}$ \\
\hline 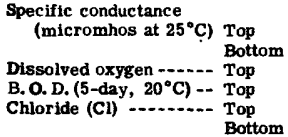 & $\begin{array}{r}205 \\
1.0 \\
1.0 \\
10\end{array}$ & $\begin{array}{l}192 \\
198 \\
1.5 \\
.0 \\
8.0 \\
10\end{array}$ & $\begin{array}{r}182 \\
184 \\
2.0 \\
.0 \\
8.0 \\
8.0\end{array}$ & $\begin{array}{r}182 \\
190 \\
2.3 \\
.0 \\
8.0 \\
8.0\end{array}$ & $\begin{array}{r}187 \\
2.7 \\
.0 \\
8.0\end{array}$ & $\begin{array}{lr}\text { T. } & 190 \\
\text { B. } & 191 \\
\text { T. } & 1.9 \\
\text { T. } & .2 \\
\text { T. } & 8.4 \\
\text { B. } & 8.7\end{array}$ \\
\hline Suspended sediment --- Top & . . & & & Lite... & $\cdots \cdot$ & T. 11 \\
\hline
\end{tabular}

Location Lehigh Avenue, Philadelphia, Pa Weather Cloudy Date August 6, 195

Sampling study No. 36

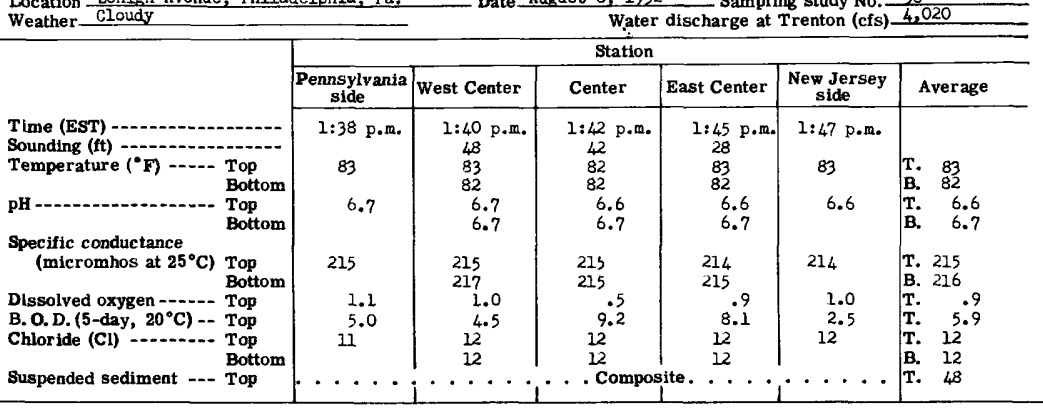


Table 9. --WATER ANALYSES OF DELAWARE RIVER BETWEen BRISTOL AND MARCUS HOOK, PA. --Contunued Analyzed by City of Philadelphia and U. S. Geological Survey; analyses in parts per million

Location Lehigh Avenue, Philadelphia, Pa.

Weather Hazy

Date September 3, 1952 Sampling study No. 37

\begin{tabular}{|c|c|c|c|c|c|c|}
\hline \multirow[b]{3}{*}{$\begin{array}{l}\text { Time (EST) } \\
\text { Sounding (ft) } \\
\text { Temperature }\left({ }^{\circ} \mathrm{F}\right) \\
\text { pH }\end{array}$} & \multicolumn{6}{|c|}{ Station } \\
\hline & $\begin{array}{c}\text { Pennsylvania } \\
\text { side }\end{array}$ & West Center & Center & East Center & $\begin{array}{c}\text { New Jersey } \\
\text { side }\end{array}$ & Average \\
\hline & $\begin{array}{c}9: 25 \text { a.m. } \\
76 \\
6.5\end{array}$ & $\begin{array}{l}9: 23 \text { a.m. } \\
41 \\
76 \\
76 \\
6.4 \\
6.5\end{array}$ & $\begin{array}{l}9: 20 \mathrm{a} . \mathrm{m} . \\
31 \\
76 \\
76 \\
6.4 \\
6.5\end{array}$ & $\begin{array}{l}9: 17 \text { a.m. } \\
21 \\
76 \\
76 \\
6.5 \\
6.4\end{array}$ & $\begin{array}{l}9: 15 \text { a.m. } \\
76 \\
6.5\end{array}$ & $\begin{array}{ll}\text { T. } & 76 \\
\text { B. } & 76 \\
\text { T. } & 6.5 \\
\text { B. } & 6.5\end{array}$ \\
\hline 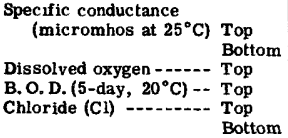 & $\begin{array}{l}183 \\
6.8 \\
3.7 \\
8.5\end{array}$ & $\begin{array}{r}190 \\
181 \\
5.6 \\
1.8 \\
9.5 \\
8.5\end{array}$ & $\begin{array}{l}176 \\
173 \\
7.0 \\
1.6 \\
7.5 \\
7.0\end{array}$ & $\begin{array}{r}175 \\
182 \\
6.8 \\
2.4 \\
7.5 \\
8.5\end{array}$ & $\begin{array}{r}176 \\
6.9 \\
1.6 \\
7.5\end{array}$ & $\begin{array}{lr}\text { T. } & 180 \\
\text { B. } & 179 \\
\text { T. } & 6.6 \\
\text { T. } & 2.2 \\
\text { T. } & 8.1 \\
\text { B. } & 8.0\end{array}$ \\
\hline Suspended sediment --- Top & $\cdots \cdot$ & & Comn & te... & $\cdots \cdot$ & T. \\
\hline
\end{tabular}

Location Lehigh Avenue, Philadelphia, Pa,

Date October 6, 1952 Sampling study No. 38

\begin{tabular}{|c|c|c|c|c|c|c|}
\hline \multirow[b]{3}{*}{$\begin{array}{l}\text { Time (EST) } \\
\text { Sounding (t) } \\
\text { Temperature }\left({ }^{\circ} \mathrm{F}\right) \\
\text { pH }\end{array}$} & \multicolumn{6}{|c|}{ Station } \\
\hline & \begin{tabular}{|c|}
$\begin{array}{c}\text { Pennsylvania } \\
\text { side }\end{array}$ \\
\end{tabular} & West Center & Center & East Center & \begin{tabular}{|c|}
$\begin{array}{c}\text { New Jersey } \\
\text { side }\end{array}$ \\
\end{tabular} & Average \\
\hline & $\begin{array}{c}11: 00 \text { a.m. } \\
68 \\
6.4\end{array}$ & $\begin{array}{c}10: 57 \text { a.m. } \\
39 \\
67 \\
67 \\
6.6 \\
6.5\end{array}$ & $\begin{array}{c}10: 55 \text { a.m. } \\
29 \\
67 \\
67 \\
6.6 \\
6.6\end{array}$ & $\begin{array}{c}10: 52 \text { a.m. } \\
17 \\
67 \\
67 \\
6.6 \\
6.7\end{array}$ & $\begin{array}{c}10: 50 \text { a.m. } \\
68 \\
6.6\end{array}$ & $\begin{array}{ll}\text { T. } & 67 \\
\text { B. } & 67 \\
\text { T. } & 6.6 \\
\text { B. } & 6.6\end{array}$ \\
\hline 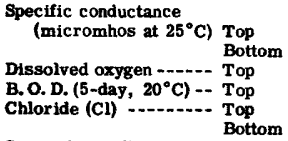 & $\begin{array}{l}221 \\
2.0 \\
1.8 \\
11\end{array}$ & $\begin{array}{r}201 \\
199 \\
4.4 \\
2.1 \\
9.5 \\
10\end{array}$ & $\begin{array}{r}195 \\
195 \\
4.9 \\
1.9 \\
9.5 \\
9.5\end{array}$ & $\begin{array}{l}201 \\
204 \\
4 \cdot 8 \\
2.7 \\
10 \\
10\end{array}$ & $\begin{array}{l}218 \\
3.3 \\
3.1 \\
12\end{array}$ & $\begin{array}{lc} & \\
\text { T. } & 207 \\
\text { B. } & 199 \\
\text { T. } & 3.9 \\
\text { T. } & 2.3 \\
\text { T. } & 10 \\
\text { B. } & 9.8\end{array}$ \\
\hline Suspended sediment $\ldots$... Top & & & & & $\cdots \cdot \cdot$ & $\mathbf{T}$ \\
\hline
\end{tabular}

Location Lehigh Avenue, Philadelphia, $\mathrm{Pa}$.

Date November 6, 1952 Sampling study No. 39 Weather Clear

\begin{tabular}{|c|c|c|c|c|c|c|}
\hline \multicolumn{3}{|l|}{ Weather Clear } & \multicolumn{4}{|c|}{ Water discharge at Trenton (cfs) $-3,130$} \\
\hline & \multicolumn{6}{|c|}{ Station } \\
\hline & $\begin{array}{c}\text { Pernsylvania } \\
\text { side }\end{array}$ & West Center & Center & East Center & $\begin{array}{c}\text { New Jersey } \\
\text { side }\end{array}$ & Average \\
\hline $\begin{array}{ll}\text { Time (EST) } & \\
\text { Sounding (ft) } \\
\text { Temperature ("F) }\end{array}$ & $\begin{array}{c}10: 04 \text { a.m. } \\
55 \\
6.4\end{array}$ & $\begin{array}{c}10: 01 \text { a.m. } \\
41 \\
55 \\
53 \\
6.5 \\
6.5\end{array}$ & $\begin{array}{l}9: 58 \text { a.m. } \\
32 \\
55 \\
53 \\
7.6 \\
6.6\end{array}$ & $\begin{array}{c}9: 55 \text { a.m. } \\
23 \\
53 \\
53 \\
6.6 \\
6.7\end{array}$ & $\begin{array}{l}9: 52 \text { a.m. } \\
53 \\
6.7\end{array}$ & $\begin{array}{ll}\text { T. } & 54 \\
\text { B. } & 53 \\
\text { T. } & 6.8 \\
\text { B. } & 6.6\end{array}$ \\
\hline 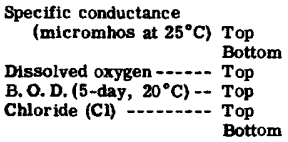 & $\begin{array}{l}306 \\
2.4 \\
9.8 \\
20\end{array}$ & $\begin{array}{l}285 \\
290 \\
2.8 \\
7.6 \\
17 \\
18\end{array}$ & $\begin{array}{l}276 \\
278 \\
3.5 \\
6.5 \\
17 \\
18\end{array}$ & $\begin{array}{l}278 \\
290 \\
4.0 \\
3.0 \\
18 \\
18\end{array}$ & $\begin{array}{l}304 \\
2.7 \\
6.5 \\
19\end{array}$ & $\begin{array}{ll}\text { T. } & 290 \\
\text { B. } & 286 \\
\text { T. } & 3.1 \\
\text { T. } & 6.7 \\
\text { T. } & 18 \\
\text { B. } & 18\end{array}$ \\
\hline Suspended sediment -.- Top & & & Comp & e. & 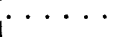 & T. 8 \\
\hline
\end{tabular}

Location Lehigh Avenue, Philadelphia, $\mathrm{Pa}$ Weather Clear

Date December 4,1952 Sampling study No._- 60 Weather Clear

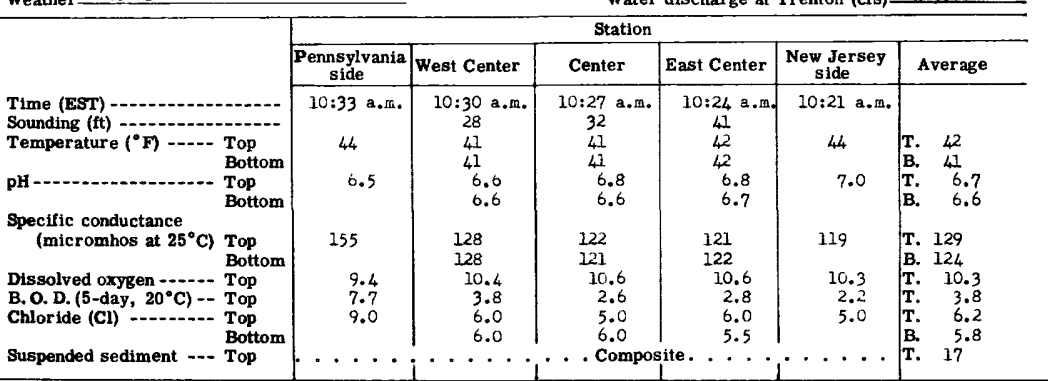


Table 10. -- WATER ANALYSES OF DELAWARE RIVER BETWEEN BRISTOL AND MARCUS HOOK, PA. Analyzed by City of Philadelphia and U. S. Geological Survey; analyses in parts per million

Location Philadelphia, Pa.-Camien, N. J. Bridge Date August 2, 1949 Sampling study No. 1 Weather Cloudy

\begin{tabular}{|c|c|c|c|c|c|c|}
\hline \multirow[b]{3}{*}{ 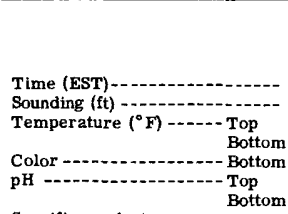 } & \multicolumn{6}{|c|}{ Station } \\
\hline & $\begin{array}{c}\text { Pennsylvania } \\
\text { side }\end{array}$ & West Center & Center & East Center & $\begin{array}{c}\text { New Jersey } \\
\text { side }\end{array}$ & Average \\
\hline & $\begin{array}{l}\overline{27} \\
84 \\
84 \\
25 \\
6.1 \\
6.1\end{array}$ & $\begin{array}{l}34 \\
84 \\
84 \\
30 \\
0.0 \\
6.1\end{array}$ & $\begin{array}{l}9: 30 \text { a.m. } \\
40 \\
84 \\
84 \\
25 \\
6.1 \\
6.1\end{array}$ & $\begin{array}{l}- \\
84 \\
84 \\
45 \\
0.1 \\
6.3\end{array}$ & $\begin{array}{l}\overline{17} \\
84 \\
84 \\
30 \\
6.0 \\
6.2\end{array}$ & $\begin{array}{ll}\text { T. } & 84 \\
\text { B. } & 84 . \\
\text { B. } & 31 \\
\text { T. } & 6.1 \\
\text { B. } & 6.2\end{array}$ \\
\hline $\begin{array}{l}\begin{array}{l}\text { Specific conductance } \\
\text { (micromhos at } 25^{\circ} \mathrm{C} \text { ) }\end{array} \\
\begin{array}{l}\text { Top } \\
\text { Bottom }\end{array} \\
\text { Dissolved oxygen }- \\
\text { B. O.D. }\left(5 \text {-day, } 20^{\circ} \mathrm{C} \text { ) }- \text { Top }\right. \\
\text { Top }\end{array}$ & ${ }_{18}^{285} \cdot 0$ & ${ }_{17}^{287} .0$ & ${ }_{15}^{283} .1$ & $20^{277} .0$ & ${ }_{20}^{277} \cdot 0$ & $\begin{array}{ll}\text { T. } & 282 \\
\text { B. } & 277 \\
\text { T. } & \\
\text { T. } & 18\end{array}$ \\
\hline 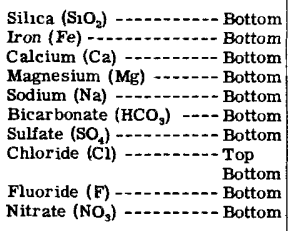 & $\begin{array}{l}2.6 \\
.04 \\
20^{.04} \\
7.6 \\
20 \\
58 \\
40 \\
18 \\
17 \\
8.5\end{array}$ & $\begin{array}{l}2.9 \\
21.06 \\
7.5 \\
17 \\
55 \\
42 \\
18 \\
18 \\
.2 .1\end{array}$ & $\begin{array}{l}2.9 \\
.04 \\
20 \\
7.6 \\
17 \\
55 \\
41 \\
17 \\
16 \\
.2 \\
7.8\end{array}$ & $\begin{array}{l}2.6 \\
.29 \\
7.3 \\
19 \\
55 \\
42 \\
17 \\
16 \\
8.0\end{array}$ & $\begin{array}{l}2.6 \\
20 \\
7.07 \\
7.4 \\
54 \\
40 \\
17 \\
17 \\
7.2 \\
.8\end{array}$ & $\begin{array}{l}2.7 \\
20 \\
7.08 \\
7.5 \\
55 \\
41 \\
17 \\
17 \\
8.2\end{array}$ \\
\hline $\begin{array}{l}\text { Dissolved solids } \\
\text { Hardness as } \mathrm{CaCO}_{3} \ldots-\text { Bottom } \\
\text { Suspended sediment }-\ldots-\text { Top }\end{array}$ & $\begin{array}{r}162 \\
81 \\
\cdots\end{array}$ & $\begin{array}{r}105 \\
84 \\
. \\
\end{array}$ & $\begin{array}{r}160 \\
81 \\
. \quad \text { Comp }\end{array}$ & $\begin{array}{r}201 \\
80 \\
\text { site. . . . }\end{array}$ & $\begin{array}{r}159 \\
80 \\
+\cdots \\
\end{array}$ & $\begin{array}{rr}\text { B. } & 161 \\
\text { B. } & 81 \\
\text { T. } & - \\
\end{array}$ \\
\hline
\end{tabular}

Location Philadelphia, Fa.-Camden, N. J. Bridge Date -September 6, 1949 Sampling study No. 2 Weather Cloudy Water discharge at Trenton (cfs) 5,310

\begin{tabular}{|c|c|c|c|c|c|c|}
\hline \multicolumn{3}{|l|}{ Weather Cloudy } & \multicolumn{4}{|c|}{ Water discharge at Trenton (cfs) 5,310} \\
\hline & \multicolumn{6}{|c|}{ Station } \\
\hline & \begin{tabular}{|c|}
$\begin{array}{c}\text { Pennsylvania } \\
\text { side }\end{array}$ \\
\end{tabular} & West Center & Center & East Center & \begin{tabular}{|c|}
$\begin{array}{c}\text { New Jersey } \\
\text { side }\end{array}$ \\
\end{tabular} & Average \\
\hline $\begin{array}{l}\text { Time (BST) } \\
\begin{array}{l}\text { Sounding }(\mathrm{ft}) \\
\text { Temperature }\left({ }^{\circ} \mathrm{F}\right)\end{array} \\
\begin{array}{l}\text { Bottom } \\
\text { Color }\end{array} \\
\text { pH }-\begin{array}{l}\text { Bottom } \\
\text { Specif }\end{array} \\
\text { Bot conductance }\end{array}$ & $\begin{array}{l}-29 \\
78 \\
78 \\
17 \\
6.5 \\
6.6\end{array}$ & $\begin{array}{l}-\overline{45} \\
78 \\
78 \\
20 \\
6.5 \\
7.5\end{array}$ & $\begin{array}{l}2: 15 \mathrm{p} . \mathrm{m} \\
47 \\
78 \\
77 \\
17 \\
6.6 \\
7.2\end{array}$ & $\begin{array}{l}32 \\
78 \\
77 \\
17 \\
6.5 \\
6.3\end{array}$ & $\begin{array}{l}-7 \\
78 \\
78 \\
17 \\
6.5 \\
7.2\end{array}$ & $\begin{array}{l}78 \\
78 \\
18 \\
6.5 \\
7.0\end{array}$ \\
\hline $\begin{array}{ll}\left.\quad \text { (micromhos at } 25^{\circ} \mathrm{C}\right) & \text { Top } \\
\text { Bottom } \\
\text { Dissolved oxygen } \cdots & \text { Top } \\
\text { B, O.D. (5-day, } 20^{\circ} \mathrm{C} \text { ) } \cdots \text { Top }\end{array}$ & $\begin{array}{l}361 \\
351 \\
3.7\end{array}$ & $\begin{array}{l}401 \\
377 \\
.2 \\
2.2\end{array}$ & $\begin{array}{r}394 \\
372 \\
3.8\end{array}$ & $\begin{array}{r}382 \\
359 \\
.3 \\
3.3\end{array}$ & $\begin{array}{l}363 \\
349 \\
3.7\end{array}$ & \begin{tabular}{lr} 
T. & 380 \\
B. & \multicolumn{1}{c}{362} \\
T. & .5 \\
T. & 3.3
\end{tabular} \\
\hline 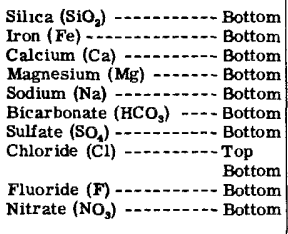 & $\begin{array}{l}4.0 \\
.06 \\
24 \\
9.0 \\
27 \\
60 \\
54 \\
33 \\
32 \\
.2 \\
9.1\end{array}$ & $\begin{array}{l}4.1 \\
.06 \\
24.5 \\
9.5 \\
33 \\
58 \\
55 \\
42 \\
39 \\
.2 \\
9.3\end{array}$ & $\begin{array}{l}4.4 \\
.06 \\
24 \\
9.1 \\
32 \\
57 \\
56 \\
40 \\
39 \\
.2 \\
8.9\end{array}$ & $\begin{array}{l}3.8 \\
2.06 \\
9.0 \\
29 \\
58 \\
55 \\
37 \\
34.2 \\
8.4\end{array}$ & $\begin{array}{l}3.7 \\
.06 \\
24 \\
8.7 \\
26 \\
60 \\
52 \\
33 \\
32 \\
.0 \\
7.4\end{array}$ & $\begin{array}{l}4.0 \\
2.06 \\
9.1 \\
29 \\
59 \\
54 \\
37 \\
35 \\
8.2\end{array}$ \\
\hline $\begin{array}{l}\text { Dissolved solids }-\cdots-\text { Bottom } \\
\text { Hardness as } \mathrm{CaCO}_{3}-\ldots \text { Bottom } \\
\text { Suspended sediment }-\cdots \text { Top }\end{array}$ & $\begin{array}{r}219 \\
97 \\
. \quad . \\
\end{array}$ & $\begin{array}{r}233 \\
99 \\
\cdots \cdot \\
\end{array}$ & $\begin{array}{l}233 \\
97 \\
\cdots \\
\end{array}$ & $\begin{array}{r}224 \\
97 \\
\text { site. . . }\end{array}$ & $\begin{array}{r}215 \\
96 \\
\cdots\end{array}$ & $\begin{array}{lr}\text { B. } & 225 \\
\text { B. } & 97 \\
\text { T. } & 22 \\
\end{array}$ \\
\hline
\end{tabular}


Table 10. -- WATER ANALYSES OF DELAWARE RIVER BETWEEN BRISTOL AND MARCUS HOOK, PA. --Continued Analyzed by City of Philadelphia and U. S. Geological Survey; analyses in parts per million

Location Fhiladelphia, Fa,-Camden, N. J. Bridge Date October 4, 1949 Sampling study No. - 3 Weather Uccaszong I Kain $\quad$ Water discharge at Trenton (cfs) 3,520

\begin{tabular}{|c|c|c|c|c|c|c|}
\hline & \multicolumn{6}{|c|}{ Station } \\
\hline & $\begin{array}{c}\text { Pennsylvania } \\
\text { side }\end{array}$ & West Center & Center & East Center & $\begin{array}{c}\text { New Jersey } \\
\text { side } \\
\end{array}$ & Average \\
\hline 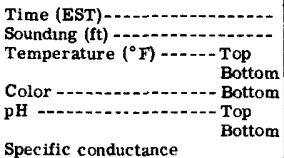 & $\begin{array}{l}\overrightarrow{41} \\
70 \\
70 \\
13 \\
6.4 \\
0.6\end{array}$ & $\begin{array}{l}- \\
45 \\
70 \\
70 \\
15 \\
6.5 \\
0.4\end{array}$ & $\begin{array}{l}2: 30 \text { p.m. } \\
45 \\
70 \\
70 \\
16 \\
6.4 \\
0.5\end{array}$ & $\begin{array}{l}-32 \\
70 \\
70 \\
16 \\
6.6 \\
0.4\end{array}$ & $\begin{array}{l}-13 \\
70 \\
71 \\
18 \\
6.6 \\
6.5\end{array}$ & $\begin{array}{ll}\text { T. } & 70 \\
\text { B. } & 70 \\
\text { B. } & 16 \\
\text { T. } & 6.5 \\
\text { B. } & 6.5\end{array}$ \\
\hline $\begin{array}{l}\text { (micromhos at } 25^{\circ} \mathrm{C} \text { ) } \begin{array}{l}\text { Top } \\
\text { Bottom }\end{array} \\
\text { Dissolved oxygen - - }- \text { Top } \\
\text { B. O. D. (5-day, } 20^{\circ} \mathrm{C} \text { ) -- Top }\end{array}$ & $\begin{array}{l}434 \\
429 \\
1.4 \\
5.1\end{array}$ & $\begin{array}{l}430 \\
412 \\
1.5 \\
5.2\end{array}$ & $\begin{array}{l}4.2 \\
430 \\
1.6 \\
5.3\end{array}$ & $\begin{array}{r}405 \\
400 \\
1.5 \\
.4\end{array}$ & $\begin{array}{l}397 \\
385 \\
2.0 \\
1.8\end{array}$ & $\begin{array}{lr}\text { T. } & 422 \\
\text { B. } & 418 \\
\text { T. } & 1.6 \\
\text { T. } & 3.6\end{array}$ \\
\hline 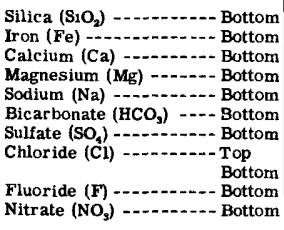 & $\begin{array}{l}2.8 \\
.12 \\
24 \\
11 \\
36 \\
50 \\
62 \\
52 \\
49 \\
.2 \\
9.7\end{array}$ & $\begin{array}{l}2.6 \\
.19 \\
24 \\
11 \\
37 \\
48 \\
64 \\
50 \\
51 \\
.3 \\
9.1\end{array}$ & $\begin{array}{l}2.7 \\
.12 \\
24 \\
11 \\
38 \\
49 \\
64 \\
52 \\
52 \\
.3 \\
9.8\end{array}$ & $\begin{array}{l}2.2 \\
23^{.11} \\
11 \\
33 \\
49 \\
61 \\
46 \\
45 \\
8.3\end{array}$ & $\begin{array}{l}2.5 \\
24 \\
10 \\
30 \\
53 \\
58 \\
42 \\
40 \\
9.3\end{array}$ & $\begin{array}{ll}\text { B. } & 2.6 \\
\text { B. } & .15 \\
\text { B. } & 24 \\
\text { B. } & 11 \\
\text { B. } & 35 \\
\text { B. } & 50 \\
\text { B. } & 62 \\
\text { T. } & 48 \\
\text { B. } & 47 \\
\text { B. } & .3 \\
\text { B. } & 9.2\end{array}$ \\
\hline $\begin{array}{l}\text { Dissolved solids - }-\ldots \text { - Bottom } \\
\text { Hardness as } \mathrm{CaCO}_{3}-\ldots \text { Bottom } \\
\text { Suspended sediment }-\ldots \text { Top }\end{array}$ & $\begin{array}{l}242 \\
105 \\
\cdots\end{array}$ & $\begin{array}{l}248 \\
105 \\
.\end{array}$ & $\begin{array}{l}246 \\
105 \\
. \quad \text { Con }\end{array}$ & $\begin{array}{l}230 \\
103 \\
\text { te.... }\end{array}$ & $\begin{array}{l}223 \\
101 \\
\cdots\end{array}$ & $\begin{array}{lr}\text { B. } & 238 \\
\text { B. } & 104 \\
\text { T. } & 23\end{array}$ \\
\hline
\end{tabular}

Location Fhiladelphia, Pa.-Cauden, N. J. Bridge Date November 1, 1949 Sampling study No. $\frac{4}{3,340}$

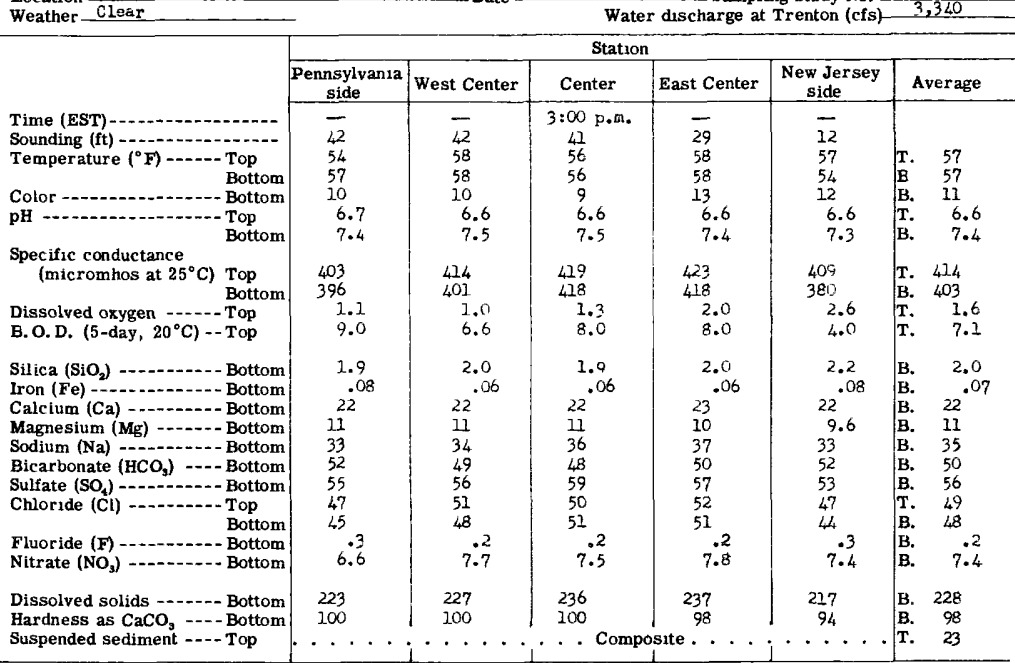


Table 10. --WATER ANALYSES OF DELAWARE RIVER BETWEEN BRISTOL AND MARCUS HOOK, PA. --Continued Analyzed by City of Philadelphia and U. S. Geological Survey; analyses in parts per million

Location Philadelphia, $\mathrm{Pa}$, -Camden, N. J, Bridge Date December 1, 1949 Sampling study No. 5 Weather clear

\begin{tabular}{|c|c|c|c|c|c|c|}
\hline \multirow[b]{3}{*}{$\begin{array}{l}\text { Time (EST) } \\
\text { Sounding (ft) } \\
\text { Temperature }\left({ }^{\circ} \mathrm{F}\right)-1 \\
\begin{array}{c}\text { Bottom } \\
\text { Color } \\
\text { pH }\end{array}\end{array}$} & \multicolumn{6}{|c|}{ Station } \\
\hline & $\begin{array}{c}\text { Pennsylvania } \\
\text { side }\end{array}$ & West Center & Center & East Center & \begin{tabular}{|c|}
$\begin{array}{c}\text { New Jersey } \\
\text { side }\end{array}$ \\
\end{tabular} & Average \\
\hline & $\begin{array}{l}-\overline{41} \\
46 \\
45 \\
10 \\
6.3 \\
6.4\end{array}$ & $\begin{array}{l}-\overline{41} \\
46 \\
44 \\
8 \\
6.4 \\
6.5\end{array}$ & $\begin{array}{l}2: 30 \mathrm{p} . \mathrm{m} \\
26 \\
43 \\
42 \\
9 \\
6.5 \\
6.5\end{array}$ & $\begin{array}{l}-7 \\
43 \\
43 \\
10 \\
6.5 \\
6.6\end{array}$ & $\begin{array}{l}\overline{12} \\
43 \\
43 \\
9 \\
6.5 \\
6.6\end{array}$ & $\begin{array}{ll}\text { T. } & 44 \\
\text { B. } & 43 \\
\text { B. } & 9 \\
\text { T. } & 6.4 \\
\text { B. } & 6.5\end{array}$ \\
\hline $\begin{array}{l}\begin{array}{l}\text { Specific conductance } \\
\left.\text { (micromhos at } 25^{\circ} \mathrm{C}\right)\end{array} \\
\begin{array}{l}\text { Top } \\
\text { Bottom }\end{array} \\
\text { Dissolved oxygen } \\
\text { B.O.D. (5-day, } 20^{\circ} \mathrm{C} \text { )-- Top } \\
\end{array}$ & $\begin{array}{r}236 \\
227 \\
3.8 \\
15.0\end{array}$ & $\begin{array}{l}222 \\
221 \\
4.2 \\
8.7\end{array}$ & $\begin{array}{l}218 \\
215 \\
4.3 \\
9.5\end{array}$ & $\begin{array}{r}219 \\
212 \\
4.5 \\
8.5\end{array}$ & $\begin{array}{l}261 \\
230 \\
4.3 \\
11.3\end{array}$ & $\begin{array}{ll}\text { T. } & 231 \\
\text { B. } & 221 \\
\text { T. } & 4.2 \\
\text { T. } & 10.6\end{array}$ \\
\hline 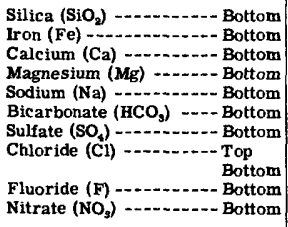 & $\begin{array}{l}4.2 \\
16 \\
5.05 \\
16 \\
35 \\
41 \\
16 \\
16 \\
.1 \\
6.8\end{array}$ & $\begin{array}{l}3.6 \\
16^{.06} \\
6.0 \\
15 \\
34 \\
39 \\
16 \\
17 \\
7.0\end{array}$ & $\begin{array}{l}4.1 \\
16 \\
5.05 \\
13 \\
34 \\
38 \\
14 \\
15 \\
6.1 \\
6.2\end{array}$ & $\begin{array}{l}4.1 \\
16 \\
5.05 \\
14 \\
34 \\
38 \\
15 \\
15 \\
.1 \\
6.8\end{array}$ & $\begin{array}{l}4.8 \\
18^{.05} \\
7.5 \\
15 \\
36 \\
37 \\
22 \\
18 \\
7.1\end{array}$ & $\begin{array}{ll}\text { B. } & 4.2 \\
\text { B. } & .05 \\
\text { B. } & 16 \\
\text { B. } & 6.2 \\
\text { B. } & 15 \\
\text { B. } & 35 \\
\text { B. } & 39 \\
\text { T. } & 17 \\
\text { B. } & 16 \\
\text { B. } & .1 \\
\text { B. } & 7.0\end{array}$ \\
\hline $\begin{array}{l}\text { Dissolved solids } \ldots \ldots \text { Bottom } \\
\text { Hardness as } \mathrm{CaCO}_{3} \ldots \text { Bottom } \\
\text { Suspended sediment } \ldots \ldots \text { Top }\end{array}$ & $\begin{array}{r}135 \\
64 \\
.\end{array}$ & $\begin{array}{r}130 \\
65 \\
\cdots\end{array}$ & $\begin{array}{r}126 \\
64 \\
. \\
\end{array}$ & $\begin{array}{r}124 \\
64 \\
\text { site. } . .\end{array}$ & $\begin{array}{r}137 \\
76 \\
. \\
\end{array}$ & $\begin{array}{lr}\text { B. } & 130 \\
\text { B. } & 67 \\
\text { T. } & 24 \\
\end{array}$ \\
\hline
\end{tabular}

Location Philadelphia, Pa.-Camden, N. J. Bridge Date January 3, 1950 Sampling study No. 6

\begin{tabular}{|c|c|c|c|c|c|c|}
\hline \multirow{3}{*}{ Weather Occasional Rain } & \multicolumn{6}{|c|}{ Water discharge at Trenton (cfs) 11,100} \\
\hline & \multicolumn{6}{|c|}{ Station } \\
\hline & $\begin{array}{c}\text { Pennsylvania } \\
\text { side }\end{array}$ & West Center & Center & East Center & $\begin{array}{l}\text { New Jersey } \\
\text { side }\end{array}$ & Average \\
\hline 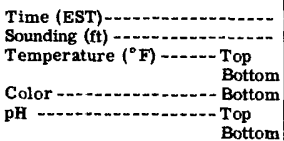 & $\begin{array}{c}2: 25 \text { p.m. } \\
45 \\
-- \\
\overline{10} \\
6.2 \\
6.7\end{array}$ & $\begin{array}{l}2: 23 \mathrm{p} \cdot \mathrm{m} . \\
45 \\
\overline{-} \\
\overline{15} \\
6.5 \\
7.0\end{array}$ & $\begin{array}{c}2: 18 \text { p.m. } \\
36 \\
- \\
-14 \\
6.5 \\
6.2\end{array}$ & $\begin{array}{l}2: 10 \mathrm{p} . \mathrm{m} . \\
\frac{23}{15} \\
6.5 \\
6.7\end{array}$ & $\begin{array}{c}2: 05 \text { p.m. } \\
26 \\
- \\
\overline{14} \\
6.4 \\
6.2\end{array}$ & $\begin{array}{l}\overline{-} \\
14 \\
6.4 \\
6.6\end{array}$ \\
\hline 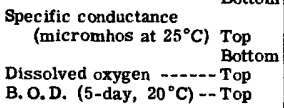 & $\begin{array}{r}166 \\
135 \\
8.3 \\
.0\end{array}$ & $\begin{array}{r}143 \\
133 \\
8.3 \\
.0\end{array}$ & $\begin{array}{r}140 \\
138 \\
8.2 \\
.0\end{array}$ & $\begin{array}{r}135 \\
132 \\
8.8 \\
.0\end{array}$ & $\begin{array}{r}135 \\
132 \\
9.0 \\
.0\end{array}$ & $\begin{array}{lr}\text { T. } & 144 \\
\text { B. } & 134 \\
\text { T. } & 8.5 \\
\text { T. } & .0\end{array}$ \\
\hline 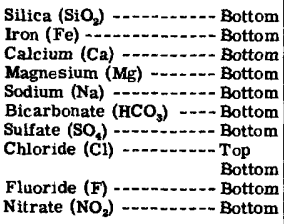 & $\begin{array}{l}5.5 \\
.12 \\
12 \\
4.1 \\
5.4 \\
17 \\
28 \\
12 \\
7.0 \\
.0 \\
6.8\end{array}$ & $\begin{array}{l}5.6 \\
.16 \\
12 \\
4.2 \\
4.3 \\
16 \\
29 \\
9.0 \\
6.0 \\
.0 \\
6.0\end{array}$ & $\begin{array}{l}5.6 \\
12.13 \\
4.5 \\
4.5 \\
15 \\
31 \\
7.0 \\
6.2 \\
.0 \\
6.1\end{array}$ & $\begin{array}{l}5.5 \\
.14 \\
12 \\
4.2 \\
4.3 \\
13 \\
32 \\
7.0 \\
5.8 \\
.0 \\
5.3\end{array}$ & $\begin{array}{l}5.4 \\
12.15 \\
4.2 \\
5.5 \\
14 \\
31 \\
8.0 \\
5.8 \\
.0 \\
5.8\end{array}$ & $\begin{array}{l}5.5 \\
12.14 \\
4.2 \\
4.8 \\
15 \\
30 \\
8.6 \\
6.2 \\
.0 \\
6.0\end{array}$ \\
\hline $\begin{array}{l}\text { Dissolved solids }-1 .-- \text { Bottom } \\
\text { Hardness as } \mathrm{CaCO}_{3}-\ldots \text { Bottom } \\
\text { Suspended sediment } \ldots \text { - Top }\end{array}$ & $\begin{array}{r}85 \\
47 \\
.\end{array}$ & $\begin{array}{l}86 \\
47 \\
.\end{array}$ & $\begin{array}{l}89 \\
48 \\
. \quad \mathrm{Cor}\end{array}$ & $\begin{array}{r}86 \\
47 \\
.\end{array}$ & $\begin{array}{l}84 \\
45 \\
.\end{array}$ & $\begin{array}{l}86 \\
47 \\
22\end{array}$ \\
\hline
\end{tabular}


Table 10. -- WATER ANALYSES OF DELAWARE RIVER BETWEEN BRISTOL AND MARCUS HOOK, PA. --Cont inued Analyzed by City of Philadelphia and U. S. Geological Survey; analyses in parts per million

Location-Philadelphia, Pa.-Canden, N._L_Exidge Date Februsxy 2, 1950 Sampling study No. 7 Weather Cloudy

\begin{tabular}{|c|c|c|c|c|c|c|}
\hline \multirow[t]{3}{*}{ Weather Cloudy } & & & Wate & discharge at & Trenton (cfs) & 13,200 \\
\hline & \multicolumn{6}{|c|}{ Station } \\
\hline & $\begin{array}{c}\text { Pennsylvania } \\
\text { side }\end{array}$ & West Center & Center & East Center & \begin{tabular}{|c|}
$\begin{array}{c}\text { New Jersey } \\
\text { side }\end{array}$ \\
\end{tabular} & Average \\
\hline $\begin{array}{l}\text { Time (EST) } \\
\text { Sounding (ft) } \\
\text { Temperature }\left({ }^{\circ} \mathrm{F}\right)-{ }^{-} \\
\text {Color } \\
\text { pH }- \text { Bottom }\end{array}$ & $\begin{array}{l}1: 44 \mathrm{p} \cdot \mathrm{m} . \\
45 \\
43 \\
42 \\
17 \\
6.6 \\
6.6\end{array}$ & $\begin{array}{l}1: 40 \mathrm{p.m} . \\
46 \\
43 \\
42 \\
18 \\
6.6 \\
6.5\end{array}$ & $\begin{array}{l}1: 35 \mathrm{p.m.} \\
46 \\
43 \\
42 \\
17 \\
6.5 \\
6.6\end{array}$ & $\begin{array}{c}1: 30 \text { p.m. } \\
34 \\
43 \\
41 \\
23 \\
6.6 \\
6.7\end{array}$ & $\begin{array}{c}1: 25 \text { p.m. } \\
8 \\
43 \\
42 \\
24 \\
6.5 \\
6.4\end{array}$ & $\begin{array}{l}43 \\
42 \\
20 \\
6.6 \\
6.6\end{array}$ \\
\hline 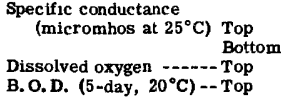 & $\begin{array}{l}152 \\
163 \\
6.6 \\
5.2\end{array}$ & $\begin{array}{l}154 \\
162 \\
7.3 \\
4.8\end{array}$ & $\begin{array}{l}147 \\
149 \\
7.8 \\
3.6\end{array}$ & $\begin{array}{l}143 \\
142 \\
8.3 \\
3.6\end{array}$ & $\begin{array}{r}139 \\
145 \\
8.4 \\
5.6\end{array}$ & $\begin{array}{lc}\text { T. } & 147 \\
\text { B. } & 152 \\
\text { T. } & 7.7 \\
\text { T. } & 4.6\end{array}$ \\
\hline 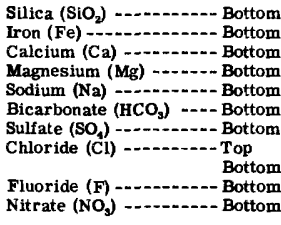 & $\begin{array}{l}5.5 \\
13 \\
13 \\
4.1 \\
14 \\
34 \\
32 \\
10 \\
9.2 \\
.1 \\
6.0\end{array}$ & $\begin{array}{l}5.7 \\
13 \\
4.12 \\
13 \\
31 \\
32 \\
9.0 \\
8.8 \\
.1 \\
7.8\end{array}$ & $\begin{array}{l}5.5 \\
12.21 \\
12 \\
3.8 \\
11 \\
28 \\
30 \\
8.0 \\
8.0 \\
.2 \\
5.7\end{array}$ & $\begin{array}{l}5.7 \\
.30 \\
12 \\
3.7 \\
11 \\
29 \\
30 \\
8.0 \\
7.5 \\
.1 \\
3.6\end{array}$ & $\begin{array}{l}5.5 \\
.21 \\
12^{3.8} \\
11 \\
28 \\
30 \\
8.0 \\
7.8 \\
.1 \\
6.0\end{array}$ & $\begin{array}{l}5.6 \\
12.19 \\
3.9 \\
12 \\
30 \\
31 \\
8.6 \\
8.3 \\
.1 \\
5.8\end{array}$ \\
\hline $\begin{array}{l}\text { Dissolved solids }-\ldots-1 \text { Bottom } \\
\text { Hardness as } \mathrm{CaCO}, \ldots \text { Bottom } \\
\text { Suspended sediment }-\cdots \text { Top }\end{array}$ & $\begin{array}{r}106 \\
49 \\
+ \\
\end{array}$ & $\begin{array}{l}96 \\
50 \\
. \\
\end{array}$ & $\begin{array}{l}95 \\
46 \\
. \mathrm{Cor} \\
\end{array}$ & $\begin{array}{r}91 \\
45 \\
\text { site. . . }\end{array}$ & $\begin{array}{l}96 \\
46 \\
\cdots\end{array}$ & $\begin{array}{l}\text { B. } \\
\text { B. } \\
\text { T. }\end{array}$ \\
\hline
\end{tabular}

Location Philadelphia, Pa.-Camden, N. J. Bridge Date March 6, 1950

Sampling study No. 8 Weather Clear

\begin{tabular}{|c|c|c|c|c|c|c|}
\hline \multirow[b]{3}{*}{ 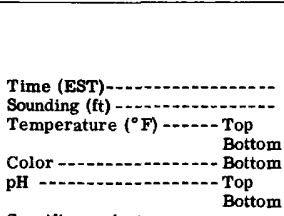 } & \multicolumn{6}{|c|}{ Station } \\
\hline & \begin{tabular}{|c|}
$\begin{array}{c}\text { Pennsylvania } \\
\text { side }\end{array}$ \\
\end{tabular} & West Center & Center & East Center & \begin{tabular}{|c|}
$\begin{array}{c}\text { New Jersey } \\
\text { side }\end{array}$ \\
\end{tabular} & Average \\
\hline & $\begin{array}{c}4: 20 \text { p.m. } \\
40 \\
36 \\
36 \\
15 \\
6.5 \\
6.7\end{array}$ & $\begin{array}{l}4: 15 \mathrm{p.m} . \\
26 \\
36 \\
36 \\
17 \\
6.6 \\
6.7\end{array}$ & $\begin{array}{l}4: 10 \mathrm{p} . \mathrm{m} . \\
45 \\
36 \\
35 \\
14 \\
6.5 \\
6.7\end{array}$ & $\begin{array}{c}4: 05 \text { p.m. } \\
29 \\
36 \\
36 \\
12 \\
6.6 \\
6.6\end{array}$ & $\begin{array}{l}4: 00 \mathrm{p} \cdot \mathrm{m} . \\
7 \\
37 \\
36 \\
12 \\
6.7 \\
6.7\end{array}$ & $\begin{array}{ll}\text { T. } & 36 \\
\text { B. } & 36 \\
\text { B. } & 14 \\
\text { T. } & 6.6 \\
\text { B. } & 6.7\end{array}$ \\
\hline $\begin{array}{l}\begin{array}{l}\text { Specific conductance } \\
\quad \text { (micromhos at } 25^{\circ} \mathrm{C} \text { ) } \\
\text { Top } \\
\text { Bottom }\end{array} \\
\text { Dissolved oxygen - - - Top } \\
\text { B. O. D. (5-day, } 20^{\circ} \mathrm{C} \text { ) -- Top }\end{array}$ & $\begin{array}{l}182 \\
185 \\
9.1 \\
8.4\end{array}$ & $\begin{array}{l}185 \\
188 \\
8.7 \\
6.0\end{array}$ & $\begin{array}{r}182 \\
185 \\
8.9 \\
5.2\end{array}$ & $\begin{array}{l}181 \\
184 \\
9.1 \\
5.4\end{array}$ & $\begin{array}{l}174 \\
177 \\
9.9 \\
6.7\end{array}$ & $\begin{array}{lc} & \\
\text { T. } & 181 \\
\text { B. } & 184 \\
\text { T. } & 9.1 \\
\text { T. } & 6.3\end{array}$ \\
\hline 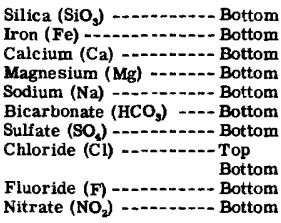 & $\begin{array}{l}6.1 \\
.01 \\
15 \\
5.3 \\
9.4 \\
26 \\
35 \\
10 \\
10 \\
.2 \\
9.1\end{array}$ & $\begin{array}{l}5.8 \\
.01 \\
15 \\
5.4 \\
9.7 \\
24 \\
38 \\
10 \\
10 \\
.2 \\
8.6\end{array}$ & $\begin{array}{l}5.8 \\
.01 \\
15 \\
5.3 \\
10 \\
25 \\
38 \\
10 \\
10 \\
.2 \\
8.2\end{array}$ & $\begin{array}{l}5.7 \\
.01 \\
15 \\
5.4 \\
9.1 \\
22 \\
38 \\
10 \\
10 \\
. .1 \\
9.1\end{array}$ & $\begin{array}{l}5.7 \\
.01 \\
15 \\
5.5 \\
8.5 \\
28 \\
34 \\
10 \\
10 \\
7.1\end{array}$ & $\begin{array}{ll}\text { B. } & 5.8 \\
\text { B. } & .01 \\
\text { B. } & 15 \\
\text { B. } & 5.4 \\
\text { B. } & 9.3 \\
\text { B. } & 25 \\
\text { B. } & 37 \\
\text { T. } & 10 \\
\text { B. } & 10 \\
\text { B. } & .2 \\
\text { B. } & 8.5\end{array}$ \\
\hline $\begin{array}{l}\text { Dissolved solids }-\ldots-\text { Bottom } \\
\text { Hardness as } \mathrm{CaCO}_{3}-\cdots \text { Bottom } \\
\text { Suspended sediment }-\ldots \text { Top }\end{array}$ & $\begin{array}{r}113 \\
59 \\
.\end{array}$ & $\begin{array}{r}117 \\
60\end{array}$ & $\begin{array}{l}111 \\
59 \\
\text {. Con }\end{array}$ & $\begin{array}{r}110 \\
60\end{array}$ & $\begin{array}{r}107 \\
60 \\
. \quad .\end{array}$ & $\begin{array}{lr}\text { B. } & 112 \\
\text { B. } & 60 \\
\text { T. } & 22\end{array}$ \\
\hline
\end{tabular}


Table 10. -- WATER ANALYSES OF DELAWARE RIVER BETWEEN BRISTOL AND MARCUS HOOK, PA. --COnt inued Analyzed by City of Phladelphia and U. S. Geological Survey; analyses in parts per million

Location Phi]edelphia, Pa.-Canden, N. J. Bridge Date April 4, 1950 Sampling study No. Weather Clear $\quad$ Water discharge at Trenton (cfs) $35,600 \cdots$

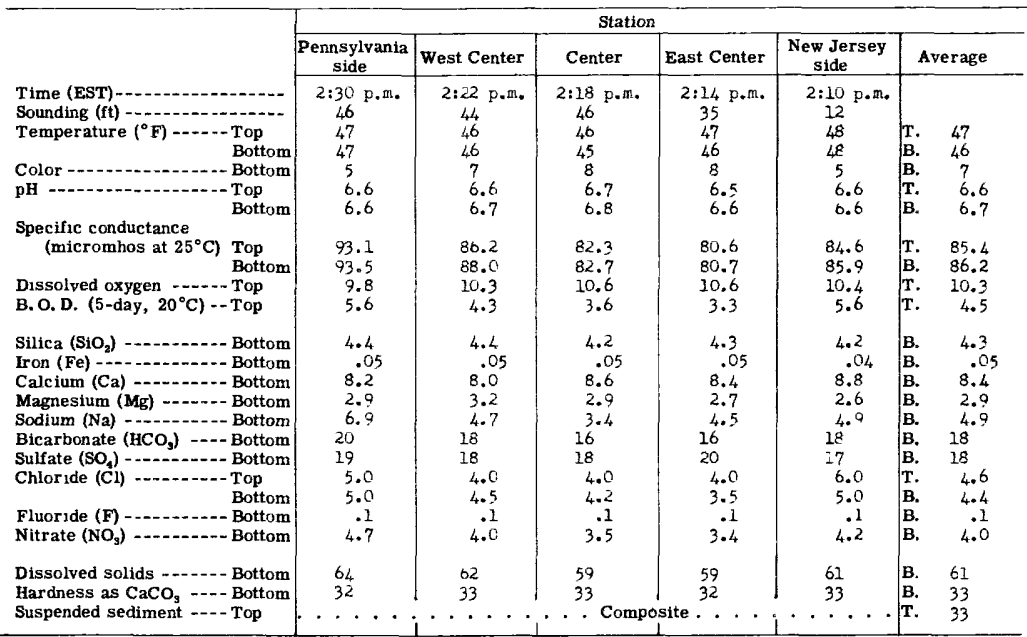

Location Philadelphia, Pa.-Canden, N. J. Bridge Date May 1, 1950

Sampling study No. 10 Weather Fain _- Water discharge at Trenton (cis) 12,300

\begin{tabular}{|c|c|c|c|c|c|c|}
\hline \multirow[b]{3}{*}{ 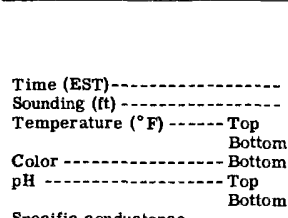 } & \multicolumn{6}{|c|}{ Station } \\
\hline & $\begin{array}{c}\text { Pennsylvania } \\
\text { side }\end{array}$ & West Center & Center & East Center & \begin{tabular}{|c|}
$\begin{array}{c}\text { New Jersey } \\
\text { side }\end{array}$ \\
\end{tabular} & Average \\
\hline & $\begin{array}{l}9: 00 \text { a.m. } \\
36 \\
53 \\
53 \\
5 \\
6.6 \\
6.7\end{array}$ & $\begin{array}{l}9: 04 \text { a.m. } \\
42 \\
52 \\
52 \\
6 \\
6.7 \\
6.7\end{array}$ & $\begin{array}{l}9: 09 \text { a.m. } \\
40 \\
52 \\
51 \\
6 \\
6.9 \\
6.7\end{array}$ & $\begin{array}{l}9: 15 \text { a.m. } \\
32 \\
52 \\
51 \\
6 \\
6.9 \\
6.6\end{array}$ & $\begin{array}{c}9: 20 \text { :.m. } \\
5 \\
52 \\
51 \\
6 \\
6.6 \\
6.6\end{array}$ & $\begin{array}{l}52 \\
52 \\
6 \\
6.7 \\
6.7\end{array}$ \\
\hline $\begin{array}{l}\text { (micromhos at } 25^{\circ} \mathrm{C} \text { ) } \begin{array}{l}\text { Top } \\
\text { Bottom }\end{array} \\
\text { Dissolved oxygen - - }- \text { Top } \\
\text { B. O.D. (5-day, } 20^{\circ} \mathrm{C} \text { ) - - Top }\end{array}$ & $\begin{array}{l}127 \\
127 \\
6.5 \\
4.6\end{array}$ & $\begin{array}{l}117 \\
109 \\
8.0 \\
2.8\end{array}$ & $\begin{array}{l}104 \\
104 \\
9.0 \\
1.4\end{array}$ & $\begin{array}{r}103 \\
102 \\
8.8 \\
1.2\end{array}$ & $\begin{array}{l}124 \\
121 \\
7.6 \\
7.3\end{array}$ & $\begin{array}{lr}\text { T. } & 115 \\
\text { B. } & 113 \\
\text { T. } & 8.0 \\
\text { T. } & 3.5\end{array}$ \\
\hline 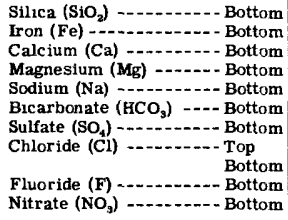 & $\begin{array}{l}4.0 \\
11.05 \\
4.1 \\
7.3 \\
31 \\
23 \\
8.0 \\
6.8 \\
.0 \\
1.4\end{array}$ & $\begin{array}{l}4.0 \\
.10 \\
10 \\
3.6 \\
6.2 \\
25 \\
21 \\
7.0 \\
5.5 \\
.0 \\
3.9\end{array}$ & $\begin{array}{l}3.0 \\
.00 \\
10^{\circ} \\
3.4 \\
5.1 \\
25 \\
20 \\
0.0 \\
4.5 \\
.0 \\
3.0\end{array}$ & $\begin{array}{l}3.7 \\
.00 \\
10 \\
3.3 \\
6.0 \\
27 \\
20 \\
6.0 \\
4.5 \\
.0 \\
3.0\end{array}$ & $\begin{array}{l}5.0 \\
.05 \\
21 \\
3.6 \\
9.0 \\
33 \\
21 \\
3.0 \\
7.0 \\
.1 \\
3.6\end{array}$ & $\begin{array}{l}4.1 \\
10.06 \\
3.6 \\
6.7 \\
28 \\
21 \\
7.0 \\
5.7 \\
.0 \\
3.0\end{array}$ \\
\hline $\begin{array}{l}\text { Dissolved solids }-\ldots \text { Bottom } \\
\text { Hardness as } \mathrm{CaCO}_{3} \ldots-\text { Bottom } \\
\text { Suspended sediment }-\ldots \text { Top }\end{array}$ & $\begin{array}{l}82 \\
44 \\
\cdots\end{array}$ & $\begin{array}{l}73 \\
40 \\
. \\
\end{array}$ & $\begin{array}{l}71 \\
39 \\
.\end{array}$ & 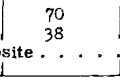 & $\begin{array}{r}81 \\
42 \\
. . \cdots\end{array}$ & $\begin{array}{l}\text { B. } \\
\text { B. } \\
\text { T. }\end{array}$ \\
\hline
\end{tabular}


Table 10. - - WATER ANALYSES OF DELAWARE RIVER BETWEEN BRISTOL AND MARCUS HOOK, PA. --Continued Analyzed by City of Philadelphia and U. S. Geological Survey; analyses in parts per million Location Philadelphia, Pa.-Camden, N. J. Bridge Date June 6, 1950 Sampling study No. II

\begin{tabular}{|c|c|c|c|c|c|c|}
\hline \multirow[t]{3}{*}{ Weather Clear } & \multicolumn{6}{|c|}{ Water discharge at Trenton (cfs) 18,900} \\
\hline & \multicolumn{6}{|c|}{ Station } \\
\hline & $\begin{array}{c}\text { Pennsylvania } \\
\text { side }\end{array}$ & West Center & Center & East Center & $\begin{array}{l}\text { New Jersey } \\
\text { side }\end{array}$ & Average \\
\hline 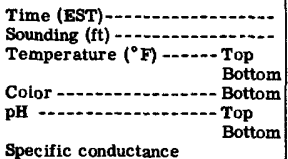 & $\begin{array}{l}8: 27 \mathrm{a} \cdot \mathrm{m} . \\
38 \\
68 \\
67 \\
22 \\
6.4 \\
6.6\end{array}$ & $\begin{array}{l}8: 34 \text { a.m. } \\
44 \\
69 \\
67 \\
23 \\
6.4 \\
6.5\end{array}$ & $\begin{array}{l}8: 40 \mathrm{a} . \mathrm{m} . \\
44 \\
68 \\
67 \\
17 \\
6.4 \\
6.9\end{array}$ & $\begin{array}{c}8: 45 \text { a.m. } \\
41 \\
68 \\
67 \\
20 \\
6.4 \\
6.8\end{array}$ & $\begin{array}{l}8: 50 \text { a.m. } \\
10 \\
68 \\
67 \\
18 \\
6.4 \\
6.7\end{array}$ & $\begin{array}{ll}\text { T. } & 68 \\
\text { B. } & 67 \\
\text { B. } & 20 \\
\text { T. } & 6.4 \\
\text { B. } & 6.7\end{array}$ \\
\hline $\begin{array}{l}\text { (micromhos at } 25^{\circ} \mathrm{C} \text { ) } \begin{array}{l}\text { Top } \\
\text { Bottom }\end{array} \\
\text { Dissolved oxygen }-\cdots \text { Top } \\
\text { B. O. D. (5-day, } 20^{\circ} \mathrm{C} \text { ) }- \text { Top }\end{array}$ & $\begin{array}{l}139 \\
139 \\
1.8 \\
1.1\end{array}$ & $\begin{array}{r}137 \\
133 \\
2.2 \\
.0\end{array}$ & $\begin{array}{r}126 \\
126 \\
3.3 \\
.0\end{array}$ & $\begin{array}{r}124 \\
223 \\
4.2 \\
.0\end{array}$ & $\begin{array}{r}154 \\
154 \\
3.4 \\
.0\end{array}$ & $\begin{array}{lr}\text { T. } & 136 \\
\text { B. } & 135 \\
\text { T. } & 3.0 \\
\text { T. } & .2\end{array}$ \\
\hline 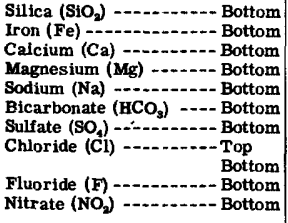 & $\begin{array}{l}5.5 \\
.06 \\
13 \\
3.5 \\
5.6 \\
27 \\
24 \\
8.0 \\
6.9 \\
.1 \\
5.6\end{array}$ & $\begin{array}{l}4.8 \\
13 \\
13 \\
3.5 \\
5.9 \\
27 \\
24 \\
6.0 \\
5.8 \\
.1 \\
4.9\end{array}$ & $\begin{array}{l}4.7 \\
12.04 \\
3.1 \\
2.8 \\
25 \\
23 \\
7.0 \\
5.4 \\
.1 \\
4.0\end{array}$ & $\begin{array}{l}5.5 \\
12.05 \\
3.5 \\
3.8 \\
24 \\
23 \\
7.0 \\
5.0 \\
.2 \\
4.9\end{array}$ & $\begin{array}{l}5.5 \\
.04 \\
14.0 \\
3.3 \\
6.5 \\
27 \\
25 \\
11 \\
9.2 \\
.1 \\
6.6\end{array}$ & $\begin{array}{lc}\text { B. } & 5.2 \\
\text { B. } & .05 \\
\text { B. } & 13 \\
\text { B. } & 3.4 \\
\text { B. } & 4.9 \\
\text { B. } & 26 \\
\text { B. } & 24 \\
\text { T. } & 7.8 \\
\text { B. } & 6.5 \\
\text { B. } & .1 \\
\text { B. } & 5.2\end{array}$ \\
\hline $\begin{array}{l}\text { Dissolved solids }-\ldots-\ldots \text { Bottom } \\
\text { Hardness as } \mathrm{CaCO}, \ldots-\text { Bottom } \\
\text { Suspended sediment }-\cdots \text { Top }\end{array}$ & $\begin{array}{l}93 \\
47 \\
\cdots \\
\end{array}$ & $\begin{array}{r}86 \\
47 \\
. \\
\end{array}$ & $\begin{array}{l}80 \\
. \\
.\end{array}$ & 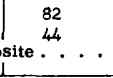 & $\begin{array}{l}98 \\
48 \\
\cdots\end{array}$ & $\begin{array}{l}\text { B. } \\
\text { B. } \\
\text { T. }\end{array}$ \\
\hline
\end{tabular}

Location Philadelphia, Pa,-Camden, N. J. Bricge Date July 6, 1950 Weather Light Rain

Sampling study No. $-\frac{12}{6}$

\begin{tabular}{|c|c|c|c|c|c|c|}
\hline \multirow[t]{3}{*}{ Weather Light Rain } & \multicolumn{6}{|c|}{ Watsr discharge at Trenton (cfs) $\quad 6.130$} \\
\hline & \multicolumn{6}{|c|}{ Station } \\
\hline & $\begin{array}{c}\text { Pennsylvania } \\
\text { side }\end{array}$ & Weat Center & Center & East Center & \begin{tabular}{|c|}
$\begin{array}{c}\text { New Jersey } \\
\text { side }\end{array}$ \\
\end{tabular} & Average \\
\hline 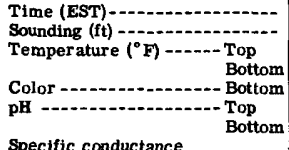 & $\begin{array}{l}1: 15 \text { p.m. } \\
39 \\
78 \\
78 \\
8 \\
6.5 \\
7.1\end{array}$ & $\begin{array}{l}1: 10 \mathrm{p} \cdot \mathrm{m} . \\
41 \\
77 \\
78 \\
7 \\
6.7 \\
7.2\end{array}$ & $\begin{array}{l}1: 08 \mathrm{p} . \mathrm{m} . \\
41 \\
78 \\
77 \\
8 \\
6.6 \\
6.8\end{array}$ & $\begin{array}{l}1: 05 \text { p.m. } \\
28 \\
77 \\
78 \\
7 \\
6.6 \\
7.0\end{array}$ & $\begin{array}{l}1: 00 \text { p.m. } \\
14 \\
77 \\
77 \\
7 \\
6.7 \\
6.3\end{array}$ & $\begin{array}{l}77 \\
78 \\
7 \\
6.6 \\
6.9\end{array}$ \\
\hline $\begin{array}{l}\text { Specific conductance } \\
\text { (micromhos at } 25^{\circ} \mathrm{C} \text { ) Top } \\
\text { Bottom } \\
\text { Dissolved oxygen --..- Top } \\
\text { B. O.D. (5-day, 20 } 20^{\circ} \mathrm{C} \text {-- Top }\end{array}$ & $\begin{array}{l}180 \\
176 \\
6.7\end{array}$ & $\begin{array}{r}164 \\
152 \\
1.0 \\
3.1\end{array}$ & $\begin{array}{r}147 \\
143 \\
2.0 \\
2.9\end{array}$ & $\begin{array}{r}158 \\
152 \\
1.4 \\
3.9\end{array}$ & $\begin{array}{l}194 \\
226 \\
1.0 \\
6.3\end{array}$ & $\begin{array}{ll}\text { T. } & 169 \\
\text { B. } & 170 \\
\text { T. } & 1.2 \\
\text { T. } & 4.5\end{array}$ \\
\hline 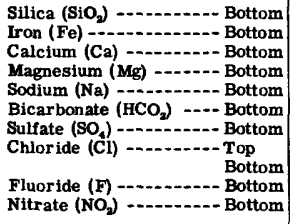 & $\begin{array}{l}4.6 \\
15 \\
5.1 \\
9.5 \\
36 \\
30 \\
10 \\
9.8 \\
.1 \\
6.0\end{array}$ & $\begin{array}{l}4.7 \\
14 \\
14.06 \\
4.8 \\
7.4 \\
27 \\
9.0 \\
7.8 \\
.1 \\
4.2\end{array}$ & $\begin{array}{l}4.5 \\
13 \\
4.04 \\
5.7 \\
34 \\
24 \\
8.0 \\
6.8 \\
.2 \\
4.5\end{array}$ & $\begin{array}{l}4.5 \\
.06 \\
14 \\
4.9 \\
5.7 \\
33 \\
26 \\
9.0 \\
7.8 \\
. .2 \\
4.6\end{array}$ & $\begin{array}{l}7.3 \\
.06 \\
21 \\
6.1 \\
9.3 \\
4.4 \\
32 \\
16 \\
18 \\
.12\end{array}$ & $\begin{array}{l}5.1 \\
.06 \\
15 \\
5.1 \\
7.4 \\
36 \\
28 \\
10 \\
10 \\
.1 \\
4.9\end{array}$ \\
\hline $\begin{array}{l}\text { Dissolved solids - }-. .- \text { Bottom } \\
\text { Hardness as } \mathrm{CaCO}_{3} \ldots-\text { Bottom } \\
\text { Suspended sediment } \ldots-\text { Top }\end{array}$ & $\begin{array}{r}109 \\
82 \\
\cdots\end{array}$ & $\begin{array}{l}93 \\
74 \\
\end{array}$ & $\begin{array}{l}86 \\
67 \\
. \mathrm{Co}\end{array}$ & $\begin{array}{r}92 \\
72 \\
\text { ite . . . }\end{array}$ & $\begin{array}{l}137 \\
101 \\
\cdots\end{array}$ & $\begin{array}{lr}\text { B. } & 103 \\
\text { B. } & 79 \\
\text { T. } & 24 \\
\end{array}$ \\
\hline
\end{tabular}

$3153910-54-7$ 
Table 10. - WATER ANALYSES OF DELAWARE RIVER BETWEEN BRISTOL AND MARCUS hOOK, PA. - Continued Analyzed by City of Philadelphia and U. S. Geological Survey; analyses in parts per million

Location Philadelphia, Pa.-Camden, N. J. Bridge_Date August 2, $1950 \quad$ Sampling study No. 13 Weather Light Rain $\quad$ Water discharge at Trenton (cis) 5,090

\begin{tabular}{|c|c|c|c|c|c|c|}
\hline \multirow[b]{3}{*}{ 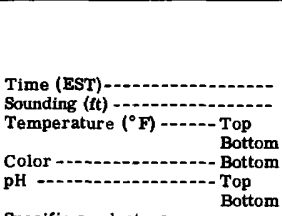 } & \multicolumn{6}{|c|}{ Station } \\
\hline & $\begin{array}{c}\text { Pennsylvania } \\
\text { side }\end{array}$ & West Center & Center & East Center & $\begin{array}{c}\begin{array}{c}\text { New Jersey } \\
\text { side }\end{array} \\
\end{array}$ & Average \\
\hline & $\begin{array}{l}8: 18 \text { a.m. } \\
44 \\
80 \\
80 \\
25 \\
6.3 \\
6.5\end{array}$ & $\begin{array}{l}8: 22 \text { a.m. } \\
44 \\
79 \\
80 \\
26 \\
6.3 \\
6.4\end{array}$ & $\begin{array}{c}8: 30 \text { a.m. } \\
41 \\
78 \\
79 \\
20 \\
6.2 \\
6.4\end{array}$ & $\begin{array}{c}8: 35 \text { a.m. } \\
30 \\
78 \\
79 \\
15 \\
6.4 \\
6.4\end{array}$ & $\begin{array}{c}8: 40 \mathrm{a} . \mathrm{m} . \\
21 \\
78 \\
79 \\
15 \\
6.5 \\
6.6\end{array}$ & $\begin{array}{lc}\text { T. } & 79 \\
\text { B. } & 79 \\
\text { B. } & 20 \\
\text { T. } & 6.3 \\
\text { B. } & 6.5\end{array}$ \\
\hline 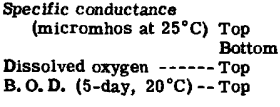 & $\begin{array}{l}220 \\
220 \\
14.8\end{array}$ & $\begin{array}{l}210 \\
209 \\
13.4\end{array}$ & $\begin{array}{r}205 \\
205 \\
9.5\end{array}$ & $\begin{array}{l}199 \\
199 \\
7.5\end{array}$ & $\begin{array}{r}201 \\
194 \\
7.8\end{array}$ & $\begin{array}{ll}\text { T. } & 207 \\
\text { B. } & 205 \\
\text { T. } & .5 \\
\text { T. } & 10.5\end{array}$ \\
\hline 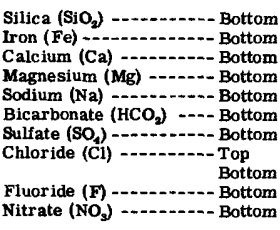 & $\begin{array}{l}6.0 \\
.31 \\
17 \\
5.5 \\
16 \\
56 \\
35 \\
12 \\
13 \\
.1 \\
.1\end{array}$ & $\begin{array}{l}6.2 \\
17^{.13} \\
5.6 \\
13 \\
53 \\
34 \\
13 \\
12 \\
.1 \\
.1\end{array}$ & $\begin{array}{l}5.5 \\
.04 \\
16 \\
5.5 \\
13 \\
51 \\
34 \\
11 \\
11 \\
.2 \\
.1\end{array}$ & $\begin{array}{l}6.6 \\
16.04 \\
5.3 \\
13 \\
50 \\
33 \\
10 \\
11 \\
.2 \\
.1\end{array}$ & $\begin{array}{l}5.5 \\
.06 \\
16 \\
5.0 \\
12 \\
48 \\
32 \\
12 \\
10 \\
.2 \\
.3\end{array}$ & $\begin{array}{ll}\text { B. } & 6.0 \\
\text { B. } & .12 \\
\text { B. } & 16 \\
\text { B. } & 5.4 \\
\text { B. } & 13 \\
\text { B. } & 52 \\
\text { B. } & 34 \\
\text { T. } & 12 \\
\text { B. } & 11 \\
\text { B. } & .2 \\
\text { B. } & .1\end{array}$ \\
\hline $\begin{array}{l}\text { Dissolved solids -- }-\cdots \text { Bottom } \\
\text { Hardness as CaCO, }-\cdots \text { Bottom } \\
\text { Suspended sediment }-\cdots \text { Top }\end{array}$ & $\begin{array}{r}128 \\
65 \\
\cdots \cdots\end{array}$ & $\begin{array}{r}122 \\
65\end{array}$ & $\begin{array}{l}119 \\
62 \\
.\end{array}$ & $\begin{array}{r}115 \\
62 \\
\text { site. . . . }\end{array}$ & $\begin{array}{l}113 \\
60 \\
\cdots \cdots\end{array}$ & $\begin{array}{lr}\text { B. } & 119 \\
\text { B. } & 63 \\
\text { T. } & 39 \\
\end{array}$ \\
\hline
\end{tabular}

Location Philadelphia, Pa.-Camden, Ne, J, Bridge Date September 6, 1950 Sampling study No. 14 Weather Clear Water discharge at Trenton (cfs) 4.860

\begin{tabular}{|c|c|c|c|c|c|c|}
\hline \multirow[t]{3}{*}{ Weather Clear } & \multicolumn{6}{|c|}{ Water discharge at Trenton (cfs) 4,860} \\
\hline & \multicolumn{6}{|c|}{ Station } \\
\hline & \begin{tabular}{|c|}
$\begin{array}{c}\text { Pennsylvania } \\
\text { side }\end{array}$ \\
\end{tabular} & West Center & Center & East Center & \begin{tabular}{|c|}
$\begin{array}{c}\text { New Jersey } \\
\text { sids }\end{array}$ \\
\end{tabular} & Average \\
\hline 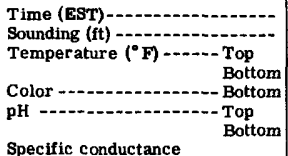 & $\begin{array}{l}8: 55 \text { a.m. } \\
22 \\
77 \\
77 \\
12 \\
6.6 \\
6.7\end{array}$ & $\begin{array}{l}8: 50 \text { a.m. } \\
44 \\
76 \\
76 \\
18 \\
6.5 \\
6.8\end{array}$ & $\begin{array}{l}9: 00 \text { a.m. } \\
46 \\
76 \\
76 \\
15 \\
6.4 \\
6.7\end{array}$ & $\begin{array}{l}9: 05 \text { a.m. } \\
32 \\
77 \\
76 \\
15 \\
7.3 \\
6.6\end{array}$ & $\begin{array}{l}9: 10 \text { a.m. } \\
24 \\
76 \\
76 \\
10 \\
6.4 \\
6.8\end{array}$ & $\begin{array}{l}76 \\
76 \\
14 \\
6.6 \\
6.7\end{array}$ \\
\hline $\begin{array}{l}\begin{array}{l}\text { Specific conductance } \\
\text { (micromhos at } 25^{\circ} \mathrm{C} \text { ) }\end{array} \\
\begin{array}{l}\text { Top } \\
\text { Bottom }\end{array} \\
\text { Dissolved oxygen }-1-\text { - Top } \\
\text { B. O. D. (5-day, } 20^{\circ} \mathrm{C} \text { ) -- Top }\end{array}$ & $\begin{array}{r}239 \\
237 \\
12.9\end{array}$ & $\begin{array}{r}247 \\
240 \\
13.5\end{array}$ & $\begin{array}{r}235 \\
233 \\
9.6\end{array}$ & $\begin{array}{r}233 \\
.9 \\
.9\end{array}$ & $\begin{array}{r}233 \\
231 \\
9.3\end{array}$ & \begin{tabular}{ll} 
T. & 237 \\
B. & 235 \\
T. & \multicolumn{1}{l}{.0} \\
T. & 11
\end{tabular} \\
\hline 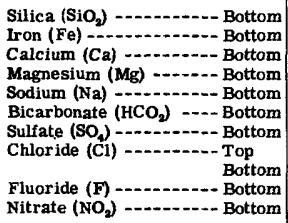 & $\begin{array}{l}4.2 \\
.07 \\
18 \\
6.6 \\
15 \\
60 \\
36 \\
14 \\
14 \\
.2 \\
.1\end{array}$ & $\begin{array}{l}4.3 \\
18^{.32} \\
7.0 \\
15 \\
64 \\
35 \\
13 \\
14 \\
.2 \\
.1\end{array}$ & $\begin{array}{l}4.0 \\
18 \\
6.15 \\
6.5 \\
14 \\
57 \\
37 \\
13 \\
13 \\
.2 \\
.1\end{array}$ & $\begin{array}{l}4.1 \\
18 \\
6.05 \\
6.6 \\
14 \\
59 \\
35 \\
13 \\
14.2 \\
.2\end{array}$ & $\begin{array}{l}4.1 \\
17.13 \\
6.8 \\
14 \\
62 \\
33 \\
13 \\
13 \\
.2 \\
.1\end{array}$ & $\begin{array}{ll}\text { B. } & 4.1 \\
\text { B. } & .14 \\
\text { B. } & 18 \\
\text { B. } & 6.7 \\
\text { B. } & 14 \\
\text { B. } & 60 \\
\text { B. } & 35 \\
\text { T. } & 13 \\
\text { B. } & 14 \\
\text { B. } & .2 \\
\text { B. } & .1\end{array}$ \\
\hline $\begin{array}{l}\text { Dissolved solids ----- Bottom } \\
\text { Hardness as } \mathrm{CaCO}_{3}--- \text { Bottom } \\
\text { Suspended sediment }---- \text { Top }\end{array}$ & $\begin{array}{r}140 \\
72 \\
. \cdot\end{array}$ & $\begin{array}{r}145 \\
74 \\
\cdots\end{array}$ & $\begin{array}{l}136 \\
72 \\
\cdot \quad \cdot \text { Cor }\end{array}$ & $\begin{array}{r}136 \\
72 \\
\text { site. . . }\end{array}$ & $\begin{array}{r}131 \\
70 \\
.\end{array}$ & $\begin{array}{lr}\text { B. } & 138 \\
\text { B. } & 72 \\
\text { T. } & 38 \\
\end{array}$ \\
\hline
\end{tabular}


Table 10. -- WATER ANAL YSES OF DELAWARE RIVER BETWEEN BRISTOL AND MARCUS HOOK, PA. --Continued Analyzed by City of Philadelphia and U. S. Geological Survey; analyses in parts per million

Location Philadelphia, Pa.-Camien, N. J. Bridge Date October 3, 1950 Sampling study No. 15 Weather Misty

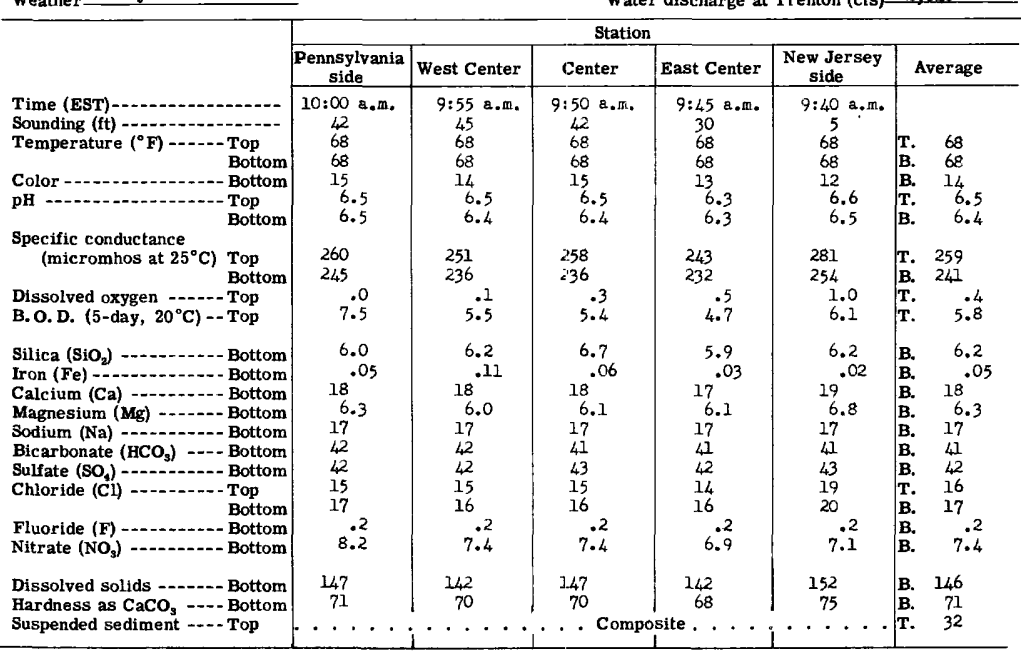

Location Philadelphia, Pa.-Camden, N. J. Bridge Date November 2, 1950 Sampling study No. 16

\begin{tabular}{|c|c|c|c|c|c|c|}
\hline \multirow[t]{3}{*}{ Weather Clear } & \multicolumn{6}{|c|}{ Water discharge at Trenton (cfs) 2.550} \\
\hline & \multicolumn{6}{|c|}{ Station } \\
\hline & $\begin{array}{c}\text { Pennsylvania } \\
\text { side }\end{array}$ & West Center & Center & East Center & $\begin{array}{c}\begin{array}{c}\text { New Jersey } \\
\text { side }\end{array} \\
\end{array}$ & Average \\
\hline 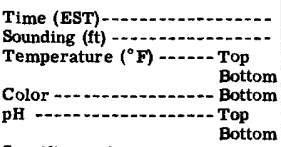 & $\begin{array}{c}8: 53 \text { a.m. } \\
4 \\
64 \\
63 \\
22 \\
6.5 \\
6.8\end{array}$ & $\begin{array}{l}8: 58 \text { a.m. } \\
30 \\
64 \\
63 \\
10 \\
6.5 \\
6.8\end{array}$ & $\begin{array}{l}9: 04 \text { a.m. } \\
42 \\
64 \\
63 \\
12 \\
6.5 \\
6.6\end{array}$ & $\begin{array}{c}9: 09 \text { a.m. } \\
42 \\
64 \\
64 \\
9 \\
6.6 \\
6.4\end{array}$ & $\begin{array}{l}9: 15 \text { a.m. } \\
38 \\
64 \\
65 \\
8 \\
6.6 \\
6.4\end{array}$ & $\begin{array}{l}64 \\
64 \\
12 \\
6.5 \\
6.6\end{array}$ \\
\hline $\begin{array}{l}\text { Specific conductance } \\
\quad \text { (micromhos at } 25^{\circ} \mathrm{C} \text { ) } \begin{array}{l}\text { Top } \\
\text { Bottom }\end{array} \\
\begin{array}{c}\text { Dissolved oxygen --- Top } \\
\left.\text { B. O.D. (5-day, 20 } 20^{\circ} \mathrm{C}\right)- \text { Top }\end{array}\end{array}$ & $\begin{array}{r}306 \\
328 \\
3.9\end{array}$ & $\begin{array}{r}300 \\
287 \\
2.4\end{array}$ & $\begin{array}{r}303 \\
287 \\
.0 \\
.6\end{array}$ & $\begin{array}{r}300 \\
292 \\
.0 \\
.3\end{array}$ & $\begin{array}{r}337 \\
292 \\
.7 \\
4.5\end{array}$ & $\begin{array}{lr}\text { T. } & 309 \\
\text { B. } & 297 \\
\text { T. } & .1 \\
\text { T. } & 2.3\end{array}$ \\
\hline 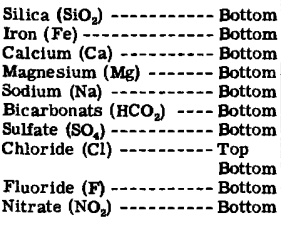 & $\begin{array}{l}4.3 \\
.07 \\
24 \\
8.7 \\
22 \\
47 \\
53 \\
20 \\
30 \\
.2 \\
8.8\end{array}$ & $\begin{array}{l}5.5 \\
.04 \\
21 \\
7.9 \\
19 \\
42 \\
53 \\
20 \\
21 \\
8.2\end{array}$ & $\begin{array}{l}6.1 \\
.03 \\
20 \\
8.2 \\
20 \\
41 \\
56 \\
20 \\
21.2 \\
8.4\end{array}$ & $\begin{array}{l}4.2 \\
.09 \\
21 \\
7.7 \\
21 \\
4.7 \\
55 \\
20 \\
21.2 \\
9.3\end{array}$ & $\begin{array}{l}5.9 \\
21.02 \\
7.8 \\
20 \\
44 \\
52 \\
28 \\
21.2 \\
11^{.2}\end{array}$ & $\begin{array}{l}5.2 \\
.05 \\
21.0 \\
8.1 \\
44 \\
54 \\
22 \\
23 \\
.2 \\
9.2\end{array}$ \\
\hline $\begin{array}{l}\text { Dissolved solids - }-1 .- \text { Bottom } \\
\text { Hardness as } \mathrm{CaCO}_{3}-\ldots \text { Bottom } \\
\text { Suspended sediment --- Top }\end{array}$ & $\begin{array}{r}193 \\
96 \\
. \\
\end{array}$ & $\begin{array}{r}171 \\
85\end{array}$ & $\begin{array}{r}171 \\
84 \\
+ \text { Com }\end{array}$ & $\begin{array}{r}174 \\
84 \\
\text { site. . . . }\end{array}$ & $\begin{array}{r}174 \\
84 \\
. \\
\end{array}$ & $\begin{array}{lr}\text { B. } & 177 \\
\text { B. } & 87 \\
\text { T. } & 49 \\
\end{array}$ \\
\hline
\end{tabular}


Table 10. -- WATER ANALYSES OF DELAWARE RIVER BETWEEN BRISTOL AND MARCUS HOOK, PA. --Continued Analyzed by City of Philadelphia and U. S. Geological Survey; analyses in parts per million

Location Philadelphia, Pa.-Canden, N. J. Bridge Date December 4, 1950 Sampling study No. 17

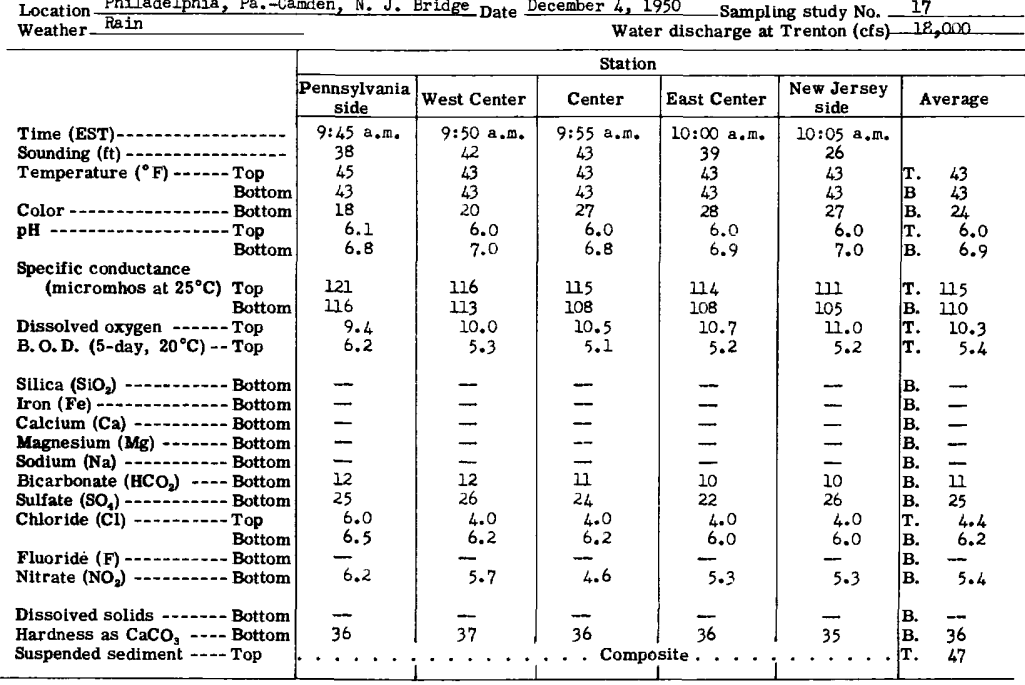

Location Philadelphia, Pa.-Camden, N. J. Bridge-Date January 3, 1951 Sampling study No. 18 Water discharge at Trenton (cfs) $\frac{18}{8,210}$

\begin{tabular}{|c|c|c|c|c|c|c|}
\hline Neat & & & & & & \\
\hline & \multicolumn{6}{|c|}{ Station } \\
\hline & \begin{tabular}{|c|}
$\begin{array}{c}\text { Pennsylvania } \\
\text { side }\end{array}$ \\
\end{tabular} & West Center & Center & East Center & $\begin{array}{c}\text { New Jersey } \\
\text { slde }\end{array}$ & Average \\
\hline $\begin{array}{l}\text { Time (EST) } \\
\begin{array}{l}\text { Sounding }(\mathrm{ft}) \\
\text { Temperature }\left({ }^{\circ} \mathrm{F}\right)\end{array} \\
\begin{array}{l}\text { Bop } \\
\text { Color }\end{array} \\
\text { pH }-\begin{array}{l}\text { Bottom } \\
\text { Specific conductance }\end{array} \quad \begin{array}{l}\text { Top } \\
\text { Bottom }\end{array}\end{array}$ & $\begin{array}{c}8: 45 \text { a.m. } \\
45 \\
37 \\
37 \\
8 \\
6.4 \\
6.7\end{array}$ & $\begin{array}{l}8: 48 \mathrm{a} . \mathrm{m} . \\
44 \\
36 \\
36 \\
\\
6.5 \\
6.8\end{array}$ & $\begin{array}{l}8: 52 \text { a.m. } \\
48 \\
35 \\
36 \\
10 \\
6.5 \\
6.5\end{array}$ & $\begin{array}{l}8: 55 \text { a.m. } \\
32 \\
35 \\
35 \\
\\
6.5 \\
6.8\end{array}$ & $\begin{array}{l}8: 58 \text { a.m. } \\
26 \\
35 \\
35 \\
12 \\
6.5 \\
6.7\end{array}$ & $\begin{array}{lc}\text { T. } & 36 \\
\text { B. } & 36 \\
\text { B. } & 10 \\
\text { T. } & 6.5 \\
\text { B. } & 6.7\end{array}$ \\
\hline $\begin{array}{l}\text { Specific conductance } \\
\quad\left(\text { micromhos at } 25^{\circ} \mathrm{C}\right) \\
\begin{array}{l}\text { Top } \\
\text { Bottom }\end{array} \\
\begin{array}{c}\left.\text { Dissolved oxygen }-10^{\circ} \mathrm{C}\right)- \text { - Top } \\
\text { B. O. D. (5-day, }\end{array}\end{array}$ & $\begin{array}{r}179 \\
172 \\
6.7 \\
5.5\end{array}$ & $\begin{array}{r}174 \\
180 \\
7.5 \\
7.0\end{array}$ & $\begin{array}{r}170 \\
164 \\
8.6 \\
6.3\end{array}$ & $\begin{array}{l}170 \\
175 \\
8.5 \\
5.6\end{array}$ & $\begin{array}{r}169 \\
165 \\
8.1 \\
4.5\end{array}$ & $\begin{array}{lr}\text { T. } & 172 \\
\text { B. } & 171 \\
\text { T. } & 7.9 \\
\text { T. } & 5.8\end{array}$ \\
\hline 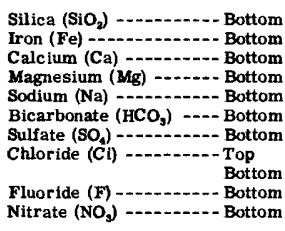 & $\begin{array}{l}7.4 \\
.07 \\
14 \\
5.4 \\
8.7 \\
25 \\
34 \\
8.0 \\
9.8 \\
.02 \\
7.5\end{array}$ & $\begin{array}{l}8.0 \\
6.0\end{array}$ & $\begin{array}{c}6.9 \\
.14 \\
14 \\
5.2 \\
8.5 \\
25 \\
34 \\
8.0 \\
9.2 \\
.2 \\
7.4\end{array}$ & $\begin{array}{l}8.0 \\
6.0\end{array}$ & $\begin{array}{l}5.2 \\
. .30 \\
14 \\
5.3 \\
8.8 \\
27 \\
35 \\
7.0 \\
9.2 \\
.2 \\
5.4\end{array}$ & $\begin{array}{lc}\text { B. } & 6.5 \\
\text { B. } & .17 \\
\text { B. } & 14 \\
\text { B. } & 5.3 \\
\text { B. } & 8.7 \\
\text { B. } & 26 \\
\text { B. } & 34 \\
\text { T. } & 7.8 \\
\text { B. } & 8.0 \\
\text { B. } & .2 \\
\text { B. } & 6.8\end{array}$ \\
\hline $\begin{array}{l}\text { Dissolved solids }-1-- \text { Bottom } \\
\text { Hardness as } \mathrm{CaCO}_{3}-\cdots-\text { Bottom } \\
\text { Suspended sediment }-\cdots-\text { Top }\end{array}$ & $\begin{array}{r}108 \\
57 \\
. \\
\end{array}$ & & $\begin{array}{r}106 \\
56 \\
. \quad \text { Com }\end{array}$ & site. . & $\begin{array}{c}103 \\
57 \\
\cdots\end{array}$ & $\begin{array}{rr}\text { B. } & 106 \\
\text { B. } & 57 \\
\text { T. } & 23 \\
\end{array}$ \\
\hline
\end{tabular}


Table 10. --WATER ANALYSES OF DELAWARE RIVER BETWEEN BRISTOL AND MarCuS hOOK, PA. --Continued Analyzed by City of Philadelphia and U. S. Geological Survey; analyses in parts per million

Location Philadelphia, Pa.-Camden, N. J. Bridge Date March 2, 1951 Sampling study No. 19

\begin{tabular}{|c|c|c|c|c|c|c|}
\hline \multirow[t]{3}{*}{ Weather Clear } & \multicolumn{6}{|c|}{ Water discharge at Trenton (cfs) 19,600} \\
\hline & \multicolumn{6}{|c|}{ Station } \\
\hline & $\begin{array}{c}\text { Pennsylvania } \\
\text { side }\end{array}$ & West Center & Center. & East Center & $\begin{array}{l}\text { New Jersey } \\
\text { side }\end{array}$ & Average \\
\hline $\begin{array}{l}\begin{array}{l}\text { Time (EST) } \\
\text { Sounding (ft) } \\
\text { Temperature }\left({ }^{\circ} \mathrm{F}\right)\end{array} \\
\text { Color } \\
\text { pH }\end{array}$ & $\begin{array}{l}1: 20 \text { p.m. } \\
42 \\
44 \\
44 \\
12 \\
6.4 \\
7.1\end{array}$ & $\begin{array}{l}1: 24 \mathrm{p} . \mathrm{m} . \\
43 \\
43 \\
42 \\
\\
6.7 \\
6.7\end{array}$ & $\begin{array}{l}1: 28 \mathrm{p} \cdot \mathrm{m} . \\
43 \\
43 \\
42 \\
9 \\
6.8 \\
7.2\end{array}$ & $\begin{array}{l}1: 32 \mathrm{p} . \mathrm{m} . \\
34 \\
43 \\
42 \\
\\
6.7 \\
6.9\end{array}$ & $\begin{array}{l}1: 36 \text { p.m. } \\
25 \\
43 \\
42 \\
10 \\
6.8 \\
7.1\end{array}$ & $\begin{array}{ll}\text { T. } & 43 \\
\text { B. } & 42 \\
\text { B. } & 10 \\
\text { T. } & 6.7 \\
\text { B. } & 7.0\end{array}$ \\
\hline 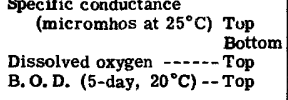 & $\begin{array}{l}138 \\
137 \\
10.8 \\
\text { bl1 }\end{array}$ & $\begin{array}{r}111 \\
114 \\
12.9 \\
5.9\end{array}$ & $\begin{array}{l}108 \\
106 \\
13.1 \\
5.7\end{array}$ & $\begin{array}{l}106 \\
107 \\
12.6 \\
5.3\end{array}$ & $\begin{array}{l}106 \\
105 \\
12.6 \\
5.9\end{array}$ & $\begin{array}{ll}\text { T. } & 114 \\
\text { B. } & 114 \\
\text { T. } & 12.4 \\
\text { T. } & \text { b6.8 }\end{array}$ \\
\hline 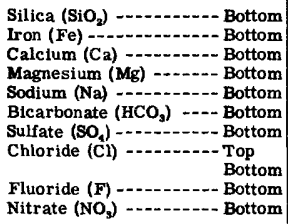 & $\begin{array}{l}4.6 \\
.14 \\
12.1 \\
6.9 \\
27 \\
25 \\
6.0 \\
8.0 \\
.2 \\
3.0\end{array}$ & $\begin{array}{l}5.0 \\
6.0\end{array}$ & $\begin{array}{l}4.8 \\
10 \\
3.18 \\
3.9 \\
22 \\
20 \\
4.0 \\
5.2 \\
.2 \\
3.0\end{array}$ & $\begin{array}{l}4.0 \\
6.0\end{array}$ & $\begin{array}{l}4.4 \\
10.14 \\
3.6 \\
3.4 \\
21 \\
20 \\
4.0 \\
5.0 \\
.2 \\
2.5\end{array}$ & $\begin{array}{lc}\text { B. } & 4.6 \\
\text { B. } & .15 \\
\text { B. } & 11 \\
\text { B. } & 3.8 \\
\text { B. } & 4.7 \\
\text { B. } & 23 \\
\text { B. } & 22 \\
\text { T. } & 4.6 \\
\text { B. } & 6.0 \\
\text { B. } & .2 \\
\text { B. } & 2.8\end{array}$ \\
\hline $\begin{array}{l}\text { Dissolved solids }-1 .- \text { Bottom } \\
\text { Hardness as } \mathrm{CaCO}_{9} \ldots-\text { Bottom } \\
\text { Suspended sediment }-\cdots-\text { Top }\end{array}$ & $\begin{array}{l}83 \\
47 \\
\cdots\end{array}$ & & $\begin{array}{l}69 \\
41 \\
\text { C Con }\end{array}$ & 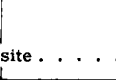 & $\begin{array}{l}65 \\
40 \\
. .\end{array}$ & $\begin{array}{l}\text { B. } \\
\text { B. } \\
\text { T. }\end{array}$ \\
\hline
\end{tabular}

b Exceeded this value.

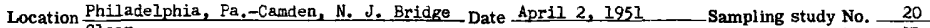

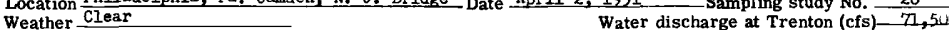

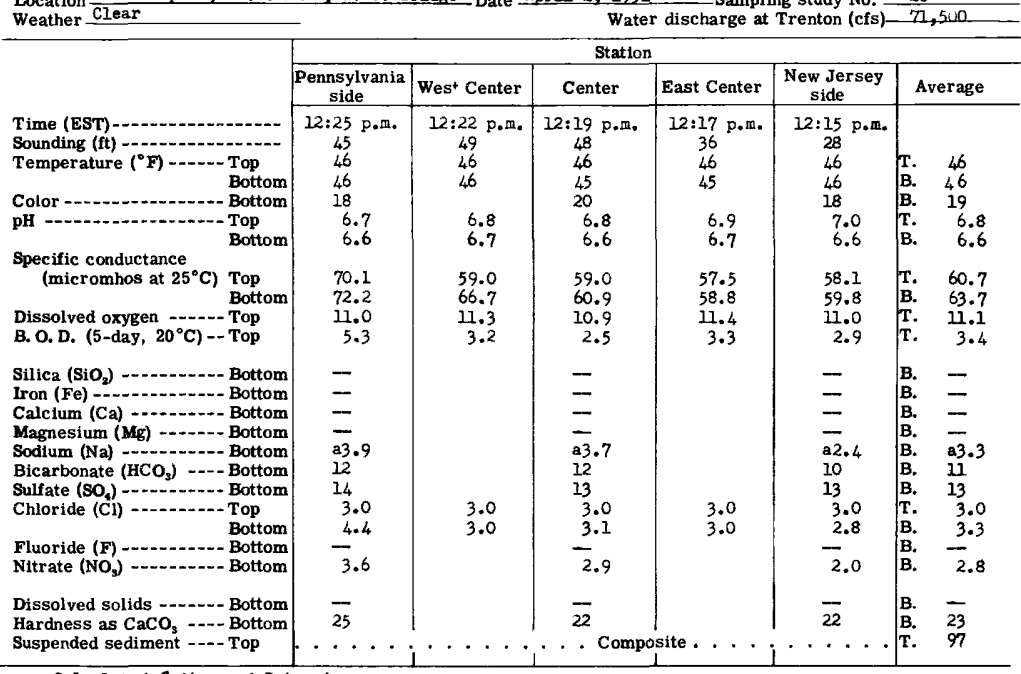

a Calculated Sodium and Potassium. 
Table 10. --WATER ANALYSES OF DELAWARE RIVER BETWEEN BRISTOL AND MARCUS hOOK, PA. --Continued Anaiyzed by City of Philadelphia and U. S. Geological Survey; analyses in parts per million

Location Philadelphia, Pa.-Camden, N. J, Bridge Date Mag 1, 1951 ._. Sampling study No. 2] Weather Clear $\quad$ Water discharge at Trenton (cfs) 13,0 10

\begin{tabular}{|c|c|c|c|c|c|c|}
\hline \multirow[b]{3}{*}{$\begin{array}{l}\text { Tlme (EST) } \\
\begin{array}{l}\text { Sounding (ft) } \\
\text { Temperature }\left({ }^{\circ} \mathrm{F}\right)-1\end{array} \\
\begin{array}{l}\text { Bop } \\
\text { Color } \\
\text { pH }-10 m\end{array} \\
\end{array}$} & \multicolumn{6}{|c|}{ Station } \\
\hline & $\begin{array}{c}\begin{array}{c}\text { Pennsylvania } \\
\text { side }\end{array} \\
\end{array}$ & West Center & Center & East Center & $\begin{array}{c}\text { New Jersey } \\
\text { side }\end{array}$ & Average \\
\hline & $\begin{array}{l}8: 20 \text { a.m. } \\
45 \\
62 \\
61 \\
12 \\
6.7 \\
7.1\end{array}$ & $\begin{array}{c}8: 25 \text { a.m. } \\
46 \\
62 \\
61 \\
6.7 \\
6.9\end{array}$ & $\begin{array}{l}8: 31 \text { a.m. } \\
48 \\
61 \\
61 \\
14 \\
6.8 \\
6.8\end{array}$ & $\begin{array}{l}8: 35 \mathrm{a} \cdot \mathrm{m} . \\
39 \\
61 \\
61 \\
6.9 \\
7.0\end{array}$ & $\begin{array}{l}8: 39 \text { a.m. } \\
27 \\
61 \\
61 \\
12 \\
6.8 \\
6.7\end{array}$ & $\begin{array}{l}61 \\
61 \\
13 \\
6.8 \\
6.9\end{array}$ \\
\hline $\begin{array}{l}\text { (micromhos at } 25^{\circ} \mathrm{C} \text { ) } \begin{array}{l}\text { Top } \\
\text { Bottom }\end{array} \\
\text { Dissolved oxygen -1-.- Top } \\
\text { B. O. D. (5-day, } 20^{\circ} \mathrm{C} \text { ) -- Top }\end{array}$ & $\begin{array}{l}142 \\
141 \\
4.3 \\
9.3\end{array}$ & $\begin{array}{r}138 \\
134 \\
4.2 \\
8.0\end{array}$ & $\begin{array}{l}126 \\
126 \\
6.1 \\
3.3\end{array}$ & $\begin{array}{l}123 \\
127 \\
6.2 \\
2.9\end{array}$ & $\begin{array}{l}129 \\
128 \\
5.7 \\
3.6\end{array}$ & $\begin{array}{lr}\text { T. } & 132 \\
\text { B. } & 131 \\
\text { T. } & 5.3 \\
\text { T. } & 5.4\end{array}$ \\
\hline 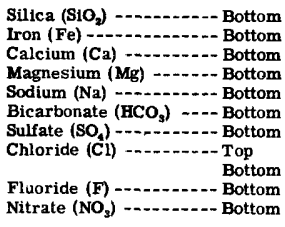 & $\begin{array}{l}6.2 \\
.05 \\
12 \\
4.8 \\
4.6 \\
26 \\
24 \\
8.0 \\
7.5 \\
.0 \\
4.0\end{array}$ & $\begin{array}{l}8.0 \\
6.0\end{array}$ & $\begin{array}{l}5.7 \\
.27 \\
11 \\
4.0 \\
5.5 \\
27 \\
22 \\
6.0 \\
6.5 \\
.0 \\
3.0\end{array}$ & $\begin{array}{l}6.0 \\
6.0\end{array}$ & $\begin{array}{l}5.6 \\
.11 \\
11 \\
4.0 \\
4.6 \\
24 \\
22 \\
7.0 \\
6.8 \\
.0 \\
2.9\end{array}$ & $\begin{array}{l}5.8 \\
.11 \\
11 \\
4.3 \\
4.9 \\
26 \\
23 \\
7.0 \\
6.6 \\
.0 \\
3.3\end{array}$ \\
\hline $\begin{array}{l}\text { Dissolved solids - }-1-\text { Bottom } \\
\text { Hardness as } \mathrm{CaCO}_{\mathrm{g}}-\ldots \text { Bottom } \\
\text { Suspended sediment --- Top }\end{array}$ & $\begin{array}{l}86 \\
50 \\
.\end{array}$ & & $\begin{array}{l}78 \\
44 \\
. \\
\end{array}$ & e. & $\begin{array}{l}80 \\
44 \\
.\end{array}$ & $\begin{array}{l}\text { B. } \\
\text { B. } \\
\text { T. }\end{array}$ \\
\hline
\end{tabular}

Location Philadelphia, Fa.-Camden, N, J. Bridge Date June 7, 1951

Sampling study No. 22 Weather cloudy

\begin{tabular}{|c|c|c|c|c|c|c|}
\hline \multirow[b]{3}{*}{ 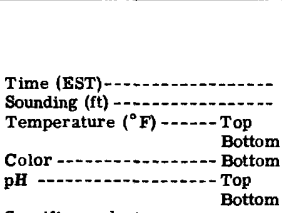 } & \multicolumn{6}{|c|}{ Station } \\
\hline & $\begin{array}{c}\text { Pennsylvania } \\
\text { side }\end{array}$ & West Center & Center & East Center & \begin{tabular}{|c|}
$\begin{array}{c}\text { New Jersey } \\
\text { side }\end{array}$ \\
\end{tabular} & Average \\
\hline & $\begin{array}{c}8: 30 \text { a.m. } \\
38 \\
73 \\
72 \\
20 \\
6.9 \\
6.8\end{array}$ & $\begin{array}{l}8: 32 \text { a.m. } \\
41 \\
72 \\
72 \\
6.6 \\
6.8\end{array}$ & $\begin{array}{l}8: 34 \mathrm{a} . \mathrm{m} . \\
44 \\
72 \\
72 \\
15 \\
6.6 \\
7.1\end{array}$ & $\begin{array}{c}8: 36 \text { a.m. } \\
30 \\
72 \\
72 \\
6.6 \\
6.8\end{array}$ & $\begin{array}{c}8: 38 \text { a.m. } \\
25 \\
72 \\
72 \\
10 \\
\varepsilon .6 \\
7.0\end{array}$ & $\begin{array}{l}72 \\
72 \\
15 \\
6.7 \\
6.9\end{array}$ \\
\hline 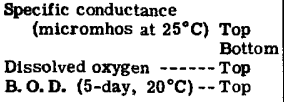 & $\begin{array}{l}204 \\
194 \\
1.5 \\
6.2\end{array}$ & $\begin{array}{l}173 \\
174 \\
1.4 \\
1.1\end{array}$ & $\begin{array}{r}170 \\
171 \\
1.4 \\
.0\end{array}$ & $\begin{array}{l}175 \\
172 \\
1.4 \\
.7\end{array}$ & $\begin{array}{l}176 \\
175 \\
1.7 \\
1.4\end{array}$ & $\begin{array}{lr}\text { T. } & 180 \\
\text { B. } & 177 \\
\text { T. } & 1.5 \\
\text { T. } & 1.9\end{array}$ \\
\hline 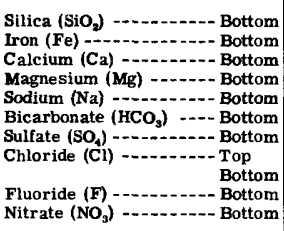 & $\begin{array}{l}4.7 \\
.07 \\
15 \\
5.5 \\
10 \\
41 \\
30 \\
\frac{11}{10} \\
.1 \\
4.9\end{array}$ & $\begin{array}{l}9.0 \\
9.0\end{array}$ & $\begin{array}{l}4.1 \\
.07 \\
14 \\
5.3 \\
8.2 \\
38 \\
28 \\
9.0 \\
8.5 \\
.1 \\
4.1\end{array}$ & $\begin{array}{l}8.0 \\
9.0\end{array}$ & $\begin{array}{l}5.0 \\
.08 \\
14 \\
5.4 \\
9.0 \\
40 \\
28 \\
8.0 \\
8.5 \\
.1 \\
4.3\end{array}$ & $\begin{array}{l}4.6 \\
.07 \\
14 \\
5.4 \\
9.1 \\
40 \\
29 \\
9.0 \\
9.0 \\
.1 \\
4.4\end{array}$ \\
\hline $\begin{array}{l}\text { Dissolved solids }-1 .- \text { Bottom } \\
\text { Hardness as CaCO } \mathrm{Ca}_{3}-\cdots \text { Bottom } \\
\text { Suspended sediment }-\ldots \text { Top }\end{array}$ & $\begin{array}{r}110 \\
60 \\
.\end{array}$ & & $\begin{array}{l}100 \\
57 \\
. \quad \mathrm{Co}\end{array}$ & 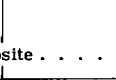 & $\begin{array}{r}105 \\
57 \\
.\end{array}$ & $\begin{array}{lr}\text { B. } & 105 \\
\text { B. } & 58 \\
\text { T. } & 33\end{array}$ \\
\hline
\end{tabular}


Table 10. --WATER ANALYSES OF DELAWARE RIVER BETWEEN BRISTOL AND MARCUS HOOK, PA. - -COntinued Analyzed by City of Philadelphia and U. S. Geological Survey; analyses in parts per million

Location Philadelphia, Pa.-Camden, N. J. Bridge Date July 5, 1951 _ Sampting study No. $\frac{23}{9}$ Weather cloudy Water discharge at Trenton (cfs) $-\frac{23}{9,190}$

\begin{tabular}{|c|c|c|c|c|c|c|}
\hline & \multicolumn{6}{|c|}{ Station } \\
\hline & \begin{tabular}{|c|} 
Pennsylvania \\
side \\
\end{tabular} & West Center & Center & East Center & \begin{tabular}{|c|}
$\begin{array}{c}\text { New Jersey } \\
\text { side }\end{array}$ \\
\end{tabular} & Average \\
\hline 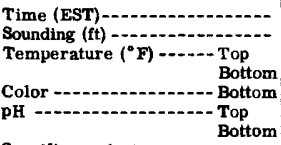 & $\begin{array}{l}8: 25 \text { a.m. } \\
34 \\
79 \\
78 \\
12 \\
6.9 \\
6.9\end{array}$ & $\begin{array}{c}8: 29 \text { a.m. } \\
41 \\
78 \\
78 \\
6.8 \\
6.9\end{array}$ & $\begin{array}{c}8: 33 \mathrm{a} \cdot \mathrm{m} . \\
42 \\
78 \\
78 \\
14 \\
6.8 \\
7.0\end{array}$ & $\begin{array}{c}8: 37 \text { a.m. } \\
33 \\
78 \\
78 \\
\\
6.8 \\
6.8\end{array}$ & $\begin{array}{c}8: 42 \text { a.m. } \\
19 \\
78 \\
77 \\
10 \\
6.5 \\
7.0\end{array}$ & $\begin{array}{lc}\text { T. } & 78 \\
\text { B. } & 78 \\
\text { B. } & 12 \\
\text { T. } & 6.8 \\
\text { B. } & 6.9\end{array}$ \\
\hline $\begin{array}{l}\begin{array}{l}\text { Specific conductance } \\
\quad\left(\text { micromhos at } 25^{\circ} \mathrm{C} \text { ) }\right.\end{array} \\
\begin{array}{l}\text { Top } \\
\text { Bottom }\end{array} \\
\text { Dissolved oxygen -a- } \\
\text { B. O. D. (5-day, } 20^{\circ} \mathrm{C} \text { ) - Top } \\
\text { - Top }\end{array}$ & $\begin{array}{r}199 \\
199 \\
.9 \\
1.8\end{array}$ & $\begin{array}{r}177 \\
175 \\
1.5 \\
.4\end{array}$ & $\begin{array}{r}168 \\
171 \\
1.9 \\
.3\end{array}$ & $\begin{array}{r}169 \\
166 \\
2.0 \\
.4\end{array}$ & $\begin{array}{l}223 \\
227 \\
1.5 \\
1.0\end{array}$ & $\begin{array}{lr}\text { T. } & 187 \\
\text { B. } & 188 \\
\text { T. } & 1.6 \\
\text { T. } & .8\end{array}$ \\
\hline 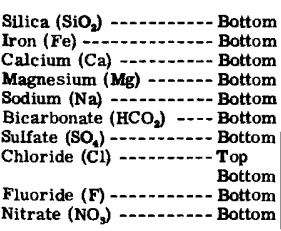 & $\begin{array}{l}5.3 \\
18 \\
5.06 \\
9.7 \\
48 \\
32 \\
10 \\
9.8 \\
.2 \\
4.0\end{array}$ & $\begin{array}{l}9.0 \\
9.0\end{array}$ & $\begin{array}{l}5.5 \\
.08 \\
17 \\
5.7 \\
4.3 \\
40 \\
28 \\
9.0 \\
7.2 \\
.1 \\
3.6\end{array}$ & $\begin{array}{l}9.0 \\
9.0\end{array}$ & $\begin{array}{l}5.8 \\
.07 \\
19^{.07} \\
6.2 \\
13 \\
47 \\
31 \\
21 \\
19 \\
.12\end{array}$ & $\begin{array}{ll}\text { B. } & 5.5 \\
\text { B. } & .07 \\
\text { B. } & 18 \\
\text { B. } & 5.9 \\
\text { B. } & 9.0 \\
\text { B. } & 45 \\
\text { B. } & 30 \\
\text { T. } & 12 \\
\text { B. } & 11 \\
\text { B. } & .1 \\
\text { B. } & 4.3\end{array}$ \\
\hline $\begin{array}{l}\text { Dissolved solids - }-1-\text { Bottom } \\
\text { Hardness as } \mathrm{CaCO}_{3}-\mathrm{C}_{\text {B }} \text { Bottom } \\
\text { Suspended sediment }--- \text { Top }\end{array}$ & $\begin{array}{r}124 \\
69 \\
.\end{array}$ & & $\begin{array}{r}114 \\
66 \\
. \\
.\end{array}$ & $\cdot$ & $\begin{array}{r}142 \\
73 \\
\cdots\end{array}$ & $\begin{array}{rr}\text { B. } & 127 \\
\text { B. } & 69 \\
\text { T. } & 19\end{array}$ \\
\hline
\end{tabular}

Location Philadelphia, Pa,-Camien, N. J. Bridge Date August 1, 1957

Sampling study No. 24 Weather Cloudy Dater discharge at Trenton (cfs) 12,400

\begin{tabular}{|c|c|c|c|c|c|c|}
\hline & \multicolumn{6}{|c|}{ Station } \\
\hline & $\begin{array}{c}\text { Pennsplvania } \\
\text { side }\end{array}$ & West Center & Center & East Center & \begin{tabular}{|c|c|}
$\begin{array}{c}\text { New Jersey } \\
\text { side }\end{array}$ \\
\end{tabular} & Average \\
\hline 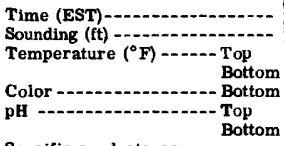 & $\begin{array}{l}8: 25 \text { a.m. } \\
37 \\
79 \\
78 \\
7 \\
6.9 \\
7.8\end{array}$ & $\begin{array}{l}8: 27 \mathrm{a} . \mathrm{m} . \\
42 \\
79 \\
78 \\
6.9 \\
7.0\end{array}$ & $\begin{array}{c}8: 29 \text { a.m. } \\
41 \\
79 \\
78 \\
7 \\
6.9 \\
7.8\end{array}$ & $\begin{array}{c}8: 31 \text { a.m. } \\
30 \\
79 \\
78 \\
\\
6.9 \\
7.0\end{array}$ & $\begin{array}{l}8: 32 \mathrm{a}, \mathrm{m} . \\
18 \\
79 \\
78 \\
7 \\
6.9 \\
7.8\end{array}$ & $\begin{array}{ll}\text { T. } & 79 \\
\text { B. } & 78 \\
\text { B. } & 7 \\
\text { T. } & 6.9 \\
\text { B. } & 7.5\end{array}$ \\
\hline $\begin{array}{l}\begin{array}{l}\text { Specific conductance } \\
\quad \text { (micromhos at } 25^{\circ} \mathrm{C} \text { ) }\end{array} \\
\begin{array}{l}\text { Top } \\
\text { Bottom }\end{array} \\
\text { Dissolved oxygen - }--- \text { Top } \\
\text { B. O.D. (5-day, 20 } 20^{\circ} \mathrm{C} \text { )- Top }\end{array}$ & $\begin{array}{l}178 \\
169 \\
2.4 \\
1.8\end{array}$ & $\begin{array}{r}178 \\
172 \\
3.0 \\
.0\end{array}$ & $\begin{array}{l}164 \\
163 \\
4.2 \\
1.5\end{array}$ & $\begin{array}{r}161 \\
164 \\
4.0 \\
3.3\end{array}$ & $\begin{array}{r}170 \\
161 \\
3.5 \\
3.3\end{array}$ & $\begin{array}{lr}\text { T. } & 170 \\
\text { B. } & 166 \\
\text { T. } & 3.4 \\
\text { T. } & 2.0\end{array}$ \\
\hline 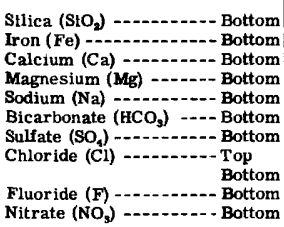 & $\begin{array}{l}5.8 \\
.01 \\
16 \\
5.2 \\
7.2 \\
42 \\
29 \\
8.0 \\
6.5 \\
.1 \\
5.3\end{array}$ & $\begin{array}{l}8.0 \\
8.0\end{array}$ & $\begin{array}{l}6.2 \\
16 \\
4.01 \\
6.1 \\
42 \\
29 \\
8.0 \\
5.6 \\
. .1 \\
5.1\end{array}$ & $\begin{array}{l}8.0 \\
8.0\end{array}$ & $\begin{array}{l}5.8 \\
.01 \\
15 \\
4.9 \\
6.6 \\
42 \\
27 \\
8.0 \\
5.8 \\
.1 \\
4.8\end{array}$ & $\begin{array}{lc}\text { B. } & 5.9 \\
\text { B. } & .01 \\
\text { B. } & 16 \\
\text { B. } & 5.0 \\
\text { B. } & 6.6 \\
\text { B. } & 42 \\
\text { B. } & 28 \\
\text { T. } & 8.0 \\
\text { B. } & 6.8 \\
\text { B. } & .1 \\
\text { B. } & 5.1\end{array}$ \\
\hline $\begin{array}{l}\text { Dissolved solids ----- Bottom } \\
\text { Hardness as } \mathrm{CaCO}_{3}--- \text { Bottom } \\
\text { Suspended sediment --- Top }\end{array}$ & $\begin{array}{r}106 \\
61 \\
. \\
\end{array}$ & 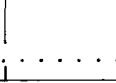 & $\begin{array}{l}105 \\
60 \\
. \quad \text { Cor } \\
\end{array}$ & site. & $\begin{array}{r}100 \\
58 \\
. \\
\end{array}$ & $\begin{array}{lr}\text { B. } & 104 \\
\text { B. } & 60 \\
\text { T. } & 15 \\
\end{array}$ \\
\hline
\end{tabular}


Table 10. --WATER ANALYSES OF DELAWARE RIVER BETWEEN BRISTOL AND MARCUS HOOK, PA. --Continued Analyzed by City of Philadelphia ard U. S. Geological Survey; analyses in parts per million

Location Philadelphia, Pa,-Camden, N. J, Bridge Date September 6, 1951 Sampling study No. 25 Weather Rain

Time (EST)

Temperature $\left({ }^{\circ} \mathrm{F}\right)$-.....

Color -

pH -

Specific conductance

(micromhos at $25^{\circ} \mathrm{C}$ ) Top

Bottom

B. O.D. (5-day, $\left.20^{\circ} \mathrm{C}\right)-$ Top

Silica $\left(\mathrm{SiO}_{2}\right)$-....-.-- Bottom

Iron (Fe) --

Calcium (Ca) -........- Bottom

Magneslum (Mg) ------- Bottom

Sodium (Na) - ......-- Bottom

Bicarbonate (HCO, ...- Bottom

Sulfate $\left(\mathrm{SO}_{4}\right)$-...-.-- Bottom

Chloride (C1) -

Fluoride (F) Nitrate $\left(\mathrm{NO}_{2}\right)$-....- Bottom

Dissolved solids - ......- Bottom Hardness as $\mathrm{CaCO}_{3}$-.-- Bottom

Suspended sediment -.-.- Top

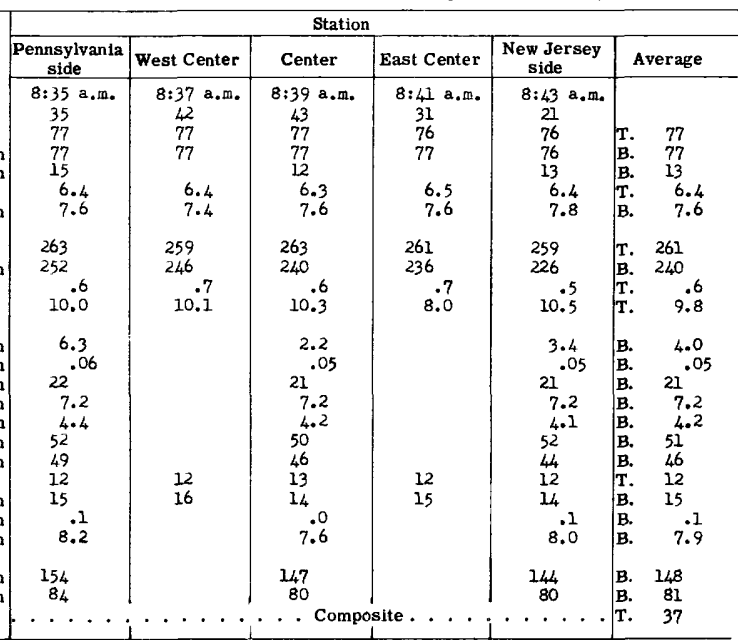

Location Philadelphia, Pa.-Canden, N. J. Eridge Date October 2, 1951 - Sampling study No. 26 Weather Clear $\quad$ Water discharge at Trenton (cfs) 2,990

\begin{tabular}{|c|c|c|c|c|c|c|}
\hline \multirow[b]{3}{*}{$\begin{array}{l}\text { Time (EST) } \\
\text { Sounding (ft) } \\
\text { Temperature ( }\end{array}$} & \multicolumn{6}{|c|}{ Station } \\
\hline & \begin{tabular}{|c|} 
Pennsylvania \\
side
\end{tabular} & West Center & Center & East Center & $\begin{array}{c}\text { New Jersey } \\
\text { side }\end{array}$ & Average \\
\hline & $\begin{array}{l}9: 22 \text { a.m. } \\
36 \\
71 \\
70 \\
8 \\
6.2 \\
7.8\end{array}$ & $\begin{array}{l}9: 24 \text { a.m. } \\
39 \\
71 \\
70 \\
\\
6.2 \\
6.6\end{array}$ & $\begin{array}{l}9: 27 \mathrm{a} \cdot \mathrm{m} . \\
40 \\
69 \\
69 \\
9 \\
6.5 \\
7.8\end{array}$ & $\begin{array}{l}9: 29 \text { a.m. } \\
29 \\
69 \\
69 \\
6.3 \\
7.1\end{array}$ & $\begin{array}{l}9: 32 \mathrm{a} . \mathrm{m} . \\
28 \\
70 \\
69 \\
9 \\
6.3 \\
7.8\end{array}$ & $\begin{array}{l}70 \\
69 \\
9 \\
6.3 \\
7.4\end{array}$ \\
\hline $\begin{array}{l}\text { Specific conductance } \\
\quad \text { (micromhos at } 25^{\circ} \mathrm{C} \text { ) } \begin{array}{l}\text { Top } \\
\text { Bottom }\end{array} \\
\left.\text { Dissolved oxygen }-10^{\circ} \mathrm{C}\right)- \text { Top } \\
\text { B. To. D. (5-day, }\end{array}$ & $\begin{array}{l}267 \\
266 \\
1.1 \\
4.5\end{array}$ & $\begin{array}{l}261 \\
229 \\
1.5 \\
3.3\end{array}$ & $\begin{array}{r}261 \\
234 \\
2.5 \\
.3\end{array}$ & $\begin{array}{r}247 \\
229 \\
2.0 \\
.0\end{array}$ & $\begin{array}{r}256 \\
24.4 \\
1.8 \\
2.4\end{array}$ & $\begin{array}{ll}\text { T. } & 258 \\
\text { B. } & 240 \\
\text { T. } & 1.8 \\
\text { T. } & 2.1\end{array}$ \\
\hline 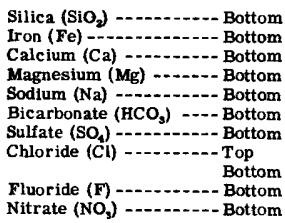 & $\begin{array}{l}2.0 \\
.05 \\
22 \\
7.6 \\
17 \\
54 \\
50 \\
16 \\
16 \\
.1 \\
7.8\end{array}$ & $\begin{array}{l}16 \\
15\end{array}$ & $\begin{array}{l}2.0 \\
20 \\
6.05 \\
14 \\
54 \\
43 \\
16 \\
14 \\
7.1\end{array}$ & $\begin{array}{l}14 \\
15\end{array}$ & $\begin{array}{l}2.0 \\
21.05 \\
7.1 \\
16 \\
50 \\
45 \\
15 \\
16 \\
12.1\end{array}$ & $\begin{array}{l}2.0 \\
.05 \\
21.0 \\
7.2 \\
16 \\
53 \\
46 \\
15 \\
15 \\
.1 \\
8.9\end{array}$ \\
\hline $\begin{array}{l}\text { Dissolved solids --- }-- \text { Bottom } \\
\text { Hardness as } \mathrm{CaCO}_{3}--- \text { Bottom } \\
\text { Suspended sediment }-\cdots-\text { Top }\end{array}$ & $\begin{array}{r}160 \\
86 \\
. .\end{array}$ & & $\begin{array}{l}\frac{142}{78} \\
. \quad \text { Com }\end{array}$ & site... & $\begin{array}{r}155 \\
82 \\
\cdots \cdot .\end{array}$ & $\begin{array}{lr}\text { B. } & 152 \\
\text { B. } & 82 \\
\text { T. } & 24\end{array}$ \\
\hline
\end{tabular}


Table 10. --WATER ANALYSES OF DELAWARE RIVER BETWEEN BRISTOL AND MARCUS HOOK, PA. --Continued Analyzed by City of Philadelpha and U. S. Geological Survey; analyses in parts per million

Location Philajelphia, Pa.-Camden, N. J. Bridge Date November 7, 1951 Sampling study No. 27

\begin{tabular}{|c|c|c|c|c|c|c|}
\hline \multirow[t]{3}{*}{ Weather Cloudy } & \multicolumn{6}{|c|}{ Water discharge at Trenton (cfs) 37,700} \\
\hline & \multicolumn{6}{|c|}{ Station } \\
\hline & $\begin{array}{c}\text { Pennsylvania } \\
\text { side }\end{array}$ & West Center & Center & East Center & $\begin{array}{l}\text { New Jersey } \\
\text { side }\end{array}$ & Average \\
\hline $\begin{array}{l}\text { Time (EST) } \\
\text { Sounding (ft) } \\
\text { Temperature ( }\end{array}$ & $\begin{array}{l}3: 29 p \cdot m . \\
18 \\
47 \\
48 \\
12 \\
6.3 \\
7.2\end{array}$ & $\begin{array}{l}3: 26 \mathrm{pm} . \mathrm{m} \\
18 \\
46 \\
47 \\
6.5 \\
6.7\end{array}$ & $\begin{array}{l}3: 23 \text { p.in. } \\
46 \\
47 \\
48 \\
17 \\
6.5 \\
7.3\end{array}$ & $\begin{array}{l}3: 21 \mathrm{p} \cdot \mathrm{m} . \\
45 \\
47 \\
49 \\
\\
6.6 \\
6.7\end{array}$ & $\begin{array}{l}3: 18 \text { p.m. } \\
20 \\
49 \\
50 \\
12 \\
6.5 \\
7.3\end{array}$ & $\begin{array}{l}47 \\
48 \\
12 \\
6.5 \\
7.0\end{array}$ \\
\hline $\begin{array}{l}\begin{array}{l}\text { Specific conductance } \\
\text { (micromhos at } 25^{\circ} \mathrm{C} \text { ) }\end{array} \\
\begin{array}{l}\text { Top } \\
\text { Bottom }\end{array} \\
\text { Dissolved oxygen }-1-\text { Top }^{\circ} \mathrm{C} \text { ) - Top } \\
\text { B. O. D. (5-day, } 20^{\circ} \text { - }\end{array}$ & $\begin{array}{l}149 \\
147 \\
9.1 \\
8.4\end{array}$ & $\begin{array}{l}113 \\
105 \\
10.2 \\
3.2\end{array}$ & $\begin{array}{l}108 \\
107 \\
10.0 \\
1.8\end{array}$ & $\begin{array}{r}114 \\
105 \\
10.6 \\
2.8\end{array}$ & $\begin{array}{l}136 \\
121 \\
9.4 \\
2.9\end{array}$ & \begin{tabular}{lr} 
& \multicolumn{1}{c}{124} \\
T. & 117 \\
T. & 9.9 \\
T. & 3.8
\end{tabular} \\
\hline 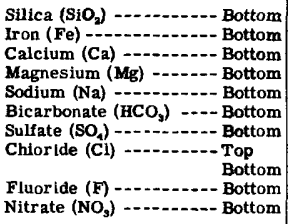 & $\begin{array}{l}5.3 \\
12.01 \\
3.9 \\
7.3 \\
21 \\
29 \\
9.0 \\
9.0 \\
.1 \\
4.1\end{array}$ & $\begin{array}{l}4.0 \\
5.0\end{array}$ & $\begin{array}{l}5.1 \\
.01 \\
10 \\
3.1 \\
3.3 \\
17 \\
23 \\
4.0 \\
4.0 \\
.1 \\
3.3\end{array}$ & $\begin{array}{l}4.0 \\
5.0\end{array}$ & $\begin{array}{l}3.9 \\
.01 \\
13 \\
3.4 \\
3.4 \\
23 \\
25 \\
8.0 \\
6.0 \\
.1 \\
3.7\end{array}$ & $\begin{array}{l}4.8 \\
12.01 \\
3.5 \\
4.7 \\
20 \\
26 \\
5.8 \\
5.8 \\
. .1 \\
3.7\end{array}$ \\
\hline $\begin{array}{l}\text { Dissolved solids } \\
\text { Hardness as } \mathrm{CaCO}_{3} \\
\text { Suspended sediment }- \text { - }- \text { Tottom }\end{array}$ & $\begin{array}{r}111 \\
46 \\
.\end{array}$ & & $\begin{array}{l}79 \\
38 \\
\text { C Con }\end{array}$ & e. & $\begin{array}{l}86 \\
47 \\
.\end{array}$ & $\begin{array}{l}\text { B. } \\
\text { B. } \\
\text { T. }\end{array}$ \\
\hline
\end{tabular}

Location Philadelphis, Pa.-Camden, N. J. Bridge Date December 5, 195I_Sampling study No. 28

\begin{tabular}{|c|c|c|c|c|c|c|}
\hline eather Rain & & & Wa & discharge & renton (cis) & 12,500 \\
\hline & & & Statio & & & \\
\hline & \begin{tabular}{|c}
$\begin{array}{c}\text { Pennsylvania } \\
\text { side }\end{array}$ \\
\end{tabular} & West Center & Center & East Center & $\begin{array}{c}\text { New Jersey } \\
\text { side }\end{array}$ & Average \\
\hline 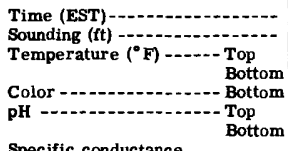 & $\begin{array}{l}9: 37 \text { a.m. } \\
42 \\
44 \\
43 \\
10 \\
6.2 \\
7.5\end{array}$ & $\begin{array}{l}9: 39 \text { a.m. } \\
44 \\
43 \\
43 \\
6.2 \\
6.8\end{array}$ & $\begin{array}{l}9: 42 \text { a.m. } \\
45 \\
43 \\
43 \\
17 \\
6.4 \\
7.5\end{array}$ & $\begin{array}{l}9: 46 \mathrm{a}, \mathrm{m} . \\
33 \\
42 \\
42 \\
6.4 \\
6.7\end{array}$ & $\begin{array}{l}9: 49 \text { a.m. } \\
23 \\
43 \\
42 \\
11 \\
6.4 \\
7.4\end{array}$ & $\begin{array}{ll}\text { T. } & 43 \\
\text { B. } & 43 \\
\text { B. } & 11 \\
\text { T. } & 6.3 \\
\text { B. } & 7.2\end{array}$ \\
\hline $\begin{array}{l}\begin{array}{l}\text { Specific conductance } \\
\left.\text { (micromhos at } 25^{\circ} \mathrm{C}\right) \\
\text { Top } \\
\text { Bottom }\end{array} \\
\text { Dissolved oxygen }- \\
\text { B. O. D. (5-day, } 20^{\circ} \mathrm{C} \text { ) - Top }\end{array}$ & $\begin{array}{l}167 \\
165 \\
7.8 \\
7.5\end{array}$ & $\begin{array}{r}166 \\
159 \\
8.3 \\
6.8\end{array}$ & $\begin{array}{r}151 \\
151 \\
9.0 \\
5.0\end{array}$ & $\begin{array}{r}145 \\
138 \\
9.3 \\
4.6\end{array}$ & $\begin{array}{r}164 \\
148 \\
9.2 \\
5.2\end{array}$ & $\begin{array}{lr} & \\
\text { T. } & 159 \\
\text { B. } & 152 \\
\text { T. } & 8.7 \\
\text { T. } & 5.8\end{array}$ \\
\hline 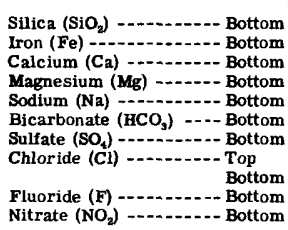 & $\begin{array}{l}6.5 \\
.01 \\
14 \\
4.4 \\
8.8 \\
30 \\
31 \\
10 \\
10 \\
6.1\end{array}$ & $\begin{array}{l}8.0 \\
8.0\end{array}$ & $\begin{array}{l}7.2 \\
13.01 \\
4.1 \\
7.2 \\
25 \\
30 \\
7.0 \\
7.5 \\
.01 \\
5.1\end{array}$ & $\begin{array}{l}5.0 \\
8.0\end{array}$ & $\begin{array}{l}6.6 \\
13 \\
4.01 \\
6.1 \\
24 \\
28 \\
11 \\
8.5 \\
.1 \\
5.5\end{array}$ & 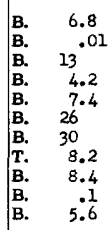 \\
\hline $\begin{array}{l}\text { Dissolved solids }-1-\text { Bottom } \\
\text { Hardness as } \mathrm{CaCO}_{3}-\ldots-\text { Bottom } \\
\text { Suspended sediment }-\ldots \text { Top }\end{array}$ & $\begin{array}{r}108 \\
53 \\
. \quad . \\
\end{array}$ & & $\begin{array}{r}105 \\
49 \\
\quad . \quad \mathrm{C} \\
\end{array}$ & & $\begin{array}{r}100 \\
49 \\
. \quad \cdot . \\
\end{array}$ & $\begin{array}{lr}\text { B. } & 104 \\
\text { B. } & 50 \\
\text { T. } & 42 \\
\end{array}$ \\
\hline
\end{tabular}


Table 10. --WATER ANALYSES OF DELAWARE RIVER BETWEEN BRISTOL AND MARCUS HOOK, PA. --ContinUed Analyzed by City of Philadelphia and $U$. S. Geological Survey; analyses in parts per million

Location Philadelphia, Pa.-Camden, N. J. Bridge Date January 3, 1952 __ Sampling study No. 29 Weather-Overcast

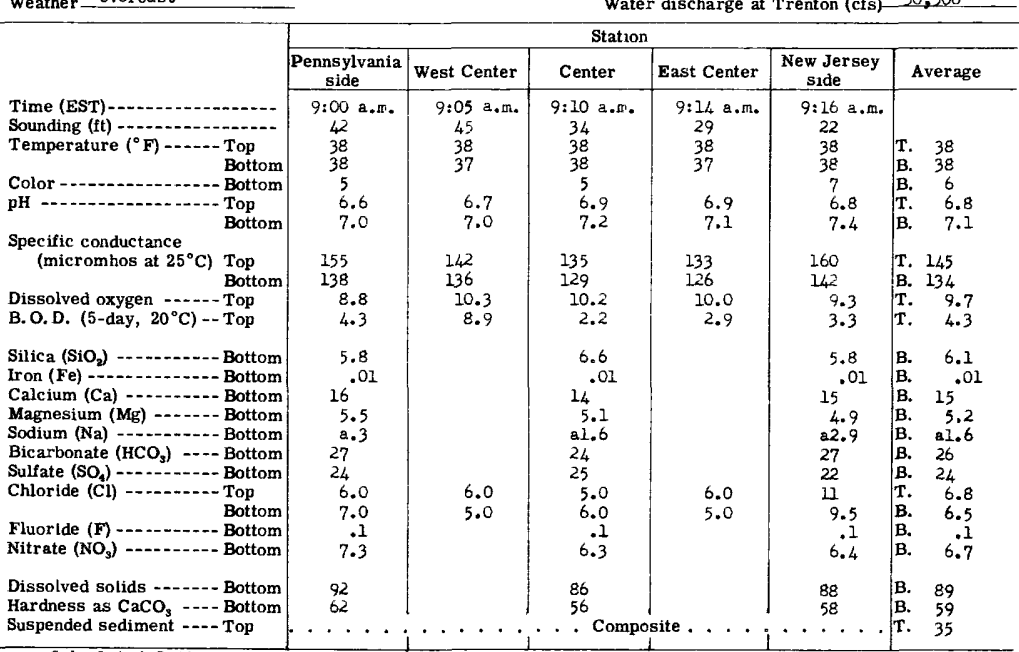

a Calculated Sodium and Potassium

Location Philadelphia, PQ,-Camden, N, J. Bridge Date February 5, 1952 Sampling study No. 30

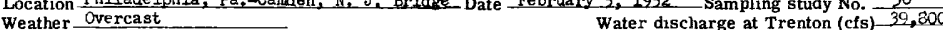

\begin{tabular}{|c|c|c|c|c|c|c|}
\hline \multirow{3}{*}{ Weather Overcast } & \multicolumn{6}{|c|}{ Water discliarge at Trenton (cfs) 39,300} \\
\hline & \multicolumn{6}{|c|}{ Station } \\
\hline & $\begin{array}{c}\text { Penns ylvania } \\
\text { side }\end{array}$ & West Center & Center & East Center & $\begin{array}{l}\text { New Jersey } \\
\text { side }\end{array}$ & Average \\
\hline 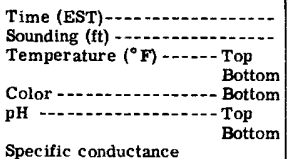 & $\begin{array}{c}12: 00 \mathrm{n} \\
45 \\
39 \\
39 \\
7 \\
6.3 \\
6.7\end{array}$ & $\begin{array}{c}11: 57 \mathrm{a} . \mathrm{m} \\
45 \\
39 \\
38 \\
7.1 \\
7.8\end{array}$ & $\begin{array}{c}11: 55 \text { a.m. } \\
37 \\
39 \\
38 \\
7 \\
6.6 \\
6.6\end{array}$ & $\begin{array}{c}11: 52 \text { a.m. } \\
35 \\
40 \\
38 \\
\\
6.5 \\
7.7\end{array}$ & $\begin{array}{c}11: 50 \text { a.m. } \\
39 \\
39 \\
38 \\
7 \\
6.9 \\
6.5\end{array}$ & $\begin{array}{lc}\text { T. } & 39 \\
\text { B. } & 38 \\
\text { B. } & 7 \\
\text { T. } & 6.7 \\
\text { B. } & 7.1\end{array}$ \\
\hline $\begin{array}{ll}\text { (micromhos at } 25^{\circ} \mathrm{C} \text { ) } & \begin{array}{l}\text { Top } \\
\text { Bottom }\end{array} \\
\text { Dissolved oxygen }-10^{\circ} \mathrm{C} \text { ) } & \text { Top } \\
\text { B. O. D. (5-day, }\end{array}$ & $\begin{array}{r}132 \\
132 \\
13.0 \\
6.4\end{array}$ & $\begin{array}{r}120 \\
132 \\
12.7 \\
4.6\end{array}$ & $\begin{array}{r}120 \\
120 \\
12.5 \\
4.5\end{array}$ & $\begin{array}{l}126 \\
121 \\
12.1 \\
4.3\end{array}$ & $\begin{array}{l}119 \\
121 \\
12.3 \\
5.2\end{array}$ & $\begin{array}{lr}\text { T. } & 123 \\
\text { B. } & 125 \\
\text { T. } & 12.5 \\
\text { T. } & 5.0\end{array}$ \\
\hline 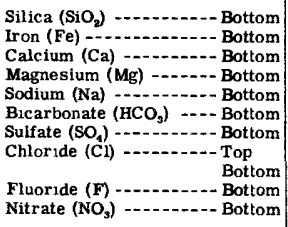 & $\begin{array}{l}6.4 \\
12.06 \\
4.5 \\
3.2 \\
25 \\
24 \\
5.0 \\
5.0 \\
.1 \\
4.4\end{array}$ & $\begin{array}{l}4.0 \\
4.0\end{array}$ & $\begin{array}{l}6.2 \\
.06 \\
11.6 \\
2.3 \\
2.5 \\
22 \\
4.0 \\
4.0 \\
.1 \\
3.8\end{array}$ & $\begin{array}{l}5.0 \\
4.0\end{array}$ & $\begin{array}{l}6.4 \\
.07 \\
11 \\
4.5 \\
2.4 \\
23 \\
22 \\
4.0 \\
4.2 \\
.1 \\
4.2\end{array}$ & $\begin{array}{ll}\text { B. } & 6.3 \\
\text { B. } & .06 \\
\text { B. } & 11 \\
\text { B. } & 4.5 \\
\text { B. } & 2.6 \\
\text { B. } & 24 \\
\text { B. } & 23 \\
\text { T. } & 4.4 \\
\text { B. } & 4.2 \\
\text { B. } & .1 \\
\text { B. } & 4.1\end{array}$ \\
\hline 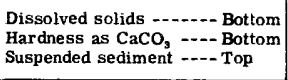 & $\begin{array}{r}107 \\
48 \\
. \quad .\end{array}$ & & $\begin{array}{l}94 \\
\quad 46 \\
+\quad \text { Com }\end{array}$ & 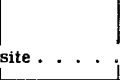 & $\begin{array}{l}92 \\
46 \\
.\end{array}$ & $\begin{array}{ll}\text { B. } & 98 \\
\text { B. } & 47 \\
\text { T. } & 35\end{array}$ \\
\hline
\end{tabular}


Table 10. - WATER ANALYSES OF DELAWARE RIVER BETWEEN BRISTOL AND MARCUS HOOK, PA. --Continued Analyzed by City of Philadelphia and U. S. Geological Survey: analyses in parts per million

\begin{tabular}{|c|c|c|c|c|c|c|}
\hline \multirow[t]{3}{*}{$\begin{array}{l}\text { Location Philadelphla, Pa.-Can } \\
\text { Weather Slightly Cloudy }\end{array}$} & \multicolumn{6}{|c|}{$\begin{aligned} \operatorname{arch} 5,1752 & \text { Sampling study No. } \frac{31}{9,730} \\
\text { Water discharge at Trenton (cfs) } & \frac{9}{2}\end{aligned}$} \\
\hline & \multicolumn{6}{|c|}{ Station } \\
\hline & \begin{tabular}{|c|}
$\begin{array}{c}\text { Pennsylvania } \\
\text { Side }\end{array}$ \\
\end{tabular} & West Center & Center & East Center & \begin{tabular}{|c|} 
New Jersey \\
side
\end{tabular} & Average \\
\hline 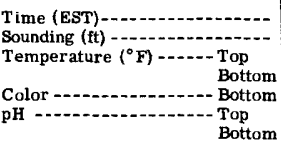 & $\begin{array}{c}12: 10 \text { p.m. } \\
46 \\
41 \\
41 \\
3 \\
7.6 \\
7.8\end{array}$ & $\begin{array}{c}12: 13 \mathrm{p.m} . \\
41 \\
41 \\
40 \\
\\
0.4 \\
7.5\end{array}$ & $\begin{array}{c}12: 16 \mathrm{p} . \mathrm{m} . \\
38 \\
41 \\
40 \\
4 \\
0.5 \\
7.9\end{array}$ & $\begin{array}{c}12: 20 \mathrm{p} . \mathrm{m} . \\
20 \\
40 \\
40 \\
\\
6.4 \\
7.5\end{array}$ & $\begin{array}{c}12: 23 \text { p.m. } \\
17 \\
40 \\
39 \\
4 \\
6.5 \\
7.7\end{array}$ & $\begin{array}{ll}\text { T. } & 41 \\
\text { B. } & 40 \\
\text { B. } & 4 \\
\text { T. } & 6.7 \\
\text { B. } & 7.7\end{array}$ \\
\hline $\begin{array}{l}\text { Specific conductance } \\
\quad \text { (micromhos at } 25^{\circ} \mathrm{C} \text { ) } \begin{array}{l}\text { Top } \\
\text { Bottom }\end{array} \\
\text { Dissolved oxygen -...- Top } \\
\text { B. O. D. (5-day, } 20^{\circ} \mathrm{C} \text { ) -- Top }\end{array}$ & $\begin{array}{l}191 \\
190 \\
7.7 \\
5.9\end{array}$ & $\begin{array}{r}186 \\
182 \\
8.6 \\
5.6\end{array}$ & $\begin{array}{r}177 \\
181 \\
9.2 \\
1.5\end{array}$ & $\begin{array}{r}170 \\
166 \\
8.6 \\
3.5\end{array}$ & $\begin{array}{r}190 \\
186 \\
9.7 \\
6.3\end{array}$ & $\begin{array}{lr} & \\
\text { T. } & 183 \\
\text { B. } & 181 \\
\text { T. } & 8.7 \\
\text { T. } & 4.6\end{array}$ \\
\hline 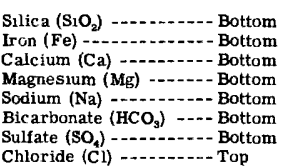 & $\begin{array}{l}6.2 \\
16 \\
16 \\
5.4 \\
10 \\
38 \\
33 \\
10\end{array}$ & 10 & $\begin{array}{l}7.0 \\
.08 \\
17^{.08} \\
5.2 \\
8.3 \\
44 \\
30 \\
8.0\end{array}$ & 8.0 & $\begin{array}{l}7.4 \\
.06 \\
17^{5.4} \\
7.6 \\
32 \\
31 \\
12\end{array}$ & $\begin{array}{ll}\text { B. } & 6.9 \\
\text { B. } & .07 \\
\text { B. } & 17 \\
\text { B. } & 5.3 \\
\text { B. } & 8.6 \\
\text { B. } & 38 \\
\text { B. } & 31 \\
\text { T. } & 9.6\end{array}$ \\
\hline $\begin{array}{l}\text { Fluoride }\langle\mathrm{F} \text { ) } \\
\text { Nitrate }\left(\mathrm{NO}_{3}\right)-\text { Bottom } \\
\end{array}$ & $\begin{array}{r}10 \\
9.9\end{array}$ & 10 & $\begin{array}{r}8.2 \\
.1 \\
7.6\end{array}$ & 9.0 & 8.12 & $\begin{array}{ll}\text { B. } & 9.8 \\
\text { B. } & .1 \\
\text { B. } & 8.6\end{array}$ \\
\hline $\begin{array}{l}\text { Dissolved solids - }---- \text { Bottom } \\
\text { Hardness as } \mathrm{CaCO}_{3}--- \text { Bottom } \\
\text { Suspended sediment ---- Top }\end{array}$ & $\begin{array}{r}115 \\
62 \\
.\end{array}$ & & $\begin{array}{r}108 \\
64 \\
. \quad C o r\end{array}$ & $0^{\circ}$ & $\begin{array}{l}109 \\
65 \\
.\end{array}$ & $\begin{array}{lr}\text { B. } & 111 \\
\text { B. } & 64 \\
\text { T. } & 29\end{array}$ \\
\hline
\end{tabular}

Location Philadelphia, Pa.-Camien, N. J. Bridge Date April 2, 1952 Sampling study No. 32

\begin{tabular}{|c|c|c|c|c|c|c|}
\hline \multirow{3}{*}{ Weather Clear } & \multicolumn{6}{|c|}{ Water discharge at Trenton (cfs) $-2,70$} \\
\hline & \multicolumn{6}{|c|}{ Station } \\
\hline & $\begin{array}{c}\text { Pennsylvania } \\
\text { side }\end{array}$ & West Center & Center & East Center & $\begin{array}{l}\text { Nev Jersey } \\
\text { side }\end{array}$ & Average \\
\hline $\begin{array}{l}\text { Time (EST) } \\
\text { Sounding (ft) } \\
\text { Temperature }\left({ }^{\circ} \mathrm{F}\right)- \\
\text { Color } \\
\text { pH }\end{array}$ & $\begin{array}{c}12: 22 \text { p.th. } \\
44 \\
50 \\
49 \\
6 \\
6.5 \\
7.0\end{array}$ & $\begin{array}{c}12: 19 \text { p.m. } \\
43 \\
49 \\
49 \\
\\
6.6 \\
7.7\end{array}$ & $\begin{array}{c}12: 16 \mathrm{p.m} \\
32 \\
50 \\
49 \\
6 \\
6.5 \\
6.9\end{array}$ & $\begin{array}{c}12: 13 \mathrm{p} \cdot \mathrm{m} . \\
23 \\
51 \\
50 \\
\\
6.4 \\
7.7\end{array}$ & $\begin{array}{c}12: 10 \mathrm{p} \cdot \mathrm{m} . \\
15 \\
51 \\
50 \\
7 \\
7.4 \\
6.7\end{array}$ & $\begin{array}{ll}\text { T. } & 50 \\
\text { B. } & 49 \\
\text { R. } & 6 \\
\mathbf{T .} & 6.7 \\
\mathbf{B} & 7.2\end{array}$ \\
\hline $\begin{array}{l}\text { Specific conductance } \\
\quad \text { (micromhos at } 25^{\circ} \mathrm{C} \text { ) Top } \\
\text { Bottom } \\
\text { Dissolved oxygen - }- \text { Top } \\
\text { B. O. D. (5-day, } 20^{\circ} \mathrm{C} \text { ) - Top }\end{array}$ & $\begin{array}{l}107 \\
105 \\
8.5 \\
4.6\end{array}$ & $\begin{array}{r}96.5 \\
95.4 \\
9.2 \\
1.7\end{array}$ & $\begin{array}{r}96.0 \\
91.6 \\
8.3 \\
1.0\end{array}$ & $\begin{array}{r}100 \\
95.4 \\
8.2 \\
2.1\end{array}$ & $\begin{array}{r}106 \\
99.1 \\
8.2 \\
2.2\end{array}$ & $\begin{array}{lr} & \\
\text { r. } & 101 \\
\text { R. } & 97.3 \\
\text { T. } & 8.5 \\
\text { r. } & 2.3\end{array}$ \\
\hline 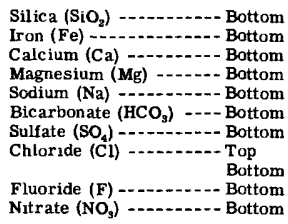 & $\begin{array}{l}4.3 \\
.06 \\
9.2 \\
2.9 \\
6.6 \\
22 \\
21 \\
5.0 \\
4.6 \\
.1 \\
5.2\end{array}$ & $\begin{array}{l}4.0 \\
3.0\end{array}$ & $\begin{array}{l}5.0 \\
.05 \\
9.2 \\
2.7 \\
6.4 \\
21 \\
22 \\
4.0 \\
3.8 \\
.1 \\
4.0\end{array}$ & $\begin{array}{l}4.0 \\
4.0\end{array}$ & $\begin{array}{l}5.9 \\
.06 \\
9.3 \\
3.0 \\
3.5 \\
18 \\
20 \\
4.0 \\
4.2 \\
.1 \\
5.3\end{array}$ & $\begin{array}{ll}\mathbf{R} & 5.1 \\
\mathbf{R} & .06 \\
\mathbf{R} & 9.2 \\
\mathbf{R} & 2.9 \\
\mathbf{R} & 5.5 \\
\mathbf{R} & 20 \\
\mathbf{R} & 21 \\
\mathbf{T} . & 4.2 \\
\mathbf{R} & 3.9 \\
\mathbf{R} & .1 \\
\mathbf{R} & 4.8\end{array}$ \\
\hline $\begin{array}{l}\text { Dissolved solids - }-1-\text { Bottom } \\
\text { Hardness as } \mathrm{CaCO}_{3}-\cdots \text { Bottom } \\
\text { Suspended sediment }-\cdots \text { Top }\end{array}$ & $\begin{array}{l}75 \\
35 \\
\cdots\end{array}$ & & $\begin{array}{l}72 \\
34 \\
\cdot \quad \mathrm{Co}\end{array}$ & site. . & $\begin{array}{r}70 \\
36 \\
.\end{array}$ & $\begin{array}{ll}\mathbf{R} & 72 \\
\mathbf{R} & 35 \\
\mathbf{T} & 24\end{array}$ \\
\hline
\end{tabular}


Table 10. --WATER ANALYSES OF DELAWARB RIVER BETWEEN BRISTOL AND MARCUS HOOK, PA. --Continuea Analyzed by City of Philadelphia and U. S. Geological Survey; analyses in parts per million

Location Philadelphia, Pa.-Ganden, N. J. Bridge_Date May 5, 1952

Sampling study No, 33

Weather Cloud

Water discharge at Trenton (cfs)- 18,700

\begin{tabular}{|c|c|c|c|c|c|c|}
\hline \multirow{3}{*}{ 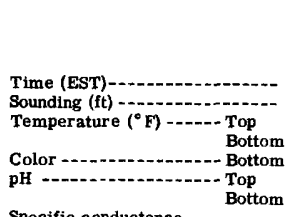 } & \multicolumn{6}{|c|}{ Station } \\
\hline & \begin{tabular}{|c} 
Pennsylvania \\
side
\end{tabular} & West Center & Center & East Center & $\begin{array}{c}\text { New Jersey } \\
\text { side } \\
\end{array}$ & Average \\
\hline & $\begin{array}{c}8: 53 \text { a.m. } \\
45 \\
58 \\
59 \\
7 \\
6.4 \\
6.9\end{array}$ & $\begin{array}{c}8: 50 \text { a.m. } \\
45 \\
58 \\
58 \\
\\
6.5 \\
6.3\end{array}$ & $\begin{array}{c}8: 46 \text { a.m. } \\
44 \\
58 \\
58 \\
7 \\
6.5 \\
6.8\end{array}$ & $\begin{array}{c}8: 43 \text { a.m. } \\
33 \\
58 \\
58 \\
\\
6.3 \\
6.3\end{array}$ & $\begin{array}{c}8: 40 \text { a.m. } \\
22 \\
58 \\
58 \\
5 \\
6.4 \\
7.0\end{array}$ & $\begin{array}{ll}\text { T. } & 58 \\
\text { B. } & 58 \\
\text { B. } & 6 \\
\text { T. } & 6.4 \\
\text { B. } & 6.7\end{array}$ \\
\hline $\begin{array}{l}\begin{array}{l}\text { Specific conductance } \\
\text { (micromhos at } 25^{\circ} \mathrm{C} \text { ) }\end{array} \\
\begin{array}{l}\text { Top } \\
\text { Bottom }\end{array} \\
\text { Dissolved oxygen - - - - Top } \\
\text { B. O. D. (5-day, } 20^{\circ} \mathrm{C} \text { ) - } \text { - Top }\end{array}$ & $\begin{array}{r}125 \\
114 \\
6.7 \\
1.6\end{array}$ & $\begin{array}{l}117 \\
122 \\
7.5 \\
1.6\end{array}$ & $\begin{array}{l}114 \\
112 \\
7.6 \\
1.8\end{array}$ & $\begin{array}{l}115 \\
115 \\
7.1 \\
2.4\end{array}$ & $\begin{array}{l}113 \\
113 \\
6.8 \\
3.1\end{array}$ & $\begin{array}{lr}\text { T. } & 117 \\
\text { B. } & 115 \\
\text { T. } & 7.1 \\
\text { T. } & 2.4\end{array}$ \\
\hline 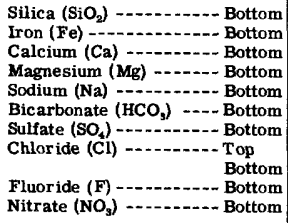 & $\begin{array}{l}5.9 \\
.03 \\
11 \\
3.6 \\
4.0 \\
25 \\
22 \\
6.0 \\
4.0 \\
.1 \\
4.6\end{array}$ & $\begin{array}{l}4.0 \\
5.0\end{array}$ & $\begin{array}{l}6.0 \\
11.06 \\
3.6 \\
3.1 \\
18 \\
26 \\
3.0 \\
3.4 \\
.1 \\
5.4\end{array}$ & $\begin{array}{l}4.0 \\
4.0\end{array}$ & $\begin{array}{l}5.6 \\
12.02 \\
3.8 \\
3.1 \\
32 \\
21 \\
3.0 \\
3.5 \\
.0 \\
2.9\end{array}$ & $\begin{array}{lc}\text { B. } & 5.8 \\
\text { B. } & .04 \\
\text { B. } & 11 \\
\text { B. } & 3.7 \\
\text { B. } & 3.4 \\
\text { B. } & 25 \\
\text { B. } & 23 \\
\text { T. } & 4.0 \\
\text { B. } & 4.0 \\
\text { B. } & .1 \\
\text { B. } & 4.3\end{array}$ \\
\hline $\begin{array}{l}\text { Dissolved solids } \\
\text { Hardness as } \mathrm{CaCO}_{3} \ldots \ldots \text { Bottom } \\
\text { Suspended sediment } \ldots \text { Top }\end{array}$ & $\begin{array}{r}69 \\
42 \\
. \\
\end{array}$ & & $\begin{array}{l}78 \\
42 \\
\text { Con }\end{array}$ & 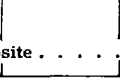 & $\begin{array}{r}74 \\
46 \\
\therefore\end{array}$ & $\begin{array}{ll}\text { B. } & 74 \\
\text { B. } & 43 \\
\text { T. } & 32 \\
\end{array}$ \\
\hline
\end{tabular}

Location Philadelphia, Pa,-Canden, N. J. Bxidge Date June 4, 1952

Sampling study No. 34 Weather Cloudy

Water discharge at Trenton (cfs) 22,600

\begin{tabular}{|c|c|c|c|c|c|c|}
\hline \multirow[b]{3}{*}{$\begin{array}{l}\text { Time (EST) } \\
\text { Sounding (ft) } \\
\text { Temperature ( }{ }^{\circ} \mathrm{F} \text { ) }-\begin{array}{l}\text { Top } \\
\text { Bottom }\end{array} \\
\text { Color } \\
\text { pH }\end{array}$} & \multicolumn{6}{|c|}{ Station } \\
\hline & \begin{tabular}{|c|}
$\begin{array}{c}\text { Pennsylvania } \\
\text { side }\end{array}$ \\
\end{tabular} & West Center & Center & East Center & $\begin{array}{c}\text { New Jersey } \\
\text { syde }\end{array}$ & Aver age \\
\hline & $\begin{array}{c}8: 32 \text { a. } \pi . \\
37 \\
67 \\
66 \\
12 \\
6.4 \\
6.9\end{array}$ & $\begin{array}{c}8: 33 \text { a.m. } \\
33 \\
66 \\
67 \\
6.4 \\
6.5\end{array}$ & $\begin{array}{l}8: 35 \text { a.m. } \\
44 \\
67 \\
67 \\
12 \\
6.7^{-} \\
6.9\end{array}$ & $\begin{array}{c}8: 38 \text { a. } 5 . \\
22 \\
66 \\
67 \\
6.6 \\
6.5\end{array}$ & $\begin{array}{l}8: 40 \mathrm{a}, \mathrm{m} . \\
19 \\
66 \\
66 \\
12 \\
6.5 \\
6.8\end{array}$ & $\begin{array}{ll}\text { T. } & 66 \\
\text { B. } & 67 \\
\text { B. } & 12 \\
\text { T. } & 6.5 \\
\text { B. } & 6.7\end{array}$ \\
\hline $\begin{array}{l}\begin{array}{l}\text { Specific conductance } \\
\quad\left(\text { micromhos at } 25^{\circ} \mathrm{C}\right)\end{array} \\
\begin{array}{l}\text { Top } \\
\text { Bottom }\end{array} \\
\begin{array}{l}\text { Dissolved oxygen } \\
\text { B. O.D. (5-day, } 20^{\circ} \mathrm{C} \text { ) - Top }\end{array} \\
\text { - Top }\end{array}$ & $\begin{array}{l}118 \\
122 \\
4.7 \\
1.0\end{array}$ & $\begin{array}{l}118 \\
120 \\
6.1 \\
3.5\end{array}$ & $\begin{array}{l}112 \\
110 \\
6.7 \\
2.2\end{array}$ & $\begin{array}{l}106 \\
109 \\
7.0 \\
1.9\end{array}$ & $\begin{array}{l}110 \\
108 \\
6.8 \\
2.6\end{array}$ & $\begin{array}{l}\text { T. } 113 \\
\text { B. } 114 \\
\text { T. } 6.3 \\
\text { T. } \quad 2.2\end{array}$ \\
\hline $\begin{array}{l}\text { Silica }\left(\mathrm{SiO}_{2}\right) \\
\text { Iron }(\mathrm{Fe}) \\
\text { Calcium }(\mathrm{Ca}) \\
\text { Magnesium }(\mathrm{Mg}) \text { - Bottom } \\
\text { Sodium (Na) } \\
\text { Bicarbonate (HCO, }) \text { - Bottom } \\
\text { Sulfate (SO, }\end{array}$ & $\begin{array}{l}5.7 \\
11.06 \\
3.9 \\
4.7 \\
28 \\
22\end{array}$ & & $\begin{array}{l}5.8 \\
.04 \\
11^{3} \\
3.7 \\
3.6 \\
26 \\
20\end{array}$ & & $\begin{array}{l}5.7 \\
.04 \\
11 \\
3.6 \\
2.0 \\
20 \\
20\end{array}$ & $\begin{array}{ll}\text { B. } & 5.7 \\
\text { B. } & .05 \\
\text { B. } & 11 \\
\text { B. } & 3.7 \\
\text { B. } & 3.4 \\
\text { B. } & 25 \\
\text { B. } & 21\end{array}$ \\
\hline 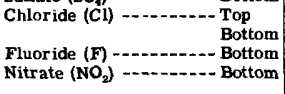 & $\begin{array}{r}4.0 \\
5.0 \\
.1 \\
5.1\end{array}$ & $\begin{array}{l}4.0 \\
4.0\end{array}$ & $\begin{array}{r}4.0 \\
3.8 \\
.1 \\
4.9\end{array}$ & $\begin{array}{l}3.0 \\
4.0\end{array}$ & $\begin{array}{r}4.0 \\
3.5 \\
.1 \\
5.6\end{array}$ & $\begin{array}{lr}\text { T. } & 3.8 \\
\text { B. } & 4.1 \\
\text { B. } & .1 \\
\text { B. } & 5.2\end{array}$ \\
\hline 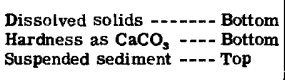 & $\begin{array}{l}73 \\
44 \\
.\end{array}$ & & $\begin{array}{l}68 \\
43 \\
.\end{array}$ & . & $\begin{array}{r}68 \\
42 \\
\therefore\end{array}$ & $\begin{array}{ll}\text { B. } & 70 \\
\text { B. } & 43 \\
\text { T. } & 36\end{array}$ \\
\hline
\end{tabular}


Table 10. -- WATER ANALYSES OF DELAWARE RIVER BETWEEN BRISTOL AND MARCUS HOOK, PA. --Continued Analyzed by City of Philadelphia and U. S. Geological Survey; analyses in parts per million

Location Philadelphia, Pa.-Camden, N. J. Bridge Date July 7, 1952

Sampling study No, Weather Light Clouds

\begin{tabular}{|c|c|c|c|c|c|c|}
\hline \multirow[b]{3}{*}{ 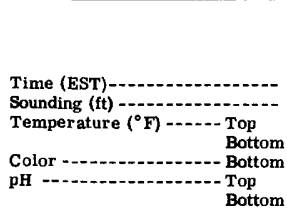 } & \multicolumn{6}{|c|}{ Station } \\
\hline & \begin{tabular}{|c} 
Pennsylvania \\
side
\end{tabular} & West Center & Center & East Center & $\begin{array}{c}\text { New Jersey } \\
\text { side }\end{array}$ & Average \\
\hline & $\begin{array}{c}8: 50 \text { a.m. } \\
35 \\
80 \\
80 \\
8 \\
6.7 \\
7.4\end{array}$ & $\begin{array}{c}8: 52 \text { a.m. } \\
39 \\
80 \\
80 \\
6.6 \\
6.8\end{array}$ & $\begin{array}{c}8: 54 \text { s.m. } \\
38 \\
79 \\
79 \\
6 \\
6.7 \\
7.4\end{array}$ & $\begin{array}{c}9: 00 \text { a.m. } \\
37 \\
79 \\
80 \\
6.7 \\
6.8\end{array}$ & $\begin{array}{c}9: 02 \text { a.r. } \\
19 \\
79 \\
79 \\
8 \\
6.6 \\
7.3\end{array}$ & $\begin{array}{ll}\text { T. } & 79 \\
\text { B. } & 80 \\
\text { B. } & 7 \\
\text { T. } & 6.7 \\
\text { B. } & 7.1\end{array}$ \\
\hline $\begin{array}{l}\text { Specific conductance } \\
\quad \text { (micromhos at } 25^{\circ} \mathrm{C} \text { ) } \begin{array}{l}\text { Top } \\
\text { Bottom }\end{array} \\
\text { Dissolved oxygen }-10^{\circ} \mathrm{C} \text { )- Top } \\
\text { B. Top }\end{array}$ & $\begin{array}{r}245 \\
223 \\
1.7\end{array}$ & $\begin{array}{r}202 \\
214 . \\
1.0\end{array}$ & $\begin{array}{r}198 \\
194 \\
1.0 \\
.0\end{array}$ & $\begin{array}{r}194 \\
198 \\
1.1 \\
.0\end{array}$ & $\begin{array}{r}210 \\
199 \\
1.5 \\
.3\end{array}$ & $\begin{array}{lr}\text { T. } & 210 \\
\text { B. } & 206 \\
\text { T. } & 1.0 \\
\text { T. } & .4\end{array}$ \\
\hline 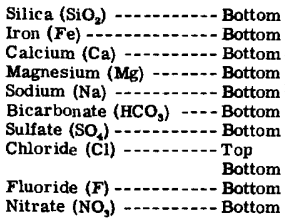 & $\begin{array}{l}6.0 \\
19 \\
6.02 \\
13 \\
52 \\
37 \\
12 \\
12 \\
12.1\end{array}$ & $10^{9.0}$ & $\begin{array}{l}6.4 \\
.02 \\
18 \\
6.2 \\
9.0 \\
51 \\
33 \\
9.0 \\
8.2 \\
.1 \\
3.0\end{array}$ & $\begin{array}{l}9.0 \\
9.0\end{array}$ & $\begin{array}{l}6.7 \\
.03 \\
17 \\
6.3 \\
10 \\
51 \\
33 \\
10 \\
8.8 \\
.1 \\
6.2\end{array}$ & $\begin{array}{ll}\text { B. } & 6.4 \\
\text { B. } & .02 \\
\text { B. } & 18 \\
\text { B. } & 6.4 \\
\text { B. } & 11 \\
\text { B. } & 51 \\
\text { B. } & 34 \\
\text { T. } & 9.8 \\
\text { B. } & 9.6 \\
\text { B. } & .1 \\
\text { B. } & 6.7\end{array}$ \\
\hline $\begin{array}{l}\text { Dissolved solids -- }--- \text { Bottom } \\
\text { Hardness as CaCO, }-- \text { Bottom } \\
\text { Suspended sediment }--- \text { Top }\end{array}$ & $\begin{array}{r}134 \\
75 \\
. \\
\end{array}$ & & $\begin{array}{l}116 \\
70 \\
.\end{array}$ & ite . . & $\begin{array}{r}119 \\
68 \\
.\end{array}$ & $\begin{array}{rr}\text { B. } & 123 \\
\text { B. } & 71 \\
\text { T. } & 18 \\
\end{array}$ \\
\hline
\end{tabular}

Location Philadelphia, Pa,-Camden, N. J. Bridge Date August 7, 1952 Sampling study No. _ 36 Weather Razn

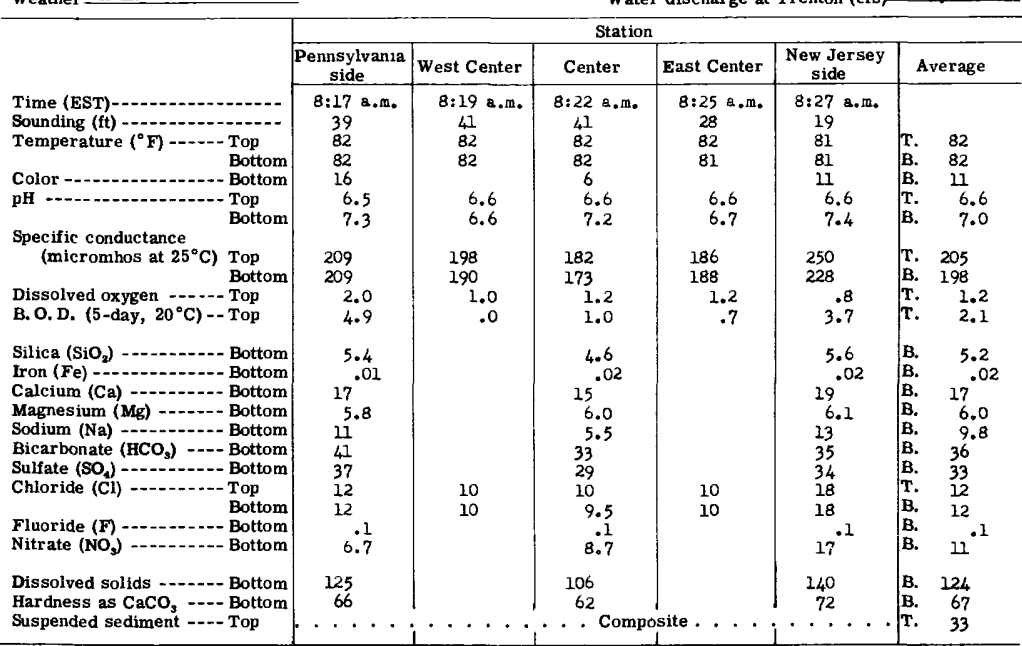


Table 10. -- WATER ANALYSES OF DELAWARE RIVER BETWEEN BRISTOL AND MARCUS HOOK, PA. --Cont InUEd Analyzed by City of Philadelphia and U. S. Geological Survey; analyses in parts per million

Location-Fhiladelphia, Fa.-Canden, Ne J. Bridge Date September 3, 1952 Sampling study No. 3 Weather Cloudy Water discharge at Trenton (cfs) 32,000

\begin{tabular}{|c|c|c|c|c|c|c|}
\hline \multirow[b]{3}{*}{$\begin{array}{l}\text { Time (EST) } \\
\text { Sounding (ft) } \\
\text { Temperature }\left({ }^{\circ} \mathrm{F}\right)-\begin{array}{l}\text { Top } \\
\text { Bottom }\end{array} \\
\text { Color } \\
\text { pH }-\begin{array}{l}\text { Bottom } \\
\text { Bottom }\end{array}\end{array}$} & \multicolumn{6}{|c|}{ Station } \\
\hline & $\begin{array}{c}\text { Pennsylvania } \\
\text { side }\end{array}$ & West Center & Center & East Center & \begin{tabular}{|c|} 
New Jersey \\
side
\end{tabular} & Average \\
\hline & $\begin{array}{c}8: 52 \text { a. } \mathrm{m} . \\
38 \\
78 \\
76 \\
1 \\
6.6 \\
7.6\end{array}$ & $\begin{array}{l}8: 54 \text { a.m. } \\
42 \\
76 \\
76 \\
\\
6.7 \\
6.7\end{array}$ & $\begin{array}{l}8: 58 \text { a.m. } \\
47 \\
76 \\
76 \\
2 \\
6.7 \\
7.5\end{array}$ & $\begin{array}{l}9: 00 \text { a.m. } \\
31 \\
76 \\
75 \\
6.5 \\
6.7\end{array}$ & $\begin{array}{l}9: 03 \text { a.m. } \\
21 \\
76 \\
76 \\
4 \\
6.7 \\
7.4\end{array}$ & $\begin{array}{l}76 \\
76 \\
2 \\
6.6 \\
7.2\end{array}$ \\
\hline 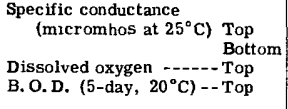 & $\begin{array}{l}204 \\
193 \\
4.7 \\
3.3\end{array}$ & $\begin{array}{l}182 \\
182 \\
6.7 \\
1.1\end{array}$ & $\begin{array}{l}182 \\
181 \\
6.7 \\
1.0\end{array}$ & $\begin{array}{r}178 \\
180 \\
6.8 \\
.7\end{array}$ & $\begin{array}{l}186 \\
201 \\
4.0 \\
3.0\end{array}$ & $\begin{array}{lr}\text { T. } & 186 \\
\text { B. } & 187 \\
\text { T. } & 5.8 \\
\text { T. } & 1.8\end{array}$ \\
\hline 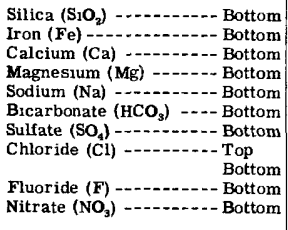 & $\begin{array}{l}2.0 \\
.01 \\
18 \\
8.0 \\
04.8 \\
50 \\
32 \\
11 \\
8.0 \\
.1 \\
2.9\end{array}$ & $\begin{array}{l}8.5 \\
8.0\end{array}$ & $\begin{array}{l}2.1 \\
.01 \\
17 \\
7.9 \\
2.9 .3 \\
47 \\
28 \\
8.5 \\
7.0 \\
.2 \\
7.8\end{array}$ & $\begin{array}{l}8.0 \\
8.0\end{array}$ & $\begin{array}{l}2.9 \\
.01 \\
17 \\
7.9 \\
a 6.1 \\
38 \\
33 \\
12 \\
12 \\
.2 \\
0.4\end{array}$ & $\begin{array}{l}2.3 \\
1.01 \\
17 \\
7.9 \\
05.1 \\
45 \\
31 \\
9.6 \\
8.6 \\
.2 \\
5.7\end{array}$ \\
\hline 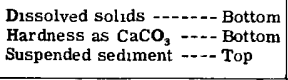 & $\begin{array}{r}116 \\
78 \\
. \quad .\end{array}$ & & $\begin{array}{l}108 \\
75 \\
.\end{array}$ & site. . & $\begin{array}{r}118 \\
75 \\
.\end{array}$ & $\begin{array}{lr}\text { B. } & 114 \\
\text { B. } & 76 \\
\text { T. } & 13\end{array}$ \\
\hline
\end{tabular}

a Calculated Sodiun and Potassiun.

Location Fhilgdelphia, Fa.-Camden, N. J. Bridge_Date Cctober 6, 1952 Sampling study No. 38 Weather Clear . . . .....

\begin{tabular}{|c|c|c|c|c|c|c|}
\hline & \multicolumn{6}{|c|}{ Station } \\
\hline & \begin{tabular}{|c} 
Pennsylvania \\
side
\end{tabular} & West Center & Center & East Center & \begin{tabular}{|c|}
$\begin{array}{c}\text { New Jersey } \\
\text { side }\end{array}$ \\
\end{tabular} & Average \\
\hline 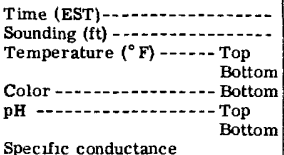 & $\begin{array}{c}10: 32 \text { a.mo } \\
39 \\
68 \\
67 \\
5 \\
6.7 \\
7.0\end{array}$ & $\begin{array}{c}10: 30 \mathrm{a}, \mathrm{m} . \\
39 \\
67 \\
07 \\
6.6 \\
6.6\end{array}$ & $\begin{array}{c}10: 21 \text { a.m. } \\
47 \\
67 \\
66 \\
8 \\
6.6 \\
7.7\end{array}$ & $\begin{array}{c}10: 25 \mathrm{a} . \mathrm{m} . \\
29 \\
67 \\
67 \\
6.5 \\
6.5\end{array}$ & $\begin{array}{c}10: 28 \text { a.m. } \\
29 \\
67 \\
66 \\
5 \\
6.6 \\
7.7\end{array}$ & $\begin{array}{l}67 \\
67 \\
6 \\
6.6 \\
7.1\end{array}$ \\
\hline $\begin{array}{ll}\text { (mpeclic conductance } & \\
\text { Dissolved oxygen } & \text { Bottom } \\
\left.\text { B. O. D. (5-day, } 20^{\circ} \mathrm{C}\right) & - \text { Top } \\
\text { Bop }\end{array}$ & $\begin{array}{l}237 \\
227 \\
1.6 \\
2.6\end{array}$ & $\begin{array}{r}215 \\
218 \\
2.0 \\
2.4\end{array}$ & $\begin{array}{r}206 \\
204 \\
3.6 \\
1.8\end{array}$ & $\begin{array}{r}210 \\
207 \\
2.6 \\
.8\end{array}$ & $\begin{array}{r}224 \\
214 \\
2.6 \\
1.2\end{array}$ & $\begin{array}{ll}\text { T. } & 218 \\
\text { B. } & 214 \\
\text { T. } & 2.5 \\
\text { T. } & 1.8\end{array}$ \\
\hline 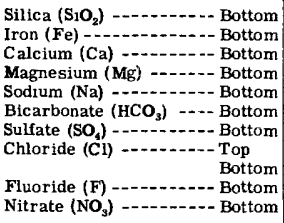 & $\begin{array}{l}2.7 \\
.03 \\
18 \\
6.9 \\
11 \\
43 \\
35 \\
14 \\
14 \\
11.1\end{array}$ & $\begin{array}{l}12 \\
11\end{array}$ & $\begin{array}{l}3.8 \\
17^{.03} \\
6.8 \\
9.6 \\
42 \\
32 \\
10 \\
11 \\
17^{.1}\end{array}$ & $\frac{11}{10}$ & $\begin{array}{l}3.2 \\
18.04 \\
6.6 \\
10 \\
42 \\
34 \\
13 \\
12 \\
12.1\end{array}$ & $\begin{array}{l}3.2 \\
18^{.03} \\
6.8 \\
10 \\
42 \\
34 \\
12 \\
12 \\
13^{.1}\end{array}$ \\
\hline $\begin{array}{l}\text { Dissolved solids }-1 .-- \text { Bottom } \\
\text { Hardness as } \mathrm{CaCO}_{3}-\ldots \text { Bottom } \\
\text { Suspended sediment }---- \text { Top }\end{array}$ & $\begin{array}{r}137 \\
73 \\
.\end{array}$ & & $\begin{array}{l}124 \\
70 \\
. \mathrm{Cc}\end{array}$ & & $\begin{array}{r}128 \\
72 \\
\quad .\end{array}$ & $\begin{array}{lr}\text { B. } & 130 \\
\text { B. } & 72 \\
\text { T. } & 9\end{array}$ \\
\hline
\end{tabular}


Table 10. - -WATER ANALYSES OF DELAWARE RIVER BETWEEN BRISTOL AND MARCUS HOOK, PA. --Continued Analyzed by City of Philadeiphia and U. S. Geological Survey; analyses in parts per million

Location Philadelphia, Pa.-Camden, N. J. Eridge Date November 6, 1952___. Sampling study No. - 39 Weather clear

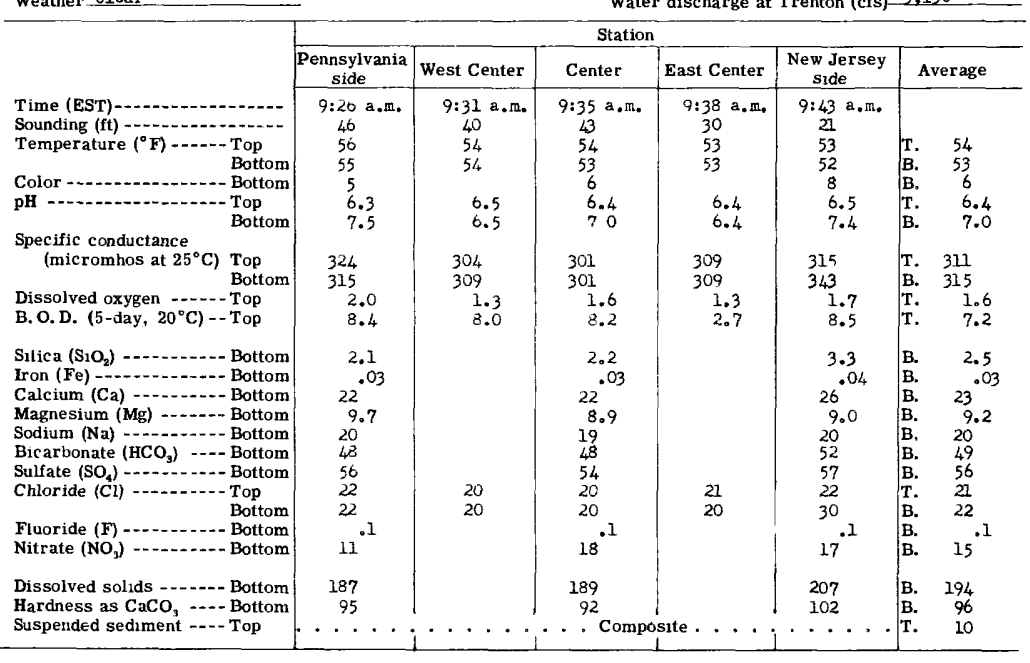

Location Philadelphia, Pa。-Camden, N, J, Bridge Date December 4, 1952 _- Sampling study No. - 40 Weather Clear

\begin{tabular}{|c|c|c|c|c|c|c|}
\hline \multirow{3}{*}{ Weather } & & & & & & \\
\hline & \multicolumn{6}{|c|}{ Station } \\
\hline & \begin{tabular}{|c|}
$\begin{array}{c}\text { Pennsylvania } \\
\text { side }\end{array}$ \\
\end{tabular} & West Center & Center & East Center & $\begin{array}{l}\text { New Jersey } \\
\text { side }\end{array}$ & Average \\
\hline 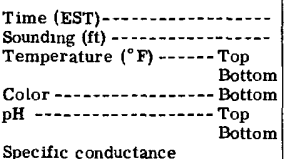 & $\begin{array}{c}9: 58 \text { a.m. } \\
42 \\
45 \\
44 \\
5 \\
6.2 \\
6.5\end{array}$ & $\begin{array}{c}10: 01 \text { a.m. } \\
45 \\
43 \\
4.4 \\
6.4 \\
6.3\end{array}$ & $\begin{array}{c}10: 04 \text { a.m. } \\
42 \\
42 \\
42 \\
8 \\
6.4 \\
6.9\end{array}$ & $\begin{array}{c}10: 07 \text { a.m. } \\
28 \\
42 \\
42 \\
\\
6.4 \\
6.4\end{array}$ & $\begin{array}{c}10: 10 \mathrm{a} . \mathrm{m} . \\
24 \\
1.2 \\
42 \\
5 \\
6.3 \\
6.6\end{array}$ & $\begin{array}{l}43 \\
43 \\
6 \\
6.3 \\
6.5\end{array}$ \\
\hline $\begin{array}{l}\text { Specific conductance } \\
\quad\left(\text { mic romhos at } 25^{\circ} \mathrm{C}\right) \\
\begin{array}{l}\text { Top } \\
\text { Bottom }\end{array} \\
\begin{aligned} \text { Dissolved oxygen } \\
\left.\text { B. O. D. (5-day, } 20^{\circ} \mathrm{C}\right)- \text { Top } \\
- \text { Top }\end{aligned}\end{array}$ & $\begin{array}{l}163 \\
163 \\
7.8 \\
7.7\end{array}$ & $\begin{array}{l}140 \\
142 \\
9.5 \\
5.4\end{array}$ & $\begin{array}{r}124 \\
124 \\
10.5 \\
3.1\end{array}$ & $\begin{array}{l}127 \\
126 \\
11.1 \\
3.8\end{array}$ & $\begin{array}{l}129 \\
126 \\
9.3 \\
3.8\end{array}$ & $\begin{array}{ll}\text { T. } & 137 \\
\text { B. } & 136 \\
\text { T. } & 9.6 \\
\text { T. } & 4.8\end{array}$ \\
\hline 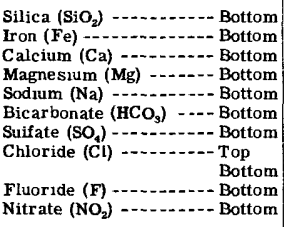 & $\begin{array}{l}5.0 \\
11.06 \\
5.2 \\
9.2 \\
22 \\
29 \\
9.5 \\
10^{.2} \\
10^{.1}\end{array}$ & $\begin{array}{l}7.5 \\
7.0\end{array}$ & $\begin{array}{l}4.7 \\
.09 \\
11 \\
4.3 \\
4.4 \\
21 \\
29 \\
6.0 \\
6.0 \\
.1 \\
6.5\end{array}$ & $\begin{array}{l}6.5 \\
6.0\end{array}$ & $\begin{array}{l}4.9 \\
.09 \\
11 \\
4.3 \\
4.4 \\
18 \\
24 \\
6.5 \\
6.0 \\
.1 \\
8.0\end{array}$ & $\begin{array}{ll}\text { B. } & 4.9 \\
\text { B. } & .08 \\
\text { B. } & 11 \\
\text { B. } & 4.6 \\
\text { B. } & 6.0 \\
\text { B. } & 20 \\
\text { B. } & 27 \\
\text { T. } & 7.2 \\
\text { B. } & 7.0 \\
\text { B. } & .1 \\
\text { B. } & 8.2\end{array}$ \\
\hline $\begin{array}{l}\text { Dissolved solids - }-1-\text { Bottom } \\
\text { Hardness as } \mathrm{CaCO}_{3}-\ldots-\text { Bottom } \\
\text { Suspended sediment --- Top }\end{array}$ & $\begin{array}{r}105 \\
49 \\
.\end{array}$ & & $\begin{array}{l}82 \\
45 \\
.\end{array}$ & ite . · & $\begin{array}{r}85 \\
45 \\
. \quad . \\
\end{array}$ & $\begin{array}{l}\mathrm{B} . \\
\mathrm{B} . \\
\mathbf{T}\end{array}$ \\
\hline
\end{tabular}


Table 11. -- WATER ANALYSES OF DELAWARE RIVER BETWEEN BRISTOL AND MARCUS HOOK, PA. Analyzed by City of Philadelphia and U. S. Geological Survey; analyses in parts per million

Location Wharton Street, Philadelphia, Pa, Date August 2, 1949___ Sampling study No. 1 Weather Cloudy

\begin{tabular}{|c|c|c|c|c|c|c|}
\hline \multicolumn{3}{|c|}{ Weather Cloudy } & \multicolumn{4}{|c|}{ Water discharge at Trenton (cfs) $-2,9<0$} \\
\hline & \multicolumn{6}{|c|}{ Station } \\
\hline & $\begin{array}{c}\text { Pennsylvania } \\
\text { side }\end{array}$ & West Center & Center & East Center & $\begin{array}{c}\text { New Jersey } \\
\text { side }\end{array}$ & Average \\
\hline $\begin{array}{ll}\text { Time (EST) } & \\
\text { Sounding (ft) } & \\
\text { Temperature }\left({ }^{\circ} \mathrm{F}\right) & \begin{array}{l}\text { Top } \\
\text { Bottom }\end{array} \\
\text { pH } & \text { Top } \\
\text { Bottom }\end{array}$ & 84 & $\begin{array}{l}\overline{45} \\
84 \\
84 \\
6.0 \\
6.0\end{array}$ & $\begin{array}{c}10: 45 \mathrm{a} . \mathrm{m} . \\
43 \\
83 \\
84 \\
5.9 \\
5.9\end{array}$ & $\begin{array}{l}-5 \\
84 \\
84 \\
6.1 \\
5.8\end{array}$ & $\begin{array}{l}- \\
84 \\
6.0\end{array}$ & $\begin{array}{lc}\text { T. } & 84 \\
\text { B. } & 84 \\
\text { T. } & 6.0 \\
\text { B. } & 5.9\end{array}$ \\
\hline 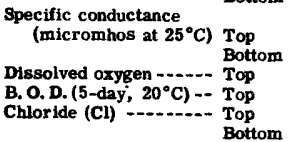 & $\begin{array}{l}283 \\
20.0 \\
18\end{array}$ & $\begin{array}{l}284 \\
285 \\
14.0 \\
18 \\
17\end{array}$ & $\begin{array}{l}283 \\
286 \\
17.0 \\
17 \\
18\end{array}$ & $\begin{array}{l}280 \\
277 \\
16.0 \\
17 \\
18\end{array}$ & $\begin{array}{l}280 \\
18.0 \\
17\end{array}$ & $\begin{array}{ll}\text { T. } & 282 \\
\text { B. } & 283 \\
\text { T. } & .0 \\
\text { T. } & 17.0 \\
\text { T. } & 17 \\
\text { B. } & 18\end{array}$ \\
\hline Suspended sediment ... Top & . & & . Com & ite. . & . & - \\
\hline
\end{tabular}

Location Whart on Street, Philadelphia, Pa. Date_September 7, 1949 Sampling study No._- 2 Weather Slight Rain

\begin{tabular}{|c|c|c|c|c|c|c|}
\hline \multicolumn{2}{|c|}{ Weather Slight Rain } & \multicolumn{5}{|c|}{ Water discharge at Trenton (cfs) $-3,810$} \\
\hline & \multicolumn{6}{|c|}{ Station } \\
\hline & \begin{tabular}{|c|}
$\begin{array}{c}\text { Pennsylvania } \\
\text { side }\end{array}$ \\
\end{tabular} & West Center & Center & East Center & $\begin{array}{c}\text { New Jersey } \\
\text { side }\end{array}$ & Average \\
\hline $\begin{array}{l}\text { Time (EST) } \\
\text { Sounding (ft) } \\
\text { Temperature ( }{ }^{\circ} \text { F) } \\
\text { pH }\end{array}$ & 78 & $\begin{array}{l}\overline{43} \\
78 \\
77 \\
6.5 \\
6.6\end{array}$ & $\begin{array}{l}2: 00 \text { p.m. } \\
45 \\
77 \\
77 \\
6.2 \\
6.5\end{array}$ & $\begin{array}{l}\overline{47} \\
78 \\
76 \\
6.3 \\
6.7\end{array}$ & $\begin{array}{l}- \\
77 \\
6.4\end{array}$ & $\begin{array}{ll}\text { T. } & 78 \\
\text { B. } & 77 \\
\text { T. } & 6.4 \\
\text { B. } & 6.6\end{array}$ \\
\hline $\begin{array}{ll}\text { Specific conductance } & \\
\left.\text { (micromhos at } 25^{\circ} \mathrm{C}\right) & \text { Top } \\
& \text { Bottom } \\
\text { Dissolved oxygen - } & \text { Top } \\
\text { B. O. D. (5-day, 20 } \mathrm{C} \text { ) - } & \text { Top } \\
\text { Chloride (Cl) } & \text { Top } \\
& \text { Bottom }\end{array}$ & $\begin{array}{l}366 \\
10.0 \\
35\end{array}$ & $\begin{array}{l}423 \\
401 \\
16.7 \\
47 \\
43\end{array}$ & $\begin{array}{l}428 \\
426 \\
.5 \\
12.0 \\
45 \\
48\end{array}$ & $\begin{array}{l}436 \\
436 \\
12.3 \\
51 \\
50\end{array}$ & $\begin{array}{l}420 \\
1.0 \\
10.5 \\
45\end{array}$ & $\begin{array}{l}\text { T. } 417 \\
\text { B. } 421 \\
\text { T. } .7 \\
\text { T. } 12.2 \\
\text { T. } 44 \\
\text { B. } 47\end{array}$ \\
\hline Suspended sediment -.. Top & . . . & . . &. Con & ite. . & $\cdot \cdot \cdot$ & T. 28 \\
\hline
\end{tabular}

Location Wharton Street, Philadelphia, Pa,

Date October 5, 1949 Sampling study No. 3

Water discharge at Trenton (cfs) $-3,280$

\begin{tabular}{|c|c|c|c|c|c|c|}
\hline & \multicolumn{6}{|c|}{ Station } \\
\hline & \begin{tabular}{|c|}
$\begin{array}{c}\text { Pennsylvania } \\
\text { side }\end{array}$ \\
\end{tabular} & West Center & Center & East Center & $\begin{array}{c}\text { New Jersey } \\
\text { side }\end{array}$ & Average \\
\hline $\begin{array}{ll}\text { Time (EST) } & \\
\text { Sounding (ft) } & \\
\text { Temperature }\left({ }^{\circ} \mathrm{F}\right) & \begin{array}{l}\text { Top } \\
\text { Bottom }\end{array} \\
\text { pH } & \begin{array}{l}\text { Top } \\
\text { Bottom }\end{array}\end{array}$ & $\begin{array}{l}-- \\
69 \\
6.7\end{array}$ & $\begin{array}{l}-75 \\
70 \\
70 \\
6.6 \\
6.7\end{array}$ & $\begin{array}{l}9: 30 \mathrm{a} . \mathrm{m} . \\
40 \\
69 \\
69 \\
6.6 \\
6.6\end{array}$ & $\begin{array}{l}-\overline{36} \\
70 \\
69 \\
6.5 \\
6.6\end{array}$ & $\begin{array}{l}- \\
69 \\
6.6\end{array}$ & $\begin{array}{ll}\text { T. } & 69 \\
\text { B. } & 69 \\
\text { T. } & 6.6 \\
\text { B. } & 6.6\end{array}$ \\
\hline 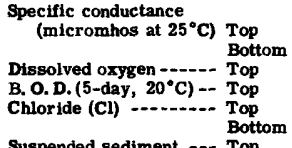 & $\begin{array}{l}381 \\
1.5 \\
8.3 \\
35\end{array}$ & $\begin{array}{l}386 \\
357 \\
1.5 \\
4.3 \\
36 \\
33\end{array}$ & $\begin{array}{l}362 \\
353 \\
1.7 \\
6.7 \\
32 \\
32\end{array}$ & $\begin{array}{l}363 \\
367 \\
1.6 \\
3.4 \\
34 \\
36\end{array}$ & $\begin{array}{l}389 \\
1.3 \\
5.3 \\
37\end{array}$ & $\begin{array}{ll}\text { T. } & 376 \\
\text { B. } & 359 \\
\text { T. } & 1.5 \\
\text { T. } & 5.6 \\
\text { T. } & 35 \\
\text { B. } & 34 \\
\text { T. } & 28\end{array}$ \\
\hline Suspended sediment & $\cdots$ & & . Comp & site. . * & $\cdots \cdot$ & T. 28 \\
\hline
\end{tabular}

Location Wharton Street, Philadelphia, Pa Weather Clear

Date November 2, 1949 Sampling study No.

\begin{tabular}{|c|c|c|c|c|c|c|}
\hline eather Clear & 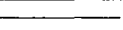 & & Wat & ischarge a & renton (cfs) & 3,430 \\
\hline & & & Station & & & \\
\hline & $\begin{array}{c}\text { Pennsylvania } \\
\text { side }\end{array}$ & West Center & Center & East Center & \begin{tabular}{|c|} 
New Jersey \\
side
\end{tabular} & Average \\
\hline $\begin{array}{ll}\text { Time (EST) } & \\
\text { Sounding (ft) } \\
\text { Temperature }\left({ }^{\circ} \mathrm{F} \text { ) }\right.\end{array} \begin{array}{l}\text { Top } \\
\text { Bottom } \\
\text { pH }\end{array}$ & 62 & $\begin{array}{l}\overline{43} \\
62 \\
60 \\
6.6 \\
6.6\end{array}$ & $\begin{array}{l}9: 30 \mathrm{a} . \mathrm{m} . \\
4.4 \\
60 \\
60 \\
6.5 \\
6.6\end{array}$ & $\begin{array}{l}\overline{38} \\
60 \\
60 \\
6.6 \\
6.6\end{array}$ & $\begin{array}{l}- \\
60 \\
6.6\end{array}$ & $\begin{array}{ll}\text { T. } & 61 \\
\text { B. } & 60 \\
\text { T. } & 6.6 \\
\text { B. } & 6.6\end{array}$ \\
\hline $\begin{array}{ll}\begin{array}{l}\text { Specific conductance } \\
\left.\text { (micromhos at } 25^{\circ} \mathrm{C}\right)\end{array} & \begin{array}{l}\text { Top } \\
\text { Bottom }\end{array} \\
\text { Dissolved oxygen - }-1 & \text { Top } \\
\text { B. O. D. (5-day, 20 C) -- Top } \\
\text { Chloride (Cl) }\end{array}$ & $\begin{array}{l}461 \\
1.0 \\
15.3 \\
59\end{array}$ & $\begin{array}{l}523 \\
509 \\
1.4 \\
11.7 \\
74 \\
72\end{array}$ & $\begin{array}{l}497 \\
493 \\
11.3 \\
11.7 \\
69 \\
71\end{array}$ & $\begin{array}{l}477 \\
468 \\
1.0 \\
8.1 \\
65 \\
64\end{array}$ & $\begin{array}{l}457 \\
1.6 \\
10.5 \\
60\end{array}$ & $\begin{array}{ll}\text { T. } & 483 \\
\text { B. } & 490 \\
\text { T. } & 1.3 \\
\text { T. } & 11.5 \\
\text { T. } & 65 \\
\text { B. } & 69\end{array}$ \\
\hline Suspended sediment -.- Top & & & . Com & ite. . . & 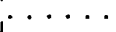 & $\mathrm{T}$. \\
\hline
\end{tabular}


Table 11. -- WATER ANALYSES OF DELAWARE RIVER BETWEEN BRISTOL AND MARCUS HOOK, PA, --COntinued Analyzed by City of Philadelphia and $U$. $\mathbf{S}$. Geological Survey; analyses in parts per million

Location Wharton Street, Philadelphia, Pa.___ Date Decenber 2, 1949 _ Sampling study No. 5 Weather Occasional Rain_ Water discharge at Trenton (cfs) 6.750

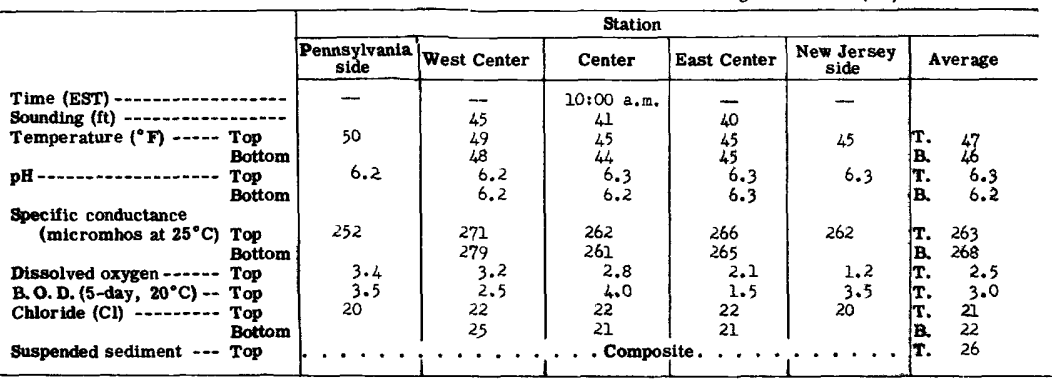

Location Wharton Street, Philadelohia, $\mathrm{Pa}$ Weather Cloudy

Date_January 5, 1950 Sampling study No. 6

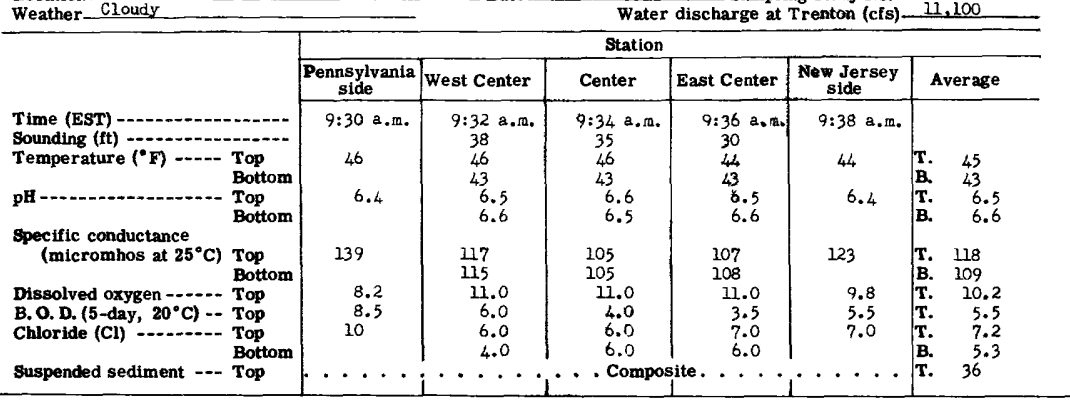

Location Wharton Street, Philadelphia, Pa. Weather clear

Date February 3, 1950 Sampling study No. 7

\begin{tabular}{|c|c|c|c|c|c|c|}
\hline \multicolumn{2}{|l|}{ Weather clear. } & \multicolumn{5}{|c|}{ Water discharge at Trenton (cfs) 14,300} \\
\hline & \multicolumn{6}{|c|}{ Station } \\
\hline & $\begin{array}{c}\text { Pennsylvania } \\
\text { side }\end{array}$ & West Center & Center & East Center & $\begin{array}{c}\text { New Jersey } \\
\text { side }\end{array}$ & Average \\
\hline $\begin{array}{ll}\text { Time (EST) } & \\
\text { Sounding }(\mathrm{ft}) & \\
\text { Temperature }\left({ }^{\circ} \mathrm{F}\right) & \begin{array}{l}\text { Top } \\
\text { Bottom }\end{array} \\
\text { pH } & \text { Top } \\
& \text { Bottom }\end{array}$ & $\begin{array}{c}9: 45 \text { a.m. } \\
43 \\
6.5\end{array}$ & $\begin{array}{l}9: 50 \text { a.m. } \\
41 \\
47 \\
41 \\
6.7 \\
6.6\end{array}$ & $\begin{array}{c}9: 52 \text { a.m. } \\
38 \\
39 \\
40 \\
6.8 \\
6.7\end{array}$ & $\begin{array}{l}9: 55 \text { a.m. } \\
34 \\
39 \\
40 \\
6.8 \\
6.7\end{array}$ & $\begin{array}{c}10: 00 \mathrm{a} . \mathrm{m} . \\
39 \\
6.7\end{array}$ & $\begin{array}{ll}\text { T. } & 40 \\
\text { B. } & 40 \\
\text { T. } & 6.7 \\
\text { B. } & 6.7\end{array}$ \\
\hline 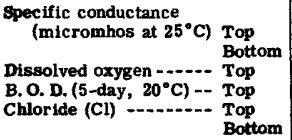 & $\begin{array}{l}151 \\
7.5 \\
7.5 \\
10\end{array}$ & $\begin{array}{l}132 \\
136 \\
9.5 \\
5.0 \\
9.0 \\
10\end{array}$ & $\begin{array}{r}218 \\
119 \\
10.0 \\
3.0 \\
8.0 \\
6.0\end{array}$ & $\begin{array}{r}111 \\
113 \\
10.7 \\
4.0 \\
7.0 \\
7.0\end{array}$ & $\begin{array}{r}126 \\
10.0 \\
6.5 \\
8.0\end{array}$ & $\begin{array}{ll}\text { T. } & 128 \\
\text { B. } & 123 \\
\text { T. } & 9.5 \\
\text { T. } & 5.2 \\
\text { T. } & 8.4 \\
\text { B. } & 7.7\end{array}$ \\
\hline Suspended sediment & 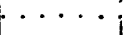 & & . Com & te... & $\cdots \cdot$ & T. \\
\hline
\end{tabular}

Location Wharton Street, Philadelphia, Pa, Weather Cloudy

Date March 7, 1950

Sampling study No. $\frac{8}{9}$

\begin{tabular}{|c|c|c|c|c|c|c|}
\hline \multicolumn{3}{|c|}{ Weather Cloudy } & \multicolumn{4}{|c|}{ Water discharge at Trenton (cfs) 7,260} \\
\hline & \multicolumn{6}{|c|}{ Station } \\
\hline & $\begin{array}{c}\text { Pennsylvania } \\
\text { side }\end{array}$ & West Center & Center & East Center & $\begin{array}{c}\text { New Jersey } \\
\text { side }\end{array}$ & Average \\
\hline $\begin{array}{ll}\text { Time (EST) } & \\
\text { Sounding (ft) } & \\
\text { Temperature }(" \mathrm{~F}) & \begin{array}{l}\text { Top } \\
\text { Bottom }\end{array} \\
\text { pH } & \begin{array}{l}\text { Top } \\
\text { Bottom }\end{array}\end{array}$ & $\begin{array}{c}3: 42 \text { p.m. } \\
37 \\
6.6\end{array}$ & $\begin{array}{l}3: 40 \text { p.ra. } \\
47 \\
37 \\
37 \\
6.6 \\
6.5\end{array}$ & $\begin{array}{l}3: 35 \text { p.m. } \\
43 \\
37 \\
36 \\
6.6 \\
6.5\end{array}$ & $\begin{array}{l}3: 34 p \cdot m . \\
41 \\
37 \\
37 \\
6.5 \\
6.5\end{array}$ & $\begin{array}{l}3: 30 \text { p.m. } \\
37 \\
6.5\end{array}$ & $\begin{array}{ll}\text { T. } & 37 \\
\text { B. } & 37 \\
\text { T. } & 6.6 \\
\text { B. } & 6.5\end{array}$ \\
\hline 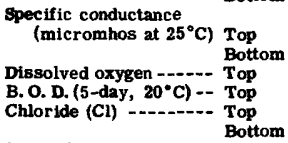 & $\begin{array}{l}189 \\
7.8 \\
8.4 \\
13\end{array}$ & $\begin{array}{l}187 \\
190 \\
8.0 \\
5.1 \\
10 \\
11\end{array}$ & $\begin{array}{l}186 \\
187 \\
8.5 \\
6.1 \\
10 \\
10\end{array}$ & $\begin{array}{l}188 \\
188 \\
8.3 \\
4 \cdot 4 \\
10 \\
10\end{array}$ & $\begin{array}{l}184 \\
8.4 \\
6.0 \\
10\end{array}$ & $\begin{array}{ll}\text { D. } & \\
\text { T. } & 187 \\
\text { B. } & 188 \\
\text { T. } & 8.2 \\
\text { T. } & 6.0 \\
\text { T. } & 10 \\
\text { B. } & 10\end{array}$ \\
\hline Suspended sediment --- Top & & $\cdots \cdot$ & Com & ite. . . & 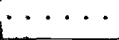 & $\mathbf{T}$ \\
\hline
\end{tabular}


Table 11. --WATER ANALYSES OF DELAWARE RIVER BETWEEN BRISTOL AND MARCUS HOOK, PA. --Continued Analyzed by City of Philadelphia and U. S. Geological Survey; analyses in parts per million

Location Tharton Street, Philadelphia, Pa, Weather Clouay

Date Apri1 3, 1250

Sampling study No. 9

\begin{tabular}{|c|c|c|c|c|c|c|}
\hline \multicolumn{3}{|c|}{ Weather Clouay } & \multicolumn{4}{|c|}{ Water discharge at Trenton (cfs) 32,300} \\
\hline & \multicolumn{6}{|c|}{ Station } \\
\hline & $\begin{array}{c}\text { Pennsylvania } \\
\text { side }\end{array}$ & West Center & Center & East Center & $\begin{array}{c}\text { New Jersey } \\
\text { side }\end{array}$ & Average \\
\hline $\begin{array}{ll}\text { Time (EST) } & \\
\text { Sounding }(f t) & \text { Top } \\
\text { Temperature }\left({ }^{\circ} \mathrm{F}\right) & \begin{array}{l}\text { Bottom } \\
\text { Top } \\
\text { BH }\end{array}\end{array}$ & $\begin{array}{c}3: 48 \text { p.m. } \\
44 \\
6.0\end{array}$ & $\begin{array}{l}3: 45 \mathrm{p}, \mathrm{m} . \\
49 \\
4.4 \\
44 \\
0.5 \\
5.6\end{array}$ & $\begin{array}{l}3: 43 \mathrm{p} . \mathrm{m} . \\
40 \\
44 \\
43 \\
6.5 \\
6.5\end{array}$ & $\begin{array}{l}3: 40 \mathrm{p} . \mathrm{m} . \\
37 \\
44 \\
44 \\
6.4 \\
6.4\end{array}$ & $\begin{array}{c}3: 37 \text { p.m. } \\
45 \\
6.4\end{array}$ & $\begin{array}{l}44 \\
44 \\
6.5 \\
6.5\end{array}$ \\
\hline 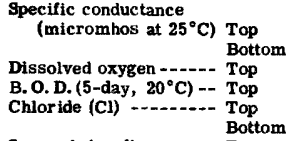 & $\begin{array}{r}83.9 \\
10.8 \\
4.6 \\
4.0\end{array}$ & $\begin{array}{r}83.0 \\
81.2 \\
10.5 \\
3.5 \\
4.0 \\
6.0\end{array}$ & $\begin{array}{r}79.8 \\
77.9 \\
11.0 \\
4.4 \\
4.0 \\
4.0\end{array}$ & $\begin{array}{r}78.6 \\
78.1 \\
10.9 \\
3.1 \\
4.0 \\
4.0\end{array}$ & $\begin{array}{r}79.4 \\
10.6 \\
4.6 \\
4.0\end{array}$ & $\begin{array}{r}80.9 \\
79.1 \\
10.8 \\
4.0 \\
4.0 \\
4.7\end{array}$ \\
\hline Suspended sediment --- Top & & & . Com & ite. . & $\dot{L} \cdot \cdots$ & 23 \\
\hline
\end{tabular}

Location Fharton Street, Philajelphia, $\mathrm{Pa}$.

Sampling study No. 10

\begin{tabular}{|c|c|c|c|c|c|c|}
\hline \multicolumn{3}{|c|}{ Weather Cloudy } & \multicolumn{4}{|c|}{ Water discharge at Trenton (cfs) 12,000} \\
\hline & \multicolumn{6}{|c|}{ Station } \\
\hline & \begin{tabular}{|c}
$\begin{array}{c}\text { Pennsylvania } \\
\text { side }\end{array}$ \\
\end{tabular} & West Center & Center & East Center & $\begin{array}{c}\text { New Jersey } \\
\text { side }\end{array}$ & Average \\
\hline $\begin{array}{l}\text { Time (EST) } \\
\text { Sounding (ft) } \\
\text { Temperature ( }{ }^{\circ} \mathrm{F} \text { ) }\end{array}$ & $\begin{array}{l}4: 40 \text { p.ra. } \\
55 \\
3.4\end{array}$ & $\begin{array}{l}4: 35 \mathrm{p} . \mathrm{m} . \\
45 \\
55 \\
54 \\
6.5 \\
6.5\end{array}$ & $\begin{array}{l}4: 32 \text { p.n. } \\
41 \\
55 \\
55 \\
6.5 \\
0.4\end{array}$ & $\begin{array}{l}4: 23 \text { p.m. } \\
37 \\
55 \\
54 \\
6.5 \\
6.4\end{array}$ & $\begin{array}{l}4: 25 \text { p.m. } \\
55 \\
6.5\end{array}$ & $\begin{array}{c}55 \\
54 \\
6.5 \\
6.4\end{array}$ \\
\hline 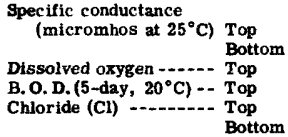 & $\begin{array}{l}140 \\
3.8 \\
8.3 \\
8.0\end{array}$ & $\begin{array}{r}135 \\
135 \\
4.4 \\
7.3 \\
7.0 \\
7.0\end{array}$ & $\begin{array}{l}136 \\
136 \\
4.9 \\
7.5 \\
7.0 \\
6.0\end{array}$ & $\begin{array}{r}135 \\
133 \\
4.9 \\
7.0 \\
7.0 \\
6.0\end{array}$ & $\begin{array}{r}134 \\
5.0 \\
4.5 \\
8.0\end{array}$ & $\begin{array}{lr}\text { T. } & 136 \\
\text { B. } & 135 \\
\text { T. } & 4.6 \\
\text { T. } & 6.9 \\
\text { T. } & 7.4 \\
\text { B. } & 6.3\end{array}$ \\
\hline Suspended sediment -- Top & 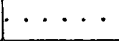 & $\cdots$ &. Com & site. ... & $\cdots \cdot$ & T. \\
\hline
\end{tabular}

Location Wharton otreet, Fhiladelphia, Pa. Weather clear Date June 5, 1950 Sampling study No. 11

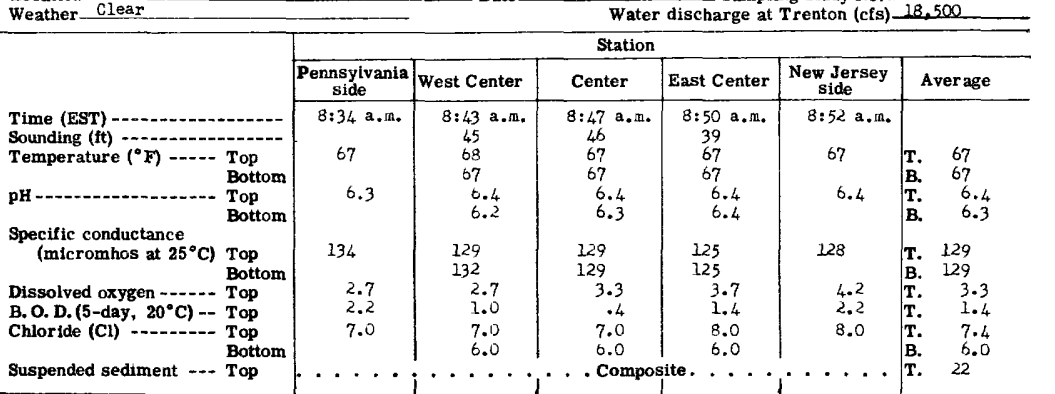

Location wharton street, PhIladelphia, Fa. Weather Cloudy Date July 5, 1950

Sampling study No. 12

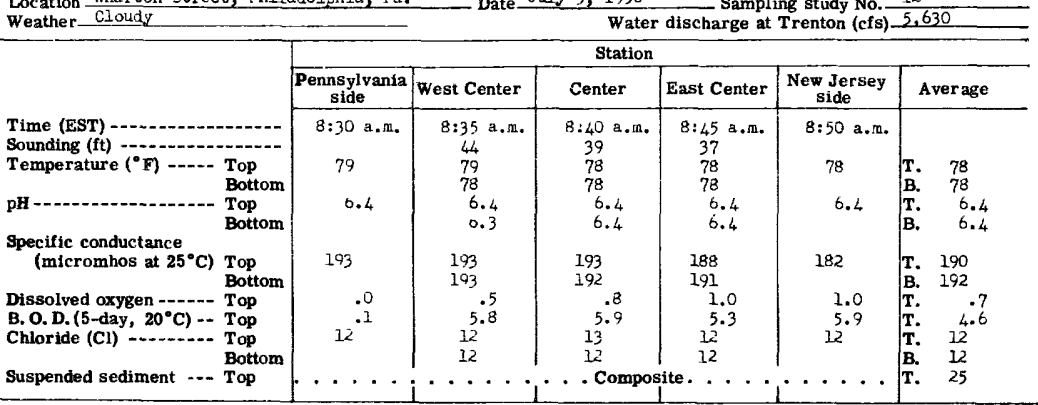


Table 11. --WATER ANALYSES OF DELAWARE RIVER BETWEEN BRISTOL AND MARC US HOOK, PA, --Continued Analyzed by City of Phladelphia and U. S. Geological Survey; analyses in parts per million

Location Wharton utreet, Philadelphia, Fa. Weather Light Rain

Date August 1, $1950 \quad$ Sampling study No 13

\begin{tabular}{|c|c|c|c|c|c|c|}
\hline \multicolumn{3}{|l|}{ Weather_Light Rain } & \multicolumn{4}{|c|}{ Water discharge at Trenton (cfs) 4 . 710} \\
\hline & \multicolumn{6}{|c|}{ Station } \\
\hline & \begin{tabular}{|c|}
$\begin{array}{c}\text { Pennsylvania } \\
\text { side }\end{array}$ \\
\end{tabular} & West Center & Center & East Center & $\begin{array}{l}\text { New Jersey } \\
\text { side }\end{array}$ & Average \\
\hline $\begin{array}{l}\text { Time (EST) } \\
\text { Sounding (ft) } \\
\text { Temperature }\left({ }^{\circ} \mathrm{F}\right) \\
\text { pH }\end{array}$ & $\begin{array}{c}8: 30 \text { a.m. } \\
80 \\
t .2\end{array}$ & $\begin{array}{l}8: 35 \mathrm{a} . \mathrm{m} . \\
43 \\
80 \\
80 \\
6.3 \\
6.4\end{array}$ & $\begin{array}{c}8: 40 \text { a.m. } \\
38 \\
80 \\
80 \\
6.2 \\
6.3\end{array}$ & $\begin{array}{l}8: 42 \text { a..... } \\
33 \\
80 \\
80 \\
6.1 \\
0.3\end{array}$ & $\begin{array}{c}8: 45 \text { a.m. } \\
80 \\
6.2\end{array}$ & $\begin{array}{lc}\text { T. } & 80 \\
\text { B. } & 80 \\
\text { T. } & 6.2 \\
\text { B. } & 6.3\end{array}$ \\
\hline 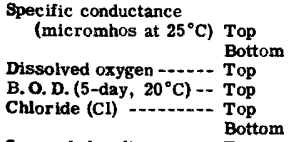 & $\begin{array}{l}199 \\
8.2 \\
12\end{array}$ & $\begin{array}{l}205 \\
211 \\
5.3 \\
10 \\
12\end{array}$ & $\begin{array}{l}203 \\
207 \\
2.3 \\
\frac{12}{12}\end{array}$ & $\begin{array}{l}192 \\
201 \\
11.0 \\
11\end{array}$ & $\begin{array}{l}217 \\
1.4 \\
12\end{array}$ & $\begin{array}{ll} & \\
\text { T. } & 203 \\
\text { B. } & 206 \\
\text { T. } & .3 \\
\text { T. } & 4.0 \\
\text { T. } & 11 \\
\text { B. } & 12\end{array}$ \\
\hline Suspended sediment & & &. $\mathrm{Com}$ & - & $\cdot \bullet \cdot$ & 28 \\
\hline
\end{tabular}

Location Wharton Street, Fh1ladelphis, Pa. Weather Clear

Date_Eeptember 5, 1950 Sampling study No. 14

\begin{tabular}{|c|c|c|c|c|c|c|}
\hline \multicolumn{3}{|c|}{ Weather clear } & \multicolumn{4}{|c|}{ Water discharge at Trenton (cfs) 5,800} \\
\hline & \multicolumn{6}{|c|}{ Station } \\
\hline & \begin{tabular}{|c|}
$\begin{array}{c}\text { Pennsylvania } \\
\text { side }\end{array}$ \\
\end{tabular} & West Center & Center & East Center & $\begin{array}{c}\text { New Jersey } \\
\text { side }\end{array}$ & Average \\
\hline $\begin{array}{ll}\text { Time (EST) } & \\
\text { Sounding (ft) } \\
\text { Temperature }\left({ }^{\circ} \mathrm{F}\right) \\
\text { pH }\end{array} \begin{array}{l}\text { Top } \\
\text { Bottom } \\
\text { Top } \\
\text { Bottom }\end{array}$ & $\begin{array}{c}8: 55 \text { a.m. } \\
78 \\
6.4\end{array}$ & $\begin{array}{l}9: 00 \text { a.m. } \\
46 \\
78 \\
77 \\
6.4 \\
6.4\end{array}$ & $\begin{array}{l}9: 05 \text { a.ja. } \\
42 \\
78 \\
77 \\
6.4 \\
0.4\end{array}$ & $\begin{array}{l}9: 10 \mathrm{a} . \mathrm{m} . \\
38 \\
78 \\
78 \\
6.4 \\
6.4\end{array}$ & $\begin{array}{l}9: 15 \mathrm{a} \cdot \mathrm{m} . \\
78 \\
6.4\end{array}$ & $\begin{array}{l}78 \\
77 \\
6.4 \\
6.4\end{array}$ \\
\hline 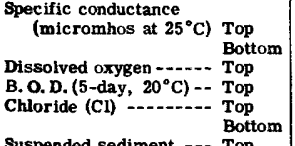 & $\overrightarrow{10}^{255}$ & $\begin{array}{l}255 \\
255 \\
7.0 \\
76 \\
10\end{array}$ & $\begin{array}{l}253 \\
251 \\
.0 \\
8.7 \\
16 \\
16\end{array}$ & $\begin{array}{l}251 \\
249 \\
7.5 \\
16 \\
16\end{array}$ & $\begin{array}{l}\therefore 45 \\
\quad .0 \\
6.9\end{array}$ & $\begin{array}{ll}\text { T. } & 252 \\
\text { B. } & 252 \\
\text { T. } & .0 \\
\text { T. } & 7.6 \\
\text { T. } & 16 \\
\text { B. } & 16 \\
\text { T. } & 19\end{array}$ \\
\hline Suspended sediment --- Top & & $\cdot \cdot \cdot$ & Comp & ite. . . & $\cdot \cdots \cdot$ & T. \\
\hline
\end{tabular}

Location Wherton otreet, Fhiladelphia, Pa. Weather-Misty

Date October 2, 1950 Sampling study No. 15

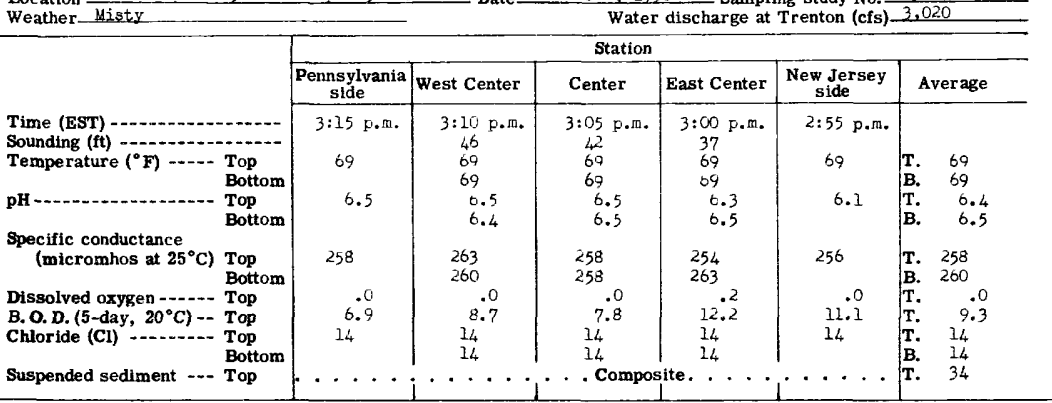

Location Wharton Street, Philadelphia, Pa. Weather Clear

\begin{tabular}{|c|c|c|c|c|c|}
\hline \multicolumn{2}{|l|}{-} & \multicolumn{4}{|c|}{ Water discharge at Trenton (cfs) $\underline{2}, 600$} \\
\hline \multicolumn{6}{|c|}{ Station } \\
\hline \begin{tabular}{|c} 
Pennsylvania \\
side \\
\end{tabular} & West Center & Center & East Center & $\begin{array}{l}\text { New Jersey } \\
\text { side }\end{array}$ & Average \\
\hline $3: 37$ p.m. & $3: 35 \mathrm{~F} \cdot \mathrm{m}$. & $3: 33$ p.m. & $3: 31 \mathrm{p} \cdot \mathrm{m}$. & $3: 29$ p.m. & \\
\hline 65 & $\begin{array}{l}65 \\
66\end{array}$ & $\begin{array}{l}05 \\
65\end{array}$ & $\begin{array}{l}64 \\
66\end{array}$ & 65 & $\begin{array}{l}65 \\
66\end{array}$ \\
\hline 6.6 & $\begin{array}{l}0.5 \\
6.5\end{array}$ & $\begin{array}{l}6.6 \\
6.2\end{array}$ & $\begin{array}{l}6.5 \\
6.2\end{array}$ & 6.5 & $\begin{array}{l}0.5 \\
0.3\end{array}$ \\
\hline 298 & $\begin{array}{l}310 \\
308\end{array}$ & $\begin{array}{l}308 \\
295\end{array}$ & $\begin{array}{l}304 \\
298\end{array}$ & 307 & $\begin{array}{ll}\text { T. } & 305 \\
\text { B. } & 300\end{array}$ \\
\hline .0 & .0 & .0 & .0 & .0 & T. \\
\hline $\begin{array}{l}12.0 \\
20\end{array}$ & $\begin{array}{l}10.5 \\
21 \\
21\end{array}$ & $\begin{array}{l}9.3 \\
21\end{array}$ & $\begin{array}{l}9.1 \\
20 \\
21\end{array}$ & $20^{7.8}$ & $\begin{array}{ll}\text { T. } & 9.5 \\
\text { T. } & 20 \\
\text { B. } & 21\end{array}$ \\
\hline . . & . & . Compos & te. . & $\cdots$ & $\mathbf{T}$. \\
\hline
\end{tabular}


Table 11. --WATER ANALYSES OF DELAWARE RIVER BETWEEN BRISTOL AND MARCUS HOOK, PA. --Continued Analyzed by City of Philadelphia and U. S. Geological Survey; analyses in parts per million

Location Wharton Street, Philadelphia, Fa. Weather Clear Date December 5, 1950 Sampling study No. 17

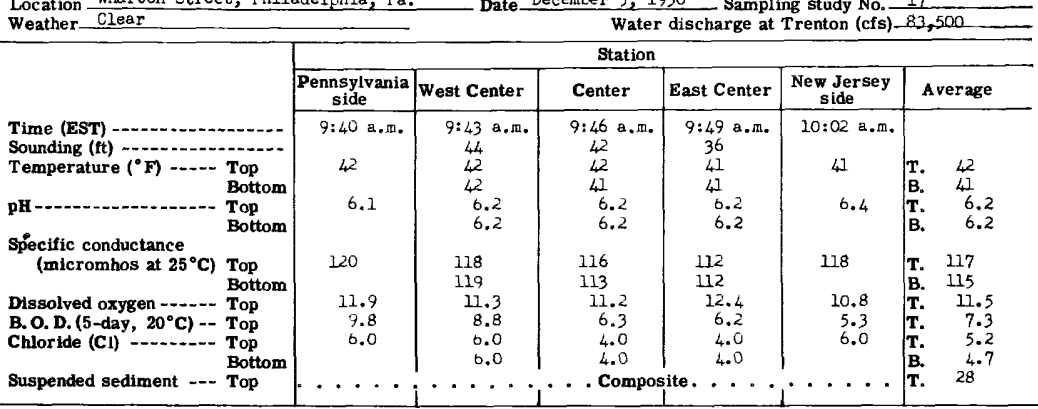

Location Wharton Street, Pniiadelphia, Fa. Weather Uvercast

Date January 2, 1951

Sampling study No. 18

\begin{tabular}{|c|c|c|c|c|c|c|}
\hline \multicolumn{3}{|c|}{ Weather Uvercast } & \multicolumn{4}{|c|}{ Water discharge at Trenton (cfs) $-8,670$} \\
\hline & \multicolumn{6}{|c|}{ Station } \\
\hline & $\begin{array}{c}\text { Pennsylvania } \\
\text { side }\end{array}$ & West Center & Center & East Center & $\begin{array}{c}\text { New Jersey } \\
\text { side }\end{array}$ & Average \\
\hline $\begin{array}{ll}\text { Time (EST) } \\
\text { Sounding (ft) } \\
\text { Temperature ( }{ }^{\circ} \mathrm{F} \text { ) } & \begin{array}{l}\text { Top } \\
\text { Bottom } \\
\text { Top }\end{array} \\
\text { Bottom }\end{array}$ & $\begin{array}{l}9: 45 \mathrm{am} . \\
36 \\
6.4\end{array}$ & $\begin{array}{l}9: 50 \text { a.m. } \\
45 \\
36 \\
36 \\
6.4 \\
6.3\end{array}$ & $\begin{array}{c}9: 55 \text { a.m. } \\
43 \\
36 \\
36 \\
6.4 \\
6.4\end{array}$ & $\begin{array}{c}10: 00 \text { a.m. } \\
38 \\
35 \\
35 \\
6.5 \\
6.3\end{array}$ & $\begin{array}{c}10: 05 \text { a.m. } \\
35 \\
6.4\end{array}$ & $\begin{array}{c}36 \\
36 \\
6.4 \\
6.3\end{array}$ \\
\hline 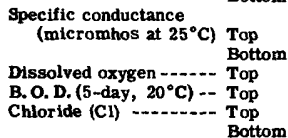 & $\begin{array}{c}186 \\
6.5 \\
67 \\
8.0\end{array}$ & $\begin{array}{l}194 \\
196 \\
6.7 \\
67 \\
8.0 \\
8.0\end{array}$ & $\begin{array}{l}196 \\
203 \\
6.4 \\
\mathrm{~b} 7 \\
8.0 \\
10\end{array}$ & $\begin{array}{r}182 \\
194 \\
6.5 \\
5.1 \\
8.0 \\
9.0\end{array}$ & $\begin{array}{l}181 \\
6.4 \\
4.3 \\
8.0\end{array}$ & $\begin{array}{lr}\text { T. } & 188 \\
\text { B. } & 198 \\
\text { T. } & 6.5 \\
\text { T. } & 66.1 \\
\text { T. } & 8.0 \\
\text { B. } & 9.0\end{array}$ \\
\hline Suspended sediment _-_ Top & $\cdot \cdot$ & $\therefore$ & . Com & site. . . & . . & $\mathbf{T}$ \\
\hline
\end{tabular}

b Exceeded this value.

Location Wharton Street, Philadelphia, Pa. Weather Rain

Date March 1, 1951

Sampling study No. 19

\begin{tabular}{|c|c|c|c|c|c|c|}
\hline Weather Rain & & & & ischarge & ( & 27,000 \\
\hline & & & Station & & & \\
\hline & $\begin{array}{c}\text { Pennsylvania } \\
\text { side }\end{array}$ & West Center & Center & East Center & $\begin{array}{c}\text { New Jersey } \\
\text { side }\end{array}$ & Average \\
\hline 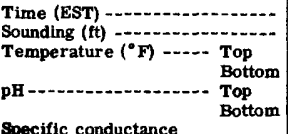 & $\begin{array}{c}10: 35 \mathrm{a} . \mathrm{m} \\
43 \\
6.5\end{array}$ & $\begin{array}{c}10: 40 \text { a.m. } \\
46 \\
42 \\
41 \\
0.5 \\
6.4\end{array}$ & $\begin{array}{c}10: 45 \mathrm{a} . \mathrm{m} . \\
41 \\
41 \\
40 \\
6.5 \\
6.4\end{array}$ & $\begin{array}{c}10: 50 \mathrm{a} . \mathrm{m} \\
41 \\
40 \\
40 \\
6.5 \\
6.5\end{array}$ & $\begin{array}{c}10: 53 \mathrm{a} . \mathrm{m} . \\
40 \\
6.5\end{array}$ & $\begin{array}{l}\text { T. } \\
\text { B. } \\
\text { T. } \\
\text { B. }\end{array}$ \\
\hline 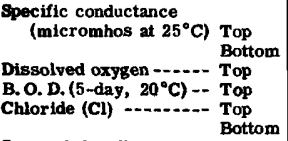 & $\begin{array}{l}126 \\
9.7 \\
b 10 \\
7.0\end{array}$ & $\begin{array}{l}115 \\
118 \\
10.9 \\
6.6 \\
5.0 \\
5.0\end{array}$ & $\begin{array}{l}116 \\
116 \\
10.1 \\
7.6 \\
6.0 \\
5.0\end{array}$ & $\begin{array}{r}113 \\
114 \\
11.6 \\
6.2 \\
5.0 \\
6.0\end{array}$ & $\begin{array}{r}108 \\
11.5 \\
5.4 \\
5.0\end{array}$ & $\begin{array}{lr} & \\
\text { T. } & 116 \\
\text { B. } & 116 \\
\text { T. } & 10.8 \\
\text { T. } & b 7.2 \\
\text { T. } & 5.6 \\
\text { B. } & 5.3\end{array}$ \\
\hline Suspended sediment -.. Top & & & . Comp & te. . . & $\cdots \cdot \cdot$ & T. \\
\hline
\end{tabular}

b Exceeded this value.

Location Wharton street, Philadelphia, $\mathrm{Pa}$. Weather Overcast

Date Apri1 3, $1251 \quad$ Sampling study No, 20

\begin{tabular}{|c|c|c|c|c|c|c|}
\hline \multirow[b]{3}{*}{ 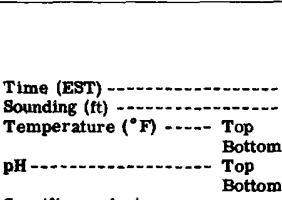 } & \multicolumn{6}{|c|}{ Station } \\
\hline & \begin{tabular}{|c|} 
Pennsylvania \\
side
\end{tabular} & West Center & Center & East Center & $\begin{array}{c}\text { New Jersey } \\
\text { side }\end{array}$ & Aver age \\
\hline & $\begin{array}{c}9: 30 \text { a.m. } \\
46 \\
6.7\end{array}$ & $\begin{array}{l}9: 33 \text { a.m. } \\
46 \\
46 \\
45 \\
6.8 \\
6.6\end{array}$ & $\begin{array}{l}9: 35 \mathrm{a} . \mathrm{m} . \\
44 \\
45 \\
45 \\
6.8 \\
6.8\end{array}$ & $\begin{array}{l}9: 38 \mathrm{a}, \mathrm{m} . \\
41 \\
45 \\
45 \\
6.7 \\
6.8\end{array}$ & $\begin{array}{c}9: 41 \text { a.m. } \\
45 \\
6.6\end{array}$ & $\begin{array}{l}45 \\
45 \\
6.7 \\
6.7\end{array}$ \\
\hline 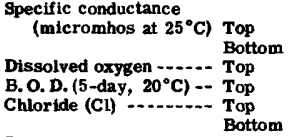 & $\begin{array}{r}78.2 \\
10.5 \\
1.9 \\
3.0\end{array}$ & $\begin{array}{r}71.0 \\
69.6 \\
10.4 \\
6.0 \\
3.0 \\
3.0\end{array}$ & $\begin{array}{r}66.0 \\
68.8 \\
11.0 \\
3.9 \\
3.0 \\
3.0\end{array}$ & $\begin{array}{r}66.0 \\
66.6 \\
10.9 \\
3.1 \\
3.0 \\
3.0\end{array}$ & $\begin{array}{r}67.6 \\
11.4 \\
4.0 \\
3.0\end{array}$ & $\begin{array}{lr}\text { T. } & 69.8 \\
\text { B. } & 68.3 \\
\text { T. } & 10.8 \\
\text { T. } & 3.8 \\
\text { T. } & 3.0 \\
\text { B. } & 3.0\end{array}$ \\
\hline Suspended sediment --- Top & 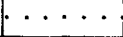 & & Com & ite. . . & 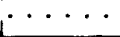 & $\mathbf{T}$ \\
\hline
\end{tabular}


Table 11. -- WATER ANALYSES OF DELAwARE RIVER BETWEen BRISTOL AND MARCUS HOOK, PA. --Continued Analyzed by City of Philadelphia and U. S. Geological Survey; analyses in parts per million

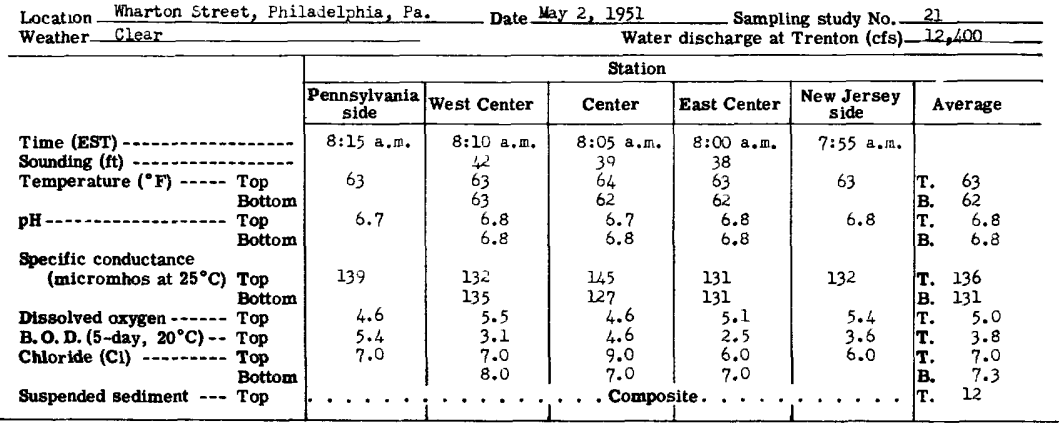

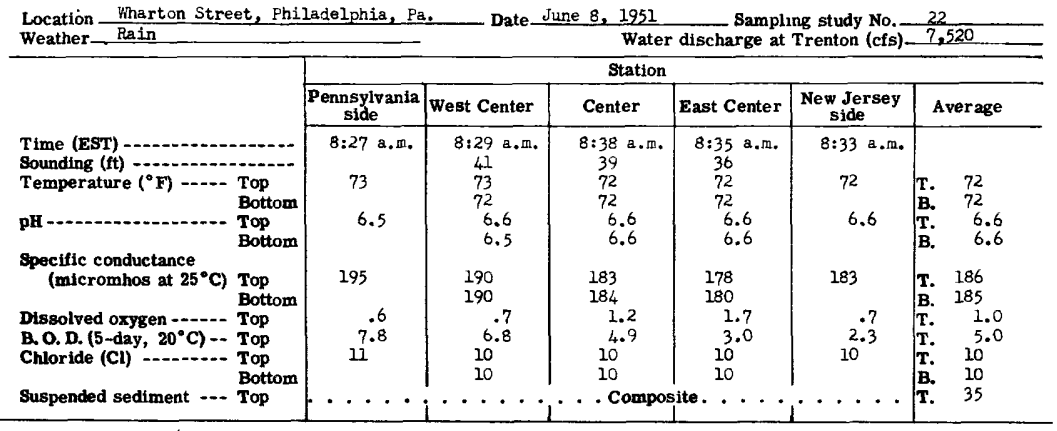

Location Tharton Street, Philadelphia, $\mathrm{Pa}$, Weather clear

\begin{tabular}{l|c|c|c|c|c|c} 
Location \\
Weather Clear.
\end{tabular}

Location Wharton Street, Philadelphia, Pa. Weather Clear

Date August 2, 1951

Sampling study No. 24

\begin{tabular}{|c|c|c|c|c|c|c|}
\hline \multicolumn{3}{|c|}{ Weather Clear } & \multicolumn{4}{|c|}{ Water discharge at Trenton (cfs) 10,300} \\
\hline & \multicolumn{6}{|c|}{ Station } \\
\hline & \begin{tabular}{|c|}
$\begin{array}{c}\text { Pennsylvania } \\
\text { side }\end{array}$ \\
\end{tabular} & West Center & Center & East Center & \begin{tabular}{|c|}
$\begin{array}{c}\text { New Jersey } \\
\text { side }\end{array}$ \\
\end{tabular} & Aver age \\
\hline 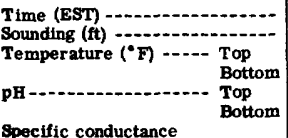 & $\begin{array}{c}1: 48 \text { p.m. } \\
80 \\
6.6\end{array}$ & $\begin{array}{l}1: 46 \text { p.m. } \\
46 \\
80 \\
81 \\
6.7 \\
6.8\end{array}$ & $\begin{array}{l}1: 44 \text { p.m. } \\
43 \\
80 \\
81 \\
7.1 \\
6.8\end{array}$ & $\begin{array}{l}1: 42 \mathrm{p} . \mathrm{m} . \\
42 \\
80 \\
81 \\
6.8 \\
6.7\end{array}$ & $\begin{array}{c}1: 40 \text { p.n. } \\
80 \\
6.8\end{array}$ & $\begin{array}{l}80 \\
81 \\
6.8 \\
6.8\end{array}$ \\
\hline 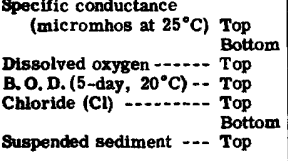 & $\begin{array}{r}300 \\
10^{-9} \\
\ldots\end{array}$ & $\begin{array}{l}199 \\
193 \\
2.3 \\
10.3 \\
10 \\
.\end{array}$ & $\begin{array}{l}230 \\
197 \\
2.4 \\
11 \\
10 \\
. \text { Com }\end{array}$ & $\begin{array}{r}197 \\
196 \\
3.0 \\
1.2 \\
10 \\
10 \\
\text { te. }\end{array}$ & $\begin{array}{r}195 \\
2.8 \\
1.2 \\
10\end{array}$ & $\begin{array}{ll}\text { T. } & 204 \\
\text { B. } & 195 \\
\text { T. } & 2.7 \\
\text { T. } & \bullet 9 \\
\text { T. } & 10 \\
\text { B. } & 10 \\
\text { T. } & 11\end{array}$ \\
\hline
\end{tabular}


Table 11. - WATER ANALYSES OF DELAWARE RIVER BETWEEN BRISTOL AND MARCUS HOOK, PA.--COntinued Analyzed by City of Philadelphia and U. S. Geological Survey; analyses in parts per million

Location Wharton Street, Philadelphia, Pa. Weather Cloudy

Date. September 5, 1951 Sampling study No. 25

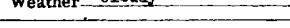

Time (EST) Temperature $\left({ }^{\circ} \mathrm{F}\right.$ - -1

$\begin{array}{ll} & \text { Bottom } \\ \text { pH-1 } & \text { Top }\end{array}$

(mieromhos at $25^{\circ} \mathrm{C}$ ) Top

Bottom

Dissolved oxygen -.....- To

B. O. D. (5-day, $\left.20^{\circ} \mathrm{C}\right)$-- Top

Chloride (Cl) $-\ldots$ Top

Suspended sediment --- Top

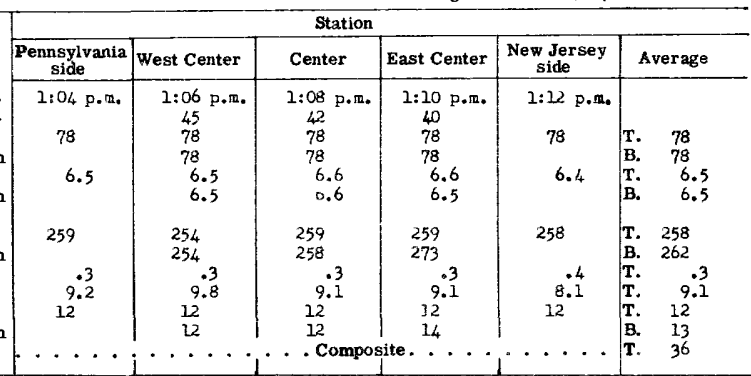

Location Thartion Stroet, Philadelphia, Pa. Weather Clear

Date October 1, 1951 Sampling study No._-26

\begin{tabular}{|c|c|c|c|c|c|c|}
\hline \multicolumn{2}{|c|}{ Weather Clear } & \multicolumn{5}{|c|}{ Water discharge at Trenton (cfs) 3,040} \\
\hline & \multicolumn{6}{|c|}{ Station } \\
\hline & $\begin{array}{c}\text { Pennsylvania } \\
\text { side }\end{array}$ & West Center & Center & East Center & $\begin{array}{l}\text { New Jersey } \\
\text { side }\end{array}$ & Aver age \\
\hline $\begin{array}{l}\text { Time (EST) } \\
\text { Sounding (ft) } \\
\text { Temperature }\left({ }^{\circ} \mathrm{F}\right)-\begin{array}{l}\text { Top } \\
\text { Bottom } \\
\text { pH }\end{array} \\
\begin{array}{l}\text { Top } \\
\text { Bottom }\end{array}\end{array}$ & $\begin{array}{c}3: 4.4+\mathrm{p} \cdot \mathrm{me} \\
72 \\
7.8\end{array}$ & $\begin{array}{l}3: 42 \mathrm{p} . \mathrm{m} . \\
46 \\
72 \\
72 \\
0.5 \\
6.4\end{array}$ & $\begin{array}{l}3: 40 \text { p.m. } \\
41 \\
72 \\
72 \\
6.5 \\
6.5\end{array}$ & $\begin{array}{l}3: 39 \text { p.m. } \\
38 \\
72 \\
72 \\
6.4 \\
6.6\end{array}$ & $\begin{array}{c}3: 38 \text { p.m. } \\
72 \\
6.5\end{array}$ & $\begin{array}{l}72 \\
72 \\
6.7 \\
6.5\end{array}$ \\
\hline 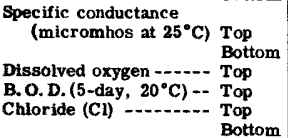 & $\begin{array}{l}306 \\
7.7 \\
7.2\end{array}$ & $\begin{array}{l}330 \\
327 \\
1.3 \\
4 \cdot 8 \\
22 \\
22\end{array}$ & $\begin{array}{l}336 \\
351 \\
1.2 \\
5.1 \\
22 \\
25\end{array}$ & $\begin{array}{l}330 \\
355 \\
2.1 \\
22 \\
25\end{array}$ & $\begin{array}{l}333 \\
3.9 \\
22\end{array}$ & $\begin{array}{ll}\text { T. } & 327 \\
\text { B. } & 344 \\
\text { T. } & .9 \\
\text { T. } & 4.6 \\
\text { T. } & 21 \\
\text { B. } & 24\end{array}$ \\
\hline Suspended sediment --- Top & $\cdots \cdot$ & $\cdots \cdot$ & . Comp & te. . . & . . & $\begin{array}{l}\text { D. } \\
\text { T. }\end{array}$ \\
\hline
\end{tabular}

Location Wharton Street, Philadelphia, Pa. Weather Clear

Date November 5, 1951 Sampling study No. 27

\begin{tabular}{|c|c|c|c|c|c|c|}
\hline \multirow[b]{3}{*}{$\begin{array}{l}\text { Time (EST) } \\
\text { Sounding (ft) } \\
\text { Temperature }\left({ }^{\circ} \mathrm{F}\right) \\
\text { pH }\end{array} \begin{array}{l}\text { Top } \\
\text { Bottom }\end{array}$} & \multicolumn{6}{|c|}{ Station } \\
\hline & $\begin{array}{c}\text { Pennsyivania } \\
\text { side }\end{array}$ & West Center & Center & East Center & $\begin{array}{c}\text { New Jersey } \\
\text { side }\end{array}$ & Average \\
\hline & $\begin{array}{c}3: 15 \mathrm{p} \cdot \mathrm{m} . \\
48 \\
6.3\end{array}$ & $\begin{array}{l}3: 18 \text { p.m. } \\
47 \\
48 \\
47 \\
6.5 \\
6.3\end{array}$ & $\begin{array}{l}3: 21 \mathrm{p} . \pi . \\
42 \\
48 \\
48 \\
6.5 \\
6.5\end{array}$ & $\begin{array}{l}3: 24 \mathrm{p} \cdot \mathrm{m} . \\
39 \\
47 \\
47 \\
6.5 \\
6.5\end{array}$ & $\begin{array}{c}3: 27 \mathrm{p} \cdot \mathrm{m} . \\
47 \\
6.6\end{array}$ & $\begin{array}{l}48 \\
47 \\
6.5 \\
6.4\end{array}$ \\
\hline 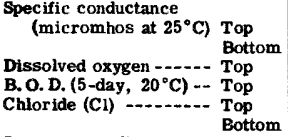 & $\begin{array}{l}161 \\
8.7 \\
6.7 \\
8.0\end{array}$ & $\begin{array}{r}151 \\
164 \\
8.9 \\
4.9 \\
6.0 \\
6.0\end{array}$ & $\begin{array}{l}149 \\
148 \\
9.3 \\
4.3 \\
6.0 \\
6.0\end{array}$ & $\begin{array}{r}146 \\
146 \\
9.2 \\
3.0 \\
6.0 \\
6.0\end{array}$ & $\begin{array}{l}48 \\
9.4 \\
3.1 \\
6.0\end{array}$ & $\begin{array}{ll}\text { T. } & 151 \\
\text { B. } & 153 \\
\text { T. } & 9.1 \\
\text { T. } & 4.4 \\
\text { T. } & 6.4 \\
\text { B. } & 6.0\end{array}$ \\
\hline Suspended sediment & & & Com & te... & & T. 32 \\
\hline
\end{tabular}

Location Wharton Street, Pniladelphia, Pa. Weather_Cloudy _......... Date December 4, 1951 Sampling study No. 28

\begin{tabular}{|c|c|c|c|c|c|c|}
\hline \multicolumn{7}{|c|}{ Weather Cloudy } \\
\hline \multirow[b]{2}{*}{$\begin{array}{ll}\text { Time (EST) } & \\
\text { Sounding }(\mathrm{ft}) & \\
\text { Temperature }\left({ }^{\circ} \mathrm{F}\right) & \begin{array}{l}\text { Top } \\
\text { Bottom }\end{array} \\
\text { pH } & \text { Bop } \\
& \text { Bottom }\end{array}$} & $\begin{array}{c}\text { Pennsylvania } \\
\text { side }\end{array}$ & West Center & Center & East Center & $\begin{array}{c}\text { New Jersey } \\
\text { side }\end{array}$ & Average \\
\hline & $\begin{array}{c}3: 20 \text { p.m. } \\
43 \\
6.4\end{array}$ & $\begin{array}{c}3: 16 \text { p.m. } \\
45 \\
42 \\
43 \\
6.4 \\
6.5\end{array}$ & $\begin{array}{l}3: 14 \mathrm{p} . \mathrm{m} . \\
43 \\
42 \\
42 \\
6.5 \\
6.5\end{array}$ & $\begin{array}{l}3: 12 \mathrm{p} . \mathrm{m} . \\
41 \\
4.2 \\
43 \\
6.6 \\
7.3\end{array}$ & $\begin{array}{c}3: 10 \mathrm{p.m} \\
4.2 \\
6.5\end{array}$ & $\begin{array}{l}42 \\
43 \\
6.5 \\
6.8\end{array}$ \\
\hline 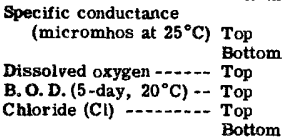 & $\begin{array}{l}161 \\
7.5 \\
7.2 \\
9.0\end{array}$ & $\begin{array}{r}155 \\
155 \\
8.2 \\
5.6 \\
8.0 \\
7.0\end{array}$ & $\begin{array}{r}144 \\
146 \\
9.6 \\
4.8 \\
6.0 \\
6.0\end{array}$ & $\begin{array}{r}145 \\
145 \\
9.4 \\
4.3 \\
6.0 \\
6.0\end{array}$ & $\begin{array}{r}147 \\
9.0 \\
2.0 \\
7.0\end{array}$ & $\begin{array}{lr}\text { T. } & 150 \\
\text { B. } & 149 \\
\text { T. } & 8.7 \\
\text { T. } & 4.8 \\
\text { T. } & 7.2 \\
\text { B. } & 6.3\end{array}$ \\
\hline Suspended sediment --- Top & - & & . Comp & te. . . & $\cdots \cdots$ & 36 \\
\hline
\end{tabular}


Table 11. - - WATER ANALYSES OF DE L.AWARE RIVER BETWEEN BRISTOL AND MARCUS HOOK, PA. - -Continued Analyzed by City of Philadelphia and U. S. Geological Survey; analyses in parts per million

Location Wharton Street, Philadelphia, Pa. Date January 2, 1952 Sampling study No. 29 Weather Gvercast Water discharge at Trenton (cfs) 21,800

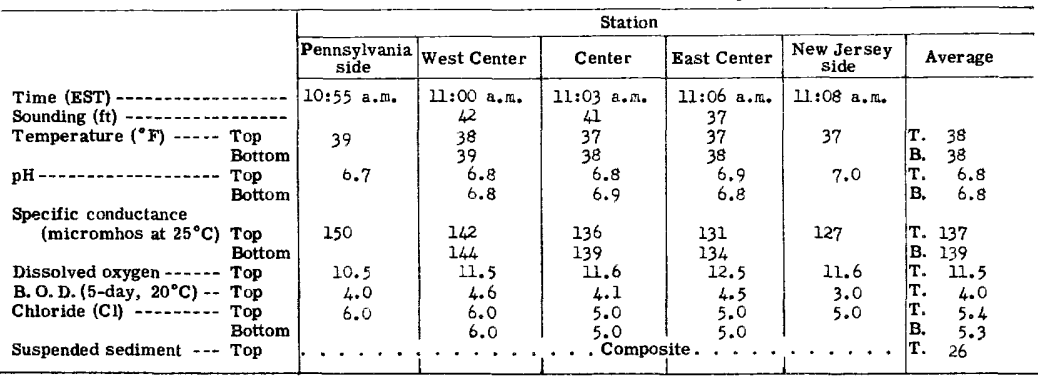

Location Wharton Street, Philadelphia, Pa, Weather_Clear

Date February 6. 1952 - Sampling study No. 30 Water discharge at Trenton (cfs) $-1,3,400$

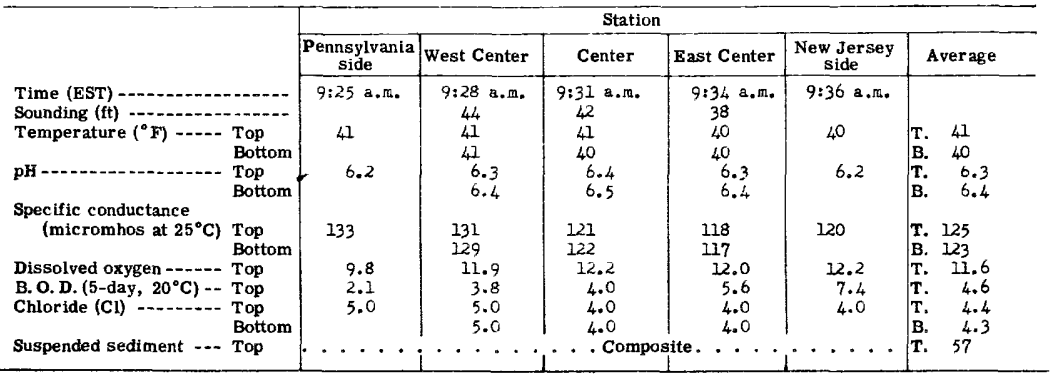

Location Wharton Street, Pniladelphia, Pa. Weather Trace of Rain

Date March 3, 1952

Sampling study No. 3$]$ Water discharge at Trenton (cfs) $-8,410$

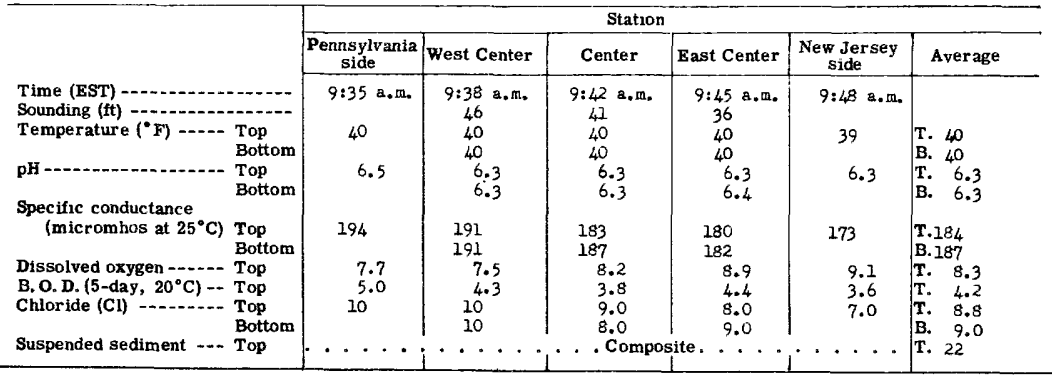

Location Tharton Street, Philadolphia, $\mathrm{Pa}$. Weather Cleas

Date April 1, 1952 Sampling study No. 32

\begin{tabular}{|c|c|c|c|c|c|c|}
\hline \multicolumn{2}{|c|}{ Weather Clear } & \multicolumn{5}{|c|}{ Water discharge at Trenton (cfs) 20,800} \\
\hline & \multicolumn{6}{|c|}{ Station } \\
\hline & $\begin{array}{c}\text { Pennsylvania } \\
\text { side }\end{array}$ & West Center & Center & East Center & $\begin{array}{c}\text { New Jersey } \\
\text { side }\end{array}$ & Average \\
\hline $\begin{array}{l}\text { Time (EST) } \\
\text { Sounding }(\mathrm{ft}) \\
\text { Temperature }(\bullet F) \\
\text { pH }\end{array}$ & $\begin{array}{c}1: 15 \text { p.m. } \\
50 \\
6.2\end{array}$ & $\begin{array}{l}1: 18 \mathrm{p.m} . \\
40 \\
49 \\
51 \\
6.2 \\
6.3\end{array}$ & $\begin{array}{l}1: 21 \mathrm{p.m} . \\
38 \\
49 \\
50 \\
6.5 \\
6.5\end{array}$ & $\begin{array}{c}1: 24 \mathrm{p} . \mathrm{m} . \\
35 \\
49 \\
50 \\
6.5 \\
6.5\end{array}$ & $\begin{array}{c}1.28 \mathrm{p.sin} . \\
50 \\
6.5\end{array}$ & $\begin{array}{ll}\text { T. } & 49 \\
\text { B. } & 50 \\
\text { T. } & 6.4 \\
\text { B. } & 6.4\end{array}$ \\
\hline 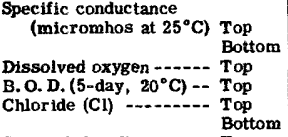 & $\begin{array}{l}90.9 \\
7.5 \\
5.3 \\
6.0\end{array}$ & $\begin{array}{r}116 \\
109 \\
8.0 \\
1.6 \\
6.0 \\
4.0\end{array}$ & $\begin{array}{l}96.0 \\
96.0 \\
9.1 \\
1.2 \\
3.0 \\
3.0\end{array}$ & $\begin{array}{r}91.8 \\
91.6 \\
9.3 \\
.9 \\
2.0 \\
2.0\end{array}$ & $\begin{array}{r}96.8 \\
9.1 \\
.4 \\
4.0\end{array}$ & $\begin{array}{lr}\text { T. } & 98.3 \\
\text { B. } & 98.9 \\
\text { T. } & 8.6 \\
\text { T. } & 1.9 \\
\text { T. } & 4.2 \\
\text { B. } & 3.0\end{array}$ \\
\hline Suspended sediment --- Top & & 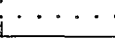 & . Compo & ite. . . & $\cdots \cdot$ & T. 14 \\
\hline
\end{tabular}


Table 11. --WATER ANALYSES OF DELAWARE RIVER BETWEEN BRISTOL AND MARCUS HOOK, PA. --Continued Analyzed by City of Philadelphia and U. S. Geological Survey; analyses in parts per million

Location Wharton Street, Philadelphia, Pa,_Date May 6, 1952 Sampling study No. 33 Weather Overcast $\quad$ Water discharge at Trenton (cfs) $-16,600$

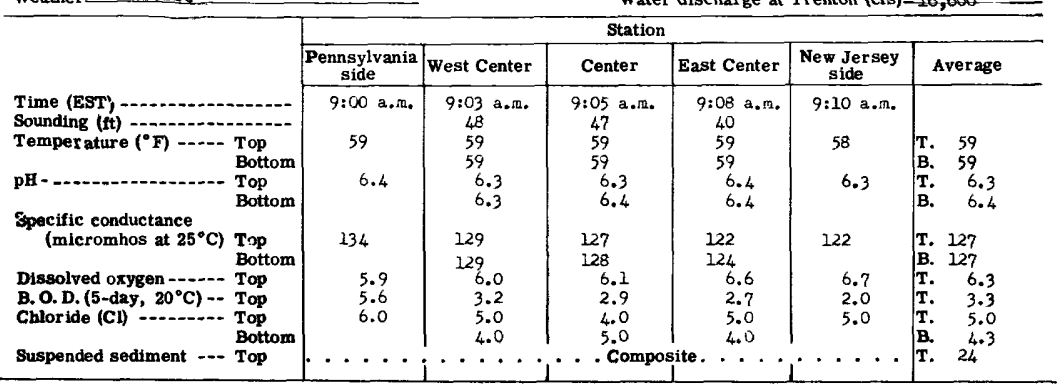

Location Wharton Street, Philadelphia, Pa.

Sampling study No. 34 Weather_Clear. Water discharge at Trenton (cfs) 35,600

\begin{tabular}{|c|c|c|c|c|c|c|}
\hline \multirow[b]{3}{*}{$\begin{array}{l}\text { Time (EST) } \\
\text { Sounding (ft) } \\
\text { Temperature }\left({ }^{\circ} \mathrm{F}\right) \\
\text { pH }\end{array}$} & \multicolumn{6}{|c|}{ Station } \\
\hline & \begin{tabular}{|c|} 
Pennsylvania \\
side \\
\end{tabular} & West Center & Center & East Center & $\begin{array}{c}\text { New Jersey } \\
\text { side }\end{array}$ & Average \\
\hline & $\begin{array}{c}8: 38 \text { a.m. } \\
66 \\
6.5\end{array}$ & $\begin{array}{c}9 \div 42 \times 12 . \\
47 \\
67 \\
68 \\
6.4 \\
6.4\end{array}$ & $\begin{array}{l}8: 43 \text { a.m. } \\
41 \\
66 \\
68 \\
6.5 \\
6.5\end{array}$ & $\begin{array}{l}8: 45 \text { a.m. } \\
41 \\
66 \\
67 \\
6.6 \\
6.5\end{array}$ & $\begin{array}{c}8: 46 \text { a.m. } \\
66 \\
6.5\end{array}$ & $\begin{array}{ll}\text { T. } & 66 \\
\text { B. } & 68 \\
\text { T. } & 6.5 \\
\text { B. } & 6.5\end{array}$ \\
\hline 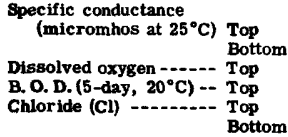 & $\begin{array}{l}129 \\
5.7 \\
5.5 \\
6.0\end{array}$ & $\begin{array}{l}127 \\
119 \\
5.6 \\
4.0 \\
6.0 \\
4.0\end{array}$ & $\begin{array}{l}119 \\
118 \\
5.5 \\
2.2 \\
5.0 \\
4.0\end{array}$ & $\begin{array}{l}215 \\
215 \\
6.6 \\
1.8 \\
4.0 \\
4.0\end{array}$ & $\begin{array}{r}112 \\
6.9 \\
2.4 \\
4.0\end{array}$ & $\begin{array}{lr}\text { T. } & 120 \\
\text { B. } & 217 \\
\text { T. } & 6.1 \\
\text { T. } & 3.2 \\
\text { T. } & 5.0 \\
\text { B. } & 4.0\end{array}$ \\
\hline Suspended sediment & $\cdots$ & i. . . & . Cor & & $\cdots$ & T. 26 \\
\hline
\end{tabular}

Location Wharton Street, Philadelphia, Pa. Weather Overcast

\begin{tabular}{|c|c|c|c|c|c|c|}
\hline \multicolumn{2}{|c|}{$\begin{array}{l}\text { Location - fharron Street, Fhiladelph12, Pa. } \\
\text { Weather Overcast }\end{array}$} & \multicolumn{5}{|c|}{ Water discharge at Trenton (cfs) 3,280} \\
\hline & \multicolumn{6}{|c|}{ Station } \\
\hline & \begin{tabular}{|c|}
$\begin{array}{c}\text { Pennsylvania } \\
\text { side }\end{array}$ \\
\end{tabular} & West Center & Center & East Center & $\begin{array}{l}\text { New Jersey } \\
\text { side }\end{array}$ & Average \\
\hline $\begin{array}{l}\text { Time (EST) } \\
\text { Sounding (ft) } \\
\text { Temperature }\left({ }^{\circ} \mathrm{F}\right)-\begin{array}{l}\text { Top } \\
\text { Bottom }\end{array} \\
\text { pH } \\
\begin{array}{l}\text { Top } \\
\text { Bottom }\end{array}\end{array}$ & $\begin{array}{c}1: 50 \text { p.m. } \\
79 \\
7.0\end{array}$ & $\begin{array}{l}1: 52 \mathrm{p} . \mathrm{m} . \\
41 \\
80 \\
79 \\
6.8 \\
6.8\end{array}$ & $\begin{array}{l}1: 54 \mathrm{p} \cdot \mathrm{m} . \\
44 \\
80 \\
79 \\
6.8 \\
6.8\end{array}$ & $\begin{array}{c}1: 56 \mathrm{p.m} . \\
46 \\
80 \\
79 \\
6.8 \\
6.8\end{array}$ & $\begin{array}{c}1: 58 \mathrm{p} \cdot \mathrm{m} . \\
80 \\
6.8\end{array}$ & $\begin{array}{lc}\text { T. } & 80 \\
\text { B. } & 79 \\
\text { T. } & 6.8 \\
\text { B. } & 6.8\end{array}$ \\
\hline 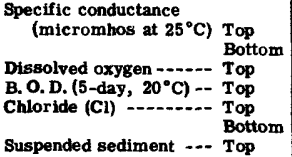 & $\begin{array}{l}276 \\
20.0 \\
15\end{array}$ & $\begin{array}{l}251 \\
247 \\
13.1 \\
14 \\
12\end{array}$ & $\begin{array}{l}253 \\
249.1 \\
8.4 \\
13\end{array}$ & $\begin{array}{l}253 \\
251 \\
8.1 \\
13 \\
13\end{array}$ & $\frac{.1}{6.0}$ & $\begin{array}{ll}\text { T. } & 257 \\
\text { B. } & 249 \\
\text { T. } & .1 \\
\text { T. } & 11.3 \\
\text { T. } & 14 \\
\text { B. } & 13 \\
\text { T. } & 56\end{array}$ \\
\hline
\end{tabular}

Location Whart on Street, Philadelphia, Pa. Weather Cloudy.

Date_August 7. 1952 Sampling study No. 36

\begin{tabular}{|c|c|c|c|c|c|c|}
\hline \multicolumn{3}{|c|}{ Weather Cloudy } & \multicolumn{4}{|c|}{ Water discharge at Trenton (cfs) $-5,920$} \\
\hline & \multicolumn{6}{|c|}{ Station } \\
\hline & \begin{tabular}{|c} 
Pennsylvania \\
side
\end{tabular} & West Center & Center & East Center & \begin{tabular}{|c|c|}
$\begin{array}{c}\text { New Jersey } \\
\text { side }\end{array}$ \\
\end{tabular} & Average \\
\hline $\begin{array}{ll}\text { Time (EST) } & \\
\text { Sounding (ft) } \\
\text { Temperature }\left({ }^{\circ} \mathrm{F}\right) \\
\text { pH }\end{array} \begin{array}{l}\text { Bop } \\
\text { Top } \\
\text { Bottom }\end{array}$ & $\begin{array}{c}12: 20 \text { p.m. } \\
83 \\
6.7\end{array}$ & $\begin{array}{c}12: 22 \text { p.m. } \\
42 \\
83 \\
82 \\
6.7 \\
6.7\end{array}$ & $\begin{array}{c}12: 24 \text { p.m. } \\
42 \\
82 \\
81 \\
6.7 \\
6.7\end{array}$ & $\begin{array}{c}12: 26 \text { p.m. } \\
38 \\
81 \\
82 \\
6.7 \\
6.7\end{array}$ & $\begin{array}{c}12: 28 \mathrm{p.m} \\
82 \\
6.7\end{array}$ & $\begin{array}{lc}\text { T. } & 82 \\
\text { B. } & 82 \\
\text { T. } & 6.7 \\
\text { B. } & 6.7\end{array}$ \\
\hline 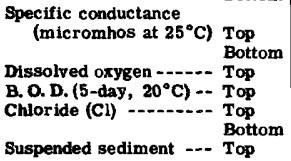 & $\begin{array}{l}215 \\
1.1 \\
10.6 \\
12 \\
\ldots\end{array}$ & $\begin{array}{l}213 \\
210 \\
1.1 \\
5.3 \\
12 \\
11 \\
\cdots\end{array}$ & $\begin{array}{l}209 \\
210 \\
5.2 \\
\frac{12}{11} \\
. \text { Com }\end{array}$ & $\begin{array}{l}207 \\
209 \\
.4 \\
10 \\
11\end{array}$ & 206 & $\begin{array}{ll}\text { T. } & 210 \\
\text { B. } & 210 \\
\text { T. } & .7 \\
\text { T. } & 6.5 \\
\text { T. } & 11 \\
\text { B. } & 11 \\
\text { T. } & 54\end{array}$ \\
\hline
\end{tabular}


Table 11. -- WATER ANALYSES OF DELAWARE RIVER BETWEEN BRISTOL AND MARCUS HOOK, PA. --Continued Analyzed by City of Philadelphia and U. S. Geological Survey; analyses in parts per million

Location Wharton Street, Philadelphia Date_September 4, 1952 Sampling study No. 37

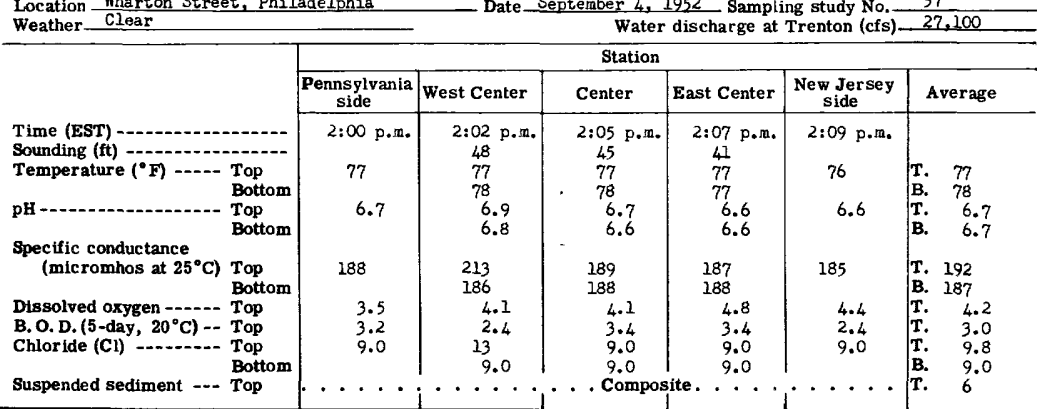

Location Wharton Street, Philadelphia, Pa. Weather S1 i ght ly Cloudy

Date October 7, $1952 \quad$ Sampling study No. 38 Water discharge at Trenton (cfs) - 4,120

\begin{tabular}{|c|c|c|c|c|c|c|}
\hline \multirow[b]{3}{*}{$\begin{array}{ll}\text { Time (EST) } & \\
\text { Sounding }(\mathrm{ft}) \\
\text { Temperature }\left({ }^{\circ} \mathrm{F}\right)-\begin{array}{l}\text { Top } \\
\text { Bottom }\end{array} \\
\text { pH } \\
\text { Bop } \\
\text { Bottom }\end{array}$} & \multicolumn{6}{|c|}{ Station } \\
\hline & \begin{tabular}{|c} 
Pennsylvania \\
side \\
\end{tabular} & West Center & Center & East Center & $\begin{array}{c}\text { New Jersey } \\
\text { side }\end{array}$ & Average \\
\hline & $\begin{array}{c}2: 45 \text { p.m. } \\
69 \\
6.8\end{array}$ & $\begin{array}{l}2: 48 \text { p.m. } \\
42 \\
69 \\
69 \\
6.8 \\
6.7\end{array}$ & $\begin{array}{l}2: 51 \text { p.m. } \\
44 \\
69 \\
68 \\
7.2 \\
6.9\end{array}$ & $\begin{array}{l}2: 54 \text { p.m. } \\
40 \\
68 \\
68 \\
6.8 \\
6.9\end{array}$ & $\begin{array}{l}2: 57 \text { p.m. } \\
66 \\
6.6\end{array}$ & $\begin{array}{lc}\text { T. } & 68 \\
\text { B. } & 68 \\
\text { T. } & 6.8 \\
\text { B. } & 6.8\end{array}$ \\
\hline 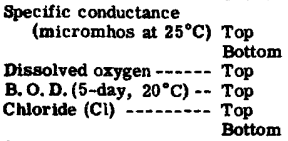 & $\begin{array}{l}257 \\
1.4 \\
9.6 \\
15\end{array}$ & $\begin{array}{l}257 \\
261 \\
1.8 \\
9.8 \\
16 \\
15\end{array}$ & $\begin{array}{l}249 \\
255 \\
1.0 \\
5.8 \\
15 \\
16\end{array}$ & $\begin{array}{l}263 \\
244 \\
1.5 \\
5.3 \\
15 \\
15\end{array}$ & $\begin{array}{l}246 \\
1.8 \\
5.2 \\
15\end{array}$ & $\begin{array}{ll} & \\
\text { T. } & 254 \\
\text { B. } & 253 \\
\text { T. } & 1.5 \\
\text { T. } & 7.1 \\
\text { T. } & 15 \\
\text { B. } & 15\end{array}$ \\
\hline Suspended sediment $\ldots$ Top & & $\cdot \cdot \cdot$ & Comp & ite. . . & $\cdot \cdot$ & T. 15 \\
\hline
\end{tabular}

Location Wharton Street, Philadelphia, Pa. Weather Clear Date November 7, 1952 Sampling study No. 39

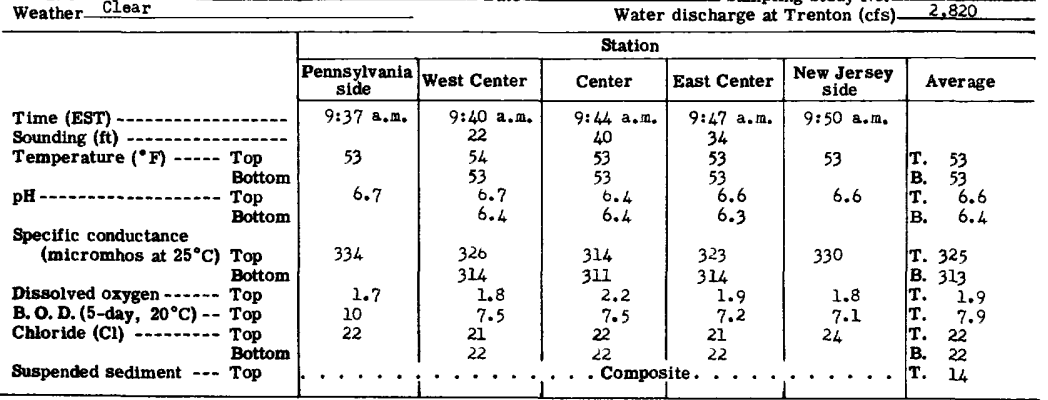

Location Tharton Street, Philadelphia, Pa. Weather-Rain Date December 5, 1952 Sampling studg No, 10

\begin{tabular}{|c|c|c|c|c|c|c|}
\hline \multicolumn{3}{|c|}{ Weather Rain } & \multicolumn{4}{|c|}{ Water discharge at Trenton (cfs) 12,600} \\
\hline & \multicolumn{6}{|c|}{ Station } \\
\hline & \begin{tabular}{|c} 
Pennsylvania \\
side
\end{tabular} & West Center & Center & East Center & $\begin{array}{c}\text { New Jersey } \\
\text { side }\end{array}$ & Average \\
\hline $\begin{array}{l}\text { Time (EST) } \\
\text { Sounding (ft) } \\
\text { Temperature }\left({ }^{\circ} \mathrm{F}\right)-\begin{array}{l}\text { Top } \\
\text { Bottom }\end{array} \\
\text { pH }\end{array}$ & $\begin{array}{c}2: 58 \text { p.m. } \\
47 \\
6.1\end{array}$ & $\begin{array}{l}3: 01 \text { p.n. } \\
48 \\
47 \\
47 \\
6.1 \\
6.2\end{array}$ & $\begin{array}{l}3: 04 \mathrm{p.m} . \\
45 \\
46 \\
47 \\
6.1 \\
6.2\end{array}$ & $\begin{array}{c}3: 07 \mathrm{p} . \mathrm{m} \\
43 \\
45 \\
47 \\
5.9 \\
6.2\end{array}$ & $\begin{array}{c}3: 10 \text { p.m. } \\
45 \\
-\end{array}$ & $\begin{array}{lc}\text { T. } & 46 \\
\text { B. } & 47 \\
\text { T. } & 6.0 \\
\text { B. } & 6.2\end{array}$ \\
\hline 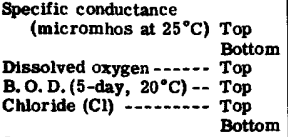 & $\begin{array}{l}173 \\
5.2 \\
5.2 \\
10\end{array}$ & $\begin{array}{r}171 \\
171 \\
5.7 \\
5.5 \\
9.5 \\
9.0\end{array}$ & $\begin{array}{r}165 \\
162 \\
6.3 \\
6.2 \\
9.5 \\
9.0\end{array}$ & $\begin{array}{r}153 \\
151 \\
6.9 \\
6.5 \\
9.0 \\
9.0\end{array}$ & $\begin{array}{l}- \\
7.3 \\
4.5 \\
-\end{array}$ & $\begin{array}{lr}\text { T. } & 165 \\
\text { B. } & 161 \\
\text { T. } & 6.3 \\
\text { T. } & 5.6 \\
\text { T. } & 9.5 \\
\text { B. } & 9.0\end{array}$ \\
\hline Suspended sediment $\ldots$ Top & $\cdot$ & & & . . . & $\cdot \cdot \cdot$ & T. 18 \\
\hline
\end{tabular}


Table 12. --WATER ANALYSES OF DELAWARE RIVER BETWEEN BRISTOL AND MARCUS HOOK, PA. Analyzed by Clty of Philadelphia and U. S. Geological Survey; analyses in parts per million

Location League Isiand, Fhiladelphia, Fa. Date August 2, 1949 Sampling study No. 1

\begin{tabular}{|c|c|c|c|c|c|c|}
\hline \multicolumn{3}{|c|}{ 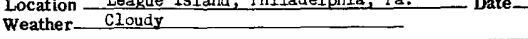 } & \multicolumn{4}{|c|}{ Water discharge at Trenton (cfs) 2,940} \\
\hline & \multicolumn{6}{|c|}{ Station } \\
\hline & $\begin{array}{c}\text { Pennsylvania } \\
\text { side }\end{array}$ & West Center & Center & East Center & \begin{tabular}{|c|}
$\begin{array}{c}\text { New Jersey } \\
\text { side }\end{array}$ \\
\end{tabular} & Average \\
\hline $\begin{array}{ll}\text { Time (EST) } & \\
\text { Sounding (ft) } & \\
\text { Temperature }\left({ }^{\circ} \mathrm{F}\right) & \begin{array}{l}\text { Top } \\
\text { Bottom }\end{array} \\
\text { pH } & \begin{array}{l}\text { Top } \\
\text { Bottom }\end{array}\end{array}$ & $\begin{array}{l}-- \\
86 \\
6.1\end{array}$ & $\begin{array}{l}45 \\
86 \\
86 \\
0.0 \\
0.0\end{array}$ & $\begin{array}{c}12: 00 \mathrm{n} \\
45 \\
84 \\
84 \\
5.9 \\
6.1\end{array}$ & $\begin{array}{l}-- \\
21 \\
86 \\
86 \\
6.0 \\
5.8\end{array}$ & $\begin{array}{l}- \\
86 \\
6.0\end{array}$ & $\begin{array}{ll}\text { T. } & 86 \\
\text { B. } & 85 \\
\text { T. } & 6.0 \\
\text { B. } & 6.0\end{array}$ \\
\hline 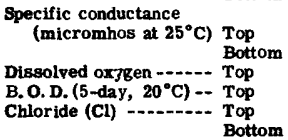 & $\begin{array}{l}298 \\
20.0 \\
20\end{array}$ & $\begin{array}{l}279 \\
302 \\
17 \cdot 0 \\
19 \\
19\end{array}$ & $\begin{array}{l}291 \\
291 \\
15.0 \\
18 \\
18\end{array}$ & $\begin{array}{l}295 \\
294 \\
15.0 \\
18 \\
20\end{array}$ & $\begin{array}{l}303 \\
1.0 \\
15.0 \\
21\end{array}$ & $\begin{array}{ll}\text { T. } & 293 \\
\text { B. } & 296 \\
\text { T. } & .4 \\
\text { T. } & 16.4 \\
\text { T. } & 19 \\
\text { B. } & 19\end{array}$ \\
\hline Suspended sediment -.. Top & . & $\cdots$ & . Com & te. . . & $\cdots \cdot \cdot$ & -- \\
\hline
\end{tabular}

Location Leaque Island, Philadelohia. Pa. _ Date September 7. 1949 Sampling study No. 2 Weather Occasional Rain Water discharge at Trenton (cfs) - 3,810

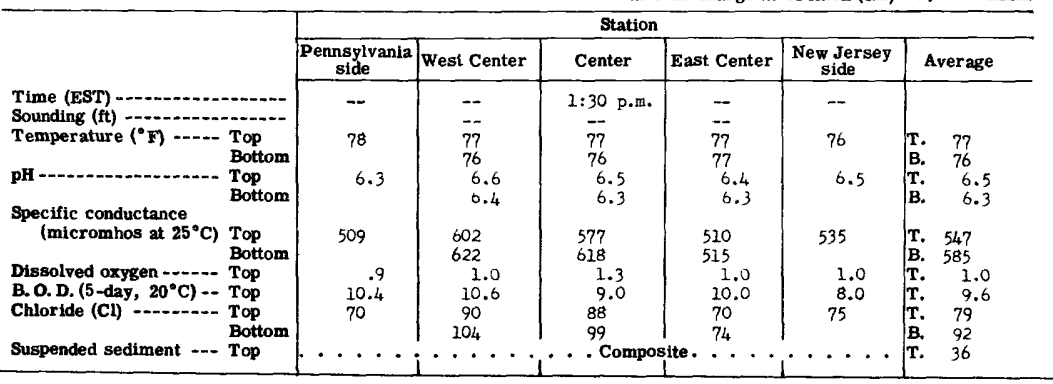

Location League Is land. Philadel phia, $\mathrm{Fa}$. Date October 5, 1949 Sampling study No. 3 Weather_Occasional Rain Water discharge at Trenton (cfs) - 3,280

\begin{tabular}{|c|c|c|c|c|c|c|}
\hline \multicolumn{3}{|c|}{ Weather_Occasional Rain } & \multicolumn{4}{|c|}{ Water discharge at Trenton $(\mathrm{cfs})-3,280$} \\
\hline & \multicolumn{6}{|c|}{ Station } \\
\hline & $\begin{array}{c}\text { Pennsylvania } \\
\text { side }\end{array}$ & West Center & Center & East Center & $\begin{array}{c}\text { New Jersey } \\
\text { side }\end{array}$ & Average \\
\hline $\begin{array}{l}\text { Time (BST) } \\
\text { Sounding (ft) } \\
\text { Temperature ( }{ }^{\circ} \mathrm{F} \text { ) } \\
\text { pH }\end{array}$ & 69 & $\begin{array}{l}-- \\
b 50 \\
69 \\
70 \\
6.4 \\
6.6\end{array}$ & $\begin{array}{c}11: 00 \text { a.m. } \\
-0 \\
69 \\
70 \\
6.4 \\
6.6\end{array}$ & $\begin{array}{l}-\overline{23} \\
69 \\
70 \\
6.4 \\
6.6\end{array}$ & $\begin{array}{l}-- \\
69 \\
0.3\end{array}$ & $\begin{array}{ll}\text { T. } & 69 \\
\text { B. } & 70 \\
\text { T. } & 6.4 \\
\text { B. } & 6.6\end{array}$ \\
\hline 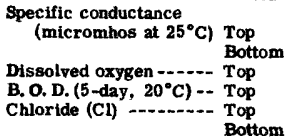 & $\begin{array}{l}632 \\
1.5 \\
6.3 \\
96\end{array}$ & $\begin{array}{l}625 \\
594 \\
1.0 \\
6.0 \\
94 \\
87\end{array}$ & $\begin{array}{l}644 \\
618 \\
1.4 \\
4.6 \\
99 \\
95\end{array}$ & $\begin{array}{l}763 \\
729 \\
1.3 \\
4.1 \\
128 \\
125\end{array}$ & $\begin{array}{r}721 \\
2.3 \\
4.3 \\
123\end{array}$ & $\begin{array}{lc}\text { T. } & 677 \\
\text { B. } & 647 \\
\text { T. } & 1.5 \\
\text { T. } & 5.1 \\
\text { T. } & 108 \\
\text { B. } & 102\end{array}$ \\
\hline Suspended sediment.- Top & & & Con & . & .. & T. 49 \\
\hline
\end{tabular}

b Exceeded this value.

Location League Island, Philadelphla, $\mathrm{Pa}$. weather Cleas

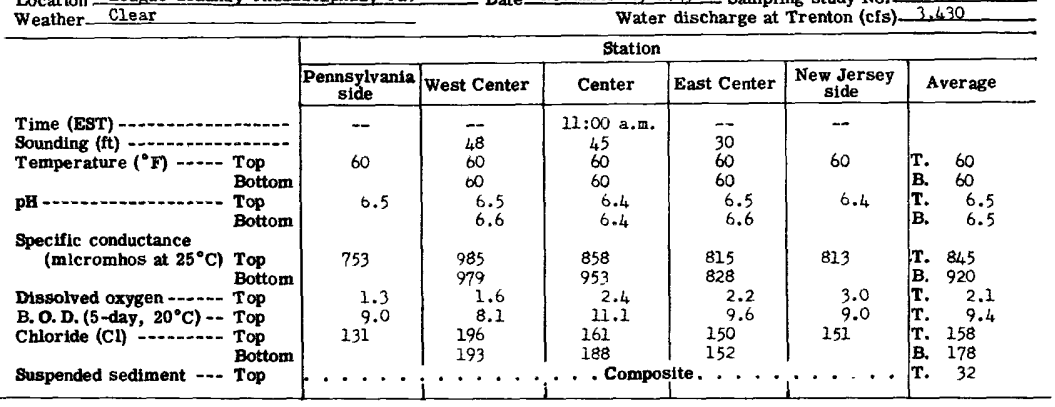


Table 12. -- WATER ANALYSES OF DELAWARE RTVER BETWEEN BRISTOL AND MARCUS HOOK, PA. --Continued Analyzed by City of Philadelphia and U. S. Geological Survey; analyses in parts per million

Location Leaque Island, Philadelphia, Pa. Weather Occasional Rain

Date December 2, 1949 Sampling study No. -5

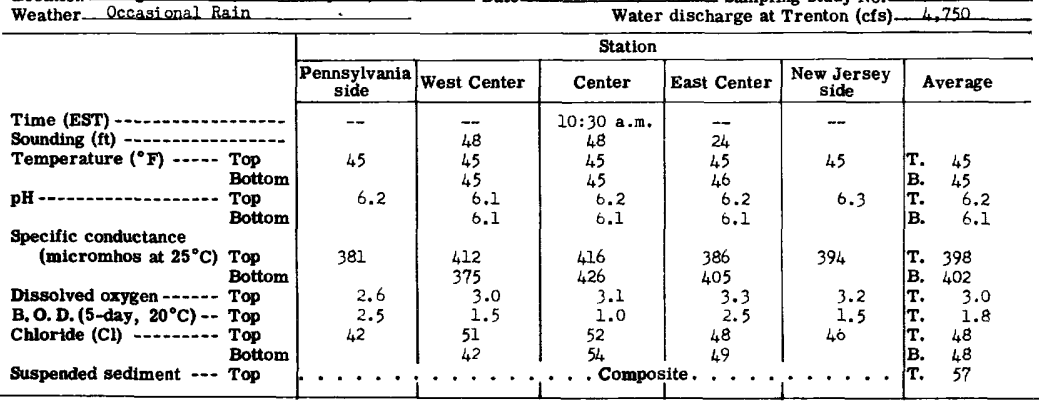

Location League Is land, Philadel phia, Pa. Weather_cloudy

- Date_Jamary 5, 1950 _ Sampling study No._

\begin{tabular}{|c|c|c|c|c|c|c|}
\hline \multicolumn{2}{|l|}{ Weather_Sloudy } & \multicolumn{5}{|c|}{ Water } \\
\hline & \multicolumn{6}{|c|}{ Station } \\
\hline & \begin{tabular}{|c|}
$\begin{array}{c}\text { Pennsylvania } \\
\text { side }\end{array}$ \\
\end{tabular} & West Center & Center & East Center & \begin{tabular}{|c|} 
New Jersey \\
side
\end{tabular} & Average \\
\hline $\begin{array}{ll}\text { Time (EST) } & \\
\text { Sounding (ft) } \\
\text { Temperature ("F) }\end{array} \begin{array}{l}\text { Top } \\
\text { Bottom } \\
\text { pH }\end{array}$ & $\begin{array}{c}10: 21 \text { a.m. } \\
45 \\
6.4\end{array}$ & $\begin{array}{c}10: 19 \text { a.m. } \\
46 \\
45 \\
45 \\
6.3 \\
6.0\end{array}$ & $\begin{array}{c}10: 17 \text { a.m. } \\
45 \\
45 \\
45 \\
6.3 \\
6.1\end{array}$ & $\begin{array}{c}10: 15 \mathrm{a} \cdot \mathrm{m} \\
23 \\
48 \\
45 \\
6.3 \\
6.0\end{array}$ & $\begin{array}{c}10: 13 \text { a.m. } \\
48 \\
6.3\end{array}$ & $\begin{array}{ll}\text { T. } & 46 \\
\text { B. } & 45 \\
\text { T. } & 6.3 \\
\text { B. } & 6.0\end{array}$ \\
\hline 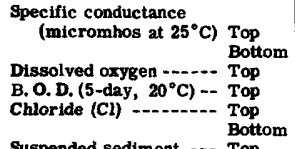 & $\begin{array}{l}157 \\
7.5 \\
4.8 \\
10\end{array}$ & $\begin{array}{l}137 \\
129 \\
8.1 \\
5.0 \\
10 \\
9.0\end{array}$ & $\begin{array}{r}138 \\
125 \\
8.5 \\
4.0 \\
10 \\
8.0\end{array}$ & $\begin{array}{l}139 \\
135 \\
8.5 \\
5.5 \\
10 \\
9.0\end{array}$ & $\begin{array}{l}140 \\
8.3 \\
4.5 \\
8.0\end{array}$ & $\begin{array}{lr} & \\
\text { T. } & 142 \\
\text { B. } & 130 \\
\text { T. } & 8.2 \\
\text { T. } & 4.8 \\
\text { T. } & 9.6 \\
\text { B. } & 8.7\end{array}$ \\
\hline Suspended sediment -.- Top & 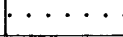 & $\cdots \cdot$ & . Comp & te. . . & 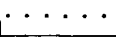 & T. \\
\hline
\end{tabular}

Location League Island, Fhiladelphia, Pa, Weather Clear.

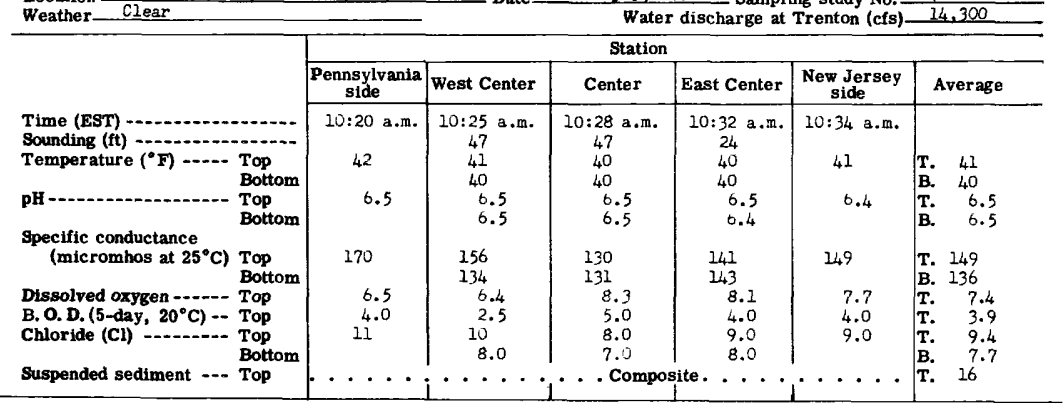

Location League Island, Philadel phia, $\mathrm{Pa}$. Date_Karch 7. 1950

Sampling study No. 8

\begin{tabular}{|c|c|c|c|c|c|c|}
\hline \multicolumn{3}{|c|}{ Weather cloudy } & \multicolumn{4}{|c|}{ Water discharge at Trenton (cfs) 7,260} \\
\hline & \multicolumn{6}{|c|}{ Station } \\
\hline & $\begin{array}{c}\text { Pennsylvania } \\
\text { side }\end{array}$ & West Center & Center & East Center & \begin{tabular}{|c|} 
New Jersey \\
side
\end{tabular} & Average \\
\hline $\begin{array}{l}\text { Time (EST) } \\
\text { Sounding (ft) } \\
\text { Temperature }\left({ }^{\circ} \mathrm{F}\right) \\
\begin{array}{l}\text { Top } \\
\text { pH }\end{array} \\
\begin{array}{l}\text { Bottom } \\
\text { Bottom }\end{array}\end{array}$ & $\begin{array}{c}3: 03 \mathrm{p} . \mathrm{m} . \\
38 \\
6.6\end{array}$ & $\begin{array}{l}3: 00 \mathrm{p} . \mathrm{m} \\
47 \\
38 \\
38 \\
0.6 \\
6.5\end{array}$ & $\begin{array}{l}2: 58 \text { p.m. } \\
45 \\
38 \\
37 \\
b .6 \\
0.5\end{array}$ & $\begin{array}{c}2: 55 \mathrm{p} \cdot \mathrm{m} . \\
28 \\
38 \\
38 \\
6.5 \\
0.5\end{array}$ & $\begin{array}{c}2: 50 \text { p.m. } \\
38 \\
6.5\end{array}$ & $\begin{array}{ll}\text { T. } & 38 \\
\text { B. } & 38 \\
\text { T. } & 6.6 \\
\text { B. } & 6.5\end{array}$ \\
\hline 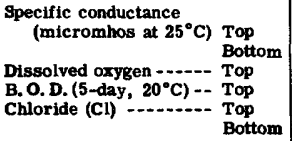 & $\begin{array}{c}207 \\
6.8 \\
3.4 \\
13\end{array}$ & $\begin{array}{l}203 \\
206 \\
8.3 \\
4.9 \\
12 \\
13\end{array}$ & $\begin{array}{l}201 \\
201 \\
7.3 \\
4.4 \\
12 \\
12\end{array}$ & $\begin{array}{l}201 \\
200 \\
8.3 \\
4 \cdot 4 \\
12 \\
12\end{array}$ & $\begin{array}{l}202 \\
8.8 \\
3.4 \\
12\end{array}$ & $\begin{array}{ll}\text { T. } & 203 \\
\text { B. } & 202 \\
\text { T. } & 7.9 \\
\text { T. } & 4.1 \\
\text { T. } & 12 \\
\text { B. } & 12\end{array}$ \\
\hline Suspended sediment --- Top & & & . Com & te. . . & - & T. \\
\hline
\end{tabular}


Table 12. -- WATER ANALYSES OF DE LAWARE RIVER BETWEEN BRISTOL AND MARCUS HOOK, PA.--Continued Analyzed by City of Philadelphia and U. S. Geological Survey; analyses in parts per million

\begin{tabular}{|c|c|c|c|c|c|c|}
\hline \multicolumn{2}{|c|}{$\begin{array}{l}\text { Location } \frac{\text { Loague Island, Philadelphia, } \mathrm{Pa} \text {. }}{\text { Wloudy }} \\
\text { Weather }\end{array}$} & \multicolumn{5}{|c|}{ 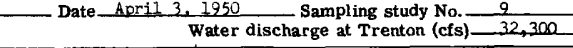 } \\
\hline & \multicolumn{6}{|c|}{ Station } \\
\hline & $\begin{array}{c}\text { Pennsylvania } \\
\text { side }\end{array}$ & West Center & Center & East Center & $\begin{array}{l}\text { New Jersey } \\
\text { side }\end{array}$ & Average \\
\hline $\begin{array}{ll}\text { Time (EST) } & \\
\text { Sounding }(\mathrm{ft}) & \\
\text { Temperature }\left({ }^{\circ} \mathbf{F}\right) & \begin{array}{l}\text { Top } \\
\text { Bottom }\end{array} \\
\text { pH } & \begin{array}{l}\text { Top } \\
\text { Bottom }\end{array}\end{array}$ & $\begin{array}{c}3: 07 \text { p.m. } \\
45 \\
6.6\end{array}$ & $\begin{array}{l}3: 05 \mathrm{p} . \mathrm{m} . \\
47 \\
44 \\
45 \\
6.5 \\
6.6\end{array}$ & $\begin{array}{l}3: 00 \mathrm{p} \cdot \mathrm{m} . \\
\mathrm{b} 50 \\
44 \\
44 \\
6.5 \\
6.5\end{array}$ & $\begin{array}{r}2: 55 \text { p.m. } \\
27 \\
44 \\
44 \\
6.5 \\
6.5\end{array}$ & $\begin{array}{c}2: 50 \text { p.m. } \\
47 \\
0.4\end{array}$ & $\begin{array}{ll}\text { T. } & 45 \\
\text { B. } & 44 \\
\text { T. } & 6.5 \\
\text { B. } & 6.5\end{array}$ \\
\hline 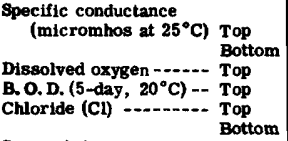 & $\begin{array}{r}107 \\
9.7 \\
2.8 \\
6.0\end{array}$ & $\begin{array}{r}95.0 \\
94.0 \\
10.5 \\
3.8 \\
5.0 \\
6.0\end{array}$ & $\begin{array}{r}81.0 \\
85.7 \\
10.3 \\
3.1 \\
5.0 \\
6.0\end{array}$ & $\begin{array}{r}80.0 \\
79.1 \\
10.5 \\
2.9 \\
4.0 \\
4.0\end{array}$ & $\begin{array}{r}88.1 \\
10.0 \\
3.0 \\
4.0\end{array}$ & $\begin{array}{lr}\text { T. } & 90.2 \\
\text { B. } & 86.3 \\
\text { T. } & 10.2 \\
\text { T. } & 3.1 \\
\text { T. } & 4.8 \\
\text { B. } & 5.3\end{array}$ \\
\hline Suspended sediment $\ldots$-.. Top & & & & & $\bullet \cdot \bullet$ & 28 \\
\hline
\end{tabular}

b Exceeded this value.

Location Loague Isiand, Philadelphia, $\mathrm{Pa}$ Weather Cloudy

Date_May 3, 1950 Sampling study No. 10

\begin{tabular}{|c|c|c|c|c|c|c|}
\hline \multicolumn{3}{|c|}{ Weather Cloudy } & \multicolumn{4}{|c|}{ Water discharge at Trenton (cts) $12,0,00$} \\
\hline & \multicolumn{6}{|c|}{ Station } \\
\hline & \begin{tabular}{|c|}
$\begin{array}{c}\text { Pennsylvania } \\
\text { side }\end{array}$ \\
\end{tabular} & West Center & Center & East Center & $\begin{array}{c}\begin{array}{c}\text { New Jersey } \\
\text { side }\end{array} \\
\end{array}$ & Average \\
\hline $\begin{array}{l}\text { Tlme (EST) } \\
\text { Sounding (ft) } \\
\text { Temperature }\left({ }^{\bullet} \mathbf{F}\right)=\begin{array}{l}\text { Top } \\
\text { Bottom }\end{array} \\
\text { pH } \\
\begin{array}{l}\text { Top } \\
\text { Bottom }\end{array}\end{array}$ & $\begin{array}{c}3: 45 \text { p.m. } \\
56 \\
6.4\end{array}$ & $\begin{array}{l}3: 48 \mathrm{p} . \mathrm{m} . \\
\mathrm{b} 50 \\
55 \\
55 \\
0.5 \\
6.4\end{array}$ & $\begin{array}{l}3: 50 \text { p.m. } \\
\text { b50 } \\
55 \\
55 \\
6.5 \\
6.4\end{array}$ & $\begin{array}{l}3: 52 \mathrm{p} . \mathrm{m} \\
47 \\
55 \\
55 \\
0.4 \\
0.4\end{array}$ & $\begin{array}{l}3: 55 \text { p.m. } \\
55 \\
6.4\end{array}$ & $\begin{array}{ll}\text { T. } & 55 \\
\text { B. } & 55 \\
\text { T. } & 6.4 \\
\text { B. } & 6.4\end{array}$ \\
\hline 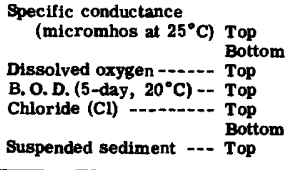 & $\begin{array}{r}163 \\
2.9 \\
10.2 \\
8.0\end{array}$ & $\begin{array}{r}171 \\
173 \\
3.6 \\
9.3 \\
8.0 \\
8.0 \\
\cdots\end{array}$ & $\begin{array}{r}169 \\
168 \\
3.3 \\
7.2 \\
8.0 \\
9.0 \\
. \text { Con }\end{array}$ & $\begin{array}{c}150 \\
155 \\
4.0 \\
5.9 \\
7.0 \\
8.0 \\
. . .\end{array}$ & $\begin{array}{l}148 \\
4.2 \\
6.7 \\
8.0 \\
\ldots .\end{array}$ & $\begin{array}{ll}\text { T. } & 160 \\
\text { B. } & 165 \\
\text { T. } & 3.6 \\
\text { T. } & 7.9 \\
\text { T. } & 7.8 \\
\text { B. } & 8.3 \\
\text { T. } & 16\end{array}$ \\
\hline
\end{tabular}

b Exceeded this value.

Location League Island, Philadel phia, $\mathrm{Pa}$._Date June 5, 1950 Sampling study No. 11 Weather-Clear $\quad$ Water discharge at Trenton (cfs) 18,500

\begin{tabular}{|c|c|c|c|c|c|c|}
\hline \multicolumn{2}{|l|}{ Weather__ Clear } & \multicolumn{5}{|c|}{ Water discharge at Trenton (cfs) 18,500} \\
\hline & \multicolumn{6}{|c|}{ Station } \\
\hline & $\begin{array}{c}\text { Pennsylvania } \\
\text { side }\end{array}$ & West Center & Center & East Center & $\begin{array}{c}\begin{array}{c}\text { New Jersey } \\
\text { side }\end{array} \\
\end{array}$ & Aver age \\
\hline $\begin{array}{l}\text { Time (EST) } \\
\text { Sounding (ft) } \\
\text { Temperature }\left({ }^{\circ} \mathrm{F}\right) \\
\text { pH }\end{array} \begin{array}{l}\text { Top } \\
\text { Bottom }\end{array}$ & $\begin{array}{c}9: 35 \mathrm{a} . \mathrm{m} . \\
68 \\
6.4\end{array}$ & $\begin{array}{l}9: 31 \mathrm{a} \cdot \mathrm{m} . \\
46 \\
68 \\
68 \\
6.3 \\
6.2\end{array}$ & $\begin{array}{l}9: 28 \mathrm{a} \cdot \mathrm{m} . \\
\mathrm{b} 50 \\
67 \\
68 \\
6.3 \\
6.2\end{array}$ & $\begin{array}{l}9: 25 \text { a.m. } \\
25 \\
\text { o? } \\
69 \\
6.2 \\
6.2\end{array}$ & $\begin{array}{l}9: 22 \text { a.m. } \\
67 \\
6.3\end{array}$ & $\begin{array}{lc}\text { T. } & 67 \\
\text { B. } & 68 \\
\text { T. } & 6.3 \\
\text { B. } & 6.2\end{array}$ \\
\hline 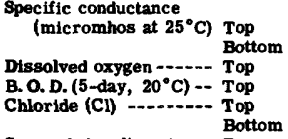 & $\begin{array}{r}146 \\
2.0 \\
1.5 \\
6.0\end{array}$ & $\begin{array}{r}145 \\
147 \\
3.1 \\
1.7 \\
7.0 \\
0.0\end{array}$ & $\begin{array}{r}143 \\
145 \\
2.9 \\
.6 \\
6.0 \\
7.0\end{array}$ & $\begin{array}{r}143 \\
143 \\
2.9 \\
.0 \\
7.0 \\
7.0\end{array}$ & $\begin{array}{r}145 \\
3.0 \\
.9 \\
7.0\end{array}$ & $\begin{array}{lr}\text { T. } & 144 \\
\text { B. } & 145 \\
\text { T. } & 2.9 \\
\text { T. } & .9 \\
\text { T. } & 6.7 \\
\text { B. } & 6.7\end{array}$ \\
\hline Suspended sediment ... Top & & $\cdots$ & Com & e... & - & 28 \\
\hline
\end{tabular}

b Exceeded this value.

Location Loague Island, Philadelphia, Pa,_ Date July 5, 1950_ Sampling study No, 12 Weather-Cloudy Water discharge at Trenton (cfs) 5.630

\begin{tabular}{|c|c|c|c|c|c|c|}
\hline \multirow[b]{3}{*}{$\begin{array}{l}\text { The (EST) } \\
\text { Sounding (ft) } \\
\text { Temperature }\left({ }^{\circ} \mathrm{F}\right) \\
\text { pH }\end{array}$} & \multicolumn{6}{|c|}{ Station } \\
\hline & $\begin{array}{c}\text { Pennsylvania } \\
\text { side }\end{array}$ & West Center & Center & East Center & \begin{tabular}{|c|}
$\begin{array}{c}\text { New Jersey } \\
\text { side }\end{array}$ \\
\end{tabular} & Average \\
\hline & $\begin{array}{c}9: 10 \mathrm{a} . \mathrm{m} . \\
78 \\
6.4\end{array}$ & $\begin{array}{l}9: 15 \text { a. 目. } \\
48 \\
78 \\
78 \\
6.4 \\
6.4\end{array}$ & $\begin{array}{l}9: 20 \mathrm{a} \cdot \mathrm{m} . \\
49 \\
78 \\
78 \\
6.4 \\
0.3\end{array}$ & $\begin{array}{l}9: 25 \text { a.m. } \\
26 \\
78 \\
78 \\
6.4 \\
6.1\end{array}$ & $\begin{array}{l}9: 30 \text { a.m. } \\
78 \\
6.4\end{array}$ & $\begin{array}{lc}\text { T. } & 78 \\
\text { B. } & 78 \\
\text { T. } & 6.4 \\
\text { B. } & 6.3\end{array}$ \\
\hline 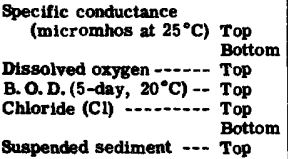 & $\begin{array}{c}209 \\
5.9 \\
13 \\
\ldots \ldots\end{array}$ & $\begin{array}{l}211 \\
211 \\
5.9 \\
12 \\
12 \\
\ldots .\end{array}$ & $\begin{array}{l}209 \\
211 \\
.9 \\
5.8 \\
13 \\
13 \\
. \text { Com }\end{array}$ & $\begin{array}{l}208 \\
208 \\
3.9 \\
12 \\
13 \\
.9 \\
\text { ite. . . . }\end{array}$ & $\begin{array}{l}209 \\
5.9 \\
13\end{array}$ & $\begin{array}{ll}\text { T. } & 209 \\
\text { B. } & 210 \\
\text { T. } & .8 \\
\text { T. } & 5.4 \\
\text { T. } & 13 \\
\text { B. } & 13 \\
\text { T. } & 29\end{array}$ \\
\hline
\end{tabular}


Table 12. -- WATER ANALYSES OF DELAWARE RIVER BETW EEN BRISTOL AND MARC US HOOK, PA. --Continued Analyzed by City of Philadelphia and U. S. Geological Survey; analyses in parts per million

Location League Igland. Philadelphia, Pa._Date August 1, 1950 __ Sampling study No. 13

\begin{tabular}{|c|c|c|c|c|c|c|}
\hline \multicolumn{3}{|c|}{ Weather Cloudy } & \multicolumn{4}{|c|}{ Water discharge at Trenton (cfs) -4.710} \\
\hline & \multicolumn{6}{|c|}{ Station } \\
\hline & \begin{tabular}{|c|} 
Pennsylvania \\
side
\end{tabular} & West Center & Center & East Center & $\begin{array}{c}\text { New Jersey } \\
\text { side }\end{array}$ & Average \\
\hline $\begin{array}{l}\text { Time (EST) } \\
\text { Sounding (ft) } \\
\text { Temperature }\left({ }^{\circ} \mathrm{F}\right)-\begin{array}{l}\text { Top } \\
\text { Bottom }\end{array} \\
\text { pH } \\
\begin{array}{l}\text { Top } \\
\text { Bottom }\end{array}\end{array}$ & $\begin{array}{c}9: 22 \text { a.m. } \\
80 \\
6.3\end{array}$ & $\begin{array}{l}9: 20 \text { a.m. } \\
49 \\
80 \\
80 \\
6.3 \\
6.3\end{array}$ & $\begin{array}{c}9: 17 \text { a.m. } \\
49 \\
80 \\
80 \\
0.2 \\
6.1\end{array}$ & $\begin{array}{l}9: 14 \text { a.m. } \\
21 \\
80 \\
80 \\
0.3 \\
0.3\end{array}$ & $\begin{array}{l}7: 10 \text { a.m. } \\
80 \\
6.3\end{array}$ & $\begin{array}{ll}\text { T. } & 80 \\
\text { B. } & 80 \\
\text { T. } & 6.3 \\
\text { B. } & 6.2\end{array}$ \\
\hline 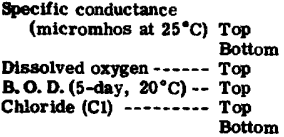 & $\begin{array}{l}205 \\
6.0 \\
25\end{array}$ & $\begin{array}{l}180 \\
199 \\
5.2 \\
13 \\
12\end{array}$ & $\begin{array}{l}191 \\
191 \\
4.2 \\
12 \\
13\end{array}$ & $\begin{array}{l}186 \\
191 \\
3.1 \\
13 \\
12\end{array}$ & 290 & $\begin{array}{ll}\text { T. } & 192 \\
\text { B. } & 194 \\
\text { T. } & .2 \\
\text { T. } & 4.3 \\
\text { T. } & 13 \\
\text { B. } & 12\end{array}$ \\
\hline Suspended sediment --- Top & & & $\mathrm{Com}$ & e. & & 26 \\
\hline
\end{tabular}

Location Leaque Island. Fhiladelphia, Pa.

Date 3eptember 5, 1950 Sampling study No. $-u_{4}$

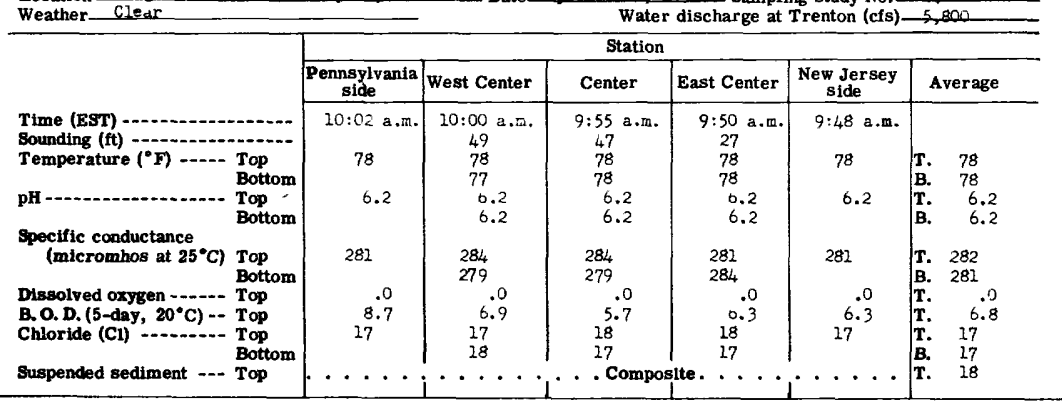

Locatiòn League Island, Philadelphia. Pa. Date October 2, 1950 Sampling study No. 25 Water discharge at Trenton (cfs) 3.320

\begin{tabular}{|c|c|c|c|c|c|c|}
\hline \multirow[b]{3}{*}{$\begin{array}{l}\text { Time (EST) } \\
\text { Sounding }(\mathrm{ft}) \\
\text { Temperature }\left({ }^{\bullet} \mathrm{F}\right) \\
\text { pH }\end{array}$} & \multicolumn{6}{|c|}{ Station } \\
\hline & \begin{tabular}{|c|}
$\begin{array}{c}\text { Pennsylvania } \\
\text { side }\end{array}$ \\
\end{tabular} & West Center & Center & East Center & $\begin{array}{c}\text { New Jersey } \\
\text { side }\end{array}$ & Average \\
\hline & $\begin{array}{c}2: 25 \text { p.m. } \\
69 \\
6.5\end{array}$ & $\begin{array}{c}2: 20 \text { p.m. } \\
\text { b50 } \\
69 \\
69 \\
6.5 \\
6.2\end{array}$ & $\begin{array}{l}2: 15 \mathrm{p} \cdot \mathrm{m} . \\
\mathrm{b} 50 \\
69 \\
69 \\
6.4 \\
6.8\end{array}$ & $\begin{array}{l}2: 10 \text { p.m. } \\
26 \\
69 \\
69 \\
6.1 \\
6.2\end{array}$ & $\begin{array}{c}2: 05 \text { p.m. } \\
69 \\
6.2\end{array}$ & $\begin{array}{ll}\text { T. } & 69 \\
\text { B. } & 69 \\
\text { T. } & 6.3 \\
\text { B. } & 6.4\end{array}$ \\
\hline 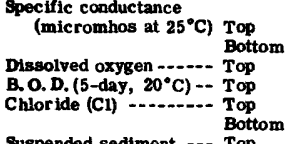 & $\begin{array}{r}288 \\
2.0 \\
2.3\end{array}$ & $\begin{array}{l}280 \\
265 \\
.0 \\
16 \\
14\end{array}$ & $\begin{array}{l}275 \\
258 \\
5.2 \\
16 \\
14\end{array}$ & $\begin{array}{l}275 \\
263 \\
7.4 \\
26 \\
14\end{array}$ & $\begin{array}{l}263 \\
6.7 \\
14\end{array}$ & $\begin{array}{ll}\text { T. } & 276 \\
\text { B. } & 262 \\
\text { T. } & .1 \\
\text { T. } & 5.3 \\
\text { T. } & 16 \\
\text { B. } & 14 \\
\text { T. } & 45\end{array}$ \\
\hline Suspended sediment -.- Top & $\cdot \cdot \cdot$ & $\cdots \cdot$ & . Comp & te. ... & - & T. 45 \\
\hline
\end{tabular}

b Exceeded this value.

Location League Is land. Philadelphia, Pa. Weather Clear

Date November 2. 2950 Sampling study No. 16

\begin{tabular}{|c|c|c|c|c|c|c|}
\hline \multicolumn{7}{|c|}{ Weather Clear } \\
\hline & \multicolumn{6}{|c|}{ Station } \\
\hline & \begin{tabular}{|c|}
$\begin{array}{c}\text { Pennsylvania } \\
\text { side }\end{array}$ \\
\end{tabular} & West Center & Center & East Center & $\begin{array}{c}\text { New Jersey } \\
\text { side }\end{array}$ & Average \\
\hline $\begin{array}{l}\text { Time (EST) } \\
\text { Soundlng (ft) } \\
\text { Temperature ("F) }\end{array}$ & $\begin{array}{c}2: 53 \text { p.m. } \\
65 \\
6.5\end{array}$ & $\begin{array}{l}2: 49 \mathrm{p} . \mathrm{m} \\
48 \\
64 \\
66 \\
6.5 \\
6.5\end{array}$ & $\begin{array}{l}2: 45 \mathrm{p} . \mathrm{m} . \\
\mathrm{b} 50 \\
65 \\
65 \\
6.5 \\
6.5\end{array}$ & $\begin{array}{l}2: 43 \text { p.m. } \\
24 \\
65 \\
66 \\
6.5 \\
6.5\end{array}$ & $\begin{array}{l}2: 40 \text { p.m. } \\
66 \\
6.6\end{array}$ & $\begin{array}{ll}\text { T. } & 65 \\
\text { B. } & 66 \\
\text { T. } & 6.5 \\
\text { B. } & 6.5\end{array}$ \\
\hline 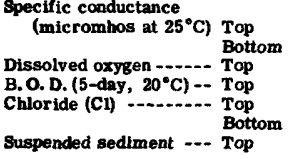 & $\begin{array}{l}343 \\
0.6 \\
24 \\
\ldots \ldots\end{array}$ & $\begin{array}{l}346 \\
352 \\
.0 \\
6.9 \\
25 \\
25 \\
.\end{array}$ & $\begin{array}{l}348 \\
351 \\
.0 \\
6.3 \\
26 \\
25 \\
. \text { Compo }\end{array}$ & $\begin{array}{r}348 \\
362 \\
.5 \\
8.0 \\
26 \\
28 \\
. .\end{array}$ & $\begin{array}{l}370 \\
4.6 \\
30\end{array}$ & $\begin{array}{ll}\text { T. } & 351 \\
\text { B. } & 355 \\
\text { T. } & .2 \\
\text { T. } & 6.5 \\
\text { T. } & 26 \\
\text { B. } & 26 \\
\text { T. } & 55\end{array}$ \\
\hline
\end{tabular}

b Exceeded this value, 
Table 12. --WATER ANALYSES OF DELAWARE RIVER BETWEEN BRISTOL AND MARCUS HOOK, PA. --Continued Analyzed by City of Philadelphia and U. S. Geological Survey; analyses in parts per million

Locatiòn League Island, Fhiladelphic, Pa. Weather_Clear

Date Necember 5,1950 Sampling study No. 17

\begin{tabular}{|c|c|c|c|c|c|c|}
\hline Weather Clear & +2 & & Water & discharge at & Trenton (cfs) & 83,500 \\
\hline & \multicolumn{6}{|c|}{ Station } \\
\hline & $\begin{array}{c}\begin{array}{c}\text { Pennsylvania } \\
\text { side }\end{array} \\
\end{array}$ & West Center & Center & East Center & \begin{tabular}{|c|c|}
$\begin{array}{c}\text { New Jersey } \\
\text { side }\end{array}$ \\
\end{tabular} & A ver age \\
\hline $\begin{array}{l}\text { Time (EST) } \\
\text { Souncing (ft) } \\
\text { Temperature ( }{ }^{\circ} \mathrm{F} \text { ) } \\
\begin{array}{l}\text { Top } \\
\text { PH }\end{array} \\
\end{array}$ & $\begin{array}{c}2: 48 \text { p.m. } \\
42 \\
6.2\end{array}$ & $\begin{array}{l}2: 45 \text { p.m. } \\
\text { b50 } \\
42 \\
42 \\
0.2 \\
0.3\end{array}$ & $\begin{array}{l}2: 43 \text { p.m. } \\
\text { b50 } \\
42 \\
42 \\
6.3 \\
0.3\end{array}$ & $\begin{array}{l}2: 40 \text { p.m. } \\
28 \\
42 \\
42 \\
6.2 \\
0.2\end{array}$ & $\begin{array}{c}2: 37 \text { p.m. } \\
41 \\
0.1\end{array}$ & $\begin{array}{ll}\text { T. } & 42 \\
\text { B. } & 42 \\
\text { T. } & 6.2 \\
\text { B. } & 6.3\end{array}$ \\
\hline 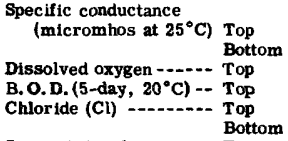 & $\begin{array}{r}118 \\
11.3 \\
8.6 \\
0.0\end{array}$ & $\begin{array}{l}121 \\
117 \\
11.4 \\
7.1 \\
6.0 \\
4.0\end{array}$ & $\begin{array}{r}114 \\
116 \\
12.3 \\
5.6 \\
4.0 \\
4.0\end{array}$ & $\begin{array}{r}117 \\
116 \\
12.0 \\
5.8 \\
4.0 \\
4.0\end{array}$ & $\begin{array}{r}118 \\
12.1 \\
5.9 \\
6.0\end{array}$ & $\begin{array}{lr} & \\
\text { T. } & 118 \\
\text { B. } & 116 \\
\text { T. } & 11.9 \\
\text { T. } & 6.6 \\
\text { T. } & 5.2 \\
\text { B. } & 4.0\end{array}$ \\
\hline Suspended sediment -- Top & & & & $\therefore \cdot$ & 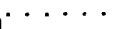 & T. 45 \\
\hline
\end{tabular}

b Exceeled this vilue.

Location League Is land. Fhilazdelphia, Pa. Weather Overcast

Date January 2, 1951 Sampling study No. - 18

\begin{tabular}{|c|c|c|c|c|c|c|}
\hline \multicolumn{7}{|c|}{ Weather Overcast } \\
\hline \multirow[b]{2}{*}{$\begin{array}{l}\text { Time (EST) } \\
\text { Sounding (ft) } \\
\left.\text { Temperature ( }{ }^{\circ} \mathrm{F}\right) \\
\text { pH }\end{array}$} & \begin{tabular}{|c|}
$\begin{array}{c}\text { Pennsylvania } \\
\text { side }\end{array}$ \\
\end{tabular} & West Center & Center & East Center & $\begin{array}{c}\text { New Jersey } \\
\text { side }\end{array}$ & Average \\
\hline & $\begin{array}{c}10: 41 \text { a.m. } \\
36 \\
6.6\end{array}$ & $\begin{array}{l}10: 38 \text { a.m. } \\
\text { b } 50 \\
35 \\
36 \\
0.3 \\
0.2\end{array}$ & $\begin{array}{c}10: 35 \mathrm{a} \cdot \mathrm{m} \\
45 \\
35 \\
36 \\
0.3 \\
0.2\end{array}$ & $\begin{array}{c}10: 33 \text { a.m. } \\
27 \\
35 \\
35 \\
6.3 \\
6.2\end{array}$ & $\begin{array}{c}10: 30 \text { a.m. } \\
34 \\
6.3\end{array}$ & $\begin{array}{lc}\text { T. } & 35 \\
\text { B. } & 36 \\
\text { T. } & 6.4 \\
\text { B. } & 6.2\end{array}$ \\
\hline \multirow[t]{2}{*}{ 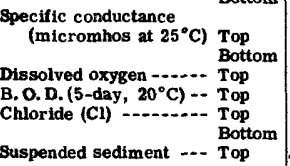 } & $\begin{array}{l}214 \\
5.4 \\
b 6 \\
10\end{array}$ & $\begin{array}{l}213 \\
217 \\
5.0 \\
b 6 \\
10 \\
10\end{array}$ & $\begin{array}{l}209 \\
213 \\
5.5 \\
b 6 \\
9 \\
10\end{array}$ & $\begin{array}{l}208 \\
209 \\
5.4 \\
06 \\
10 \\
10\end{array}$ & $\begin{array}{c}212 \\
5.9 \\
b 6 \\
8\end{array}$ & $\begin{array}{ll}\text { T. } & 211 \\
\text { B. } & 213 \\
\text { T. } & 5.4 \\
\text { T. } & 66.0 \\
\text { T. } & 9.4 \\
\text { B. } & 10\end{array}$ \\
\hline & & & . Com & e... & & T. 22 \\
\hline
\end{tabular}

b Exceeded this value.

Location League Island, Fhiladelphia, Pa. Weather Rain Date March 1, 1951

Sampling study No. 19

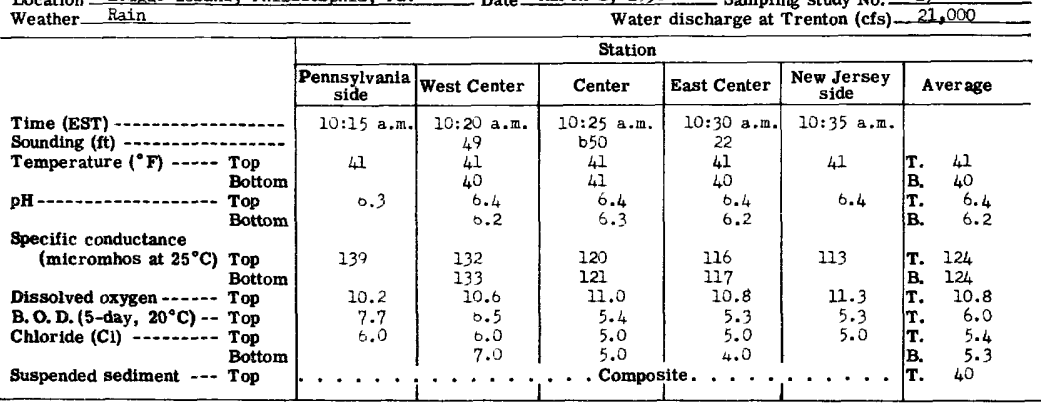

b Jxeeeded this value.

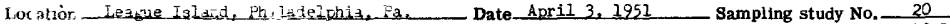

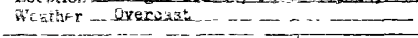
Water discharge at Trenton (cfs) $-53,100$

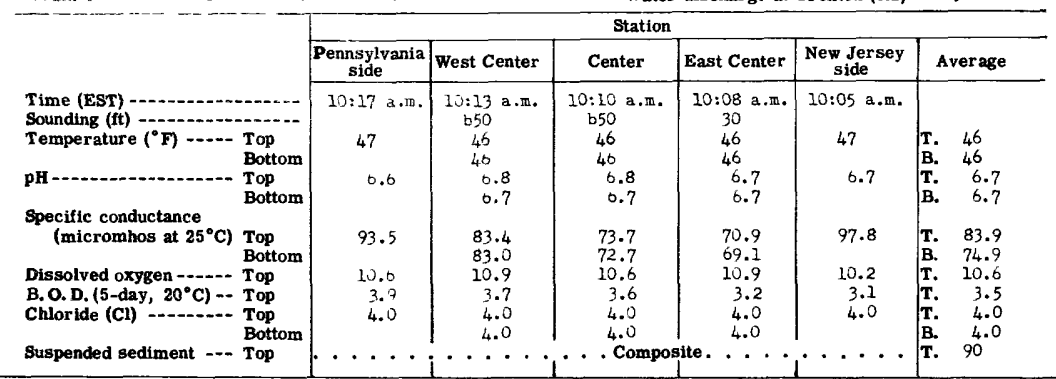


Table 12. -- WATER ANALYSES OF DELAWARE RTVER BETWEEN BRISTOL AND MARCUS HOOK, PA. --Continued Analyzed by City of Philadelphia and $U$. S. Geological Survey; analyses in parts per million

Location League Island, Philadelphia, Pa. _ Date May 2, 1951

Sampling study No. Weather Clear Water discharge at Trenton (cfs) $-12,400$

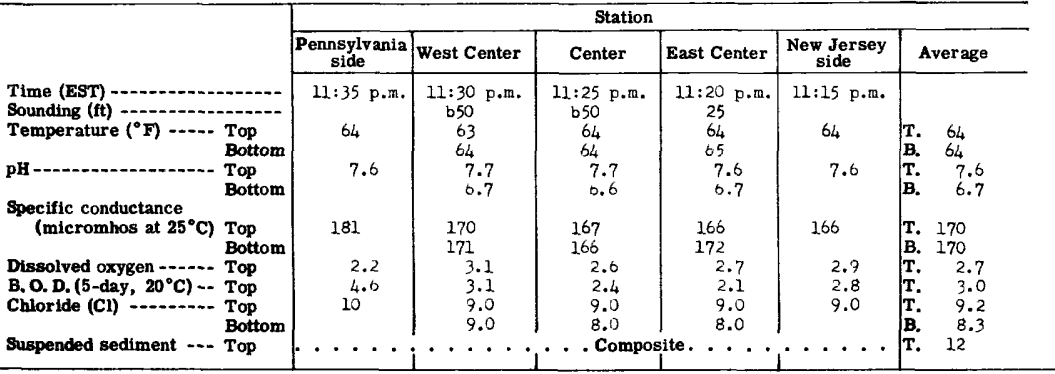

b Exceeded this value.

Location League Island, Fhiladelphia, Fa. Weather Rain

Date_June 8, 1951

Sampling study No. 22

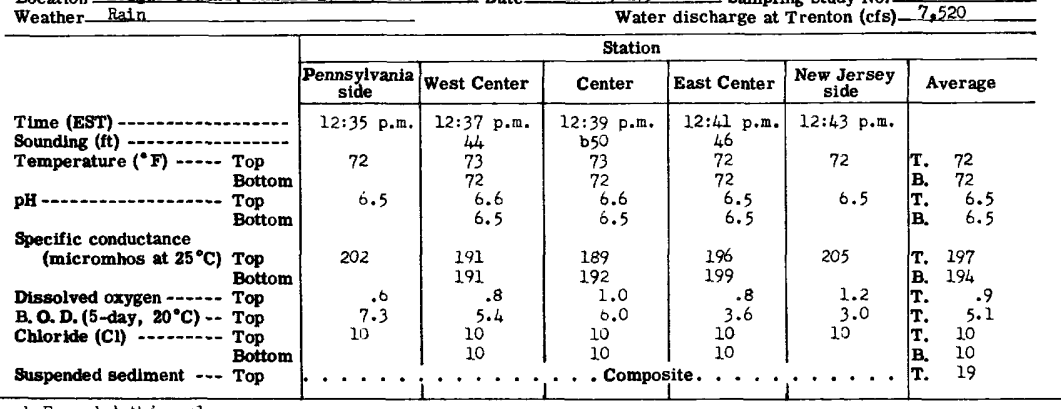

b Exceerled this value.

Location League Island, Phsladel phia, Pa, Weather Clear

Date_July 6, 1951

Sampling study No. 23

\begin{tabular}{|c|c|c|c|c|c|c|}
\hline \multicolumn{3}{|c|}{ Weather Clear } & \multicolumn{4}{|c|}{ Water discharge at Trenton (cfs) $-9,250$} \\
\hline & \multicolumn{6}{|c|}{ Station } \\
\hline & \begin{tabular}{|c|} 
Pennsylvania \\
side
\end{tabular} & West Center & Center & East Center & $\begin{array}{l}\text { New Jersey } \\
\text { side }\end{array}$ & Average \\
\hline $\begin{array}{ll}\text { Time (EST) } & \\
\text { Sounding (ft) } & \\
\text { Temperature ("F) } & \begin{array}{l}\text { Top } \\
\text { Bottom } \\
\text { pH }\end{array} \\
& \text { Top } \\
\text { Bottom }\end{array}$ & $\begin{array}{c}12: 36 \text { p.m. } \\
79 \\
6.6\end{array}$ & $\begin{array}{r}12: 39 \text { p.m. } \\
\text { b } 50 \\
79 \\
79 \\
6.6 \\
6.5\end{array}$ & $\begin{array}{c}12: 41 \mathrm{p} . \mathrm{m} . \\
\mathrm{b} 50 \\
78 \\
79 \\
6.6 \\
6.4\end{array}$ & $\begin{array}{c}12: 44 \mathrm{p} \cdot \mathrm{m} . \\
35 \\
78 \\
78 \\
6.6 \\
6.4\end{array}$ & $\begin{array}{c}12: 47 \text { p.m. } \\
77 \\
6.6\end{array}$ & $\begin{array}{ll}\text { T. } & 78 \\
\text { B. } & 79 \\
\text { T. } & 6.6 \\
\text { B. } & 6.4\end{array}$ \\
\hline $\begin{array}{ll}\begin{array}{l}\text { Specific conductance } \\
\text { (micromhos at } 25^{\circ} \mathrm{C} \text { ) }\end{array} & \begin{array}{l}\text { Top } \\
\text { Bottom }\end{array} \\
& \\
\text { Dissolved oxygen -...- } & \text { Top } \\
\text { B. O. D. (5-day, } 20^{\circ} \mathrm{C} \text { ) - } & \text { Top } \\
\text { Chloride (C1) } & \text { Bottom }\end{array}$ & $\begin{array}{r}218 \\
1.6 \\
12^{.4}\end{array}$ & $\begin{array}{l}208 \\
210 \\
1.0 \\
12.0 \\
12\end{array}$ & $\begin{array}{l}207 \\
201 \\
1.6 \\
12 \\
12\end{array}$ & $\begin{array}{l}202 \\
205 \\
2.0 \\
12 \\
12\end{array}$ & $\begin{array}{r}199 \\
3.9 \\
12^{.5}\end{array}$ & $\begin{array}{ll}\text { T. } & 207 \\
\text { B. } & 205 \\
\text { T. } & 2.0 \\
\text { T. } & \cdot 3 \\
\text { T. } & 12 \\
\text { B. } & 12\end{array}$ \\
\hline Suspended sediment $\ldots$-.. Top & & & . Comp & ite. . . & & $\mathbf{T}$ \\
\hline
\end{tabular}

b Exceeded this value.

Location League Igland, Philadel phia, Pa. Weather Clear

Date August 2, 1951 Sampling study No. 24 Water discharge at

\begin{tabular}{|c|c|c|c|c|c|c|}
\hline & \multicolumn{6}{|c|}{ Station } \\
\hline & \begin{tabular}{|c|} 
Pennsylvania \\
side
\end{tabular} & West Center & Center & East Center & $\begin{array}{c}\text { New Jersey } \\
\text { side }\end{array}$ & Average \\
\hline $\begin{array}{l}\text { Time (EST) } \\
\text { Sounding (ft) } \\
\text { Temperature }\left({ }^{\circ} \mathrm{F}\right) \\
\text { pH }\end{array} \begin{array}{l}\text { Top } \\
\text { Bottom }\end{array}$ & $\begin{array}{c}12: 55 \text { p.to. } \\
80 \\
6.7\end{array}$ & $\begin{array}{c}12: 57 \mathrm{p.m} . \\
\mathrm{b} 50 \\
80 \\
80 \\
6.8 \\
0.8\end{array}$ & $\begin{array}{c}12: 59 \mathrm{p.m} \\
\mathrm{b} 50 \\
80 \\
80 \\
6.7 \\
0.7\end{array}$ & $\begin{array}{l}1.01 \mathrm{p} . \mathrm{m} . \\
28 \\
80 \\
80 \\
0.7 \\
6.8\end{array}$ & $\begin{array}{c}1: 03 \mathrm{p.m} . \\
89 \\
7.1\end{array}$ & $\begin{array}{ll}\text { T. } & 80 \\
\text { B. } & 80 \\
\text { T. } & 6.8 \\
\text { B. } & 6.8\end{array}$ \\
\hline 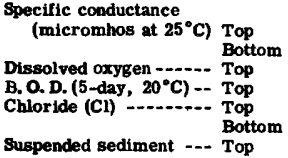 & $\begin{array}{r}231.2 \\
12^{.0}\end{array}$ & $\begin{array}{l}224 \\
231 \\
2.4 \\
11 \\
11.0\end{array}$ & $\frac{11}{216}{ }^{221} .6$ & $\begin{array}{l}213 \\
219 \\
3.6 \\
1.2 \\
\frac{11}{11}\end{array}$ & $\begin{array}{l}209 \\
4.6 \\
2.7 \\
11\end{array}$ & $\begin{array}{ll}\text { T. } & 219 \\
\text { B. } & 224 \\
\text { T. } & 3.3 \\
\text { T. } & .9 \\
\text { T. } & 11 \\
\text { B. } & 11 \\
\text { T. } & 10\end{array}$ \\
\hline
\end{tabular}

b Exceeded this value. 
Table 12. - WATER ANALYSES OF DELAWARE RIVER BETWEEN BRISTOL AND MARCUS HOOK, PA. --Contînued Analyzed by City of Philadelphia and $U$. S. Geological Survey; analyses in parts per million

Location _League Island, Philadel phia, Pa.____ Date_September 5, 1951 Sampling study No. 25

\begin{tabular}{|c|c|c|c|c|c|c|}
\hline \multicolumn{3}{|c|}{ Weather Cloudy } & \multicolumn{4}{|c|}{ Water discharge at Trenton (cfs) 3,680} \\
\hline & \multicolumn{6}{|c|}{ Station } \\
\hline & $\begin{array}{c}\text { Pennsylvania } \\
\text { side }\end{array}$ & West Center & Center & East Center & $\begin{array}{c}\text { New Jersey } \\
\text { side }\end{array}$ & Average \\
\hline $\begin{array}{ll}\text { Time (EST) } & \\
\text { Sounding (ft) } & \\
\text { Temperature }\left({ }^{\circ} \mathrm{F}\right) & \begin{array}{l}\text { Top } \\
\text { Bottom }\end{array} \\
\text { pH } & \begin{array}{l}\text { Top } \\
\text { Bottom }\end{array}\end{array}$ & $\begin{array}{c}12: 22 \mathrm{p} \cdot \mathrm{m} . \\
79 \\
6.4\end{array}$ & $\begin{array}{c}12: 24 \text { p.m. } \\
35 \\
79 \\
78 \\
6.4 \\
6.4\end{array}$ & $\begin{array}{c}12: 26 \mathrm{p} . \mathrm{m} \\
45 \\
78 \\
78 \\
6.3 \\
6.4\end{array}$ & $\begin{array}{c}12: 28 \mathrm{p} . \mathrm{m} . \\
35 \\
78 \\
78 \\
6.4 \\
6.4\end{array}$ & $\begin{array}{c}12: 30 \text { p.m. } \\
78 \\
6.4\end{array}$ & $\begin{array}{lc}\text { T. } & 78 \\
\text { B. } & 78 \\
\text { T. } & 6.4 \\
\text { B. } & 6.4\end{array}$ \\
\hline 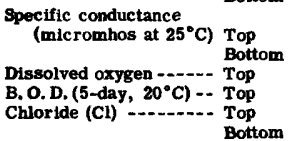 & $\begin{array}{l}301 \\
12.9 \\
15\end{array}$ & $\begin{array}{l}284 \\
275 \\
11.8 \\
14 \\
13\end{array}$ & $\begin{array}{l}282 \\
28.4 \\
9.2 \\
14.2 \\
14\end{array}$ & $\begin{array}{l}291 \\
293 \\
9.1 \\
14 \\
14\end{array}$ & $\begin{array}{l}298 \\
.6 \\
8.6 \\
15\end{array}$ & $\begin{array}{ll}\text { T. } & 291 \\
\text { B. } & 284 \\
\text { T. } & .3 \\
\text { T. } & 10.3 \\
\text { T. } & 14 \\
\text { B. } & 14\end{array}$ \\
\hline Suspended sediment -.- Top & & & & e. . & 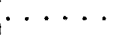 & $\mathbf{T}$ \\
\hline
\end{tabular}

Location League Island, Philadelphia, $\mathrm{Pa}$. Weather Clear

Date October 1, 1951 Sampling study No. 26

\begin{tabular}{|c|c|c|c|c|c|c|}
\hline \multicolumn{3}{|c|}{ Weather Clear } & \multicolumn{4}{|c|}{ Water discharge at Trenton (cfs) 3.040} \\
\hline & \multicolumn{6}{|c|}{ Station } \\
\hline & $\begin{array}{c}\text { Pennsylvania } \\
\text { side }\end{array}$ & West Center & Center & East Center & $\begin{array}{c}\text { New Jersey } \\
\text { side }\end{array}$ & Average \\
\hline $\begin{array}{ll}\text { Time (EST) } \\
\text { Sounding (ft) } \\
\text { Temperature }\left({ }^{\circ} \mathrm{F}\right) \\
\text { pH }\end{array}$ & $\begin{array}{c}2: 56 \mathrm{p} . \mathrm{m} . \\
71 \\
6.5\end{array}$ & $\begin{array}{l}2: 54 \mathrm{p} \cdot \mathrm{m} . \\
\mathrm{b} 50 \\
71 \\
72 \\
6.4 \\
6.5\end{array}$ & $\begin{array}{l}2: 52 \text { p.m. } \\
\text { b50 } \\
71 \\
72 \\
6.5 \\
7.6\end{array}$ & $\begin{array}{l}2: 50 \mathrm{p} \cdot \mathrm{m} . \\
37 \\
72 \\
71 \\
6.5 \\
6.4\end{array}$ & $\begin{array}{c}2: 48 \mathrm{p} \cdot \mathrm{m} . \\
72 \\
6.5\end{array}$ & $\begin{array}{ll}\text { T. } & 71 \\
\text { B. } & 72 \\
\text { T. } & 6.5 \\
\text { B. } & 6.8\end{array}$ \\
\hline 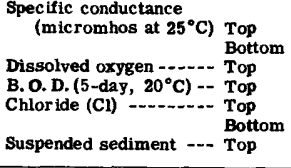 & $\begin{array}{l}362 \\
2.0 \\
6.9 \\
25 \\
\ldots\end{array}$ & $\begin{array}{c}390 \\
384 \\
2.2 \\
3.3 \\
28 \\
28 \\
\ldots\end{array}$ & $\begin{array}{l}376 \\
396 \\
.9 \\
3.0 \\
25 \\
38 \\
\therefore \text {. Con }\end{array}$ & $\begin{array}{c}365 \\
425 \\
1.7 \\
3.3 \\
25 \\
40 \\
\text { site. }\end{array}$ & $\begin{array}{l}377 \\
1.4 \\
2.1 \\
25\end{array}$ & $\begin{array}{ll}\text { T. } & 374 \\
\text { B. } & 402 \\
\text { T. } & 1.6 \\
\text { T. } & 3.7 \\
\text { T. } & 26 \\
\text { B. } & 35 \\
\text { T. } & 30\end{array}$ \\
\hline
\end{tabular}

b Exceeded this value.

Location League Igland, Philadel phia, Pa.___ Date November 5, 2951_ Sampling study No._27 Weather Clear _... Water discharge at Trenton (cfs)_- 39,800

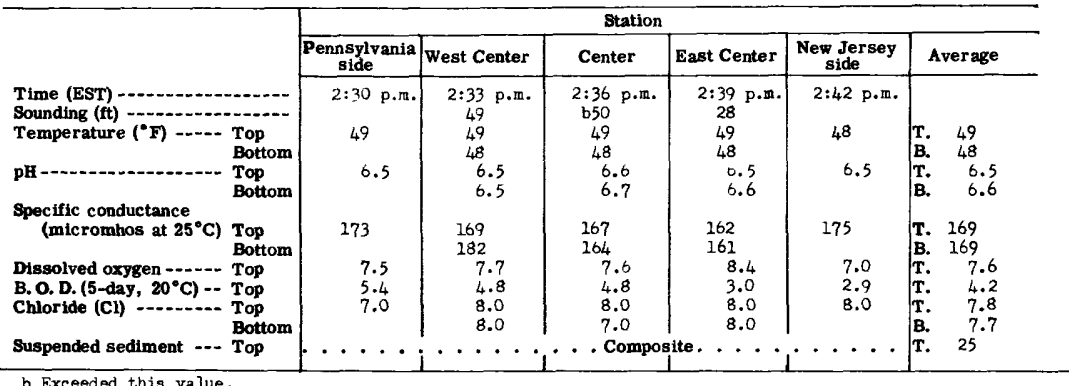

b Exceeded this value.

Location League [s] and, Philadelphia, Pa. Weather-Cloudy

Date December 4,2951 Sampling study No. 28

\begin{tabular}{|c|c|c|c|c|c|c|}
\hline \multicolumn{7}{|c|}{ Weather Cloudy } \\
\hline & \multicolumn{6}{|c|}{ Station } \\
\hline & \begin{tabular}{|c|} 
Pennsylvania \\
side
\end{tabular} & West Center & Center & East Center & $\begin{array}{c}\text { New Jersey } \\
\text { side }\end{array}$ & Aver age \\
\hline $\begin{array}{l}\text { Time (EST) } \\
\text { Sounding (ft) } \\
\text { Temperature }\left({ }^{\circ} \mathrm{F}\right) \\
\text { pH }\end{array}$ & $\begin{array}{c}2: 37 \text { p.r. } \\
43 \\
6.5\end{array}$ & $\begin{array}{r}2.34 \text { p.m. } \\
\text { b } 50 \\
43 \\
44 \\
6.3 \\
6.3\end{array}$ & $\begin{array}{l}2: 30 \text { p.m. } \\
\text { b50 } \\
43 \\
45 \\
6.3 \\
0.3\end{array}$ & $\begin{array}{l}2: 28 \text { p.n. } \\
25 \\
43 \\
45 \\
-- \\
6.3\end{array}$ & $\begin{array}{c}2: 26 \text { p.m. } \\
44 \\
0.2\end{array}$ & $\begin{array}{ll}\text { T. } & 43 \\
\text { B. } & 45 \\
\text { T. } & 6.3 \\
\text { B. } & 6.3\end{array}$ \\
\hline 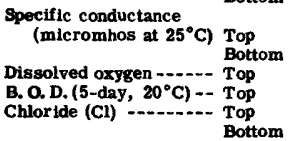 & $\begin{array}{l}160 \\
7.7 \\
5.2 \\
7.0\end{array}$ & $\begin{array}{r}168 \\
164 \\
8.0 \\
5.1 \\
8.0 \\
8.0\end{array}$ & $\begin{array}{r}273 \\
170 \\
7.4 \\
4.2 \\
8.0 \\
8.0\end{array}$ & $\begin{array}{r}17 \overline{6} \\
7.2 \\
4.4 \\
-8.0\end{array}$ & $\begin{array}{r}177 \\
7.2 \\
4.2 \\
8.0\end{array}$ & $\begin{array}{lr} & \\
\text { T. } & 169 \\
\text { B. } & 270 \\
\text { T. } & 7.5 \\
\text { T. } & 4.6 \\
\text { T. } & 7.7 \\
\text { B. } & 8.0\end{array}$ \\
\hline Suspended sediment -.- Top & & . & . Com & . . & $\because \cdot$ & T. 19 \\
\hline
\end{tabular}

b Exceeded this value. 
Table 12. -- WATER ANALYSES OF DELAWARE RIVER BETWEEN BRISTOL AND MARCUS HOOK, PA. --COntinued Analyzed by City of Philadelphia and U. S. Geological Survey; analyses in parts per million

Location Legque Island, PhiladeIuhia, Pa._Date January 2, 1952 __ Sampling study No, 29

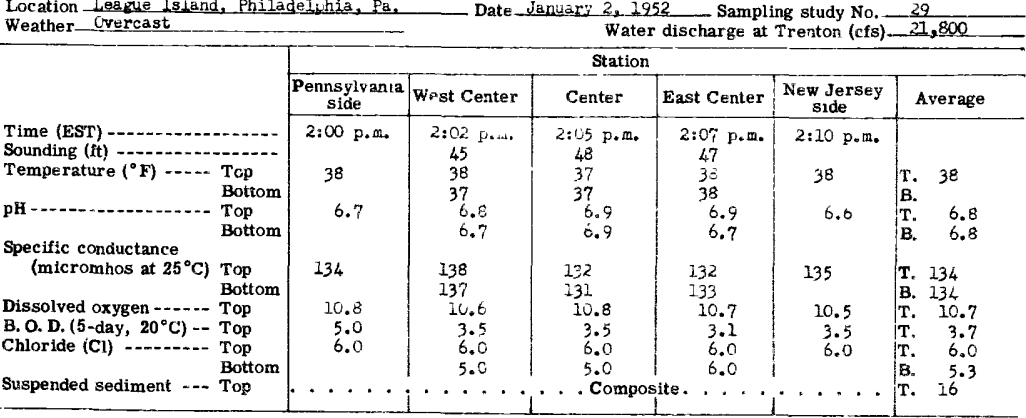

Location Leagle Islend, Philsdelphia, Pa. Weather Clear.

Date February 6, 2952___ Samping study No. 30

\begin{tabular}{|c|c|c|c|c|c|c|}
\hline \multicolumn{7}{|c|}{ Weather Clear } \\
\hline & $\begin{array}{c}\text { Pennsylvania } \\
\text { side }\end{array}$ & West Center & Center & East Center & $\begin{array}{c}\text { New Jersey } \\
\text { side }\end{array}$ & Average \\
\hline $\begin{array}{l}\text { Time (EST) } \\
\text { Sounding (ft) } \\
\text { Temperature }\left({ }^{\circ} \mathrm{F}\right) \\
\text { pH } \\
\begin{array}{l}\text { Top } \\
\text { Bottom } \\
\text { Specific conductance }\end{array} \quad \begin{array}{l}\text { Top } \\
\text { Bottom }\end{array}\end{array}$ & $\begin{array}{c}10: 00 \mathrm{~B} . \mathrm{m} \\
41 \\
6.2\end{array}$ & $\begin{array}{c}10: 03 \mathrm{a} . \mathrm{m} \\
48 \\
41 \\
41 \\
0.4 \\
0.4\end{array}$ & $\begin{array}{c}10: 05 \text { a.m. } \\
50 \\
40 \\
40 \\
6.5 \\
6.3\end{array}$ & $\begin{array}{c}10: 07 \text { a.m. } \\
25 \\
40 \\
39 \\
6.4 \\
6.4\end{array}$ & $\begin{array}{l}10: 10 \text { a.m. } \\
39 \\
6.3\end{array}$ & $\begin{array}{ll}\text { T. } & 40 \\
\text { B. } & 40 \\
\text { T. } & 6.4 \\
\text { B. } & 6.4\end{array}$ \\
\hline $\begin{array}{l}\text { Specific conductance } \\
\text { (mleromhos at } 25^{\circ} \mathrm{C} \text { ) Top } \\
\text { Bottom }\end{array}$ & 159 & $\begin{array}{l}102 \\
169\end{array}$ & $\begin{array}{l}128 \\
129\end{array}$ & $\begin{array}{l}126 \\
126\end{array}$ & 127 & $\begin{array}{ll}\text { T. } & 140 \\
\text { B. } 141\end{array}$ \\
\hline \multirow[t]{2}{*}{ 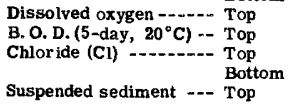 } & $\begin{array}{r}1.8 \\
4.5 \\
5.0\end{array}$ & $\begin{array}{r}12.2 \\
3.9 \\
6.0 \\
8.0\end{array}$ & $\begin{array}{r}12.2 \\
4.2 \\
4.0 \\
5.0\end{array}$ & $\begin{array}{r}12.2 \\
4.5 \\
4.0 \\
4.0\end{array}$ & $\begin{array}{r}12.0 \\
5.4 \\
4.0\end{array}$ & $\begin{array}{lr}\text { T. } & 12.1 \\
\text { T. } & 4.5 \\
\text { T. } & 4.6 \\
\text { B. } & 5.7\end{array}$ \\
\hline & $\cdot \cdot \cdot \cdot$ & $\cdot \ldots$ & . C Com & & & T. 49 \\
\hline
\end{tabular}

Location Leazue Island, Philadelchia, $\mathrm{Pa}$. Weather Overcast. Date_March 3,1952 Sampling study No. 31

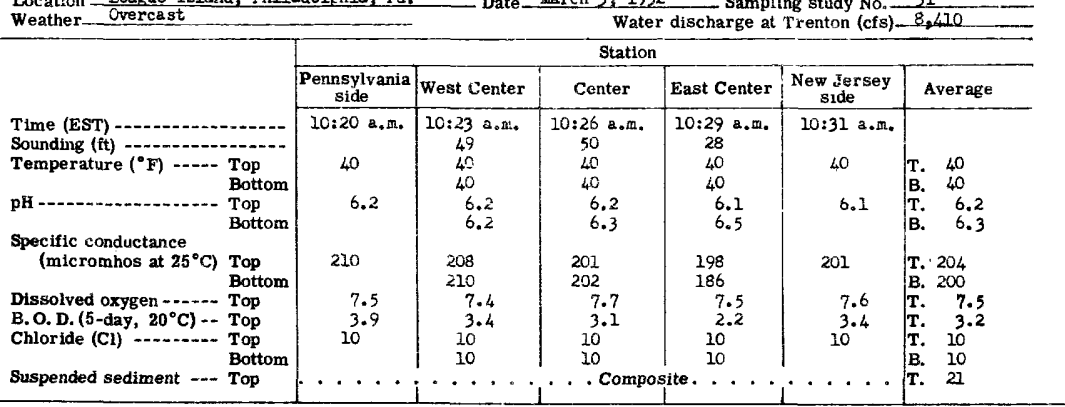

Location League Islard, Philadelphia, Pa. Weather-Clear Date April 1, 1952

Sampling study No, 32

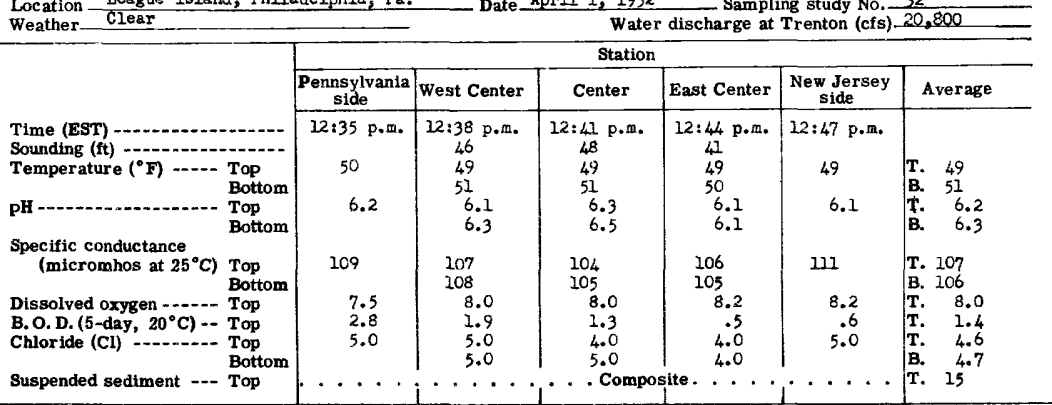


Table 12. --WATER ANALYSES Of DE LAWARE RIVER BETWEEN BRISTOL AND MARCUS hOOK, PA. --Contunued Analyzed by City of Phlladelphia and U. S. Geological Survey, analyses in parts per million

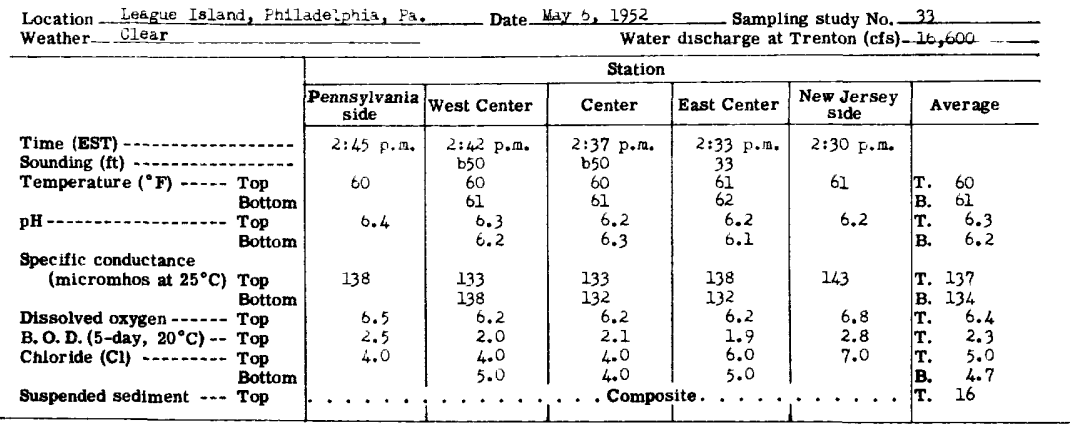

b ixceeded this valtie.

Location League Island, Phlladelphia, Pa. Weather clear

Date_June 3, 1952 Sampling study No. 34

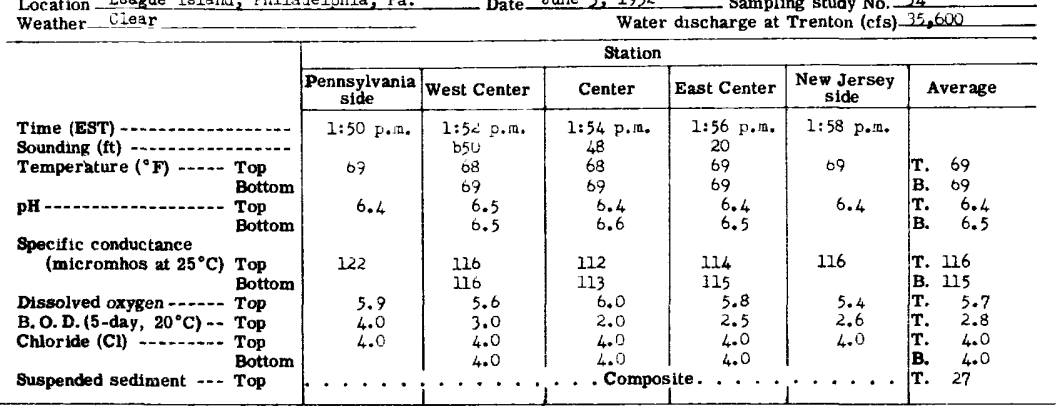

Location Loasue IsIand, Pniladolphia, Ps, Weather ivercast

Date JuIV B, 1352

- Sampling study No. -35 Water discharge at Trenton (cfs) 3,280

\begin{tabular}{|c|c|c|c|c|c|c|}
\hline \\
\hline & \multicolumn{6}{|c|}{ Station } \\
\hline & $\begin{array}{c}\text { Pennsyivania } \\
\text { side }\end{array}$ & West Center & Center & East Center & $\begin{array}{c}\text { New Jersey } \\
\text { side }\end{array}$ & Average \\
\hline $\begin{array}{l}\text { Time (EST) } \\
\text { Sounding (ft) } \\
\text { Temperature }\left({ }^{\circ} \mathrm{F}\right) \\
\text { pH }\end{array} \begin{array}{l}\text { Top } \\
\text { Bottom } \\
\text { Top } \\
\text { Bottom }\end{array}$ & $\begin{array}{c}1: 06 \text { p.m. } \\
80 \\
0.8\end{array}$ & $\begin{array}{l}1: 08 \text { p.u. } \\
\text { b50 } \\
30 \\
79 \\
6.7 \\
6.8\end{array}$ & $\begin{array}{l}1: 10 \text { p.m. } \\
\text { b50 } \\
80 \\
79 \\
6.8 \\
0.8\end{array}$ & $\begin{array}{l}1: 12 \text { p.m. } \\
\text { b50 } \\
80 \\
79 \\
0.8 \\
6.8\end{array}$ & $\begin{array}{l}1: 14 \mathrm{p} . \mathrm{m} \\
30 \\
0.8\end{array}$ & $\begin{array}{ll}\text { T. } & 80 \\
\text { B. } & 79 \\
\text { T. } & 6.8 \\
\text { B. } & 6.8\end{array}$ \\
\hline 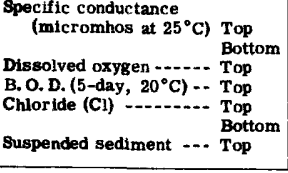 & $\begin{array}{l}.0 \\
14.4 \\
\ldots \ldots\end{array}$ & $\begin{array}{l}276 \\
264 \\
.1 \\
7.2 \\
14 \\
14 \\
\cdots\end{array}$ & $\begin{array}{l}267 \\
26.4 \\
.2 \\
7.2 \\
1.2 \\
.13 \\
. \text { Compo }\end{array}$ & $\begin{array}{l}267 \\
264 \\
.1 \\
4.8 \\
14 \\
14 \\
\text { te. }\end{array}$ & $\begin{array}{l}267 \\
1.1 .2 \\
. \cdots\end{array}$ & $\begin{array}{ll}\text { T. } & 270 \\
\text { B. } & 264 \\
\text { T. } & \cdot 1 \\
\text { T. } & 5.8 \\
\text { T. } & 14 \\
\text { B. } & 14 \\
\text { T. } & 82\end{array}$ \\
\hline
\end{tabular}

b Exceeded this value.

Location League Island, Philladelphia, $\mathrm{Pa}$. Weather Cloudy .... Date August 7,1952 Sampling study No. 36

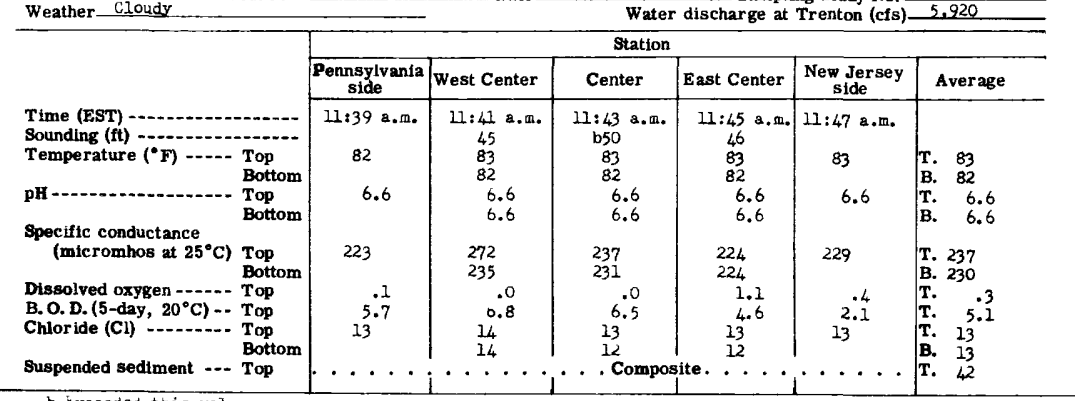

b Exceeded this value. 
Table 12. - WATER ANALYSES OF DELAWARE RIVER BETWEEN BRISTOL AND MARCUS HOOK, PA. --Contunued Analyzed by City of Philadelphia and U. S. Geological Survey; analyses in parts per million

Location _- League Island, Philadelfnia, Pa. Weather Clear Date Eeptember $4,195 \%$ Sampling study No. 37

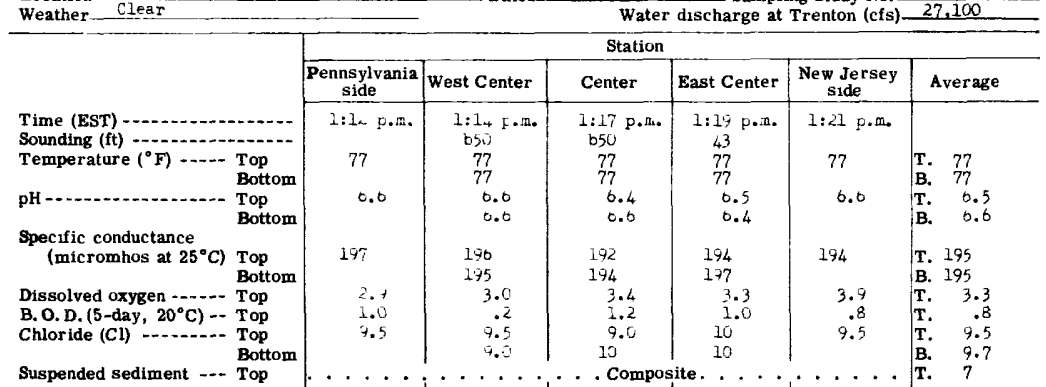

\footnotetext{
b exeeeded tris valut.
}

Location Leaque Island, Philadelphias Pa, Weather Slight ly Cloud

\begin{tabular}{|c|c|c|c|c|c|c|}
\hline \multicolumn{3}{|l|}{ Weather Slightly Cloudy } & \multicolumn{4}{|c|}{ Water discharge at Trenton (cfs) -4.120} \\
\hline & \multicolumn{6}{|c|}{ Station } \\
\hline & \begin{tabular}{|c|}
$\begin{array}{c}\text { Pennsylvania } \\
\text { side }\end{array}$ \\
\end{tabular} & West Center & Center & East Center & \begin{tabular}{|c|} 
New Jersey \\
side
\end{tabular} & Average \\
\hline $\begin{array}{ll}\text { Time (EST) } & \\
\text { Sounding (ft) } & \\
\text { Temperature }\left({ }^{\bullet} \mathrm{F}\right) & \text { Top } \\
\text { pH } & \text { Bottom } \\
& \text { Bottom }\end{array}$ & $\begin{array}{c}2: 00 \text { p.m. } \\
69 \\
6.6\end{array}$ & $\begin{array}{l}2: 03 \text { p. an. } \\
46 \\
69 \\
68 \\
6.4 \\
6.5\end{array}$ & $\begin{array}{l}2: 06 \text { p.m. } \\
\text { b50 } \\
69 \\
68 \\
6.7 \\
6.7\end{array}$ & $\begin{array}{l}2: 09 \text { p.m. } \\
\text { b50 } \\
68 \\
68 \\
6.7 \\
6.7\end{array}$ & $\begin{array}{l}2: 12 \text { p.m. } \\
67 \\
6.4\end{array}$ & $\begin{array}{ll}\text { T. } & 68 \\
\text { B. } & 68 \\
\text { T. } & 6.6 \\
\text { B. } & 6.6\end{array}$ \\
\hline 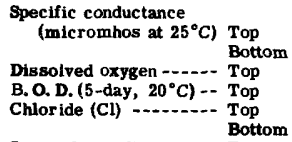 & $\begin{array}{r}285 \\
2.7 \\
3.5 \\
17\end{array}$ & $\begin{array}{l}265 \\
272 \\
1.7 \\
3.7 \\
16 \\
16\end{array}$ & $\begin{array}{l}285 \\
283 \\
1.5 \\
6.7 \\
16 \\
16\end{array}$ & $\begin{array}{l}285 \\
293 \\
1.5 \\
4.9 \\
16 \\
16\end{array}$ & $\begin{array}{l}274 \\
1.6 \\
6.6 \\
16\end{array}$ & $\begin{array}{ll}\text { T. } & 279 \\
\text { B. } & 283 \\
\text { T. } & 1.8 \\
\text { T. } & 5.1 \\
\text { T. } & 16 \\
\text { B. } & 16\end{array}$ \\
\hline Suspended sediment -.. Top & $\cdots \cdot$ & & . Com & site. . . & & T. 14 \\
\hline
\end{tabular}

b exceeded this value.

Location League Island, Fhiladelphia, $\mathrm{Pa}$. Weather Cloar

adelphia, $\mathrm{Pa}$. Date November 72 1952_- Sampling study No. 39

\begin{tabular}{|c|c|c|c|c|c|c|}
\hline \multicolumn{7}{|c|}{ Weather - Cloar } \\
\hline & \multicolumn{6}{|c|}{ Station } \\
\hline & \begin{tabular}{|c} 
Pennsylvania \\
side
\end{tabular} & Weat Center & Center & East Center & $\begin{array}{c}\begin{array}{c}\text { New Jersey } \\
\text { side }\end{array} \\
\end{array}$ & Average \\
\hline $\begin{array}{ll}\text { Time (EST) } \\
\text { Sounding (ft) } \\
\text { Temperature }\left({ }^{\circ} \mathrm{F}\right)\end{array}$ & $\begin{array}{c}10: 55 \text { a. } 3 . \\
51 \\
6.0\end{array}$ & $\begin{array}{c}10: 50 \text { a.m. } \\
44 \\
51 \\
51 \\
6.7 \\
6.5\end{array}$ & $\begin{array}{c}10: 40 \text { a.m. } \\
48 \\
51 \\
51 \\
6.8 \\
6.4\end{array}$ & $\begin{array}{c}10: 30 \text { a.m. } \\
16 \\
51 \\
51 \\
6.5 \\
6.3\end{array}$ & $\begin{array}{c}10: 25 \mathrm{a} \cdot \mathrm{m} . \\
51 \\
0.3\end{array}$ & $\begin{array}{ll}\text { T. } & 51 \\
\text { B. } & 51 \\
\text { T. } & 6.6 \\
\text { B. } & 6.4\end{array}$ \\
\hline 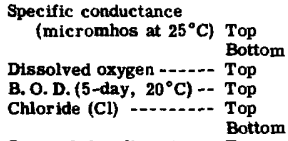 & $\begin{array}{l}369 \\
2.2 \\
10.1 \\
26\end{array}$ & $\begin{array}{l}372 \\
362 \\
1.8 \\
7.7 \\
28 \\
28\end{array}$ & $\begin{array}{l}343 \\
353 \\
2.0 \\
6.9 \\
26 \\
27\end{array}$ & $\begin{array}{l}366 \\
356 \\
4.7 \\
20.0 \\
30 \\
30\end{array}$ & $\begin{array}{l}360 \\
3.0 \\
7.5 \\
30\end{array}$ & $\begin{array}{ll}\text { T. } & 362 \\
\text { B. } & 357 \\
\text { T. } & 2.7 \\
\text { T. } & 8.4 \\
\text { T. } & 28 \\
\text { B. } & 28\end{array}$ \\
\hline Suspended sediment -.. Top & & & . Com & te. . . & . . & T. 12 \\
\hline
\end{tabular}
Location League Island, PhijadeIphis, Fa.
Weather hain Date December 5, 1952 Sampling study No. 40 Water discharge at Trenton (cfs) 12,600

\begin{tabular}{|c|c|c|c|c|c|c|}
\hline & \multicolumn{6}{|c|}{ Station } \\
\hline & \begin{tabular}{|c|}
$\begin{array}{c}\text { Pennsylvanial } \\
\text { side }\end{array}$ \\
\end{tabular} & West Center & Center & East Center & $\begin{array}{c}\text { New Jersey } \\
\text { side }\end{array}$ & Average \\
\hline $\begin{array}{l}\text { Time (EST) } \\
\text { Sounding (ft) } \\
\text { Temperature ( } \\
\text { pH }\end{array}$ & $\begin{array}{c}2: 18 \text { p. m. } \\
48 \\
6.4\end{array}$ & $\begin{array}{c}2: 21 \text { p.m. } \\
\text { b50 } \\
48 \\
48 \\
6.3 \\
6.3\end{array}$ & $\begin{array}{l}2: 24 \mathrm{p} . \mathrm{m} \\
\mathrm{b} 50 \\
46 \\
47 \\
6.2 \\
6.2\end{array}$ & $\begin{array}{c}2: 27 p+m \\
49 \\
48 \\
48 \\
6.2 \\
--\end{array}$ & $\begin{array}{c}2: 30 \text { p.m. } \\
47 \\
6.1\end{array}$ & $\begin{array}{ll}\text { T. } & 47 \\
\text { B. } & 48 \\
\text { T. } & 6.2 \\
\text { B. } & 6.2\end{array}$ \\
\hline 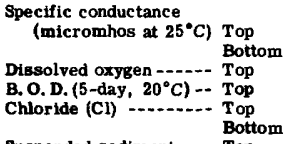 & $\begin{array}{l}191 \\
0.2 \\
0.1 \\
9.5\end{array}$ & $\begin{array}{l}179 \\
188 \\
6.3 \\
6.2 \\
9.0 \\
7.0\end{array}$ & $\begin{array}{l}173 \\
173 \\
5.7 \\
5.3 \\
9.0 \\
8.5\end{array}$ & $\begin{array}{r}167 \\
6.3 \\
6.0 \\
9.0\end{array}$ & $\begin{array}{l}162 \\
6.1 \\
4.6 \\
8.5\end{array}$ & $\begin{array}{lr}\text { T. } & 174 \\
\text { B. } & 180 \\
\text { T. } & 6.1 \\
\text { T. } & 5.6 \\
\text { T. } & 9.0 \\
\text { B. } & 8.8\end{array}$ \\
\hline Suspended sediment $\ldots$ Top & & & Com & site. . . & 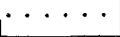 & T. 20 \\
\hline
\end{tabular}

b Exceeded this ralue.

$3153910-54-10$ 
Table 13. - - WATER ANALYSES OF DELAWARE RIVER BETWEEN BRISTOL AND MARCUS HOOK, PA. Analyzed by City of Philadelphia and U. S. Geological Survey; analyses in parts per million Location Eddystone, $\mathrm{Pa}$. Date_August 3, 3949
Weather-Trace of Rain

\begin{tabular}{|c|c|c|c|c|c|c|}
\hline & \multicolumn{6}{|c|}{ Station } \\
\hline & $\begin{array}{c}\text { Pennsylvania } \\
\text { side }\end{array}$ & West Center & Center & Kast Center & $\begin{array}{l}\text { New Jersey } \\
\text { side }\end{array}$ & Average \\
\hline $\begin{array}{l}\text { Time (EST) } \\
\text { Sounding (ft) } \\
\text { Temperature }\left({ }^{\circ} \mathrm{F}\right) \\
\text { pH }\end{array}$ & $\begin{array}{l}-- \\
86 \\
6.1\end{array}$ & $\begin{array}{l}-5 \\
45 \\
85 \\
84 \\
5.9 \\
6.1\end{array}$ & $\begin{array}{l}-- \\
\overline{84} \\
-\overline{6.1} \\
--\end{array}$ & $\begin{array}{l}38 \\
84 \\
84 \\
5.9 \\
6.0\end{array}$ & 84 & $\begin{array}{ll}\text { T. } & 85 \\
\text { B. } & 84 \\
\text { T. } & 6.0 \\
\text { B. } & 6.0\end{array}$ \\
\hline 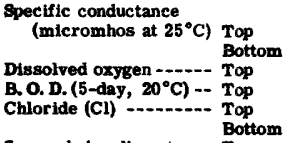 & $\begin{array}{l}400 \\
.7 \\
6.0\end{array}$ & $\begin{array}{l}405 \\
303 \\
1.0 \\
5.0 \\
47 \\
40\end{array}$ & $\begin{array}{l}382 \\
-3.0 \\
6.0 \\
40\end{array}$ & $\begin{array}{l}397 \\
397 \\
2.1 \\
4.0 \\
44 \\
45\end{array}$ & $\begin{array}{l}397 \\
5.8 \\
47\end{array}$ & $\begin{array}{ll}\text { T. } & 396 \\
\text { B. } & 380 \\
\text { T. } & .9 \\
\text { T. } & 5.2 \\
\text { T. } & 44 \\
\text { B. } & 42\end{array}$ \\
\hline Suspended sediment $\ldots$ Top & $\cdots$ & $\cdot$ & . Com & ite. . . & $\cdots \cdot$ & $\mathbf{T}$ \\
\hline
\end{tabular}

Location Eddystone, Pa

Date September 7, 1949 Sampling study No. 2

Weather Trace of Bsin Water discharge at Trenton (cfs) -3.810 .

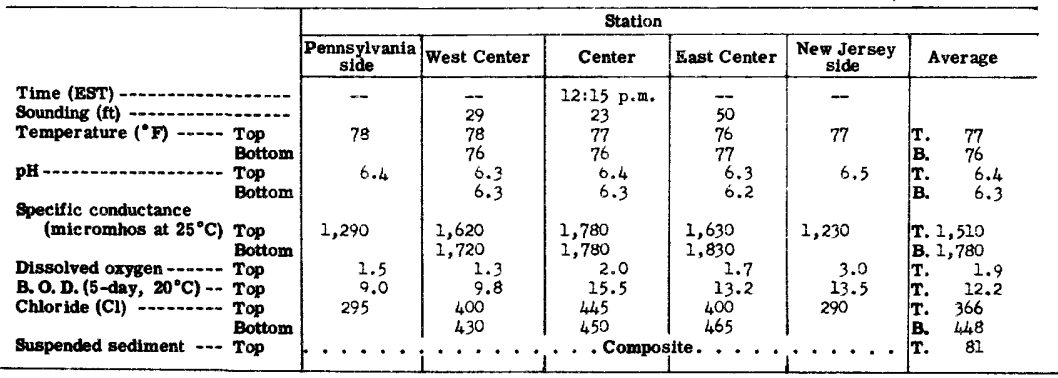

Location Eddyatone, Pa. Weather Occasional Rain

Date Oetober 5,1949 Bampling study No. 3 Water discharge at Trenton (cfs) 3,280

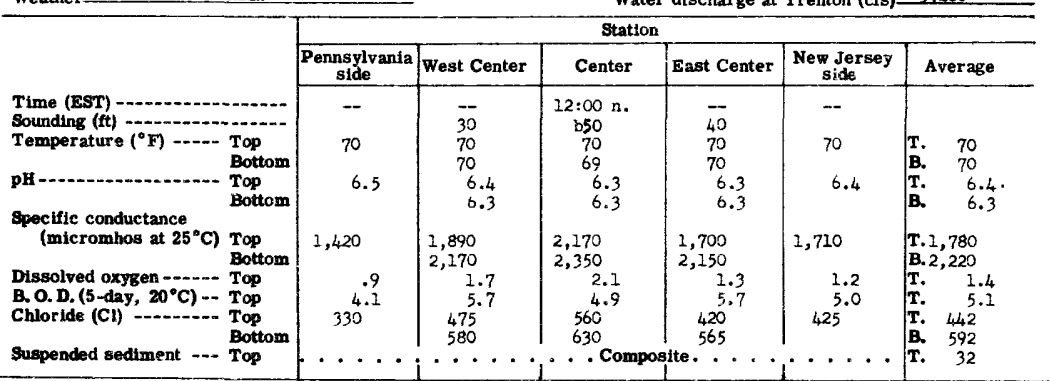

b Exceeded this value.

Location Edidystone, $\mathrm{Pa}$.

Weather... Clear.

Date November 2, 1249 Sampling study No.

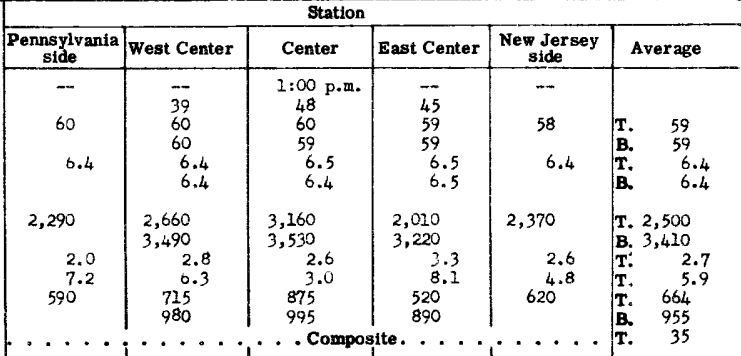


Table 13. -- WATER ANALYSES OF DBLAWARE FIVER BETWEEN BRISTOL AND MARCUS HOOK, PA. - Continued Analyzed by City of Phizdelphia and $U$. $\$$. Geological Survey; analyses in parts per million

Location Eddystone, $\mathrm{Pa}$. Date December 2, 1949 _________nompling study No. 5

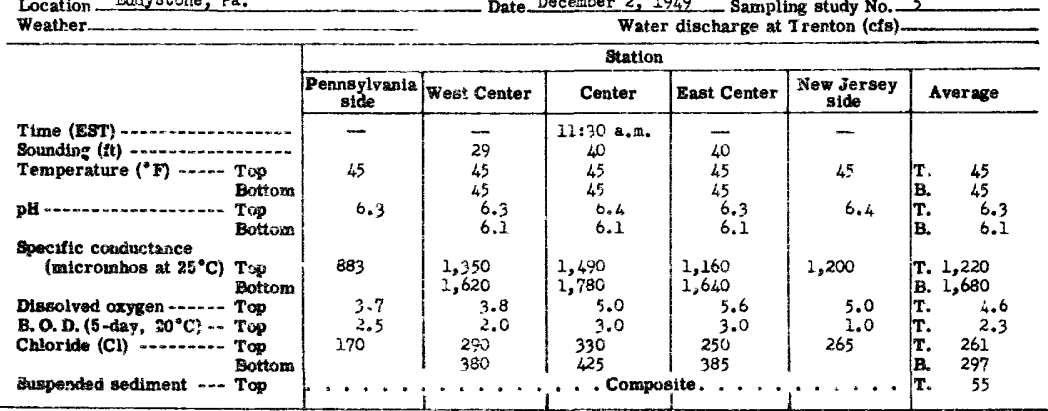

Location - Eqdystone, Pa. Weather Cloucy

Date_January 5, 1950 Bampling study No. 6

\begin{tabular}{|c|c|c|c|c|c|c|}
\hline & \multicolumn{6}{|c|}{ Station } \\
\hline & $\begin{array}{c}\text { Pennsylyania } \\
\text { sife }\end{array}$ & Wes' Center & Center & Erast Conter & $\begin{array}{c}\text { New Jersey } \\
\text { side }\end{array}$ & Ayerage \\
\hline $\begin{array}{l}\text { Time (EST) } \\
\text { Sounding (ft) } \\
\text { Temperature ('F) } \\
\text { pH } \\
\begin{array}{l}\text { Bottow } \\
\text { Botem }\end{array}\end{array}$ & $\begin{array}{l}2: 25 \text { p.m. } \\
50 \\
6.3\end{array}$ & $\begin{array}{l}2.29 \mathrm{p.O1} . \\
30 \\
49 \\
43 \\
6.2 \\
5.1\end{array}$ & $\begin{array}{l}2: 31 \text { p.m. } \\
45 \\
48 \\
46 \\
6.2 \\
6.0\end{array}$ & $\begin{array}{l}2: 35 p \cdot 0 . \\
\frac{47}{6.2} \\
-\end{array}$ & $\begin{array}{l}2: 35 \text { p.m. } \\
49 \\
6.2\end{array}$ & $\begin{array}{ll}\text { R. } & 49 \\
\text { B. } & 47 \\
\text { T. } & 6.2 \\
\text { B. } & 6.0\end{array}$ \\
\hline 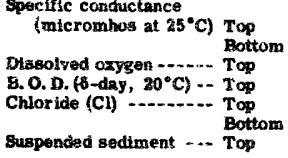 & $\begin{array}{l}293 \\
6.7 \\
4.5 \\
10\end{array}$ & $\begin{array}{l}192 \\
176 \\
6.7 \\
400 \\
12 \\
12\end{array}$ & $\begin{array}{l}168 \\
171 \\
7.0 \\
3.0 \\
10 \\
10 \\
. \mathrm{Co}\end{array}$ & $\begin{array}{l}162 \\
7.5 \\
10.0 \\
10 \\
-\ldots\end{array}$ & $\begin{array}{r}164 \\
7.3 \\
4.0 \\
9.0\end{array}$ & $\begin{array}{ll}\text { T. } & 276 \\
\text { B. } & 174 \\
\text { T. } & 7.0 \\
\text { T. } & 3.9 \\
\text { T. } & 10 \\
\text { B. } & 11 \\
\text { T. } & 60\end{array}$ \\
\hline
\end{tabular}

Location . Eddratone, $P$. Weather_Clear

Date Pobrucr.3.1950 Saropling study No._.

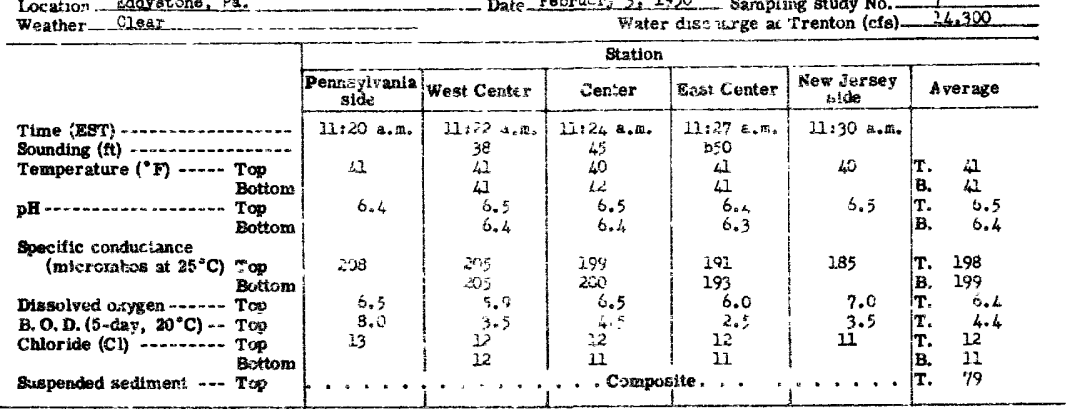

b Exceeded this veluo.

\begin{tabular}{|c|c|c|c|c|c|c|}
\hline $\begin{array}{l}\text { Location Eddystone Pa. } \\
\text { Weather Partly Cloudy }\end{array}$ & & - Date 1 & $\frac{\operatorname{sech} 7,195}{\text { Wat }}$ & discharge & $\begin{array}{l}\text { gg study No. } \\
\text { renton (cfs) }\end{array}$ & $\frac{8}{7.260}$ \\
\hline & & & Stat Io & & & \\
\hline & $\begin{array}{c}\text { Pennsylvania } \\
\text { sijas }\end{array}$ & West Center & Center & East Center & $\begin{array}{c}\text { Ne' Jersey } \\
\text { side }\end{array}$ & Aver age \\
\hline 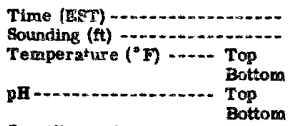 & $\begin{array}{c}11: 1.8 \text { a.m. } \\
36 \\
6.6\end{array}$ & $\begin{array}{c}1: 45 \text { B.m. } \\
29 \\
37 \\
36 \\
6.5 \\
6.5\end{array}$ & $\begin{array}{c}11: 41 \mathrm{a}+\mathrm{m} . \\
47 \\
36 \\
37 \\
6.6 \\
5.5\end{array}$ & $\begin{array}{c}11: 40 \text { a.m. } \\
33 \\
38 \\
37 \\
6.6 \\
6.5\end{array}$ & $\begin{array}{c}11: 35 \mathrm{a} . \mathrm{m} . \\
38 \\
6.7\end{array}$ & $\begin{array}{ll}\text { T. } & 37 \\
\text { B. } & 37 \\
\text { T. } & 6.6 \\
\text { B. } & 6.5\end{array}$ \\
\hline 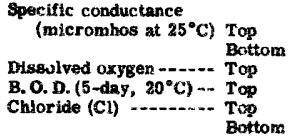 & $\begin{array}{l}212 \\
8.5 \\
4.6 \\
22\end{array}$ & $\begin{array}{l}204 \\
206 \\
8.7 \\
3.8 \\
12 \\
12\end{array}$ & $\begin{array}{l}202 \\
204 \\
9.2 \\
4.3 \\
12 \\
22\end{array}$ & $\begin{array}{l}202 \\
204 \\
9.2 \\
3.8 \\
12 \\
12\end{array}$ & $\begin{array}{l}202 \\
9.1 \\
3.7 \\
12\end{array}$ & $\begin{array}{ll}\text { T. } & 204 \\
\text { B. } & 205 \\
\text { T. } & 8.9 \\
\text { T. } & 4.0 \\
\text { T. } & 12 \\
\text { B. } & 12\end{array}$ \\
\hline uspended sediment -... Top & & & S & & $=\cdot \cdot$ & $r$ \\
\hline
\end{tabular}


Table 13. -- WATER ANALYSES OF DELAWARE RIVER BETWEEN BRISTOL AND MARC US HOOK, EA - -COntInUed Analyzed by City of Philadeiphia and U. S. Geological Survey; analyses in parts per million

Location Eddystone, Pa. Weather Light Rain

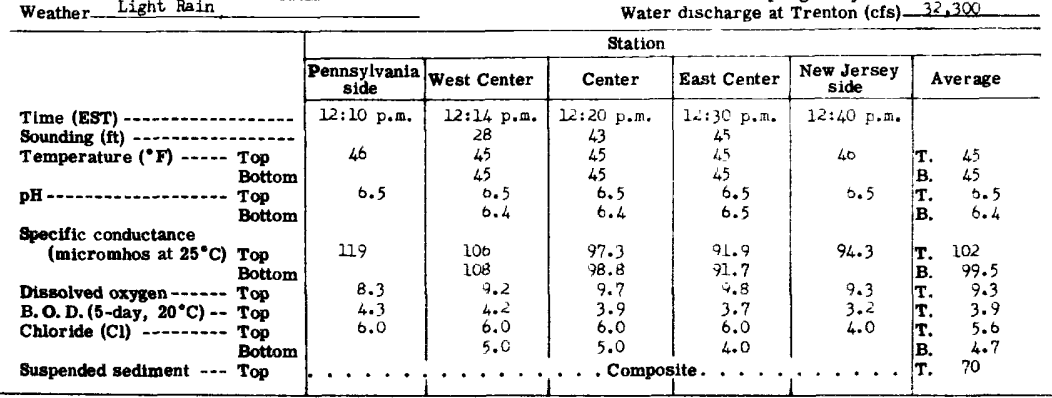

Location Eddystone, $\mathrm{Pa}$. Date Hay 3, 1950 Sampling study No. Weather Cloudy Water discharge at Trenton (cfs) 17,000 Station

\begin{tabular}{|c|c|c|c|c|c|c|}
\hline & \multicolumn{6}{|c|}{ Station } \\
\hline & \begin{tabular}{|c} 
Pennsylvania \\
side
\end{tabular} & West Center & Center & East Center & $\begin{array}{c}\text { New Jersey } \\
\text { side }\end{array}$ & Average \\
\hline $\begin{array}{l}\text { Time (EST) } \\
\text { Sounding (ft) } \\
\text { Temperature }\left({ }^{\circ} \text { F) }\right. \\
\text { pH }\end{array}$ & $\begin{array}{c}2: 40 \text { p.m. } \\
57 \\
6.6\end{array}$ & $\begin{array}{l}2: 43 \text { p.m. } \\
30 \\
57 \\
55 \\
6.5 \\
6.5\end{array}$ & $\begin{array}{l}2: 48 \text { p.m. } \\
\text { b50 } \\
55 \\
55 \\
6.5 \\
\text { b. } 4\end{array}$ & $\begin{array}{l}2: 55 \mathrm{p} . \mathrm{m} . \\
43 \\
56 \\
55 \\
6.5 \\
6.5\end{array}$ & $\begin{array}{c}3: 00 \mathrm{~F}, \mathrm{~m} . \\
56 \\
6.5\end{array}$ & $\begin{array}{l}56 \\
55 \\
6.5 \\
6.5\end{array}$ \\
\hline $\begin{array}{ll}\text { (micromhos at } 25^{\circ} \mathrm{C} \text { ) } & \begin{array}{l}\text { Top } \\
\text { Bottom }\end{array} \\
\text { Dissolved oxygen } \ldots . . .- & \text { Top } \\
\text { B. O. D. (5-day, 20 } 20^{\circ} \mathrm{C} \text { ) - Top } \\
\text { Chloride (Cl) }\end{array}$ & $\begin{array}{l}186 \\
3.3 \\
9.4 \\
10\end{array}$ & $\begin{array}{l}175 \\
176 \\
3.4 \\
0.9 \\
10 \\
8.0\end{array}$ & $\begin{array}{l}174 \\
174 \\
3.9 \\
7.2 \\
10 \\
9.0\end{array}$ & $\begin{array}{r}171 \\
173 \\
3.6 \\
6.7 \\
9.0 \\
9.0\end{array}$ & $\begin{array}{l}174 \\
4.2 \\
6.5 \\
9.0\end{array}$ & $\begin{array}{lr}\text { T. } & 176 \\
\text { B. } & 174 \\
\text { T. } & 3.0 \\
\text { T. } & 7.3 \\
\text { T. } & 9.6 \\
\text { B. } & 8.7\end{array}$ \\
\hline Suspended sediment $\ldots$ Top & & $\cdots$ & Com & te. . & . & 38 \\
\hline
\end{tabular}

b Exceeded this value.

Location Eddystone, Pa. Weather Clear

\begin{tabular}{|c|c|c|c|c|c|c|}
\hline $\begin{array}{l}\text { Location Ladyst } \\
\text { Weather Clear }\end{array}$ & & & wat & discharge & $\begin{array}{l}\text { g study No } \\
\text { renton (cf }\end{array}$ & $\frac{11}{18}, 500$ \\
\hline & & & Statio & & & \\
\hline & $\begin{array}{c}\text { Pennsylvania } \\
\text { side }\end{array}$ & West Center & Center & East Center & $\begin{array}{c}\text { New Jersey } \\
\text { side }\end{array}$ & Average \\
\hline $\begin{array}{l}\text { Time (EST) } \\
\text { Sounding (ft) } \\
\text { Temperature }\left({ }^{\bullet} \mathrm{F}\right) \\
\text { pH }-1\end{array}$ & $\begin{array}{c}10: 30 \text { a.m. } \\
08 \\
6.2\end{array}$ & $\begin{array}{c}10: 35 \text { a.m. } \\
45 \\
68 \\
69 \\
6.4 \\
6.2\end{array}$ & $\begin{array}{c}10: 37 \text { a.m. } \\
43 \\
68 \\
68 \\
6.3 \\
6.2\end{array}$ & $\begin{array}{c}10: 41 \mathrm{a} . \mathrm{m} \\
38 \\
68 \\
68 \\
6.4 \\
6.1\end{array}$ & $\begin{array}{c}10: 44 \mathrm{a} . \mathrm{m} . \\
68 \\
6 . \overline{3}\end{array}$ & $\begin{array}{ll}\text { T. } & 68 \\
\text { B. } & 68 \\
\text { T. } & 6.3 \\
\text { B. } & 0.2\end{array}$ \\
\hline 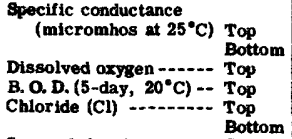 & $\begin{array}{l}170 \\
\\
2.3 \\
2.1 \\
8.0\end{array}$ & $\begin{array}{r}157 \\
160 \\
2.3 \\
.3 \\
8.0 \\
8.0\end{array}$ & $\begin{array}{r}156 \\
157 \\
2.2 \\
1.8 \\
7.0 \\
8.0\end{array}$ & $\begin{array}{r}151 \\
155 \\
3.1 \\
.5 \\
7.0 \\
8.0\end{array}$ & $\begin{array}{r}153 \\
3.0 \\
.6 \\
7.0\end{array}$ & $\begin{array}{lr}\text { T. } & 257 \\
\text { B. } & 157 \\
\text { T. } & 2.6 \\
\text { T. } & 1.1 \\
\text { T. } & 7.4 \\
\text { B. } & 9.0\end{array}$ \\
\hline Suspended sediment _..- Top & $\cdots \cdot$ & & Co & e. . & . & T. 25 \\
\hline
\end{tabular}

Location Eddystone, Pa. Weather Cloudy 
Table 13. -- WATER ANALYSES OF DELAWARE RIVER BETWEEN BRISTOL AND MARCUS HOOK, PA. - Continued Analyzed by City of Philadelphia and U. S. Geological Survey; analyses in parts per million

Location Eddystone, $\mathrm{Pa}$.

Weather. Cloudy Water discharge at Trenton (cfs) $-4,210$

\begin{tabular}{|c|c|c|c|c|c|c|}
\hline & \multicolumn{6}{|c|}{ Station } \\
\hline & \begin{tabular}{|c|} 
Pennsylvania \\
side
\end{tabular} & West Center & Center & East Center & $\begin{array}{c}\text { New Jersey } \\
\text { side }\end{array}$ & Average \\
\hline $\begin{array}{ll}\text { Time (EST) } & \\
\text { Sounding (ft) } \\
\text { Temperature }(" \mathrm{~F})\end{array}$ & $\begin{array}{c}10: 15 \mathrm{a} . \mathrm{ml} . \\
80 \\
6.2\end{array}$ & $\begin{array}{c}10: 17 \text { a.m. } \\
24 \\
80 \\
80 \\
6.3 \\
6.4\end{array}$ & $\begin{array}{c}10: 20 \text { a.m. } \\
46 \\
80 \\
80 \\
6.2 \\
6.3\end{array}$ & $\begin{array}{c}10: 22 \text { a.m. } \\
30 \\
80 \\
80 \\
6.3 \\
0.4\end{array}$ & $\begin{array}{c}10: 25 \text { a. } . \text {. } \\
80 \\
6.2\end{array}$ & $\begin{array}{l}80 \\
80 \\
6.2 \\
6.4\end{array}$ \\
\hline 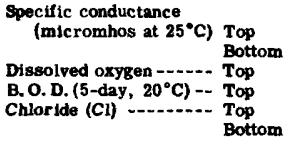 & $\begin{array}{l}234 \\
3.8 \\
3.8\end{array}$ & $\begin{array}{l}236 \\
244 \\
3.9 \\
15 \\
13\end{array}$ & $\begin{array}{l}225 \\
234 \\
2.3 \\
16 \\
14\end{array}$ & $\begin{array}{r}224 \\
232 \\
1.9 \\
1.9 \\
14\end{array}$ & $\begin{array}{l}229 \\
1.0 \\
2.2 \\
26\end{array}$ & $\begin{array}{ll}\text { T. } & 230 \\
\text { B. } & 237 \\
\text { T. } & .8 \\
\text { T. } & 2.6 \\
\text { T. } & 15 \\
\text { B. } & 14\end{array}$ \\
\hline Suspended sediment $\ldots$ Top & & $\cdots \cdot$ & & ... & $\cdots \cdot$ & 47 \\
\hline
\end{tabular}

Location Eddystone, $\mathrm{Pa}$.

Date September 5,1950 Sampling study No. 14

Weather-Fair $\quad$ Water discharge at Trenton (cfs) 5,800

\begin{tabular}{|c|c|c|c|c|c|c|}
\hline \\
\hline & \multicolumn{6}{|c|}{ Station } \\
\hline & $\begin{array}{c}\text { Pennsylvania } \\
\text { side }\end{array}$ & West Center & Center & East Center & $\begin{array}{c}\text { New Jersey } \\
\text { side }\end{array}$ & Average \\
\hline 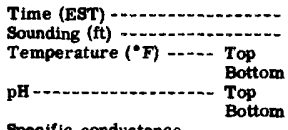 & $\begin{array}{c}12: 35 \text { p.m. } \\
78 \\
6.4\end{array}$ & $\begin{array}{c}12: 32 \mathrm{p} . \mathrm{m} . \\
47 \\
78 \\
78 \\
6.4 \\
6.3\end{array}$ & $\begin{array}{c}12: 30 \text { p.m. } \\
28 \\
78 \\
78 \\
0.5 \\
6.4\end{array}$ & $\begin{array}{c}12: 25 \mathrm{p} . \mathrm{m} \\
18 \\
78 \\
78 \\
6.5 \\
6.4\end{array}$ & $\begin{array}{c}12: 20 \text { p.m. } \\
78 \\
6.6\end{array}$ & $\begin{array}{ll}\text { T. } & 78 \\
\text { B. } & 78 \\
\text { T. } & 6.5 \\
\text { B. } & 6.4\end{array}$ \\
\hline 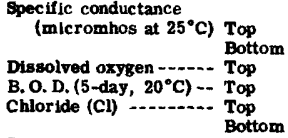 & $\begin{array}{r}304 \\
.5 \\
6.0\end{array}$ & $\begin{array}{l}305 \\
305 \\
1.0 \\
7.8 \\
22 \\
23\end{array}$ & $\begin{array}{l}313 \\
316 \\
1.0 \\
6.9 \\
24 \\
25\end{array}$ & $\begin{array}{l}318 \\
319 \\
1.0 \\
6.0 \\
25 \\
26\end{array}$ & $\begin{array}{l}320 \\
1.1 \\
4.8 \\
26\end{array}$ & $\begin{array}{ll}\text { T. } & 312 \\
\text { B. } & 313 \\
\text { T. } & .9 \\
\text { T. } & 6.3 \\
\text { T. } & 2.4 \\
\text { B. } & 25\end{array}$ \\
\hline Suspended sediment $\ldots$ Top & -4 & . & $\cdot \operatorname{Cos}$ & te. . . & ${ }^{\circ}$ & T. 34 \\
\hline
\end{tabular}

Location Eddystone, $\mathrm{Pa}$.

Date Detober 2e 1950 Sampling study No. $\frac{15}{30}$

\begin{tabular}{|c|c|c|c|c|c|c|}
\hline Weather Clear & & & & lischarge & renton (cf: & 3,020 \\
\hline & & & Statio & & & \\
\hline & $\begin{array}{c}\text { Pennsylvania } \\
\text { side } \\
\end{array}$ & West Center & Center & East Center & $\begin{array}{c}\text { New Jersey } \\
\text { side }\end{array}$ & Average \\
\hline $\begin{array}{l}\text { Time (EST) } \\
\text { Sounding (ft) } \\
\text { Temperature }\left({ }^{\circ} \mathrm{F}\right) \\
\text { gH }\end{array}$ & $\begin{array}{l}12: 55 \text { p.m. } \\
69 \\
6.2\end{array}$ & $\begin{array}{l}1: 00 \text { p. m. } \\
24 \\
68 \\
68 \\
6.3 \\
6.3\end{array}$ & $\begin{array}{l}1: 05 \mathrm{p} . \mathrm{m} . \\
42 \\
68 \\
68 \\
6.3 \\
0.2\end{array}$ & $\begin{array}{c}1: 10 \text { p.m. } \\
\text { b50 } \\
68 \\
68 \\
6.4 \\
6.2\end{array}$ & $\begin{array}{l}1: 15 \mathrm{p} . \mathrm{m} . \\
68 \\
6.4\end{array}$ & $\begin{array}{ll}\text { T. } & 68 \\
\text { B. } & 68 \\
\text { T. } & 6.3 \\
\text { B. } & 0.2\end{array}$ \\
\hline 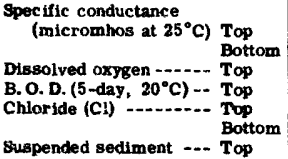 & $\begin{array}{l}330 \\
3.0 \\
28\end{array}$ & $\begin{array}{l}311 \\
310 \\
.4 \\
1.0 \\
24 \\
24\end{array}$ & $\begin{array}{l}305 \\
296 \\
2.7 \\
24 \\
24\end{array}$ & $\begin{array}{l}314 \\
299 \\
2.8 \\
26 \\
2.9\end{array}$ & $\begin{array}{l}325 \\
\quad .8 \\
26.8\end{array}$ & $\begin{array}{ll}\text { T. } & 317 \\
\text { B. } & 302 \\
\text { T. } & .7 \\
\text { T. } & 2.7 \\
\text { T. } & 26 \\
\text { B. } & 24 \\
\text { T. } & 77\end{array}$ \\
\hline
\end{tabular}

b Excoeded this vaiue.

Location Ëddystone, Pa.

Date November 1, 1950 Sampling study No. 16

\begin{tabular}{|c|c|c|c|c|c|c|}
\hline \multicolumn{7}{|c|}{ Weather Cloar } \\
\hline & \multicolumn{6}{|c|}{ Station } \\
\hline & $\begin{array}{c}\text { Pennsylvania } \\
\text { side }\end{array}$ & West Center & Center & East Center & $\begin{array}{c}\text { New Jersey } \\
\text { side } \\
\end{array}$ & Average \\
\hline $\begin{array}{l}\text { Time (EST) } \\
\text { Sounding (ft) } \\
\text { Temperature ("F) }\end{array}$ & $\begin{array}{c}1: 43 \text { p.m. } \\
64 \\
6.0\end{array}$ & $\begin{array}{l}1: 40 \mathrm{p} . \mathrm{m} . \\
20 \\
64 \\
65 \\
6.6 \\
0.5\end{array}$ & $\begin{array}{l}1: 37 \mathrm{p} . \mathrm{m} . \\
49 \\
64 \\
65 \\
6.5 \\
6.2\end{array}$ & $\begin{array}{l}1: 34 \text { p.m. } \\
22 \\
65 \\
65 \\
6.4 \\
6.1\end{array}$ & $\begin{array}{c}1: 30 \text { p.m. } \\
66 \\
6.3\end{array}$ & $\begin{array}{ll}\text { T. } & 65 \\
\text { B. } & 65 \\
\text { T. } & 6.5 \\
\text { B. } & 6.3\end{array}$ \\
\hline 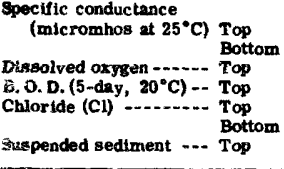 & $\begin{array}{l}531 \\
60^{.6} \\
\ldots\end{array}$ & $\begin{array}{l}484 \\
483 \\
.6 \\
6.6 \\
50 \\
50 \\
. \ldots .\end{array}$ & $\begin{array}{l}509 \\
495 \\
1.2 \\
7.5 \\
55 \\
55 \\
. \text { Con }\end{array}$ & $\begin{array}{l}603 \\
581 \\
6.7 \\
80 \\
80 \\
\text { tte. }\end{array}$ & $\begin{array}{l}652 \\
1.8 \\
6.6 \\
100\end{array}$ & $\begin{array}{ll}\text { T. } & 556 \\
\text { B. } & 520 \\
\text { T. } & 1.0 \\
\text { T. } & 6.6 \\
\text { T. } & 69 \\
\text { B. } & 62 \\
\text { T. } & 77\end{array}$ \\
\hline
\end{tabular}


Table 13. - WATER ANALYSES OF DELAWARE RIVER BETWEEN BRISTOL AND MARC GS HOOK, PA. - COntinued Analyzed by City of Philadelphia and U. S. Geological Survevi analyses in parts per million

Location Eddystone, Fs.

Date Decembsr is 1950 Sampling study No. 17

Time (EST)

Temperature ( $\mathrm{D} F$ ) -... Top

Bottom

Boton

Specific conductance

(micromhos at $25^{\circ} \mathrm{C}$ ) Top

Botfom

Chloride (Cl) $\ldots 0^{\circ} \mathrm{C}$ ) - Top

Suspended sediment ... Bottom

b Exceeded this value.

Location Eddystone, Fa. Weather_erercast

Date Jarivary 2, 1951___ sampling study No. $\quad 18$

\begin{tabular}{|c|c|c|c|c|c|c|}
\hline \multicolumn{2}{|c|}{ Weather Grercast } & \multicolumn{5}{|c|}{ Water discharge at Trenton (cfs) $-8,670$} \\
\hline & \multicolumn{6}{|c|}{ Station } \\
\hline & $\begin{array}{l}\text { Pennsylvania } \\
\text { side }\end{array}$ & West Center & Center & East Center & $\begin{array}{c}\begin{array}{c}\text { New Jersey } \\
\text { side }\end{array} \\
\end{array}$ & Average \\
\hline $\begin{array}{ll}\text { Time (EST) } \\
\text { Sounding (ft) } \\
\text { Temperature }\left({ }^{\circ} \mathrm{g}\right) \\
\text { pH } & \begin{array}{l}\text { Top } \\
\text { Bottom }\end{array} \\
& \begin{array}{l}\text { Top } \\
\text { Bottom }\end{array}\end{array}$ & $\begin{array}{c}11: 34=. \mathrm{m} . \\
36 \\
6.5\end{array}$ & $\begin{array}{c}1 \mathrm{i}: 30 . .1 \mathrm{~A} . \\
35 \\
36 \\
36 \\
6.4 \\
6.3\end{array}$ & $\begin{array}{c}11: 26 \text { a. } 50 . \\
47 \\
35 \\
36 \\
6.3 \\
5.6\end{array}$ & $\begin{array}{c}11.23 \text { a.m. } \\
39 \\
36 \\
36 \\
6.4 \\
5.3\end{array}$ & $\begin{array}{l}22: 20 \mathrm{a} \cdot \mathrm{m} . \\
30 \\
6.4\end{array}$ & $\begin{array}{ll}\text { T. } & 36 \\
\text { B. } & 36 \\
\text { T. } & 6.4 \\
\text { B. } & 6.4\end{array}$ \\
\hline 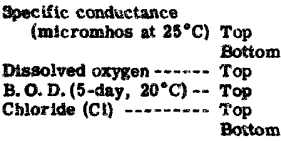 & $\begin{array}{l}212 \\
6.0 \\
5.7 \\
10\end{array}$ & $\begin{array}{l}212 \\
210 \\
6.2 \\
6.0 \\
9.0 \\
10\end{array}$ & $\begin{array}{r}208 \\
209 \\
6.3 \\
6.1 \\
8.0 \\
8.0\end{array}$ & $\begin{array}{l}205 \\
207 \\
0.5 \\
4.7 \\
8.0 \\
10\end{array}$ & $\begin{array}{l}204 \\
5.4 \\
5.0 \\
8.0\end{array}$ & $\begin{array}{lr}\text { T. } & 208 \\
\text { B. } & 209 \\
\text { T. } & 6.3 \\
\text { T. } & 5.5 \\
\text { T. } & 8.6 \\
\text { B. } & 9.3\end{array}$ \\
\hline Suspended sediment $\ldots$ Top & & &. Com & ite. . & - & ? \\
\hline
\end{tabular}

Location Eddystone, Fa.

Weatter Eain

Date Warct 3 - 1951

Samping study No. ..... 19 Water discharge ad Trenton (cis) - 21, 00

\begin{tabular}{|c|c|c|c|c|c|c|}
\hline & \multicolumn{6}{|c|}{ Statlet } \\
\hline & $\begin{array}{c}\text { Penneylvents } \\
\text { side }\end{array}$ & West Cenzer & Center & Isat Center & $\begin{array}{l}\text { New Jersey } \\
\text { side }\end{array}$ & Average \\
\hline 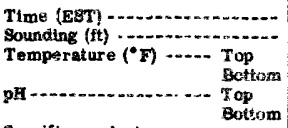 & $\begin{array}{c}21: 15.9 .0 . \\
41 \\
b .4\end{array}$ & $\begin{array}{c}12.20 \\
28 \\
4.1 \\
41 \\
6.5 \\
0.3\end{array}$ & $\begin{array}{c}21: 258.30 \\
49 \\
41 \\
40 \\
6.5 \\
0.4\end{array}$ & $\begin{array}{l}11: 30 \mathrm{a} . \mathrm{an} . \\
30 \\
41 \\
40 \\
6.5 \\
6.4\end{array}$ & $\begin{array}{l}11: 35 \mathrm{a} . \mathrm{x} . \\
\text { 4. } \\
0.5\end{array}$ & $\begin{array}{ll}r & 41 \\
\mathbf{C} & 40 \\
\mathbf{T} . & 6.5 \\
\text { B. } & 6.4\end{array}$ \\
\hline 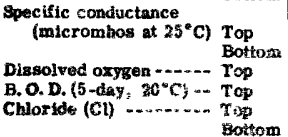 & $\begin{array}{l}15.4 \\
20.2 \\
6.7 \\
8.0\end{array}$ & $\begin{array}{r}134 \\
137 \\
10.6 \\
5.5 \\
6.0 \\
7.0\end{array}$ & $\begin{array}{l}132 \\
135 \\
10.9 \\
6.1 \\
0.0 \\
6.0\end{array}$ & $\begin{array}{l}128 \\
129 \\
10.9 \\
5.9 \\
6.0 \\
0.0\end{array}$ & $\begin{array}{r}129 \\
10.5 \\
6.0 \\
6.0\end{array}$ & $\begin{array}{lr} & \\
\text { T. } & 135 \\
\text { B. } & 134 \\
\text { r. } & 10.7 \\
\text { r. } & 6.0 \\
\text { r. } & 6.4 \\
\text { B. } & 6.3\end{array}$ \\
\hline susponded sediment ... Top & $+\cdots$ & & . Coxap & ito. . . & 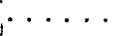 & T. 84 \\
\hline
\end{tabular}

Locatica Eddjetone, Pe.

Date Agr 11 3, 3951 _ Sampling stuty Ko._- 20

\begin{tabular}{|c|c|c|c|c|c|c|}
\hline \multirow[b]{3}{*}{ 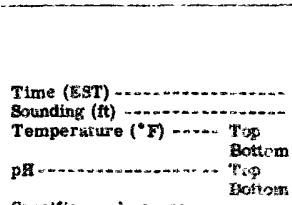 } & \multicolumn{6}{|c|}{ Station } \\
\hline & $\begin{array}{c}\text { Pennsylvania } \\
\text { side }\end{array}$ & Weat Ceater & Center & East Center & $\begin{array}{c}\text { New Jersey } \\
\text { side }\end{array}$ & Average \\
\hline & $\begin{array}{c}12: 10 \% . m . \\
40 \\
0,8\end{array}$ & $\begin{array}{c}11: 08 \text { a.... } \\
30 \\
48 \\
46 \\
0.8 \\
6.8\end{array}$ & $\begin{array}{c}11: 06 \mathrm{a}, \mathrm{m} . \\
650 \\
4 ? \\
=? \\
5.8 \\
0.5\end{array}$ & $\begin{array}{c}11: 03 \mathrm{a}, \mathrm{m} \\
31 \\
47 \\
17 \\
0.9 \\
6.8\end{array}$ & $\begin{array}{c}11: \infty 0 \mathrm{~s} \cdot \mathrm{m} . \\
47 \\
5.8\end{array}$ & $\begin{array}{l}47 \\
47 \\
6.6 \\
0.8\end{array}$ \\
\hline 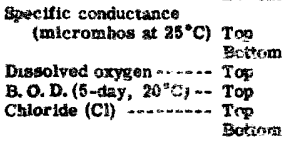 & $\begin{array}{l}104 \\
9.9 \\
4.2 \\
4.0\end{array}$ & $\begin{array}{r}87.2 \\
86.1 \\
10.0 \\
2.3 \\
4.2 \\
4.0\end{array}$ & $\begin{array}{l}74.3 \\
74.0 \\
10.6 \\
3.2 \\
4.0 \\
4.0\end{array}$ & $\begin{array}{l}74.3 \\
74.3 \\
10.0 \\
2.2 \\
4.0 \\
4.0\end{array}$ & $\begin{array}{l}5.7 \\
10.4 \\
3.2 \\
4.5\end{array}$ & $\begin{array}{lr}\text { T. } & 52,9 \\
\text { B. } & 73.1 \\
\text { T. } & 10.2 \\
\text { T. } & 3.2 \\
\text { T. } & 6.0 \\
\text { B. } & 4.0\end{array}$ \\
\hline Buzpended redimzint $\rightarrow$ Tos & & & C Con & 1 & 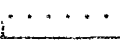 & $\mathrm{T}$ \\
\hline
\end{tabular}

t. Exceeded tin's vajue. 
Table 13. - WATER ANALYSES OF DELAWARE RIVER BETWEEN BRISTOL AND MARCUS HOOK, PA. --CONTINUEd Analyzed by City of Philadelphia and U. S. Geological Survey; analyses in parts per million

Location Eddystone, $\mathrm{Pa}$. Weather Cles

W - _ _ -

Time (EST)

Sounding (ft) -

Temperature $\left({ }^{\circ} \mathrm{F}\right)$

Pottom
Top

Bottom

(micrombos at $25^{\circ} \mathrm{C}$ ) Top

Bottom

Dissolved oxygen -.....- Top

B. O. D. (5-day, $20^{\circ} \mathrm{C}$ ) -- Top

Chloride (Cl) -.....- TOP

Suspended sediment .... Top

b Exceeded tnis value.
Date May 2, 195

Sampling study No.

Water discharge at Trenton (cfs) $12,4,00$

Station

\begin{tabular}{|c|c|c|c|c|c|}
\hline $\begin{array}{c}\text { Pennsylvania } \\
\text { side }\end{array}$ & West Center & Center & East Center & $\begin{array}{c}\text { New Jersey } \\
\text { Side }\end{array}$ & Aver age \\
\hline $10: 45$ a.m. & $\begin{array}{c}10: 40 \text { a.m. } \\
28\end{array}$ & $\begin{array}{c}10: 35 \text { a.m. } \\
\text { b50 }\end{array}$ & $\begin{array}{c}10: 30 \text { a.m. } \\
46\end{array}$ & $10: 25$ a.m. & \\
\hline
\end{tabular}

6.6

195

\begin{tabular}{l|l}
28 & 650 \\
63 & 63
\end{tabular}

6.6

63

6.6
6.6

18

2.4
2.8

11.8
6.6

\begin{tabular}{l|l}
81 & 177 \\
82 & 177
\end{tabular}

$2.2 \quad 3.4$

\begin{tabular}{l|l}
11 & 2.6 \\
10 & 9.0
\end{tabular}
46

63
63
0.6

0.6
6.6

177
177
3.4
2.6
9.0
9.0

6.6

B. 63

179

176
177

3.0

3.0

9.0
3.5 T 179

$3.5 \quad$ T. 2.8

$\begin{array}{ll}9.0 & \text { T. } \\ \text { T. } & 9.8 \\ \text { B. } & 9.3\end{array}$

Location Eddystone, Fa. Weather Rayn

Date_June 8, 1951 _____ampling study No. 22

\begin{tabular}{|c|c|c|c|c|c|c|}
\hline \multicolumn{2}{|c|}{ Weather Rain } & \multicolumn{5}{|c|}{ Water discharge at Trenton (cfs) $-7,520$} \\
\hline & \multicolumn{6}{|c|}{ Station } \\
\hline & \begin{tabular}{|c|}
$\begin{array}{c}\text { Pennsylvania } \\
\text { side }\end{array}$ \\
\end{tabular} & West Center & Center & East Center & \begin{tabular}{|c|} 
New Jersey \\
side
\end{tabular} & Average \\
\hline 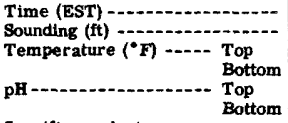 & $\begin{array}{c}11: 30 \text { a.m. } \\
72 \\
6.0\end{array}$ & $\begin{array}{c}11: 32 \text { a.m. } \\
22 \\
72 \\
72 \\
6.6 \\
6.5\end{array}$ & $\begin{array}{c}11: 34 \text { a.m. } \\
47 \\
72 \\
72 \\
0.6 \\
0.5\end{array}$ & $\begin{array}{c}11: 36 \text { a.m. } \\
44 \\
72 \\
72 \\
6.6 \\
6.5\end{array}$ & $\begin{array}{c}11: 38 \mathrm{a} . \mathrm{m} . \\
72 \\
6.6\end{array}$ & $\begin{array}{ll}\text { T. } & 72 \\
\text { B. } & 72 \\
\text { T. } & 6.6 \\
\text { B. } & 6.5\end{array}$ \\
\hline 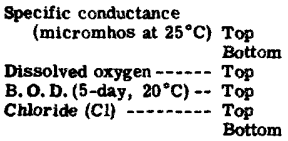 & $\begin{array}{l}229 \\
1.5 \\
3.0 \\
12\end{array}$ & $\begin{array}{l}229 \\
232 \\
2.7 \\
13 \\
12\end{array}$ & $\begin{array}{l}223 \\
22.4 \\
12.8 \\
12\end{array}$ & $\begin{array}{l}223 \\
224 \\
1.0 \\
2.0 \\
13 \\
12\end{array}$ & $\begin{array}{l}226 \\
1.1 \\
1.5 \\
12\end{array}$ & $\begin{array}{ll}\text { T. } & 226 \\
\text { B. } & 227 \\
\text { T. } & 1.0 \\
\text { T. } & 2.3 \\
\text { T. } & 12 \\
\text { B. } & 12\end{array}$ \\
\hline uspended sediment.-- Top & & &. Cor & te... & & 41 \\
\hline
\end{tabular}

Location Eddystone, $\mathrm{Pa}$

Date_Juiy 6, 1951 Sampling study No._23

Weather Clear

Water discharge at Trenton (cfs) 9,250

\begin{tabular}{|c|c|c|c|c|c|c|}
\hline \\
\hline \multirow{2}{*}{ Time (EST) } & \multicolumn{6}{|c|}{ Station } \\
\hline & $\begin{array}{c}\text { Pennsylvania } \\
\text { side }\end{array}$ & West Center & Center & East Center & $\begin{array}{l}\text { New Jersey } \\
\text { side }\end{array}$ & Average \\
\hline $\begin{array}{ll}\text { Time (EST) } & \\
\text { Sounding (ft) } & \\
\text { Temperature }\left({ }^{\circ} \mathrm{F}\right) & \begin{array}{l}\text { Top } \\
\text { Bottom }\end{array} \\
& \text { Pop } \\
\text { Bottom }\end{array}$ & $\begin{array}{c}11: 35 \mathrm{a} . \mathrm{m} . \\
78 \\
6.6\end{array}$ & $\begin{array}{c}11: 37 \text { a.m. } \\
35 \\
78 \\
77 \\
6.5 \\
6.6\end{array}$ & $\begin{array}{c}11: 39 \text { a.m. } \\
48 \\
79 \\
77 \\
6.9 \\
6.5\end{array}$ & $\begin{array}{c}11: 41 \mathrm{a} . \mathrm{m} \\
45 \\
78 \\
77 \\
6.6 \\
6.6\end{array}$ & $\begin{array}{c}11: 43 \mathrm{a} \cdot \mathrm{m} . \\
78 \\
6.7\end{array}$ & $\begin{array}{ll}\text { T. } & 78 \\
\text { B. } & 77 \\
\text { T. } & 6.7 \\
\text { B. } & 0.6\end{array}$ \\
\hline 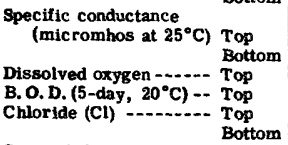 & 235 & $\begin{array}{l}225 \\
224 \\
2.1 \\
13 \\
13\end{array}$ & $\begin{array}{l}256 \\
224 \\
2.1 \\
13 \\
13\end{array}$ & $\begin{array}{l}224 \\
231 \\
2.4 \\
13 \\
13\end{array}$ & $\begin{array}{r}239 \\
2.5 \\
13\end{array}$ & \begin{tabular}{ll} 
D. & \\
T. & 236 \\
B. & 226 \\
T. & 2.2 \\
T. & \multicolumn{2}{c}{$\cdot 2$} \\
T. & 13 \\
B. & 13
\end{tabular} \\
\hline Suspended sediment --- Top & $\cdots$ & &. $\mathrm{Co}$ & & & $\mathrm{T}$. \\
\hline
\end{tabular}

Location Eddystone, $\mathrm{Pa}$. Weather Clear

Date Algust 2, 1951 ___ Sampling study No 24

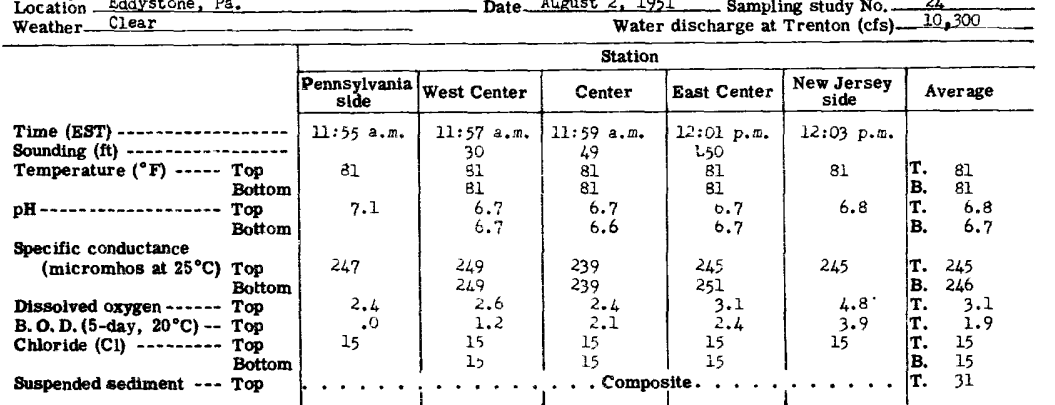

b uxceeded this value. 
Table 13, -- WATER ANALYSES OF DELAWARE RIVER BETWEeN BRISTOL AND MARCUS HOOK, PA. --Continued Analyzed by City of Philadelphia and U. S. Geological Survey; analyses in parts per million

Location - Eddystone, Fa.

Date Septemiter 5, 175 - Sampling study No. 25 Water discharge at Trenton (cfs) $3,68 \mathrm{C}$

Weather_._Louds

Time (EST)

Temperature ( $\mathrm{F}$ ) -... Top

Botton

Top

Specific conductance

(micromhos at $25^{\circ} \mathrm{C}$ ) $\mathrm{Top}$

Bottom
(micromhos at $\left.25^{\circ} \mathrm{C}\right)$ Top

Dissolved oxygen -...... Top

B. O. D. (5-day, $20^{\circ} \mathrm{C}$ ) -- Top

Chloride (C1) ........ Top

Suspended sediment ... Top

Station

\begin{tabular}{|c|c|c|c|c|c|}
\hline \multicolumn{6}{|c|}{ Station } \\
\hline \begin{tabular}{|c|} 
Pennsylvania \\
side
\end{tabular} & West Center & Center & East Center & $\begin{array}{c}\text { New Jersey } \\
\text { side }\end{array}$ & Average \\
\hline $\begin{array}{c}\text { il: } 4 \text { a.s. } \\
\text { r8 } \\
0.5\end{array}$ & $\begin{array}{c}11: \text { a.t a. Al. } \\
24 \\
71 \\
78 \\
0.3 \\
6.5\end{array}$ & $\begin{array}{c}11:-4 \text { a. } 5.1 . \\
17 \\
\because 2 \\
78 \\
0.4 \\
0.3\end{array}$ & $\begin{array}{c}11:<t a .15 . \\
48 \\
-7 \\
70 \\
b .5 \\
t .+\end{array}$ & $\begin{array}{c}11: 2 E \text { a. . . } \\
n \\
0.4\end{array}$ & $\begin{array}{l}79 \\
79 \\
0.4 \\
0.4\end{array}$ \\
\hline $\begin{array}{l}31 i \\
.3 \\
19 \\
3.4\end{array}$ & $\begin{array}{c}32+ \\
325 \\
8.0 \\
12 \\
18\end{array}$ & $\begin{array}{l}3.7 \\
3.5 \\
.5 \\
0.5 \\
18 \\
19\end{array}$ & $\begin{array}{l}3 \% \\
298 \\
18 \\
0.4 \\
10\end{array}$ & $\begin{aligned} 3-3 \\
2.7 \\
18\end{aligned}$ & $\begin{array}{ll}\text { T. } & 319 \\
\text { B. } & 316 \\
\text { T. } & .5 \\
\text { T. } & 7.3 \\
\text { T. } & 18 \\
\text { B. } & 17\end{array}$ \\
\hline & & . Com & & $\cdots \cdot$ & $\mathbf{T}$ \\
\hline
\end{tabular}

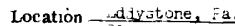

Weather- Clear

Date xtober $1,1+51$ Sampling study No. 26

\begin{tabular}{|c|c|c|c|c|c|c|}
\hline Weather Clear & - & & & & & 140 \\
\hline & \multicolumn{6}{|c|}{ Station } \\
\hline & $\begin{array}{c}\text { Pennsylvania } \\
\text { side }\end{array}$ & West Center & Center & East Center & $\begin{array}{c}\text { New Jersey } \\
\text { side }\end{array}$ & Average \\
\hline $\begin{array}{l}\text { Time (EST) } \\
\text { Sounding (ft) } \\
\text { Temperature ("F) }\end{array} \begin{array}{l}\text { Top } \\
\text { Bottom }\end{array}$ & $\begin{array}{c}1: 47 \text { F. } \\
72 \\
0.5\end{array}$ & $\begin{array}{l}1: 4 . F \cdot \Omega . \\
35 \\
7 . \\
71 \\
b .4 \\
6.3\end{array}$ & $\begin{array}{l}1: i \text { s. } \\
\text { b5 } \\
7.2 \\
71 \\
0.4 \\
6.5\end{array}$ & $\begin{array}{l}1: 40 \text { r.o.h. } \\
36 \\
72 \\
71 \\
6.3 \\
5.4\end{array}$ & $\begin{array}{l}1: 38 \text { p.m. } \\
72 \\
6.1\end{array}$ & $\begin{array}{ll}\text { T. } & 72 \\
\text { B. } & 71 \\
\text { T. } & 5.5 \\
\text { B. } & 0.4\end{array}$ \\
\hline $\begin{array}{ll}\begin{array}{l}\text { Specific conductance } \\
\text { (micromhos at } 25^{\circ} \mathrm{C} \text { ) }\end{array} & \begin{array}{l}\text { Top } \\
\text { Bottom }\end{array} \\
& \text { Dissolved oxygen - - - Top } \\
\text { B. O. D. (5-day, 20 } \mathrm{C} \text { ) - } & \text { Top } \\
\text { Chloride (Cl) - } & \text { Top } \\
& \text { Bottom }\end{array}$ & $\begin{array}{r}6.54 \\
1.7 \\
5.4 \\
110\end{array}$ & $\begin{array}{l}1,210 \\
1,5+10 \\
1.6 \\
8.7 \\
255 \\
330\end{array}$ & $\begin{array}{r}1,140 \\
1,140 \\
1 . ? \\
10.2 \\
205 \\
225\end{array}$ & $\begin{array}{c}866 \\
+, 190 \\
2.0 \\
5.4 \\
150 \\
230\end{array}$ & $\begin{array}{l}865 \\
2.0 \\
0.9 \\
.55\end{array}$ & $\begin{array}{l}\text { T. } \\
\text { B. } 1,283 \\
\text { T. } \quad 1.8 \\
\text { T. } \quad 7.3 \\
\text { T. } \quad 174 \\
\text { B. } 262\end{array}$ \\
\hline Suspended sediment $\ldots$-.- Top & & & & & & $\mathbf{T}$ \\
\hline
\end{tabular}

b Exceede 1 this value.

Location didystcne, Fä.

Date November 5,1951 Sampling study No, 27

Weather ilear

Water discharge at Trenton (cfs) $-29, F O$

\begin{tabular}{|c|c|c|c|c|c|c|}
\hline \multicolumn{7}{|c|}{ Weather blear } \\
\hline & \multicolumn{6}{|c|}{ Station } \\
\hline & $\begin{array}{c}\text { Pennsylvania } \\
\text { side }\end{array}$ & West Center & Center & East Center & $\begin{array}{l}\text { New Jersey } \\
\text { side }\end{array}$ & Average \\
\hline $\begin{array}{l}\text { Time (EST) } \\
\text { Sounding (ft) } \\
\text { Temperature ("F) }\end{array}$ & $\begin{array}{c}2: 15 \text { f.m. } \\
5 \alpha \\
0.5\end{array}$ & $\begin{array}{l}1: 18 \text { P.w... } \\
25 \\
51 \\
51 \\
6.6 \\
0.1\end{array}$ & $\begin{array}{l}1: 21 \text { p.13. } \\
40 \\
51 \\
51 \\
6.5 \\
7.5\end{array}$ & $\begin{array}{c}1: 24 . \\
b 50 \\
50 \\
50 \\
0.6 \\
b .6\end{array}$ & $\begin{array}{c}2.27 \text { [..n. } \\
50 \\
6.5\end{array}$ & $\begin{array}{l}51 \\
51 \\
6.5 \\
6.8\end{array}$ \\
\hline 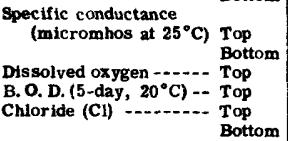 & $\begin{array}{l}223 \\
4.6 \\
3.3 \\
11\end{array}$ & $\begin{array}{l}204 \\
207 \\
0.1 \\
3.0 \\
10 \\
7.0\end{array}$ & $\begin{array}{l}176 \\
178 \\
0.6 \\
3.2 \\
10 \\
10\end{array}$ & $\begin{array}{l}172 \\
170 \\
6.1 \\
2.8 \\
7.0 \\
10\end{array}$ & $\begin{array}{l}211 \\
5.0 \\
3.0 \\
12\end{array}$ & $\begin{array}{ll} & \\
\text { T. } & 205 \\
\text { B. } & 198 \\
\text { T. } & 5.7 \\
\text { T. } & 3.1 \\
\text { T. } & 10 \\
\text { B. } & 9.7\end{array}$ \\
\hline Suspended sediment -.- Top & ( & & - Con & te. . . & . & $\mathbf{T}$ \\
\hline
\end{tabular}

t Exceeded th_3 value.

Location Eadystone, Fa. Weather clousi

Date Secember 42 1951___ Sampling study No. 28

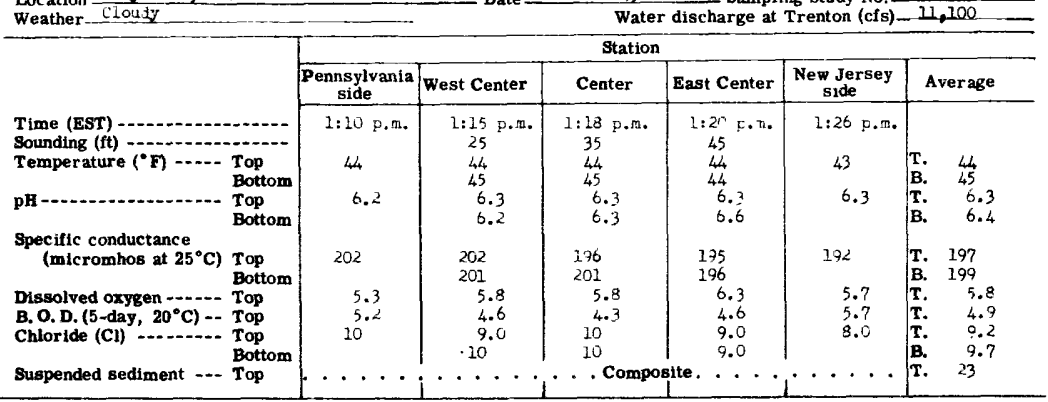


Table 13. -- WATER ANALYSES OF DELAWARE RIVER BETWEEN BRISTOL AND MARCUS hOOK, PA. --Continued Analyzed by City of Philadelphia and $U$. S. Geological Survey; analyses in parts per milion

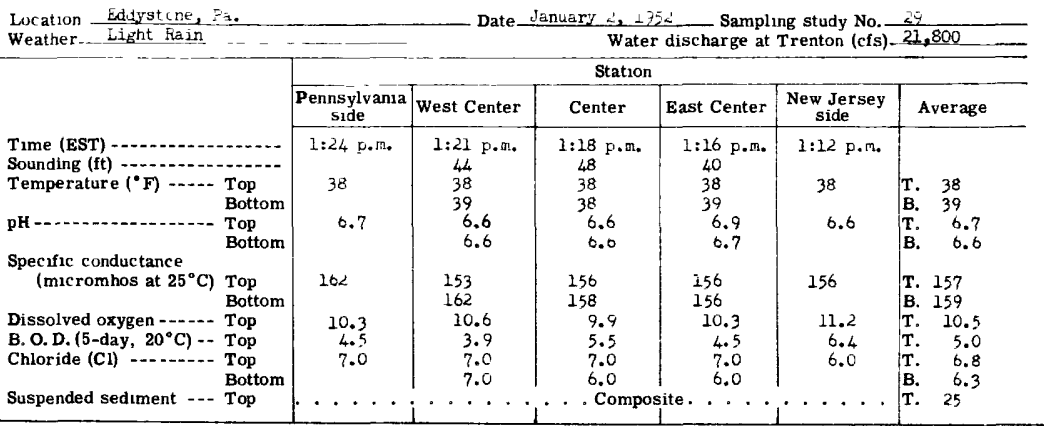

Location Lddystone, F8.

Date February 6, 1952 Sampling study No. 30

\begin{tabular}{|c|c|c|c|c|c|c|}
\hline \multicolumn{3}{|c|}{ Weather Clear } & \multirow{2}{*}{\multicolumn{4}{|c|}{$\begin{array}{l}\text { Water discharge at Trenton (cfs) } 13,400 \\
\text { Station }\end{array}$}} \\
\hline & & & & & & \\
\hline & $\begin{array}{c}\text { Pennsylvania } \\
\text { side }\end{array}$ & West Center & Center & East Center & $\begin{array}{c}\text { New Jersey } \\
\text { side }\end{array}$ & Average \\
\hline $\begin{array}{l}\text { Time (EST) } \\
\text { Sounding (ft) } \\
\text { Temperature }\left({ }^{\circ} \text { F) }\right. \\
\text { pH }\end{array} \begin{array}{l}\text { Top } \\
\text { Bottom } \\
\text { Top } \\
\text { Bottom }\end{array}$ & $\begin{array}{c}11: 00 \text { a.m. } \\
40 \\
6.3\end{array}$ & $\begin{array}{c}11: 03 \text { a. . . } \\
30 \\
40 \\
39 \\
6.3 \\
6.3\end{array}$ & $\begin{array}{l}11: 05 \text { a.m. } \\
48 \\
38 \\
38 \\
7.6 \\
6.2\end{array}$ & $\begin{array}{c}11: 07 \text { a.m. } \\
47 \\
38 \\
38 \\
6.2 \\
6.3\end{array}$ & $\begin{array}{c}11: 12 \text { a.m. } \\
39 \\
6.2\end{array}$ & $\begin{array}{ll}\text { T. } & 39 \\
\text { B. } & 38 \\
\text { T. } & 6.5 \\
\text { B. } & 6.3\end{array}$ \\
\hline 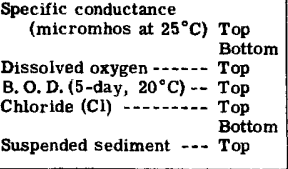 & $\begin{array}{r}161 \\
10.7 \\
3.5 \\
6.0\end{array}$ & $\begin{array}{r}166 \\
158 \\
11.0 \\
4.3 \\
8.0 \\
7.0 \\
.\end{array}$ & $\begin{array}{r}138 \\
137 \\
12.6 \\
4.0 \\
5.0 \\
6.0 \\
. \text { Con }\end{array}$ & $\begin{array}{r}137 \\
140 \\
11.8 \\
4.1 \\
5.0 \\
5.0 \\
. . .\end{array}$ & $\begin{array}{r}137 \\
11.7 \\
4.5 \\
5.0\end{array}$ & $\begin{array}{ll}\text { T. } & 148 \\
\text { B. } & 145 \\
\text { T. } & 11.4 \\
\text { T. } & 4.1 \\
\text { T. } & 5.8 \\
\text { B. } & 6.0 \\
\text { T. } & 44\end{array}$ \\
\hline
\end{tabular}

Location Eddystone, Pa. Weather uvercast

Date March 3, 1952

Sampling study No. 31

\begin{tabular}{|c|c|c|c|c|c|c|}
\hline $\begin{array}{l}\text { Location Eddystone, } \mathrm{Pa} . \\
\text { Weather Uvercast }\end{array}$ & $\ldots-\ldots$ & 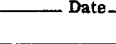 & Wate & discharge a & $\begin{array}{l}\text { ing study No. } \\
\text { Trenton (cts) }\end{array}$ & 2,410 \\
\hline & & & Station & & & \\
\hline & \begin{tabular}{|c|}
$\begin{array}{c}\text { Pennsylvania } \\
\text { side }\end{array}$ \\
\end{tabular} & West Center & Center & East Center & $\begin{array}{c}\text { New Jersey } \\
\text { side }\end{array}$ & Average \\
\hline $\begin{array}{ll}\text { Time (EST) } & \\
\text { Sounding (ft) } & \\
\text { Temperature }\left({ }^{\circ} \mathrm{F}\right) & \begin{array}{l}\text { Top } \\
\text { Bottom }\end{array} \\
\text { pH } & \text { Top } \\
& \text { Bottom }\end{array}$ & $\begin{array}{c}11: 10 \mathrm{a} \cdot \mathrm{m} . \\
40 \\
6.2\end{array}$ & $\begin{array}{c}11: 13 \text { a.m. } \\
27 \\
40 \\
40 \\
6.2 \\
6.3\end{array}$ & $\begin{array}{c}11: 15 \text { a.m. } \\
32 \\
40 \\
40 \\
6.3 \\
6.2\end{array}$ & $\begin{array}{c}11: 18 \text { a.m. } \\
50 \\
40 \\
40 \\
6.2 \\
6.2\end{array}$ & $\begin{array}{c}11: 21 \text { a.m. } \\
39 \\
6.2\end{array}$ & $\begin{array}{ll}\text { T. } & 40 \\
\text { B. } & 40 \\
\text { T. } & 6.2 \\
\text { B. } & 6.2\end{array}$ \\
\hline $\begin{array}{ll}\begin{array}{l}\text { Specific conductance } \\
\text { (micromhos at } 25^{\circ} \mathrm{C} \text { ) }\end{array} & \begin{array}{l}\text { Top } \\
\text { Bottom }\end{array} \\
\text { Dissolved oxygen }-\ldots-\text { Top } \\
\text { B. O. D. (5-day, } 20^{\circ} \mathrm{C} \text { ) } & \text { Top } \\
\text { Chloride (C) } & \text { Top } \\
& \text { Bottom }\end{array}$ & $\begin{array}{l}215 \\
7.3 \\
10\end{array}$ & $\begin{array}{l}211 \\
212 \\
7.5 \\
2.8 \\
10 \\
10\end{array}$ & $\begin{array}{l}208 \\
212 \\
8.0 \\
3.0 \\
10 \\
10\end{array}$ & $\begin{array}{l}210 \\
211 \\
8.3 \\
3.2 \\
10 \\
10\end{array}$ & $\begin{array}{l}210 \\
8.2 \\
3.2 \\
10\end{array}$ & $\begin{array}{ll}\text { T. } & 211 \\
\text { B. } & 212 \\
\text { T. } & 7.9 \\
\text { T. } & 2.8 \\
\text { T. } & 10 \\
\text { B. } & 10\end{array}$ \\
\hline Suspended sediment.-- Top & 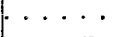 & . & . Con & te. . & . . . & T. 40 \\
\hline
\end{tabular}

Location Eddystone, Pa. Weather-Overcsst

Date April 1, 1952____ Sampling study No. 32

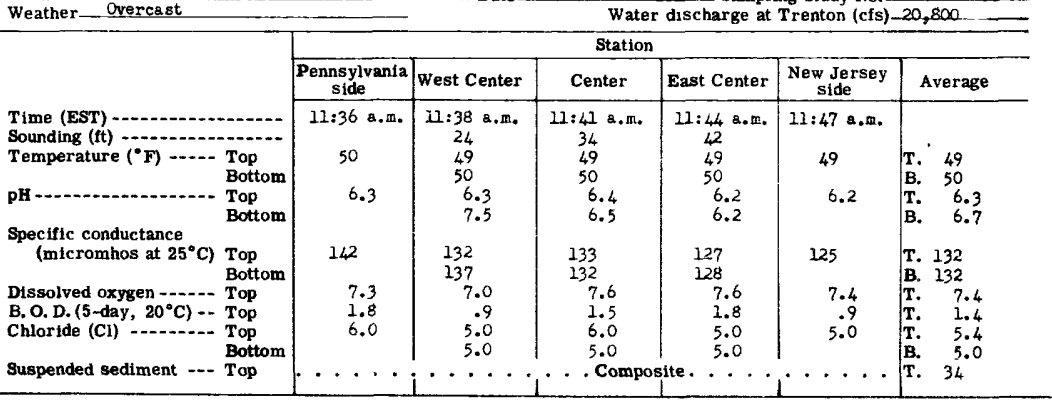


Table 13, --WATER ANALYSES OF DELAWARE RIVER BETWEEN BRISTOL AND MARCUS HOOK, PA. --CONtinued Analyzed by City of Philadelphia and U. S. Geological Survey, analyses in parts per million

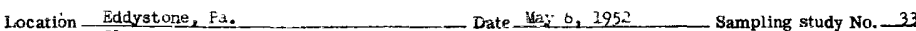
Weather Clear $\quad$ Water discharge at Trenton (cts). 16,600 -

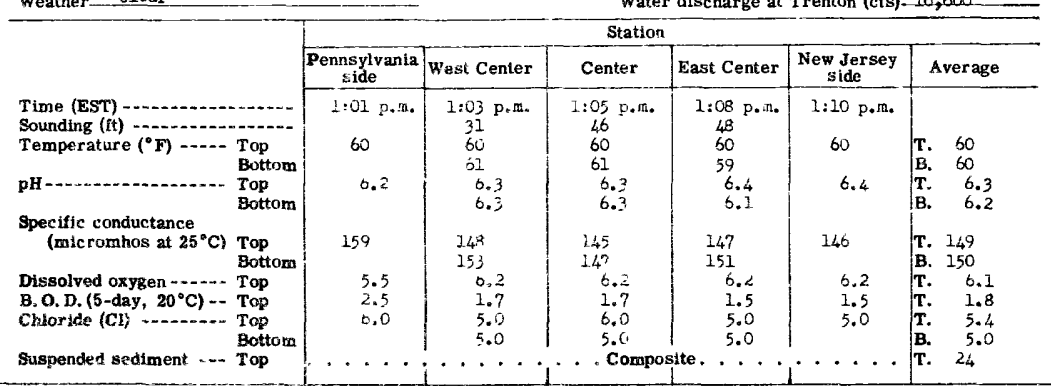

Location - bddystorie, Fa,

\begin{tabular}{|c|c|c|c|c|c|c|}
\hline Weather Cisar & -- & & Wate & discharge at & Trenton (cfs) & 35,600 \\
\hline & & & Station & & & \\
\hline & $\begin{array}{c}\text { Pennsyly ania } \\
\text { side }\end{array}$ & West Center & Center & East Center & $\begin{array}{c}\text { New Jersey } \\
\text { side }\end{array}$ & Average \\
\hline $\begin{array}{l}\text { Time (EST) } \\
\text { Sounding }(\mathrm{It}) \\
\left.\text { Temperature } f^{\circ} \mathrm{F}\right)\end{array} \begin{array}{l}\text { Top } \\
\text { Bottom } \\
\text { Top } \\
\text { Bottom }\end{array}$ & $\begin{array}{l}12: 25 \mathrm{P} . \mathrm{*} . \\
69 \\
6.5\end{array}$ & $\begin{array}{c}12: 27 \text { p.m. } \\
31 \\
68 \\
70 \\
6.5 \\
6.5\end{array}$ & $\begin{array}{c}12: 20 \text { p.int } \\
44 \\
63 \\
09 \\
6.5 \\
6.5\end{array}$ & $\begin{array}{c}12: 32 \mathrm{pe} n \\
40 \\
68 \\
59 \\
6.5 \\
6.0\end{array}$ & $\begin{array}{c}12: 34 \text { p.m. } \\
68 \\
6.6\end{array}$ & $\begin{array}{ll}\text { T. } & 68 \\
\text { B. } & 69 \\
\text { T. } & 6.5 \\
\text { B. } & 6.5\end{array}$ \\
\hline 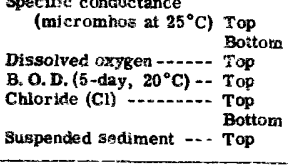 & $\begin{array}{r}142 \\
4.5 \\
2.3 \\
5.17 \\
\\
\end{array}$ & $\begin{array}{r}123 \\
131 \\
5.7 \\
1.9 \\
4.0 \\
4.0 \\
\cdots\end{array}$ & $\begin{array}{r}122 \\
123 \\
5.3 \\
1.9 \\
4.0 \\
4.0 \\
. \quad \text { Con }\end{array}$ & $\begin{array}{r}121 \\
147 \\
5.8 \\
2.0 \\
4.0 \\
4.0 \\
\text { te. }\end{array}$ & $\begin{array}{r}124 \\
5.1 \\
1.6 \\
4.0\end{array}$ & $\begin{array}{ll}\text { T. } & 126 \\
\text { B. } & 134 \\
\text { T. } & 5.2 \\
\text { T. } & 2.0 \\
\text { T. } & 4.2 \\
\text { B. } & 4.0 \\
\text { T. } & 36\end{array}$ \\
\hline
\end{tabular}

Location Ẽdystone, Pa. Date July 8, 1952

Sampling study No. -35 Weather_overcest__ Water discharge at Trenton (cfs) 3,280

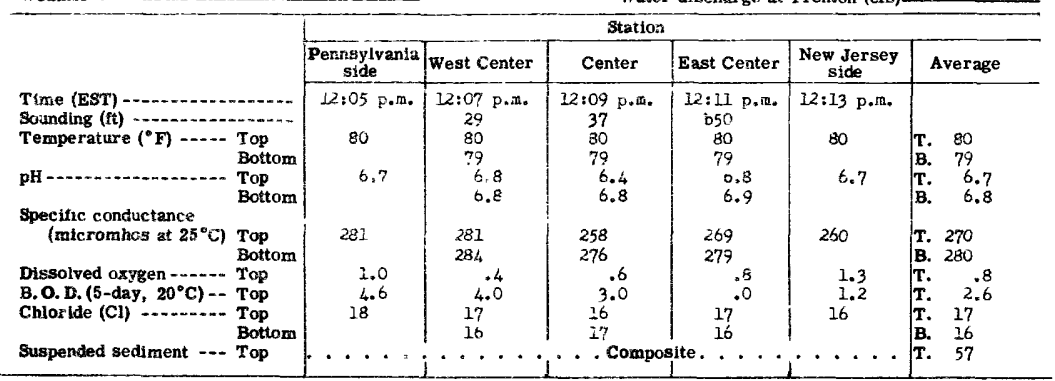

b Exceeded this value.

Location Eddystone, Pa. Weather_Cloudy

Date August Ie 2952 _ Sampling study No. 36

\begin{tabular}{|c|c|c|c|c|c|c|}
\hline \multicolumn{3}{|c|}{ Weather_Cloudy } & \multicolumn{4}{|c|}{ Water discharge at Trenton (cfs) $-5,920$} \\
\hline & \multicolumn{6}{|c|}{ Station } \\
\hline & $\begin{array}{c}\text { Pennsylvania } \\
\text { side }\end{array}$ & West Center & Center & East Center & $\begin{array}{c}\text { New Jersey } \\
\text { side }\end{array}$ & Average \\
\hline $\begin{array}{l}\text { Time (EST) } \\
\text { Sounding (ft) } \\
\text { Temperature }\left({ }^{\circ} \mathrm{F}\right) \\
\begin{array}{l}\text { pH } \\
\text { pottom }\end{array} \\
\begin{array}{l}\text { Bop } \\
\text { Bottom }\end{array}\end{array}$ & $\begin{array}{c}10: 35 \text { a.m. } \\
32 \\
0.8\end{array}$ & $\begin{array}{c}10: 37 \text {. m. } \\
27 \\
82 \\
82 \\
6.8 \\
0.8\end{array}$ & $\begin{array}{c}10: 39 \text { a.m. } \\
43 \\
82 \\
81 \\
6.8 \\
6.7\end{array}$ & $\begin{array}{c}10: 41 \text { a. } \mathrm{m} . \\
40 \\
82 \\
82 \\
6.8 \\
6.8\end{array}$ & $\begin{array}{c}10: 43 \text { a.m. } \\
82 \\
0.8\end{array}$ & $\begin{array}{lc}\text { T. } & 82 \\
\text { B. } & 82 \\
\text { T. } & 6.8 \\
\text { B. } & 6.8\end{array}$ \\
\hline 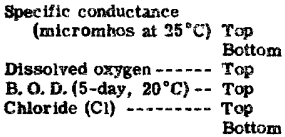 & $\begin{array}{r}243 \\
1.0 \\
13\end{array}$ & $\begin{array}{l}239 \\
245 \\
2.1 \\
13 \\
1.4\end{array}$ & $\begin{array}{l}233 \\
245 \\
1.8 \\
1.8 \\
14\end{array}$ & $\begin{array}{l}235 \\
245 \\
1.1 \\
1.2 \\
13 \\
13\end{array}$ & $\begin{array}{r}237 \\
2.6 \\
13\end{array}$ & $\begin{array}{ll}\text { T. } & 237 \\
\text { B. } & 245 \\
\text { T. } & .5 \\
\text { T. } & 1.8 \\
\text { T. } & 13 \\
\text { B. } & 14\end{array}$ \\
\hline Suspended sediment --- Top & . . & & Com & ite... & . . . & $\mathbf{T}$ \\
\hline
\end{tabular}


Table 13. --WATER ANALYSES OF DELAWARE RIVER BETWEEN BRISTOL AND MARCUS HOOK, PA. --Continued Analyzed by City of Philadelphia and U. S. Geological Survey; analyses in parts per million

Location - Eddystone, Pa.

Date_September 4 . 1952 Sampling study No. 37 Weather-Clear

Water discharge at Trenton (cfs) 27,100

\begin{tabular}{|c|c|c|c|c|c|c|}
\hline \multicolumn{3}{|c|}{ Weather-Clear } & \multicolumn{4}{|c|}{ Water discharge at Trenton (cfs) 27,100} \\
\hline & \multicolumn{6}{|c|}{ Station } \\
\hline & $\begin{array}{c}\text { Pennsylvania } \\
\text { stade }\end{array}$ & West Center & Center & East Center & $\begin{array}{c}\text { New Jersey } \\
\text { side }\end{array}$ & Average \\
\hline $\begin{array}{l}\text { Time (EST) } \\
\text { Sounding (ft) } \\
\text { Temperature }\left({ }^{\circ} \mathrm{F}\right) \\
\text { pH }\end{array} \begin{array}{l}\text { Top } \\
\text { Bottom } \\
\text { Top } \\
\text { Bottom }\end{array}$ & $\begin{array}{c}22: 08 \mathrm{p} . \mathrm{m} \\
77 \\
6.7\end{array}$ & $\begin{array}{l}12: 10 \mathrm{p} . \mathrm{m} . \\
35 \\
77 \\
78 \\
\text { b.6 } \\
\text { b. } 3\end{array}$ & $\begin{array}{c}12: 13 \mathrm{p.m} . \\
\mathrm{b} 50 \\
77 \\
77 \\
5.5 \\
5.5\end{array}$ & $\begin{array}{c}12: 15 \text { p.m. } \\
650 \\
78 \\
78 \\
6.5 \\
6,8\end{array}$ & $\begin{array}{c}12: 17 \text { p.m. } \\
76 \\
0.5\end{array}$ & $\begin{array}{ll}\text { T. } & 77 \\
\text { B. } & 78 \\
\text { T. } & 6.6 \\
\text { B. } & 6.5\end{array}$ \\
\hline 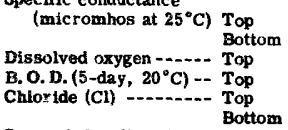 & $\begin{array}{l}245 \\
2.2 \\
1.0 \\
12\end{array}$ & $\begin{array}{l}236 \\
228 \\
1.7 \\
110\end{array}$ & $\begin{array}{l}228 \\
235 \\
1.7 \\
110^{\circ}\end{array}$ & $\begin{array}{l}236 \\
238 \\
1.4 \\
12.0 \\
11\end{array}$ & $\begin{array}{l}231 \\
2.2 \\
6.4 \\
11\end{array}$ & $\begin{array}{l}\text { T. } 235 \\
\text { B. } 234 \\
\text { T. } \quad 1.8 \\
\text { T. } \quad 1.6 \\
\text { T. } \quad 17 \\
\text { B. } \quad 11\end{array}$ \\
\hline Suspended sediment $\ldots$ Top & - & ... & . Comp & ... & 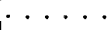 & T. 14 \\
\hline
\end{tabular}

b Exceeded tass value.

Location Eddystone, $\mathrm{Pa}$. Weather-Partly Clouar

Date October 7, 1952 Sampling study No. 38 Water discharge at Trenton (cfs) - 4 a 120

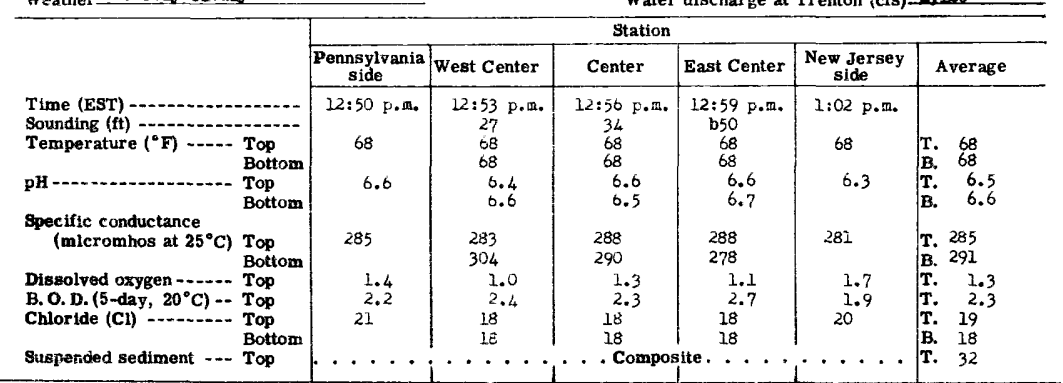

3 Exceeded this value.

Location Eddystone, $\mathrm{Pa}$ Weather Clear

Date Norember 16' $195 \%$ Sampling study No. 39

\begin{tabular}{|c|c|c|c|c|c|c|}
\hline \multicolumn{7}{|c|}{ Weather Clear } \\
\hline & \multicolumn{6}{|c|}{ Station } \\
\hline & $\begin{array}{c}\text { Pennsylvania } \\
\text { side }\end{array}$ & West Center & Center & East Center & $\begin{array}{c}\text { New Jersey } \\
\text { side }\end{array}$ & Average \\
\hline $\begin{array}{ll}\text { Time (EST) } \\
\text { Sounding (ft) } \\
\text { Temperature }\left({ }^{\circ} \mathrm{F}\right) \\
\text { pH }\end{array}$ & $\begin{array}{c}12: 15 \text { p.m. } \\
51 \\
6.4\end{array}$ & $\begin{array}{c}12: 10 \text { p.m. } \\
35 \\
51 \\
50 \\
6.3 \\
0.2\end{array}$ & $\begin{array}{c}12: 05 \mathrm{p} . \mathrm{m} . \\
\text { b50 } \\
49 \\
50 \\
6.4 \\
6.2\end{array}$ & $\begin{array}{c}12: 00 \mathrm{n} \\
8 \\
49 \\
50 \\
6.4 \\
0.1\end{array}$ & $\begin{array}{l}1]: 50 \text { a.m. } \\
50 \\
6.2\end{array}$ & $\begin{array}{ll}\text { T. } & 50 \\
\text { B. } & 50 \\
\text { T. } & 6.3 \\
\text { B. } & 6.2\end{array}$ \\
\hline 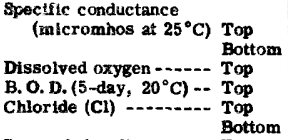 & $\begin{array}{l}770 \\
3.4 \\
4.4 \\
148\end{array}$ & $\begin{array}{l}1,070 \\
1,080 \\
3.8 \\
7.6 \\
240 \\
242\end{array}$ & $\begin{array}{l}946 \\
971 \\
4 \cdot 2 \\
7.6 \\
202 \\
215\end{array}$ & $\begin{array}{l}925 \\
935 \\
4.4 \\
7.6 \\
195 \\
202\end{array}$ & $\begin{array}{r}957 \\
4.3 \\
7.9 \\
205\end{array}$ & $\begin{array}{l}\text { T. } 935 \\
\text { B. } 995 \\
\text { T. } 4.0 \\
\text { T. } 7.0 \\
\text { T. } 198 \\
\text { B. } 220\end{array}$ \\
\hline Suspended sediment -.- Top & & $\cdots$ &. Com & e. . & 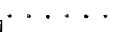 & T. 28 \\
\hline
\end{tabular}

Exceeded this value.

Location Eddystone, Pa. Woation - Lderste

Date Decamber 5, 1952 Sampling study No

\begin{tabular}{|c|c|c|c|c|c|c|}
\hline \multicolumn{3}{|c|}{$\begin{array}{l}\text { Loeation - Eddystone, } \mathrm{Pa} \text {. } \\
\text { Weather }\end{array}$} & \multicolumn{4}{|c|}{$\begin{array}{l}\text { I } 5,1952 \\
\text { Water discharge at Trenton (cfs) }\end{array}$} \\
\hline & \multicolumn{5}{|c|}{ Station } & \multirow[b]{2}{*}{ Average } \\
\hline & $\begin{array}{c}\begin{array}{c}\text { Pennsylvania } \\
\text { side }\end{array} \\
\end{array}$ & West Cencer & Center & East Center & $\begin{array}{c}\text { New Jersey } \\
\text { side }\end{array}$ & \\
\hline $\begin{array}{l}\text { Time (EST) } \\
\text { Sounding (ft) } \\
\text { Temperature ( }{ }^{\circ} \mathrm{F} \text { ) } \\
\text { pH }\end{array}$ & $\begin{array}{c}1: 23 \mathrm{~F} . \mathrm{m} . \\
48 \\
-\end{array}$ & $\begin{array}{l}1.26 \mathrm{p} \cdot \mathrm{m} . \\
32 \\
28 \\
47 \\
6.2 \\
6.2\end{array}$ & $\begin{array}{l}2.29 \text { p.m. } \\
42 \\
48 \\
47 \\
6.2 \\
6.2\end{array}$ & $\begin{array}{c}1: 32 \mathrm{p} \cdot \mathrm{m} \cdot \\
\mathrm{b} 50 \\
47 \\
47 \\
6.2 \\
6.2\end{array}$ & $\begin{array}{c}1: 35 \text { p.m. } \\
48 \\
6.2\end{array}$ & $\begin{array}{ll}\text { T. } & 48 \\
\text { B. } & 47 \\
\text { T. } & 6.2 \\
\text { B. } & 6.2\end{array}$ \\
\hline 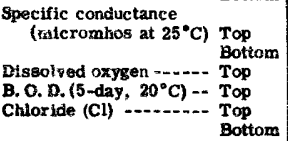 & $\begin{array}{l}- \\
7.2 \\
6.2 \\
-\end{array}$ & $\begin{array}{r}173 \\
172 \\
7.4 \\
4.7 \\
9.0 \\
9.0\end{array}$ & $\begin{array}{l}166 \\
165 \\
7.3 \\
4.5 \\
8.5 \\
8.5\end{array}$ & $\begin{array}{r}161 \\
162 \\
7.9 \\
5.9 \\
8.0 \\
8.5\end{array}$ & $\begin{array}{l}161 \\
8.1 \\
3.1 \\
8.0\end{array}$ & $\begin{array}{lr} & \\
\text { T. } & 165 \\
\text { B. } & 166 \\
\text { T. } & 7.6 \\
\text { T. } & 4.8 \\
\text { T. } & 8.4 \\
\text { B. } & 8.7\end{array}$ \\
\hline Sispended sadiment --- Top & - & & & 6. . . & & T. 28 \\
\hline
\end{tabular}


Table 14. - -WATER ANALYSES OF DELAWARE RIVER BETWEEN BRISTOL AND MARCUS HOOK, PA. Analyzed by $C$ ity of Philadelphia and U. S. Geological Survey; analyses in parts per million

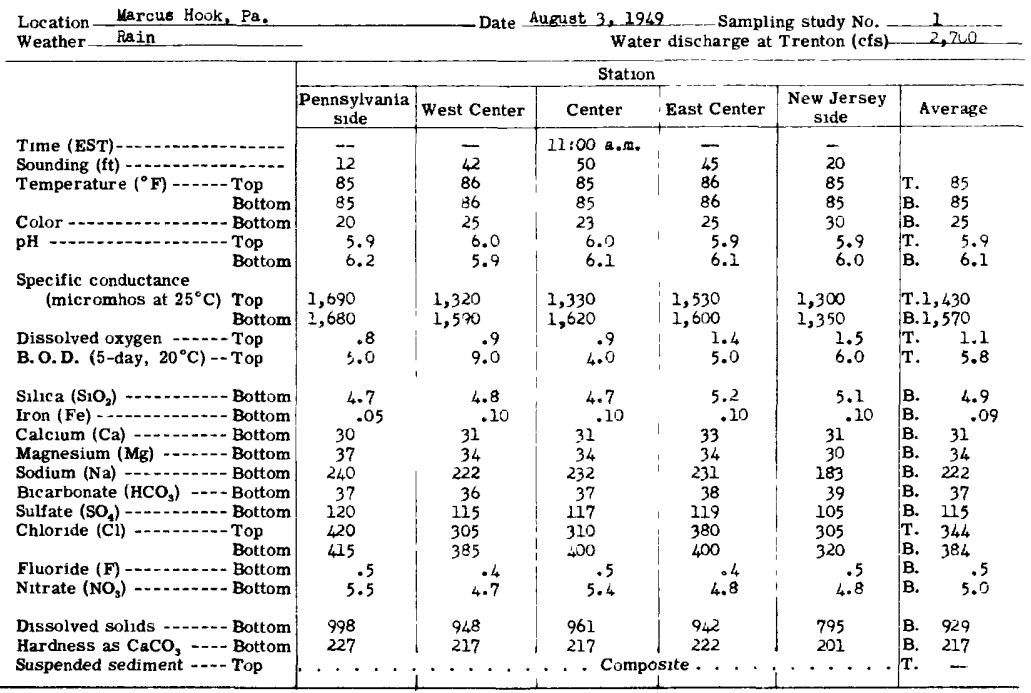

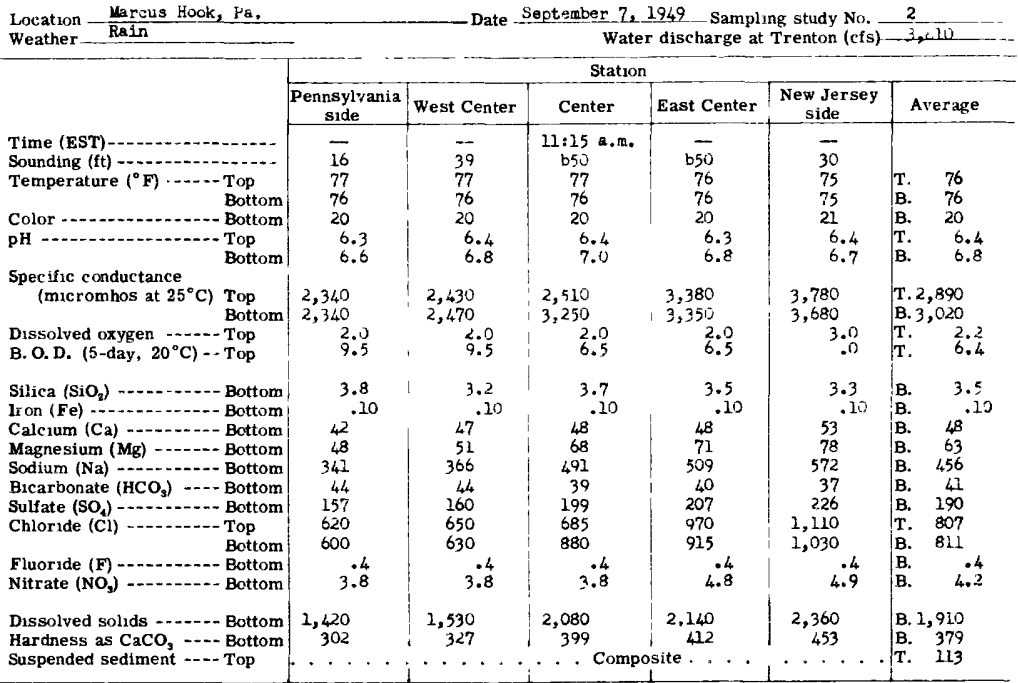

b Exceeded this ralue. 
Table 14. -- WATER ANALYSES OF DELAWARE RIVER BETWEEN BRISTOL AND MARCUS HOOK, PA. --Continued Analyzed by City of Philadelphia and U. S. Geological Survey, analyses in parts per million

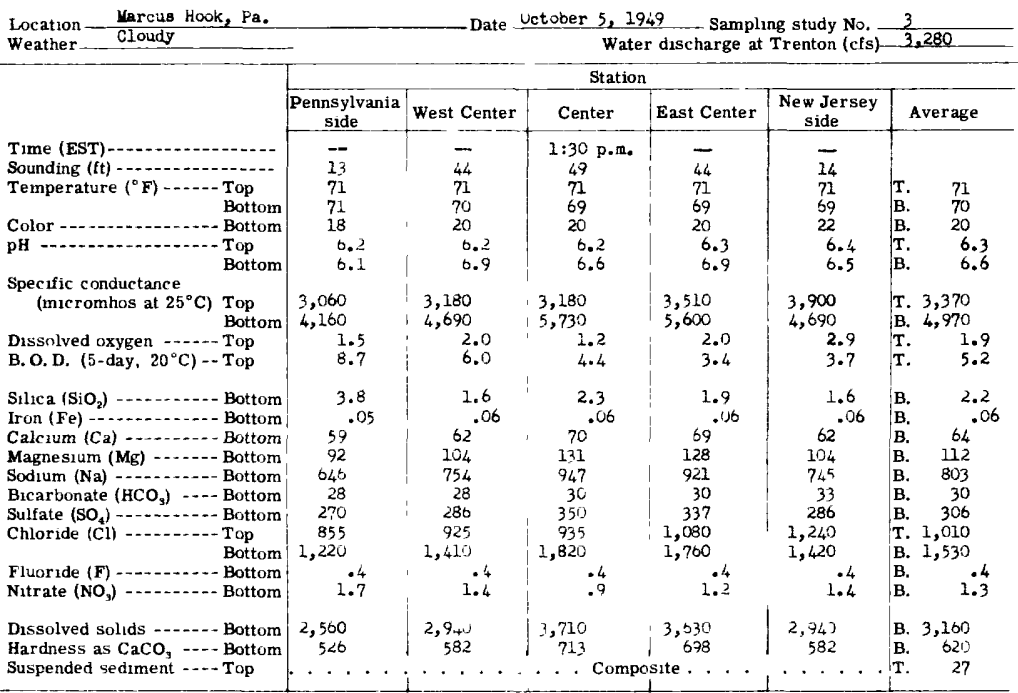

Location Marcus Hook, Pa.

Date Noveriber 2,1749 - Sampling study No. - 4

\begin{tabular}{|c|c|c|c|c|c|c|}
\hline Weather Clear & & & & discharg & enton (c & 3.430 \\
\hline & & & Statiu & & & \\
\hline & \begin{tabular}{|c|}
$\begin{array}{c}\text { Pennsylvania } \\
\text { side }\end{array}$ \\
\end{tabular} & West Center & Center & East Center & $\begin{array}{l}\text { New Jersey } \\
\text { side }\end{array}$ & Average \\
\hline Time (EST) & - & - & $2: 100$ p.m. & - & - & \\
\hline Sounding (ft) & 14 & 45 & 48 & 41 & 27 & \\
\hline Temperature $\left({ }^{\circ} \mathbf{F}\right)-\ldots-$ Top & 0,1 & 6is & 59 & 58 & 57 & 59 \\
\hline Bottom & 60 & 58 & 58 & 60 & 59 & B. \\
\hline Color - Bottom & 21 & 20 & 23 & 20 & 18 & B. \\
\hline pH 10. Top & $\begin{array}{l}6.1 \\
6.2\end{array}$ & $\begin{array}{l}6.1 \\
6.5\end{array}$ & 6.2 & $\begin{array}{l}0.3 \\
0.3\end{array}$ & $\begin{array}{l}6.4 \\
6.2\end{array}$ & $\begin{array}{l}6.2 \\
6.3\end{array}$ \\
\hline pecific conductance & & & & & & \\
\hline $\begin{array}{cl}\text { (micromhos at } 25^{\circ} \mathrm{C} \text { ) } & \mathrm{Top}_{\text {Bottom }}\end{array}$ & $\begin{array}{l}5,320 \\
5,340\end{array}$ & $\begin{array}{l}5,150 \\
6,230\end{array}$ & $\begin{array}{l}5,060 \\
6,200\end{array}$ & $\begin{array}{l}5,150 \\
6,230\end{array}$ & $\begin{array}{l}5,150 \\
5,930\end{array}$ & $\begin{array}{l}\text { T. } 5,170 \\
\text { B. } 5,970\end{array}$ \\
\hline $\begin{array}{l}\text { Dissolved oxygen - } \\
\left.\text { B. O. D. (5-day, } 20^{\circ} \mathrm{C}\right)- \text { Top }\end{array}$ & $\begin{array}{l}4.1 \\
0.1\end{array}$ & $\begin{array}{l}4.0 \\
5.7\end{array}$ & $\begin{array}{l}4.1 \\
3.3\end{array}$ & $\begin{array}{l}4.9 \\
1.8\end{array}$ & $\begin{array}{l}5.2 \\
2.4\end{array}$ & $\begin{array}{l}4.5 \\
3.8\end{array}$ \\
\hline $\begin{array}{l}\text { Silica }\left(\mathrm{S}_{1} \mathrm{O}_{2}\right) \\
\text { Iron }(\mathrm{Fe})\end{array}$ & $\begin{array}{l}2.3 \\
.06\end{array}$ & $\begin{array}{l}1.3 \\
.06\end{array}$ & $\begin{array}{l}2.3 \\
.10\end{array}$ & 1.5 & $\begin{array}{l}2.7 \\
.08\end{array}$ & $\begin{array}{l}2.0 \\
.07\end{array}$ \\
\hline Calcium (Ca) -....... Bottom & $70^{\circ}$ & $7+$ & $75^{\circ}$ & 74 & 73 & B. \\
\hline Magne sum (Mg) -..... Bottom & 122 & 144 & 147 & 147 & 138 & 140 \\
\hline Sodium (Na) - & 899 & 1,060 & 1,090 & 1,090 & 1,010 & B. 1,030 \\
\hline Bicarbonate $\left(\mathrm{HCO}_{3}\right)$ & 27 & 28 & 24 & 24 & 28 & B. \\
\hline Sulfate $\left(\mathrm{SO}_{4}\right) \ldots$ & 337 & 382 & 380 & 381 & 371 & B. 370 \\
\hline Chloride (Cl) $\ldots \ldots$ & 1,610 & 1,560 & 1,500 & 1,550 & 1,560 & T. 1,560 \\
\hline Bottom & 1,670 & 1,990 & 2,000 & 2,020 & 1,900 & B. 1,920 \\
\hline Fluoride (F) & $\because 4$ & .4 & .4 & .4 & .4 & .4 \\
\hline Nitrate $\left(\mathrm{NO}_{3}\right)-\ldots-$ Bottom & 1.0 & 1.3 & 1.0 & 1.0 & .8 & 1.0 \\
\hline Dissolved solıds - & 3,480 & $4,060^{\circ}$ & 4,130 & 4,150 & 3,900 & B. 3,940 \\
\hline Hardness as $\mathrm{CaCO}_{3}$... Bottom & 670 & 777 & 792 & 789 & 750 & B. $\quad 757$ \\
\hline Suspended sedıment $\ldots$ Top & $\cdots$ & 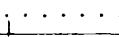 & . C Com & osite. & $\therefore$ & $\mathrm{T}$. \\
\hline
\end{tabular}


Table 14. - WATER ANA LYSES OF DELAWARE RIVER BETWEEN BRISTOL AND MARCUS HOOK, PA. --COntinued Analyzed by City of Philadelphia and U. S. Geological Survey; analyses in parts per million

Location Karcus Hook, $\mathrm{Pa}$. Weather Cloudy

Date Deceraber 2, 1949 Sampling study No. 5

\begin{tabular}{|c|c|c|c|c|c|c|}
\hline \multicolumn{2}{|l|}{ Weather Cloudy } & \multicolumn{5}{|c|}{ Water discharge at Trenton (cfs) 4.750} \\
\hline & \multicolumn{6}{|c|}{ Station } \\
\hline & \begin{tabular}{|c}
$\begin{array}{c}\text { Pennsylvania } \\
\text { side }\end{array}$ \\
\end{tabular} & West Center & Center & East Center & $\begin{array}{c}\text { New Jersey } \\
\text { side }\end{array}$ & Average \\
\hline $\begin{array}{l}\begin{array}{l}\text { Time (EST) } \\
\text { Sounding }(\mathrm{ft}) \\
\text { Temperature }\left({ }^{\circ} \mathrm{F}\right)\end{array} \\
\begin{array}{c}\text { Color } \\
\text { pH }\end{array} \\
\begin{array}{c}\text { Bottom } \\
\text { Bot }\end{array}\end{array}$ & $\begin{array}{l}16 \\
45 \\
45 \\
8 \\
6.4 \\
6.4\end{array}$ & $\begin{array}{l}-10 \\
45 \\
44 \\
10 \\
6.2 \\
6.9\end{array}$ & $\begin{array}{c}12: 30 \mathrm{p} .10 \\
\text { b50 } \\
45 \\
45 \\
12 \\
6.2 \\
6.9\end{array}$ & $\begin{array}{l}-37 \\
45 \\
45 \\
12 \\
5.2 \\
6.9\end{array}$ & $\begin{array}{l}-19 \\
45 \\
45 \\
10 \\
6.3 \\
6.3\end{array}$ & $\begin{array}{ll}\text { 1. } & 45 \\
\text { B. } & 45 \\
\text { B. } & 10 \\
\text { T. } & 6.3 \\
\text { B. } & 6.7\end{array}$ \\
\hline 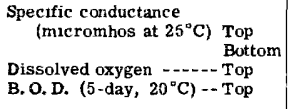 & $\begin{array}{r}2,300 \\
2,610 \\
5.4 \\
1.0\end{array}$ & $\begin{array}{r}3,270 \\
4,050 \\
5,8 \\
.5\end{array}$ & $\begin{array}{r}2,760 \\
4,420 \\
5.8 \\
2.5\end{array}$ & $\begin{array}{r}2,830 \\
4,000 \\
5.7 \\
.5\end{array}$ & $\begin{array}{r}3,270 \\
3,570 \\
6.4 \\
2.5\end{array}$ & $\begin{array}{lr}\text { T. } & 2,890 \\
\text { B. } & 3,730 \\
\text { T. } & 5.8 \\
\text { T. } & 1.4\end{array}$ \\
\hline 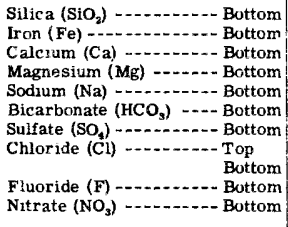 & $\begin{array}{l}3.9 \\
41^{.35} \\
59 \\
374 \\
22 \\
192 \\
575 \\
660 \\
6.3\end{array}$ & $\begin{array}{c}3.6 \\
.10 \\
54 \\
92 \\
636 \\
16 \\
271 \\
890 \\
1,130 \\
3.5\end{array}$ & $\begin{array}{c}3.6 \\
.10 \\
58 \\
681 \\
17 \\
281 \\
725 \\
1,240 \\
3.5\end{array}$ & $\begin{array}{r}3.8 \\
.10 \\
55 \\
32 \\
640 \\
17 \\
270 \\
760 \\
1,140 \\
3.1\end{array}$ & $\begin{array}{c}3.6 \\
48^{.15} \\
83 \\
562 \\
19 \\
243 \\
900 \\
1,000 \\
.5 \\
3.0\end{array}$ & $\begin{array}{lc}\text { B. } & 3.7 \\
\text { B. } & .12 \\
\text { B. } & 51 \\
\text { B. } & 85 \\
\text { B. } & 580 \\
\text { B. } & 18 \\
\text { B. } & 251 \\
\text { T. } & 770 \\
\text { B. } & 1,030 \\
\text { B. } & .5 \\
\text { B. } & 3.3\end{array}$ \\
\hline $\begin{array}{l}\text { Dissolved solids } \ldots \ldots \text { Buttom } \\
\text { Hardness as } \mathrm{CaCO}_{3} \ldots-\text { Bottom } \\
\text { Suspended sediment }-\ldots \text { Top }\end{array}$ & $\begin{array}{r}1,580 \\
34,5 \\
. \\
\end{array}$ & $\begin{array}{r}2,500 \\
5 \pm 3 \\
\cdots \\
\end{array}$ & $\begin{array}{r}2,790 \\
560 \\
. \quad C\end{array}$ & $\begin{array}{r}2,540 \\
516\end{array}$ & $\begin{array}{r}2,250 \\
461\end{array}$ & $\begin{array}{rr}\text { B. } & 2,340 \\
\text { B. } & 479 \\
\text { T. } & 40 \\
\end{array}$ \\
\hline
\end{tabular}

b Exceeded this value.

Location Yarcus Hook, Pa. Weather Clear

Date January 5, 1950 Sampling study No, 6

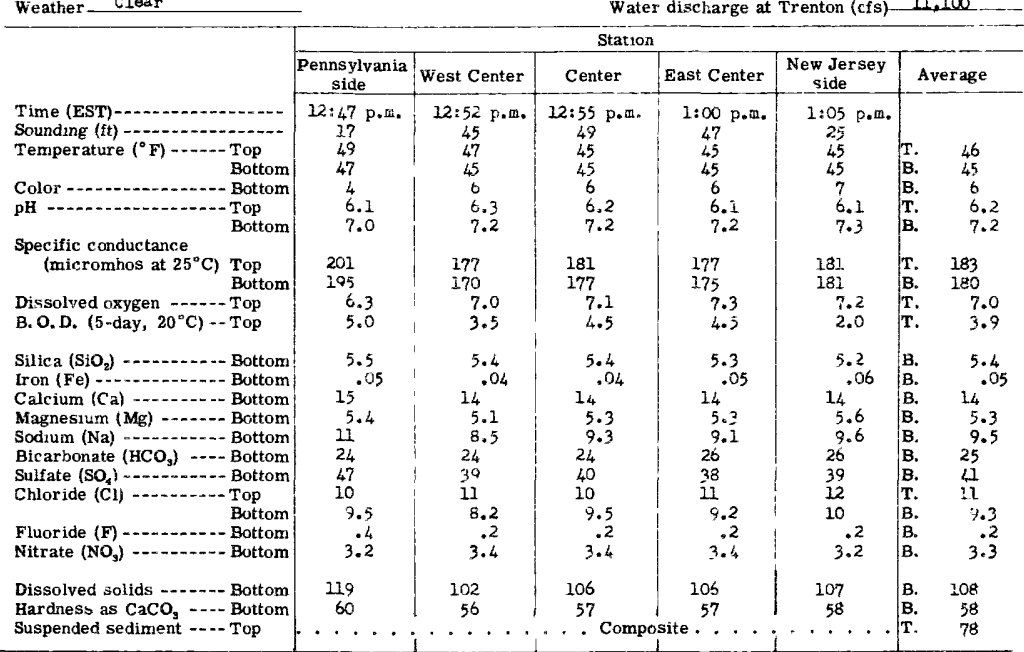


Table 14. -- WATER ANALYSES OF DELAWARE RIVER BETWEEN BRISTOL AND MARCUS HOOK, PA. --CONtinued Analyzed by City of Philadelphia and U. S. Geological Survey: analyses in parts per million

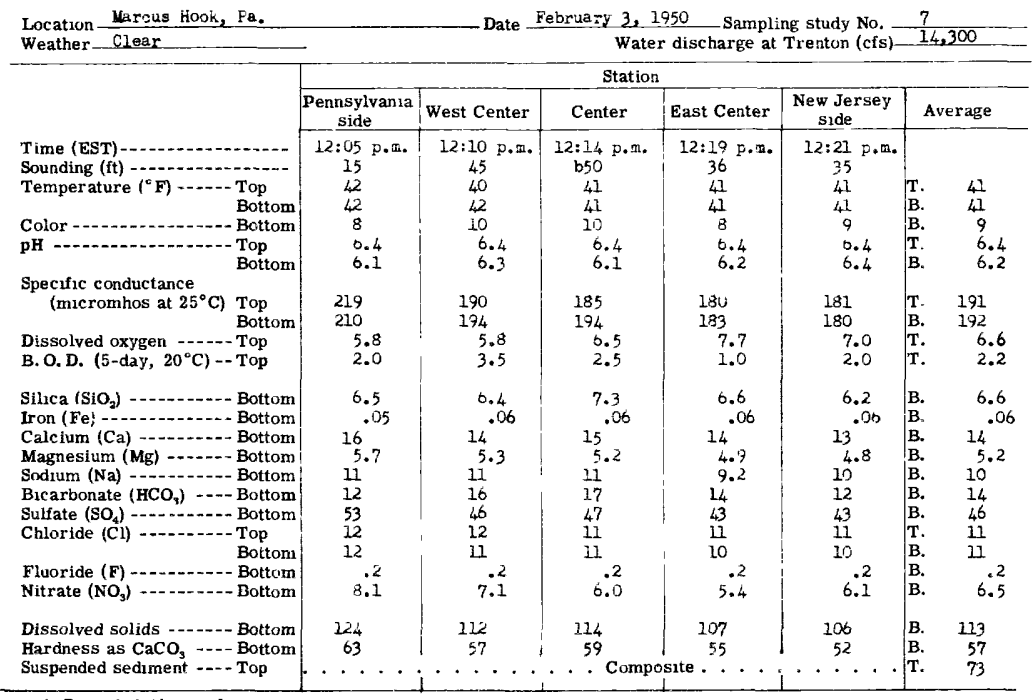

b Exceeded this value.

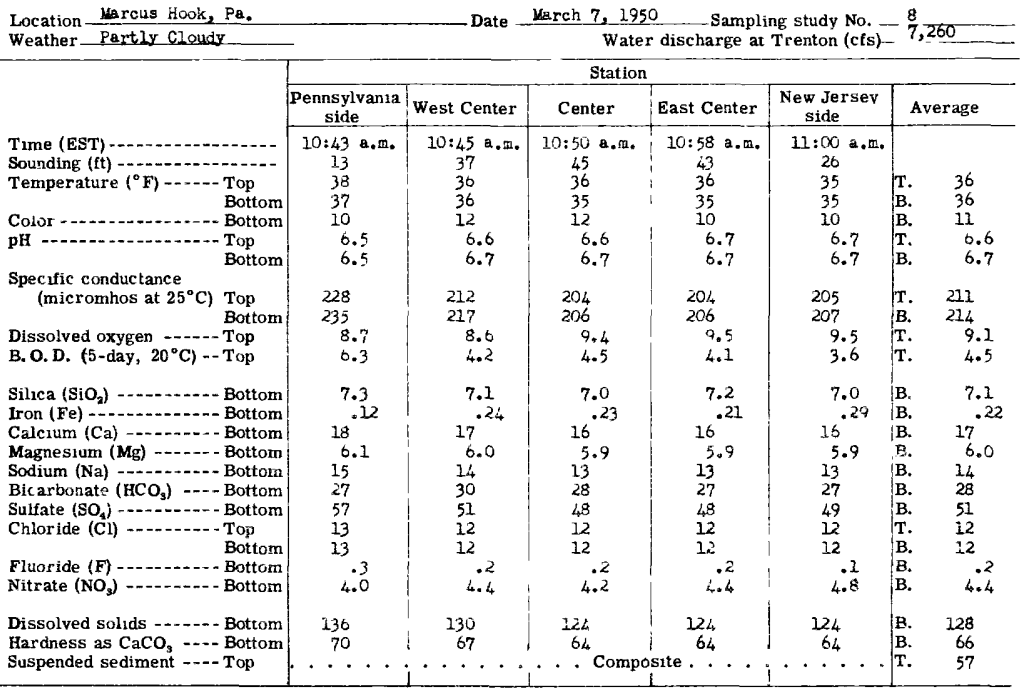


Table 14. - - WATER ANALYSES OF DELAWARE RIVER BETWEEN BRISTOL AND MARCUS HOOK, PA. --ContinUed Analyzed by City of Phladelphia and U. S. Geological Survey; analyses in parts per million

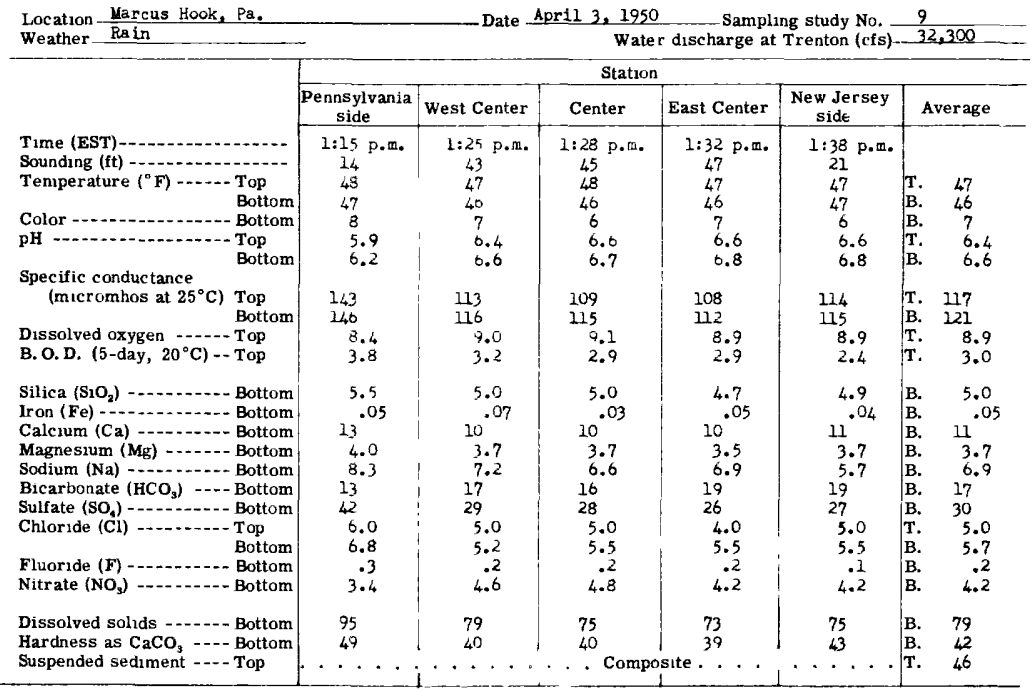

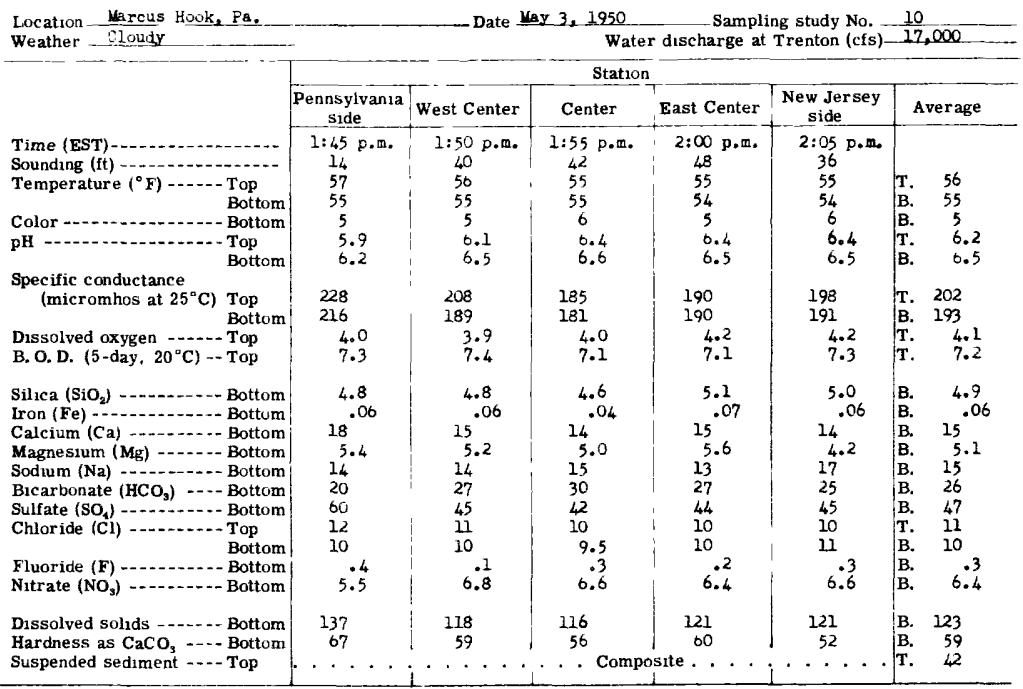


Table 14. -- WATER ANALYSES OF DELAWARE RIVER BETWEEN BRISTOL AND MARCUS hOOK, PA. --Cont inued Analyzed by City of Philadelphia and U. S. Geological Survey; analyses in parts per million

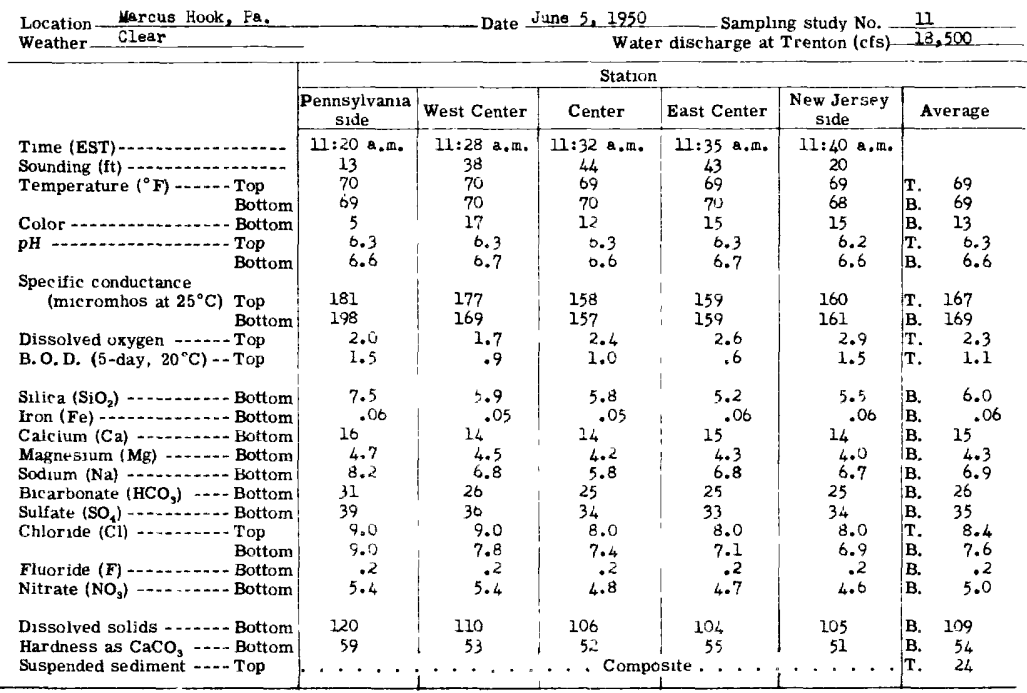

Location Karcus Hook, Fa. Date July 5, 1950 Sampling study No. 12

\begin{tabular}{|c|c|c|c|c|c|c|}
\hline \multirow[t]{3}{*}{ Weather Cloudy } & \multicolumn{6}{|c|}{ Water discharge at Trenton (cfs) $-5,630$} \\
\hline & \multicolumn{6}{|c|}{ Station } \\
\hline & $\begin{array}{c}\text { Pennsylvania } \\
\text { side }\end{array}$ & West Center & Center & East Center & $\begin{array}{l}\text { New Jersey } \\
\text { side }\end{array}$ & Average \\
\hline 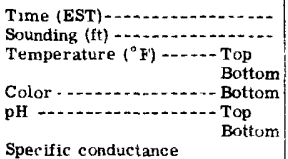 & \begin{tabular}{c|}
$11: 15$ a.17. \\
11 \\
79 \\
78 \\
8 \\
6.4 \\
6.4
\end{tabular} & $\begin{array}{c}11: 20, a, m \\
42 \\
79 \\
78 \\
6 \\
6.5 \\
6.6\end{array}$ & $\begin{array}{c}11: 25 \text { a.m. } \\
44 \\
78 \\
79 \\
4 \\
0.2 \\
6.3\end{array}$ & $\begin{array}{c}11: 30 \mathrm{a} . \mathrm{k} \\
35 \\
78 \\
78 \\
4 \\
6.3 \\
b .3\end{array}$ & $\begin{array}{c}11: 40 \text { a.m. } \\
25 \\
79 \\
78 \\
8 \\
0.2 \\
0.3\end{array}$ & $\begin{array}{l}79 \\
78 \\
6 \\
6.3 \\
6.4\end{array}$ \\
\hline $\begin{array}{l}\begin{array}{l}\text { Specific conductance } \\
\text { (micromhos at } 25^{\circ} \mathrm{C} \text { ) }\end{array} \\
\text { Top } \\
\text { Bottom } \\
\text { Dissoived oxygen - - - Top } \\
\left.\text { B. O. D. (5-day, } 20^{\circ} \mathrm{C}\right)- \text { Top }\end{array}$ & $\begin{array}{l}233 \\
227 \\
1.2 \\
3.5\end{array}$ & $\begin{array}{l}226 \\
221 \\
1.7 \\
3.2\end{array}$ & $\begin{array}{l}221 \\
222 \\
1.8 \\
3.5\end{array}$ & $\begin{array}{r}222 \\
222 \\
4.3 \\
3.6\end{array}$ & $\begin{array}{r}221 \\
224 \\
2.4 \\
5.2\end{array}$ & $\begin{array}{lr}\text { T. } & 225 \\
\text { B. } & 224 \\
\text { T. } & 1.9 \\
\text { T. } & 3.8\end{array}$ \\
\hline 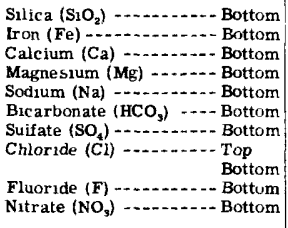 & $\begin{array}{l}0.9 \\
18 \\
.06 \\
6.0 \\
14 \\
28 \\
49 \\
16 \\
14 \\
.3 \\
5.3\end{array}$ & $\begin{array}{l}5.6 \\
.06 \\
18 \\
6.1 \\
12 \\
29 \\
47 \\
14 \\
12 \\
4.3\end{array}$ & $\begin{array}{l}7.2 \\
.06 \\
18 \\
6.3 \\
12 \\
32 \\
49 \\
14 \\
12 \\
.3 \\
2.7\end{array}$ & $\begin{array}{l}5.1 \\
18.06 \\
5.9 \\
10 \\
30 \\
48 \\
14 \\
13 \\
.3 \\
4.4\end{array}$ & $\begin{array}{l}6.4 \\
.04 \\
18 \\
6.1 \\
13 \\
32 \\
50 \\
14 \\
13 \\
.3 \\
2.8\end{array}$ & $\begin{array}{l}6.2 \\
.06 \\
18 \\
6.1 \\
12 \\
30 \\
49 \\
14 \\
13 \\
.3 \\
3.9\end{array}$ \\
\hline $\begin{array}{l}\text { Dissolved solids } \ldots \ldots \text { Bottom } \\
\text { Hardness as } \mathrm{CaCO}_{3} \ldots \ldots \text { Bottom } \\
\text { Suspended sediment } \ldots \text { Top }\end{array}$ & $\begin{array}{r}138 \\
70 \\
\cdots \\
\end{array}$ & $\begin{array}{r}131 \\
70 \\
\cdots\end{array}$ & $\begin{array}{l}134 \\
71 \\
. \mathrm{Co}\end{array}$ & $\begin{array}{r}132 \\
69 \\
.\end{array}$ & $\begin{array}{r}134 \\
70 \\
.\end{array}$ & $\begin{array}{rr}\text { B. } & 134 \\
\text { B. } & 70 \\
\text { T. } & 34 \\
\end{array}$ \\
\hline
\end{tabular}


Table 14. -- WATER ANALYSES OF DELAWARE RIVER BETWEEN BRISTOL AND MARCUS HOOK, PA. --Continned Analyzed by City of Philadelphia and U. S. Geological Survey; analyses in parts per million

Location Marcus Hook, Pa. Weather-Cloudy

Date Auguat I, 1950 Samping study No, 13

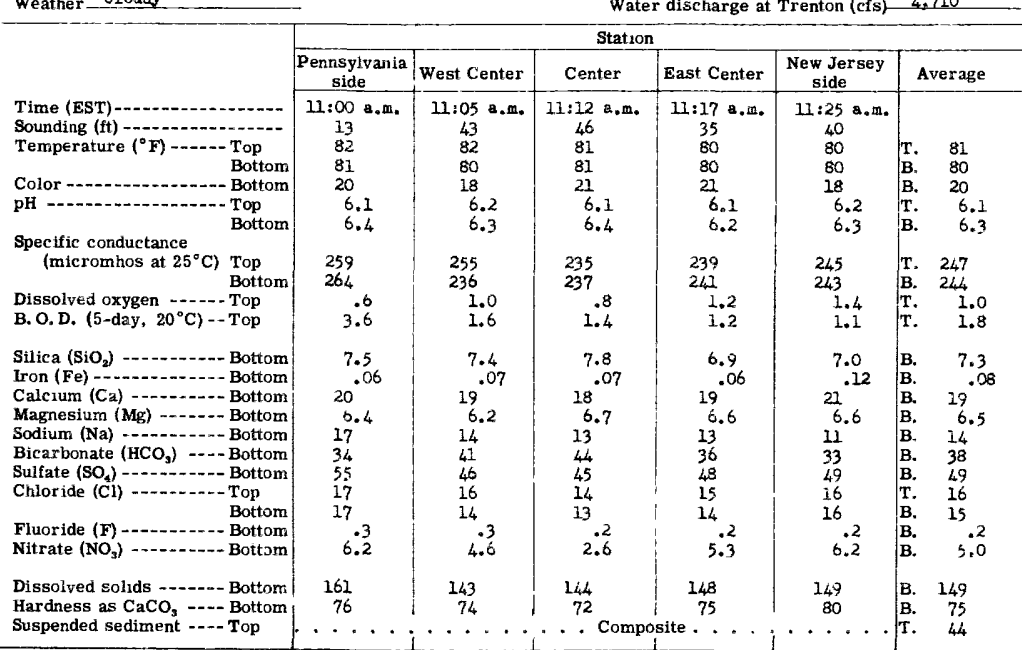

Location Narcus Hook, Pa. Weather CIear

Date September 5, 1950 Sampling study No. 14

\begin{tabular}{|c|c|c|c|c|c|c|}
\hline \multirow[t]{3}{*}{ Weather Clear } & \multicolumn{6}{|c|}{ Water discharge at Trenton (cts) $-5,800$} \\
\hline & \multicolumn{6}{|c|}{ Station } \\
\hline & $\begin{array}{c}\text { Pennsylvania } \\
\text { side }\end{array}$ & West Center & Center & East Center & $\begin{array}{l}\text { New Jersey } \\
\text { side }\end{array}$ & Average \\
\hline 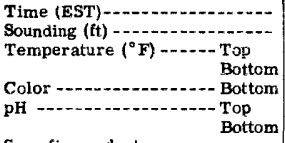 & $\begin{array}{c}10: 55 \text { a.m. } \\
39 \\
78 \\
77 \\
75 \\
6.3 \\
6.4\end{array}$ & $\begin{array}{c}11: 00 \mathrm{a} . \mathrm{m} \\
48 \\
78 \\
77 \\
12 \\
6.4 \\
6.7\end{array}$ & $\begin{array}{c}11: 05 \text { a.m. } \\
48 \\
78 \\
77 \\
15 \\
6.4 \\
6.5\end{array}$ & $\begin{array}{l}11: 15 \text { a.m. } \\
27 \\
78 \\
77 \\
27 \\
6.4 \\
6.6\end{array}$ & $\begin{array}{c}11: 20 \text { a.m. } \\
25 \\
78 \\
77 \\
15 \\
6.3 \\
6.6\end{array}$ & $\begin{array}{lc}\text { r. } & 78 \\
\text { B. } & 77 \\
\text { B. } & 17 \\
\text { T. } & 6.4 \\
\text { B. } & 6.6\end{array}$ \\
\hline $\begin{array}{l}\begin{array}{l}\text { Specific conductance } \\
\quad\left(\text { micromhos at } 25^{\circ} \mathrm{C}\right)\end{array} \\
\begin{array}{l}\text { Top } \\
\text { Bottom }\end{array} \\
\text { Dissolved oxygen } \ldots \text {...- Top } \\
\text { B. O.D. (5-day, } 20^{\circ} \mathrm{C} \text { ) -- Top }\end{array}$ & $\begin{array}{l}586 \\
577 \\
.5 \\
7.8\end{array}$ & $\begin{array}{r}525 \\
535 \\
.8 \\
6.0\end{array}$ & $\begin{array}{l}523 \\
530 \\
1.5 \\
5.4\end{array}$ & $\begin{array}{r}524 \\
534 \\
.6\end{array}$ & $\begin{array}{l}514 \\
541 \\
1.5 \\
6.6\end{array}$ & $\begin{array}{lr}\text { r. } & \\
\text { r. } & 534 \\
\text { B. } & 543 \\
\text { r. } & 1.0 \\
\text { r. } & 6.2\end{array}$ \\
\hline 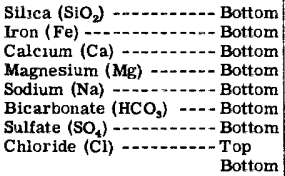 & $\begin{array}{l}5.1 \\
23 \\
14 \\
58 \\
42 \\
68 \\
96 \\
92\end{array}$ & $\begin{array}{l}5.3 \\
22 \\
12 \\
60 \\
62 \\
63 \\
84 \\
80\end{array}$ & $\begin{array}{l}5.4 \\
.14 \\
22 \\
13 \\
49 \\
42 \\
62 \\
82 \\
78\end{array}$ & $\begin{array}{l}5.4 \\
22 \\
22 \\
13 \\
52 \\
42 \\
64 \\
80 \\
82\end{array}$ & $\begin{array}{l}5.5 \\
.16 \\
22 \\
13 \\
51 \\
41 \\
63 \\
82 \\
82\end{array}$ & \begin{tabular}{ll} 
B. & 5.3 \\
B. & \multicolumn{1}{c}{.15} \\
B. & 22 \\
B. & 13 \\
B. & 54 \\
B. & 46 \\
B. & 64 \\
T. & 85 \\
B. & 83
\end{tabular} \\
\hline 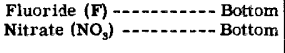 & 8.3 & 7.9 & 8.3 & 7.9 & 6.3 & 7.3 \\
\hline $\begin{array}{l}\text { Dissolved solids - } \\
\text { Hardness as } \mathrm{CaCO}_{3}-\text { Bottom }_{2}\end{array}$ & $\begin{array}{l}336 \\
115\end{array}$ & $\begin{array}{l}324 \\
104\end{array}$ & $\begin{array}{l}314 \\
108\end{array}$ & $\begin{array}{l}321 \\
108\end{array}$ & $\begin{array}{l}328 \\
108\end{array}$ & $\begin{array}{ll}\text { B. } & 325 \\
\text { B. } & 109\end{array}$ \\
\hline ed sediment -.-- To & & & - . Com & site. . & $\cdot \cdot \cdot$ & 30 \\
\hline
\end{tabular}


Table 14. --WATER ANA LYSES OF DELAWARE RIVER BETWEEN BRISTOL AND MARCUS HOOK, PA. --Continued Analyzed by City of Philadelphia and U. S. Geological Survey; analyses in parts per million

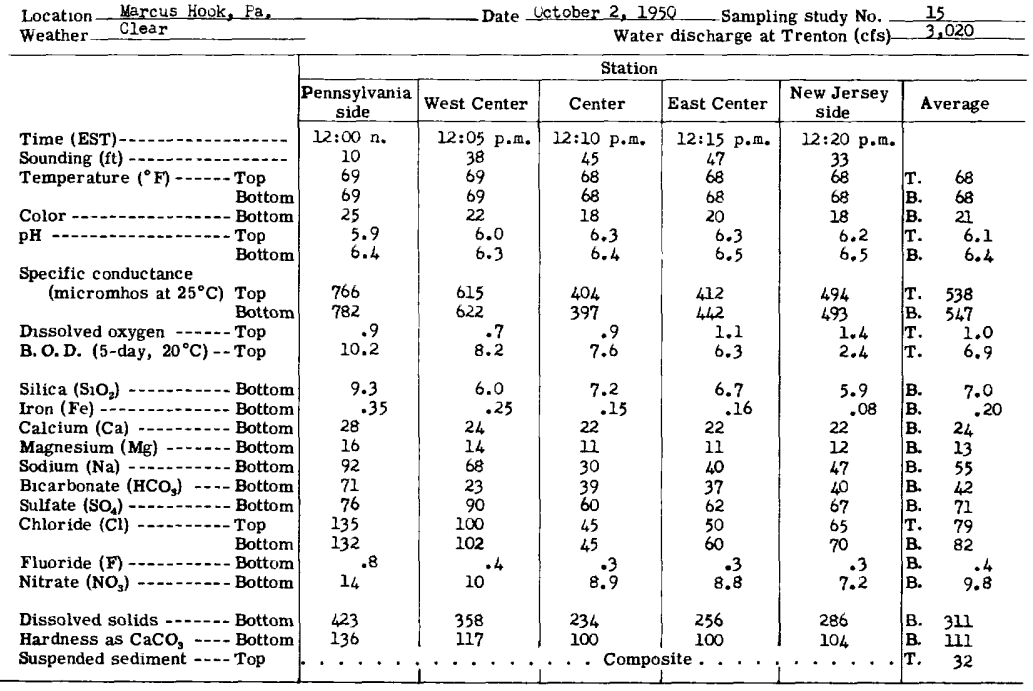

Location Marcus Hook, Pa. Date November 1,1950 Sampling study No. 16

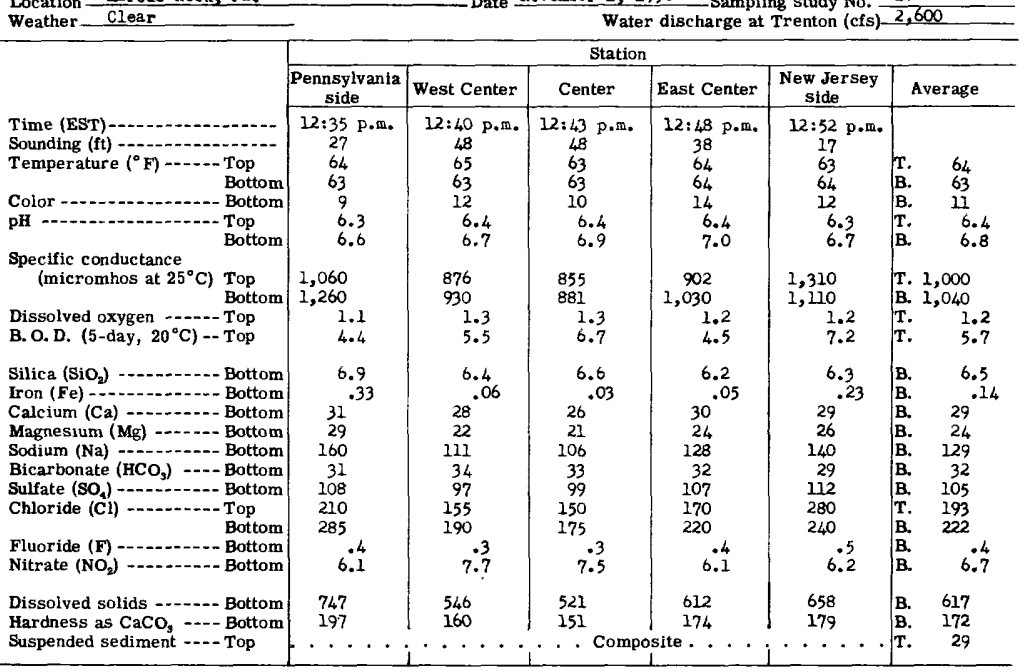

$3153910-54-11$ 
Table 14. - - WATER ANALYSES OF DELAWARE RIVER BETWEEN BRISTOL AND MARCUS HOOK, PA. --Continued Analyzed by City of Philadelphia and U. S. Geological Survey; analyses in parts per million

Location Larcus Hook, $\mathrm{Pa}$._______ Date __ecember 5, 1950 __ Sampling study No. - 17 Weather Partly Cloudy Water discharge at Trenton (cfs) - $-8,3,500$

\begin{tabular}{|c|c|c|c|c|c|c|}
\hline \multirow[b]{3}{*}{$\begin{array}{l}\text { Time (EST) } \\
\text { Sounding (ft) } \\
\text { Temperature }\left({ }^{\circ} \mathrm{F}\right) \\
\begin{array}{c}\text { Bottom } \\
\text { Color }\end{array} \\
\text { pH }- \text { Bottom } \\
\text { Bop }\end{array}$} & \multicolumn{6}{|c|}{ Station } \\
\hline & $\begin{array}{c}\text { Pennsylvania } \\
\text { side }\end{array}$ & West Center & Center & East Center & \begin{tabular}{|c} 
New Jersey \\
side
\end{tabular} & Average \\
\hline & $\begin{array}{c}11: 15 \text { a. } \text {. } \\
3 \\
43 \\
41 \\
28 \\
6.0 \\
7.1\end{array}$ & $\begin{array}{c}11: 18 \mathrm{a} . \mathrm{m} . \\
42 \\
42 \\
41 \\
28 \\
0.0 \\
0.9\end{array}$ & $\begin{array}{c}11: 22 \text { a.m. } \\
47 \\
42 \\
41 \\
26 \\
6.0 \\
7.1\end{array}$ & $\begin{array}{c}12: 25 \mathrm{a}, \mathrm{m} . \\
41 \\
41 \\
41 \\
28 \\
0.0 \\
0.6\end{array}$ & $\begin{array}{c}11: 28 \text { a. } \\
27 \\
42 \\
47 \\
28 \\
6.0 \\
6.9\end{array}$ & $\begin{array}{l}42 \\
41 \\
28 \\
6.0 \\
6.9\end{array}$ \\
\hline 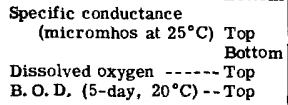 & $\begin{array}{r}157 \\
144 \\
10.0 \\
6.2\end{array}$ & $\begin{array}{r}142 \\
139 \\
10.6 \\
6.2\end{array}$ & $\begin{array}{r}139 \\
137 \\
11.0 \\
6.0\end{array}$ & $\begin{array}{r}139 \\
137 \\
10.9 \\
6.1\end{array}$ & $\begin{array}{r}140 \\
136 \\
10.4 \\
0.2\end{array}$ & $\begin{array}{r}143 \\
139 \\
10.6 \\
6.1\end{array}$ \\
\hline 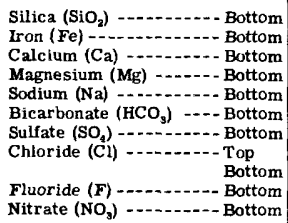 & $\begin{array}{l}- \\
\overline{-} \\
\overline{-} \\
10 \\
39 \\
8.0 \\
8.0 \\
\frac{6.4}{6}\end{array}$ & $\begin{array}{l}\overline{-} \\
\overline{-} \\
\overline{-} \\
\overline{34} \\
6.0 \\
7.2 \\
7.2\end{array}$ & $\begin{array}{l}- \\
= \\
= \\
\overline{10} \\
33 \\
6.0 \\
7.0 \\
6.4\end{array}$ & $\begin{array}{l}- \\
\overline{-} \\
\overline{-} \\
10 \\
33 \\
6.0 \\
7.2 \\
6.3\end{array}$ & $\begin{array}{l}- \\
= \\
\overline{10} \\
33 \\
6.0 \\
\overline{7.2} \\
5.8\end{array}$ & $\begin{array}{l}\overline{-} \\
\overline{-} \\
\overline{10} \\
34 \\
6.4 \\
\frac{7.3}{6.4}\end{array}$ \\
\hline $\begin{array}{l}\text { Dissolved solids }-\ldots-\text { - Bottom } \\
\text { Hardness as } \mathrm{CaCO}_{3} \ldots-\text { Bottom } \\
\text { Suspended sediment }--- \text { Top }\end{array}$ & $\overline{46}$ & $\overline{42}$ & 42 & $\overline{42}$ & $\frac{-}{42}$ & $\begin{array}{r}\overline{43} \\
123\end{array}$ \\
\hline
\end{tabular}

Location Marcus Hook, $\mathrm{Fa}$. Weather Uvercast

Date January 2, 1951 Sampling study No. 18

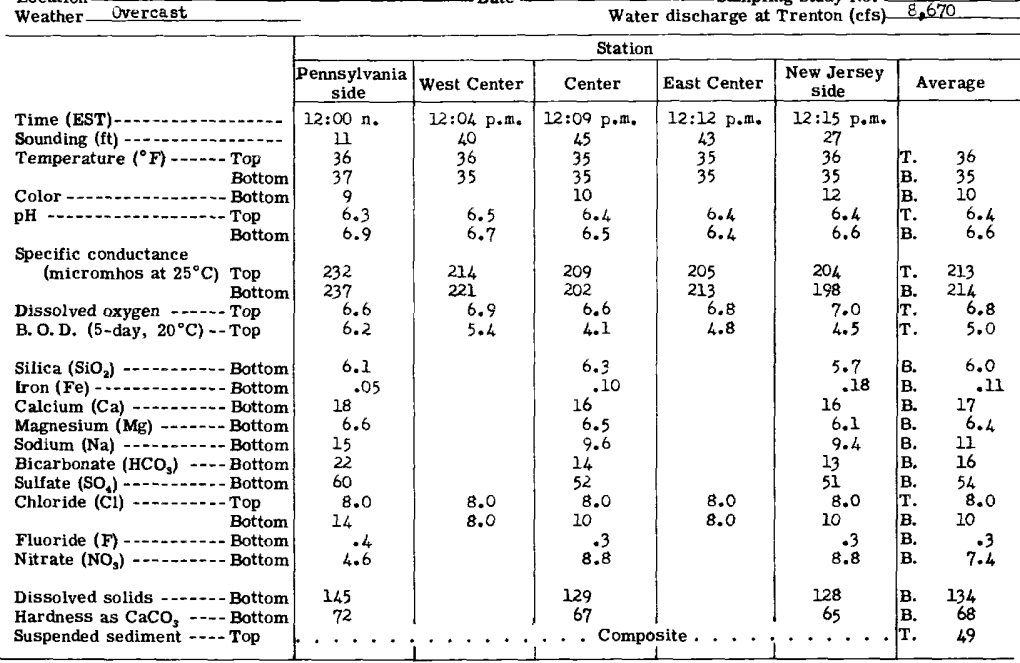


Table 14. -- WATER ANALYSES OF DELAWARE RIVER BETWEEN BRISTOL AND MARCUS HOOK, PA. --Continued Analyzed by City of Philadelphia and U. S. Geological Survey; analyses in parts per million

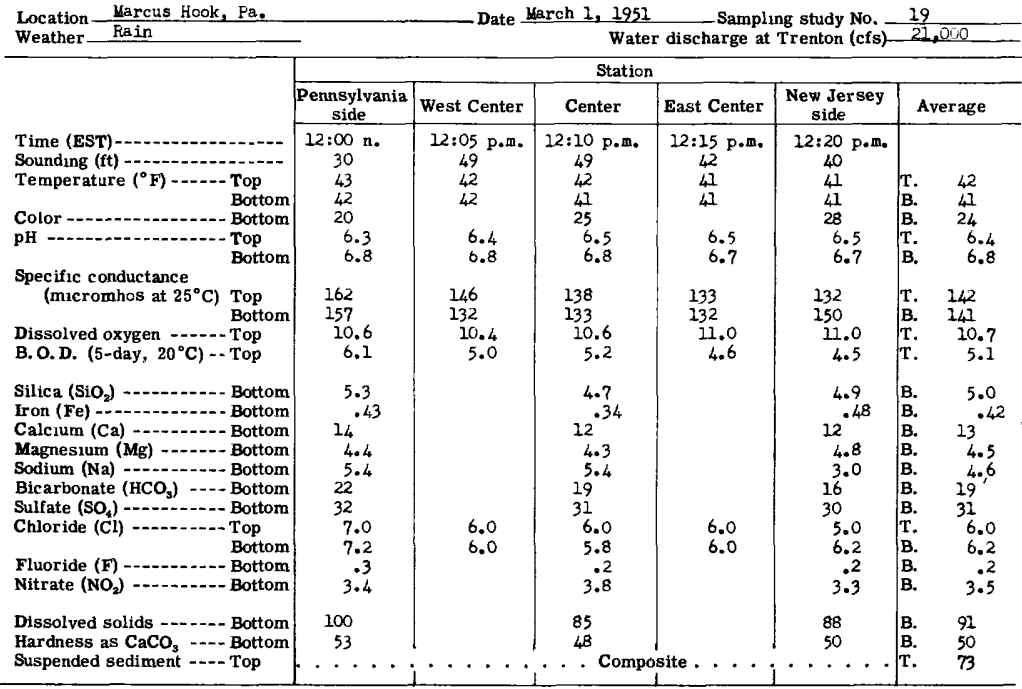

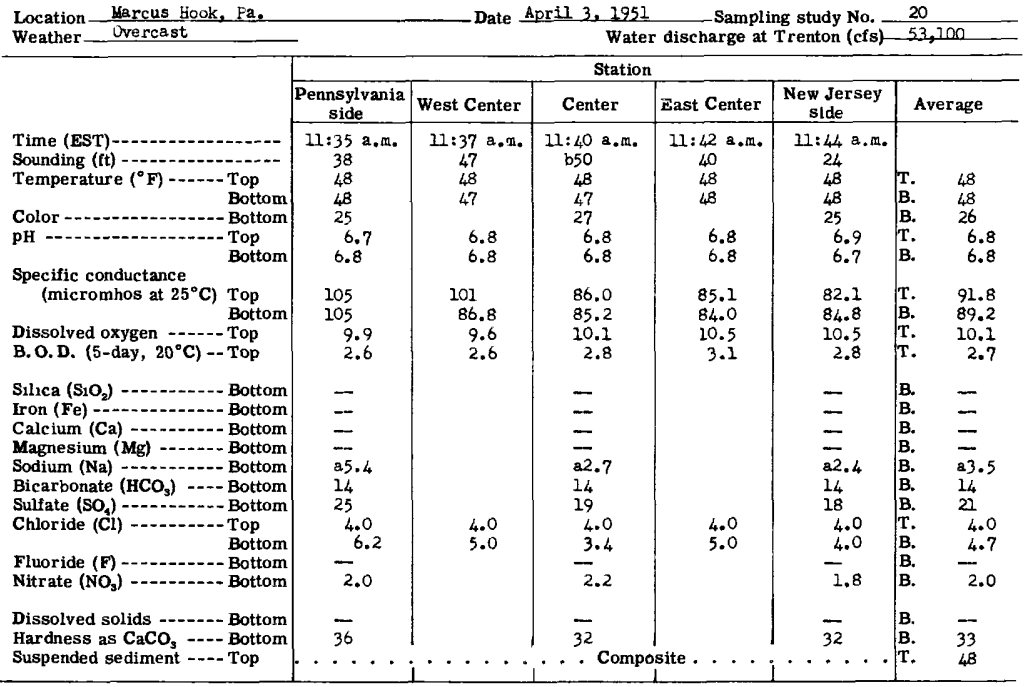

a Calculated Sodium and Potassium.

b exceeded this value. 
Table 14. --WATER ANALYSES OF DELAWARE RVER BETWEEN BRISTOL AND MARCUS HOOK, PA. --Continued Analyzed by City of Philadelphia and U. S. Geological Survey; analyses in parts per million

Location Marcus Hook, $\mathrm{Pa}$. Weather Cloar

\begin{tabular}{|c|c|c|c|c|c|c|}
\hline \multirow{3}{*}{ Weather Cloar } & \multicolumn{6}{|c|}{ Water discharge at Trenton (cfs) 72,400} \\
\hline & \multicolumn{6}{|c|}{ Station } \\
\hline & $\begin{array}{c}\text { Pennsylvania } \\
\text { side }\end{array}$ & West Center & Center & East Center & $\begin{array}{l}\text { New Jersey } \\
\text { side }\end{array}$ & Average \\
\hline $\begin{array}{l}\text { Time (EST) } \\
\text { Sounding (ft) } \\
\text { Temperature }\left({ }^{\circ} \mathbf{F}\right) \\
\begin{array}{l}\text { Bottom } \\
\text { ph }\end{array} \\
\text { Bolor } \\
\text { Bottom }\end{array}$ & $\begin{array}{l}9: 50 \mathrm{a} . \mathrm{m} . \\
36 \\
63 \\
62 \\
18 \\
6.5 \\
6.7\end{array}$ & $\begin{array}{c}9: 55 \text { a.m. } \\
48 \\
62 \\
61 \\
6.6 \\
6.8\end{array}$ & $\begin{array}{c}10: 00 \text { a.m. } \\
\text { b50 } \\
62 \\
61 \\
15 \\
6.7 \\
6.8\end{array}$ & $\begin{array}{l}10: 05 \mathrm{a} . \mathrm{m} . \\
\mathrm{b} 50 \\
61 \\
61 \\
6.7 \\
6.8\end{array}$ & $\begin{array}{c}10: 10 \mathrm{a} . \mathrm{m} . \\
39 \\
61 \\
61 \\
10 \\
6.7 \\
6.8\end{array}$ & $\begin{array}{l}62 \\
61 \\
14 \\
6.6 \\
6.8\end{array}$ \\
\hline 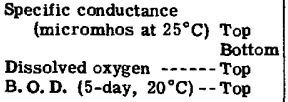 & $\begin{array}{r}196 \\
192 \\
2.5 \\
2.5\end{array}$ & $\begin{array}{l}181 \\
182 \\
2.8 \\
2.2\end{array}$ & $\begin{array}{l}181 \\
180 \\
3.0 \\
2.4\end{array}$ & $\begin{array}{l}180 \\
191 \\
2.8 \\
2.6\end{array}$ & $\begin{array}{l}181 \\
177 \\
3.5 \\
2.8\end{array}$ & $\begin{array}{r}184 \\
184 \\
2.9 \\
2.5\end{array}$ \\
\hline 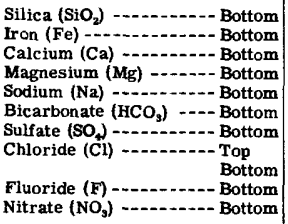 & $\begin{array}{l}7.1 \\
16 \\
.15 \\
5.6 \\
7.8 \\
16 \\
47 \\
11 \\
10 \\
.2 \\
5.9\end{array}$ & $\begin{array}{l}10 \\
10\end{array}$ & $\begin{array}{l}7.4 \\
.12 \\
15 \\
5.4 \\
6.6 \\
18 \\
40 \\
10 \\
9.8 \\
.2 \\
5.0\end{array}$ & $\begin{array}{l}10 \\
10\end{array}$ & $\begin{array}{l}7.0 \\
.12 \\
1.4 \\
5.4 \\
7.0 \\
18 \\
39 \\
10 \\
9.8 \\
.2 \\
4.2\end{array}$ & $\begin{array}{l}7.2 \\
.13 \\
15 \\
5.5 \\
7.1 \\
17 \\
42 \\
10 \\
9.9 \\
.2 \\
5.0\end{array}$ \\
\hline $\begin{array}{l}\text { Dissolved solids }-1-\text { Bottom } \\
\text { Hardness as } \mathrm{CaCO}_{3}-\cdots \text { Bottom } \\
\text { Suspended sediment }-\cdots \text { Top }\end{array}$ & $\begin{array}{r}119 \\
63 \\
.\end{array}$ & & $\begin{array}{l}109 \\
60 \\
. C o m\end{array}$ & . & $\begin{array}{r}111 \\
57 \\
.\end{array}$ & $\begin{array}{r}113 \\
60 \\
27\end{array}$ \\
\hline
\end{tabular}

b Exceeded this value.

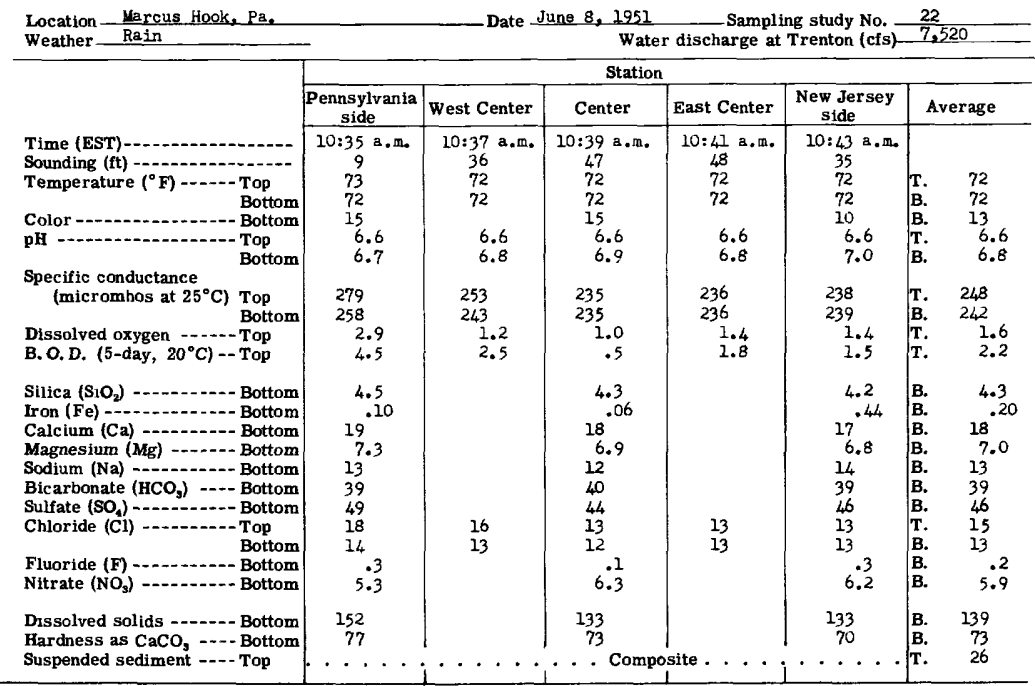


Table 14. -- WATER ANALYSES OF DELAWARE RIVER BETWEEN BRISTOL AND MARCUS HOOK, PA. --Continued Analyzed by City of Philadelphia and U. S. Geological Survey; analyses in parts per million

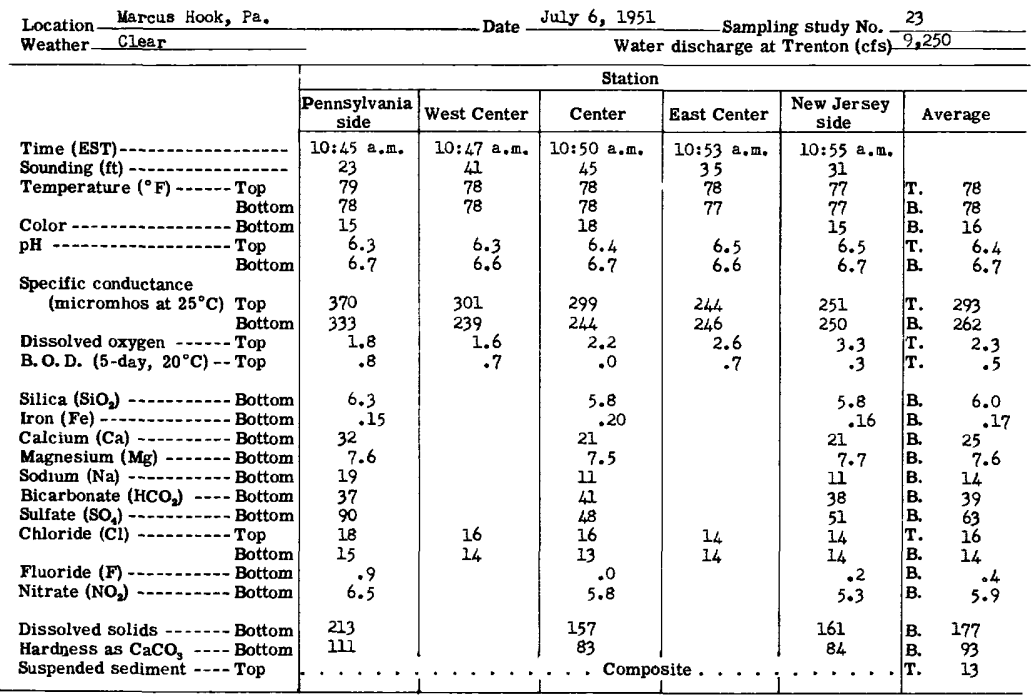

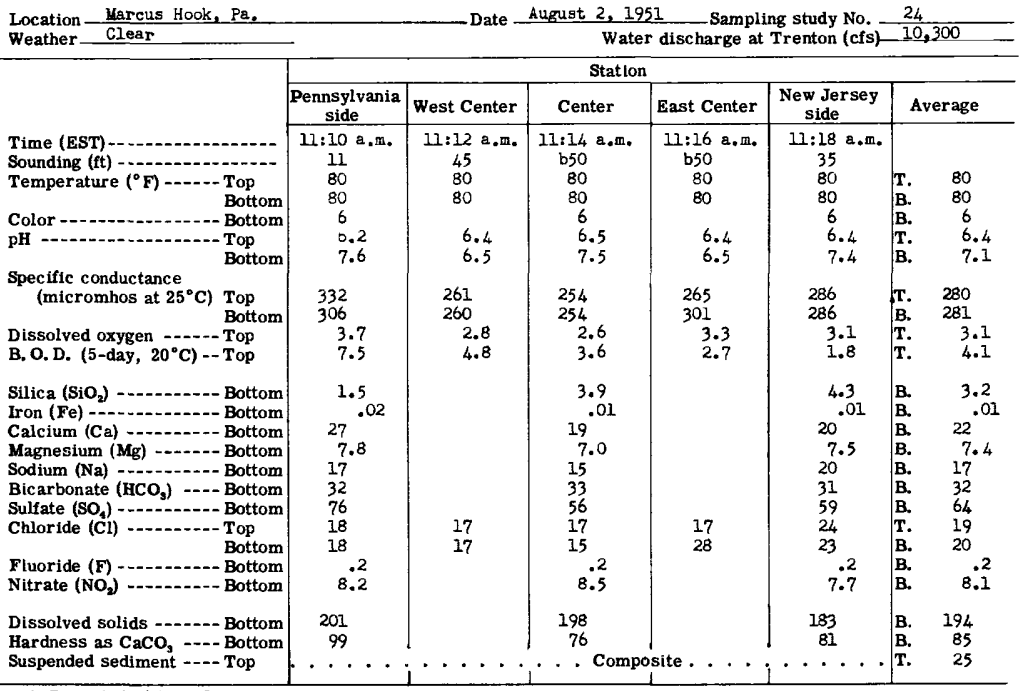

b Exceeded this value. 
Table 14. -- WATER ANALYSES OF DELAWARE RIVER BETWEEN BRISTOL AND MARCUS HOOK, PA. --Continued Analyzed by City of Philadelphia and U. S. Geological Survey; analyses in parts per million

Location Larcus Hook, Fa. Weather Cloudy Hook, Fa.

Date September 5, 1951 Sampling study No. 25 Water discharge at Trenton (cfs) 3,680

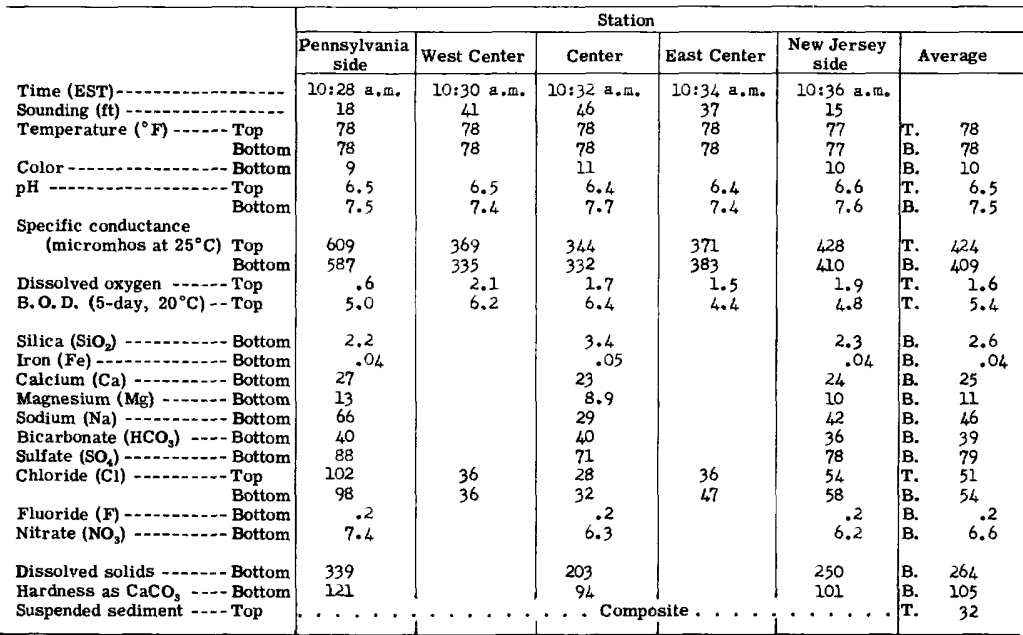

Location Marcus Hook, Pa.

\begin{tabular}{|c|c|c|c|c|c|c|}
\hline \multicolumn{7}{|l|}{ Weather Clear } \\
\hline & \multicolumn{6}{|c|}{ Station } \\
\hline & \begin{tabular}{|c|}
$\begin{array}{c}\text { Pennsylvania } \\
\text { side }\end{array}$ \\
\end{tabular} & West Center & Center & East Center & $\begin{array}{c}\text { New Jersey } \\
\text { side }\end{array}$ & Average \\
\hline 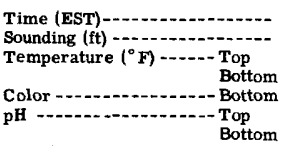 & \begin{tabular}{|c|}
$12: 45$ p.m. \\
29 \\
71 \\
70 \\
10 \\
6.2 \\
7.4
\end{tabular} & $\begin{array}{c}12: 50 \text { p.m. } \\
49 \\
70 \\
69 \\
6.3 \\
6.6\end{array}$ & $\begin{array}{c}12: 52 \mathrm{p.m} . \\
\text { b50 } \\
70 \\
70 \\
10 \\
6.2 \\
7.4\end{array}$ & $\begin{array}{c}12: 55 \mathrm{p} . \mathrm{m} . \\
48 \\
70 \\
69 \\
6.3 \\
7.1\end{array}$ & $\begin{array}{c}12: 58 \mathrm{p} . \mathrm{m} . \\
29 \\
70 \\
70 \\
10 \\
6.3 \\
7.5\end{array}$ & $\begin{array}{l}70 \\
70 \\
10 \\
6.3 \\
7.2\end{array}$ \\
\hline $\begin{array}{l}\text { Specific conductance } \\
\quad \text { (micromhos at } 25^{\circ} \mathrm{C} \text { ) } \begin{array}{l}\text { Top } \\
\text { Bottom }\end{array} \\
\text { Dissolved oxygen -.- Top } \\
\text { B. O. D. (5-day, } 20^{\circ} \mathrm{C} \text { ) - - Top }\end{array}$ & $\begin{array}{r}2,070 \\
2,560 \\
1.8 \\
24.7\end{array}$ & $\begin{array}{r}2,050 \\
3,200 \\
3.4 \\
15.9\end{array}$ & $\begin{array}{r}2,160 \\
3,330 \\
2.0 \\
12.9\end{array}$ & $\begin{array}{r}3,080 \\
3,320 \\
2.4 \\
13.5\end{array}$ & $\begin{array}{r}3,260 \\
3,020 \\
3.0 \\
23.2\end{array}$ & $\begin{array}{lr}\text { T. } & 2,520 \\
\text { B. } & 3,090 \\
\text { T. } & 2.5 \\
\text { T. } & 14.0\end{array}$ \\
\hline 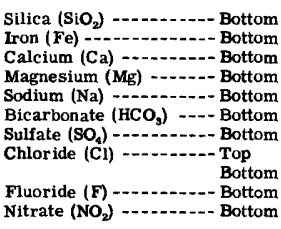 & $\begin{array}{l}3.0 \\
.05 \\
47 \\
52 \\
395 \\
24 \\
192 \\
475 \\
680 \\
.2 \\
4.9\end{array}$ & $\begin{array}{l}480 \\
900\end{array}$ & $\begin{array}{l}2.6 \\
.05 \\
50 \\
69 \\
520 \\
24 \\
228 \\
520 \\
930 \\
.2 \\
3.6\end{array}$ & $\begin{array}{l}780 \\
985\end{array}$ & $\begin{array}{l}3.2 \\
.05 \\
48 \\
62 \\
478 \\
26 \\
218 \\
830 \\
865 \\
.2 \\
4.2\end{array}$ & $\begin{array}{r}2.9 \\
48 \\
61 \\
464 \\
25 \\
213 \\
617 \\
872 \\
.05 \\
4.2\end{array}$ \\
\hline 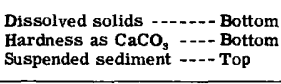 & $\begin{array}{r}1,430 \\
331 \\
.\end{array}$ & & $\begin{array}{r}1,900 \\
408 \\
. \quad \mathrm{Co} \\
\end{array}$ & e.*. & $\begin{array}{r}1,770 \\
375 \\
. \cdots \\
\end{array}$ & \begin{tabular}{|rr} 
B. & 1,700 \\
B. & 371 \\
T. & 88 \\
\end{tabular} \\
\hline
\end{tabular}

b Exceeded this value. 
Table 14. --WATER ANALYSES OF DELAWARE RIVER BETWEEN BRISTOL AND MARCUS HOOK, PA. --CONtInUEd Analyzed by City of Philadelphia and U. S. Geological Survey; analyses in parts per million

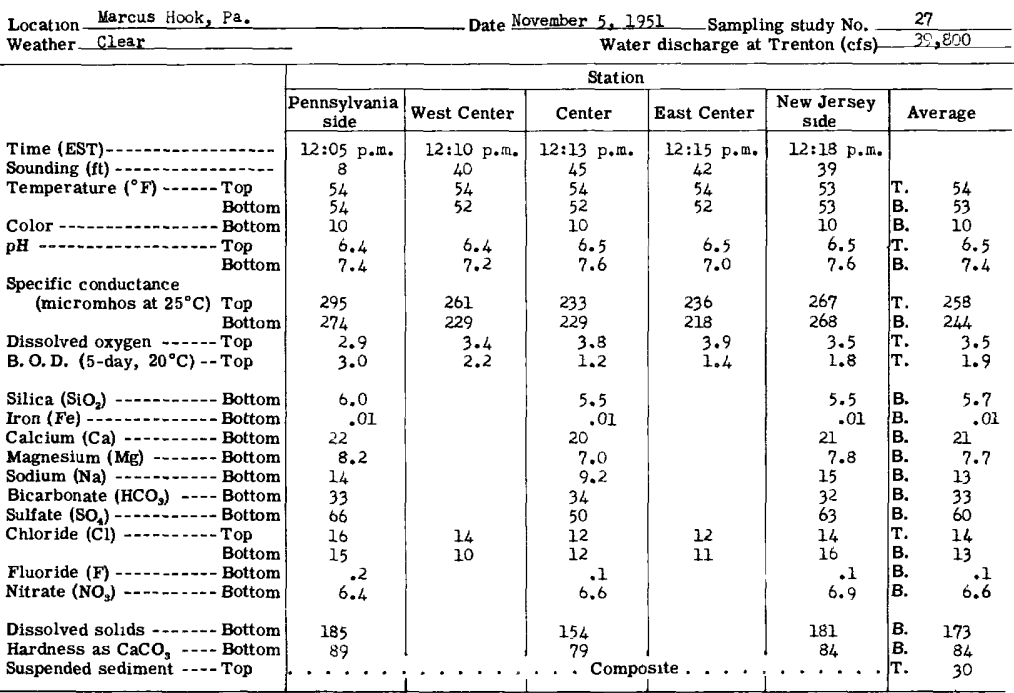

Location Karcus Hook, Pa. Weather Cloudy

Date December 4, 1951___ Sampling study No. 28

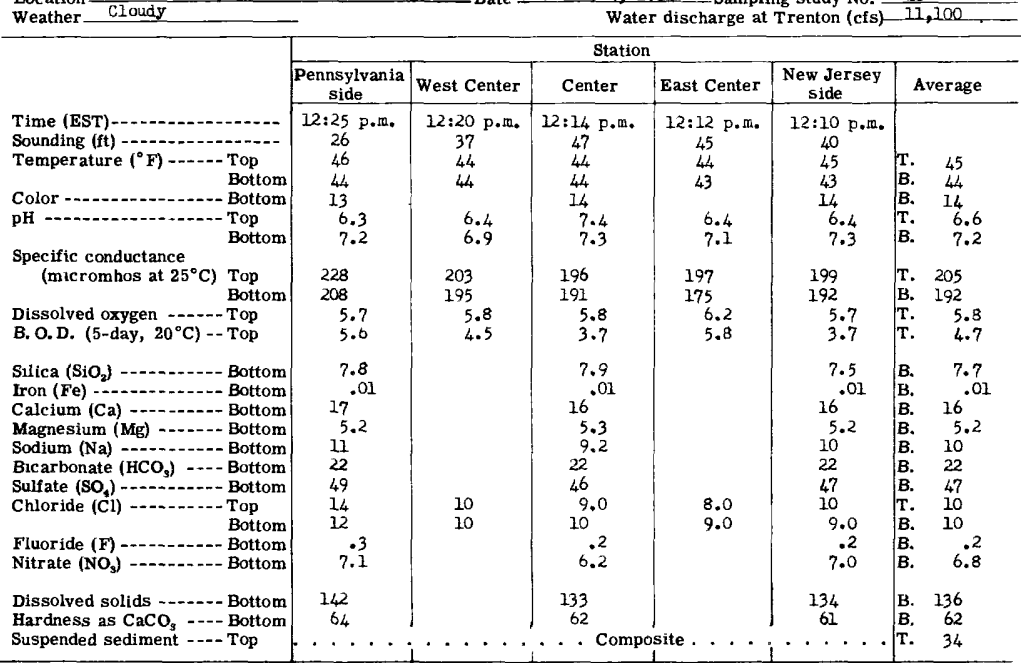


Table 14. - - WATER ANALYSES OF DELAWARE RIVER BETWEEN BRISTOL AND MARCUS hOOK, PA. --Continued Analyzed by City of Philadelphia and U. S. Geological Survey; analyses in parts per million

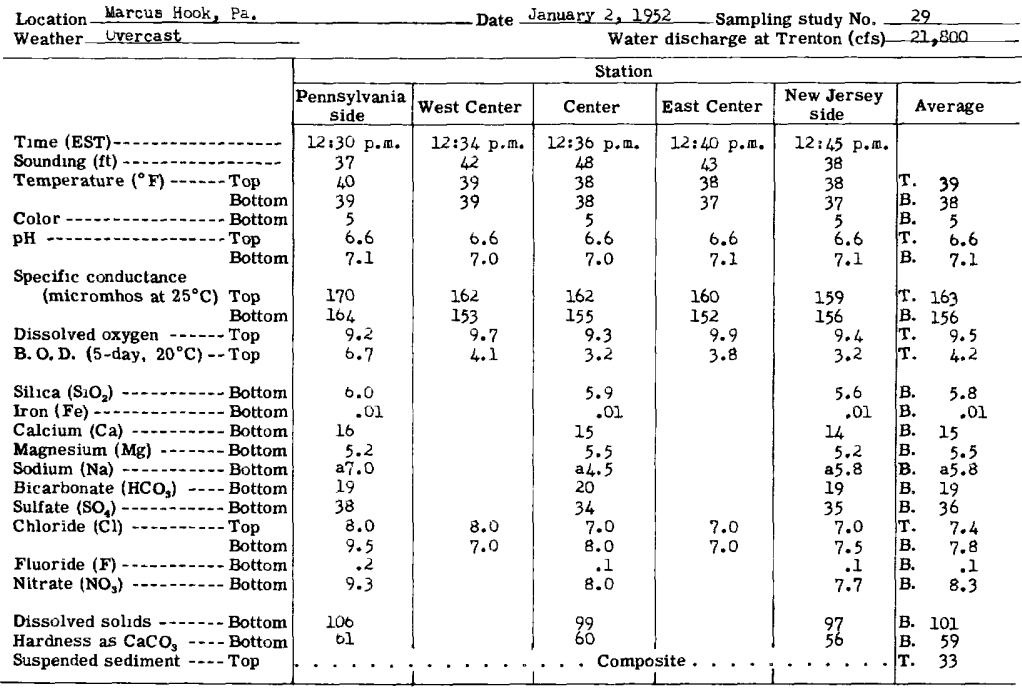

a Calculatea Sodzum and Potassium.

\begin{tabular}{|c|c|c|c|c|c|c|}
\hline \multirow[t]{3}{*}{$\begin{array}{l}\text { Location Marcus Hook, } \mathrm{Pa} \text {. } \\
\text { Weather }\end{array}$} & \multicolumn{6}{|c|}{-Date February $6,1952-$ Sampling study No. $\frac{30}{\text { Water discharge at Trenton (cfs) }-43,400}$} \\
\hline & \multicolumn{6}{|c|}{ Station } \\
\hline & $\begin{array}{c}\text { Pennsylvania } \\
\text { side }\end{array}$ & West Center & Center & East Center & $\begin{array}{l}\text { New Jersey } \\
\text { side }\end{array}$ & Average \\
\hline 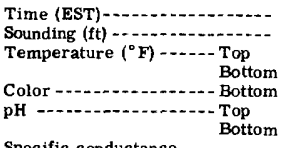 & $\begin{array}{c}11: 54 \text { a. } \pi \\
23 \\
41 \\
47 \\
9 \\
6.0 \\
6.2\end{array}$ & $\begin{array}{c}11: 50 \text { a.m. } \\
47 \\
40 \\
39 \\
6.2 \\
7.5\end{array}$ & $\begin{array}{c}11: 40 \text { a.m. } \\
49 \\
39 \\
38 \\
9 \\
6.2 \\
6.4\end{array}$ & $\begin{array}{c}11: 43 \text { a.m. } \\
44 \\
39 \\
39 \\
6.2 \\
7.4\end{array}$ & $\begin{array}{c}11:=40 \mathrm{a} \cdot \mathrm{m} . \\
42 \\
40 \\
38 \\
9 \\
6.1 \\
6.3\end{array}$ & $\begin{array}{ll}\text { T. } & 40 \\
\text { B. } & 39 \\
\text { B. } & 9 \\
\text { T. } & 6.1 \\
\text { B. } & 6.8\end{array}$ \\
\hline $\begin{array}{l}\begin{array}{l}\text { Specific conductance } \\
\left.\text { (micromhos at } 25^{\circ} \mathrm{C}\right)\end{array} \\
\begin{array}{l}\text { Top } \\
\text { Bottom }\end{array} \\
\text { Dissolved oxygen -...- Top } \\
\left.\text { B. O. D. (5-day, } 20^{\circ} \mathrm{C}\right) \text { - Top }\end{array}$ & $\begin{array}{r}192 \\
193 \\
10.0 \\
3.4\end{array}$ & $\begin{array}{r}155 \\
156 \\
10.9 \\
4.4\end{array}$ & $\begin{array}{r}150 \\
1.49 \\
10.9 \\
4.2\end{array}$ & $\begin{array}{l}150 \\
147 \\
10.7 \\
4.1\end{array}$ & $\begin{array}{r}149 \\
146 \\
10.8 \\
4.1\end{array}$ & 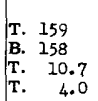 \\
\hline 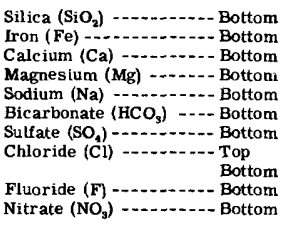 & $\begin{array}{l}8.2 \\
17 \\
17 \\
5.8 \\
6.5 \\
14 \\
50 \\
8.0 \\
7.5 \\
.3 \\
7.8\end{array}$ & $\begin{array}{l}6.0 \\
6.0\end{array}$ & $\begin{array}{l}6.7 \\
.05 \\
12 \\
5.1 \\
5.4 \\
20 \\
33 \\
6.0 \\
5.5 \\
.1 \\
5.6\end{array}$ & $\begin{array}{l}6.0 \\
6.0\end{array}$ & $\begin{array}{l}6.8 \\
12.06 \\
5.2 \\
5.4 \\
21 \\
33 \\
6.0 \\
6.0 \\
.1 \\
5.8\end{array}$ & $\begin{array}{lc}\text { B. } & 7.2 \\
\text { B. } & .05 \\
\text { B. } & 14 \\
\text { B. } & 5.4 \\
\text { B. } & 5.8 \\
\text { B. } & 18 \\
\text { B. } & 39 \\
\text { T. } & 6.4 \\
\text { B. } & 6.2 \\
\text { B. } & .2 \\
\text { B. } & 6.4\end{array}$ \\
\hline $\begin{array}{l}\text { Dissolved solids } \ldots . .- \text { Bottom } \\
\text { Hardness as } \mathrm{CaCO}, \ldots \text { Bottom } \\
\text { Suspended sediment } \ldots \text { - Top }\end{array}$ & $\begin{array}{r}150 \\
60 \\
. \quad .\end{array}$ & & $\begin{array}{l}108 \\
51 \\
. \quad \mathrm{Cor}\end{array}$ & & $\begin{array}{r}10 ? \\
51 \\
\cdots \\
\end{array}$ & $\begin{array}{lr}\text { B. } & 122 \\
\text { B. } & 56 \\
\text { T. } & 40\end{array}$ \\
\hline
\end{tabular}


Table 14. -- WATER ANALYSES OF DELAWARE RIVER BETWEEN BRISTOL AND MARCUS HOOK, PA. --Continued Analyzed by City of Philadelphia and U. S. Geological Survey; analyses in parts per million

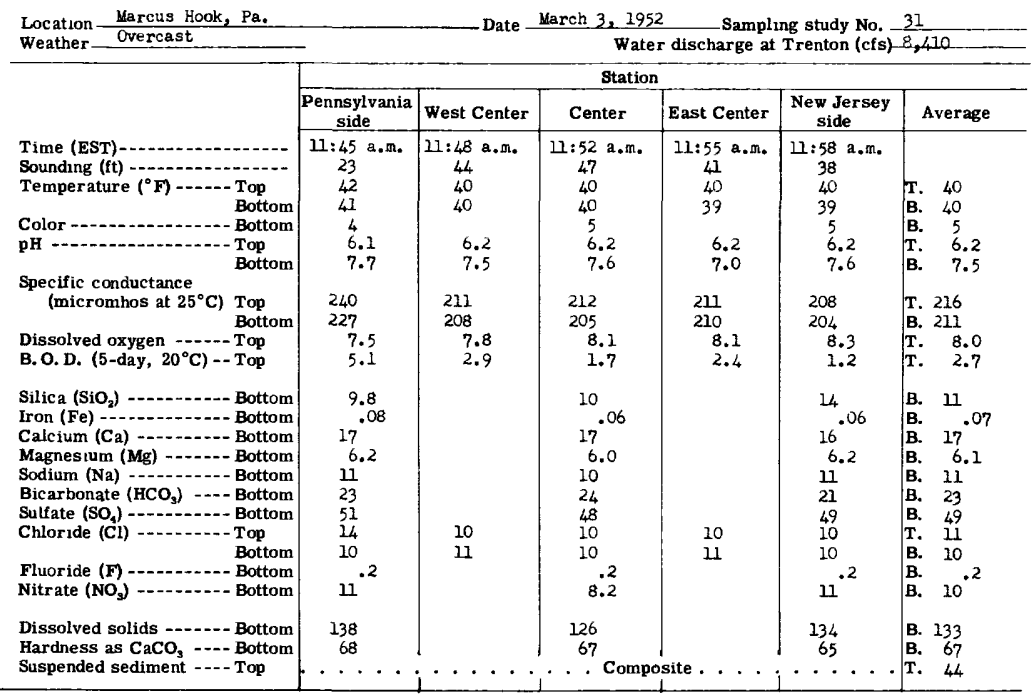

Location Marcus Hook, Pa. Weather Overcast

Date Apri1 1, 1952 Sampling study No. 32

\begin{tabular}{|c|c|c|c|c|c|c|}
\hline eather Overcast & & & & lischarge & renton ( & 0,800 \\
\hline & & & Station & & & \\
\hline & \begin{tabular}{|c|}
$\begin{array}{c}\text { Pennsylvania } \\
\text { side }\end{array}$ \\
\end{tabular} & West Center & Center & East Center & \begin{tabular}{|c} 
New Jersey \\
side
\end{tabular} & Average \\
\hline 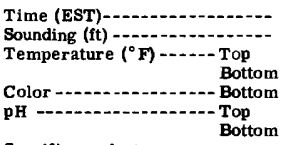 & $\begin{array}{c}10: 45 \text { a.m. } \\
26 \\
50 \\
50 \\
3 \\
6.0 \\
6.9\end{array}$ & $\begin{array}{c}10: 48 \text { a.m. } \\
39 \\
50 \\
50 \\
\\
6.0 \\
7.5\end{array}$ & $\begin{array}{c}10: 51 \text { a.m. } \\
43 \\
49 \\
49 \\
3 \\
6.3 \\
6.8\end{array}$ & $\begin{array}{c}10: 53 \mathrm{a} \cdot \mathrm{m} . \\
47 \\
49 \\
48 \\
6.1 \\
6.9\end{array}$ & $\begin{array}{c}10: 56 \text { a.m. } \\
40 \\
48 \\
48 \\
3 \\
6.2 \\
6.8\end{array}$ & $\begin{array}{ll}\text { T. } & 49 \\
\text { B. } & 49 \\
\text { B. } & 3 \\
\text { T. } & 6.1 \\
\text { B. } & 7.0\end{array}$ \\
\hline 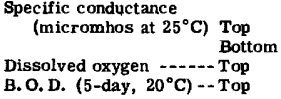 & $\begin{array}{l}151 \\
144 \\
6.4 \\
3.7\end{array}$ & $\begin{array}{r}153 \\
145 \\
6.5 \\
3.9\end{array}$ & $\begin{array}{r}141 \\
134 \\
6.5 \\
1.7\end{array}$ & $\begin{array}{l}138 \\
133 \\
6.7 \\
1.3\end{array}$ & $\begin{array}{r}132 \\
126 \\
7.3 \\
.3\end{array}$ & $\begin{array}{ll}\text { T. } & 143 \\
\text { B. } & 136 \\
\text { T. } & 6.7 \\
\text { T. } & 2.4\end{array}$ \\
\hline 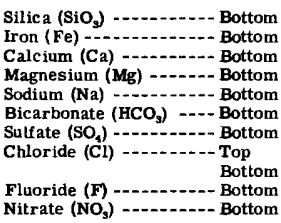 & $\begin{array}{l}7.1 \\
12.04 \\
4.1 \\
11 \\
21 \\
39 \\
6.0 \\
6.6 \\
.02 \\
7.5\end{array}$ & $\begin{array}{l}7.0 \\
6.0\end{array}$ & $\begin{array}{l}7.0 \\
.08 \\
12 \\
3.8 \\
6.6 \\
20 \\
30 \\
6.0 \\
5.9 \\
.1 \\
7.4\end{array}$ & $\begin{array}{l}5.0 \\
6.0\end{array}$ & $\begin{array}{l}6.4 \\
.06 \\
11 \\
3.7 \\
6.4 \\
18 \\
30 \\
5.0 \\
5.2 \\
.1 \\
6.8\end{array}$ & $\begin{array}{lc}\text { B. } & 6.8 \\
\text { B. } & .06 \\
\text { B. } & 12 \\
\text { B. } & 3.9 \\
\text { B. } & 8.0 \\
\text { B. } & 20 \\
\text { B. } & 33 \\
\text { T. } & 5.8 \\
\text { B. } & 5.9 \\
\text { B. } & .1 \\
\text { B. } & 7.2\end{array}$ \\
\hline $\begin{array}{l}\text { Dissolved solids -...-.- Bottom } \\
\text { Hardness as } \mathrm{CaCO}, \ldots-\text { Bottom } \\
\text { Suspended sediment } \ldots \text {-.. Top }\end{array}$ & $\begin{array}{r}111 \\
47 \\
.\end{array}$ & & $\begin{array}{l}92 \\
46 \\
.\end{array}$ & 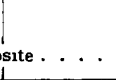 & $\begin{array}{r}90 \\
43 \\
\therefore\end{array}$ & $\begin{array}{ll}\text { B. } & 98 \\
\text { B. } & 45 \\
\text { T. } & 38\end{array}$ \\
\hline
\end{tabular}


Table 14. - - WATER ANALYSES OF DELAWARE RIVER BETWEEN BRISTOL AND MARCUS hOOK, PA. --Continued Analyzed by City of Philadelphia and U. S. Geological Survey; analyses in parts per million

Location Marcus Hook, Fa. Weather Cloudy

Date May 6, 195

Sampling study No. 33

\begin{tabular}{|c|c|c|c|c|c|c|}
\hline \multicolumn{3}{|l|}{ Weather Cloudy } & \multicolumn{4}{|c|}{ Water discharge at Trenton (cfs) 16,600} \\
\hline & \multicolumn{6}{|c|}{ Station } \\
\hline & $\begin{array}{c}\text { Pennsylvania } \\
\text { side }\end{array}$ & West Center & Center & East Center & $\begin{array}{c}\text { New Jersey } \\
\text { side }\end{array}$ & Average \\
\hline $\begin{array}{l}\text { Time (EST) } \\
\begin{array}{l}\text { Sounding }(\mathrm{ft}) \\
\text { Temperature }\left({ }^{\circ} \mathrm{F}\right)\end{array} \\
\begin{array}{l}\text { Color } \\
\text { pH }\end{array} \\
\text { Specific conductance } \quad \text { Bottom } \\
\text { Sottom }\end{array}$ & $\begin{array}{c}12: 13 \mathrm{p} . \mathrm{m} . \\
31 \\
59 \\
58 \\
6 \\
6.3 \\
6.8\end{array}$ & $\begin{array}{c}12: 10 \mathrm{p} . \mathrm{m} . \\
42 \\
59 \\
59 \\
\\
6.3 \\
6.3\end{array}$ & $\begin{array}{c}12: 06 \text { p.m. } \\
45 \\
59 \\
58 \\
6 \\
6.3 \\
6.7\end{array}$ & $\begin{array}{c}12: 03 \text { p.m. } \\
8 \\
59 \\
59 \\
6.4 \\
6.2\end{array}$ & $\begin{array}{c}12: 00 \mathrm{n} \\
6 \\
59 \\
58 \\
7 \\
6.3 \\
6.5\end{array}$ & $\begin{array}{ll}\text { T. } & 59 \\
\text { B. } & 58 \\
\text { B. } & 6 \\
\text { T. } & 6.3 \\
\text { B. } & 6.5\end{array}$ \\
\hline $\begin{array}{l}\text { (micromhos at } 25^{\circ} \mathrm{C} \text { ) } \begin{array}{l}\text { Top } \\
\text { Bottom }\end{array} \\
\text { Dissolved unygen }-10^{\circ} \mathrm{C} \text { ) - Top } \\
\text { B. O. D. (5-day, }\end{array}$ & $\begin{array}{l}167 \\
152 \\
5.8 \\
2.4\end{array}$ & $\begin{array}{l}164 \\
151 \\
5.8 \\
2.3\end{array}$ & $\begin{array}{l}151 \\
137 \\
7.2 \\
3.0\end{array}$ & $\begin{array}{r}145 \\
146 \\
6.9 \\
.1\end{array}$ & $\begin{array}{r}145 \\
150 \\
6.8 \\
3.3\end{array}$ & $\begin{array}{lr}\text { T. } & 154 \\
\text { B. } & 147 \\
\text { T. } & 6.5 \\
\text { T. } & 2.2\end{array}$ \\
\hline 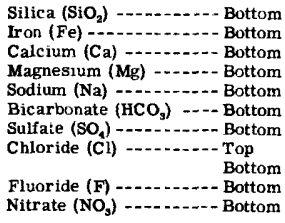 & $\begin{array}{l}7.8 \\
.04 \\
14 \\
4.5 \\
5.8 \\
22 \\
37 \\
8.0 \\
5.8 \\
.2 \\
2.8\end{array}$ & $\begin{array}{l}6.0 \\
4.0\end{array}$ & $\begin{array}{l}8.2 \\
.02 \\
13 \\
4.1 \\
5.1 \\
17 \\
35 \\
5.0 \\
5.4 \\
.1 \\
5.6\end{array}$ & $\begin{array}{l}5.0 \\
5.0\end{array}$ & $\begin{array}{l}10 \\
13 \\
4.08 \\
5.1 \\
14 \\
42 \\
5.0 \\
5.8 \\
.1 \\
3.5\end{array}$ & $\begin{array}{lc}\text { B. } & 8.7 \\
\text { B. } & .05 \\
\text { B. } & 13 \\
\text { B. } & 4.4 \\
\text { B. } & 5.3 \\
\text { B. } & 18 \\
\text { B. } & 38 \\
\text { T. } & 5.8 \\
\text { B. } & 5.2 \\
\text { B. } & .1 \\
\text { B. } & 4.0\end{array}$ \\
\hline $\begin{array}{l}\text { Dissolved solids }-\ldots-\text { Bottom } \\
\text { Hardness as } \mathrm{CaCO}_{\mathrm{g}}-\ldots-\text { Bottom } \\
\text { Suspended sediment --- Top }\end{array}$ & $\begin{array}{l}93 \\
53 \\
\cdots\end{array}$ & & $\begin{array}{l}88 \\
49 \\
\text { Con }\end{array}$ & - & $\begin{array}{r}99 \\
51 \\
.\end{array}$ & $\begin{array}{ll}\text { B. } & 93 \\
\text { B. } & 51 \\
\text { T. } & 63\end{array}$ \\
\hline
\end{tabular}

Location Marcus Hook, Pa.

Sampling study No. 34

\begin{tabular}{|c|c|c|c|c|c|c|}
\hline \multirow{3}{*}{ Weather Clear } & \multicolumn{6}{|c|}{ Water discharge at Trenton (cfs) 35,600} \\
\hline & \multicolumn{6}{|c|}{ Station } \\
\hline & \begin{tabular}{|c|}
$\begin{array}{c}\text { Pennsylvania } \\
\text { side }\end{array}$ \\
\end{tabular} & West Center & Center & East Center & $\begin{array}{l}\text { New Jersey } \\
\text { side }\end{array}$ & Average \\
\hline $\begin{array}{l}\text { Time (EST) } \\
\text { Sounding (ft) } \\
\text { Temperature }\left({ }^{\circ} \mathrm{F}\right)-1 \\
\text { Color }- \text { Top } \\
\text { pH }- \text { Bottom } \\
\end{array}$ & $\begin{array}{c}1: 15 \text { a.m. } \\
27 \\
68 \\
67 \\
7 \\
6.4 \\
6.8\end{array}$ & $\begin{array}{c}11: 18 \text { a.sl. } \\
47 \\
68 \\
69 \\
\\
6.5 \\
6.5\end{array}$ & $\begin{array}{c}\mathrm{L}: 20 \text { a.m. } \\
49 \\
69 \\
68 \\
7 \\
6.5 \\
6.8\end{array}$ & $\begin{array}{c}12.22 \text { a. } \pi \\
45 \\
70 \\
68 \\
6.6 \\
6.5\end{array}$ & $\begin{array}{c}11: 24 \text { a.m. } \\
36 \\
69 \\
68 \\
7 \\
6.7 \\
6.6\end{array}$ & $\begin{array}{lc}\text { T. } & 69 \\
\text { B. } & 68 \\
\text { B. } & 7 \\
\text { T. } & 6.5 \\
\text { B. } & 6.6\end{array}$ \\
\hline $\begin{array}{l}\begin{array}{l}\text { Specific conductance } \\
\quad\left(\text { micromhos at } 25^{\circ} \mathrm{C}\right)\end{array} \\
\begin{array}{l}\text { Top } \\
\text { Bottom }\end{array} \\
\begin{array}{c}\text { Dissolved oxygen }- \\
\text { B. O. D. }\left(5-\text { day, } 20^{\circ} \mathrm{C} \text { ) - Top }\right.\end{array} \\
\text { Top }\end{array}$ & $\begin{array}{l}152 \\
147 \\
4 * 3 \\
2.5\end{array}$ & $\begin{array}{l}143 \\
142 \\
4.4 \\
1.2\end{array}$ & $\begin{array}{l}143 \\
139 \\
4 \cdot 4 \\
1.2\end{array}$ & $\begin{array}{r}145 \\
138 \\
4.5 \\
1.3\end{array}$ & $\begin{array}{l}138 \\
130 \\
5.3 \\
1.5\end{array}$ & $\begin{array}{lr}\text { T. } & 144 \\
\text { B. } & 139 \\
\text { T. } & 4.6 \\
\text { T. } & 1.6\end{array}$ \\
\hline 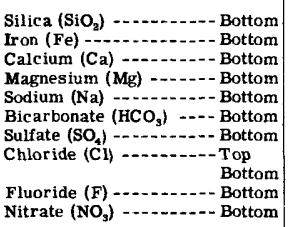 & $\begin{array}{l}7.2 \\
13.04 \\
4.7 \\
5.9 \\
23 \\
34 \\
6.0 \\
5.5 \\
.1 \\
7.2\end{array}$ & $\begin{array}{l}0.0 \\
6.0\end{array}$ & $\begin{array}{l}6.9 \\
.04 \\
13 \\
4.5 \\
4.9 \\
23 \\
32 \\
6.0 \\
5.0 \\
.1 \\
6.6\end{array}$ & $\begin{array}{l}6.0 \\
5.0\end{array}$ & $\begin{array}{l}6.9 \\
.04 \\
32 \\
4.4 \\
4.6 \\
22 \\
30 \\
5.0 \\
4.5 \\
.1 \\
6.5\end{array}$ & $\begin{array}{lc}\text { B. } & 7.0 \\
\text { B. } & .04 \\
\text { B. } & 13 \\
\text { B. } & 4.5 \\
\text { B. } & 5.1 \\
\text { B. } & 23 \\
\text { B. } & 32 \\
\text { T. } & 5.8 \\
\text { B. } & 5.2 \\
\text { B. } & .1 \\
\text { B. } & 6.8\end{array}$ \\
\hline $\begin{array}{l}\text { Dissolved solids }-\ldots-\text { Bottom } \\
\text { Hardness as } \mathrm{CaCO}_{3}-\ldots \text { Bottom } \\
\text { Suspended sediment } \ldots \text { Top }\end{array}$ & $\begin{array}{l}95 \\
52 \\
\cdot \cdots\end{array}$ & & $\begin{array}{l}90 \\
51 \\
.\end{array}$ & site. . . & $\begin{array}{l}88 \\
48 \\
\therefore\end{array}$ & $\begin{array}{ll}\text { B. } & 91 \\
\text { B. } & 50 \\
\text { T. } & 19\end{array}$ \\
\hline
\end{tabular}


Table 14. -- WATER ANALYSES OF DELAWARE RIVER BETWEEN BRISTOL AND MARCUS HOOK, PA. --Continued Analyzed by City of Philadelphia and U. S. Geological Survey; analyses in parts per million

\begin{tabular}{|c|c|c|c|c|c|c|}
\hline \multirow[t]{3}{*}{$\begin{array}{l}\text { Location Marcus Hook, Pa. } \\
\text { Weather }\end{array}$} & \multicolumn{6}{|c|}{$\frac{J_{w 2} 8,1952}{\text { Water }}$} \\
\hline & \multicolumn{6}{|c|}{ Station } \\
\hline & $\begin{array}{c}\text { Pennsylvania } \\
\text { side }\end{array}$ & West Center & Center & East Center & $\begin{array}{c}\text { New Jersey } \\
\text { side }\end{array}$ & Average \\
\hline $\begin{array}{l}\text { Time (EST) } \\
\text { Sounding (ft) } \\
\text { Temperature }\left({ }^{\circ} \mathrm{F}\right)-\mathrm{T} \\
\text { Color } \\
\text { pH }\end{array}$ & $\begin{array}{l}11: 10 \mathrm{a} . \mathrm{m} . \\
14 \\
80 \\
80 \\
10 \\
6.3 \\
7.2\end{array}$ & $\begin{array}{c}11: 13 \text { a.m. } \\
47 \\
80 \\
80 \\
\\
6.7 \\
6.7\end{array}$ & $\begin{array}{c}11: 16 \text { a.m. } \\
\text { b50 } \\
80 \\
79 \\
7 \\
6.6 \\
7.1\end{array}$ & $\begin{array}{c}11: 19 \text { a.m. } \\
\text { b50 } \\
80 \\
80 \\
6.6 \\
6.6\end{array}$ & $\begin{array}{c}11: 25 \text { a.m. } \\
38 \\
80 \\
79 \\
9 \\
6.6 \\
6.7\end{array}$ & $\begin{array}{ll}\text { T. } & 80 \\
\text { B. } & 80 \\
\text { B. } & 9 \\
\text { T. } & 6.6 \\
\text { B. } & 6.9\end{array}$ \\
\hline $\begin{array}{l}\text { Specific conductance } \\
\quad\left(\text { micromhos at } 25^{\circ} \mathrm{C} \text { ) } \begin{array}{l}\text { Top } \\
\text { Bottom }\end{array}\right. \\
\begin{array}{c}\text { Dissolved oxygen }- \\
\left.\text { B. O. D. (5-day, } 20^{\circ} \mathrm{C}\right)\end{array}-\text { - Top } \\
\text { Top }\end{array}$ & $\begin{array}{r}508 \\
348 \\
2.2\end{array}$ & $\begin{array}{r}280 \\
277 \\
1.0 \\
1.0\end{array}$ & $\begin{array}{l}268 \\
262 \\
1.0 \\
3.4\end{array}$ & $\begin{array}{r}277 \\
283 \\
1.9\end{array}$ & $\begin{array}{r}333 \\
323 \\
1.1 \\
.0\end{array}$ & $\begin{array}{lr}\text { T. } & 333 \\
\text { B. } & 299 \\
\text { T. } & 1.0 \\
\text { T. } & 1.5\end{array}$ \\
\hline 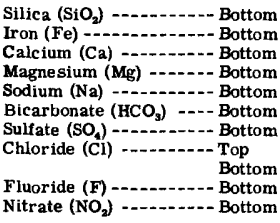 & $\begin{array}{l}5.5 \\
.01 \\
25 \\
8.4 \\
25 \\
32 \\
72 \\
92 \\
34.2 \\
10^{.2}\end{array}$ & $\begin{array}{l}22 \\
20\end{array}$ & $\begin{array}{l}6.0 \\
.03 \\
20 \\
7.3 \\
17 \\
36 \\
55 \\
20 \\
18 \\
12^{.2}\end{array}$ & $\begin{array}{l}23 \\
24\end{array}$ & $\begin{array}{l}7.3 \\
.02 \\
20 \\
8.2 \\
25 \\
29 \\
59 \\
39 \\
36 \\
.2 \\
9.3\end{array}$ & \begin{tabular}{ll} 
B. & 6.3 \\
B. & .02 \\
B. & 22 \\
B. & 8.0 \\
B. & 22 \\
B. & 32 \\
B. & 62 \\
T. & 39 \\
B. & 26 \\
B. & \multicolumn{2}{c}{.2} \\
B. & $10^{\circ}$
\end{tabular} \\
\hline $\begin{array}{l}\text { Dissolved solids - }-1-\text { Bottom } \\
\text { Hardness as } \mathrm{CaCO}_{3}-\cdots \text { Bottom } \\
\text { Suspended sediment }-\cdots-\text { Top }\end{array}$ & $\begin{array}{r}204 \\
97 \\
\cdot . \\
\end{array}$ & & $\begin{array}{r}157 \\
80 \\
. \quad \text { Con }\end{array}$ & Ite . . . & $\begin{array}{r}187 \\
84 \\
. \quad . . .\end{array}$ & $\begin{array}{lr}\text { B. } & 183 \\
\text { B. } & 87 \\
\text { T. } & 53\end{array}$ \\
\hline
\end{tabular}

b Exceeded this value.

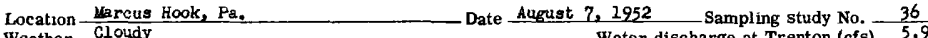

\begin{tabular}{|c|c|c|c|c|c|c|}
\hline $\begin{array}{l}\text { Location Harcus hook, } \mathrm{Pa}_{\text {. }} \text {. } \\
\text { Weather }\end{array}$ & & & Wat & discharge & $\begin{array}{l}\text { g study No. } \\
\text { renton (cfs) }\end{array}$ & 5,920 \\
\hline & & & Station & & & \\
\hline & \begin{tabular}{|c|}
$\begin{array}{c}\text { Pennsylvania } \\
\text { Side }\end{array}$ \\
\end{tabular} & West Center & Center & East Center & \begin{tabular}{|c|}
$\begin{array}{c}\text { New Jersey } \\
\text { side }\end{array}$ \\
\end{tabular} & Average \\
\hline 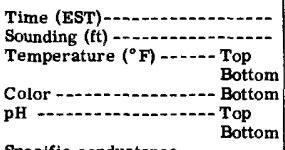 & $\begin{array}{l}9: 47 \mathrm{a} \cdot \mathrm{m} . \\
13 \\
81 \\
81 \\
8 \\
6.7 \\
7.1\end{array}$ & $\begin{array}{c}9: 49 \text { a.m. } \\
47 \\
82 \\
81 \\
6.8 \\
6.7\end{array}$ & $\begin{array}{l}9: 51 \text { a.m. } \\
46 \\
82 \\
81 \\
13 \\
6.7 \\
7.4\end{array}$ & $\begin{array}{l}9: 53 \propto .10 . \\
45 \\
82 \\
81 \\
\\
6.7 \\
6.7\end{array}$ & $\begin{array}{l}9: 55 \mathrm{a} . \mathrm{m} . \\
36 \\
81 \\
81 \\
9 \\
6.7 \\
7.3\end{array}$ & $\begin{array}{ll}\text { T. } & 82 \\
\text { B. } & 81 \\
\text { B. } & 10 \\
\text { T. } & 6.7 \\
\text { B. } & 7.0\end{array}$ \\
\hline $\begin{array}{l}\begin{array}{l}\text { Specific conductance } \\
\quad\left(\text { micromhos at } 25^{\circ} \mathrm{C}\right)\end{array} \\
\begin{array}{l}\text { Top } \\
\text { Bottom }\end{array} \\
\text { Dissolved oxygen -..-- Top } \\
\left.\text { B. O. D. (5-day, } 20^{\circ} \mathrm{C}\right) \text {-- Top }\end{array}$ & $\begin{array}{r}241 \\
225 \\
2.9\end{array}$ & $\begin{array}{r}245 \\
239 \\
.9 \\
2.0\end{array}$ & $\begin{array}{l}237 \\
219 \\
1.1 \\
2.8\end{array}$ & $\begin{array}{r}233 \\
229 \\
1.0 \\
2.1\end{array}$ & $\begin{array}{r}231 \\
217 \\
.9 \\
.5\end{array}$ & $\begin{array}{lc}\text { T. } & 237 \\
\text { B. } & 226 \\
\text { T. } & 1.0 \\
\text { T. } & 1.9\end{array}$ \\
\hline 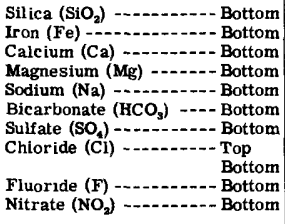 & $\begin{array}{l}6.2 \\
18^{.04} \\
6.3 \\
12 \\
33 \\
39 \\
15 \\
14.2 \\
14^{.2}\end{array}$ & $\begin{array}{l}15 \\
14\end{array}$ & $\begin{array}{l}6.2 \\
.03 \\
17^{.03} \\
6.1 \\
12 \\
32 \\
38 \\
14 \\
13 \\
14^{.3}\end{array}$ & $\begin{array}{l}14 \\
14\end{array}$ & $\begin{array}{l}5.5 \\
17^{.02} \\
6.0 \\
11 \\
31 \\
38 \\
13 \\
14 \\
16^{.2}\end{array}$ & \begin{tabular}{ll} 
B. & 6.0 \\
B. & .03 \\
B. & 17 \\
B. & 6.1 \\
B. & 12 \\
B. & 32 \\
B. & 38 \\
T. & 14 \\
B. & 14 \\
B. & \multicolumn{2}{c}{.2} \\
B. & $15^{\circ}$
\end{tabular} \\
\hline $\begin{array}{l}\text { Dissolved solids }-1-1-\text { Bottom } \\
\text { Hardness as } \mathrm{CaCO}_{3}-\cdots \text { Bottom } \\
\text { Suspended sediment }-\cdots \text { Top }\end{array}$ & $\begin{array}{r}137 \\
71 \\
\times \\
\end{array}$ & & $\begin{array}{r}135 \\
68 \\
.\end{array}$ & 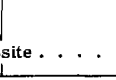 & $\begin{array}{r}131 \\
67 \\
? \\
\end{array}$ & $\begin{array}{rr}\text { B. } & 134 \\
\text { B. } & 69 \\
\text { T. } & 46 \\
\end{array}$ \\
\hline
\end{tabular}


Table 14. -- WATER ANALYSES OF DELAWARE RIVER BETWEEN BRISTOL AND MARCUS HOOK, PA. --Continued Analyzed by City of Philadelphia and U. S. Geological Survey; analyses in parts per million

Location -Marcus Hook, Pa.

Weather Clear

Date September 4 e 1952 Sampling study No. 37

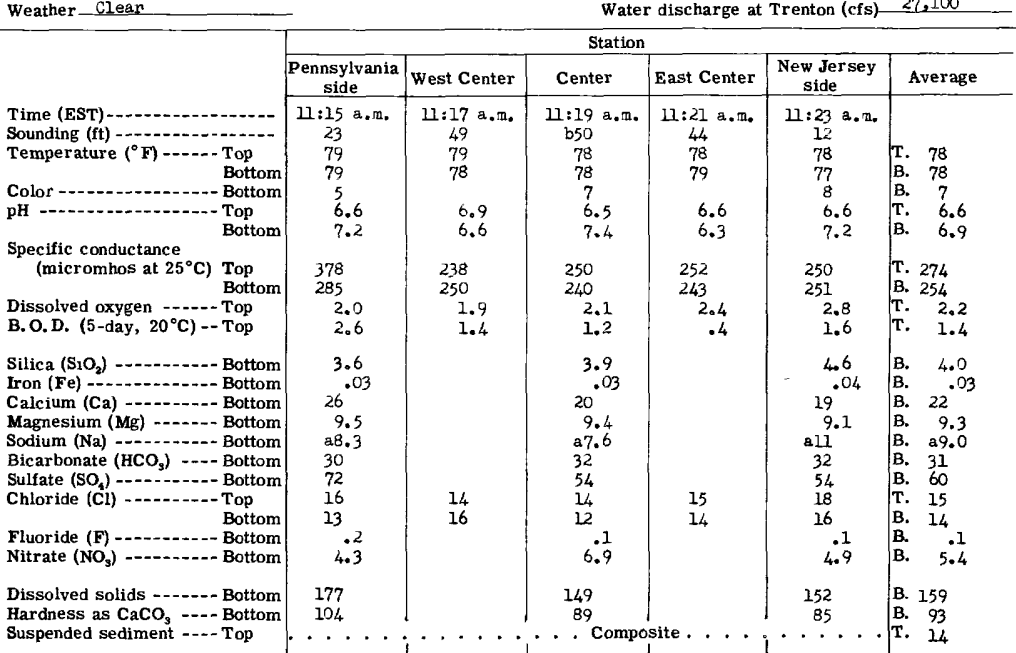

Calculated Sodium and Potassium.

b Exceeded this value.

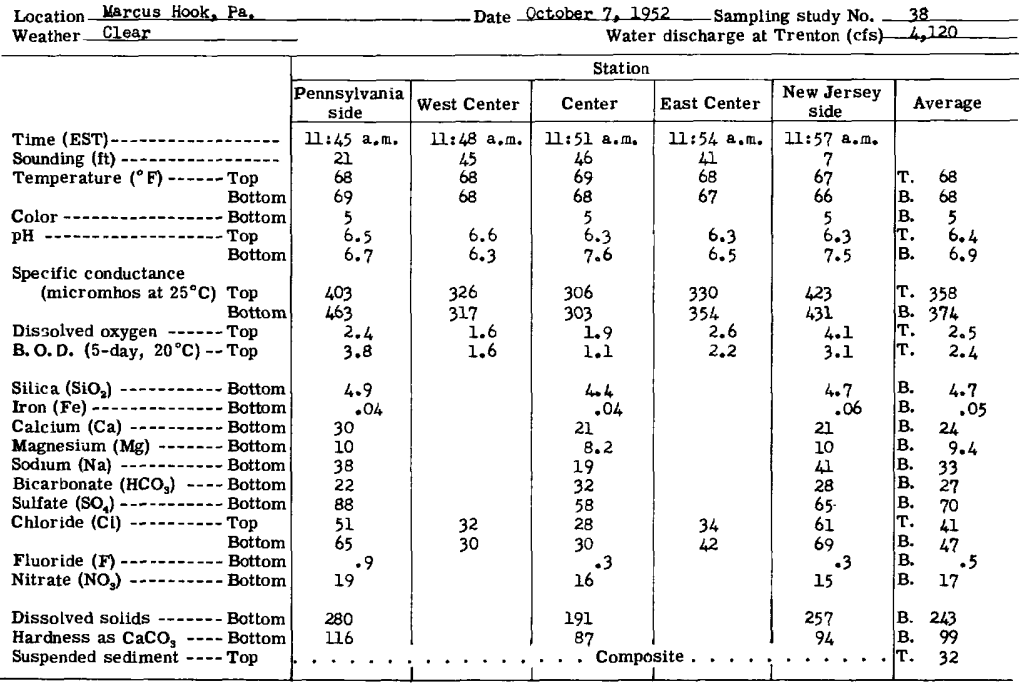


Table 14. -- WATER ANALYSES OF DELAWARE RIVER BETWEEN BRISTOL AND MARCUS HOOK, PA. --Continued Analyzed by City of Philadelphia and U. S. Geological Survey; analyses in parts per million

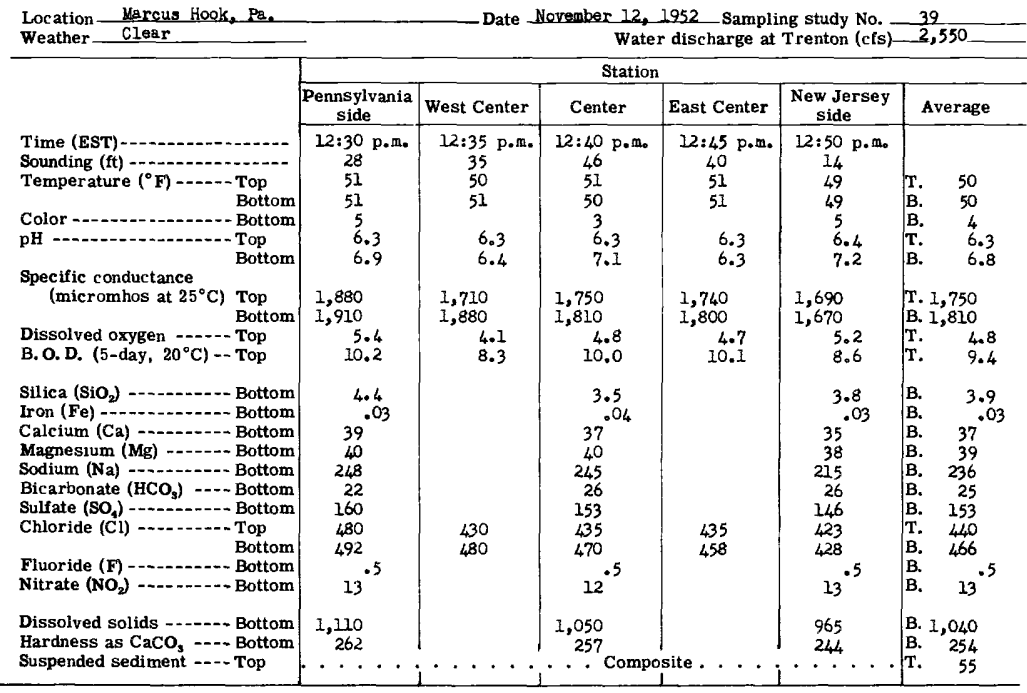

\begin{tabular}{|c|c|c|c|c|c|c|}
\hline \multicolumn{7}{|l|}{$\begin{array}{l}\text { Location Marcus Hook, } \mathrm{Ps}_{0} \\
\text { Weather Rain }\end{array}$} \\
\hline \multirow[b]{3}{*}{ 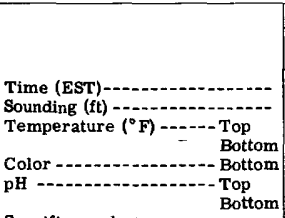 } & \multicolumn{6}{|c|}{ Station } \\
\hline & $\begin{array}{c}\text { Pennsylvania } \\
\text { side }\end{array}$ & West Center & Center & East Center & $\begin{array}{l}\text { New Jersey } \\
\text { side }\end{array}$ & Average \\
\hline & $\begin{array}{c}12: 40 \mathrm{p} . \mathrm{m} \\
15 \\
48 \\
48 \\
3 \\
5.7 \\
6.3\end{array}$ & $\begin{array}{c}12: 43 \mathrm{p} . \mathrm{m} . \\
43 \\
48 \\
48 \\
\\
5.7 \\
5.7\end{array}$ & $\begin{array}{c}12: 46 \mathrm{p}, \mathrm{m} . \\
\mathrm{b} 50 \\
48 \\
47 \\
10 \\
5.7 \\
6.6\end{array}$ & $\begin{array}{c}12: 49 \mathrm{p} \cdot \mathrm{m} . \\
35 \\
48 \\
48 \\
\\
5.7 \\
5.7\end{array}$ & $\begin{array}{c}12: 52 \text { p.mo } \\
26 \\
47 \\
47 \\
15 \\
5.7 \\
6.9\end{array}$ & $\begin{array}{lc}\text { T. } & 48 \\
\text { B. } & 48 \\
\text { B. } & 9 \\
\text { T. } & 5.7 \\
\text { B. } & 6.2\end{array}$ \\
\hline $\begin{array}{l}\begin{array}{l}\text { Specific conductance } \\
\text { (micromhos at } 25^{\circ} \mathrm{C} \text { ) }\end{array} \\
\begin{array}{l}\text { Top } \\
\text { Bottom }\end{array} \\
\text { Dissolved oxygen --- Top } \\
\text { B. O. D. (5-day, } 20^{\circ} \mathrm{C} \text { ) - Top }\end{array}$ & $\begin{array}{r}187 \\
184 \\
7.0 \\
4.7\end{array}$ & $\begin{array}{l}172 \\
175 \\
7.0 \\
4.1\end{array}$ & $\begin{array}{r}168 \\
167 \\
7.6 \\
3.1\end{array}$ & $\begin{array}{l}162 \\
165 \\
7.6 \\
4.3\end{array}$ & $\begin{array}{l}162 \\
162 \\
7.5 \\
3.0\end{array}$ & $\begin{array}{lr}\text { T. } & 170 \\
\text { B. } & 171 \\
\text { T. } & 7.3 \\
\text { T. } & 3.8\end{array}$ \\
\hline 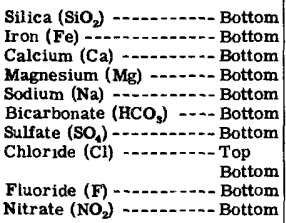 & $\begin{array}{l}5.4 \\
.06 \\
15 \\
5.0 \\
7.7 \\
13 \\
45 \\
10 \\
9.5 \\
.3 \\
6.1\end{array}$ & 10 & $\begin{array}{l}5.0 \\
13 \\
13 \\
6.06 \\
24.4 \\
14 \\
37 \\
10 \\
8.0 \\
6.1 \\
6.3\end{array}$ & 9.0 & $\begin{array}{l}6.3 \\
.08 \\
12 \\
6.0 \\
26.2 \\
12 \\
37 \\
10 \\
9.0 \\
.1 \\
8.3\end{array}$ & $\begin{array}{ll}\text { B. } & 5.6 \\
\text { B. } & .07 \\
\text { B. } & 13 \\
\text { B. } & 5.7 \\
\text { B. } & - \\
\text { B. } & 13 \\
\text { B. } & 40 \\
\text { T. } & 9.8 \\
\text { B. } & 8.8 \\
\text { B. } & .2 \\
\text { B. } & 6.9\end{array}$ \\
\hline $\begin{array}{l}\text { Dissolved solids }-\ldots-\text { Bottom } \\
\text { Hardness as } \mathrm{CaCO}_{3} \ldots-\text { Bottom } \\
\text { Suspended sediment } \ldots-\text { Top }\end{array}$ & $\begin{array}{r}124 \\
58 \\
.\end{array}$ & ( & $\begin{array}{l}111 \\
57 \\
. \quad \text { Cor }\end{array}$ & the. & $\begin{array}{r}111 \\
55 \\
. \quad .\end{array}$ & $\begin{array}{lr}\text { B. } & 115 \\
\text { B. } & 57 \\
\text { T. } & 50\end{array}$ \\
\hline
\end{tabular}

a Calculated Sodium and Potassium.

Exceeded this value. 


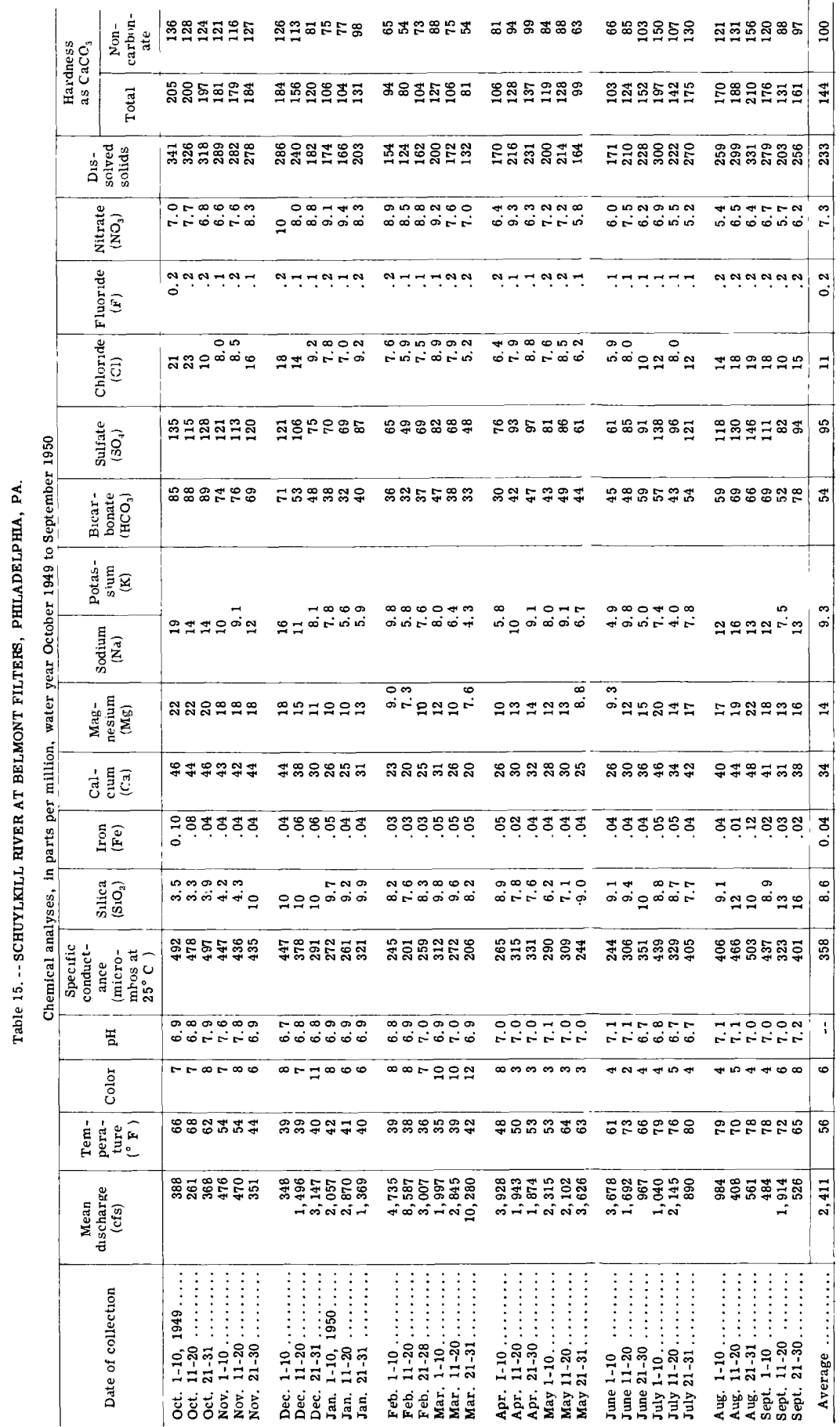




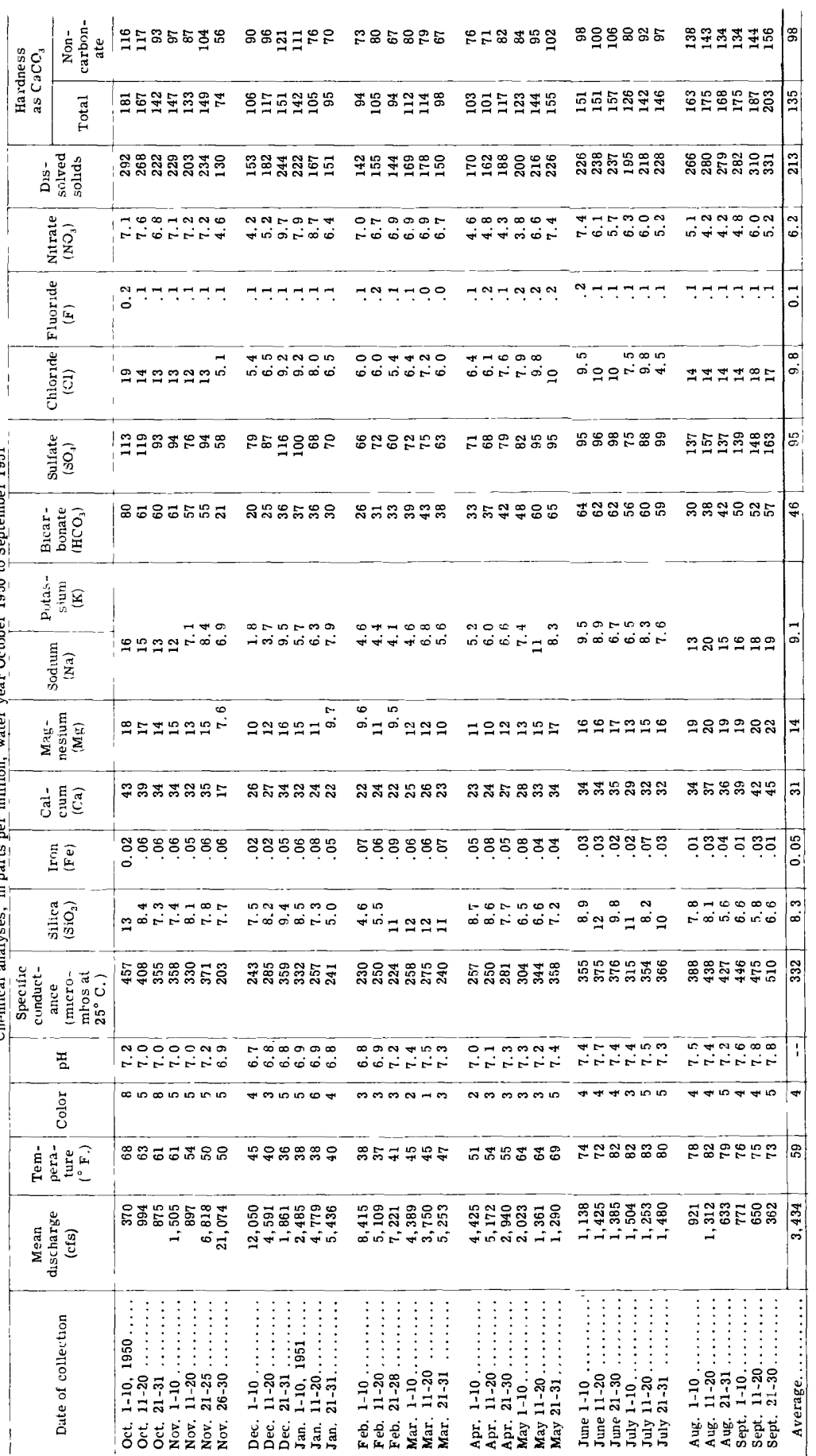




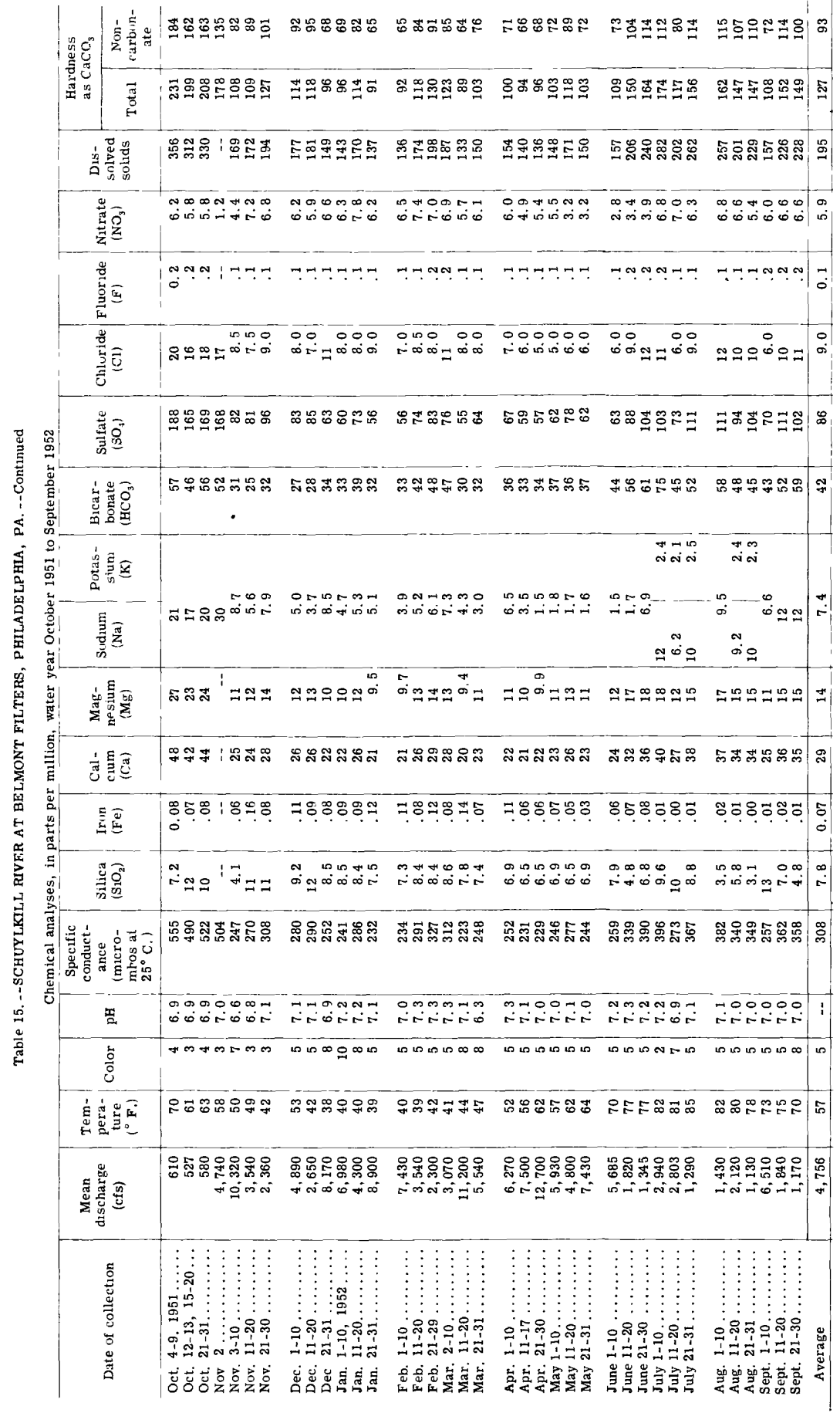


Table 16. --DELAW ARE RIVER AT TRENTON, N. J. (MORRISVILLE, PA.)

Suspended sediment, water year October 1949 to Septeniber 1950

\begin{tabular}{|c|c|c|c|c|c|c|c|c|c|}
\hline \multirow[b]{3}{*}{ Day } & \multicolumn{3}{|c|}{ October } & \multicolumn{3}{|c|}{ November } & \multicolumn{3}{|c|}{ December } \\
\hline & \multirow{2}{*}{$\begin{array}{l}\text { Mean } \\
\text { dis- } \\
\text { charge } \\
\text { (cfs) }\end{array}$} & \multicolumn{2}{|c|}{ Suspended sediment } & \multirow{2}{*}{$\begin{array}{c}\text { Mean } \\
\text { dis- } \\
\text { charge } \\
\text { (cfs) }\end{array}$} & \multicolumn{2}{|c|}{ Suspended sediment } & \multirow{2}{*}{$\begin{array}{l}\text { Mean } \\
\text { dis- } \\
\text { charge } \\
\text { (cfs) }\end{array}$} & \multicolumn{2}{|c|}{ Suspended sediment } \\
\hline & & $\begin{array}{c}\text { Mean } \\
\text { concen- } \\
\text { tration } \\
(\mathrm{ppm}) \\
\end{array}$ & $\begin{array}{l}\text { Tons } \\
\text { per } \\
\text { day }\end{array}$ & & $\begin{array}{c}\text { Mean } \\
\text { concen- } \\
\text { tration } \\
\text { (ppm) }\end{array}$ & $\begin{array}{l}\text { Tons } \\
\text { per } \\
\text { day }\end{array}$ & & $\begin{array}{l}\text { Mean } \\
\text { concen- } \\
\text { tration } \\
(\text { ppm) }\end{array}$ & $\begin{array}{l}\text { Tons } \\
\text { per } \\
\text { day }\end{array}$ \\
\hline $\begin{array}{l}1 \ldots \ldots \\
2 \cdots \\
3 \cdots \\
4 \cdots \\
5 \cdots\end{array}$ & $\begin{array}{l}3,590 \\
3,940 \\
3,680 \\
3,520 \\
3,280\end{array}$ & $\begin{array}{l}9 \\
8 \\
7 \\
5 \\
3\end{array}$ & $\begin{array}{l}87 \\
85 \\
70 \\
48 \\
27\end{array}$ & $\begin{array}{l}3,340 \\
3,430 \\
3,460 \\
3,750 \\
3,910\end{array}$ & $\left.\begin{array}{r}9 \\
8 \\
7 \\
10 \\
10\end{array}\right]$ & $\begin{array}{r}81 \\
74 \\
65 \\
101 \\
106\end{array}$ & $\begin{array}{l}4,970 \\
4,750 \\
4,860 \\
4,900 \\
4,360\end{array}$ & $\begin{array}{l}6 \\
4 \\
5 \\
6 \\
6\end{array}$ & $\begin{array}{l}80 \\
51 \\
66 \\
79 \\
71\end{array}$ \\
\hline 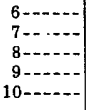 & $\begin{array}{l}2,960 \\
2,930 \\
3,020 \\
3,160 \\
3,040\end{array}$ & $\begin{array}{l}4 \\
6 \\
6 \\
6 \\
6\end{array}$ & $\begin{array}{l}32 \\
48 \\
49 \\
51 \\
49\end{array}$ & $\begin{array}{l}4,110 \\
4,220 \\
4,280 \\
3,940 \\
3,810\end{array}$ & $\begin{array}{r}14 \\
10 \\
10 \\
10 \\
9\end{array}$ & $\begin{array}{r}155 \\
114 \\
116 \\
106 \\
93\end{array}$ & $\begin{array}{l}4,040 \\
3,780 \\
4,040 \\
3,850 \\
3,370\end{array}$ & $\begin{array}{l}4 \\
6 \\
6 \\
4 \\
4\end{array}$ & $\begin{array}{l}44 \\
61 \\
65 \\
42 \\
36\end{array}$ \\
\hline $\begin{array}{l}11 \ldots \ldots \\
12 \ldots \ldots \\
13 \ldots \ldots \\
14 \ldots \ldots \\
15 \ldots-\ldots\end{array}$ & $\begin{array}{l}2,790 \\
2,630 \\
2,760 \\
3,160 \\
2,740\end{array}$ & $\begin{array}{l}5 \\
4 \\
4 \\
6 \\
4\end{array}$ & $\begin{array}{l}38 \\
28 \\
30 \\
51 \\
30\end{array}$ & $\begin{array}{l}3,590 \\
3,400 \\
3,310 \\
3,400 \\
4,710\end{array}$ & $\begin{array}{l}10 \\
10 \\
10 \\
14 \\
23\end{array}$ & $\begin{array}{r}97 \\
92 \\
89 \\
129 \\
292\end{array}$ & $\begin{array}{r}3,070 \\
3,490 \\
4,250 \\
23,900 \\
31,900\end{array}$ & $\begin{array}{r}4 \\
9 \\
14 \\
328 \\
121\end{array}$ & $\begin{array}{r}33 \\
85 \\
161 \\
\mathrm{~s} 25,200 \\
\mathrm{~s} 10,900\end{array}$ \\
\hline $\begin{array}{l}16 \ldots-\ldots \\
17-\ldots \\
18 \cdots \\
19 \\
20 \cdots\end{array}$ & $\begin{array}{l}2,530 \\
2,290 \\
2,220 \\
2,090 \\
2,390\end{array}$ & $\begin{array}{l}4 \\
5 \\
5 \\
4 \\
5\end{array}$ & $\begin{array}{l}27 \\
31 \\
30 \\
23 \\
32\end{array}$ & $\begin{array}{l}7,130 \\
8,120 \\
6,900 \\
6,290 \\
6,040\end{array}$ & $\begin{array}{r}75 \\
65 \\
27 \\
13 \\
8\end{array}$ & $\begin{array}{r}\mathrm{s} 1,520 \\
1,430 \\
503 \\
221 \\
130\end{array}$ & $\begin{array}{l}20,100 \\
14,300 \\
11,900 \\
10,600 \\
10,600\end{array}$ & $\begin{array}{r}48 \\
12 \\
4 \\
3 \\
2\end{array}$ & $\begin{array}{r}2,600 \\
463 \\
129 \\
86 \\
57\end{array}$ \\
\hline $\begin{array}{l}21 \ldots \ldots \\
22 \ldots \ldots \\
23 \ldots \ldots \\
24 \ldots \ldots \\
25 \ldots \ldots\end{array}$ & $\begin{array}{l}2,500 \\
2,320 \\
2,140 \\
2,020 \\
2,020\end{array}$ & $\begin{array}{l}6 \\
4 \\
4 \\
2 \\
2\end{array}$ & $\begin{array}{l}40 \\
25 \\
23 \\
11 \\
11\end{array}$ & $\begin{array}{l}\mathbf{5}, \mathbf{4 3 0} \\
\mathbf{5}, 120 \\
\mathbf{5}, 430 \\
\mathbf{5}, \mathbf{5 5 0} \\
\mathbf{5 , 0 9 0}\end{array}$ & $\begin{array}{r}4 \\
9 \\
10 \\
7 \\
6\end{array}$ & $\begin{array}{r}59 \\
124 \\
147 \\
105 \\
82\end{array}$ & $\begin{array}{r}11,000 \\
10,300 \\
9,710 \\
9,990 \\
13,600\end{array}$ & $\begin{array}{l}2 \\
2 \\
1 \\
1 \\
4\end{array}$ & $\begin{array}{r}59 \\
56 \\
26 \\
27 \\
147\end{array}$ \\
\hline $\begin{array}{l}26 \ldots \\
27 \\
28 \\
29 \\
30 \\
31 \\
\cdots\end{array}$ & $\begin{array}{l}2,190 \\
2,440 \\
2,960 \\
2,930 \\
2,990 \\
3,100 \\
\end{array}$ & $\begin{array}{l}2 \\
2 \\
4 \\
6 \\
5 \\
4 \\
\end{array}$ & $\begin{array}{l}12 \\
13 \\
32 \\
48 \\
40 \\
33\end{array}$ & $\begin{array}{r}4,530 \\
4,220 \\
4,460 \\
4,320 \\
4,530 \\
-- \\
\end{array}$ & $\begin{array}{r}5 \\
6 \\
4 \\
6 \\
6 \\
-- \\
\end{array}$ & $\begin{array}{l}61 \\
68 \\
48 \\
70 \\
73 \\
-- \\
\end{array}$ & $\begin{array}{l}12,600 \\
17,300 \\
20,900 \\
22,400 \\
18,900 \\
15,300 \\
\end{array}$ & $\begin{array}{r}1 \\
171 \\
117 \\
78 \\
28 \\
15 \\
\end{array}$ & $\begin{array}{r}34 \\
\text { s } 8,550 \\
6,600 \\
4,720 \\
1,430 \\
620 \\
\end{array}$ \\
\hline \multirow[t]{2}{*}{ Total- } & 86,320 & - & 1,150 & 139,820 & - & 6,350 & 339,030 & -- & 62,580 \\
\hline & \multicolumn{3}{|c|}{ January } & \multicolumn{3}{|c|}{ February } & \multicolumn{3}{|c|}{ March } \\
\hline 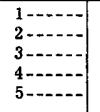 & $\begin{array}{l}12,700 \\
11,400 \\
11,100 \\
10,800 \\
11,100\end{array}$ & $\begin{array}{r}11 \\
9 \\
8 \\
13 \\
9\end{array}$ & $\begin{array}{l}377 \\
277 \\
240 \\
379 \\
270\end{array}$ & $\begin{array}{l}12,800 \\
13,200 \\
14,300 \\
12,700 \\
11,400\end{array}$ & $\begin{array}{r}8 \\
5 \\
11 \\
12 \\
6\end{array}$ & $\begin{array}{l}276 \\
178 \\
425 \\
411 \\
185\end{array}$ & $\begin{array}{l}8,570 \\
8,770 \\
8,220 \\
7,220 \\
7,400\end{array}$ & $\begin{array}{l}2 \\
3 \\
4 \\
3 \\
2\end{array}$ & $\begin{array}{l}46 \\
71 \\
89 \\
59 \\
40\end{array}$ \\
\hline 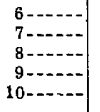 & $\begin{array}{l}11,300 \\
13,000 \\
23,200 \\
22,900 \\
18,200\end{array}$ & $\begin{array}{r}3 \\
9 \\
40 \\
32 \\
15\end{array}$ & $\begin{array}{r}92 \\
316 \\
\mathrm{~s} 2,550 \\
1,980 \\
737\end{array}$ & $\begin{array}{r}10,300 \\
9,900 \\
9,880 \\
10,600 \\
13,600\end{array}$ & $\begin{array}{r}3 \\
4 \\
6 \\
4 \\
18\end{array}$ & $\begin{array}{r}83 \\
108 \\
160 \\
114 \\
661\end{array}$ & $\begin{array}{r}7,080 \\
7,260 \\
7,690 \\
16,700 \\
34,700\end{array}$ & $\begin{array}{r}1 \\
4 \\
2 \\
59 \\
194\end{array}$ & $\begin{array}{r}19 \\
76 \\
42 \\
\text { s } 3,340 \\
18,900\end{array}$ \\
\hline $\begin{array}{l}11-\ldots \\
12 \ldots-\ldots \\
13 \ldots \\
14 \ldots-\ldots \\
15 \cdots\end{array}$ & $\begin{array}{l}21,300 \\
26,300 \\
21,800 \\
13,200 \\
17,400\end{array}$ & $\begin{array}{l}22 \\
30 \\
20 \\
10 \\
12\end{array}$ & $\begin{array}{r}1,270 \\
2,130 \\
1,180 \\
491 \\
564\end{array}$ & $\begin{array}{l}13,900 \\
15,300 \\
14,400 \\
17,200 \\
23,100\end{array}$ & $\begin{array}{r}16 \\
12 \\
8 \\
15 \\
48\end{array}$ & $\begin{array}{r}600 \\
496 \\
311 \\
697 \\
2,990\end{array}$ & $\begin{array}{l}35,700 \\
25,300 \\
23,200 \\
22,200 \\
18,700\end{array}$ & $\begin{array}{r}160 \\
40 \\
21 \\
22 \\
16\end{array}$ & $\begin{array}{r}15,400 \\
2,730 \\
1,320 \\
1,320 \\
808\end{array}$ \\
\hline $\begin{array}{l}16 \ldots \ldots \\
17 \ldots \ldots \\
18 \\
19 \\
20-\ldots\end{array}$ & $\begin{array}{l}16,700 \\
14,500 \\
13,600 \\
12,800 \\
11,600\end{array}$ & $\begin{array}{r}9 \\
13 \\
10 \\
9 \\
11\end{array}$ & $\begin{array}{l}406 \\
509 \\
367 \\
311 \\
345\end{array}$ & $\begin{array}{l}21,300 \\
19,300 \\
17,900 \\
16,000 \\
14,100\end{array}$ & $\begin{array}{l}41 \\
44 \\
22 \\
11 \\
10\end{array}$ & $\begin{array}{r}2,360 \\
2,290 \\
1,060 \\
475 \\
381\end{array}$ & $\begin{array}{l}16,200 \\
14,800 \\
14,000 \\
13,100 \\
12,000\end{array}$ & $\begin{array}{r}15 \\
12 \\
16 \\
10 \\
5\end{array}$ & $\begin{array}{l}656 \\
480 \\
605 \\
354 \\
162\end{array}$ \\
\hline $\begin{array}{l}21 \ldots \ldots \\
22 \ldots-\ldots \\
23 \ldots \ldots \\
24 \ldots \\
25 \cdots\end{array}$ & $\begin{array}{l}9,820 \\
8,820 \\
8,270 \\
8,420 \\
9,290\end{array}$ & $\begin{array}{l}8 \\
8 \\
8 \\
8 \\
6\end{array}$ & $\begin{array}{l}212 \\
191 \\
179 \\
182 \\
150\end{array}$ & $\begin{array}{l}11,700 \\
11,200 \\
10,400 \\
10,100 \\
10,900\end{array}$ & $\begin{array}{l}8 \\
7 \\
7 \\
7 \\
4\end{array}$ & $\begin{array}{l}253 \\
212 \\
197 \\
191 \\
118\end{array}$ & $\begin{array}{l}12,700 \\
19,200 \\
28,800 \\
31,200 \\
28,100\end{array}$ & $\begin{array}{r}6 \\
35 \\
209 \\
184 \\
57\end{array}$ & $\begin{array}{r}206 \\
1,810 \\
\mathrm{~s} 16,900 \\
15,500 \\
4,320\end{array}$ \\
\hline $\begin{array}{l}26-\ldots \\
27-\ldots \\
28 \\
29-\ldots \\
30 \cdots \\
31 \ldots \ldots\end{array}$ & $\begin{array}{l}10,200 \\
11,200 \\
11,400 \\
11,100 \\
10,400 \\
11,100 \\
\end{array}$ & $\begin{array}{l}5 \\
6 \\
5 \\
6 \\
8 \\
6 \\
\end{array}$ & $\begin{array}{l}138 \\
181 \\
154 \\
180 \\
225 \\
180\end{array}$ & $\begin{array}{r}10,600 \\
8,770 \\
7,930 \\
-- \\
-- \\
-- \\
\end{array}$ & $\begin{array}{r}2 \\
2 \\
2 \\
-- \\
-- \\
--\end{array}$ & $\begin{array}{l}57 \\
47 \\
43 \\
-- \\
-- \\
--\end{array}$ & $\begin{array}{l}\mathbf{2 4}, 000 \\
24,200 \\
\mathbf{2 5}, 700 \\
\mathbf{4 5}, \mathbf{2 0 0} \\
\mathbf{7 4}, 100 \\
\mathbf{5 5}, \mathbf{7 0 0}\end{array}$ & $\begin{array}{r}24 \\
19 \\
21 \\
184 \\
343 \\
99\end{array}$ & $\begin{array}{r}1,560 \\
1,240 \\
1,460 \\
\mathrm{~s} 27,600 \\
68,700 \\
\mathrm{~s} 14,900\end{array}$ \\
\hline Total- & 429,920 & $\ldots$ & 16,760 & 372,870 & -- & 15,380 & 677,710 & -- & 200,700 \\
\hline
\end{tabular}

s Computed by subdıviding day.

$3153910-54-12$ 
Table 16. -- DELAW ARE RIVER AT TRENTON, N. J. (MORRISVILLE, PA.)--Continued

Suspended sediment, water year October 1949 to September 1950--Contınued

\begin{tabular}{|c|c|c|c|c|c|c|c|c|c|}
\hline \multirow[b]{3}{*}{ Day } & \multicolumn{3}{|c|}{ April } & \multicolumn{3}{|c|}{ May } & \multicolumn{3}{|c|}{ June } \\
\hline & \multirow{2}{*}{$\begin{array}{l}\text { Mean } \\
\text { dis- } \\
\text { charge } \\
\text { (cfs) }\end{array}$} & \multicolumn{2}{|c|}{ Suspended sediment } & \multirow{2}{*}{$\begin{array}{l}\text { Mean } \\
\text { dis- } \\
\text { charge } \\
\text { (cfs) }\end{array}$} & \multicolumn{2}{|c|}{ Suspended sedument } & \multirow{2}{*}{$\begin{array}{l}\text { Mean } \\
\text { dis- } \\
\text { charge } \\
\text { (cfs) }\end{array}$} & \multicolumn{2}{|c|}{ Suspended sediment } \\
\hline & & $\begin{array}{l}\text { Mean } \\
\text { concen- } \\
\text { tration } \\
\text { (ppm) }\end{array}$ & $\begin{array}{c}\text { Tons } \\
\text { per } \\
\text { day }\end{array}$ & & $\begin{array}{l}\text { Mean } \\
\text { concen- } \\
\text { tration } \\
\text { (ppm) }\end{array}$ & $\begin{array}{l}\text { Tons } \\
\text { per } \\
\text { day }\end{array}$ & & $\begin{array}{l}\text { Mean } \\
\text { concen- } \\
\text { tration } \\
(\mathrm{ppm}) \\
\end{array}$ & $\begin{array}{l}\text { Tons } \\
\text { per } \\
\text { day }\end{array}$ \\
\hline $\begin{array}{l}1-\cdots \\
2-\cdots \\
3-\cdots \\
4-\cdots \\
5-\cdots-\end{array}$ & $\begin{array}{l}42,000 \\
35,700 \\
32,300 \\
36,600 \\
57,000\end{array}$ & $\begin{array}{l}43 \\
26 \\
18 \\
16 \\
71\end{array}$ & $\begin{array}{r}4,880 \\
2,510 \\
1,570 \\
1,580 \\
10,900\end{array}$ & $\begin{array}{l}12,300 \\
14,900 \\
17,000 \\
15,600 \\
14,100\end{array}$ & $\begin{array}{r}5 \\
5 \\
8 \\
10\end{array}$ & $\begin{array}{l}166 \\
201 \\
367 \\
421\end{array}$ & $\begin{array}{l}15,000 \\
17,700 \\
17,600 \\
16,500 \\
18,500\end{array}$ & $\begin{array}{l}12 \\
22 \\
20 \\
13 \\
22\end{array}$ & $\begin{array}{r}486 \\
1,050 \\
950 \\
579 \\
1,100\end{array}$ \\
\hline 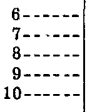 & $\begin{array}{l}76,600 \\
55,000 \\
36,700 \\
28,400 \\
23,700\end{array}$ & $\begin{array}{r}191 \\
87 \\
31 \\
20 \\
12\end{array}$ & $\begin{array}{r}39,500 \\
12,900 \\
3,070 \\
1,530 \\
768\end{array}$ & $\begin{array}{l}14,500 \\
15,900 \\
13,800 \\
12,500 \\
11,700\end{array}$ & 2 & 120 & $\begin{array}{l}18,900 \\
16,200 \\
14,000 \\
12,200 \\
11,000\end{array}$ & \begin{tabular}{r|}
24 \\
13 \\
12 \\
8 \\
10
\end{tabular} & $\begin{array}{r}1,220 \\
569 \\
454 \\
\mathbf{2 6 4} \\
297\end{array}$ \\
\hline 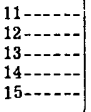 & $\begin{array}{l}20,900 \\
18,900 \\
17,700 \\
16,800 \\
15,600\end{array}$ & $\begin{array}{l}9 \\
7 \\
8 \\
7 \\
5\end{array}$ & $\begin{array}{l}508 \\
357 \\
382 \\
318 \\
211\end{array}$ & $\begin{array}{r}11,000 \\
11,600 \\
11,000 \\
9,820 \\
8,720\end{array}$ & 2 & 56 & $\begin{array}{r}10,200 \\
10,200 \\
10,200 \\
9,710 \\
10,200\end{array}$ & $\begin{array}{l}12 \\
14 \\
14 \\
12\end{array}$ & $\begin{array}{l}330 \\
386 \\
386 \\
315\end{array}$ \\
\hline $\begin{array}{l}16 \ldots \ldots \\
17 \ldots \ldots \\
18 \ldots \\
19 \ldots \ldots \\
20 \ldots\end{array}$ & $\begin{array}{l}14,400 \\
13,100 \\
12,300 \\
12,300 \\
12,500\end{array}$ & $\begin{array}{r}5 \\
3 \\
4 \\
10 \\
6\end{array}$ & $\begin{array}{l}194 \\
106 \\
133 \\
332 \\
202\end{array}$ & $\begin{array}{r}8,770 \\
9,560 \\
9,500 \\
11,900 \\
15,700\end{array}$ & 12 & $\begin{array}{l}284 \\
315\end{array}$ & $\begin{array}{r}11,700 \\
11,400 \\
10,200 \\
9,450 \\
10,200\end{array}$ & 12 & 353 \\
\hline $\begin{array}{l}21 \ldots \ldots \\
22 \ldots \ldots \\
23 \ldots \ldots \\
24 \ldots \ldots \\
25-\ldots \ldots\end{array}$ & $\begin{array}{l}14,300 \\
20,200 \\
18,100 \\
15,500 \\
15,000\end{array}$ & $\begin{array}{r}12 \\
18 \\
14 \\
8 \\
5\end{array}$ & $\begin{array}{l}463 \\
982 \\
684 \\
335 \\
202 \\
\end{array}$ & $\begin{array}{l}16,100 \\
14,900 \\
13,300 \\
14,600 \\
16,700\end{array}$ & 14 & 572 & $\begin{array}{l}9,990 \\
9,400 \\
9,290 \\
8,520 \\
7,780\end{array}$ & 8 & 180 \\
\hline 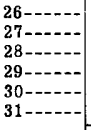 & $\begin{array}{r}15,000 \\
14,200 \\
14,400 \\
13,300 \\
12,300 \\
- \\
\end{array}$ & $\begin{array}{r}4 \\
4 \\
4 \\
6 \\
5 \\
-- \\
\end{array}$ & $\begin{array}{r}162 \\
153 \\
156 \\
215 \\
166 \\
-- \\
\end{array}$ & $\begin{array}{l}17,200 \\
15,700 \\
14,100 \\
14,000 \\
17,200 \\
16,600\end{array}$ & $\begin{array}{l}22 \\
17 \\
\end{array}$ & $\begin{array}{r}659 \\
\\
1,020 \\
762 \\
\end{array}$ & $\begin{array}{r}7,690 \\
9,290 \\
8,930 \\
7,640 \\
6,950 \\
-- \\
\end{array}$ & $\begin{array}{r}11 \\
7 \\
-- \\
\end{array}$ & $\begin{array}{l}256 \\
131 \\
--- \\
\end{array}$ \\
\hline \multirow[t]{2}{*}{ Total- } & 730,800 & -- & 85,470 & 420,270 & $\cdots$ & 10,830 & 346,540 & -- & 12,480 \\
\hline & \multicolumn{3}{|c|}{ July } & \multicolumn{3}{|c|}{ August } & \multicolumn{3}{|c|}{ September } \\
\hline 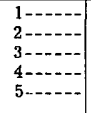 & $\begin{array}{l}6,820 \\
6,510 \\
5,590 \\
5,430 \\
5,630\end{array}$ & 7 & $\begin{array}{r}126 \\
77\end{array}$ & $\begin{array}{l}4,710 \\
5,090 \\
7,820 \\
7,220 \\
6,860\end{array}$ & $\begin{array}{r}2 \\
19\end{array}$ & $\begin{array}{r}26 \\
374\end{array}$ & $\begin{array}{l}4,460 \\
4,040 \\
4,140 \\
5,840 \\
5,800\end{array}$ & $\begin{array}{r}20 \\
6 \\
9 \\
27 \\
15\end{array}$ & $\begin{array}{r}241 \\
65 \\
101 \\
426 \\
235\end{array}$ \\
\hline 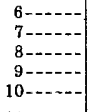 & $\begin{array}{l}6,130 \\
8,320 \\
7,690 \\
6,680 \\
6,770\end{array}$ & 10 & 199 & $\begin{array}{l}6,290 \\
5,630 \\
4,900 \\
4,780 \\
4,750\end{array}$ & $\begin{array}{r}6 \\
6 \\
9 \\
10\end{array}$ & $\begin{array}{r}97 \\
79 \\
116 \\
128\end{array}$ & $\begin{array}{l}4,860 \\
4,360 \\
4,010 \\
3,720 \\
3,700\end{array}$ & $\begin{array}{r}8 \\
8 \\
4 \\
4 \\
14\end{array}$ & $\begin{array}{r}105 \\
94 \\
43 \\
40 \\
\text { s } 215\end{array}$ \\
\hline $\begin{array}{l}11 \ldots \ldots \\
12 \ldots \ldots \\
13 \ldots \ldots \\
14 \ldots \ldots \\
15 \ldots \ldots\end{array}$ & $\begin{array}{r}6,770 \\
10,700 \\
11,000 \\
12,200 \\
12,500\end{array}$ & $\begin{array}{r}8 \\
38 \\
30\end{array}$ & $\begin{array}{r}146 \\
1,100 \\
958\end{array}$ & $\begin{array}{l}4,600 \\
4,680 \\
4,320 \\
3,880 \\
3,160\end{array}$ & $\begin{array}{r}10 \\
11 \\
9 \\
10 \\
6\end{array}$ & $\begin{array}{r}124 \\
139 \\
105 \\
105 \\
51\end{array}$ & $\begin{array}{l}7,180 \\
6,340 \\
6,290 \\
6,900 \\
6,770\end{array}$ & $\begin{array}{r}230 \\
55 \\
30 \\
32 \\
20\end{array}$ & $\begin{array}{r}4,460 \\
941 \\
509 \\
596 \\
\mathbf{3 6 6}\end{array}$ \\
\hline $\begin{array}{l}16-\ldots \\
17 \ldots \ldots- \\
18 \\
19 \\
20-\ldots\end{array}$ & $\begin{array}{r}11,600 \\
9,560 \\
8,220 \\
7,880 \\
7,360\end{array}$ & 13 & 290 & $\begin{array}{l}2,960 \\
3,430 \\
3,340 \\
3,340 \\
4,390\end{array}$ & $\begin{array}{r}6 \\
5 \\
7 \\
6 \\
28\end{array}$ & $\begin{array}{r}48 \\
46 \\
63 \\
54 \\
332\end{array}$ & $\begin{array}{l}6,170 \\
5,590 \\
4,930 \\
4,280 \\
4,180\end{array}$ & $\begin{array}{r}15 \\
8 \\
6 \\
5 \\
5\end{array}$ & $\begin{array}{r}250 \\
121 \\
80 \\
58 \\
56\end{array}$ \\
\hline 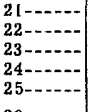 & $\begin{array}{l}7,000 \\
7,130 \\
7,500 \\
6,250 \\
5,710\end{array}$ & 8 & 151 & $\begin{array}{l}6,460 \\
6,640 \\
7,360 \\
5,960 \\
5,010\end{array}$ & $\begin{array}{r}50 \\
31 \\
30 \\
14 \\
8\end{array}$ & $\begin{array}{l}872 \\
556 \\
596 \\
225 \\
108\end{array}$ & $\begin{array}{l}4,390 \\
4,280 \\
4,040 \\
4,080 \\
3,850\end{array}$ & $\begin{array}{l}6 \\
6 \\
4 \\
3 \\
2\end{array}$ & $\begin{array}{l}71 \\
69 \\
44 \\
33 \\
21\end{array}$ \\
\hline $\begin{array}{l}26 \ldots \\
27-\ldots \\
28-\ldots \\
29-\ldots \\
30-\ldots \\
31-\ldots \ldots\end{array}$ & $\begin{array}{l}6,420 \\
7,310 \\
7,180 \\
6,290 \\
5,710 \\
4,930 \\
\end{array}$ & $\begin{array}{l}6 \\
4\end{array}$ & $\begin{array}{l}98 \\
75\end{array}$ & $\begin{array}{l}4,680 \\
4,140 \\
3,780 \\
3,280 \\
3,460 \\
5,430 \\
\end{array}$ & $\begin{array}{r}7 \\
6 \\
5 \\
5 \\
4 \\
91 \\
\end{array}$ & $\begin{array}{r}89 \\
67 \\
51 \\
44 \\
37 \\
1,330 \\
\end{array}$ & $\begin{array}{r}3,310 \\
3,220 \\
3,850 \\
3,650 \\
3,400 \\
-- \\
\end{array}$ & $\begin{array}{r}2 \\
2 \\
1 \\
1 \\
1 \\
-- \\
\end{array}$ & $\begin{array}{r}18 \\
17 \\
10 \\
9.9 \\
9.2 \\
-- \\
\end{array}$ \\
\hline Total-1 & 234,790 & -- & 8,680 & 152,350 & - & 6,730 & 141,630 & - & 9,300 \\
\hline
\end{tabular}

s Computed by subdividing day. 
Table 16, -- DELAW ARE RIVER AT TRENTON, N. J. (MORRISVILLE, F A.)--Continued

Suspended sediment, water year October 1950 to Sept $\epsilon$ mber 1951

\begin{tabular}{|c|c|c|c|c|c|c|c|c|c|}
\hline \multirow[b]{3}{*}{ Day } & \multicolumn{3}{|c|}{ October } & \multicolumn{3}{|c|}{ November } & \multicolumn{3}{|c|}{ December } \\
\hline & \multirow{2}{*}{$\begin{array}{c}\text { Mean } \\
\text { dis- } \\
\text { charge } \\
\text { (cfs) }\end{array}$} & \multicolumn{2}{|c|}{ Suspended sediment } & \multirow{2}{*}{$\begin{array}{c}\text { Mean } \\
\text { dis- } \\
\text { charge } \\
\text { (cfs) }\end{array}$} & \multicolumn{2}{|c|}{ Suspended sediment } & \multirow{2}{*}{$\begin{array}{c}\text { Mean } \\
\text { dis- } \\
\text { charge } \\
\text { (cfs) }\end{array}$} & \multicolumn{2}{|c|}{ Suspended sediment } \\
\hline & & $\begin{array}{l}\text { Mean } \\
\text { concen- } \\
\text { tration } \\
(\mathrm{ppm})\end{array}$ & $\begin{array}{l}\text { Tons } \\
\text { per } \\
\text { day }\end{array}$ & & $\begin{array}{l}\text { Mean } \\
\text { concen- } \\
\text { tration } \\
(\mathrm{ppm})\end{array}$ & $\begin{array}{l}\text { Tons } \\
\text { per } \\
\text { day }\end{array}$ & & $\begin{array}{c}\text { Mean } \\
\text { concen- } \\
\text { tration } \\
\text { (ppm) }\end{array}$ & $\begin{array}{l}\text { Tons } \\
\text { per } \\
\text { day }\end{array}$ \\
\hline 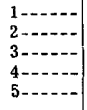 & $\begin{array}{l}3,070 \\
3,020 \\
2,840 \\
2,600 \\
2,820\end{array}$ & $\begin{array}{l}1 \\
5 \\
4 \\
2 \\
4\end{array}$ & $\begin{array}{r}8 \\
41 \\
31 \\
14 \\
31\end{array}$ & $\begin{array}{l}2,600 \\
2,550 \\
2,440 \\
2,550 \\
3,680\end{array}$ & \begin{tabular}{l|}
1 \\
2 \\
1 \\
1 \\
6
\end{tabular} & $\begin{array}{r}7 \\
14 \\
7 \\
7 \\
60\end{array}$ & $\begin{array}{l}21,700 \\
18,200 \\
15,800 \\
17,200 \\
83,000\end{array}$ & $\begin{array}{r}35 \\
25 \\
20 \\
100 \\
870\end{array}$ & $\begin{array}{r}2,050 \\
1,230 \\
853 \\
4,640 \\
195,000\end{array}$ \\
\hline 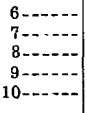 & $\begin{array}{l}3,070 \\
3,040 \\
2,740 \\
2,790 \\
2,930\end{array}$ & $\begin{array}{l}7 \\
5 \\
4 \\
6 \\
4\end{array}$ & $\begin{array}{l}58 \\
41 \\
30 \\
45 \\
32\end{array}$ & $\begin{array}{l}5,280 \\
7,180 \\
8,120 \\
6,770 \\
5,880\end{array}$ & $\begin{array}{l}24 \\
43 \\
55 \\
24 \\
13\end{array}$ & $\begin{array}{r}342 \\
833 \\
1,210 \\
439 \\
206\end{array}$ & $\begin{array}{l}94,100 \\
52,600 \\
51,800 \\
60,000 \\
50,000\end{array}$ & $\begin{array}{l}300 \\
115 \\
100 \\
175 \\
130\end{array}$ & $\begin{array}{l}76,200 \\
16,300 \\
14,000 \\
28,400 \\
17,600\end{array}$ \\
\hline $\begin{array}{l}11 \ldots \ldots \\
12 \ldots \ldots \\
13 \ldots \ldots \\
14 \ldots \ldots \\
15 \ldots \ldots\end{array}$ & $\begin{array}{l}3,160 \\
3,980 \\
3,750 \\
4,140 \\
4,010\end{array}$ & $\begin{array}{r}5 \\
9 \\
9 \\
10 \\
8\end{array}$ & $\begin{array}{r}43 \\
97 \\
91 \\
112 \\
87\end{array}$ & $\begin{array}{l}5,360 \\
4,970 \\
4,640 \\
4,360 \\
4,180\end{array}$ & $\begin{array}{l}9 \\
6 \\
4 \\
4 \\
4\end{array}$ & $\begin{array}{r}130 \\
80 \\
50 \\
47 \\
45\end{array}$ & $\begin{array}{l}37,500 \\
31,600 \\
26,600 \\
22,600 \\
19,700\end{array}$ & $\begin{array}{l}70 \\
33 \\
30 \\
17 \\
14\end{array}$ & $\begin{array}{r}7,090 \\
2,820 \\
2,150 \\
1,040 \\
745\end{array}$ \\
\hline $\begin{array}{l}16-\ldots \\
17-\ldots \\
18-\ldots \\
19-\ldots \\
20-\ldots \\
21-\ldots\end{array}$ & $\begin{array}{l}3,650 \\
3,430 \\
3,100 \\
3,190 \\
2,990 \\
2,840\end{array}$ & $\begin{array}{l}5 \\
4 \\
4 \\
3 \\
2 \\
1\end{array}$ & $\begin{array}{r}49 \\
37 \\
33 \\
26 \\
16 \\
8\end{array}$ & $\begin{array}{l}4,040 \\
3,940 \\
3,940 \\
3,810 \\
3,720 \\
3,810\end{array}$ & \begin{tabular}{l|}
3 \\
3 \\
2 \\
3 \\
4 \\
7
\end{tabular} & $\begin{array}{l}33 \\
32 \\
21 \\
31 \\
40 \\
72\end{array}$ & $\begin{array}{l}18,500 \\
16,700 \\
14,600 \\
12,700 \\
12,800 \\
11,800\end{array}$ & $\begin{array}{r}12 \\
10 \\
8 \\
8 \\
9 \\
11\end{array}$ & $\begin{array}{l}599 \\
451 \\
315 \\
274 \\
311 \\
350\end{array}$ \\
\hline $\begin{array}{l}22 \ldots \ldots \\
23-\cdots \\
24-\ldots- \\
25 \cdots\end{array}$ & $\begin{array}{l}2,520 \\
2,760 \\
2,960 \\
3,070\end{array}$ & $\begin{array}{l}1 \\
3 \\
2 \\
2\end{array}$ & $\begin{array}{r}7 \\
22 \\
16 \\
17\end{array}$ & $\begin{array}{r}4,530 \\
5,880 \\
7,310 \\
15,800\end{array}$ & $\begin{array}{r}8 \\
12 \\
18 \\
250\end{array}$ & $\begin{array}{r}98 \\
191 \\
355 \\
\text { s } 22,600\end{array}$ & $\begin{array}{r}10,800 \\
10,100 \\
10,300 \\
9,610\end{array}$ & $\begin{array}{l}8 \\
4 \\
4 \\
4\end{array}$ & $\begin{array}{l}233 \\
109 \\
111 \\
104\end{array}$ \\
\hline 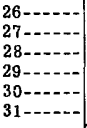 & $\begin{array}{l}3,520 \\
3,490 \\
3,220 \\
3,100 \\
2,990 \\
2,820\end{array}$ & $\begin{array}{l}4 \\
1 \\
1 \\
1 \\
2 \\
2 \\
\end{array}$ & $\begin{array}{r}38 \\
9 \\
9 \\
8 \\
16 \\
15\end{array}$ & $\begin{array}{r}65,600 \\
107,200 \\
61,100 \\
35,500 \\
26,400 \\
--\end{array}$ & $\begin{array}{r}1,720 \\
560 \\
275 \\
110 \\
40 \\
--\end{array}$ & $\begin{array}{r}s 274,000 \\
162,000 \\
45,400 \\
10,500 \\
2,850 \\
--\end{array}$ & $\begin{array}{r}9,130 \\
8,000 \\
8,000 \\
10,000 \\
13,000 \\
12,300\end{array}$ & $\begin{array}{r}4 \\
3 \\
4 \\
14 \\
12 \\
7\end{array}$ & $\begin{array}{r}99 \\
65 \\
86 \\
378 \\
421 \\
232 \\
\end{array}$ \\
\hline \multirow[t]{2}{*}{ Total - } & 97,610 & -- & 1,087 & 423,140 & --1 & 521,700 & 790,740 & -- & 374,256 \\
\hline & \multicolumn{3}{|c|}{ January } & \multicolumn{3}{|c|}{ February } & \multicolumn{3}{|c|}{ March } \\
\hline 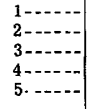 & $\begin{array}{r}9,560 \\
8,420 \\
7,980 \\
9,080 \\
12,600\end{array}$ & $\begin{array}{r}8 \\
9 \\
6 \\
8 \\
18\end{array}$ & $\begin{array}{c}207 \\
205 \\
129 \\
196 \\
\mathrm{~s} 645\end{array}$ & $\begin{array}{l}14,400 \\
22,300 \\
35,000 \\
26,700 \\
19,900\end{array}$ & \begin{tabular}{r|}
7 \\
54 \\
52 \\
24 \\
20
\end{tabular} & $\begin{array}{r}272 \\
3,250 \\
s 4,970 \\
1,730 \\
1,070\end{array}$ & $\begin{array}{l}20,100 \\
18,800 \\
17,500 \\
18,500 \\
18,600\end{array}$ & $\begin{array}{r}10 \\
3 \\
3 \\
10 \\
12\end{array}$ & $\begin{array}{l}543 \\
152 \\
142 \\
500 \\
603\end{array}$ \\
\hline $\begin{array}{r}6 \\
7 \ldots \\
8 \\
9 \\
10-\ldots \ldots\end{array}$ & $\begin{array}{l}24,300 \\
20,200 \\
16,800 \\
14,200 \\
12,900\end{array}$ & $\begin{array}{r}93 \\
50 \\
17 \\
15 \\
6\end{array}$ & $\begin{array}{r}6,100 \\
2,730 \\
771 \\
575 \\
209\end{array}$ & $\begin{array}{l}17,600 \\
23,600 \\
46,300 \\
40,000 \\
27,400\end{array}$ & $\begin{array}{r}12 \\
119 \\
272 \\
60 \\
20\end{array}$ & \begin{tabular}{|r}
570 \\
$\mathrm{~s} 11,200$ \\
34,000 \\
$6, \mathbf{4 8 0}$ \\
$1, \mathbf{4 8 0}$
\end{tabular} & $\begin{array}{l}16,700 \\
17,400 \\
17,500 \\
18,700 \\
19,700\end{array}$ & $\begin{array}{l}5 \\
4 \\
2 \\
2 \\
8\end{array}$ & $\begin{array}{r}225 \\
188 \\
94 \\
101 \\
426\end{array}$ \\
\hline $\begin{array}{l}11 \ldots \ldots \\
12 \ldots \\
13 \ldots \ldots \\
14 \ldots \ldots \\
15 \ldots\end{array}$ & $\begin{array}{l}11,800 \\
11,500 \\
11,800 \\
11,100 \\
20,200\end{array}$ & $\begin{array}{r}6 \\
5 \\
4 \\
4 \\
93\end{array}$ & $\begin{array}{r}191 \\
155 \\
127 \\
120 \\
\mathrm{~s} 5,610\end{array}$ & $\begin{array}{l}22,100 \\
18,700 \\
19,500 \\
24,800 \\
28,100\end{array}$ & $\begin{array}{l}18 \\
22 \\
22 \\
37 \\
46\end{array}$ & $\begin{array}{l}1,070 \\
1,110 \\
1,160 \\
2,480 \\
3,490\end{array}$ & $\begin{array}{l}17,200 \\
14,800 \\
14,100 \\
16,300 \\
20,400\end{array}$ & $\begin{array}{l}5 \\
3 \\
2 \\
3 \\
5\end{array}$ & $\begin{array}{r}232 \\
120 \\
76 \\
132 \\
275\end{array}$ \\
\hline $\begin{array}{l}16 \ldots \\
17 \ldots \\
18 \\
19 \\
20\end{array}$ & $\begin{array}{l}18,300 \\
17,900 \\
17,200 \\
15,100 \\
15,200\end{array}$ & $\begin{array}{r}87 \\
32 \\
12 \\
9 \\
7\end{array}$ & $\begin{array}{r}4,300 \\
1,550 \\
557 \\
367 \\
287\end{array}$ & $\begin{array}{l}24,700 \\
20,900 \\
24,100 \\
21,400 \\
22,100\end{array}$ & \begin{tabular}{r|}
14 \\
3 \\
28 \\
23 \\
16
\end{tabular} & $\begin{array}{r}934 \\
169 \\
1,820 \\
1,330 \\
955\end{array}$ & $\begin{array}{l}23,200 \\
21,300 \\
18,600 \\
16,400 \\
21,700\end{array}$ & $\begin{array}{r}6 \\
3 \\
1 \\
2 \\
51\end{array}$ & $\begin{array}{r}376 \\
173 \\
50 \\
89 \\
\mathrm{~s} 3,190\end{array}$ \\
\hline $\begin{array}{l}21 \ldots-\ldots \\
22 \ldots \ldots \\
23 \ldots \\
24 \ldots \\
25 \ldots\end{array}$ & $\begin{array}{l}16,400 \\
17,400 \\
16,500 \\
20,900 \\
42,400\end{array}$ & $\begin{array}{r}19 \\
18 \\
11 \\
62 \\
288\end{array}$ & $\begin{array}{r}841 \\
846 \\
490 \\
\text { s } 4,070 \\
33,000\end{array}$ & $\begin{array}{l}27,900 \\
41,100 \\
47,200 \\
35,200 \\
26,600\end{array}$ & $\begin{array}{r}48 \\
110 \\
138 \\
57 \\
14\end{array}$ & $\begin{array}{r}3,620 \\
12,200 \\
17,600 \\
5,420 \\
1,010\end{array}$ & $\begin{array}{l}29,300 \\
28,200 \\
24,400 \\
21,300 \\
20,500\end{array}$ & $\begin{array}{r}44 \\
13 \\
6 \\
10 \\
8\end{array}$ & $\begin{array}{r}3,480 \\
990 \\
395 \\
575 \\
443\end{array}$ \\
\hline $\begin{array}{l}26 \cdots \\
27-\ldots- \\
28-\cdots \\
29-\ldots- \\
30-\cdots \\
31-\cdots\end{array}$ & $\begin{array}{l}42,400 \\
30,200 \\
23,700 \\
20,600 \\
18,400 \\
16,200\end{array}$ & $\begin{array}{r}168 \\
60 \\
28 \\
17 \\
11 \\
6 \\
\end{array}$ & $\begin{array}{r}19,200 \\
4,890 \\
1,790 \\
946 \\
546 \\
262 \\
\end{array}$ & $\begin{array}{r}22,500 \\
21,400 \\
20,700 \\
-- \\
-- \\
-- \\
\end{array}$ & $\begin{array}{r}6 \\
8 \\
9 \\
-- \\
-- \\
--\end{array}$ & $\begin{array}{r}364 \\
462 \\
503 \\
-- \\
-- \\
-- \\
\end{array}$ & $\begin{array}{l}23,200 \\
21,600 \\
18,900 \\
18,200 \\
31,300 \\
89,200 \\
\end{array}$ & $\begin{array}{r}8 \\
11 \\
8 \\
4 \\
9 \\
308 \\
\end{array}$ & $\begin{array}{r}501 \\
642 \\
408 \\
197 \\
\mathrm{~s} 978 \\
\mathrm{~s} 80,100 \\
\end{array}$ \\
\hline Total- & 551,240 & -- & 91,912 & 742,200 & -- & 120,719 & 693,600 & -- & 96,396 \\
\hline
\end{tabular}

s Computed by subdividing day. 
Table 16. -- DELAWARE RIVER AT TRENTON, N. J. (MORRISVILLE, PA.)--Continued

Suspended sediment, water year 1950 to September 1951--Continued

\begin{tabular}{|c|c|c|c|c|c|c|c|c|c|}
\hline \multirow[b]{3}{*}{ Day } & \multicolumn{3}{|c|}{ April } & \multicolumn{3}{|c|}{ May } & \multicolumn{3}{|c|}{ June } \\
\hline & \multirow{2}{*}{$\begin{array}{c}\text { Mean } \\
\text { dis - } \\
\text { charge } \\
\text { (cfs) }\end{array}$} & \multicolumn{2}{|c|}{ Suspended sediment } & \multirow{2}{*}{$\begin{array}{c}\text { Mean } \\
\text { dis - } \\
\text { charge } \\
\text { (cfs) }\end{array}$} & \multicolumn{2}{|c|}{ Suspended sediment } & \multirow{2}{*}{$\begin{array}{c}\text { Mean } \\
\text { dis- } \\
\text { charge } \\
\text { (cfs) }\end{array}$} & \multicolumn{2}{|c|}{ Suspended sediment } \\
\hline & & $\begin{array}{c}\text { Mean } \\
\text { concen- } \\
\text { tration } \\
\text { (ppm) }\end{array}$ & $\begin{array}{l}\text { Tons } \\
\text { per } \\
\text { day }\end{array}$ & & $\begin{array}{c}\text { Mean } \\
\text { concen- } \\
\text { tration } \\
\text { (ppm) }\end{array}$ & $\begin{array}{l}\text { Tons } \\
\text { per } \\
\text { day }\end{array}$ & & $\begin{array}{c}\text { Mean } \\
\text { concen- } \\
\text { tration } \\
(\mathrm{ppm})\end{array}$ & $\begin{array}{l}\text { Tons } \\
\text { per } \\
\text { day }\end{array}$ \\
\hline 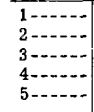 & $\begin{array}{r}120,700 \\
70,500 \\
51,600 \\
44,600 \\
37,800\end{array}$ & $\begin{array}{r}325 \\
110 \\
26 \\
13 \\
16\end{array}$ & $\begin{array}{r}106,000 \\
20,900 \\
3,620 \\
1,560 \\
1,630\end{array}$ & $\begin{array}{r}12,600 \\
12,000 \\
11,000 \\
10,300 \\
9,770\end{array}$ & $\begin{array}{l}7 \\
5 \\
8 \\
8 \\
6\end{array}$ & $\begin{array}{l}238 \\
162 \\
238 \\
222 \\
158\end{array}$ & $\begin{array}{r}10,800 \\
9,610 \\
8,620 \\
8,320 \\
9,030\end{array}$ & $\begin{array}{r}5 \\
2 \\
5 \\
4 \\
36\end{array}$ & $\begin{array}{r}146 \\
52 \\
116 \\
90 \\
878\end{array}$ \\
\hline $\begin{array}{r}6 \\
7 \ldots \\
8 \ldots \\
9 \\
10\end{array}$ & $\begin{array}{l}31,900 \\
27,300 \\
23,900 \\
20,900 \\
19,700\end{array}$ & $\begin{array}{r}12 \\
13 \\
9 \\
13 \\
16\end{array}$ & $\begin{array}{r}1,030 \\
958 \\
581 \\
734 \\
851\end{array}$ & $\begin{array}{l}9,240 \\
8,320 \\
7,780 \\
8,170 \\
7,780\end{array}$ & $\begin{array}{l}6 \\
5 \\
4 \\
6 \\
8\end{array}$ & $\begin{array}{r}150 \\
112 \\
84 \\
132 \\
168\end{array}$ & $\begin{array}{l}9,400 \\
8,420 \\
7,310 \\
6,640 \\
6,290\end{array}$ & $\begin{array}{r}28 \\
3 \\
1 \\
2 \\
3\end{array}$ & $\begin{array}{r}711 \\
68 \\
20 \\
36 \\
51\end{array}$ \\
\hline $\begin{array}{l}11 \ldots \ldots \\
12 \ldots \ldots \\
13 \ldots \ldots \\
14 \ldots \ldots\end{array}$ & $\begin{array}{l}19,800 \\
20,500 \\
27,800 \\
39,900 \\
34,800\end{array}$ & $\begin{array}{r}13 \\
16 \\
100 \\
100 \\
30\end{array}$ & $\begin{array}{r}695 \\
886 \\
7,510 \\
10,800 \\
2,820\end{array}$ & $\begin{array}{l}7,730 \\
8,270 \\
8,570 \\
7,640 \\
7,180\end{array}$ & $\begin{array}{r}7 \\
12 \\
6 \\
2 \\
2\end{array}$ & $\begin{array}{r}146 \\
268 \\
139 \\
41 \\
39\end{array}$ & $\begin{array}{l}6,040 \\
5,840 \\
5,960 \\
7,310 \\
9,240\end{array}$ & $\begin{array}{r}2 \\
3 \\
3 \\
10 \\
18\end{array}$ & $\begin{array}{r}33 \\
47 \\
48 \\
197 \\
449\end{array}$ \\
\hline $\begin{array}{l}16 \ldots \\
17 \ldots \ldots \\
18 \ldots \\
19 \\
20 \ldots \ldots\end{array}$ & $\begin{array}{l}28,200 \\
25,200 \\
22,500 \\
20,200 \\
18,500\end{array}$ & $\begin{array}{r}6 \\
11 \\
8 \\
5 \\
7\end{array}$ & $\begin{array}{l}457 \\
748 \\
486 \\
273 \\
350\end{array}$ & $\begin{array}{l}7,360 \\
7,040 \\
6,680 \\
6,510 \\
6,250\end{array}$ & $\begin{array}{l}2 \\
2 \\
4 \\
4 \\
7\end{array}$ & $\begin{array}{r}40 \\
38 \\
72 \\
70 \\
118\end{array}$ & $\begin{array}{r}10,200 \\
9,290 \\
7,730 \\
6,640 \\
6,460\end{array}$ & $\begin{array}{r}18 \\
5 \\
2 \\
2 \\
2\end{array}$ & $\begin{array}{r}496 \\
125 \\
42 \\
36 \\
35\end{array}$ \\
\hline $\begin{array}{l}21 \ldots \ldots \\
22 \ldots \ldots \\
23-\ldots \\
24 \ldots \\
25 \ldots\end{array}$ & $\begin{array}{l}16,700 \\
15,300 \\
14,800 \\
16,900 \\
17,900\end{array}$ & $\begin{array}{r}8 \\
6 \\
9 \\
12 \\
15\end{array}$ & $\begin{array}{l}361 \\
248 \\
360 \\
548 \\
725\end{array}$ & $\begin{array}{l}5,630 \\
5,400 \\
5,960 \\
7,830 \\
8,870\end{array}$ & $\begin{array}{r}7 \\
4 \\
4 \\
6 \\
11\end{array}$ & $\begin{array}{r}106 \\
58 \\
64 \\
127 \\
264\end{array}$ & $\begin{array}{r}6,170 \\
5,630 \\
9,500 \\
10,400 \\
8,320\end{array}$ & $\begin{array}{r}1 \\
1 \\
130 \\
130 \\
35\end{array}$ & $\begin{array}{r}17 \\
15 \\
\mathrm{~s} 3,650 \\
3,650 \\
786\end{array}$ \\
\hline $\begin{array}{l}26 \ldots \ldots \\
27 \ldots \ldots \\
28 \ldots \\
29 \\
30 \ldots \\
31 \ldots \ldots\end{array}$ & $\begin{array}{r}16,200 \\
15,000 \\
14,400 \\
13,400 \\
13,200 \\
\ldots\end{array}$ & $\begin{array}{r}10 \\
8 \\
9 \\
5 \\
4 \\
-\cdots \\
\end{array}$ & $\begin{array}{r}437 \\
324 \\
350 \\
181 \\
143 \\
-- \\
\end{array}$ & $\begin{array}{r}8,370 \\
7,220 \\
6,210 \\
7,260 \\
11,600 \\
12,500 \\
\end{array}$ & $\begin{array}{r}8 \\
5 \\
4 \\
14 \\
24 \\
19 \\
\end{array}$ & $\begin{array}{r}181 \\
97 \\
67 \\
274 \\
752 \\
641 \\
\end{array}$ & $\begin{array}{r}7,080 \\
7,360 \\
6,680 \\
7,080 \\
7,500 \\
-- \\
\end{array}$ & $\begin{array}{l}20 \\
22 \\
28 \\
33 \\
26 \\
-- \\
\end{array}$ & $\begin{array}{r}382 \\
437 \\
505 \\
631 \\
526 \\
-- \\
\end{array}$ \\
\hline \multirow[t]{2}{*}{ Total- } & 860,100 & -- & 166,566 & 257,040 & -- & 5,266 & 234,870 & -- & 14,275 \\
\hline & \multicolumn{3}{|c|}{ July } & \multicolumn{3}{|c|}{ August } & \multicolumn{3}{|c|}{ September } \\
\hline $\begin{array}{l}1 \ldots \\
2 \ldots \\
3 \ldots \\
4 \ldots \ldots \\
5 \ldots \ldots-\end{array}$ & $\begin{array}{l}8,820 \\
8,980 \\
9,400 \\
8,570 \\
8,930\end{array}$ & $\begin{array}{l}14 \\
33 \\
34 \\
34 \\
43\end{array}$ & $\begin{array}{r}333 \\
800 \\
863 \\
787 \\
1,040\end{array}$ & $\begin{array}{r}12,000 \\
9,990 \\
8,420 \\
7,180 \\
6,640\end{array}$ & $\begin{array}{l}29 \\
24 \\
30 \\
28 \\
28\end{array}$ & $\begin{array}{l}940 \\
647 \\
682 \\
543 \\
502 \\
\end{array}$ & $\begin{array}{l}3,940 \\
3,810 \\
4,080 \\
3,850 \\
3,680\end{array}$ & $\begin{array}{l}2 \\
7 \\
7 \\
6 \\
6\end{array}$ & $\begin{array}{l}21 \\
72 \\
77 \\
62 \\
60\end{array}$ \\
\hline $\begin{array}{r}6 \ldots \ldots \\
7 \ldots \ldots \\
9 \\
9\end{array}$ & $\begin{array}{l}8,980 \\
8,170 \\
7,080 \\
6,380 \\
5,750\end{array}$ & $\begin{array}{r}34 \\
20 \\
12 \\
24 \\
6\end{array}$ & $\begin{array}{r}824 \\
441 \\
230 \\
413 \\
93\end{array}$ & $\begin{array}{l}5,920 \\
5,360 \\
5,090 \\
5,240 \\
5,120\end{array}$ & $\begin{array}{r}27 \\
27 \\
8 \\
16 \\
6\end{array}$ & $\begin{array}{r}431 \\
391 \\
110 \\
226 \\
83\end{array}$ & $\begin{array}{l}3,810 \\
4,570 \\
5,010 \\
4,420 \\
3,940\end{array}$ & $\begin{array}{l}5 \\
3 \\
6 \\
4 \\
3\end{array}$ & $\begin{array}{l}52 \\
37 \\
81 \\
48 \\
32\end{array}$ \\
\hline $\begin{array}{l}11-\ldots \\
12 \ldots \ldots \\
13 \ldots \ldots \\
14-\ldots \\
15-\ldots\end{array}$ & $\begin{array}{l}5,320 \\
5,840 \\
6,130 \\
5,960 \\
6,000\end{array}$ & $\begin{array}{r}11 \\
4 \\
17 \\
22 \\
19\end{array}$ & $\begin{array}{r}158 \\
63 \\
281 \\
354 \\
308\end{array}$ & $\begin{array}{l}6,290 \\
6,860 \\
6,000 \\
6,730 \\
6,210\end{array}$ & $\begin{array}{r}6 \\
16 \\
41 \\
25 \\
30\end{array}$ & $\begin{array}{l}102 \\
296 \\
664 \\
454 \\
503\end{array}$ & $\begin{array}{l}3,780 \\
3,620 \\
4,010 \\
3,880 \\
4,880\end{array}$ & $\begin{array}{r}5 \\
7 \\
10 \\
9 \\
10\end{array}$ & $\begin{array}{r}51 \\
68 \\
108 \\
94 \\
132\end{array}$ \\
\hline $\begin{array}{l}16-\ldots-\ldots \\
17-\ldots \\
18-\ldots \ldots \\
19-\ldots \ldots \\
20-\ldots-\ldots\end{array}$ & $\begin{array}{l}5,630 \\
5,120 \\
5,160 \\
5,400 \\
6,730\end{array}$ & $\begin{array}{r}7 \\
1 \\
2 \\
5 \\
27\end{array}$ & $\begin{array}{r}106 \\
14 \\
28 \\
73 \\
491\end{array}$ & $\begin{array}{r}8,300 \\
10,800 \\
9,660 \\
10,100 \\
8,720\end{array}$ & $\begin{array}{r}57 \\
124 \\
61 \\
40 \\
22\end{array}$ & $\begin{array}{r}\text { s 1, 430 } \\
3,620 \\
1,590 \\
1,090 \\
518\end{array}$ & $\begin{array}{l}6,550 \\
5,400 \\
5,800 \\
5,550 \\
4,820\end{array}$ & $\begin{array}{l}15 \\
16 \\
15 \\
21 \\
18\end{array}$ & $\begin{array}{l}265 \\
233 \\
235 \\
315 \\
234\end{array}$ \\
\hline $\begin{array}{l}21 \ldots \ldots \\
22-\ldots \ldots \\
23-\ldots \ldots \\
24-\ldots \ldots \\
25-\ldots-\ldots\end{array}$ & $\begin{array}{l}6,640 \\
8,120 \\
8,320 \\
7,540 \\
6,680\end{array}$ & $\begin{array}{l}27 \\
54 \\
31 \\
22 \\
12\end{array}$ & $\begin{array}{r}484 \\
1,180 \\
696 \\
448 \\
217\end{array}$ & $\begin{array}{l}7,180 \\
6,680 \\
6,420 \\
5,750 \\
5,160\end{array}$ & $\begin{array}{r}10 \\
6 \\
2 \\
6 \\
7\end{array}$ & $\begin{array}{r}194 \\
108 \\
35 \\
93 \\
97\end{array}$ & $\begin{array}{l}4,390 \\
3,910 \\
3,460 \\
3,280 \\
3,160\end{array}$ & $\begin{array}{l}7 \\
3 \\
3 \\
3 \\
3\end{array}$ & $\begin{array}{l}83 \\
32 \\
28 \\
27 \\
26\end{array}$ \\
\hline 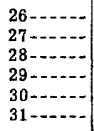 & $\begin{array}{r}6,210 \\
5,200 \\
9,020 \\
25,800 \\
15,000 \\
14,200\end{array}$ & $\begin{array}{r}14 \\
15 \\
33 \\
219 \\
127 \\
67\end{array}$ & $\begin{array}{r}235 \\
211 \\
\mathbf{s} 1,890 \\
15,300 \\
5,140 \\
2,570 \\
\end{array}$ & $\begin{array}{l}4,710 \\
4,460 \\
4,390 \\
4,040 \\
4,390 \\
4,320\end{array}$ & $\begin{array}{r}15 \\
4 \\
1 \\
2 \\
3 \\
4\end{array}$ & $\begin{array}{r}191 \\
48 \\
12 \\
22 \\
36 \\
47\end{array}$ & $\begin{array}{r}3,100 \\
3,310 \\
3,590 \\
3,940 \\
3,750 \\
--\end{array}$ & $\begin{array}{r}3 \\
7 \\
4 \\
3 \\
4 \\
--\end{array}$ & $\begin{array}{l}25 \\
63 \\
39 \\
32 \\
41 \\
--\end{array}$ \\
\hline Total- & 251,080 & - & 36,071 & 208,130 & -- & 15,705 & 125,290 & -- & 2,673 \\
\hline
\end{tabular}

s Computed by subdividing day. 
Table 16. --DELAW ARE RIVER AT TRENTON, N, J. (MORRISVILLE, PA.)--Continued

Suspended sediment, water year October 1951 to Seftember 1952

\begin{tabular}{|c|c|c|c|c|c|c|c|c|c|}
\hline \multirow[b]{3}{*}{ Day } & \multicolumn{3}{|c|}{ October } & \multicolumn{3}{|c|}{ November } & \multicolumn{3}{|c|}{ December } \\
\hline & \multirow{2}{*}{$\begin{array}{c}\text { Mean } \\
\text { dıs- } \\
\text { charge } \\
\text { (cfs) }\end{array}$} & \multicolumn{2}{|c|}{ Suspended sedıment } & \multirow{2}{*}{$\begin{array}{l}\text { Mean } \\
\text { dis- } \\
\text { charge } \\
\text { (cfs) }\end{array}$} & \multicolumn{2}{|c|}{ Suspended sedıment } & \multirow{2}{*}{$\begin{array}{c}\text { Mean } \\
\text { dis- } \\
\text { charge } \\
\text { (cfs) }\end{array}$} & \multicolumn{2}{|c|}{ Suspended sediment } \\
\hline & & $\begin{array}{l}\text { Mean } \\
\text { concen- } \\
\text { tration } \\
\text { (ppm) }\end{array}$ & $\begin{array}{l}\text { Tons } \\
\text { per } \\
\text { day }\end{array}$ & & $\begin{array}{l}\text { Mean } \\
\text { concen- } \\
\text { tration } \\
\text { (ppm) }\end{array}$ & $\begin{array}{l}\text { Tons } \\
\text { per } \\
\text { day }\end{array}$ & & $\begin{array}{l}\text { Mean } \\
\text { concen- } \\
\text { tration } \\
(\mathrm{ppm})\end{array}$ & $\begin{array}{l}\text { Tons } \\
\text { per } \\
\text { day }\end{array}$ \\
\hline $\begin{array}{l}1 \ldots \ldots \\
2 \ldots \ldots \\
3 \ldots \\
4 \cdots \\
5 \ldots \ldots-\end{array}$ & $\begin{array}{l}3,040 \\
2,990 \\
2,900 \\
3,370 \\
3,430\end{array}$ & $\begin{array}{l}2 \\
2 \\
8 \\
7 \\
5\end{array}$ & $\begin{array}{l}16 \\
16 \\
63 \\
64 \\
46\end{array}$ & $\begin{array}{r}8,820 \\
12,700 \\
21,600 \\
35,600 \\
38,300\end{array}$ & $\begin{array}{r}142 \\
79 \\
106 \\
160 \\
155\end{array}$ & $\begin{array}{r}3,380 \\
2,710 \\
6,180 \\
15,400 \\
16,000\end{array}$ & $\begin{array}{l}11,200 \\
11,100 \\
10,800 \\
10,800 \\
12,100\end{array}$ & $\begin{array}{r}8 \\
10 \\
9 \\
7 \\
10\end{array}$ & $\begin{array}{l}242 \\
300 \\
262 \\
204 \\
327\end{array}$ \\
\hline $\begin{array}{r}6 \cdots \\
7 \ldots \ldots \\
8 \cdots \ldots \\
9 \cdots \\
10 \cdots\end{array}$ & $\begin{array}{l}3,460 \\
3,620 \\
4,680 \\
5,320 \\
6,770\end{array}$ & $\begin{array}{r}5 \\
13 \\
11 \\
15 \\
15\end{array}$ & $\begin{array}{r}47 \\
127 \\
139 \\
215 \\
274\end{array}$ & $\begin{array}{l}27,100 \\
36,400 \\
62,300 \\
66,100 \\
46,300\end{array}$ & $\begin{array}{r}105 \\
483 \\
150 \\
115 \\
51\end{array}$ & $\begin{array}{r}7,680 \\
53,500 \\
25,200 \\
20,500 \\
6,370\end{array}$ & $\begin{array}{l}25,200 \\
32,200 \\
26,000 \\
21,800 \\
19,500\end{array}$ & $\begin{array}{l}35 \\
20 \\
10 \\
12 \\
11\end{array}$ & $\begin{array}{r}2,380 \\
1,740 \\
702 \\
707 \\
578\end{array}$ \\
\hline $\begin{array}{l}11 \ldots \ldots- \\
12-\ldots-n \\
13-\ldots-n \\
14-\ldots-n \\
15-\ldots-n\end{array}$ & $\begin{array}{l}7,080 \\
7,000 \\
7,260 \\
7,310 \\
7,830\end{array}$ & $\begin{array}{r}26 \\
10 \\
7 \\
8 \\
8\end{array}$ & $\begin{array}{l}497 \\
189 \\
137 \\
158 \\
169\end{array}$ & $\begin{array}{l}32,900 \\
25,800 \\
21,600 \\
19,100 \\
19,100\end{array}$ & $\begin{array}{r}50 \\
20 \\
10 \\
7 \\
10\end{array}$ & $\begin{array}{r}4,440 \\
1,390 \\
583 \\
362 \\
516\end{array}$ & $\begin{array}{l}20,800 \\
19,200 \\
17,000 \\
15,500 \\
14,700\end{array}$ & $\begin{array}{r}12 \\
6 \\
8 \\
8 \\
10\end{array}$ & $\begin{array}{l}675 \\
310 \\
367 \\
335 \\
397\end{array}$ \\
\hline 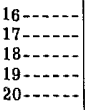 & $\begin{array}{l}6,550 \\
6,170 \\
5,590 \\
5,160 \\
5,160\end{array}$ & $\begin{array}{l}7 \\
7 \\
6 \\
4 \\
3\end{array}$ & $\begin{array}{r}124 \\
117 \\
90 \\
56 \\
42\end{array}$ & $\begin{array}{l}19,500 \\
21,400 \\
20,100 \\
18,000 \\
16,200\end{array}$ & $\begin{array}{r}29 \\
10 \\
10 \\
8 \\
9\end{array}$ & $\begin{array}{r}1,530 \\
578 \\
543 \\
389 \\
394\end{array}$ & $\begin{array}{l}13,800 \\
12,000 \\
16,000 \\
20,000 \\
28,000\end{array}$ & $\begin{array}{r}9 \\
8 \\
9 \\
10 \\
8\end{array}$ & $\begin{array}{l}335 \\
259 \\
389 \\
540 \\
605\end{array}$ \\
\hline $\begin{array}{l}21 \ldots \ldots \\
22-\ldots \\
23-\ldots- \\
24-\ldots- \\
25-\ldots-\end{array}$ & $\begin{array}{l}4,970 \\
4,530 \\
4,080 \\
4,180 \\
5,200\end{array}$ & $\begin{array}{r}3 \\
3 \\
3 \\
11 \\
12\end{array}$ & $\begin{array}{r}40 \\
37 \\
33 \\
124 \\
168\end{array}$ & $\begin{array}{l}14,800 \\
13,700 \\
12,400 \\
12,000 \\
13,100\end{array}$ & $\begin{array}{l}5 \\
6 \\
4 \\
8 \\
2\end{array}$ & $\begin{array}{r}200 \\
222 \\
134 \\
259 \\
71\end{array}$ & $\begin{array}{l}40,000 \\
32,100 \\
32,200 \\
27,600 \\
23,100\end{array}$ & $\begin{array}{r}9 \\
10 \\
10 \\
9 \\
10\end{array}$ & $\begin{array}{r}97 \\
867 \\
869 \\
670 \\
624\end{array}$ \\
\hline $\begin{array}{l}26 \ldots \ldots \\
27-\ldots \\
28-\ldots \\
29 \ldots \ldots \\
30 \ldots \\
31 \ldots \\
\end{array}$ & $\begin{array}{l}6,640 \\
7,400 \\
8,120 \\
7,690 \\
7,220 \\
6,640 \\
\end{array}$ & $\begin{array}{r}14 \\
14 \\
7 \\
6 \\
3 \\
3 \\
\end{array}$ & $\begin{array}{r}251 \\
280 \\
153 \\
124 \\
59 \\
54 \\
\end{array}$ & $\begin{array}{r}15,600 \\
15,800 \\
14,100 \\
12,800 \\
12,100 \\
--\end{array}$ & $\begin{array}{r}5 \\
10 \\
15 \\
11 \\
10 \\
--\end{array}$ & $\begin{array}{r}211 \\
427 \\
572 \\
381 \\
327 \\
--\end{array}$ & $\begin{array}{l}21,200 \\
18,200 \\
15,800 \\
14,400 \\
14,100 \\
14,400 \\
\end{array}$ & $\begin{array}{r}10 \\
9 \\
9 \\
10 \\
8 \\
11\end{array}$ & $\begin{array}{l}572 \\
443 \\
383 \\
389 \\
305 \\
427 \\
\end{array}$ \\
\hline \multirow[t]{2}{*}{ Total- } & 171,360 & $=$ & 3,909 & 705,320 & -- & 170,449 & 610,800 & -- & 17,300 \\
\hline & \multicolumn{3}{|c|}{ January } & \multicolumn{3}{|c|}{ February } & \multicolumn{3}{|c|}{ March } \\
\hline $\begin{array}{l}1 \ldots \ldots \\
2 \ldots \ldots \\
3-\ldots \\
4 \ldots-\ldots \\
5-\ldots\end{array}$ & $\begin{array}{l}15,200 \\
20,900 \\
29,400 \\
30,900 \\
25,900\end{array}$ & $\begin{array}{r}6 \\
16 \\
80 \\
160 \\
30\end{array}$ & $\begin{array}{r}246 \\
903 \\
6,350 \\
13,300 \\
2,100\end{array}$ & $\begin{array}{l}23,100 \\
21,300 \\
21,900 \\
31,400 \\
38,300\end{array}$ & $\begin{array}{r}7 \\
47 \\
63 \\
47 \\
67\end{array}$ & $\begin{array}{r}437 \\
2,700 \\
3,730 \\
3,980 \\
6,930\end{array}$ & $\begin{array}{l}9,290 \\
9,080 \\
8,170 \\
7,450 \\
9,450\end{array}$ & $\begin{array}{l}2 \\
2 \\
1 \\
2 \\
2\end{array}$ & $\begin{array}{l}50 \\
49 \\
22 \\
40 \\
51\end{array}$ \\
\hline $\begin{array}{r}6 \ldots \ldots \\
7 \ldots \ldots \\
8 \ldots \ldots \\
9 \\
10 \ldots \ldots\end{array}$ & $\begin{array}{l}22,900 \\
20,000 \\
17,400 \\
15,600 \\
14,900\end{array}$ & $\begin{array}{r}5 \\
14 \\
10 \\
12 \\
9\end{array}$ & $\begin{array}{r}3,090 \\
756 \\
470 \\
505 \\
362\end{array}$ & $\begin{array}{l}41,900 \\
35,500 \\
28,800 \\
24,600 \\
21,800\end{array}$ & $\begin{array}{r}40 \\
9 \\
13 \\
9 \\
11\end{array}$ & $\begin{array}{r}4,530 \\
863 \\
1,010 \\
598 \\
647\end{array}$ & $\begin{array}{r}10,400 \\
9,660 \\
8,980 \\
8,870 \\
8,220\end{array}$ & $\begin{array}{l}2 \\
2 \\
2 \\
1 \\
1\end{array}$ & $\begin{array}{l}56 \\
52 \\
49 \\
24 \\
22\end{array}$ \\
\hline $\begin{array}{l}11 \ldots \ldots \\
12 \ldots \ldots \\
13 \ldots-\ldots \\
14 \ldots \ldots \\
15 \ldots \ldots\end{array}$ & $\begin{array}{l}14,500 \\
13,900 \\
12,500 \\
11,700 \\
12,600\end{array}$ & $\begin{array}{r}8 \\
11 \\
9 \\
13 \\
18\end{array}$ & $\begin{array}{l}313 \\
413 \\
304 \\
411 \\
612\end{array}$ & $\begin{array}{l}19,600 \\
18,200 \\
17,000 \\
14,500 \\
13,100\end{array}$ & $\begin{array}{r}11 \\
5 \\
2 \\
3 \\
3\end{array}$ & $\begin{array}{r}582 \\
246 \\
92 \\
117 \\
106\end{array}$ & $\begin{array}{l}19,400 \\
59,900 \\
67,800 \\
44,700 \\
31,600\end{array}$ & $\begin{array}{r}128 \\
504 \\
230 \\
104 \\
50\end{array}$ & $\begin{array}{r}\text { s } 9,850 \\
81,500 \\
42,100 \\
12,600 \\
4,270\end{array}$ \\
\hline $\begin{array}{l}16 \ldots \ldots \\
17 \ldots \ldots \\
18 \ldots \ldots \\
19 \\
20 \ldots \ldots\end{array}$ & $\begin{array}{l}14,500 \\
17,300 \\
19,700 \\
20,500 \\
23,300\end{array}$ & $\begin{array}{l}14 \\
23 \\
41 \\
26 \\
22\end{array}$ & $\begin{array}{r}548 \\
1,070 \\
2,180 \\
1,440 \\
1,380\end{array}$ & $\begin{array}{l}12,100 \\
12,500 \\
12,600 \\
11,800 \\
12,000\end{array}$ & $\begin{array}{l}2 \\
2 \\
2 \\
2 \\
1\end{array}$ & $\begin{array}{l}65 \\
68 \\
68 \\
64 \\
32\end{array}$ & $\begin{array}{l}24,500 \\
20,500 \\
18,200 \\
18,900 \\
20,200\end{array}$ & $\begin{array}{r}22 \\
13 \\
8 \\
15 \\
19\end{array}$ & $\begin{array}{r}1,460 \\
718 \\
394 \\
767 \\
1,040\end{array}$ \\
\hline $\begin{array}{l}21 \ldots \ldots \\
22 \ldots \ldots \\
23 \ldots \ldots \\
24 \ldots \\
25 \ldots \ldots\end{array}$ & $\begin{array}{l}21,500 \\
20,500 \\
23,900 \\
24,200 \\
22,700\end{array}$ & $\begin{array}{r}16 \\
10 \\
23 \\
8 \\
2\end{array}$ & $\begin{array}{r}929 \\
554 \\
1,480 \\
523 \\
123\end{array}$ & $\begin{array}{r}11,400 \\
11,500 \\
11,000 \\
9,940 \\
9,500\end{array}$ & $\begin{array}{l}1 \\
2 \\
1 \\
2 \\
3\end{array}$ & $\begin{array}{l}31 \\
62 \\
30 \\
54 \\
77\end{array}$ & $\begin{array}{l}18,900 \\
19,800 \\
32,100 \\
36,500 \\
39,600\end{array}$ & $\begin{array}{r}8 \\
15 \\
46 \\
59 \\
58\end{array}$ & $\begin{array}{r}408 \\
802 \\
3,990 \\
5,820 \\
6,200\end{array}$ \\
\hline $\begin{array}{l}26 \ldots \ldots \\
27 \ldots-\ldots \\
28 \ldots \ldots \\
29 \ldots \ldots \\
30 \ldots- \\
31 \ldots\end{array}$ & $\begin{array}{l}24,300 \\
32,700 \\
58,400 \\
52,600 \\
36,200 \\
27,500\end{array}$ & $\begin{array}{r}3 \\
41 \\
51 \\
45 \\
10 \\
2\end{array}$ & $\begin{array}{r}197 \\
3,620 \\
8,040 \\
6,390 \\
977 \\
149\end{array}$ & $\begin{array}{r}9,080 \\
9,400 \\
9,190 \\
9,080 \\
=- \\
--\end{array}$ & $\begin{array}{r}2 \\
2 \\
1 \\
1 \\
-- \\
--\end{array}$ & $\begin{array}{l}49 \\
51 \\
25 \\
25 \\
-- \\
--\end{array}$ & $\begin{array}{l}34,500 \\
31,000 \\
30,500 \\
28,400 \\
24,400 \\
21,400\end{array}$ & $\begin{array}{r}27 \\
21 \\
22 \\
25 \\
12 \\
7\end{array}$ & $\begin{array}{r}2,520 \\
1,760 \\
1,810 \\
1,920 \\
791 \\
405\end{array}$ \\
\hline Total- & 718,100 & -- & 59,735 & 522,090 & -- & 27,269 & 732,370 & -- & 181,540 \\
\hline
\end{tabular}

s Computed by subdividing day. 
Table 16. -- DELAW ARE RIVER AT TRENTON, N. J. (MORRISVILLE, PA.)--Contınued

Suspended sediment, water year October 1951 to September 1952--Continued

\begin{tabular}{|c|c|c|c|c|c|c|c|c|c|}
\hline \multirow[b]{3}{*}{ Day } & \multicolumn{3}{|c|}{ April } & \multicolumn{3}{|c|}{ May } & \multicolumn{3}{|c|}{ June } \\
\hline & \multirow{2}{*}{$\begin{array}{c}\text { Mean } \\
\text { dis- } \\
\text { charge } \\
\text { (cfs) }\end{array}$} & \multicolumn{2}{|c|}{ Suspended sediment } & \multirow{2}{*}{$\begin{array}{c}\text { Mean } \\
\text { dis- } \\
\text { charge } \\
\text { (cfs) }\end{array}$} & \multicolumn{2}{|c|}{ Suspended sediment } & \multirow{2}{*}{$\begin{array}{l}\text { Mean } \\
\text { dis- } \\
\text { charge } \\
\text { (cfs) }\end{array}$} & \multicolumn{2}{|c|}{ Suspended sedimen } \\
\hline & & $\begin{array}{l}\text { Mean } \\
\text { concen- } \\
\text { tration } \\
\text { (ppm) }\end{array}$ & $\begin{array}{l}\text { Tons } \\
\text { per } \\
\text { day }\end{array}$ & & $\begin{array}{l}\text { Mean } \\
\text { concen - } \\
\text { tration } \\
\text { (ppm) }\end{array}$ & $\begin{array}{l}\text { Tons } \\
\text { per } \\
\text { day }\end{array}$ & & $\begin{array}{l}\text { Mean } \\
\text { concen- } \\
\text { tration } \\
(\mathrm{ppm})\end{array}$ & $\begin{array}{l}\text { Tons } \\
\text { per } \\
\text { day }\end{array}$ \\
\hline $\begin{array}{l}1 \ldots \\
2 \ldots \ldots \\
3 \ldots \ldots \\
4 \ldots \ldots \\
5\end{array}$ & $\begin{array}{l}19,900 \\
21,800 \\
33,900 \\
37,400 \\
39,500\end{array}$ & $\begin{array}{r}2 \\
6 \\
30 \\
78 \\
722 \\
\end{array}$ & $\begin{array}{r}107 \\
353 \\
2,750 \\
7,880 \\
\mathrm{~s} 75,600\end{array}$ & $\begin{array}{l}31,600 \\
26,000 \\
22,500 \\
19,800 \\
17,800\end{array}$ & $\begin{array}{r}22 \\
14 \\
5 \\
3 \\
20\end{array}$ & $\begin{array}{r}, 880 \\
983 \\
304 \\
160 \\
961\end{array}$ & $\begin{array}{l}\mathbf{2 5}, 500 \\
33,800 \\
34,300 \\
\mathbf{2 7}, 600 \\
\mathbf{2 3}, 800\end{array}$ & $\begin{array}{l}20 \\
63 \\
46 \\
26 \\
21\end{array}$ & $\begin{array}{l}1,380 \\
5,750 \\
4,260 \\
1,940 \\
1,350\end{array}$ \\
\hline $\begin{array}{r}6 \\
7-\ldots \\
8-\cdots \\
9-\ldots \\
10-\ldots-\end{array}$ & $\begin{array}{l}68,700 \\
73,600 \\
51,000 \\
38,200 \\
31,200\end{array}$ & $\begin{array}{r}295 \\
195 \\
90 \\
40 \\
24\end{array}$ & $\begin{array}{r}54,700 \\
38,700 \\
12,400 \\
4,130 \\
2,020\end{array}$ & $\begin{array}{l}15,900 \\
15,500 \\
14,200 \\
13,300 \\
12,700\end{array}$ & $\begin{array}{r}44 \\
7 \\
3 \\
2 \\
4\end{array}$ & $\begin{array}{r}1,890 \\
293 \\
115 \\
72 \\
137\end{array}$ & $\begin{array}{l}22,100 \\
20,100 \\
17,300 \\
15,000 \\
14,400\end{array}$ & $\begin{array}{r}23 \\
18 \\
27 \\
8 \\
12\end{array}$ & $\begin{array}{r}1,370 \\
977 \\
1,260 \\
324 \\
467\end{array}$ \\
\hline $\begin{array}{l}11 \ldots \ldots \\
12 \ldots \\
13 \ldots \\
14 \ldots \\
15 \ldots\end{array}$ & $\begin{array}{l}26,400 \\
22,800 \\
20,300 \\
24,900 \\
45,900\end{array}$ & $\begin{array}{r}17 \\
9 \\
8 \\
14 \\
134\end{array}$ & $\begin{array}{r}1,210 \\
554 \\
438 \\
941 \\
16,600\end{array}$ & $\begin{array}{l}12,200 \\
13,600 \\
20,600 \\
26,900 \\
24,400\end{array}$ & $\begin{array}{r}2 \\
4 \\
32 \\
33 \\
19\end{array}$ & $\begin{array}{r}66 \\
147 \\
1,780 \\
2,400 \\
1,250\end{array}$ & $\begin{array}{r}13,600 \\
12,300 \\
11,200 \\
10,200 \\
9,450\end{array}$ & $\begin{array}{r}12 \\
8 \\
7 \\
4 \\
6\end{array}$ & $\begin{array}{l}441 \\
266 \\
212 \\
110 \\
153\end{array}$ \\
\hline $\begin{array}{l}16 \ldots \ldots \\
17 \ldots \ldots \\
18 \ldots \ldots \\
19 \\
20 \ldots \ldots\end{array}$ & $\begin{array}{l}66,500 \\
55,700 \\
39,600 \\
31,400 \\
26,200\end{array}$ & $\begin{array}{r}183 \\
100 \\
41 \\
20 \\
13\end{array}$ & $\begin{array}{r}32,900 \\
15,000 \\
4,380 \\
1,700 \\
920\end{array}$ & $\begin{array}{l}21,400 \\
19,500 \\
17,900 \\
17,000 \\
16,500\end{array}$ & $\begin{array}{l}15 \\
11 \\
12 \\
12 \\
26\end{array}$ & $\begin{array}{r}\mathbf{8 6 7} \\
\mathbf{5 7 9} \\
\mathbf{5 8 0} \\
\mathbf{5 5 1} \\
1,160\end{array}$ & $\begin{array}{l}8,420 \\
7,780 \\
8,270 \\
7,930 \\
7,360\end{array}$ & $\begin{array}{l}4 \\
3 \\
4 \\
4 \\
2\end{array}$ & $\begin{array}{l}91 \\
63 \\
89 \\
86 \\
40\end{array}$ \\
\hline $\begin{array}{l}21 \ldots \ldots \\
22 \ldots \ldots \\
23 \ldots \\
24 \ldots \\
25-\ldots-\end{array}$ & $\begin{array}{l}22,800 \\
20,100 \\
18,400 \\
17,100 \\
16,200\end{array}$ & $\begin{array}{r}12 \\
11 \\
10 \\
7 \\
10\end{array}$ & $\begin{array}{l}739 \\
597 \\
497 \\
323 \\
437\end{array}$ & $\begin{array}{l}18,600 \\
18,900 \\
17,600 \\
15,800 \\
21,400\end{array}$ & $\begin{array}{l}12 \\
13 \\
13 \\
10 \\
46\end{array}$ & $\begin{array}{r}603 \\
663 \\
618 \\
427 \\
2,660\end{array}$ & $\begin{array}{l}6,860 \\
6,340 \\
6,340 \\
6,550 \\
7,000\end{array}$ & $\begin{array}{l}2 \\
3 \\
6 \\
8 \\
7\end{array}$ & $\begin{array}{r}37 \\
51 \\
103 \\
141 \\
132\end{array}$ \\
\hline $\begin{array}{l}26 \ldots \ldots \\
27 \ldots \ldots \\
28 \\
29 \\
30 \ldots \ldots \\
31 \ldots\end{array}$ & $\begin{array}{r}23,200 \\
31,000 \\
45,100 \\
44,100 \\
39,000 \\
--\end{array}$ & $\begin{array}{r}28 \\
50 \\
104 \\
79 \\
50 \\
--\end{array}$ & $\begin{array}{r}1,750 \\
4,180 \\
12,700 \\
9,410 \\
5,260 \\
-- \\
\end{array}$ & $\begin{array}{l}45,200 \\
51,000 \\
36,100 \\
28,000 \\
24,200 \\
21,500\end{array}$ & $\begin{array}{r}265 \\
118 \\
40 \\
28 \\
39 \\
19 \\
\end{array}$ & $\begin{array}{r}32,300 \\
16,200 \\
3,900 \\
2,120 \\
\mathbf{2}, 550 \\
1,100 \\
\end{array}$ & $\begin{array}{r}6,640 \\
6,170 \\
5,400 \\
4,900 \\
4,820 \\
-. \\
\end{array}$ & $\begin{array}{r}4 \\
3 \\
3 \\
8 \\
7 \\
-- \\
\end{array}$ & $\begin{array}{r}72 \\
50 \\
44 \\
106 \\
91 \\
--\end{array}$ \\
\hline \multirow[t]{2}{*}{ Total- } & $, 051,900$ & -- & 309,176 & 677,600 & $\cdots$ & 79,316 & 391,430 & -- & 21,456 \\
\hline & \multicolumn{3}{|c|}{ July } & \multicolumn{3}{|c|}{ August } & \multicolumn{3}{|c|}{ September } \\
\hline 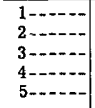 & $\begin{array}{l}4,380 \\
4,300 \\
4,270 \\
3,810 \\
3,720\end{array}$ & $\begin{array}{l}4 \\
4 \\
8 \\
2 \\
2\end{array}$ & $\begin{array}{l}47 \\
46 \\
92 \\
21 \\
20\end{array}$ & $\begin{array}{l}4,960 \\
4,880 \\
5,170 \\
4,880 \\
4,050\end{array}$ & $\begin{array}{l}1 \\
2 \\
2 \\
8 \\
1\end{array}$ & $\begin{array}{r}13 \\
26 \\
28 \\
105 \\
11\end{array}$ & $\begin{array}{r}7,160 \\
27,500 \\
32,000 \\
27,100 \\
20,200\end{array}$ & $\begin{array}{r}20 \\
390 \\
142 \\
61 \\
38\end{array}$ & $\begin{array}{r}387 \\
33,600 \\
14,200 \\
5,180 \\
2,070\end{array}$ \\
\hline $\begin{array}{r}6 \\
7 \ldots \ldots \\
8 \cdots \cdots \\
90 \cdots\end{array}$ & $\begin{array}{r}3,560 \\
3,370 \\
3,280 \\
5,120 \\
28,100\end{array}$ & $\begin{array}{r}3 \\
2 \\
2 \\
13 \\
214\end{array}$ & $\begin{array}{r}29 \\
18 \\
18 \\
\mathrm{~s} 208 \\
\mathrm{~s} 24,300\end{array}$ & $\begin{array}{l}4,020 \\
5,920 \\
6,230 \\
5,420 \\
5,420\end{array}$ & $\begin{array}{l}1 \\
3 \\
4 \\
2 \\
3\end{array}$ & $\begin{array}{l}11 \\
48 \\
67 \\
29 \\
44\end{array}$ & $\begin{array}{r}15,800 \\
13,000 \\
10,900 \\
9,410 \\
8,460\end{array}$ & $\begin{array}{r}58 \\
18 \\
10 \\
5 \\
4\end{array}$ & $\begin{array}{r}2,470 \\
632 \\
294 \\
127 \\
91\end{array}$ \\
\hline $\begin{array}{l}11 \ldots \ldots \\
12 \ldots \ldots \\
13 \ldots-\ldots \\
14 \cdots \ldots \\
15 \cdots \cdots\end{array}$ & $\begin{array}{l}81,300 \\
44,600 \\
25,800 \\
18,800 \\
14,700\end{array}$ & $\begin{array}{r}448 \\
174 \\
60 \\
22 \\
14\end{array}$ & $\begin{array}{r}98,300 \\
21,000 \\
4,180 \\
1,120 \\
\mathbf{5 5 6}\end{array}$ & $\begin{array}{l}7,760 \\
7,860 \\
7,570 \\
7,050 \\
6,140\end{array}$ & $\begin{array}{r}7 \\
20 \\
18 \\
12 \\
5\end{array}$ & $\begin{array}{r}147 \\
424 \\
368 \\
228 \\
83\end{array}$ & $\begin{array}{l}7,910 \\
7,240 \\
7,050 \\
6,870 \\
6,100\end{array}$ & $\begin{array}{l}6 \\
2 \\
2 \\
5 \\
1\end{array}$ & $\begin{array}{r}128 \\
39 \\
38 \\
93 \\
16\end{array}$ \\
\hline $\begin{array}{l}16 \ldots-\ldots \\
17 \ldots \ldots \\
18 \ldots \ldots \\
19 \\
20 \ldots \ldots\end{array}$ & $\begin{array}{r}12,400 \\
10,900 \\
9,730 \\
8,770 \\
8,010\end{array}$ & $\begin{array}{l}8 \\
6 \\
3 \\
2 \\
3\end{array}$ & $\begin{array}{r}268 \\
177 \\
79 \\
47 \\
65\end{array}$ & $\begin{array}{r}5,840 \\
8,700 \\
12,400 \\
9,030 \\
8,160\end{array}$ & $\begin{array}{r}\mathbf{2} \\
31 \\
71 \\
39 \\
12\end{array}$ & $\begin{array}{r}32 \\
728 \\
2,380 \\
951 \\
264\end{array}$ & $\begin{array}{r}5,970 \\
7,000 \\
6,500 \\
7,760 \\
10,100\end{array}$ & $\begin{array}{r}2 \\
3 \\
3 \\
22 \\
162\end{array}$ & $\begin{array}{r}32 \\
57 \\
53 \\
461 \\
4,420\end{array}$ \\
\hline $\begin{array}{l}21 \ldots \ldots \\
22 \ldots \ldots \\
23 \ldots \\
24 \ldots \\
25 \ldots\end{array}$ & $\begin{array}{l}8,160 \\
7,280 \\
8,980 \\
8,770 \\
7,570\end{array}$ & $\begin{array}{r}9 \\
29 \\
18 \\
13 \\
7\end{array}$ & $\begin{array}{l}198 \\
570 \\
436 \\
308 \\
143\end{array}$ & $\begin{array}{l}7,380 \\
8,930 \\
7,570 \\
6,960 \\
5,880\end{array}$ & $\begin{array}{r}7 \\
167 \\
29 \\
7 \\
1\end{array}$ & $\begin{array}{r}139 \\
4,030 \\
593 \\
132 \\
16\end{array}$ & $\begin{array}{l}8,560 \\
6,870 \\
6,230 \\
6,320 \\
6,010\end{array}$ & $\begin{array}{r}16 \\
8 \\
6 \\
5 \\
5\end{array}$ & $\begin{array}{r}370 \\
148 \\
101 \\
85 \\
81\end{array}$ \\
\hline $\begin{array}{l}26 \ldots \ldots \\
27 \ldots \ldots \\
28 \\
29 \\
30 \ldots \\
31 \ldots \ldots\end{array}$ & $\begin{array}{l}6,360 \\
5,540 \\
5,370 \\
4,920 \\
5,080 \\
5,420\end{array}$ & $\begin{array}{l}\mathbf{5} \\
\mathbf{2} \\
\mathbf{2} \\
\mathbf{2} \\
\mathbf{3} \\
\mathbf{3}\end{array}$ & $\begin{array}{l}86 \\
30 \\
29 \\
27 \\
41 \\
44\end{array}$ & $\begin{array}{l}4,960 \\
4,450 \\
4,380 \\
4,490 \\
4,570 \\
4,490\end{array}$ & $\begin{array}{l}1 \\
2 \\
2 \\
4 \\
4 \\
3\end{array}$ & $\begin{array}{l}13 \\
24 \\
24 \\
48 \\
49 \\
36\end{array}$ & $\begin{array}{r}5,130 \\
4,840 \\
4,680 \\
4,420 \\
4,020 \\
--\end{array}$ & $\begin{array}{r}7 \\
15 \\
6 \\
4 \\
5 \\
--\end{array}$ & $\begin{array}{r}97 \\
196 \\
76 \\
48 \\
54 \\
--\end{array}$ \\
\hline Total- & 372,370 & -- & 152,503 & 195,520 & -- & 11,091 & 301,110 & -- & 65,644 \\
\hline
\end{tabular}

s Computed by subdividing day. 
Table 17. --SCHUYLKILL RIVER AT MANAYUNK, PHILADELPHIA, PA.

Suspended sediment, water year October 1949 to September 1950

\begin{tabular}{|c|c|c|c|c|c|c|c|c|c|}
\hline \multirow[b]{3}{*}{ Day } & \multicolumn{3}{|c|}{ October } & \multicolumn{3}{|c|}{ November } & \multicolumn{3}{|c|}{ December } \\
\hline & \multirow{2}{*}{$\begin{array}{c}\text { Mean } \\
\text { dis - } \\
\text { charge } \\
\text { (cfs) }\end{array}$} & \multicolumn{2}{|c|}{ Suspended sediment } & \multirow{2}{*}{$\begin{array}{c}\text { Mean } \\
\text { dis- } \\
\text { charge } \\
\text { (cfs) }\end{array}$} & \multicolumn{2}{|c|}{ Suspendec sediment } & \multirow{2}{*}{$\begin{array}{c}\text { Mean } \\
\text { dis- } \\
\text { charge } \\
\text { (cfs) }\end{array}$} & \multicolumn{2}{|c|}{ Suspended sediment } \\
\hline & & $\begin{array}{l}\text { Mean } \\
\text { concen- } \\
\text { tration } \\
\text { (ppm) }\end{array}$ & $\begin{array}{l}\text { Tons } \\
\text { per } \\
\text { day }\end{array}$ & & $\begin{array}{l}\text { Mean } \\
\text { concen- } \\
\text { tration } \\
\text { (ppm) }\end{array}$ & $\begin{array}{l}\text { Tons } \\
\text { per } \\
\text { day }\end{array}$ & & $\begin{array}{l}\text { Mean } \\
\text { concen- } \\
\text { tration } \\
\text { (ppm) }\end{array}$ & $\begin{array}{l}\text { Tons } \\
\text { per } \\
\text { day }\end{array}$ \\
\hline $\begin{array}{l}1-\ldots \\
2 \ldots-\ldots \\
3 \ldots \\
4 \ldots \\
5 \ldots\end{array}$ & $\begin{array}{l}894 \\
833 \\
691 \\
646 \\
587\end{array}$ & $\begin{array}{r}32 \\
13 \\
13 \\
14 \\
8\end{array}$ & $\begin{array}{l}77 \\
29 \\
24 \\
24 \\
13\end{array}$ & $\begin{array}{r}949 \\
1,050 \\
838 \\
680 \\
761\end{array}$ & $\begin{array}{l}27 \\
17 \\
16 \\
20 \\
12\end{array}$ & $\begin{array}{l}69 \\
48 \\
37 \\
37 \\
25\end{array}$ & $\begin{array}{l}725 \\
724 \\
656 \\
626 \\
624\end{array}$ & $\begin{array}{l}10 \\
10 \\
11 \\
11 \\
10\end{array}$ & $\begin{array}{l}20 \\
20 \\
19 \\
19 \\
17\end{array}$ \\
\hline $\begin{array}{r}6 \ldots \ldots \\
7 \ldots \ldots \\
8 \ldots \ldots \\
9 \\
10 \ldots \ldots\end{array}$ & $\begin{array}{l}591 \\
593 \\
612 \\
619 \\
646\end{array}$ & $\begin{array}{r}14 \\
11 \\
8 \\
10 \\
12\end{array}$ & $\begin{array}{l}22 \\
18 \\
13 \\
17 \\
21\end{array}$ & $\begin{array}{l}745 \\
716 \\
655 \\
638 \\
614\end{array}$ & $\begin{array}{r}7 \\
6 \\
8 \\
10 \\
8\end{array}$ & $\begin{array}{l}14 \\
12 \\
14 \\
17 \\
13\end{array}$ & $\begin{array}{l}568 \\
596 \\
570 \\
590 \\
555\end{array}$ & $\begin{array}{l}3 \\
4 \\
5 \\
4 \\
6\end{array}$ & $\begin{array}{l}5 \\
6 \\
8 \\
6 \\
9\end{array}$ \\
\hline 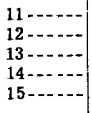 & $\begin{array}{l}622 \\
608 \\
562 \\
562 \\
571\end{array}$ & $\begin{array}{l}4 \\
7 \\
9 \\
6 \\
7\end{array}$ & $\begin{array}{r}7 \\
12 \\
14 \\
9 \\
11\end{array}$ & $\begin{array}{l}550 \\
587 \\
615 \\
690 \\
844\end{array}$ & $\begin{array}{r}10 \\
14 \\
9 \\
14 \\
16\end{array}$ & $\begin{array}{l}15 \\
22 \\
15 \\
26 \\
36\end{array}$ & $\begin{array}{r}556 \\
562 \\
1,700 \\
4,060 \\
2,920\end{array}$ & $\begin{array}{r}1 \\
6 \\
1 \\
170 \\
115\end{array}$ & $\begin{array}{r}2 \\
9 \\
5 \\
1,860 \\
907\end{array}$ \\
\hline $\begin{array}{l}16 \ldots \ldots \\
17 \ldots \ldots \\
18 \ldots \ldots \\
19 \\
20 \ldots \ldots\end{array}$ & $\begin{array}{l}556 \\
542 \\
533 \\
502 \\
500\end{array}$ & $\begin{array}{r}10 \\
14 \\
9 \\
7 \\
7\end{array}$ & $\begin{array}{r}15 \\
20 \\
13 \\
9 \\
9\end{array}$ & $\begin{array}{r}1,160 \\
868 \\
756 \\
697 \\
696\end{array}$ & $\begin{array}{r}23 \\
18 \\
12 \\
7 \\
8\end{array}$ & $\begin{array}{l}72 \\
42 \\
24 \\
13 \\
15\end{array}$ & $\begin{array}{l}2,160 \\
1,510 \\
1,300 \\
1,360 \\
1,540\end{array}$ & $\begin{array}{l}53 \\
38 \\
25 \\
16 \\
16\end{array}$ & $\begin{array}{r}309 \\
155 \\
88 \\
59 \\
66\end{array}$ \\
\hline $\begin{array}{l}21 \ldots \ldots \\
22 \ldots \ldots- \\
23 \ldots \ldots \\
24-\ldots- \\
25-\ldots-\end{array}$ & $\begin{array}{l}479 \\
499 \\
479 \\
481 \\
480\end{array}$ & $\begin{array}{r}10 \\
8 \\
12 \\
9 \\
13\end{array}$ & $\begin{array}{l}13 \\
11 \\
16 \\
12 \\
17\end{array}$ & $\begin{array}{l}688 \\
474 \\
630 \\
677 \\
682 \\
\end{array}$ & $\begin{array}{r}8 \\
10 \\
8 \\
4 \\
11\end{array}$ & $\begin{array}{r}15 \\
13 \\
14 \\
7 \\
20\end{array}$ & $\begin{array}{l}1,490 \\
1,310 \\
1,270 \\
1,240 \\
1,560\end{array}$ & $\begin{array}{l}15 \\
15 \\
21 \\
20 \\
15\end{array}$ & $\begin{array}{l}60 \\
53 \\
72 \\
67 \\
63\end{array}$ \\
\hline 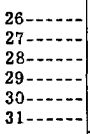 & $\begin{array}{l}747 \\
708 \\
926 \\
819 \\
650 \\
859 \\
\end{array}$ & $\begin{array}{r}18 \\
13 \\
7 \\
20 \\
14 \\
22 \\
\end{array}$ & $\begin{array}{l}36 \\
25 \\
17 \\
44 \\
25 \\
51\end{array}$ & $\begin{array}{r}689 \\
615 \\
620 \\
615 \\
634 \\
--\end{array}$ & $\begin{array}{r}17 \\
6 \\
6 \\
8 \\
10 \\
--\end{array}$ & $\begin{array}{l}32 \\
10 \\
10 \\
13 \\
17 \\
--\end{array}$ & $\begin{array}{l}1,450 \\
7,420 \\
8,420 \\
5,790 \\
4,320 \\
3,380\end{array}$ & $\begin{array}{r}60 \\
1,220 \\
675 \\
330 \\
125 \\
75\end{array}$ & $\begin{array}{r}235 \\
\text { s } 30,600 \\
15,300 \\
5,160 \\
1,460 \\
684\end{array}$ \\
\hline \multirow[t]{2}{*}{ Total - } & 19,397 & -- & 644 & 21,453 & -- & 717 & 61,552 & $\because$ & 57,340 \\
\hline & \multicolumn{3}{|c|}{ January } & \multicolumn{3}{|c|}{ February } & \multicolumn{3}{|c|}{ March } \\
\hline $\begin{array}{l}1 \ldots \ldots \\
2 \ldots \ldots \\
3 \ldots \ldots \\
4-\ldots \\
5 \cdots\end{array}$ & $\begin{array}{l}2,780 \\
2,440 \\
2,240 \\
2,320 \\
2,270\end{array}$ & $\begin{array}{l}30 \\
18 \\
16 \\
20 \\
17\end{array}$ & $\begin{array}{r}225 \\
119 \\
97 \\
125 \\
104\end{array}$ & $\begin{array}{l}4,250 \\
5,030 \\
7,170 \\
5,480 \\
4,670\end{array}$ & $\begin{array}{r}97 \\
100 \\
285 \\
140 \\
87\end{array}$ & $\begin{array}{l}1,110 \\
1,360 \\
5,520 \\
2,070 \\
1,100\end{array}$ & $\begin{array}{l}2,150 \\
2,170 \\
1,780 \\
1,540 \\
1,680\end{array}$ & $\begin{array}{l}17 \\
24 \\
17 \\
22 \\
20\end{array}$ & $\begin{array}{r}99 \\
141 \\
82 \\
92 \\
91\end{array}$ \\
\hline $\begin{array}{r}6 \ldots-\ldots \\
7 \ldots \ldots \\
8 \cdots- \\
9-\cdots \\
10-\cdots\end{array}$ & $\begin{array}{l}1,970 \\
1,950 \\
2,720 \\
2,420 \\
2,230\end{array}$ & $\begin{array}{l}18 \\
22 \\
11 \\
17 \\
22\end{array}$ & $\begin{array}{r}96 \\
116 \\
81 \\
111 \\
133\end{array}$ & $\begin{array}{l}3,920 \\
3,920 \\
3,490 \\
4,440 \\
7,800\end{array}$ & $\begin{array}{r}43 \\
36 \\
29 \\
106 \\
363\end{array}$ & $\begin{array}{r}455 \\
381 \\
273 \\
\mathrm{~s} 1,970 \\
7,640\end{array}$ & $\begin{array}{l}1,790 \\
1,670 \\
1,700 \\
4,410 \\
4,000\end{array}$ & $\begin{array}{r}14 \\
10 \\
18 \\
198 \\
156\end{array}$ & $\begin{array}{r}68 \\
45 \\
83 \\
2,360 \\
1,680\end{array}$ \\
\hline 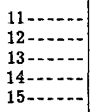 & $\begin{array}{l}4,460 \\
4,680 \\
3,830 \\
3,710 \\
3,380\end{array}$ & $\begin{array}{r}168 \\
168 \\
62 \\
54 \\
31\end{array}$ & $\begin{array}{r}2,020 \\
2,120 \\
641 \\
541 \\
283\end{array}$ & $\begin{array}{r}6,320 \\
5,360 \\
4,730 \\
11,500 \\
17,400\end{array}$ & $\begin{array}{r}188 \\
105 \\
63 \\
852 \\
1,900\end{array}$ & $\begin{array}{r}3,210 \\
1,520 \\
805 \\
s 28,500 \\
89,300\end{array}$ & $\begin{array}{l}2,800 \\
2,780 \\
3,940 \\
5,050 \\
3,630\end{array}$ & $\begin{array}{r}60 \\
34 \\
60 \\
114 \\
45\end{array}$ & $\begin{array}{r}454 \\
255 \\
638 \\
1,550 \\
441\end{array}$ \\
\hline 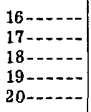 & $\begin{array}{l}2,810 \\
2,420 \\
2,160 \\
2,100 \\
1,970\end{array}$ & $\begin{array}{r}32 \\
26 \\
19 \\
17 \\
6\end{array}$ & $\begin{array}{r}243 \\
170 \\
111 \\
96 \\
32\end{array}$ & $\begin{array}{r}13,500 \\
10,300 \\
7,840 \\
6,420 \\
5,350\end{array}$ & $\begin{array}{r}1,150 \\
680 \\
370 \\
240 \\
175\end{array}$ & $\begin{array}{r}41,900 \\
18,900 \\
7,830 \\
4,160 \\
2,530\end{array}$ & $\begin{array}{l}3,040 \\
2,770 \\
2,650 \\
2,480 \\
2,200\end{array}$ & $\begin{array}{l}26 \\
23 \\
35 \\
43 \\
34\end{array}$ & $\begin{array}{l}213 \\
172 \\
251 \\
288 \\
202\end{array}$ \\
\hline $\begin{array}{l}21 \ldots \ldots \\
22 \ldots \ldots \\
23 \ldots \ldots \\
24 \ldots \ldots \\
25 \ldots-\ldots\end{array}$ & $\begin{array}{l}1,640 \\
1,570 \\
1,540 \\
1,500 \\
1,460\end{array}$ & $\begin{array}{r}12 \\
8 \\
3 \\
3 \\
6\end{array}$ & $\begin{array}{l}53 \\
34 \\
12 \\
12 \\
24\end{array}$ & $\begin{array}{l}4,140 \\
3,720 \\
3,850 \\
3,680 \\
3,330\end{array}$ & $\begin{array}{l}82 \\
59 \\
54 \\
45 \\
38\end{array}$ & $\begin{array}{l}917 \\
593 \\
561 \\
447 \\
342\end{array}$ & $\begin{array}{r}4,490 \\
8,130 \\
21,800 \\
17,400 \\
11,100\end{array}$ & $\begin{array}{r}200 \\
460 \\
2,750 \\
1,480 \\
660\end{array}$ & $\begin{array}{r}\mathrm{s} 4,170 \\
10,100 \\
\mathrm{~s} 186,600 \\
69,500 \\
19,800\end{array}$ \\
\hline $\begin{array}{l}26-\cdots- \\
27-\ldots \\
28-\cdots \\
29-\cdots- \\
30-\cdots \\
31-\cdots \\
\end{array}$ & $\begin{array}{l}1,530 \\
1,500 \\
1,560 \\
1,540 \\
1,760 \\
2,550 \\
\end{array}$ & $\begin{array}{r}7 \\
7 \\
10 \\
5 \\
8 \\
25 \\
\end{array}$ & $\begin{array}{r}29 \\
28 \\
42 \\
21 \\
38 \\
172 \\
\end{array}$ & $\begin{array}{r}2,980 \\
2,450 \\
2,080 \\
-- \\
-- \\
-- \\
\end{array}$ & $\begin{array}{l}30 \\
30 \\
15 \\
-- \\
-- \\
-- \\
\end{array}$ & $\begin{array}{r}241 \\
198 \\
84 \\
-- \\
-- \\
--\end{array}$ & $\begin{array}{l}8,670 \\
8,970 \\
9,670 \\
9,980 \\
9,000 \\
7,080 \\
\end{array}$ & $\begin{array}{l}370 \\
300 \\
408 \\
366 \\
298 \\
225 \\
\end{array}$ & $\begin{array}{r}8,660 \\
7,270 \\
10,700 \\
9,860 \\
7,240 \\
4,300\end{array}$ \\
\hline Total- & 73,010 & -- & 7,930 & 165,120 & -- & 223,900 & 170,520 & $=$ & 347,400 \\
\hline
\end{tabular}

s Computed by subdividing day. 
Table 17. --SChUYLKILL RIVER AT MANAYUNK, PHILADELPHIA, PA. --Continued

Suspended sediment, water year October 1949 to September $1950-$-Continued

\begin{tabular}{|c|c|c|c|c|c|c|c|c|c|}
\hline \multirow[b]{3}{*}{ Day } & \multicolumn{3}{|c|}{ April } & \multicolumn{3}{|c|}{ May } & \multicolumn{3}{|c|}{ June } \\
\hline & \multirow{2}{*}{$\begin{array}{l}\text { Mean } \\
\text { dis- } \\
\text { charge } \\
\text { (cfs) }\end{array}$} & \multicolumn{2}{|c|}{ Suspended sediment } & \multirow{2}{*}{$\begin{array}{c}\text { Mean } \\
\text { dis - } \\
\text { charge } \\
\text { (cfs) }\end{array}$} & \multicolumn{2}{|c|}{ Suspended sediment } & \multirow{2}{*}{$\begin{array}{c}\text { Mean } \\
\text { dis- } \\
\text { charge } \\
\text { (cfs) }\end{array}$} & \multicolumn{2}{|c|}{ Suspended sediment } \\
\hline & & $\begin{array}{l}\text { Mean } \\
\text { concen- } \\
\text { tration } \\
(\text { ppm })\end{array}$ & $\begin{array}{l}\text { Tons } \\
\text { per } \\
\text { day }\end{array}$ & & $\begin{array}{l}\text { Mean } \\
\text { concen- } \\
\text { tration } \\
(\mathrm{ppm})\end{array}$ & $\begin{array}{l}\text { Tons } \\
\text { per } \\
\text { day }\end{array}$ & & $\begin{array}{c}\text { Mean } \\
\text { concen- } \\
\text { tration } \\
\text { (ppm) }\end{array}$ & $\begin{array}{l}\text { Tons } \\
\text { per } \\
\text { day }\end{array}$ \\
\hline $\begin{array}{l}1 \ldots \ldots \\
2 \ldots \ldots \\
3 \\
4 \\
5 \\
5\end{array}$ & $\begin{array}{l}6,020 \\
5,380 \\
4,860 \\
4,810 \\
4,520\end{array}$ & $\begin{array}{r}172 \\
128 \\
100 \\
85 \\
80\end{array}$ & $\begin{array}{r}2,800 \\
1,860 \\
1,310 \\
1,100 \\
976\end{array}$ & $\begin{array}{l}2,380 \\
2,970 \\
3,470 \\
3,290 \\
2,900\end{array}$ & $\begin{array}{l}22 \\
22\end{array}$ & $\begin{array}{l}141 \\
176 \\
246\end{array}$ & $\begin{array}{l}5,080 \\
5,780 \\
4,650 \\
4,560 \\
5,120\end{array}$ & $\begin{array}{r}76 \\
169 \\
80 \\
72 \\
81\end{array}$ & $\begin{array}{r}1,040 \\
2,480 \\
1,000 \\
886 \\
1,120\end{array}$ \\
\hline $\begin{array}{r}6-\ldots \\
7-\ldots \\
8-\ldots- \\
9-\ldots- \\
10-\ldots-\end{array}$ & $\begin{array}{l}3,920 \\
3,500 \\
3,180 \\
3,020 \\
2,850\end{array}$ & $\begin{array}{l}65 \\
48 \\
39 \\
30 \\
19\end{array}$ & $\begin{array}{l}688 \\
454 \\
335 \\
245 \\
146\end{array}$ & $\begin{array}{l}2,510 \\
2,480 \\
2,190 \\
1,930 \\
1,870\end{array}$ & 16 & 92 & $\begin{array}{l}3,740 \\
3,170 \\
2,800 \\
2,510 \\
2,260\end{array}$ & $\begin{array}{l}33 \\
22 \\
20 \\
16 \\
18\end{array}$ & $\begin{array}{r}333 \\
188 \\
151 \\
11 \\
11\end{array}$ \\
\hline $\begin{array}{l}11-\ldots \\
12-\ldots \\
13-\ldots \\
14-\ldots \\
15-\ldots-\end{array}$ & $\begin{array}{l}2,700 \\
2,680 \\
2,520 \\
2,370 \\
2,210\end{array}$ & $\begin{array}{l}18 \\
18 \\
15 \\
29 \\
40\end{array}$ & $\begin{array}{l}131 \\
130 \\
102 \\
185 \\
239\end{array}$ & $\begin{array}{l}1,830 \\
1,740 \\
1,600 \\
1,540 \\
1,670\end{array}$ & 13 & 59 & $\begin{array}{l}2,250 \\
2,180 \\
1,870 \\
1,730 \\
1,950\end{array}$ & 22 & 119 \\
\hline $\begin{array}{l}16 \ldots-\ldots \\
17 \ldots \\
18 \ldots \\
19 \\
20-\ldots-\end{array}$ & $\begin{array}{l}2,110 \\
2,040 \\
1,940 \\
1,840 \\
1,860\end{array}$ & $\begin{array}{l}37 \\
19 \\
15 \\
16 \\
16\end{array}$ & $\begin{array}{r}211 \\
105 \\
79 \\
79 \\
80\end{array}$ & $\begin{array}{l}1,940 \\
2,190 \\
2,260 \\
4,180 \\
4,890\end{array}$ & $\begin{array}{r}18 \\
20 \\
96 \\
114\end{array}$ & $\begin{array}{r}94 \\
122 \\
1,080 \\
1,510\end{array}$ & $\begin{array}{l}2,480 \\
2,260 \\
1,840 \\
1,630 \\
1,630\end{array}$ & 18 & 103 \\
\hline 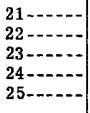 & $\begin{array}{l}2,060 \\
2,140 \\
1,920 \\
1,840 \\
2,020\end{array}$ & $\begin{array}{l}17 \\
15 \\
12 \\
13 \\
17\end{array}$ & $\begin{array}{l}94 \\
87 \\
62 \\
65 \\
93\end{array}$ & $\begin{array}{l}3,700 \\
3,040 \\
2,650 \\
4,430 \\
4,980\end{array}$ & $\begin{array}{r}78 \\
38 \\
26 \\
100 \\
102\end{array}$ & $\begin{array}{r}779 \\
312 \\
186 \\
1,200 \\
1,370\end{array}$ & $\begin{array}{l}1,810 \\
1,600 \\
1,440 \\
1,290 \\
1,340\end{array}$ & 16 & 72 \\
\hline 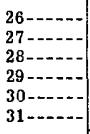 & $\begin{array}{r}2,310 \\
2,240 \\
2,410 \\
2,380 \\
2,160 \\
-- \\
\end{array}$ & $\begin{array}{l}18 \\
18 \\
24 \\
22 \\
22 \\
-- \\
\end{array}$ & $\begin{array}{r}112 \\
109 \\
156 \\
142 \\
128 \\
--\end{array}$ & $\begin{array}{l}3,700 \\
3,410 \\
2,900 \\
3,580 \\
6,100 \\
4,480\end{array}$ & $\begin{array}{r}62 \\
41 \\
31 \\
57 \\
157 \\
68 \\
\end{array}$ & $\begin{array}{r}619 \\
377 \\
243 \\
551 \\
2,590 \\
822 \\
\end{array}$ & $\begin{array}{r}1,200 \\
1,120 \\
995 \\
981 \\
967 \\
--\end{array}$ & $\begin{array}{l}15 \\
14 \\
12 \\
--\end{array}$ & $\begin{array}{l}39 \\
31 \\
--\end{array}$ \\
\hline \multirow[t]{2}{*}{ Total- } & 85,820 & $=-$ & 12,300 & 92,800 & -- & 13,950 & 72,233 & -- & 8,760 \\
\hline & \multicolumn{3}{|c|}{ July } & \multicolumn{3}{|c|}{ August } & \multicolumn{3}{|c|}{ September } \\
\hline 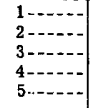 & $\begin{array}{r}965 \\
976 \\
926 \\
1,030 \\
1,070\end{array}$ & $\begin{array}{r}12 \\
14 \\
\text { e } 20 \\
319\end{array}$ & $\begin{array}{l}31 \\
36 \\
\\
56 \\
55\end{array}$ & $\begin{array}{r}927 \\
889 \\
7,790 \\
1,980 \\
1,080\end{array}$ & $\begin{array}{r}12 \\
121 \\
22\end{array}$ & $\begin{array}{r}30 \\
725 \\
64\end{array}$ & $\begin{array}{r}1,040 \\
747 \\
804 \\
949 \\
854\end{array}$ & $\begin{array}{r}18 \\
8 \\
18 \\
24 \\
19\end{array}$ & $\begin{array}{l}50 \\
16 \\
39 \\
62 \\
44\end{array}$ \\
\hline $\begin{array}{r}6 \ldots \\
7 \ldots \ldots \\
8 \ldots \\
9 \\
10 \\
\end{array}$ & $\begin{array}{r}833 \\
2,280 \\
1,690 \\
1,440 \\
2,200\end{array}$ & $\begin{array}{l}10 \\
31 \\
13 \\
12 \\
47\end{array}$ & $\begin{array}{r}22 \\
19 \\
59 \\
47 \\
279\end{array}$ & $\begin{array}{l}994 \\
850 \\
829 \\
813 \\
786\end{array}$ & $\begin{array}{r}18 \\
13 \\
14 \\
16 \\
8\end{array}$ & $\begin{array}{l}48 \\
30 \\
31 \\
35 \\
17\end{array}$ & $\begin{array}{l}690 \\
625 \\
587 \\
578 \\
942\end{array}$ & $\begin{array}{l}13 \\
14 \\
15 \\
11 \\
41\end{array}$ & $\begin{array}{r}24 \\
24 \\
24 \\
17 \\
104\end{array}$ \\
\hline $\begin{array}{l}11 \ldots \ldots \\
12 \ldots \ldots \\
13 \ldots \ldots \\
14 \ldots \ldots \\
15 \ldots-\ldots\end{array}$ & $\begin{array}{l}2,300 \\
4,120 \\
3,020 \\
2,620 \\
2,700\end{array}$ & $\begin{array}{r}38 \\
115 \\
45 \\
\\
24\end{array}$ & $\begin{array}{r}236 \\
1,280 \\
367 \\
\\
170\end{array}$ & $\begin{array}{l}864 \\
806 \\
784 \\
735 \\
690\end{array}$ & $\begin{array}{r}11 \\
9 \\
8 \\
7 \\
10\end{array}$ & $\begin{array}{l}26 \\
20 \\
17 \\
14 \\
19\end{array}$ & $\begin{array}{l}5,150 \\
3,960 \\
2,230 \\
1,840 \\
2,400\end{array}$ & $\begin{array}{r}326 \\
282 \\
62 \\
34 \\
138\end{array}$ & $\begin{array}{r}\text { s } 5,180 \\
3,020 \\
373 \\
169 \\
894\end{array}$ \\
\hline $\begin{array}{l}16 \ldots \ldots \\
17 \ldots \ldots \\
18 \ldots-\ldots \\
19 \ldots-\ldots \\
20-\ldots\end{array}$ & $\begin{array}{l}2,550 \\
2,110 \\
1,930 \\
1,610 \\
1,490\end{array}$ & 14 & 68 & $\begin{array}{l}696 \\
648 \\
621 \\
677 \\
711\end{array}$ & $\begin{array}{l}10 \\
10 \\
11 \\
13 \\
15\end{array}$ & $\begin{array}{l}19 \\
17 \\
18 \\
24 \\
29\end{array}$ & $\begin{array}{r}2,020 \\
1,400 \\
1,150 \\
1,000 \\
936\end{array}$ & $\begin{array}{l}47 \\
26 \\
15 \\
15 \\
10\end{array}$ & $\begin{array}{r}255 \\
98 \\
46 \\
40 \\
25\end{array}$ \\
\hline $\begin{array}{l}21 \ldots \ldots \\
22 \ldots \ldots \\
23 \ldots-\ldots \\
24-\ldots- \\
25 \ldots-\ldots\end{array}$ & $\begin{array}{l}1,530 \\
1,360 \\
1,200 \\
1,120 \\
1,130\end{array}$ & 10 & 35 & $\begin{array}{r}997 \\
1,200 \\
831 \\
758 \\
736\end{array}$ & $\begin{array}{r}27 \\
21 \\
20 \\
8 \\
8\end{array}$ & $\begin{array}{l}73 \\
68 \\
45 \\
16 \\
16\end{array}$ & $\begin{array}{l}912 \\
930 \\
959 \\
897 \\
827\end{array}$ & $\begin{array}{r}8 \\
5 \\
4 \\
10 \\
5\end{array}$ & $\begin{array}{l}20 \\
12 \\
16 \\
24 \\
11\end{array}$ \\
\hline $\begin{array}{l}26-\ldots \\
27-\cdots \\
28-\cdots- \\
29-\cdots- \\
30-\cdots \\
31-\cdots-\end{array}$ & $\begin{array}{r}1,250 \\
1,420 \\
1,140 \\
1,050 \\
999 \\
928 \\
\end{array}$ & 11 & 32 & $\begin{array}{r}716 \\
691 \\
660 \\
686 \\
706 \\
1,070\end{array}$ & $\begin{array}{r}10 \\
8 \\
10 \\
15 \\
18 \\
22\end{array}$ & $\begin{array}{l}19 \\
15 \\
18 \\
28 \\
34 \\
63\end{array}$ & $\begin{array}{r}777 \\
729 \\
720 \\
699 \\
717 \\
--\end{array}$ & $\begin{array}{r}6 \\
6 \\
9 \\
11 \\
14 \\
--\end{array}$ & $\begin{array}{l}13 \\
12 \\
17 \\
21 \\
27 \\
--\end{array}$ \\
\hline Total- & 50,987 & $\ldots$ & 3,690 & 33,221 & -- & 3,030 & 38,069 & -- & 10,670 \\
\hline $\begin{array}{l}\text { Total } \\
\text { Total }\end{array}$ & $\begin{array}{l}\text { scharge } \\
\text { ad for ye }\end{array}$ & $\begin{array}{l}\text { year }(\mathrm{sec} \\
\text { (tons) } \ldots\end{array}$ & $\begin{array}{l}- \text { foot } \mathrm{da} \\
\ldots \ldots \\
\end{array}$ & $\cdots \cdots$ & $\ldots \ldots$ & $\ldots \ldots$ & $\ldots \ldots \ldots$ & $\ldots \ldots \ldots$ & $\begin{array}{l}884,182 \\
690,331 \\
\end{array}$ \\
\hline
\end{tabular}


Table 17. --SCHUYLKILL RIVER AT MANAYUNK, PHILADELPHIA, FA. --Continued

Suspended sediment, water year October 1950 to Septe nber 1951

\begin{tabular}{|c|c|c|c|c|c|c|c|c|c|}
\hline \multirow[b]{3}{*}{ Day } & \multicolumn{3}{|c|}{ October } & \multicolumn{3}{|c|}{ November } & \multicolumn{3}{|c|}{ December } \\
\hline & \multirow{2}{*}{$\begin{array}{c}\text { Mean } \\
\text { dis - } \\
\text { charge } \\
\text { (efs) }\end{array}$} & \multicolumn{2}{|c|}{ Suspended sedıment } & \multirow{2}{*}{$\begin{array}{c}\text { Mean } \\
\text { dis- } \\
\text { charge } \\
\text { (cfs) }\end{array}$} & \multicolumn{2}{|c|}{ Suspended sediment } & \multirow{2}{*}{$\begin{array}{c}\text { Mean } \\
\text { dis- } \\
\text { charge } \\
\text { (cfs) }\end{array}$} & \multicolumn{2}{|c|}{ Suspended sedıment } \\
\hline & & $\begin{array}{l}\text { Mean } \\
\text { concen- } \\
\text { tration } \\
\text { (ppm) }\end{array}$ & $\begin{array}{l}\text { Tons } \\
\text { per } \\
\text { day }\end{array}$ & & $\begin{array}{l}\text { Mean } \\
\text { concen- } \\
\text { tration } \\
(p p m) \\
\end{array}$ & $\begin{array}{l}\text { Tons } \\
\text { per } \\
\text { day }\end{array}$ & & $\begin{array}{c}\text { Mean } \\
\text { concen- } \\
\text { tration } \\
\text { (ppm) }\end{array}$ & $\begin{array}{l}\text { Tons } \\
\text { per } \\
\text { day }\end{array}$ \\
\hline $\begin{array}{l}1 \ldots \ldots \\
2 \ldots-\ldots \\
3 \ldots \ldots \\
4 \\
5 \ldots-\ldots\end{array}$ & $\begin{array}{l}668 \\
670 \\
654 \\
640 \\
611\end{array}$ & $\begin{array}{l}11 \\
10 \\
13 \\
18 \\
16\end{array}$ & $\begin{array}{l}20 \\
18 \\
23 \\
31 \\
26\end{array}$ & $\begin{array}{r}837 \\
822 \\
791 \\
1,010 \\
2,290\end{array}$ & $\begin{array}{l}15 \\
14 \\
18 \\
18 \\
52\end{array}$ & $\begin{array}{r}34 \\
31 \\
38 \\
49 \\
322\end{array}$ & $\begin{array}{r}4,940 \\
4,220 \\
3,650 \\
9,270 \\
33,800\end{array}$ & $\begin{array}{r}65 \\
40 \\
25 \\
982 \\
3,540\end{array}$ & $\begin{array}{r}867 \\
456 \\
247 \\
\mathrm{~s} 56,400 \\
\mathrm{~s} 320,000\end{array}$ \\
\hline $\begin{array}{r}6 \ldots \ldots \\
7 \ldots \ldots \\
8 \ldots \ldots \\
9-\ldots- \\
10 \ldots \ldots\end{array}$ & $\begin{array}{l}598 \\
573 \\
585 \\
638 \\
928\end{array}$ & $\begin{array}{r}14 \\
12 \\
9 \\
15 \\
20\end{array}$ & $\begin{array}{l}23 \\
19 \\
14 \\
26 \\
50\end{array}$ & $\begin{array}{l}3,710 \\
2,730 \\
2,180 \\
1,850 \\
1,700\end{array}$ & $\begin{array}{r}38 \\
20 \\
13 \\
17 \\
7\end{array}$ & $\begin{array}{r}381 \\
147 \\
76 \\
85 \\
32\end{array}$ & $\begin{array}{r}15,500 \\
9,680 \\
18,760 \\
13,900 \\
9,670\end{array}$ & $\begin{array}{r}1,280 \\
400 \\
3,440 \\
910 \\
218\end{array}$ & $\begin{array}{r}53,600 \\
\mathrm{e} 10,450 \\
\mathrm{~s} 191,000 \\
34,150 \\
5,690\end{array}$ \\
\hline $\begin{array}{l}11 \ldots \ldots \\
12 \ldots \ldots \\
13 \ldots \ldots \\
14 \ldots \ldots \\
15 \ldots \ldots\end{array}$ & $\begin{array}{l}2,120 \\
1,910 \\
1,480 \\
1,430 \\
1,220\end{array}$ & $\begin{array}{l}24 \\
23 \\
20 \\
15 \\
13\end{array}$ & $\begin{array}{r}137 \\
119 \\
80 \\
58 \\
43\end{array}$ & $\begin{array}{l}1,470 \\
1,320 \\
1,230 \\
1,170 \\
1,180\end{array}$ & $\begin{array}{r}9 \\
13 \\
18 \\
18 \\
21\end{array}$ & $\begin{array}{l}36 \\
46 \\
60 \\
57 \\
67\end{array}$ & $\begin{array}{l}8,110 \\
7,440 \\
6,070 \\
5,040 \\
4,330\end{array}$ & $\begin{array}{r}132 \\
124 \\
90 \\
48 \\
24\end{array}$ & $\begin{array}{r}\text { e 2, } 890 \\
2,490 \\
\text { e } 1,480 \\
653 \\
\text { e } 281\end{array}$ \\
\hline $\begin{array}{l}16-\ldots-1 \\
17-\ldots-1 \\
18 \ldots \ldots \\
19-\ldots \ldots \\
20-\ldots-n\end{array}$ & $\begin{array}{r}1,050 \\
991 \\
923 \\
863 \\
844\end{array}$ & $\begin{array}{l}11 \\
14 \\
13 \\
10 \\
10\end{array}$ & $\begin{array}{l}31 \\
38 \\
32 \\
23 \\
23\end{array}$ & $\begin{array}{r}1,220 \\
1,100 \\
1,180 \\
1,020 \\
972\end{array}$ & $\begin{array}{l}19 \\
13 \\
13 \\
10 \\
14\end{array}$ & $\begin{array}{l}63 \\
39 \\
41 \\
28 \\
37\end{array}$ & $\begin{array}{l}4,380 \\
4,260 \\
3,520 \\
3,110 \\
2,630\end{array}$ & $\begin{array}{l}50 \\
34 \\
20 \\
19 \\
16\end{array}$ & $\begin{array}{r}591 \\
391 \\
190 \\
160 \\
\text { e } 114\end{array}$ \\
\hline $\begin{array}{l}21 \ldots \ldots \\
22-\ldots \\
23-\ldots \\
24-\ldots- \\
25-\cdots\end{array}$ & $\begin{array}{r}800 \\
794 \\
1,720 \\
2,280 \\
1,500\end{array}$ & $\begin{array}{l}12 \\
14 \\
24 \\
27 \\
17\end{array}$ & $\begin{array}{r}26 \\
30 \\
112 \\
166 \\
69\end{array}$ & $\begin{array}{r}995 \\
1,580 \\
1,830 \\
1,230 \\
29,830\end{array}$ & $\begin{array}{r}28 \\
28 \\
27 \\
16 \\
4,010\end{array}$ & $\begin{array}{r}75 \\
119 \\
133 \\
53 \\
\mathrm{~s} 483,000\end{array}$ & $\begin{array}{l}2,580 \\
2,470 \\
2,340 \\
2,340 \\
2,320\end{array}$ & $\begin{array}{r}8 \\
8 \\
10 \\
9 \\
9\end{array}$ & $\begin{array}{r}56 \\
53 \\
63 \\
\text { e } 57 \\
\text { e } 56\end{array}$ \\
\hline $\begin{array}{l}26 \ldots \ldots \\
27 \ldots \ldots \\
28 \\
29 \\
30 \\
31 \ldots \ldots\end{array}$ & $\begin{array}{r}1,120 \\
986 \\
904 \\
860 \\
872 \\
868 \\
\end{array}$ & $\begin{array}{l}20 \\
19 \\
14 \\
15 \\
17 \\
17 \\
\end{array}$ & $\begin{array}{l}60 \\
50 \\
34 \\
35 \\
40 \\
40 \\
\end{array}$ & $\begin{array}{r}59,840 \\
23,270 \\
10,290 \\
7,500 \\
5,860 \\
--\end{array}$ & $\begin{array}{r}3,180 \\
1,120 \\
560 \\
230 \\
98 \\
-- \\
\end{array}$ & $\begin{array}{r}\text { s } 537,000 \\
70,400 \\
15,600 \\
4,660 \\
1,550 \\
-- \\
\end{array}$ & $\begin{array}{l}2,140 \\
1,490 \\
1,390 \\
1,680 \\
2,480 \\
2,620 \\
\end{array}$ & $\begin{array}{r}10 \\
9 \\
4 \\
4 \\
8 \\
6\end{array}$ & $\begin{array}{r}\text { e } 58 \\
\text { e } 36 \\
15 \\
\text { e } 18 \\
53 \\
42\end{array}$ \\
\hline \multirow[t]{2}{*}{ Total- } & 32,100 & -- & 1,500 & 172,007 & -- & $1,114,000$ & 193,320 & $\cdots$ & 682,600 \\
\hline & \multicolumn{3}{|c|}{ January } & \multicolumn{3}{|c|}{ February } & \multicolumn{3}{|c|}{ March } \\
\hline 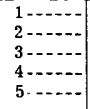 & $\begin{array}{l}2,240 \\
1,880 \\
1,810 \\
2,050 \\
3,430\end{array}$ & $\begin{array}{l}-- \\
-- \\
-- \\
-- \\
--\end{array}$ & $\begin{array}{l}-- \\
-- \\
-- \\
-- \\
--\end{array}$ & $\begin{array}{r}4,300 \\
11,500 \\
7,560 \\
5,150 \\
4,710\end{array}$ & $\begin{array}{r}49 \\
792 \\
275 \\
76 \\
47\end{array}$ & $\begin{array}{r}569 \\
\mathrm{~s} 25,800 \\
5,610 \\
1,060 \\
598\end{array}$ & $\begin{array}{l}4,110 \\
4,170 \\
4,030 \\
5,280 \\
6,450\end{array}$ & $\begin{array}{l}17 \\
28 \\
25 \\
15 \\
48\end{array}$ & $\begin{array}{l}189 \\
315 \\
272 \\
214 \\
836\end{array}$ \\
\hline $\begin{array}{r}6 \cdots \\
7-\cdots \\
8-\cdots- \\
9-\cdots \\
10-\cdots-\end{array}$ & $\begin{array}{l}3,950 \\
3,460 \\
3,640 \\
2,980 \\
2,490\end{array}$ & $\begin{array}{l}-- \\
-- \\
-- \\
-- \\
--\end{array}$ & $\begin{array}{l}-- \\
-- \\
-- \\
-- \\
--\end{array}$ & $\begin{array}{r}4,220 \\
10,400 \\
24,900 \\
8,440 \\
6,030\end{array}$ & $\begin{array}{r}40 \\
107 \\
1,490 \\
350 \\
373\end{array}$ & $\begin{array}{r}456 \\
\text { e } 30,000 \\
\text { e } 100,000 \\
7,980 \\
6,070\end{array}$ & $\begin{array}{l}5,240 \\
4,820 \\
4,680 \\
4,350 \\
3,860\end{array}$ & $\begin{array}{l}40 \\
42 \\
40 \\
14 \\
16\end{array}$ & $\begin{array}{l}566 \\
546 \\
505 \\
164 \\
167\end{array}$ \\
\hline $\begin{array}{l}11 \ldots \ldots \\
12 \ldots \ldots \\
13 \ldots-\ldots \\
14 \ldots \ldots \\
15 \ldots \ldots\end{array}$ & $\begin{array}{r}2,390 \\
2,310 \\
2,120 \\
2,120 \\
13,800\end{array}$ & $\begin{array}{l}-- \\
-- \\
-- \\
-- \\
--\end{array}$ & $\begin{array}{l}-- \\
-- \\
-- \\
-- \\
--\end{array}$ & $\begin{array}{l}4,900 \\
4,510 \\
4,950 \\
6,460 \\
5,370\end{array}$ & $\begin{array}{r}140 \\
66 \\
49 \\
104 \\
76\end{array}$ & $\begin{array}{r}1,850 \\
804 \\
655 \\
1,810 \\
1,100\end{array}$ & $\begin{array}{l}3,480 \\
3,300 \\
3,180 \\
3,700 \\
4,730\end{array}$ & $\begin{array}{r}11 \\
9 \\
13 \\
22 \\
14\end{array}$ & $\begin{array}{r}103 \\
80 \\
112 \\
220 \\
179\end{array}$ \\
\hline $\begin{array}{l}16 \ldots \ldots \\
17 \ldots \ldots \\
18 \ldots-\ldots \\
19 \ldots \ldots \\
20 \ldots \ldots\end{array}$ & $\begin{array}{l}9,660 \\
5,970 \\
4,660 \\
4,040 \\
3,720\end{array}$ & $\begin{array}{l}-- \\
-- \\
50 \\
30 \\
19\end{array}$ & $\begin{array}{l}629^{--} \\
327 \\
191\end{array}$ & $\begin{array}{l}4,260 \\
4,310 \\
7,420 \\
5,850 \\
6,190\end{array}$ & $\begin{array}{r}91 \\
133 \\
230 \\
148 \\
138\end{array}$ & $\begin{array}{l}1,050 \\
1,550 \\
4,610 \\
2,340 \\
2,310\end{array}$ & $\begin{array}{l}4,120 \\
3,530 \\
3,180 \\
3,120 \\
8,240\end{array}$ & $\begin{array}{r}8 \\
56 \\
115 \\
137 \\
299\end{array}$ & $\begin{array}{r}89 \\
534 \\
987 \\
1.150 \\
\mathrm{~s} 4,830\end{array}$ \\
\hline $\begin{array}{l}21 \ldots \ldots \\
22 \ldots-\ldots \\
23 \ldots- \\
24 \ldots- \\
25 \ldots\end{array}$ & $\begin{array}{r}3,510 \\
3,470 \\
2,970 \\
8,180 \\
12,090\end{array}$ & $\begin{array}{r}19 \\
15 \\
18 \\
468 \\
790\end{array}$ & $\begin{array}{r}180 \\
140 \\
144 \\
s 17,400 \\
s 27,500\end{array}$ & $\begin{array}{r}10,200 \\
13,700 \\
8,880 \\
6,940 \\
5,860\end{array}$ & $\begin{array}{l}661 \\
875 \\
480 \\
155 \\
104\end{array}$ & $\begin{array}{r}\mathrm{s} 21,100 \\
32,400 \\
11,500 \\
2,910 \\
1,650\end{array}$ & $\begin{array}{l}8,050 \\
6,440 \\
5,580 \\
4,920 \\
4,510\end{array}$ & $\begin{array}{r}213 \\
280 \\
72 \\
37 \\
78\end{array}$ & $\begin{array}{r}4,630 \\
4,870 \\
1,080 \\
491 \\
950\end{array}$ \\
\hline 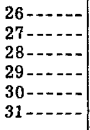 & $\begin{array}{l}8,000 \\
6,140 \\
5,170 \\
4,900 \\
4,820 \\
3,900\end{array}$ & $\begin{array}{r}275 \\
105 \\
62 \\
64 \\
42 \\
44 \\
\end{array}$ & $\begin{array}{r}5,940 \\
1,740 \\
865 \\
847 \\
546 \\
463 \\
\end{array}$ & $\begin{array}{r}5,240 \\
4,950 \\
4,510 \\
- \\
- \\
--\end{array}$ & $\begin{array}{l}58 \\
72 \\
37 \\
-- \\
-- \\
-- \\
\end{array}$ & $\begin{array}{r}821 \\
962 \\
451 \\
-- \\
-- \\
-- \\
\end{array}$ & $\begin{array}{r}3,950 \\
3,510 \\
3,260 \\
3,360 \\
6,600 \\
10,900 \\
\end{array}$ & $\begin{array}{r}93 \\
98 \\
52 \\
145 \\
442 \\
481 \\
\end{array}$ & $\begin{array}{r}992 \\
929 \\
458 \\
1,320 \\
57,840 \\
\mathrm{~s} 14,800 \\
\end{array}$ \\
\hline Total- & 141,870 & -- & 298260 & 201,710 & - & 268,000 & 148,650 & -- & 50,420 \\
\hline
\end{tabular}

e Estımated.

s Computed by subdividing day.

a Includes estimated discharge for missing days. 
Table 17. --SCHUYLKILL RIVER AT MANAYUNK, PHILADELPHIA, PA. --Continued

Suspended sediment, water year October 1950 to September 1951 --Continued

\begin{tabular}{|c|c|c|c|c|c|c|c|c|c|}
\hline \multirow[b]{3}{*}{ Day } & \multicolumn{3}{|c|}{ April } & \multicolumn{3}{|c|}{ May } & \multicolumn{3}{|c|}{ June } \\
\hline & \multirow{2}{*}{$\begin{array}{c}\text { Mean } \\
\text { dis- } \\
\text { charge } \\
\text { (cts) }\end{array}$} & \multicolumn{2}{|c|}{ Suspended sediment } & \multirow{2}{*}{$\begin{array}{c}\text { Mean } \\
\text { dis- } \\
\text { charge } \\
\text { (cfs) }\end{array}$} & \multicolumn{2}{|c|}{ Suspended sedıment } & \multirow{2}{*}{$\begin{array}{c}\text { Mean } \\
\text { dis- } \\
\text { charge } \\
\text { (cfs) }\end{array}$} & \multicolumn{2}{|c|}{ Suspended sedıment } \\
\hline & & $\begin{array}{l}\text { Mean } \\
\text { concen- } \\
\text { tration } \\
(\mathrm{ppm})\end{array}$ & $\begin{array}{l}\text { Tons } \\
\text { per } \\
\text { day }\end{array}$ & & $\begin{array}{l}\text { Mean } \\
\text { concen- } \\
\text { tration } \\
(\mathrm{ppm})\end{array}$ & $\begin{array}{c}\text { Tons } \\
\text { per } \\
\text { day }\end{array}$ & & $\begin{array}{l}\text { Mean } \\
\text { concen- } \\
\text { tration } \\
\text { (ppm) }\end{array}$ & $\begin{array}{l}\text { Tons } \\
\text { per } \\
\text { day }\end{array}$ \\
\hline $\begin{array}{l}1+\cdots \\
2-\cdots \\
3-\cdots- \\
4-\cdots- \\
5-\cdots-\end{array}$ & $\begin{array}{l}6,440 \\
5,560 \\
6,970 \\
5,900 \\
4,660\end{array}$ & $\begin{array}{r}400 \\
51 \\
174 \\
80 \\
40\end{array}$ & $\begin{array}{r}6,960 \\
766 \\
\mathrm{~s} 3,420 \\
1,270 \\
503\end{array}$ & $\begin{array}{l}3,660 \\
2,960 \\
2,620 \\
2,370 \\
2,260\end{array}$ & $\begin{array}{r}27 \\
3 \\
5 \\
8 \\
15\end{array}$ & $\begin{array}{r}267 \\
24 \\
35 \\
51 \\
92\end{array}$ & $\begin{array}{l}1,280 \\
1,130 \\
1,150 \\
2,170 \\
2,320\end{array}$ & $\begin{array}{r}9 \\
11 \\
17 \\
26 \\
32\end{array}$ & $\begin{array}{r}31 \\
33 \\
53 \\
152 \\
200\end{array}$ \\
\hline $\begin{array}{r}6-\cdots-- \\
7-\cdots \\
8 \cdots-\cdots \\
9 \cdots-\cdots \\
10 \cdots\end{array}$ & $\begin{array}{l}4,100 \\
3,750 \\
3,460 \\
3,270 \\
3,110\end{array}$ & $\begin{array}{l}12 \\
31 \\
15 \\
10 \\
25\end{array}$ & $\begin{array}{r}133 \\
314 \\
140 \\
88 \\
210\end{array}$ & $\begin{array}{l}2,150 \\
2,020 \\
1,920 \\
1,760 \\
1,570\end{array}$ & $\begin{array}{r}19 \\
10 \\
7 \\
22 \\
22\end{array}$ & $\begin{array}{r}110 \\
55 \\
36 \\
104 \\
93\end{array}$ & $\begin{array}{l}1,650 \\
1,360 \\
1,150 \\
1,090 \\
1,180\end{array}$ & $\begin{array}{r}18 \\
14 \\
8 \\
15 \\
12\end{array}$ & $\begin{array}{l}80 \\
51 \\
25 \\
44 \\
38\end{array}$ \\
\hline $\begin{array}{l}11 \ldots \ldots \\
12 \cdots \cdots \\
13 \cdots-\cdots \\
14 \cdots \cdots \\
15 \cdots-\cdots\end{array}$ & $\begin{array}{r}3,470 \\
3,510 \\
10,100 \\
8,540 \\
6,700\end{array}$ & $\begin{array}{r}36 \\
25 \\
600 \\
180 \\
85\end{array}$ & $\begin{array}{r}337 \\
237 \\
\mathrm{~s} 18,000 \\
4,150 \\
1,540\end{array}$ & $\begin{array}{l}1,880 \\
2,440 \\
2,220 \\
1,750 \\
1,570\end{array}$ & $\begin{array}{r}32 \\
20 \\
3 \\
6 \\
15\end{array}$ & $\begin{array}{r}163 \\
132 \\
18 \\
28 \\
64\end{array}$ & $\begin{array}{l}1,500 \\
1,690 \\
1,420 \\
2,510 \\
2,750\end{array}$ & $\begin{array}{r}20 \\
25 \\
9 \\
2 \\
8\end{array}$ & $\begin{array}{r}81 \\
114 \\
35 \\
14 \\
59\end{array}$ \\
\hline $\begin{array}{l}16 \ldots \\
17 \ldots \ldots \\
18 \ldots \ldots \\
19 \ldots \ldots \\
20 \ldots \ldots\end{array}$ & $\begin{array}{l}5,470 \\
4,970 \\
4,390 \\
3,910 \\
3,690\end{array}$ & $\begin{array}{l}35 \\
27 \\
31 \\
35 \\
29\end{array}$ & $\begin{array}{l}517 \\
362 \\
367 \\
369 \\
289\end{array}$ & $\begin{array}{l}1,450 \\
1,390 \\
1,310 \\
1,320 \\
1,350\end{array}$ & $\begin{array}{r}20 \\
20 \\
11 \\
9 \\
28\end{array}$ & $\begin{array}{r}78 \\
75 \\
39 \\
32 \\
102\end{array}$ & $\begin{array}{l}2,010 \\
1,600 \\
1,390 \\
1,310 \\
1,140\end{array}$ & $\begin{array}{r}18 \\
19 \\
8 \\
7 \\
2\end{array}$ & $\begin{array}{r}98 \\
82 \\
30 \\
25 \\
6.2\end{array}$ \\
\hline $\begin{array}{l}21 \ldots \ldots \\
22 \ldots \ldots \\
23 \ldots \ldots \\
24 \ldots \ldots \\
25 \ldots \ldots\end{array}$ & $\begin{array}{l}3,350 \\
3,000 \\
3,400 \\
4,310 \\
3,510\end{array}$ & $\begin{array}{l}11 \\
25 \\
20 \\
31 \\
30\end{array}$ & $\begin{array}{r}99 \\
202 \\
184 \\
361 \\
284\end{array}$ & $\begin{array}{l}1,310 \\
1,340 \\
1,480 \\
2,240 \\
2,140\end{array}$ & $\begin{array}{l}16 \\
30 \\
29 \\
21 \\
18\end{array}$ & $\begin{array}{r}57 \\
109 \\
116 \\
127 \\
104\end{array}$ & $\begin{array}{l}1,140 \\
1,020 \\
1,220 \\
1,740 \\
2,010\end{array}$ & $\begin{array}{r}9 \\
15 \\
6 \\
10 \\
19\end{array}$ & $\begin{array}{r}28 \\
41 \\
20 \\
47 \\
103\end{array}$ \\
\hline $\begin{array}{l}26 \ldots \\
27 \ldots \\
28 \cdots \\
29 \cdots \\
30 \ldots \\
31 \ldots \\
\end{array}$ & $\begin{array}{r}3,180 \\
2,980 \\
2,750 \\
2,650 \\
3,280 \\
--\end{array}$ & $\begin{array}{r}17 \\
9 \\
9 \\
9 \\
21 \\
--\end{array}$ & $\begin{array}{r}146 \\
72 \\
67 \\
64 \\
186 \\
--\end{array}$ & $\begin{array}{l}1,520 \\
1,270 \\
1,230 \\
1,390 \\
2,050 \\
1,560\end{array}$ & $\begin{array}{r}20 \\
28 \\
8 \\
15 \\
19 \\
10\end{array}$ & $\begin{array}{r}82 \\
96 \\
27 \\
56 \\
105 \\
42 \\
\end{array}$ & $\begin{array}{r}2,000 \\
1,720 \\
1,390 \\
1,510 \\
3,340 \\
-- \\
\end{array}$ & $\begin{array}{l}31 \\
21 \\
16 \\
30 \\
42 \\
--\end{array}$ & $\begin{array}{r}167 \\
97 \\
60 \\
122 \\
379 \\
--\end{array}$ \\
\hline \multirow[t]{2}{*}{ Total- } & 134,380 & -- & 41,640 & 57,500 & -- & 2,520 & 48,890 & -- & 2,320 \\
\hline & \multicolumn{3}{|c|}{ July } & \multicolumn{3}{|c|}{ August } & \multicolumn{3}{|c|}{ September } \\
\hline $\begin{array}{l}1 \ldots \ldots \\
2 \ldots \ldots \\
3 \ldots-\ldots \\
4 \ldots-\ldots\end{array}$ & $\begin{array}{l}2,890 \\
2,190 \\
1,860 \\
1,530 \\
1,870\end{array}$ & $\begin{array}{l}19 \\
29 \\
30 \\
28 \\
30\end{array}$ & $\begin{array}{l}148 \\
171 \\
151 \\
116 \\
151\end{array}$ & $\begin{array}{l}1,940 \\
1,620 \\
1,410 \\
1,250 \\
1,150\end{array}$ & $\begin{array}{r}10 \\
8 \\
25 \\
32 \\
20\end{array}$ & $\begin{array}{r}52 \\
35 \\
95 \\
108 \\
62\end{array}$ & $\begin{array}{r}705 \\
661 \\
1,600 \\
1,780 \\
1,100\end{array}$ & $\begin{array}{r}3 \\
1 \\
8 \\
18 \\
45\end{array}$ & $\begin{array}{r}5.7 \\
1.8 \\
35 \\
86 \\
134\end{array}$ \\
\hline $\begin{array}{r}6 \\
7 \ldots \ldots \\
8 \ldots \ldots \\
9 \\
90 \ldots\end{array}$ & $\begin{array}{l}2,490 \\
1,730 \\
1,350 \\
1,170 \\
1,080\end{array}$ & $\begin{array}{r}31 \\
35 \\
20 \\
12 \\
8\end{array}$ & $\begin{array}{r}208 \\
164 \\
73 \\
38 \\
23\end{array}$ & $\begin{array}{r}1,070 \\
999 \\
950 \\
983 \\
1,020\end{array}$ & $\begin{array}{r}3 \\
2 \\
12 \\
35 \\
12\end{array}$ & $\begin{array}{l}8.7 \\
5.4 \\
31 \\
93 \\
33\end{array}$ & $\begin{array}{r}903 \\
922 \\
1,120 \\
1,120 \\
864\end{array}$ & $\begin{array}{l}5 \\
8 \\
9 \\
3 \\
4\end{array}$ & $\begin{array}{r}12 \\
20 \\
27 \\
9.1 \\
9.3\end{array}$ \\
\hline $\begin{array}{l}11 \ldots \ldots \\
12 \ldots \ldots \\
13 \ldots \ldots \\
14 \ldots \ldots \\
15 \ldots \ldots\end{array}$ & $\begin{array}{r}989 \\
961 \\
1,730 \\
2,240 \\
1,220\end{array}$ & $\begin{array}{r}5 \\
7 \\
20 \\
19 \\
5\end{array}$ & $\begin{array}{r}13 \\
18 \\
93 \\
115 \\
16\end{array}$ & $\begin{array}{l}1,480 \\
1,680 \\
1,600 \\
2,640 \\
1,670\end{array}$ & $\begin{array}{l}26 \\
25 \\
25 \\
55 \\
23\end{array}$ & $\begin{array}{l}104 \\
113 \\
108 \\
392 \\
104\end{array}$ & $\begin{array}{r}788 \\
760 \\
767 \\
755 \\
1,140\end{array}$ & $\begin{array}{l}3 \\
2 \\
6 \\
6 \\
7\end{array}$ & $\begin{array}{l}6.4 \\
4.1 \\
12 \\
12 \\
22\end{array}$ \\
\hline $\begin{array}{l}16 \ldots \\
17 \ldots \ldots \\
18 \ldots \\
19 \ldots \\
20 \ldots \ldots\end{array}$ & $\begin{array}{r}1,020 \\
941 \\
1,490 \\
1,790 \\
3,430\end{array}$ & $\begin{array}{r}9 \\
9 \\
20 \\
10 \\
10\end{array}$ & $\begin{array}{l}25 \\
23 \\
80 \\
48 \\
93\end{array}$ & $\begin{array}{l}1,390 \\
1,360 \\
1,780 \\
1,420 \\
1,350\end{array}$ & $\begin{array}{r}21 \\
6 \\
7 \\
13 \\
48\end{array}$ & $\begin{array}{r}79 \\
22 \\
34 \\
50 \\
175\end{array}$ & $\begin{array}{r}1,670 \\
1,190 \\
949 \\
848 \\
794\end{array}$ & $\begin{array}{l}8 \\
2 \\
2 \\
1 \\
3\end{array}$ & $\begin{array}{l}36 \\
6.4 \\
5.1 \\
2.3 \\
6.4\end{array}$ \\
\hline $\begin{array}{l}21 \ldots \\
22 \ldots \ldots \\
23 \ldots \\
24 \ldots \\
25 \ldots\end{array}$ & $\begin{array}{l}2,170 \\
1,390 \\
1,190 \\
1,230 \\
1,300\end{array}$ & $\begin{array}{r}5 \\
7 \\
22 \\
18 \\
30\end{array}$ & $\begin{array}{r}29 \\
26 \\
71 \\
60 \\
105\end{array}$ & $\begin{array}{r}1,520 \\
1,250 \\
1,080 \\
993 \\
926\end{array}$ & $\begin{array}{r}12 \\
2 \\
9 \\
10 \\
12\end{array}$ & $\begin{array}{l}49 \\
6.8 \\
26 \\
27 \\
30\end{array}$ & $\begin{array}{l}744 \\
703 \\
713 \\
631 \\
678\end{array}$ & $\begin{array}{l}5 \\
6 \\
1 \\
1 \\
5\end{array}$ & $\begin{array}{r}10 \\
11 \\
1.9 \\
1.7 \\
9.2\end{array}$ \\
\hline 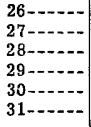 & $\begin{array}{r}1,110 \\
971 \\
860 \\
3,580 \\
3,530 \\
2,520 \\
\end{array}$ & $\begin{array}{r}8 \\
2 \\
2 \\
160 \\
50 \\
19 \\
\end{array}$ & $\begin{array}{c}24 \\
5.2 \\
4.6 \\
1,550 \\
477 \\
129\end{array}$ & $\begin{array}{l}894 \\
846 \\
816 \\
785 \\
733 \\
724 \\
\end{array}$ & $\begin{array}{r}3 \\
1 \\
2 \\
10 \\
39 \\
10 \\
\end{array}$ & $\begin{array}{l}7.2 \\
2.3 \\
4.4 \\
21 \\
81 \\
20\end{array}$ & $\begin{array}{r}659 \\
653 \\
649 \\
607 \\
694 \\
-- \\
\end{array}$ & $\begin{array}{r}4 \\
5 \\
5 \\
4 \\
6 \\
--\end{array}$ & $\begin{array}{r}7.1 \\
8.8 \\
8.7 \\
6.6 \\
11 \\
\end{array}$ \\
\hline Total- & 53,822 & - & 4.250 & 39,369 & -- & 1,980 & 27,167 & -- & 529 \\
\hline
\end{tabular}

s Computed by subdividing day. 
Table 17, --SCHUYL.KILL RIVER AT MANAYUNK, PHILADELPHIA, PA. --Continued

Suspended sediment, water year October 1951 to Septem ber 1952

\begin{tabular}{|c|c|c|c|c|c|c|c|c|c|}
\hline \multirow[b]{3}{*}{ Day } & \multicolumn{3}{|c|}{ October } & \multicolumn{3}{|c|}{ November } & \multicolumn{3}{|c|}{ December } \\
\hline & \multirow{2}{*}{$\begin{array}{c}\text { Mean } \\
\text { dis- } \\
\text { charge } \\
\text { (cfs) }\end{array}$} & \multicolumn{2}{|c|}{ Suspended sediment } & \multirow{2}{*}{$\begin{array}{c}\text { Mean } \\
\text { dis- } \\
\text { charge } \\
\text { (cfs) }\end{array}$} & \multicolumn{2}{|c|}{ Suspended sediment } & \multirow{2}{*}{$\begin{array}{l}\text { Mean } \\
\text { dis- } \\
\text { charge } \\
\text { (cfs) }\end{array}$} & \multicolumn{2}{|c|}{ Suspended sedıment } \\
\hline & & $\begin{array}{c}\text { Mean } \\
\text { concen- } \\
\text { tration } \\
\text { (ppm) }\end{array}$ & $\begin{array}{c}\text { Tons } \\
\text { per } \\
\text { day }\end{array}$ & & $\begin{array}{c}\text { Mean } \\
\text { concen- } \\
\text { tration } \\
\text { (ppm) }\end{array}$ & $\begin{array}{l}\text { Tons } \\
\text { per } \\
\text { day }\end{array}$ & & $\begin{array}{c}\text { Mean } \\
\text { concen- } \\
\text { tration } \\
(\text { ppm) }\end{array}$ & $\begin{array}{l}\text { Tons } \\
\text { per } \\
\text { day }\end{array}$ \\
\hline $\begin{array}{l}1 \\
2 \\
3 \\
4 \\
5 \\
5\end{array} \ldots$ & $\begin{array}{l}651 \\
643 \\
653 \\
619 \\
600\end{array}$ & $\begin{array}{r}2 \\
5 \\
6 \\
7 \\
13\end{array}$ & $\begin{array}{r}4 \\
9 \\
11 \\
12 \\
21\end{array}$ & $\begin{array}{r}3,110 \\
5,060 \\
9,740 \\
11,400 \\
6,630\end{array}$ & $\begin{array}{l}125 \\
192 \\
880 \\
910 \\
260\end{array}$ & $\begin{array}{r}1,050 \\
2,620 \\
23,100 \\
28,000 \\
4,650\end{array}$ & $\begin{array}{l}2,120 \\
2,060 \\
1,960 \\
1,890 \\
3,250\end{array}$ & $\begin{array}{r}10 \\
5 \\
6 \\
7 \\
16\end{array}$ & $\begin{array}{r}57 \\
28 \\
32 \\
36 \\
140\end{array}$ \\
\hline $\begin{array}{r}6 \ldots-\ldots \\
7 \\
8 \\
9 \\
10 \\
\end{array}$ & $\begin{array}{r}598 \\
662 \\
1,230 \\
1,860 \\
1,170\end{array}$ & $\begin{array}{l}10 \\
28 \\
40 \\
30 \\
15\end{array}$ & $\begin{array}{r}16 \\
50 \\
133 \\
151 \\
47\end{array}$ & $\begin{array}{r}4,570 \\
16,500 \\
19,900 \\
9,830 \\
6,480\end{array}$ & $\begin{array}{r}50 \\
630 \\
1,220 \\
420 \\
118\end{array}$ & $\begin{array}{r}617 \\
28,100 \\
65,600 \\
11,100 \\
2,060\end{array}$ & $\begin{array}{r}14,300 \\
9,520 \\
6,870 \\
5,460 \\
4,480\end{array}$ & $\begin{array}{r}1,080 \\
380 \\
100 \\
52 \\
43\end{array}$ & $\begin{array}{r}41,700 \\
9,770 \\
1,860 \\
767 \\
520\end{array}$ \\
\hline $\begin{array}{l}11 \ldots \ldots \\
12 \ldots \ldots \\
13 \ldots \ldots \\
14 \ldots \ldots \\
15 \ldots \ldots\end{array}$ & $\begin{array}{r}1,100 \\
1,100 \\
1,100 \\
905 \\
804\end{array}$ & $\begin{array}{l}15 \\
11 \\
14 \\
12 \\
10\end{array}$ & $\begin{array}{l}45 \\
33 \\
42 \\
29 \\
22\end{array}$ & $\begin{array}{l}4,970 \\
4,100 \\
3,470 \\
3,170 \\
3,660\end{array}$ & $\begin{array}{l}50 \\
30 \\
20 \\
15 \\
15\end{array}$ & $\begin{array}{l}671 \\
332 \\
187 \\
128 \\
148\end{array}$ & $\begin{array}{l}3,800 \\
3,420 \\
3,130 \\
2,750 \\
2,890\end{array}$ & $\begin{array}{l}28 \\
20 \\
15 \\
10 \\
11\end{array}$ & $\begin{array}{r}287 \\
185 \\
127 \\
74 \\
86\end{array}$ \\
\hline $\begin{array}{l}16 \ldots-\ldots \\
17 \ldots \\
18 \\
19 \\
20\end{array}$ & $\begin{array}{l}794 \\
770 \\
727 \\
735 \\
704\end{array}$ & $\begin{array}{l}18 \\
18 \\
15 \\
15 \\
12\end{array}$ & $\begin{array}{l}39 \\
37 \\
29 \\
30 \\
23\end{array}$ & $\begin{array}{l}3,760 \\
4,250 \\
4,170 \\
3,650 \\
3,220\end{array}$ & $\begin{array}{l}18 \\
25 \\
32 \\
30 \\
20\end{array}$ & $\begin{array}{l}183 \\
287 \\
360 \\
296 \\
174\end{array}$ & $\begin{array}{l}3,300 \\
2,310 \\
2,190 \\
2,760 \\
3,080\end{array}$ & $\begin{array}{r}18 \\
11 \\
9 \\
14 \\
11\end{array}$ & $\begin{array}{r}160 \\
69 \\
53 \\
104 \\
\mathrm{a} 91\end{array}$ \\
\hline $\begin{array}{l}21 \ldots \ldots \\
22 \ldots \ldots \\
23 \ldots \ldots \\
24 \ldots \ldots \\
25 \ldots \ldots \\
\end{array}$ & $\begin{array}{r}668 \\
678 \\
682 \\
758 \\
1,010\end{array}$ & $\begin{array}{r}9 \\
10 \\
11 \\
20 \\
19\end{array}$ & $\begin{array}{l}16 \\
18 \\
20 \\
41 \\
52\end{array}$ & $\begin{array}{l}2,830 \\
2,550 \\
2,370 \\
2,350 \\
2,420\end{array}$ & $\begin{array}{r}10 \\
5 \\
5 \\
2 \\
2\end{array}$ & $\begin{array}{l}76 \\
34 \\
32 \\
13 \\
13\end{array}$ & $\begin{array}{r}26,000 \\
16,900 \\
9,290 \\
6,870 \\
5,720\end{array}$ & $\begin{array}{r}1,280 \\
830 \\
220 \\
55 \\
40\end{array}$ & $\begin{array}{r}\text { a } 89,900 \\
\text { a } 37,900 \\
5,520 \\
1,020 \\
\text { a } 618\end{array}$ \\
\hline $\begin{array}{l}26 \ldots \ldots \\
27 \ldots \ldots \\
28 \\
29 \\
30 \\
31 \ldots\end{array}$ & $\begin{array}{r}1,120 \\
1,060 \\
870 \\
868 \\
1,110 \\
941 \\
\end{array}$ & $\begin{array}{l}20 \\
15 \\
16 \\
10 \\
12 \\
28 \\
\end{array}$ & $\begin{array}{l}60 \\
43 \\
38 \\
23 \\
36 \\
71 \\
\end{array}$ & $\begin{array}{r}3,270 \\
3,520 \\
2,760 \\
2,310 \\
2,200 \\
- \\
\end{array}$ & $\begin{array}{l}20 \\
30 \\
10 \\
10 \\
20 \\
-- \\
\end{array}$ & $\begin{array}{r}177 \\
285 \\
75 \\
62 \\
119 \\
-- \\
\end{array}$ & $\begin{array}{l}6,230 \\
6,040 \\
4,470 \\
3,900 \\
3,730 \\
4,160 \\
\end{array}$ & $\begin{array}{l}42 \\
40 \\
28 \\
20 \\
15 \\
20 \\
\end{array}$ & $\begin{array}{l}706 \\
652 \\
338 \\
211 \\
151 \\
\mathbf{2 2 5} \\
\end{array}$ \\
\hline \multirow{2}{*}{ Total- } & 27,190 & -- & 1,201 & 158,220 & -- & 170,549 & 174,850 & $\because$ & 193,387 \\
\hline & \multicolumn{3}{|c|}{ January } & \multicolumn{3}{|c|}{ February } & \multicolumn{3}{|c|}{ March } \\
\hline $\begin{array}{l}1 \ldots \\
2 \ldots \\
3 \\
4-\ldots \\
5 \\
\end{array}$ & $\begin{array}{r}4,850 \\
7,880 \\
11,100 \\
11,100 \\
9,350\end{array}$ & $\begin{array}{r}38 \\
95 \\
188 \\
210 \\
106\end{array}$ & $\begin{array}{r}498 \\
2,020 \\
5,630 \\
6,290 \\
2,680\end{array}$ & $\begin{array}{r}6,240 \\
5,930 \\
5,690 \\
13,900 \\
12,800\end{array}$ & $\begin{array}{r}40 \\
30 \\
28 \\
531 \\
420\end{array}$ & $\begin{array}{r}674 \\
480 \\
430 \\
\mathrm{~s} 25,300 \\
14,500\end{array}$ & $\begin{array}{l}2,280 \\
2,310 \\
2,240 \\
2,300 \\
4,680\end{array}$ & $\begin{array}{r}5 \\
6 \\
4 \\
8 \\
45\end{array}$ & $\begin{array}{r}31 \\
37 \\
24 \\
50 \\
569\end{array}$ \\
\hline $\begin{array}{r}6-\ldots \ldots \\
7 \\
8 \ldots \ldots \\
9 \\
10-\ldots \ldots\end{array}$ & $\begin{array}{l}7,920 \\
6,270 \\
5,160 \\
4,610 \\
4,590\end{array}$ & $\begin{array}{l}65 \\
55 \\
35 \\
25 \\
29\end{array}$ & $\begin{array}{r}1,390 \\
931 \\
488 \\
311 \\
359\end{array}$ & $\begin{array}{l}8,490 \\
7,280 \\
6,260 \\
5,630 \\
5,100\end{array}$ & $\begin{array}{r}130 \\
58 \\
50 \\
35 \\
38\end{array}$ & $\begin{array}{r}2,980 \\
1,140 \\
845 \\
532 \\
523\end{array}$ & $\begin{array}{l}5,200 \\
3,940 \\
3,410 \\
3,140 \\
3,050\end{array}$ & $\begin{array}{r}45 \\
16 \\
7 \\
5 \\
5\end{array}$ & $\begin{array}{r}632 \\
170 \\
64 \\
42 \\
41\end{array}$ \\
\hline $\begin{array}{l}11-\ldots \\
12 \\
13 \ldots \\
14 \ldots \\
15-\ldots\end{array}$ & $\begin{array}{l}4,900 \\
4,200 \\
3,600 \\
3,390 \\
3,600\end{array}$ & $\begin{array}{l}42 \\
26 \\
15 \\
16 \\
15\end{array}$ & $\begin{array}{l}556 \\
295 \\
146 \\
146 \\
146\end{array}$ & $\begin{array}{l}4,710 \\
4,450 \\
4,000 \\
3,480 \\
3,200\end{array}$ & $\begin{array}{l}25 \\
25 \\
10 \\
15 \\
25\end{array}$ & $\begin{array}{l}318 \\
300 \\
108 \\
141 \\
216\end{array}$ & $\begin{array}{r}20,400 \\
29,500 \\
14,700 \\
10,300 \\
7,690\end{array}$ & $\begin{array}{r}1,210 \\
1,750 \\
450 \\
175 \\
85\end{array}$ & $\begin{array}{r}112,000 \\
139,400 \\
17,900 \\
4,870 \\
1,760\end{array}$ \\
\hline $\begin{array}{l}16 \ldots \ldots \\
17 \ldots \ldots \\
18 \ldots \ldots \\
19 \ldots \ldots\end{array}$ & $\begin{array}{l}4,100 \\
3,800 \\
6,300 \\
6,490 \\
5,640\end{array}$ & $\begin{array}{l}17 \\
15 \\
70 \\
88 \\
55\end{array}$ & $\begin{array}{r}188 \\
154 \\
1,190 \\
1,540 \\
838\end{array}$ & $\begin{array}{l}3,160 \\
3,960 \\
4,500 \\
3,780 \\
3,220\end{array}$ & $\begin{array}{r}10 \\
20 \\
35 \\
10 \\
5\end{array}$ & $\begin{array}{r}85 \\
214 \\
425 \\
102 \\
43\end{array}$ & $\begin{array}{l}6,290 \\
5,490 \\
4,880 \\
6,440 \\
9,520\end{array}$ & $\begin{array}{r}68 \\
42 \\
38 \\
92 \\
178\end{array}$ & $\begin{array}{r}1,150 \\
623 \\
501 \\
1,600 \\
4,580\end{array}$ \\
\hline $\begin{array}{l}21 \ldots \ldots \\
22 \ldots \ldots \\
23 \ldots \ldots \\
24 \ldots \ldots \\
25 \ldots\end{array}$ & $\begin{array}{l}5,480 \\
4,890 \\
8,150 \\
6,740 \\
5,160\end{array}$ & $\begin{array}{r}40 \\
25 \\
100 \\
75 \\
31\end{array}$ & $\begin{array}{r}592 \\
330 \\
2,200 \\
1,360 \\
432\end{array}$ & $\begin{array}{l}3,010 \\
2,970 \\
2,760 \\
2,610 \\
2,620\end{array}$ & $\begin{array}{r}4 \\
7 \\
4 \\
4 \\
10\end{array}$ & $\begin{array}{l}33 \\
56 \\
30 \\
28 \\
71\end{array}$ & $\begin{array}{l}6,370 \\
5,960 \\
6,820 \\
8,310 \\
6,940\end{array}$ & $\begin{array}{l}53 \\
35 \\
59 \\
65 \\
37\end{array}$ & $\begin{array}{r}912 \\
563 \\
1,090 \\
1,460 \\
693\end{array}$ \\
\hline $\begin{array}{l}26 \ldots \ldots \\
27 \ldots \\
28 \ldots \\
29 \\
30 \ldots \ldots \\
31 \ldots \ldots\end{array}$ & $\begin{array}{r}9,700 \\
14,200 \\
17,000 \\
13,500 \\
9,230 \\
7,220 \\
\end{array}$ & $\begin{array}{r}310 \\
510 \\
640 \\
325 \\
120 \\
70 \\
\end{array}$ & $\begin{array}{r}8,120 \\
19,600 \\
29,400 \\
11,800 \\
2,990 \\
1,360 \\
\end{array}$ & $\begin{array}{r}2,500 \\
2,390 \\
2,330 \\
2,280 \\
-. \\
-- \\
\end{array}$ & $\begin{array}{r}5 \\
5 \\
5 \\
6 \\
-- \\
-- \\
\end{array}$ & $\begin{array}{l}34 \\
32 \\
31 \\
37 \\
-- \\
-- \\
\end{array}$ & $\begin{array}{l}6,240 \\
5,590 \\
5,040 \\
4,560 \\
4,320 \\
3,990 \\
\end{array}$ & $\begin{array}{r}26 \\
20 \\
15 \\
11 \\
12 \\
8 \\
\end{array}$ & $\begin{array}{r}438 \\
302 \\
204 \\
135 \\
140 \\
86 \\
\end{array}$ \\
\hline Total- & 220,120 & -- & 103,980 & 139,250 & -- & 49,708 & 211,900 & $\cdots$ & 292,067 \\
\hline
\end{tabular}

s Computed by subdividing day.

a Computed from estimated concentration graph. 
Table 17. --SCHUYLKILL RIVER aT MANAYUNK, PHILADELPHIA, PA. --Continued

Suspended sediment, water year October 1951 to September $1952--$ Continued

\begin{tabular}{|c|c|c|c|c|c|c|c|c|c|}
\hline \multirow[b]{3}{*}{ Day } & \multicolumn{3}{|c|}{ April } & \multicolumn{3}{|c|}{ May } & \multicolumn{3}{|c|}{ June } \\
\hline & \multirow{2}{*}{$\begin{array}{l}\text { Mean } \\
\text { dis:- } \\
\text { charge } \\
\text { (cfs) }\end{array}$} & \multicolumn{2}{|c|}{ Suspended sediment } & \multirow{2}{*}{$\begin{array}{l}\text { Mean } \\
\text { dis- } \\
\text { charge } \\
\text { (cfs) }\end{array}$} & \multicolumn{2}{|c|}{ Suspended sediment } & \multirow{2}{*}{$\begin{array}{c}\text { Mean } \\
\text { dis - } \\
\text { charge } \\
\text { (cfs) }\end{array}$} & \multicolumn{2}{|c|}{ Suspended sediment } \\
\hline & & $\begin{array}{c}\text { Mean } \\
\text { concen- } \\
\text { tration } \\
(\mathrm{ppm})\end{array}$ & $\begin{array}{l}\text { Tons } \\
\text { per } \\
\text { day }\end{array}$ & & $\begin{array}{c}\text { Mean } \\
\text { concen- } \\
\text { tration } \\
(\mathrm{ppm})\end{array}$ & $\begin{array}{l}\text { Tons } \\
\text { per } \\
\text { day }\end{array}$ & & $\begin{array}{c}\text { Mean } \\
\text { concen- } \\
\text { tration } \\
(\mathrm{ppm}) \\
\end{array}$ & $\begin{array}{l}\text { Tons } \\
\text { per } \\
\text { day }\end{array}$ \\
\hline $\begin{array}{l}1 \\
2 \ldots+ \\
3 \\
3 \\
4 \\
5 \\
-\end{array}$ & $\begin{array}{l}3,590 \\
3,830 \\
5,760 \\
4,680 \\
8,740\end{array}$ & $\begin{array}{r}9 \\
8 \\
42 \\
20 \\
226\end{array}$ & $\begin{array}{r}87 \\
83 \\
653 \\
253 \\
\mathrm{~s} 8,040\end{array}$ & $\begin{array}{r}11,400 \\
8,990 \\
7,410 \\
6,440 \\
5,660\end{array}$ & $\begin{array}{r}136 \\
75 \\
50 \\
39 \\
25\end{array}$ & $\begin{array}{r}4,190 \\
1,820 \\
1,000 \\
678 \\
382\end{array}$ & $\begin{array}{r}14,800 \\
11,000 \\
6,130 \\
4,970 \\
4,710\end{array}$ & $\begin{array}{r}340 \\
180 \\
40 \\
30 \\
16\end{array}$ & $\begin{array}{r}13,600 \\
5,350 \\
662 \\
403 \\
203\end{array}$ \\
\hline $\begin{array}{r}6-\cdots \\
7-\cdots \\
8 \cdots \\
9-\cdots \\
10 \cdots\end{array}$ & $\begin{array}{r}12,100 \\
8,670 \\
7,070 \\
5,920 \\
5,190\end{array}$ & $\begin{array}{r}270 \\
120 \\
45 \\
28 \\
25\end{array}$ & $\begin{array}{r}8,820 \\
2,810 \\
859 \\
448 \\
350\end{array}$ & $\begin{array}{l}5,200 \\
4,790 \\
4,310 \\
4,010 \\
3,820\end{array}$ & $\begin{array}{l}24 \\
22 \\
35 \\
20 \\
18\end{array}$ & $\begin{array}{l}337 \\
285 \\
407 \\
217 \\
186\end{array}$ & $\begin{array}{l}4,550 \\
3,950 \\
3,460 \\
3,210 \\
2,980\end{array}$ & $\begin{array}{l}15 \\
18 \\
13 \\
10 \\
10\end{array}$ & $\begin{array}{r}184 \\
192 \\
121 \\
87 \\
80\end{array}$ \\
\hline $\begin{array}{l}11 \cdots-\cdots \\
12 \cdots- \\
13 \cdots- \\
14 \cdots \\
15 \cdots-\end{array}$ & $\begin{array}{r}4,700 \\
4,240 \\
3,920 \\
6,460 \\
12,800\end{array}$ & $\begin{array}{r}21 \\
15 \\
9 \\
41 \\
355\end{array}$ & $\begin{array}{r}266 \\
172 \\
95 \\
715 \\
12,300\end{array}$ & $\begin{array}{l}3,960 \\
5,520 \\
7,860 \\
6,240 \\
5,310\end{array}$ & $\begin{array}{r}19 \\
60 \\
115 \\
52 \\
25\end{array}$ & $\begin{array}{r}203 \\
894 \\
2,440 \\
876 \\
358\end{array}$ & $\begin{array}{l}2,830 \\
2,530 \\
2,330 \\
2,220 \\
2,110\end{array}$ & $\begin{array}{r}15 \\
8 \\
14 \\
8 \\
8\end{array}$ & $\begin{array}{r}115 \\
55 \\
88 \\
48 \\
46\end{array}$ \\
\hline $\begin{array}{l}16 \ldots \ldots \\
17 \ldots \ldots \\
18 \ldots \ldots \\
19 \ldots \ldots \\
20 \ldots \ldots\end{array}$ & $\begin{array}{r}13,100 \\
9,340 \\
7,620 \\
6,360 \\
5,580\end{array}$ & $\begin{array}{r}260 \\
110 \\
55 \\
35 \\
30\end{array}$ & $\begin{array}{r}9,200 \\
2,770 \\
1,130 \\
601 \\
452\end{array}$ & $\begin{array}{l}4,750 \\
4,130 \\
4,140 \\
4,580 \\
4,300\end{array}$ & $\begin{array}{l}20 \\
10 \\
10 \\
21 \\
25\end{array}$ & $\begin{array}{l}257 \\
112 \\
112 \\
260 \\
290\end{array}$ & $\begin{array}{l}1,990 \\
1,940 \\
1,830 \\
1,830 \\
1,820\end{array}$ & $\begin{array}{r}9 \\
7 \\
6 \\
8 \\
11\end{array}$ & $\begin{array}{l}48 \\
37 \\
30 \\
40 \\
54\end{array}$ \\
\hline $\begin{array}{l}21 \ldots \ldots- \\
22 \ldots \ldots \\
23 \ldots \ldots \\
24 \ldots \ldots- \\
25-\ldots\end{array}$ & $\begin{array}{l}5,020 \\
4,530 \\
4,160 \\
3,920 \\
4,260\end{array}$ & $\begin{array}{r}26 \\
25 \\
15 \\
5 \\
19\end{array}$ & $\begin{array}{r}352 \\
306 \\
168 \\
53 \\
219\end{array}$ & $\begin{array}{l}5,620 \\
4,480 \\
3,690 \\
3,410 \\
9,100\end{array}$ & $\begin{array}{r}42 \\
18 \\
10 \\
10 \\
326\end{array}$ & $\begin{array}{r}637 \\
218 \\
100 \\
92 \\
\mathrm{~s} 14,000\end{array}$ & $\begin{array}{l}1,800 \\
1,750 \\
1,920 \\
2,060 \\
2,010\end{array}$ & $\begin{array}{l}15 \\
19 \\
15 \\
15 \\
11\end{array}$ & $\begin{array}{l}73 \\
90 \\
78 \\
83 \\
60\end{array}$ \\
\hline $\begin{array}{l}26 \ldots \ldots \\
27 \ldots \ldots \\
28 \ldots \\
29-\ldots \\
30 \\
31 \ldots \ldots\end{array}$ & $\begin{array}{r}11,100 \\
14,200 \\
40,400 \\
26,200 \\
16,200 \\
\ldots\end{array}$ & $\begin{array}{r}192 \\
280 \\
2,040 \\
801 \\
450 \\
-- \\
\end{array}$ & $\begin{array}{r}5,750 \\
10,700 \\
\mathrm{~s} 244,000 \\
56,700 \\
19,700 \\
-- \\
\end{array}$ & $\begin{array}{r}21,700 \\
11,400 \\
7,690 \\
6,250 \\
5,790 \\
5,730 \\
\end{array}$ & $\begin{array}{r}952 \\
190 \\
55 \\
36 \\
35 \\
32 \\
\end{array}$ & $\begin{array}{r}\mathrm{s} 57,700 \\
5,850 \\
1,140 \\
608 \\
547 \\
495 \\
\end{array}$ & $\begin{array}{r}1,730 \\
1,510 \\
1,380 \\
1,280 \\
1,230 \\
-- \\
\end{array}$ & $\begin{array}{l}12 \\
11 \\
12 \\
16 \\
16 \\
-- \\
\end{array}$ & $\begin{array}{l}56 \\
45 \\
45 \\
55 \\
53 \\
--\end{array}$ \\
\hline \multirow[t]{2}{*}{ Total- } & 269,660 & -- & 388,052 & 197,680 & $\ldots$ & 96,691 & 97,860 & -- & 22,081 \\
\hline & \multicolumn{3}{|c|}{ July } & \multicolumn{3}{|c|}{ August } & \multicolumn{3}{|c|}{ September } \\
\hline 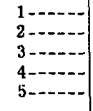 & $\begin{array}{l}1,190 \\
1,120 \\
1,080 \\
1,110 \\
1,100\end{array}$ & $\begin{array}{r}12 \\
4 \\
5 \\
4 \\
7\end{array}$ & $\begin{array}{l}39 \\
12 \\
15 \\
12 \\
21\end{array}$ & $\begin{array}{l}1,220 \\
1,220 \\
1,800 \\
1,640 \\
1,270\end{array}$ & $\begin{array}{r}8 \\
9 \\
10 \\
8 \\
8\end{array}$ & $\begin{array}{l}26 \\
30 \\
49 \\
35 \\
27\end{array}$ & $\begin{array}{r}6,460 \\
18,100 \\
13,400 \\
8,340 \\
5,440\end{array}$ & $\begin{array}{r}540 \\
1,400 \\
410 \\
140 \\
59\end{array}$ & $\begin{array}{r}9,420 \\
68,400 \\
14,800 \\
3,150 \\
867\end{array}$ \\
\hline $\begin{array}{r}6 \\
7 \\
8 \\
8 \\
9 \\
10 \\
\end{array}$ & $\begin{array}{r}1,010 \\
980 \\
1,330 \\
4,030 \\
19,600\end{array}$ & $\begin{array}{r}10 \\
7 \\
14 \\
120 \\
1,480\end{array}$ & $\begin{array}{r}27 \\
19 \\
50 \\
131 \\
78,300\end{array}$ & $\begin{array}{l}1,540 \\
2,010 \\
2,340 \\
2,120 \\
2,320\end{array}$ & $\begin{array}{l}20 \\
22 \\
26 \\
34 \\
35\end{array}$ & $\begin{array}{r}83 \\
119 \\
164 \\
195 \\
219\end{array}$ & $\begin{array}{l}4,290 \\
3,580 \\
3,280 \\
2,840 \\
2,470\end{array}$ & $\begin{array}{l}32 \\
21 \\
18 \\
13 \\
10\end{array}$ & $\begin{array}{r}371 \\
203 \\
159 \\
100 \\
67\end{array}$ \\
\hline $\begin{array}{l}11 \ldots-\ldots \\
12 \ldots- \\
13 \cdots \\
14-\ldots \\
15 \cdots\end{array}$ & $\begin{array}{l}9,150 \\
4,770 \\
3,400 \\
2,740 \\
2,280\end{array}$ & $\begin{array}{r}320 \\
48 \\
22 \\
17 \\
10\end{array}$ & $\begin{array}{r}7,910 \\
618 \\
202 \\
126 \\
62\end{array}$ & $\begin{array}{l}2,520 \\
2,790 \\
2,120 \\
2,040 \\
1,720\end{array}$ & $\begin{array}{l}29 \\
30 \\
24 \\
16 \\
14\end{array}$ & $\begin{array}{r}197 \\
226 \\
137 \\
88 \\
65\end{array}$ & $\begin{array}{l}2,250 \\
2,110 \\
1,940 \\
1,730 \\
1,670\end{array}$ & $\begin{array}{l}12 \\
11 \\
10 \\
10 \\
11\end{array}$ & $\begin{array}{l}73 \\
63 \\
52 \\
47 \\
50\end{array}$ \\
\hline $\begin{array}{l}16 \ldots \\
17 \ldots \\
18 \ldots \\
19 \\
20 \ldots\end{array}$ & $\begin{array}{l}2,050 \\
1,900 \\
1,780 \\
1,650 \\
1,630\end{array}$ & $\begin{array}{r}18 \\
8 \\
9 \\
9 \\
14\end{array}$ & $\begin{array}{r}100 \\
41 \\
43 \\
40 \\
62\end{array}$ & $\begin{array}{l}1,870 \\
3,050 \\
3,640 \\
2,590 \\
2,060\end{array}$ & $\begin{array}{l}22 \\
34 \\
56 \\
28 \\
20\end{array}$ & $\begin{array}{l}111 \\
280 \\
550 \\
196 \\
111\end{array}$ & $\begin{array}{l}1,720 \\
2,340 \\
1,760 \\
2,870 \\
3,130\end{array}$ & $\begin{array}{l}18 \\
14 \\
12 \\
50 \\
60\end{array}$ & $\begin{array}{r}84 \\
88 \\
57 \\
387 \\
507\end{array}$ \\
\hline $\begin{array}{l}21 \ldots \ldots \\
22 \ldots \ldots \\
23 \ldots \\
24 \ldots \ldots \\
25 \ldots\end{array}$ & $\begin{array}{l}1,720 \\
2,380 \\
2,320 \\
1,920 \\
1,510\end{array}$ & $\begin{array}{r}12 \\
13 \\
9 \\
8 \\
11\end{array}$ & $\begin{array}{l}56 \\
84 \\
56 \\
41 \\
45\end{array}$ & $\begin{array}{l}1,810 \\
1,630 \\
1,750 \\
1,520 \\
1,310\end{array}$ & $\begin{array}{l}20 \\
13 \\
16 \\
12 \\
16\end{array}$ & $\begin{array}{l}98 \\
57 \\
76 \\
49 \\
57\end{array}$ & $\begin{array}{l}2,090 \\
1,650 \\
1,610 \\
1,620 \\
1,470\end{array}$ & $\begin{array}{l}34 \\
25 \\
19 \\
14 \\
14\end{array}$ & $\begin{array}{r}192 \\
111 \\
83 \\
61 \\
56\end{array}$ \\
\hline $\begin{array}{l}26 \ldots \ldots \\
27 \ldots \ldots \\
28 \ldots \\
29 \\
30 \ldots \\
31 \ldots \ldots\end{array}$ & $\begin{array}{l}1,360 \\
1,320 \\
1,360 \\
1,400 \\
1,410 \\
1,250 \\
\end{array}$ & $\begin{array}{l}8 \\
7 \\
6 \\
8 \\
9 \\
7 \\
\end{array}$ & $\begin{array}{l}29 \\
25 \\
22 \\
30 \\
34 \\
24 \\
\end{array}$ & $\begin{array}{l}1,260 \\
1,110 \\
1,090 \\
1,090 \\
1,310 \\
2,020 \\
\end{array}$ & $\begin{array}{l}12 \\
13 \\
10 \\
12 \\
22 \\
47 \\
\end{array}$ & $\begin{array}{r}41 \\
39 \\
29 \\
35 \\
78 \\
256 \\
\end{array}$ & $\begin{array}{r}1,380 \\
1,270 \\
1,220 \\
1,190 \\
1,180 \\
-- \\
\end{array}$ & $\begin{array}{r}11 \\
10 \\
8 \\
9 \\
10 \\
-- \\
\end{array}$ & $\begin{array}{l}41 \\
34 \\
26 \\
29 \\
32 \\
--\end{array}$ \\
\hline Total- & 81,850 & $\cdots$ & 88,276 & 57,780 & -- & 3,723 & 104,400 & -- & 99,610 \\
\hline
\end{tabular}

$\mathrm{s}$ Computed by subdıviding day. 


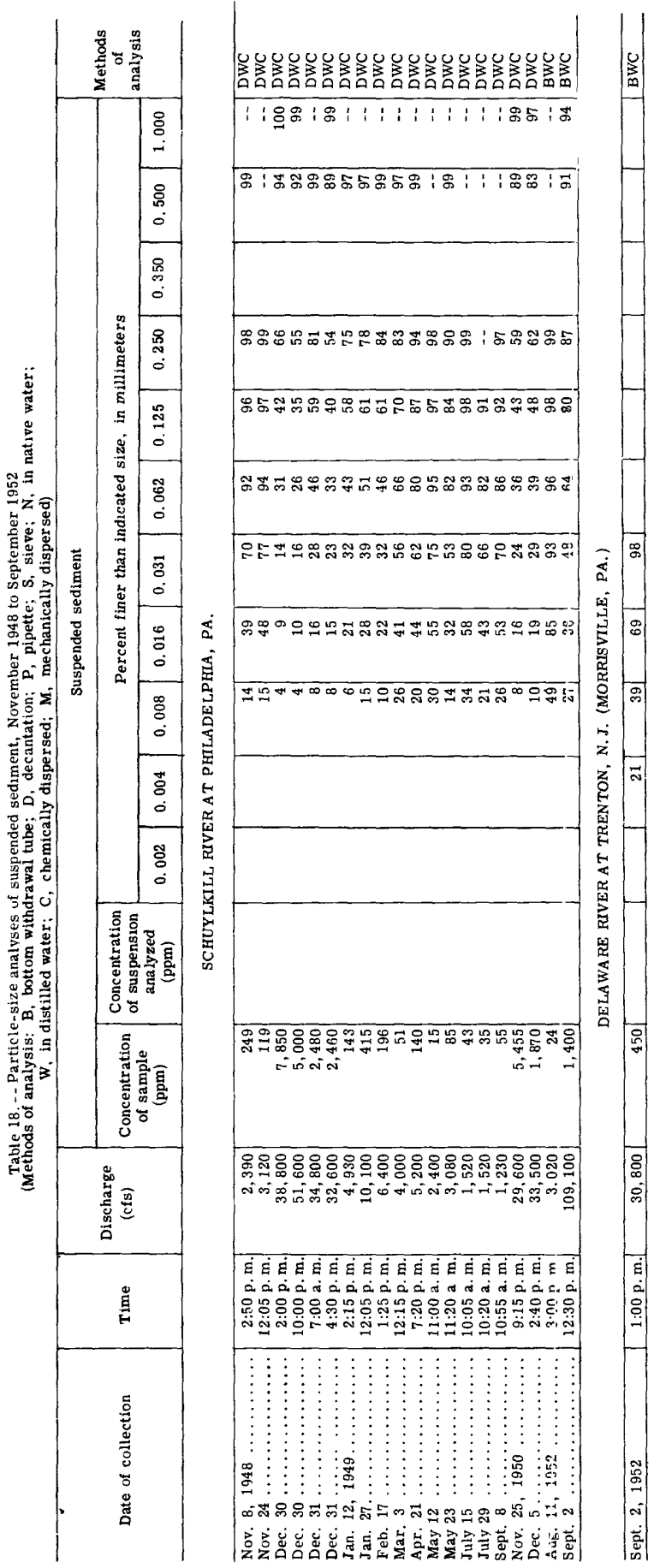

U. S. GO VERNMENT PRINTING OFFICE : O-1954 


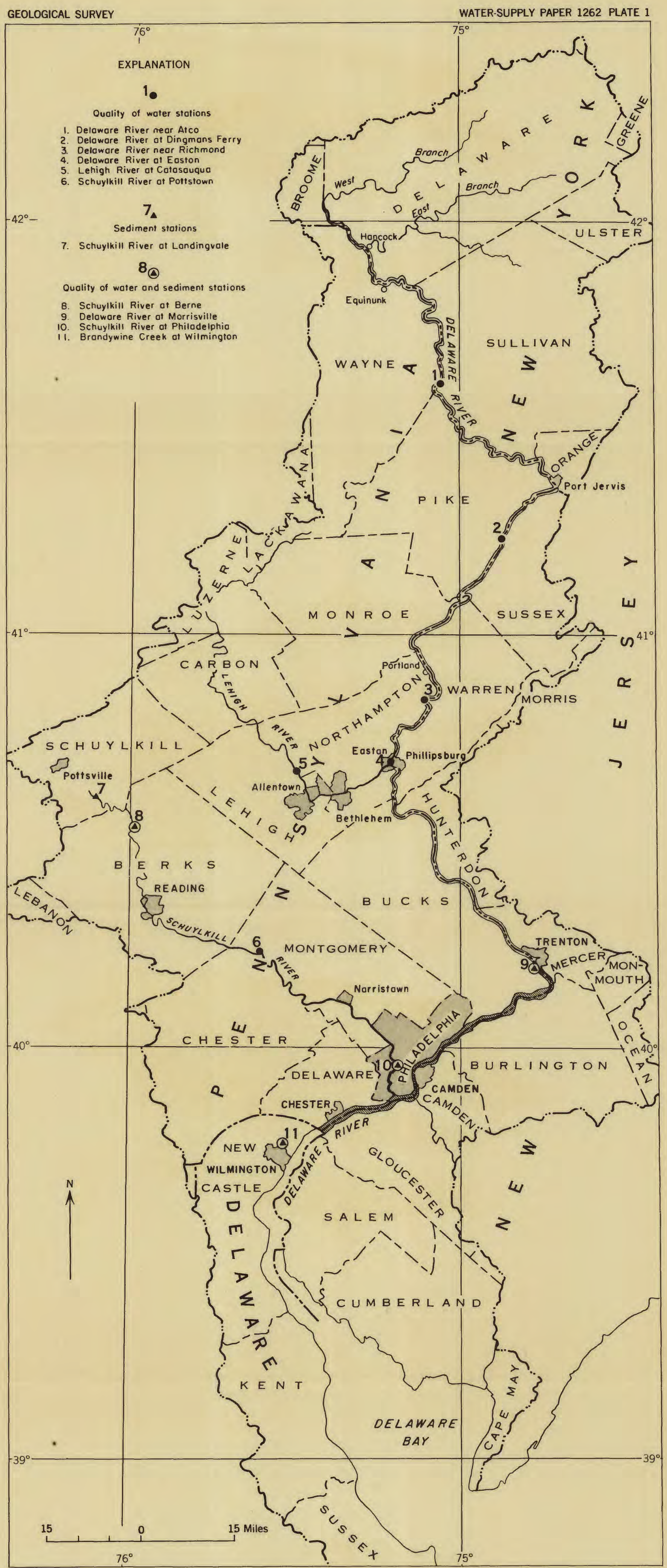

MAP OF DELAWARE RIVER BASIN, SHOWING SAMPLING LOCATIONS FOR CHEMICAL QUALITY AND SEDIMENT 